NASA SP-105

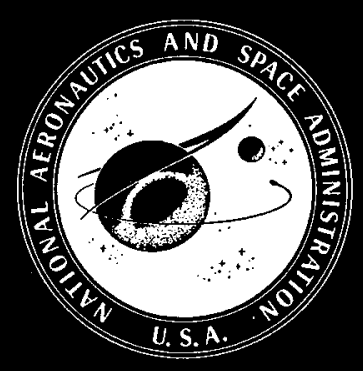

\title{
VACUUM
}

TECHNOLOGY

AND SPACE

SIMULATION

Santeler

Holkeboèr
Jones

Pagano 

NASA SP-105

\section{VACUUM TECHNOLOGY AND SPACE SIMULATION}

Donald J. Santeler

David H. Holkeboer

Prepared under contract NASw -680

by Aero Vac Corporation
Donald W. Jones

Frank Pagano 
For Sale by the Superintendent of Documents,

U.S. Government Printing Office, Washington, D. C. 20402

Library of Congress Catalog Card Number 66.60047 


\title{
Foreword
}

\begin{abstract}
$\Delta{ }^{L}$
THOUGH SPACE VEHICLES encounter many environments during their various missions, vacuum is the most persistent condition affecting design and operation. The study of vacuum effects on materials, components, or systems, alone or in combination with other environments, has grown into a distinct technology during the past several years to the point where vacuum test chambers of many shapes, sizes, and capabilities are now in use. The research and development activities carried out in such vacuum facilities are highly important to progress in the space exploration program. As this program advances, the sophistication and number of vacuum systems in our Nation's laboratories steadily increase and more and more individuals are exposed to the subject of vacuum, as designers, operators, or experimenters.

As the utilization of vacuum technology has increased, so has the need for an authoritative technical manual which will introduce interested personnel to the subject, allow an engineer or scientist skilled in vacuum to probe the field in depth, or furnish the technician with information on equipment characteristics and practical vacuum techniques. Although there are many excellent reference books, reports, and papers dealing with the vacuum field, no specific text is available which combines material on space vacuum simulation, technology, and the related problem of pressure measurement in space chambers.

Therefore, to fill this information gap, the National Aeronautics and Space Administration has had a comprehensive vacuum manual prepared under contract. Its content and format are the result of consultation between the authors and NASA technical personnel and review by NASA staff members at Headquarters and at several Research Centers. This final document should serve as a useful reference volume on the state-ofthe-art of vacuum technology applied to space simulation.
\end{abstract}

Mac C. Adams Associate Administrator for Advanced Research and Technology National Aeronautics and Space Administration 



\section{Preface}

C PACE-SIMULATION FACILITIES have proven their worth in (1) several technological areas: basic research, development, and engineering. Countless hours of testing have been and will be undertaken to advance our knowledge of environmental effects on space vehicle systems and components. As our space flight capabilities increase, our needs for more definitive preflight and post flight test information will also grow. Both preflight and postflight studies in vehicle and component reliability, vacuum heat transfer effects, materials degradation, and bearing and metal fatigue can be performed, under simulated conditions, in the laboratory.

The special facilities and vacuum levels required for these studies created a host of new technical problems in a variety of scientific and engineering areas. To solve the difficulties which arose in vacuum technology, new and ingenious solutions were developed. Many of these developments have reached the general scientific community through technical papers, delivered at symposia and published in journals, dealing with space simulation versus space environment duplication, level of vacuum required, test procedures, and evaluation techniques. However, there is no single source which consolidates the numerous interacting vacuum techniques required for space simulation.

Since 1960, a number of authoritative books have been published which update the published state of the art of vacuum technology and which cover a wide range of interests. For example, Dushman discusses the scientific aspects of vacuum, while Guthrie proves useful for the training of vacuum-laboratory technicians. Still another volume, Pirani and Yarwood, is concerned primarily with the engineer. However, the range of information covered in most texts tends toward a general, rather than a specific, interest. Therefore, any author will provide descriptions of various types of vacuum gages, but the specific problem of pressure measurement in space chambers is rarely discussed.

It is the intention of the following chapters, therefore, to examine to a reasonable extent the essential specifics of vacuum technology. As a result, many general aspects of vacuum technology have been omitted, and the reader is referenced to texts which treat these aspects. An attempt has been made to present new material on each topic, or a new treatment of the subject.

In conclusion, the engineer who needs to purchase, operate, maintain, or otherwise use vacuum-space-simulation equipment and who must have a thorough understanding of the principles of vacuum technology, as related to space simulation, will find "Vacuum Technology and Space Simulation" specifically designed for his particular requirements. 



\section{Contents}

chapter

page

1 VACUUM NOMENCLATURE _.......................... 1

Molecular Fundamentals _._._._._._........... 2

Gas Flow -..........-. 6

Directional Effects _............. 7

Glossary -......-.-1 8

Symbols -..-

2 PRESSURE _........ 19

Definitions of Pressure _..._._._._._._._._._._._. 19

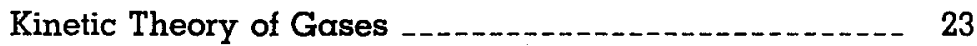

Molecular Kinetics -.-_-_-_-_-_-_-_-_-_-_-_ 28

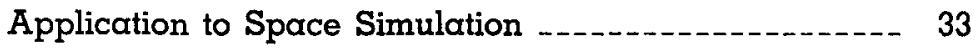

3 PRESSURE MEASUREMENT _............... 41

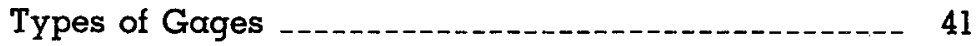

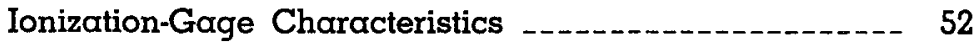

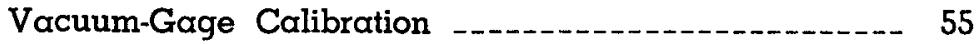

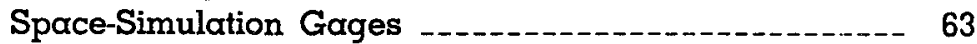

4 PUMPING SPEED

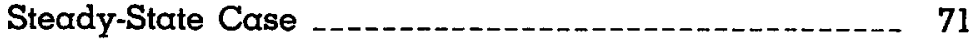

Transient Case, Pumpdown, and Repressurization _...-- 77

5 GAS FLOW IN COMPONENTS AND SYSTEMS _-_.....- 83

Molecular Flow Through an Orifice _............... 84

Molecular Flow Through a Tube of Circular

Cross Section -

Laminar Viscous Flow in a Long Circular Tube _....--- 88

Flow Characteristics of Complex Shapes-Molecular Flow -......... 94

Flow Characteristics of Complex Shapes-Transition Flow -

Flow Characteristics of Complex Shapes-Laminar Viscous Flow -

Flow Characteristics of Complex Shapes-Choked or Critical Flow -..-

Flow of Gas Through Cylindrical Tubes _._-_._._._-_. 108

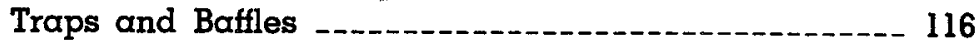


6 PUMPING SYSTEMS

Rotary Oil-Sealed Pumps -..--

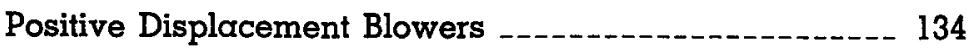

Diffusion Pumps -....... 135

Sputter Ion Pumps ....._. 142

Sorption Pumping - -.......- 143

7 CRYOGENIC PUMPING _._. 151

Perfect Cryogenic Pumping Surfaces _._._-_._._._-_._- 151

Extended Cryogenic Pumping Surfaces _............. 154

Liquid Nitrogen Traps _..-_..- 166

8 GAS LOAD _......... 173

Definition of $Q_{L}$, Gas Load on Systems _._._._._._. 173

Sources of the Gas Load _._-_._-_._- 175

9 OUTGASSING OF MATERIALS _._._-_. 197

Metals _-

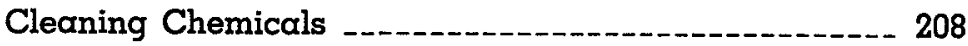

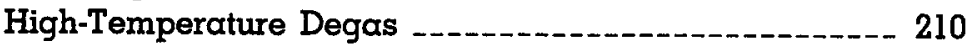

Low Temperature -....-_. 213

Exposure --.--_-

Polymers -......... 213

10 SYSTEMS -

Space Simulation -

Types of Vacuum Systems _._. 229

High-Vacuum Systems-General Considerations _.-.-..- 234

System Design -

Troubleshooting Diffusion-Pumped Systems _._._._._. 267

11 LEAK DETECTION _-_._. 275

Gas Flow Through Leaks _._.

Basic Equations of Steady-State Leak Detection _._-_._-_ 279

Basic Equations of Transient Leak Detection -...-...-_-_ 283

Other Aspects of Leak Detection -..--_-_-_-_-_-_..- 288

Vacuum-Gage Methods of Leak Detection _._._._._._.-. 291

Leak-Detection Techniques for Systems _..._..._._._._. 300 


\section{Vacuum Nomenclature}

\section{INTRODUCTION}

THE FIRST STEPS into any new venture generally require a guide to insure that the correct path is being followed. Thus, an engineer assuming new responsibilities in activities concerned with vacuum technology and space simulation will need aid in becoming acclimatized to the language, terminology, and units peculiar to vacuum.

It is the intent of chapter 1 to serve as a map to the charted courses of vacuum technology. Terms and units defined in this chapter will be eiaborated upon in later chapters. All symbols used in the text, their descriptions and units have been alphabetically listed at the conclusion of this chapter.

Since popular usage has established atmospheric pressure as the upper limit of vacuum, a vacuum may be regarded as any pressure less than standard atmospheric pressure. When scientists first became interested in vacuum-level measurements, a value of 28 or $29 \mathrm{~mm} \mathrm{Hg}$ below atmospheric pressure was considered a respectable vacuum. As research improved vacuum levels, it became necessary to convert to an absolute scale to give meaning to engineering equations, in much the same manner as an absolute scale is used in temperature calculations. Under the traditional measurement system, normal atmospheric pressure is expressed in millimeters of mercury; that is, $760 \mathrm{~mm} \mathrm{Hg}$ equals atmospheric pressure.

In recent years the traditional pressure units have given way to the torr which is defined as 1/760 of a standard atmosphere, and is now the measure of vacuum level accepted by those working with vacuum. It should be noted, however, that the standard atmosphere is not quite $760 \mathrm{~mm} \mathrm{Hg}$. There is a discrepancy of approximately 1 part in 7 million between $\mathrm{mm} \mathrm{Hg}$ and the torr; but, for practical considerations, the terms "torr" and "mm Hg" may be interchanged. The following table lists the conversion factors between the torr and other pressure units popularly used today:

$$
1 \text { torr equals }\left\{\begin{array}{l}
1 \mathrm{~mm} \mathrm{Hg} \\
10^{3} \text { microns } \\
1.33 \times 10^{3} \text { microbars } \\
1.33 \times 10^{3} \mathrm{dyn} / \mathrm{cm}^{2} \\
133 \text { newton } / \mathrm{m}^{2} \\
1 / 760 \mathrm{std} . \mathrm{atm} \\
0.01934 \mathrm{lb} / \mathrm{in}^{2}
\end{array}\right.
$$


The following divisions of the region below atmospheric pressure indicate the generally accepted degrees of vacuum:

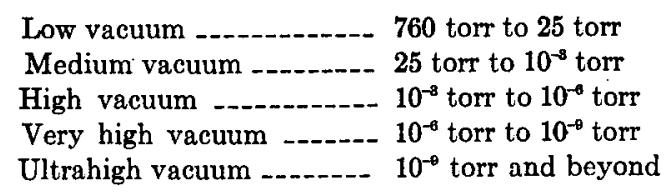

The following sections will introduce several concepts in order to provide a foundation for some basic nomenclature. However, the basic equations of pressure and the ideal gas laws, which have been derived from kinetic theory, will be considered further in chapter 2.

\section{MOLECULAR FUNDAMENTALS}

At conditions of standard atmospheric pressure and temperature, there are approximately $2.5 \times 10^{19}$ molecules $/ \mathrm{cm}^{3}$. Each molecule will have an average mean free path, $\lambda$, of $6.6 \times 10^{-6} \mathrm{~cm}$ between collisions. Equations (1.1) and (1.2) point out the effect of decreased pressure on the molecular density and the mean free path. The density, in units of molecules $/ \mathrm{cm}^{3}$, decreases by

$$
n=9.656 \times 10^{18} \frac{P}{T}
$$

while the mean free path, $\mathrm{cm}$, increases as

$$
\lambda=2.331 \times 10^{-20} \frac{T}{P \delta^{2}}
$$

where $P$ is the pressure in torr, $T$ is the absolute temperature in ${ }^{\circ} \mathrm{K}$, and $\delta$ is the molecular diameter in $\mathrm{cm}$. Molecular density depends on the gas temperature and pressure, while the mean free path also depends on the gas type. Table 1.1 lists values of $n$ and $\lambda$ for different values of air pressure at $25^{\circ} \mathrm{C}$; table 1.2 gives various molecular properties for different gases at $25^{\circ} \mathrm{C}$ and $10^{-3}$ torr. The extremely small size of molecules may be seen from a comparison of molecular density and mean free path. That is, there are over $3 \times 10^{6}$ molecules $/ \mathrm{cm}^{3}$ at a pressure of $10^{-10}$ torr; yet a molecule will travel, on the average, over 483 kilometers between collisions.

The concept of mean free path is extremely important in vacuum engineering in that it defines the boundaries of two different types of gaseous flow. At atmospheric-pressure or low-vacuum conditions, the mean free path is exceedingly small. Hence, the molecules are in a constant state of intercollision, transferring energy, or momentum, 
through the gas. The momentum transfer from molecule to molecule leads to the concept of gas viscosity, and the flow itself is called viscous flow. When dealing with the gaseous flow in a tube which is long relative to its diameter, the viscous flow is governed by the Poiseuille equation. Chapter 5, which discusses the various flow regimes, shows that Poiseuille flow is seldom encountered in vacuum systems.

As the pressure decreases to the high- and very-high-vacuum regions, the mean free path becomes much longer than the dimensions of the confining chamber, with the result that the molecules will collide more frequently with the walls of the chamber than with each other. Gas flow in such a molecular flow regime is no longer dependent on momentum transfer between molecules, but now depends only on the statistical motion of the independently moving molecules.

There is a third region of gas flow which ranges between the viscous and molecular regions. The molecules in the intermediate region make an appreciable number of collisions, both with each other and with the confining walls. First identified with limitations of the viscous flow region, this region became known as slip flow. It is known also as Knudsen flow after the man who first established the mathematical relationship for the apparent changes in viscosity as pressure decreases. The conventional equations of Knudsen flow do not apply to many vacuum systems due to the limitations on the ratio of chamber length to diameter. Therefore, it is more appropriate to use the generalized term of transition flow to cover the flow region between viscous and molecular. Figure 1.1 illustrates the relationship between pressure, chamber dimensions, and flow regimes for nitrogen gas at $25^{\circ} \mathrm{C}$. That is, at high pressure (low vacuum) the flow will be viscous with molecular interactions; while at low pressure (high vacuum) the flow is molecular in nature and is governed by the statistics of interaction with the system walls. Another commonly used term in vacuum technology is molecular incidence or impingement rate. A mathematical relationship for molecular incidence, defined as the rate at which molecules strike a unit area of surface, molecules $/ \mathrm{cm}^{2}$ sec, can be derived from kinetic theory. That is

$$
\Phi=3.513 \times 10^{22} \frac{P}{(M T)^{1 / 2}}
$$

Langmuir used a modification of equation (1.3) to deduce equilibrium vapor pressure (evaporation rate equals incidence rate) from the rate of evaporation. In addition, the length of time required to contaminate a clean surface in an ultra-high-vacuum environment can be determined from the molecular incidence rate, $\Phi$.

Various experimenters have observed that the unsaturated surfaces of materials such as metal and glass have a high affinity for gas molecules. Nitrogen, oxygen, and other permanent gases may be physically 


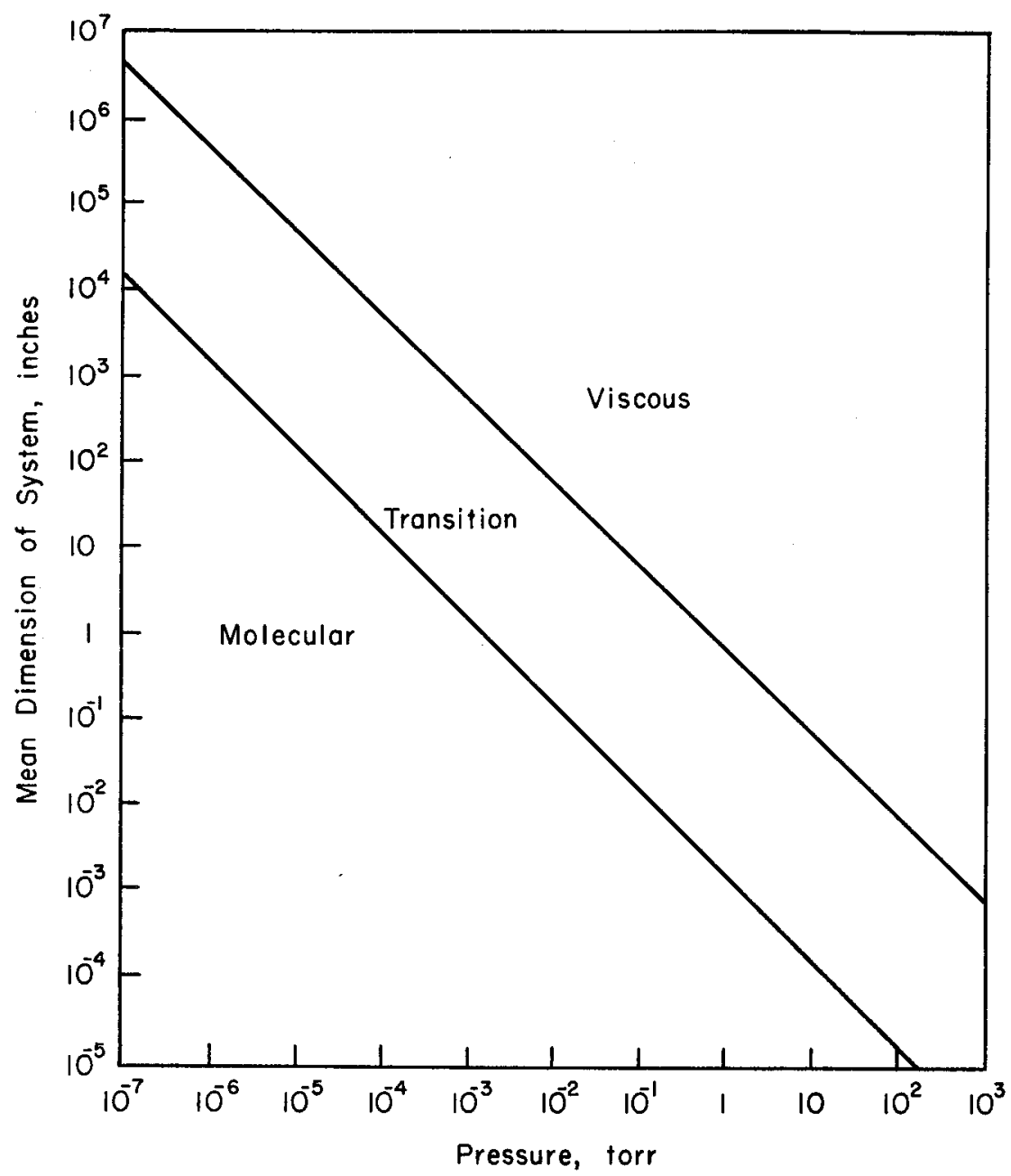

Figure 1.1.-Relationship between pressure, chamber dimensions, and flow regimes for nitrogen gas at $25^{\circ} \mathrm{C}$.

or chemically adsorbed in an amount approaching one complete molecular layer. (See chapter 8.) A freshly cleaved surface, or one which has just undergone a high-temperature bakeout, is free initially of such contaminating gases. As molecules from the vacuum region collide with the surface according to equation (1.3) for molecular incidence, they are taken up by the surface and begin to form a contaminating layer. To obtain the time required to form a particular molecular coverage, divide the molecular coverage by the molecular incidence rate and the probability of adsorption upon collision with the wall. Of course, the probability of adhering or sticking will depend on the amount of coverage 
which already exists. Assuming that every molecule striking the surface will adhere, table 1.1 lists values of the molecular incidence rate and time to form a monolayer for air at $25^{\circ} \mathrm{C}$.

TABLE 1.1.-Molecular Incidence Rate and Time To Form a Monolayer for Air at $25^{\circ} \mathrm{C}$

\begin{tabular}{|c|c|c|c|c|}
\hline $\begin{array}{c}\text { Pressure, } \\
\text { torr }\end{array}$ & $\begin{array}{l}\text { Molecular density, } \\
n, \text { molecule } / \mathrm{cm}^{3}\end{array}$ & $\begin{array}{c}\text { Mean free } \\
\text { path, } \\
\lambda, \mathrm{cm}\end{array}$ & $\begin{array}{c}\text { Molecular } \\
\text { incidence } \\
\text { rate, } \Phi, \\
\text { molecule } / \mathrm{cm}^{2} \mathrm{sec}\end{array}$ & $\begin{array}{l}\text { Time to } \\
\text { form } 1 \\
\text { monolayer, } \\
t, \text { sec }\end{array}$ \\
\hline 760 & $2.46 \times 10^{10}$ & $6.69 \times 10^{-8}$ & $3.14 \times 10^{28}$ & $2.63 \times 10^{-8}$ \\
\hline 1 & $324 \times 10^{16}$ & $5.09 \times 10^{-3}$ & $4.13 \times 10^{20}$ & $2.00 \times 10^{-8}$ \\
\hline $10^{-8}$ & $3.24 \times 10^{18}$ & $5.09 \times 10^{\circ}$ & $4.13 \times 10^{17}$ & $2.00 \times 10^{-8}$ \\
\hline $10^{-6}$ & $3.24 \times 10^{20}$ & $5.09 \times 10^{8}$ & $4.13 \times 10^{14}$ & $2.00 \times 10^{0}$ \\
\hline $10^{-9}$ & $3.24 \times 10^{7}$ & $5.09 \times 10^{8}$ & $4.13 \times 10^{11}$ & $2.00 \times 10^{9}$ \\
\hline $10^{-12}$ & $3.24 \times 10^{4}$ & $5.09 \times 10^{9}$ & $4.13 \times 10^{8}$ & $2.00 \times 10^{8}$ \\
\hline $10^{-15}$ & $3.24 \times 10^{1}$ & $5.09 \times 10^{12}$ & $4.13 \times 10^{5}$ & $2.00 \times 10^{9}$ \\
\hline
\end{tabular}

Table 1.2 gives similar data for different gases at a pressure of $10^{-3}$ torr.

$\mathrm{T}_{\mathrm{ABLE}}$ 1.2.-Molecular Incidence Rate and Time To Form a Monolayer for Some Common Gases at $25^{\circ} \mathrm{C}$ and $10^{-5}$ Torr

\begin{tabular}{|c|c|c|c|c|c|}
\hline Gas & $\begin{array}{l}\text { Molecular } \\
\text { weight, } M \\
\text { gram/mole }\end{array}$ & $\begin{array}{c}\text { Molecular } \\
\text { diameter, } \\
\delta, \mathrm{cm}\end{array}$ & $\begin{array}{c}\text { Mean free } \\
\text { path, } \\
\lambda, \mathrm{cm}\end{array}$ & $\begin{array}{c}\text { Molecular } \\
\text { incidence } \\
\text { rate, } \Phi, \\
\text { molecule } / \mathrm{cm}^{2} \text { sec }\end{array}$ & $\begin{array}{c}\text { Time to } \\
\text { form } 1 \\
\text { monolayer, } \\
t \text {, sec }\end{array}$ \\
\hline Air & 29 & $3.74 \times 10^{-8}$ & 5.09 & $4.13 \times 10^{17}$ & $1.99 \times 10^{-8}$ \\
\hline $\mathrm{N}_{2}$ & 28 & 3.76 & 5.01 & 4.19 & 1.93 \\
\hline $\mathrm{O}_{2}$ & 32 & 3.64 & 5.40 & 3.93 & 2.22 \\
\hline $\mathrm{H}_{2}$ & 2 & 2.75 & 9.31 & 15.64 & 0.975 \\
\hline $\mathrm{He}$ & 4 & 2.18 & 14.72 & 11.11 & 2.18 \\
\hline $\mathrm{H}_{2} \mathrm{O}$ & 18 & 4.68 & 3.37 & 5.21 & 1.01 \\
\hline $\mathrm{CO}_{2}$ & 44 & 4.65 & 3.34 & 3.35 & 1.59 \\
\hline A & 40 & 3.67 & 5.31 & 3.51 & 2.43 \\
\hline
\end{tabular}

An interesting corollary to molecular surface concentration is the ratio of molecules on the surface to those in the vacuum environment. At normal temperatures the number of molecules on the surface is relatively independent of the pressure until the pressure is reduced to extremely low values which correspond to the equilibrium pressure of a partial layer of contamination. Since the molecules in the vacuum region have a linear dependency on pressure, a pressure will exist such that there are as many molecules on the surface as there are in the vacuum region. 
Below this particular value of pressure there will be more surface molecules than vacuum space molecules. The exact value of the ratio of surface to vacuum region molecules will depend on the surface to volume ratio of the confining chamber, pressure, and temperature. Table 1.3 gives values of this ratio for air at $25^{\circ} \mathrm{C}$ in a 1 -foot-diameter spherical chamber.

TABLE 1.3.-Values of the Ratio of Surface to Vacuum Region Molecules in a Smooth 1.Foot-Diameter Sphere for Air at $25^{\circ} \mathrm{C}$ with $2.4 \times 10^{17}$ Molecules in 1 Monolayer

\begin{tabular}{|c|c|c|}
\hline $\begin{array}{c}\text { Pressure, } \\
\text { torr }\end{array}$ & Molecules in volume & Ratio, $\frac{\text { molecules on surface }}{\text { molecules in volume }}$ \\
\hline $10^{-2}$ & $4.8 \times 10^{18}$ & 0.05 \\
$10^{-8}$ & $4.8 \times 10^{17}$ & .5 \\
$10^{-4}$ & $4.8 \times 10^{18}$ & 5.0 \\
$10^{-5}$ & $4.8 \times 10^{15}$ & 50.0 \\
\hline
\end{tabular}

The data indicate equal numbers of molecules at both locations at a pressure of only $5 \times 10^{-4}$ torr. Thus, it may be concluded that for most high-vacuum applications, the molecules in the system are located principally on the surface. If it is assumed that no other gas sources are present, a clean surface in a high-vacuum system could readily adsorb the molecules to an extremely low value of equilibrium pressure. This factor becomes increasingly important when ultrahigh vacuums are desired.

\section{GAS FLOW}

When an incompressible fluid, such as water, flows through a tube, it is customary to speak of the flow in units of $\mathrm{ft}^{3} / \mathrm{min}$, which define both the volumetric flow rate and the mass flow rate (at a specified value of temperature). In the case of compressible gases, the volumetric flow rate and the mass flow rate are not the same. That is, the volumetric flow rate indicates nothing about the quantity of gas flowing unless the pressure and temperature are specified.

Conventional vacuum nomenclature uses the term "speed," $S$, liter/sec, in place of volumetric flow. The product of speed at a particular point in a system and the pressure at the same point yields the gas throughput $Q$. One of the most basic and important equations in vacuum technology is the relationship $Q=P S$. Throughput $Q$, normally measured in torr liter/sec, is a mass flow quantity equivalent to a volumetric flow of 1 liter/sec at a pressure of 1 torr (at a standard temperature, usually $20^{\circ} \mathrm{C}$ ). The conversion to mass flow may be obtained from the relationship $1.7 \times 10^{4}$ torr liter $=1 \mathrm{gram} \mathrm{mol}$ wt. 
Equally important to the vacuum engineer is the concept of conductance which is the throughput/unit pressure drop; i.e., $Q / \Delta P$. The units of conductance are torr liter/sec $\Delta$ torr which, by popular convention, reduce to liter/sec. The symbols $U, F$, and $C$ have been used by various authors to signify conductance. This text will use the symbol $F$. Frequently, conductance is confused with speed, also expressed as liter/sec. To avoid misuse of the two terms, note that speed occurs at a point and defines the throughput/unit pressure at that point, while conductance occurs between two points and defines the throughput/unit pressure gradient between the two points. The conductance between two points may be combined with the speed at a point to obtain the speed at the second point. Details of this procedure will be discussed in chapter 5 . Conversion factors between the various units of speed and throughput are given in table 1.4 .

\begin{tabular}{|c|c|}
\hline SPEED & THROUGHPUT \\
\hline $\begin{array}{l}1 \text { liter } / \mathrm{sec}: \\
2.12 \mathrm{ft}^{8} / \mathrm{min} \\
1000 \mathrm{~cm}^{2} / \mathrm{sec} \\
60 \mathrm{liter} / \mathrm{min} \\
3.6 \mathrm{~m}^{8} / \mathrm{hr}\end{array}$ & $\begin{array}{l}1 \text { torr liter } / \mathrm{sec}: \\
1.316 \mathrm{~atm} \mathrm{~cm}^{8} / \mathrm{sec} \\
1.27 \times 10^{5} \mathrm{micron} \mathrm{ft}^{3} / \mathrm{hr} \\
10^{\mathrm{a}} \mathrm{micron} \mathrm{liter} / \mathrm{sec}\end{array}$ \\
\hline
\end{tabular}

Outgassing, which includes all forms of gas entering the vacuum space from the solid space, is examined in detail in chapters 8 and 9 . Gases on the surface or interior of materials, vapors of a solid or liquid, decomposition, or chemical reactions all may contribute to the outgassing in a system. Outgassing is measured in the same units as throughput; i.e., torr liter/sec. Since outgassing represents a major limitation on the performance of such items as pumps, gages, and traps, its importance in the design of vacuum systems cannot be minimized.

\section{DIRECTIONAL EFFECTS}

If a uniform pressure distribution throughout the confining chamber is assumed, the normal equations for pressure, density, speed, conductance, and molecular incidence rate have been derived from kinetic theory. The above assumption is valid for most vacuum system applications. Care should be exercised to use properly either the static pressure or the total pressure (depending on the particular application) for the cases of gas flow in tubes or pressure measurements taken near the inlet to an efficient pumping system. The problem of directional effects and their interpretation acquires an increased importance when the dis- 
cussion turns to space-simulation chambers. In particular, the streaming of molecules in outer space can hardly be considered a uniform pressure distribution. In addition, the space-vehicle velocity considerably exceeds the thermal velocity of the surrounding molecules, and the molecules leaving the space vehicle travel in paths away from the vehicle. As a result, conventional kinetic theory approaches cannot be applied to the interactions of gas molecules with a space vehicle. Spacesimulation chambers designed to simulate the high pumping speed of space will experience considerable directional gas flows because of the high pumping speed of the containing walls. One solution to the problem of directional effects replaces pressure with molecular incidence rate, and pumping speed and conductance with probability. Recent developments in probability and molecular incidence theory have progressed to the point where they are used in conventional calculations as an alternate approach to kinetic theory. Later chapters discuss the importance of molecular incidence rates on pressure measurements and pumping-speed calculations.

\section{GLOSSARY}

As a scientific discipline develops and becomes more refined, a language is evolved which characterizes that discipline. The following terms form the foundation upon which the language of vacuum technology rests. A complete familiarity with this most basic listing of the terminology is essential to the beginning vacuum engineer who wishes to proceed with research, perform tests, or otherwise work with vacuum systems.

A complete listing of vacuum terminology will be found in the handbook, "Glossary of Terms Used in Vacuum Technology," published by Pergamon Press for the American Vacuum Society which officially approved the listing as the most definitive for the varied applications of vacuum technology.

The following list of terms has been selected from the "Glossary."

\section{Pressure Terminology}

Pressure (as measured in a vacuum)-The term pressure as used in vacuum technology usually must be interpreted as the quantity measured at a specified time by a socalled vacuum gage, whose sensing element is located in a cavity (gage tube) with an opening oriented in a specified direction at a specified point within the system, assuming a specified calibration factor. The sensitivity of the sensing element is in general not the same for all molecular species, but the gage reading is frequently reported using the calibration factor for air regardless of the composition of the gas.

Ultimate pressure-The limiting pressure approached in the vacuum 
system after sufficient pumping time to establish that further reductions will be negligible.

Forepressure-The total pressure on the outlet side of a pump measured near the outlet port. Sometimes called the backing pressure. In discussing the action of a vapor jet, the term forepressure may be used to designate the total pressure of the gas against which the jet impinges.

Partial pressure-The pressure of a designated component of a gaseous mixture.

Absolute pressure-A term used in engineering literature to indicate pressure above the absolute zero value corresponding to empty space or the absolute zero of temperature as distinguished from gage pressure. In vacuum technology, pressure always corresponds to absolute pressure, not gage pressure, and therefore the term absolute pressure is not required.

Gas-Gas is defined as the state of matter in which the molecules are practically unrestricted by intermolecular forces, so that the molecules are free to occupy any space within an enclosure. In vacuum techrology, the word gas has been loosely applied to the noncondensable gas and vapor within a vacuum system.

Noncondensable gas-A gas whose temperature is above its critical temperature, so that it cannot be liquefied by increase of pressure alone.

Vapor-A gas whose temperature is below its critical temperature, so that it can be condensed to the liquid or solid state by increase of pressure alone.

Manometer-An instrument for measuring pressure of gases and vapors whether above or below atmospheric pressure.

Absolute manometer-A manometer whose calibration can be calculated from the measurable physical constants of the instrument and which is the same for all ideal gases.

Vacuum gage-A manometer for pressures below 760 torr. Terms such as vacuum gage, Pirani gage, ionization gage, etc., should be used only when referring to the complete gage, not merely to the gage tube.

Mass spectrometer-An instrument which is capable of separating ionized molecules of different mass/charge ratio and measuring the respective ion currents. The mass spectrometer may be used as a vacuum gage to measure the partial pressure of a specified gas, as a leak detector sensitive to a particular tracer gas, or as an analytical instrument to determine the percentage composition of a gas mixture. Various types are distinguished by the method of separating the ions.

Vacuum-gage tube-The part of a vacuum gage which contains the elements exposed directly to the vacuum system, including the pressure sensing means, the envelope or means for supporting the operating elements, and any connecting tube attached permanently to the envelope. External connecting means, such as electrical connectors, may be in- 
cluded in referring to a particular gage tube. Sometimes called vacuum gage head. Certain contractions are commonly used in referring to specific types of tubes; e.g., Pirani tube instead of Pirani gage tube, ion tube instead of ionization gage tube.

Vacuum gage control circuit-The part of an electrically operated vacuum gage which comprises the electrical circuit necessary to energize the tube, control and measure currents or voltages, and in some cases to supply power for degassing the tube elements. It does not include the vacuum gage tube or any meter or recorder which is not an integral part of the cabinet or panel assembly in which the circuit is mounted. This is also referred to as the vacuum gage power supply, the vacuum gage power rack, the vacuum gage control box, or simply the vacuum gage control. Various terms are formed by substituting in the above in place of the word vacuum, the words Pirani, thermocouple, ionization, Philips, etc., referring to particular types of vacuum gages.

Thermal conductivity vacuum gage-A vacuum gage containing two surfaces at different temperatures between which heat can be transported by the gas molecules so that changes in the temperature (or in the heating power required to maintain constant temperature) of one of the surfaces can be correlated with the gas pressure by calibration against a McLeod gage. Various types of thermal conductivity gages are distinguished according to the method of indicating the temperature change. The common types are listed below:

(1) Pirani gage-An increase of pressure from the zero point causes a decrease in the temperature of a heated filament of material having a large temperature coefficient of resistance, thus unbalancing a Wheatstone bridge circuit (or the circuit is adjusted to maintain the filament temperature constant).

(2) Thermocouple gage-The decrease in temperature of a heated filament as the pressure rises is indicated by decreased electromotive force in a thermocouple circuit having the junction in thermal contact with the center of the heated filament.

(3) Thermistor gage-A form of Pirani gage employing a thermistor as the heated element.

(4) Bimetallic strip gage--Deflection of bimetallic strip with changing temperature indicates the changes in pressure.

Ionization vacuum gage-A vacuum gage comprising a means of ionizing the gas molecules and a means of correlating the number and type of ions produced with the pressure of the gas. Various types of ionization gages are distinguished according to the method of producing the ionization. The common types are as follows:

(1) Hot cathode ionization gage-The ions are produced by collisions with electrons emitted from a hot filament (or cathode) and 
accelerated by an electric field. Also called hot filament ionization gage, ionization gage, or simply ion gage. The Bayard-Alpert ionization gage employs a tube with an electrode structure designed to minimize X-ray-induced electron emission from the ion collector.

(2) Cold cathode ionization gage-The'ions are produced by a cold cathode discharge, usually in the presence of a magnetic field which lengthens the path of the electrons between cathode and anode. The discharge tube is a transparent tube in which the color and form of a cold cathode discharge (without the presence of a magnetic field) gives an indication of the pressure and the nature of the gas. The Philips ionization gage or Penning gage, is a cold cathode ionization gage in which a magnetic field is directed parallel to the axis of an annular electrode, normally the anode, located between two plate electrodes perpendicular to the axis. Various modifications of the Penning gage are named after the inventors, and certain types are referred to as magnetron vacuum gages.

(3) Radioactive ionization gage-The ions are produced by radiations (usually alpha particles) emitted from a radioactive source.

McLeod gage-A liquid-level vacuum gage in which a known volume of the gas, at the pressure to be measured, is compressed by the movement of a liquid colımn to a much smaller known volume, at which the resulting higher pressure is measured. Particular designs are named after the inventors or various trade names.

\section{Speed Terminology}

Speed-The speed of a pump for a given gas is the ratio of the throughput of that gas to the partial pressure of that gas at a specified point near the mouth (or inlet port) of a pump.

Throttling-Reducing the net pumping speed of a pumping system by partially closing a valve or installing a section of pipeline with low conductance.

Mechanical pump-A pump which moves the gas by the cyclic motion of a system of mechanical parts, such as pistons, eccentric rotors, vanes, valves, etc.

Fore pump-The pump which produces the necessary fore vacuum for a pump which is incapable of discharging gases at atmospheric pressure. Sometimes called the backing pump.

Roughing pump-The pump used to reduce the system pressure to the point at which a vapor pump (or other pump requiring a fore vacuum) can take hold and operate efficiently. The roughing pump may then also be used as the fore pump for the vapor pump, or the roughing pump may be shut off and a smaller pump used as fore pump when the gas load is relatively small. 
Vapor pump -Any pump employing a vapor jet as the pumping means. Applies to ejector pumps as well as diffusion pumps.

Booster pump-A vapor pump or a specially designed mechanical pump used between a vapor pump and a fore pump to increase the maximum gas throughput which can be handled. The limiting or breaking forepressure of the booster at this maximum throughput must be appreciably greater than that of the vapor pump which it backs.

Trap, adsorption-A chamber containing activated charcoal, or other medium for adsorbing gases and vapors (usually at room temperature), designed to reduce the partial pressure of adsorbable gases in the system.

Accommodation coefficient, for free-molecule heat transfer-The ratio of the energy actually transferred between impinging gas molecules and a surface and the energy which would be theoretically transferred if the impinging molecules reached complete thermal equilibrium with the surface.

Baffle-A system of cooled walls, plates, or tubing placed near the inlet of a vapor pump to condense backstreaming vapor at a temperature below that of the room and return the fluid to the boiler.

Trap, refrigerated-A system of cooled walls, plates, or tubing placed beyond the baffle or the condenser to reduce the partial pressure of vapor migrating from the pump, or from the vacuum system, and from which the condensed vapor cannot return to the pump boiler.

Pump fluid-The operating fluid used in vapor pumps or in liquidsealed mechanical pumps. Sometimes called working medium, working fluid, or pump oil.

Ion pump-An electrical device for pumping gas comprising a means for ionizing the gas and a system of electrodes at suitable potentials, and in some cases also a magnetic field, which causes the ions formed to move toward the auxiliary pump or trap.

Sorption pump-A pump with a renewable trapping surface which reduces the partial pressure of gases by adsorption, absorption, or chemisorption.

Getter (noun) - A material which is included in a vacuum system or device for removing gas by sorption.

Getter (verb) - To remove gas by sorption.

Ion sorption pump-A pump which combines the action of an ion pump and a sorption pump by driving the ions into the sorbent.

Getter ion pump-An ion-sorption pump in which a getter is continuously or intermittently vaporized and condensed on the trapping surface to give a fresh deposit of sorbent.

\section{Throughput Terminology}

Throughput-The quantity of gas in pressure-volume units at a specified temperature flowing per unit time across a specified open cross sec- 
tion of a pump or pipeline. The specified temperature may be the actual temperature of the gas or a standard reference temperature. It is recommended that throughput be referred to standard room temperature.

Outgassing-The spontaneous evolution of gas from a material in a vacuum.

Degassing-The deliberate removal of gas from a material, usually by application of heat under high vacuum.

Adsorption-The condensing of gas on the surface of a solid.

Absorption-The binding of gas in the interior of a solid (liquid).

Chemisorption--The binding of gas on the surface or in the interior of a solid (or liquid) by chemical action.

Sorption-The taking up of gas by absorption, adsorption, chemisorption, or any combination of these processes.

Permeation-As applied to gas flow through solids, permeation is the passage of gas into, through, and out of a solid barrier having no holes large enough to permit more than a small fraction of the gas to pass through any one hole. The process always involves diffusion through the solid and may involve various surface phenomena, such as sorption, dissociation, migration, and desorption of the gas molecules.

Evaporation rate--The mass of material evaporated per unit time from unit surface of a liquid or solid; or, the number of molecules of a given substance evaporated per second per square centimeter from the free surface of the condensed phase.

Molecular flow-The flow of gas through a duct under conditions such that the mean free path is greater than the largest dimensions of a transverse section of the duct.

Viscous flow-The flow of gas through a duct under conditions such that the mean free path is very small in comparison with the smallest dimension of a transverse section of the duct. This flow may be either laminar or turbulent.

Poiseuille flow-The particular case of laminar viscous flow through a long pipe of circular section.

Knudsen flow-The flow of gases through ducts and tubes under conditions intermediate between laminar viscous flow and molecular flow. Also called transition flow. The Knudsen equation of flow applies to this intermediate range for the case of pipes with circular cross section.

Reynolds number-As applied to the flow of gas through a circular tube, the Reynolds number is a dimensionless quantity equal to the product of the gas density in grams per cubic centimeter times the flow velocity in centimeters per second times the tube diameter in centimeters divided by the viscosity coefficient in poise. That is, $R_{e}=\rho v d / \eta$.

Back migration-The passage of vapor molecules from a vapor into a 
vacuum system by reevaporation from baffles and the inside wall of the pump casing and connections to the system.

Back streaming-The direct flight of vapor molecules by scattering from the hot vapor jet or evaporation from hot nozzle parts in the direction of the mouth or intake part of a vapor pump. May be spelled as one word, backstreaming.

Leak-In vacuum technology, a hole, or porosity, in the wall of an enclosure capable of passing gas from one side of the wall to the other under action of a pressure or concentration differential existing across the wall.

Leak rate-In leak detection practice, leak rate is defined as the rate of flow (in pressure-volume units per unit time) through a leak with gas at a specified high pressure (usually atmospheric pressure) on the inlet side and gas at a pressure on the exit side which is low enough to have negligible effect on the rate of flow.

Virtual leak-(1) The evolution of vapor from a cold trap on which the vapor had previously condensed at higher pressures or lower trap temperatures.

(2) The semblance of a leak in a vacuum system caused by slow release of sorbed or occluded gas. The term is sometimes used to include volatilization or disintegration of a material unstable under vacuum.

Leak detector-A device for detecting and locating leaks, and indicating the magnitude thereof.

Tracer gas-A gas which, passing through a leak, can then be detected by a specific leak detector and thus disclose the presence of a leak. Also called search gas.

Minimum detectable leak-(1) The size of the smallest leak, expressed in terms of mass flow per unit time, that can be unambiguously detected by a given leak detector in the presence of noise and background.

(2) The product of the minimum detectable pressure change and the pumping speed at the detector.

Sensitivity-(1) In the case of a leak detector, the smallest concentration of tracer gas in air that can be detected by the device.

(2) The sensitivity of a leak-sensing element is the minimum partial pressure of air which, if changed to the same pressure of search gas, produces the minimum detectable indication; that is, an indication of three times random noise in 3 seconds.

Response time-The time for a leak detector or a leak-detection system to yield a signal output equal to 63 percent of the maximum signal attained when tracer gas is applied indefinitely to the system under test.

Standard leak-(1) A device which permits leakage through it, at a specified rate, of a specified gas, with atmospheric pressure at one end 
of the device and a pressure on the other side sufficiently low to have negligible effect on the leak rate.

(2) A capillary or porous wall leak, usually in a glass or metal tube, whose dimensions have been adjusted to give a conductance within specified limits for a specified gas at a standard reference temperature with specified inlet and exit pressures. Standard leaks for attaching to vacuum test manifolds with air at atmospheric pressure exposed to the inlet are usually protected by filters to avoid clogging by dust particles. Standard leaks for calibrating mass spectrometers are usually fused to a glass reservoir containing the specified gas at a known high temperature.

(3) A device providing a known throughput into a vacuum system. Also referred to as a calibrated leak.

\section{SYMBOLS}

A surface or cross-sectional area, $\mathrm{cm}^{2}$

$a \quad$ radius, centimeters

$B \quad$ a view factor

$C \quad$ a proportionality factor

$C_{0} \quad$ bulk concentration of a gas, torr liter $/ \mathrm{cm}^{3}$

$c \quad$ velocity of sound or acoustic velocity, $\mathrm{cm} / \mathrm{sec}$

$c_{v} \quad$ specific heat at constant volume, cal $/ \mathrm{gram}^{\circ} \mathrm{K}$

$D$ diffusion coefficient, $\mathrm{cm}^{2} / \mathrm{sec}$

$d$ diameter, centimeters

$E \quad$ kinetic energy of a molecule, ergs

$\Delta E$ characteristic activation energy, cal/gram mole

$F \quad$ conductance, $\frac{Q}{\Delta P}$ liter $/$ sec

$f \quad$ wall incidence rate, liter $/ \mathrm{cm}^{2} \mathrm{sec}$

$G$ geometrical factor defining the fraction of molecules having a single-contact trajectory with the condensing surface, dimensionless

$\Delta H \quad$ heat of reaction, cal/gram mole

$h_{c} \quad$ distance of liquid in closed capillary, centimeters

$h_{o} \quad$ distance of liquid in open capillary, centimeters

I current, amperes

$K \quad$ a proportionality factor

$K_{p} \quad$ permeation rate, $\left(\mathrm{atm}^{1 / 2} \mathrm{~cm}\right)(\mathrm{mm}$ thick $/ \mathrm{sec})$ for metals, $\frac{\mathrm{atm} \mathrm{\textrm {cm } ^ { 3 }}}{\mathrm{cm}^{2} \mathrm{sec}}$ for $1 \frac{\mathrm{atm}}{\mathrm{mm}}$ for nonmetals

$k \quad$ Boltzmann's constant:

$1.38 \times 10^{-16} \mathrm{erg} / \mathrm{molecule}^{\circ} \mathrm{K}$

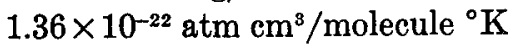

$1.04 \times 10^{-22}$ torr liter $/$ molecule $^{\circ} \mathrm{K}$

$3.30 \times 10^{-24} \mathrm{cal} / \mathrm{molecule}{ }^{\circ} \mathrm{K}$ 
$k$ thermal conductivity, watt $/ \mathrm{cm}{ }^{\circ} \mathrm{C}$

$\& \quad$ length, centimeters

$M \quad$ gram molecular weight, gram/mole

$m \quad$ mass of a molecule, grams

in mass flow rate, gram/sec

$N \quad$ number of molecules

$n \quad$ molecular density or concentration, molecules $/ \mathrm{cm}^{3}$

$P \quad$ pressure, torr or dyne $/ \mathrm{cm}^{2}$

$P_{e q} \quad$ equilibrium pressure, torr

$P_{V} \quad$ vapor pressure of a condensed phase, torr

$Q \quad$ gas throughput, torr liter/sec

$Q_{H} \quad$ thermal flux, watt

$Q_{L} \quad$ leak rate, torr liter/sec

$q \quad$ outgassing rate, torr liter $/ \mathrm{cm}^{2}$ sec

$q_{H} \quad$ thermal flux density, watt $/ \mathrm{cm}^{2}$

$q_{L} \quad$ quantity of gas leaving a surface per unit time, torr liter $/ \mathrm{cm}^{2}$ sec

$q_{R} \quad$ radiant flux density, watt $/ \mathrm{cm}^{2}$

$q_{e} \quad$ equilibrium rate of gas striking or leaving a surface, torr liter/ $\mathrm{cm}^{2} \mathrm{sec}$

$q_{s} \quad$ quantity of gas striking a surface per unit time, torr liter $/ \mathrm{cm}^{2} \mathrm{sec}$

$R \quad=82.05 \mathrm{~atm} \mathrm{~cm}^{3} / \mathrm{gram} \mathrm{mole}^{\circ} \mathrm{K}$

$=62.36$ torr liter $/$ gram mole ${ }^{\circ} \mathrm{K}$

$=8.314 \times 10^{7} \mathrm{erg} / \mathrm{gram}$ mole ${ }^{\circ} \mathrm{K}$

$=1.987 \mathrm{cal} / \mathrm{mole}{ }^{\circ} \mathrm{K}$

$R^{*} \quad$ ratio of critical to molecular orifice flow

$\Delta R \quad$ change in detector reading

$R_{c} \quad$ compression ratio

$R_{e} \quad$ Reynolds number

$R_{t} \quad$ detector reading for any gas specie

$r$

ratio of system speed for air and tracer gas, $\frac{S_{a}}{S_{t}}$

$S$

speed, $\frac{Q}{P}$, liter/sec

gas concentration on a surface at any time $t$, torr liter $/ \mathrm{cm}^{2}$

$s_{0} \quad$ original gas concentration on a surface, torr liter $/ \mathrm{cm}^{2}$

T. absolute temperature, ${ }^{\circ} \mathrm{K}$

$\dot{T} \quad$ heating rate, ${ }^{\circ} \mathrm{K} / \mathrm{sec}$

$t \quad$ time, seconds

$u \quad$ root mean square velocity of gas molecules, $\mathrm{cm} / \mathrm{sec}$

$V \quad$ volume, liters

$V_{s} \quad$ volume of sample, liters 
$v \quad$ velocity, centimeters/second

$\bar{v} \quad$ average velocity of gas molecule, centimeters/second

$v_{s} \quad$ velocity of spacecraft, $\mathrm{cm} / \mathrm{sec}$

$W \quad$ mass, grams

$x \quad$ distance, centimeters

$y \quad$ thickness, centimeters

$Z \quad$ a factor in equation (5.8)

$z \quad$ space simulator pumping effectiveness

$\Phi$ molecular flux density or molecular incidence rate, molecule/ $\mathrm{cm}^{2} \mathrm{sec}$

a molecular capture coefficient of a surface, sticking fraction

$a^{\prime} \quad$ molecular capture coefficient for an array

$a_{E} \quad$ thermal absorbtivity

$\beta \quad$ a plane angle, degrees

$\beta^{\prime} \quad$ angle defined in figure 7.3

$\gamma \quad$ ratio of specific heats

$\delta \quad$ molecular diameter, centimeters

$\epsilon \quad$ base of natural logarithms

$\eta \quad$ viscosity, poise

$\theta$ angle measured from chamber wall normal, degrees

$\lambda \quad$ mean free path, centimeters

$\mu \quad=\left(\frac{M_{a}}{M_{t}}\right)^{1 / 2}$ where $M_{a}$ is molecular weight of air and $M_{t}$ is molecular weight of tracer gas

$v \quad=\left(\frac{\eta_{a}}{\eta_{t}}\right), \eta_{a}$ is viscosity for air, $\eta_{t}$ is viscosity for tracer gas

$\rho \quad$ density, grams/cubic centimeter

$\rho_{M} \quad$ density of mercury

$\sigma \quad$ gage sensitivity factor

$\tau$ time constant, seconds

$\tau_{1 / 2}$ half-life, time to reduce variable to $1 / 2$, seconds

$\tau_{1 / 10}$ time to reduce variable to $1 / 10$, seconds

$\tau_{v} \approx 10^{-13}$ second, vibrational period of a lattice

$\tau_{R}$ residence time, second

$\phi$ molecular flux rate, molecule/second

$\psi \quad$ a solid angle

$\omega \quad$ signifies either $\mu$ or $v$ 



\section{2}

\section{Pressure}

\section{INTRODUCTION}

$\mathrm{P}$ RESSURE IS UNDOUBTEDLY the most widely quoted parameter in vacuum technology. While this term is used generally to express the degree of vacuum, often it serves as a measure of vacuum system merit, cleanliness of the vacuum environment, and as a means of comparing system vacuum to space. In addition, the vacuum engineer who is working in the high- and ultra-high-vacuum regions and encounters the difficulty of measuring actual pressure at these levels will use pressure to refer to some related quantity rather than pressure itself. Therefore, both pressure and the related quantities substituted for pressure at high-vacuum levels are discussed in this chapter, particularly with respect to their meaning in space and in space-simulation-system vacuum chambers. The four sections of the chapter present, in order, a qualitative discussion of the various meanings of pressure as applied to vacuum technology, the conventional treatment of the kinetic theory of gases which explains the quantitative relationships between pressure, flux density, molecular concentration and mean free path as functions of the degree of vacuum, a special treatment of the kinetic theory of gases involving boundary conditions particularly pertinent to space simulation, and the application of the above approaches to space vacuum and space simulators.

\section{DEFINITIONS OF PRESSURE}

Pressure in a gas, defined in terms of gas impingement on a surface, is the time rate of change of the normal component of momentum of the impinging gas molecules per unit area of surface.

The definitions of absolute pressure (as presented in chapter 1 and reference 1), are repeated here to illustrate that of necessity pressure is associated with direction, if it is assumed that these strict definitions are applied.

(1) The net rate of transfer of momentum in the direction of the positive normal to an imaginary plane surface located in a specified position in the gas by molecules crossing the surface in both directions, momentum transmitted in the opposite direction being counted as negative, divided by the area of the surface... 
(2) The pressure exerted by a gas on a real surface is defined as the force applied per unit area. . . .

As mentioned above, the usage of the term "pressure" often extends beyond the exact definitions given to include other measures of the degree of vacuum which are not related to the force concept of absolute pressure. For example, space simulators which generally operate in the $10^{-3}$ to $10^{-9}$ torr range experience pressures which are between onemillionth to one-trillionth of an atmosphere and thus constitute completely negligible forces acting on the spacecraft. Additionally, atmospheric forces in space ordinarily are of a similar or even lower magnitude. Under such conditions the molecular concentration of a gas and the closely allied parameter of mean free path are frequently more important considerations than forces due to gas pressure. Since gas molecules at high vacuum have mean free paths of inches, feet, or miles, the gas molecules rarely collide in flight, and in an enclosed system, nearly all molecular collisions occur with the system walls. Gas behavior at high vacuum may be likened to a collection of independent mobile particles, as opposed to the continuous fluid medium discussed in chapter 5 .

When defining mean free path, concentration (more precisely, molecular concentration or number density), which is the average number of nolecules per unit volume at a point in a gas at a given time and assumed to be nondirectional, is the important parameter to consider. Effectively, the mean free path is the controlling parameter in space and space simulator properties: thermal conductance, viscosity, and electrical breakdown. However, the vacuum engineer seldom expresses vacuum levels in terms of concentration, but rather speaks of a corresponding pressure.

Since a surface in a vacuum environment may adsorb one or more layers of gas molecules, as discussed in chapter 8, an additional group of gas parameters must be considered. Surface properties such as cold welding, emissivity, and work function are affected by the adsorbed layers of gas. Surface coverage and surface-dependent phenomena are affected by the flux density of incident gas molecules; i.e., the number of molecules incident per unit area per unit time. In addition, the factors of chemical species, energy, and direction of incidence also have a bearing on surface-dependent phenomena, whereas pressure as such does not. However, the vacuum engineer may use the term "pressure" when speaking about a comparison of the flux densities of space and a space simulator.

Although it is imprecise to speak of pressure when dealing with vacuum-gage readings, since hardly any commonly used vacuum gage measures the actual pressure in a vacuum chamber, this usage is recognized by the American Vacuum Society (chapter 1). For example, the 
McLeod gage (chapter 3), which measures its own internal pressure, does not sense the pressure in the attached vacuum space (under conditions of molecular flow), rather it senses the molecular flux density. Furthermore, the ionization gage (chapter 3 ) measures the positive ion current created by bombarding the gas with electrons.

The reading of such a gage is affected by the parameters of the concentration of the gas molecules subjected to bombardment, the probability of ionization of the gas, and the collection efficiency of the gage for gas ions. If the gage is suitably calibrated and the gas type is known, the gage measures internal gas concentration. The concentration of gas molecules in the gage is not directly indicative of pressure or concentration in the vacuum space, with the exception of nude gages. Rather, concentration depends on the flux density in the vacuum chamber, the average temperature of the gage, and the molecular weight of the gas. Since it is difficult and, in many cases unnecessary, to consider the interactions between each of the parameters and the gage to obtain a reading, the reading is regarded simply as pressure. An alternate term used, "equivalent nitrogen pressure," arises, since ionization gages normally are furnished to read the pressure directly, in torr or $\mathrm{mm} \mathrm{Hg}$, when used with nitrogen gas at room temperature in the absence of pressure gradients (a random distribution).

Each auxiliary application of the term "pressure" contains a certain inexactitude due to the generalization and extension of the term. The kinetic theory of gases, to be discussed next, permits this inexactness to be evaluated. Thus, in the special case of an essentially stationary gas, the simple perfect gas law may be applied

$$
P=n k T
$$

where

$$
\begin{array}{ll}
P & \text { pressure, torr } \\
n & \text { concentration of molecules } \\
k & \text { Boltzmann's constant } \\
T & \text { absolute temperature, }{ }^{\circ} \mathrm{K}
\end{array}
$$

The gas constant may be expressed per molecule, $k$, or per gram mole, $R$ (the more common usage). The relationship between $k$ and $R$ is $k / m=R / M$, and $R / k=6.023 \times 10^{23}$ (Avogadro's number).

Of course, $R$ (or $k$ ) must be rendered in the appropriate units when used in any equation. The list of values of $R$ and $k$ for various units given in the nomenclature section has been found convenient in some applications.

In addition, the flux density, molecule $/ \mathrm{cm}^{2}$ sec, is

$$
\Phi=n \sqrt{\frac{k T}{2 \pi m}}
$$


where

$\Phi$ the molecular flux density, molecule $/ \mathrm{cm}^{2} \mathrm{sec}$

$m$ the mass of a molecule

Equations (2.1) and 2.2) illustrate that the relationship between pressure, concentration, and molecular flux density is dependent on temperature. The pressure error of a gage which actually measures concentration is approximately 1 percent for each $3^{\circ} \mathrm{C}$ or $5 \frac{1}{2}^{\circ} \mathrm{F}$ deviation from the calibration temperature. The pressure error of a gage which actually measures flux density is dependent on the molecular weight and also exhibits a 1-percent error for $6^{\circ} \mathrm{C}\left(11^{\circ} \mathrm{F}\right)$. Greater errors are likely to occur in space and space simulators where the gas is not stationary. In such cases, pressure and flux density become directional in nature, whereas concentration measurements do not. Directional effects may be one order of magnitude or more in typical cases. The section on applications to space simulation appearing later in this chapter will discuss directional effects further. In this connection, it must be stated that the pressures quoted as being equivalent to various altitudes in space are in terms of stationary gas, a situation which definitely does not exist relative to a spacecraft traveling at orbital velocity (ref. 2).

When considering ionization gages, the sensitivity factor constants for many gases are between 0.75 and 1.5 times that of nitrogen. The most notable exceptions are hydrogen and helium, which give even lower readings, and various hydrocarbons which give higher readings. Chapter 3 discusses a number of important considerations in the use of ionization gages.

The interpretation of gage behavior may be confused if conceptual errors exist. An ionization gage can be regarded as a pressuremeasuring device under ordinary conditions, but considerable uncertainty arises when unusual conditions prevail. For example, the cooling of an ionization gage shell generally causes the pressure in the tube to. fall at the same time that the concentration of the molecules and the resulting gage reading rise. These events do not reflect any change in pressure in the attached vacuum space.

Frequently the vacuum engineer will deal with gas atmospheres of mixed composition for which the partial pressures of the various components must be considered. The preceding discussion on pressure applies to partial pressure as well. The term "partial pressure" is used relative to concentration, flux density, and gage readings in the same manner as the term "pressure." Partial pressure, however, applies to only one gas species. Other pertinent considerations for mixed compositions are that various gas species may have different directional distributions and even different temperatures in high vacuum. For example, when a spacecraft is tested in a chamber equipped with a 
liquid-nitrogen-cooled thermal liner, the gas evolving from the spacecraft is likely to be predominantly water vapor at room temperature. However, the gas leaving the liner and impinging on the spacecraft is at the liquid nitrogen temperature, and it may be mostly nitrogen or hydrogen. The orientation of the spectrometer then becomes important.

In summary, pressure is defined as the normal force per unit area exerted by a gas on a surface. In vacuum technology the term "pressure". is also used to characterize phenomena which depend on concentration, flux density, or mean free path, and in the sense of vacuum-gage reading. The latter uses of the term are less precise than that set forth in the exact definition, particularly in space-simulation facilities which incorporate high pumping speeds. In addition, errors of concept may arise when the engineer is unaware of the sense in which the term "pressure" is being used.

\section{KINETIC THEORY OF GASES}

The kinetic theory of gases originally proposed by Bernoulli in 1738 described the mechanics of a gas consisting of particles (molecules) in motion. It has been applied to explain the quantitative relationships among pressure, concentration, flux density, and other gas properties. Many excellent publications have treated the subject in detail (refs. 3 to 7). The purpose of the following discussion is to present the basic assumptions and limitations of the kinetic theory of gases, and to outline in summary form the quantitative solutions obtained by a conventional application of the theory. A subsequent section entitled "Molecular Kinetics" will present solutions obtained under the varied conditions which are peculiar to high-vacuum applications, space-simulation facilities in particular.

\section{Nature of Kinetic Theory}

A gas is considered to consist of a collection of individual molecules in ceaseless, random motion with an average kinetic energy given by

$$
E=\frac{3}{2} k T
$$

where $k$ is Boltzmann's constant with a value of $1.381 \times 10^{-6} \mathrm{erg} / \mathrm{mole}-$ cule ${ }^{\circ} \mathrm{K}$.

The molecules in a sample of gas do not all have the same kinetic energy; rather, there is a distribution of velocities known as a Maxwellian distribution

$$
\frac{d N}{N}=4 \pi\left(\frac{m}{2 \pi k T}\right)^{3 / 2}\left[\exp \left(-\frac{m v^{2}}{2 k T}\right)\right] v^{2} d v
$$


where

$v \quad$ the molecular velocity considered

$\frac{d N}{N}$ the fraction of molecules having velocities between $v$ and $v+d v$

$m$ the mass of a molecule

An average velocity and a root mean square velocity (defining the average energy) can be derived from equation (2.4). Molecular velocities are similar to the speed of sound, somewhat over $0.34 \mathrm{~km} / \mathrm{sec}$ in air, and are considerably higher than the speed of sound in the light gases. Molecules with such velocities make numerous collisions with each other and with the walls of the container. Since the average distance that a molecule travels between collisions in air at atmospheric pressure is about three-millionths of 1 inch, gases tend to behave like continuous media at normal pressures. All gas molecules in the vicinity of a given point tend to have the same energy (temperature) and collision rate with each other. The numerous collisions among molecules give rise to the idea of a static pressure of molecules impacting on each other at a point in the gas. In addition, intermolecular collisions are mechanisms for the transport of force, momentum (viscosity), and energy (thermal conductivity) observed in gases. In high vacuum, however, intermolecular collisions are almost nonexistent with a consequent alteration in transport properties (as discussed in chapters 1 and 5).

The nature of the interaction of colliding molecules is an important part of kinetic theory. Although the collisions are elastic, and total momentum and energy are conserved, exchanges of energy between colliding molecules may occur. The size and concentration of gas molecules determine the probability of intermolecular collisions. In some cases, it is necessary to assume that a molecule has a size and that it has some particular type of force field by which it interacts (collides) with other molecules.

Ordinarily, the assumption is made that molecules occupy a negligible fraction of the total volume (except when computing collision frequency), and experience negligible interactions when not in direct collision. Special mathematical treatments, van der Waals equation, for example, have been developed for cases where the latter assumptions are not entirely valid. Under vacuum conditions, molecular interactions in the gas phase may be negligible; however, interactions with container walls, such as energy exchange and adsorption, frequently are significant.

Generally, to obtain quantitative solutions to the nature of the interaction of colliding molecules, the following assumptions are made:

(1) The gas is stationary; i.e., there is no macroscopic movement of the gas. 
(2) The gas is uniform in composition (except in diffusion calculations) .

(3) The gas is uniform in temperature.

(4) The gas is in thermal and mechanical equilibrium with the confining container, and there is neither adsorption nor desorption at the walls; the container walls are uniform in temperature.

These assumptions are frequently, but not always, applicable in vacuum systems.

\section{Quantitative Relationships}

A number of important quantitative relationships will be presented next. These relationships will not be derived, but a brief explanation of their background will be given. The assumptions presented above apply to this discussion.

The following effective molecular velocities can be derived from equation (2.4) which defines the Maxwellian distribution. The average velocity, $\mathrm{cm} / \mathrm{sec}$, is

$$
\bar{v}=\left(\frac{8 R T}{\pi M}\right)^{1 / 2}
$$

and the root mean square velocity, $\mathrm{cm} / \mathrm{sec}$, is

$$
u=\left(\frac{3 R T}{M}\right)^{1 / 2}
$$

For comparison, the speed of sound is

$$
c=\left(\frac{\gamma R T}{M}\right)^{1 / 2}
$$

where $\gamma$ is the ratio of specific heats; for air $\gamma=1.4$.

The pressure and flux density of gas molecules on any surface may be computed using the assumption that the gas molecules are contained in a chamber with a concentration of $n$ molecules $/ \mathrm{cm}^{3}$ and an average energy defined by some temperature $T$.

Additionally, it is necessary to consider the random directions exhibited by the gas molecules when approaching or leaving a surface. Molecules traveling with an average velocity, $\bar{v}$, at an angle, $\theta$, with respect to the normal to a surface have a normal component of velocity of

$$
\bar{v}_{n}=\bar{v} \cos \theta
$$

Molecules approaching a point on the surface appear to an observer to come from a hemispherical surface centered at the viewing point. If the entire visible hemisphere has an area $2 \pi \rho^{2}$, the area between angles $\theta$ and $\theta+d \theta$ will be $2 \pi \rho^{2} \sin \theta d \theta$, or a fraction $\sin \theta d \theta$ of the total visible area.

The molecular flux density on a surface may be computed as the 
product of $n / 2$ (since half of the molecules are traveling away from the surface) and the integrated average velocity of approach. That is,

$$
\Phi=\frac{n}{2} \int_{0}^{\pi} \bar{v} \cos \theta \sin \theta d \theta=\frac{n \bar{v}}{4}
$$

The pressure on the surface is the product of the number of molecules and their change in momentum, integrated over the area of view

$$
\begin{aligned}
P & =\int_{0}^{\pi}\left(\frac{n}{2} v \cos \theta \sin \theta d \theta\right)(2 m v \cos \theta) \\
& =\frac{1}{3} n m u^{2}
\end{aligned}
$$

Since the average squared velocity is being considered, the rms velocity $u$ must be used. In addition, the assumption has been made that the molecules are incident from random directions; i.e., there is no macroscopic motion of the gas. Furthermore, when computing pressure, it has been assumed that the gas molecules bounce off the surface with no adsorption and no change of energy (temperature). Thus, the change iri momentum is $(2 m v \cos \theta)$.

Since the average kinetic energy of the molecules is proportional to temperature (eq. (2.3)).

$$
\frac{m u^{2}}{2}=E=\frac{3}{2} k T
$$

it follows that

$$
P=n k T
$$

and

$$
P=\Phi(2 \pi m k T)^{1 / 2}
$$

Equation (2.9) is also known as the Efflux equation and is discussed in more detail in chapters 4 and 5. Equation (2.12) is a form of the perfect gas law. The conditions used in the kinetic theory derivation of equation (2.12) are described as "ideal gas laws." Normally, the equation is written in terms of the molar quantities, and the appropriate value of $R$ is used instead of $k$. Equation (2.13) has been applied to the evaporation process where the equilibrium vapor pressure characterizes a state at which the incident flux is equal to the evaporation rate.

The kinetic theory of gases also is applicable to the calculation of the mean free path of molecules in a gas. The mean free path for a single gas type is

$$
\lambda=\frac{1}{2 \pi \delta^{2} n}
$$

See references 2 to 6 for a development of this equation. An aid to remembering the relative scale of the mean free path is that $\lambda$ at 1 micron 
$\mathrm{Hg}$ pressure is about the length of a man's thumb. At high-vacuum levels, the mean free path tends to be longer than the largest diameter of the conventional vacuum system. There are several important consequences of the lengthy mean free paths which exist in vacuum systems under high-vacuum conditions and in space. First of all, outgassing products depart from spacecraft surfaces in straight lines and normally do not return to such surfaces except when they impact with some solid object such as the walls of a vacuum chamber. In addition, a number of molecular streams, each having different temperatures, compositions or concentrations, may coexist without significant interaction. Lastly, equilibrium of flux density and flow in response to flux gradients tends to replace pressure equilibrium and pressure gradients in flow that obtains at normal pressures.

Further development of the theory of collisions of gas molecules has been used to explain the properties of diffusion, thermal conductivity, and viscosity of gases. Again, the basic assumptions under which the solution is obtained must be kept in mind. These will not be discussed further here because the effects are normally negligible in high vacuum.

It is important to remember that the above equations and discussion apply to gases in vacuum systems in some, but not all, cases. The assumption of a stationary gas is not valid in space simulators which employ cryogenic pumping to attain high pumping speeds. Nor does the assumption apply in some parts of space where the velocity of a spacecraft is comparable to or greater than the average molecular velocity. In the above-mentioned type of space simulator, the gas is not necessarily uniform in composition or temperature. Under these conditions, pressure, flux, temperature, and concentration are related, but the relationships are quantitatively different than those indicated by conventional kinetic theory. For example, when the predominant gas in a space-simulation chamber is outgassing material from the item under test, and when this gas is being condensed on a cold thermal shroud as quickly as it is evolved by the test item, the pressure on a surface depends on the location and orientation of the surface. However, the concentration of molecules depends on location, but not orientation. As a result, equations (2.12) and (2.9) are not consistent in the above situation. In addition, equation (2.13) yields a pressure which is high by a factor of 2 if all the incident gas molecules are condensed, with the assumption that the gas is incident from random directions. When gas incidence is not random, a further error enters the calculation. Therefore, before attempting to use the equations of the kinetic theory of gases, the vacuum engineer should consider their applicability to any given situation.

Thus, the kinetic theory of gases is usually developed under the assumptions of an isotropic gas in equilibrium with the surfaces, and 
macroscopically stationary. The kinetic theory has been widely used in vacuum technology to determine the relationship between such gas properties as concentration, pressure, temperature, flux density, and mean free path. Ordinarily, the necessary conditions for application of the theory are met satisfactorily in vacuum systems with walls at room temperature and with low or moderate pumping speeds. Other situations have been treated by various modifications of the conventional theory. For example, see chapter 4 and the "Molecular Kinetics" section of this chapter.

\section{MOLECULAR KINETICS}

Molecular kinetics (ref. 8) is the branch of the kinetic theory of gases which is particularly adapted to the molecular flow regime. The basic assumptions are:

(1) A gas consists of molecules in motion.

(2) Since molecules seldom, if ever, collide with each other, molecules follow straight-line trajectories.

(3) Molecules departing from a surface (entering the gas phase) have a cosine distribution of direction and an average kinetic energy corresponding to the temperature of the surface.

(4) Separate populations of molecules having different temperatures, compositions, and flow patterns may coexist in a given gas volume.

This approach to gas distribution emphasizes the flux density pattern on the various surfaces rather than the more familiar parameters of pressure or concentration. The remainder of this chapter will be concerned principally with applications of molecular kinetic theory to gas statics, to several elementary principles of gas transfer from surface to surface, and to cases where there is an absence of flow but the temperatures are not uniform. Chapters 4 and 5 also discuss the molecular kinetics treatment of gas-flow problems, as does reference 9.

When considering the process of gas transfer from one surface to another in a high vacuum, it is useful to note that gas molecules in high vacuum behave somewhat like photons. The equations for radiant heat transfer, particularly view factor considerations, can be applied to gas molecule trajectories. Thus, the exchange of gas molecules between surfaces may be understood with reference to figure 2.1. The flow of gas from a small area $d A_{1}$ to a second small area $d A_{2}$ is

$$
\phi_{1-2}=\frac{\Phi_{1} \cos \beta_{1} \cos \beta_{2} d A_{1} d A_{2}}{\pi \ell^{2}}
$$

where $\Phi_{1}$ is the flux density leaving surface $d A_{1}$. Further discussions may be obtained from any general treatise on heat transfer, such as reference 10. Equation (2.15) may be written in terms of the solid angle $d \psi$ subtended by surface $d A_{1}$ from $d A_{2}$ 


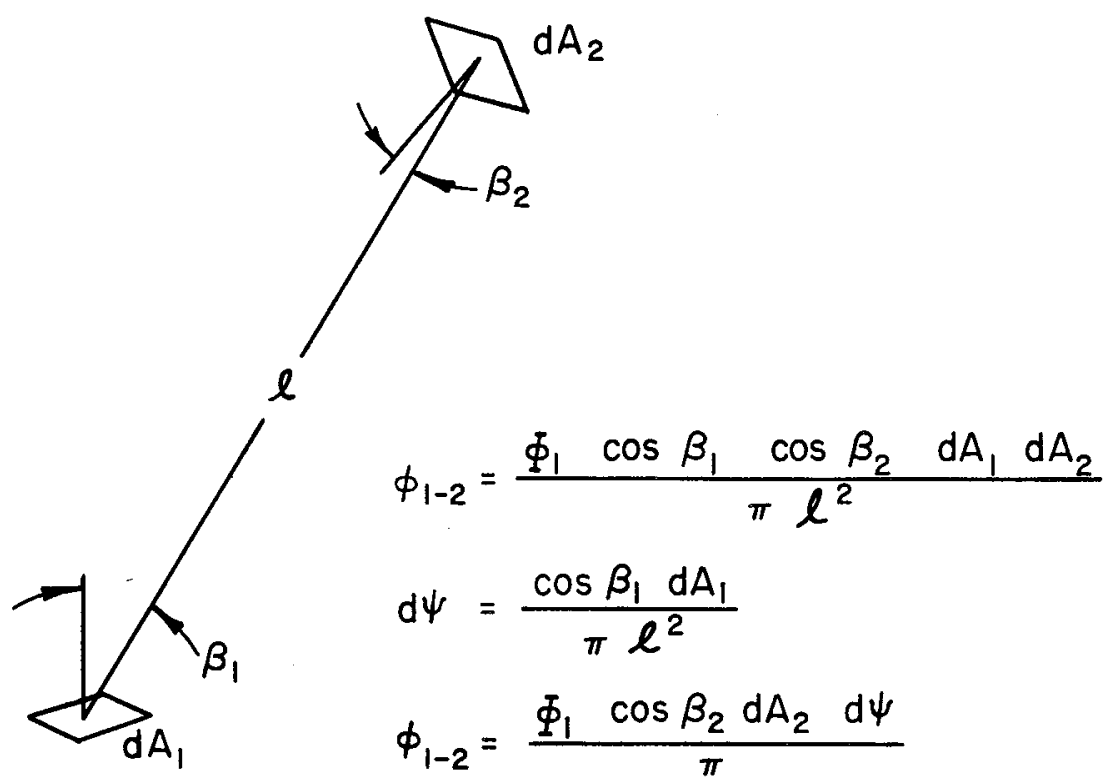

Figure 2.1.-Exchange of gas molecules between surface elements $d A_{1}$ and $d A_{2}$, where $\phi_{1-2}$ is the gas flow.

$$
\phi_{1-2}=\frac{\Phi_{1} \cos \beta_{2} d A_{2} d \psi_{1}}{\pi}
$$

'This form of the equation permits Theorem I to be stated.

Theorem I.-Any surface element occupying the identical solid angle and emitting the same flux density as $d A_{1}$ will produce exactly the same gas load on $d A_{2}$.

In the special case where $d A_{1}$ and $d A_{2}$ are small elements on the surface of a sphere of radius $a$ as shown in figure 2.2 , the application of equation (2.15) to the direct gas flow from 1 to 2 yields

$$
\phi_{1-2}=\frac{\Phi_{1} d A_{1} d A_{2}}{A}
$$

where $A$ is the area of the sphere, $\mathrm{cm}^{2}$. Since the location of $d A$ on the sphere is immaterial, Theorem II may be stated.

Theorem II.-All surface elements on a sphere are subjected to the same incident flux density when gas is emitted by any surface element.

If the emitting surface $d A_{1}$ is extended to constitute the entire area of the sphere, all the wall areas are uniform flux emitters and Theorem III may be stated. 


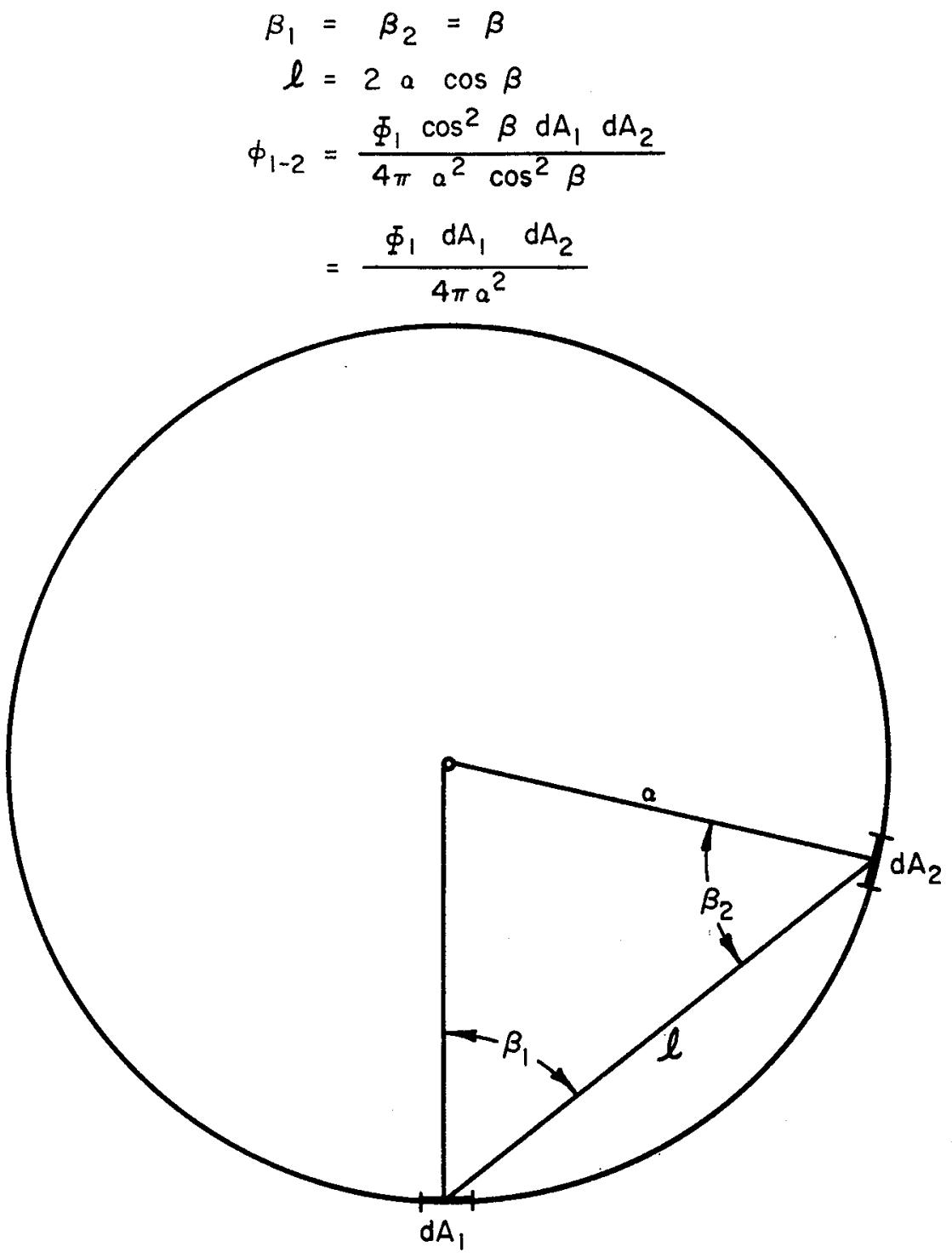

Figure 2.2.-Molecular flow between surface elements $d A_{1}$ and $d A_{2}$ on a sphere of radius $a$, where $\phi_{1-2}$ represents the gas flow.

Theorem III.--The flux density incident on $d A_{2}$ is the same as the flux density emitted by all other surfaces within view in the uniform case.

The three theorems are true, irrespective of temperature uniformity, adsorption effects, or gas flow rates. 
If a gas is stationary, each element of the surface area emits gas at the same rate at which it receives gas. Otherwise, a steady flow would exist or the quantity of flow would change with time. Under these conditions, it can be shown that all surfaces experience the same incident gas load. In the case of the sphere, the gas flux density incident on an element of the surface area has been shown to be the same for all elements.

Now, if the spherical wall is replaced by one of arbitrary shape, as indicated in figure 2.3, the direct gas flow to surface $d A_{2}$ from any element $d A_{3}$ may be studied by replacing $d A_{3}$ with an equivalent element, $d A_{s}{ }^{\prime}$, in accordance with Theorem I. Equation (2.16) shows that $d A_{3}{ }^{\prime}$ is equivalent to $d A_{3}$ if it occupies the identical solid angle and emits gas at the same flux density. A consideration of the actual surface in figure 2.3 , element by element, illustrates that the same gas incidence pattern exists on $d A_{2}$ as that in the spherical case, if, and only if, every

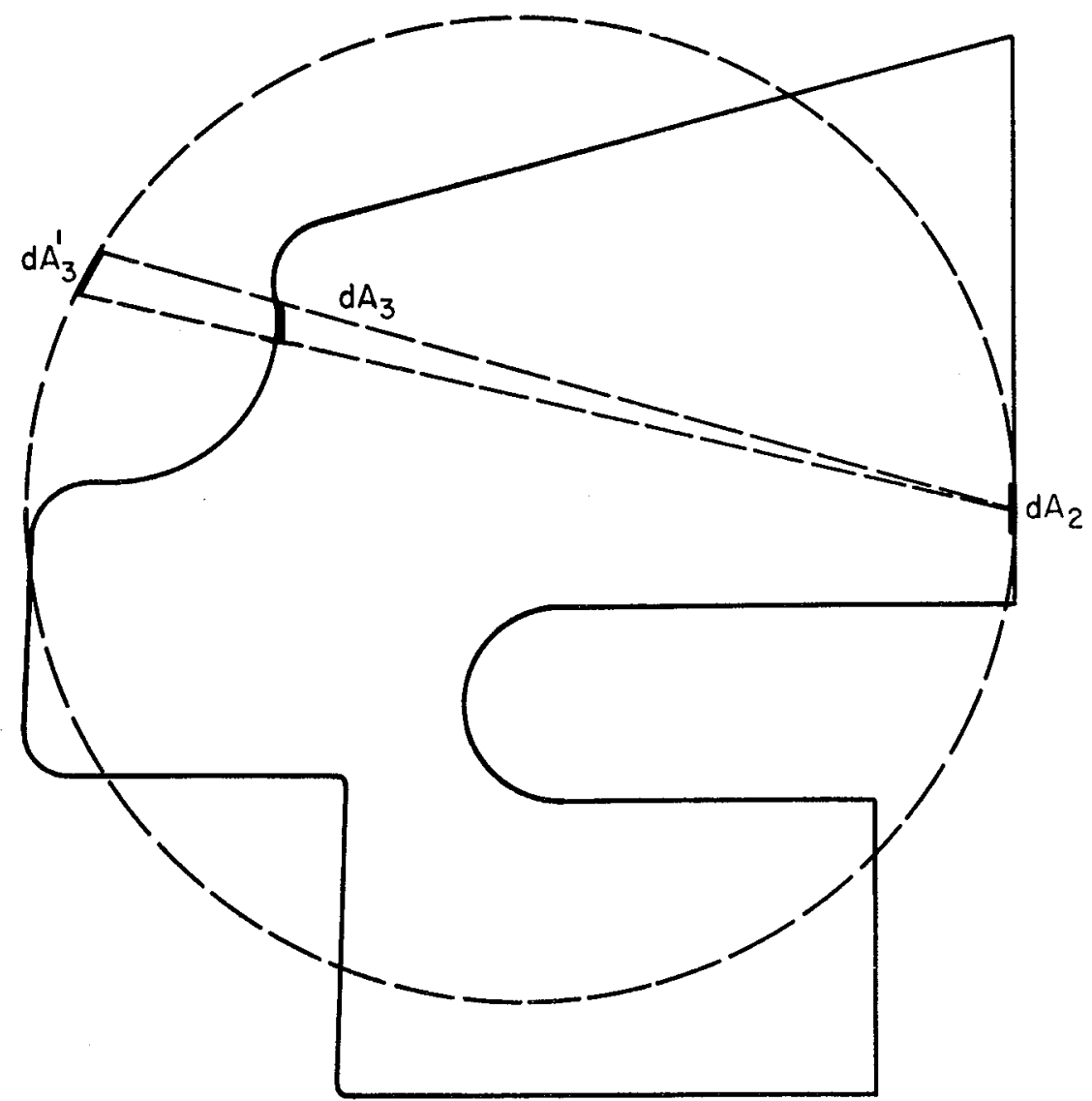

FicuRE 2.3.-Molecular flow between surface elements in a nonspherical enclosure where $d A_{8}$ has been replaced by an equivalent element, $d A_{s}{ }^{\prime}$. 
surface emitting gas to area $d A_{2}$ does so with the same flux density. This uniform emission can take place in a stationary gas only with a uniform incident flux density on all the surfaces. This, in turn, implies a uniform emitted flux from other surfaces and, hence, a uniform incident flux on these other surfaces. The argument can be extended with the resultant conclusion that the flux density in a stationary gas is the same on all surfaces. That is, uniform flux density is a possible equilibrium condition in a stationary gas.

However, uniform flux density may not be the only equilibrium condition. Suppose $d A_{2}$ is a more intense gas emitter than the average surface. In a stationary gas, it can be such only if it receives a greater incident flux density than average. Consequently, $d A_{2}$ must be exposed to some intense gas emitter, say $d A_{3}$ or its equivalent $d A_{3}{ }^{\prime}$. If the equivalent sphere is used again to define the entire gas load upon $d A_{2}$, and if the gas excess is considered to come from excess gas emission from another point $d A_{3}{ }^{\prime}$, an application of equation (2.17) to this excess amount will determine the flux density emitted by $d A_{2}$

$$
\Phi_{2}=\frac{\phi_{3-2}}{d A_{2}}=\Phi_{3} \frac{d A_{3}{ }^{\prime}}{A}
$$

Since $\frac{d A_{3}{ }^{\prime}}{A}$ is less than one, $\Phi_{3}$ is greater than $\Phi_{2}$; i.e., the source $d A_{3}$ must be more intense than $d A_{2}$. Now, it is necessary to look for the source of the gas producing the emission rate from $d A_{3}$. Since this source must be still more intense, the ultimate source of gas for the postulated nonuniformity becomes infinitely intense. The only solution is to find an area such that a net amount of gas is added to the system to account for the local intensity. Similarly, a molecular sink is required if an element is to have a lower than average incidence flux density. Neither the concept of a source nor a sink is allowed in a stationary gas.

Once the principle of uniform flux density has been accepted, the topic of thermal transpiration can be evaluated. Consider two evacuated volumes connected by a tube, as in figure 2.4. Assume that the temperatures of the two chambers are different, but each chamber by itself is uniform in temperature. Conventional kinetic theory can be applied to each chamber. Equation (2.9) for the case of the gage may be written

and, from equation (2.14)

$$
n_{G}=\Phi\left(\frac{2 \pi m}{k T_{G}}\right)^{1 / 2}
$$

$$
P_{G}=\Phi\left(2 \pi m k T_{G}\right)^{1 / 2}
$$

Similar relationships can be written for the chamber in terms of $T_{c}$.

$$
\begin{aligned}
& n_{G}=n_{C}\left(\frac{T_{G}}{T_{G}}\right)^{1 / 2} \\
& P_{Q}=P_{C}\left(\frac{T_{G}}{T_{C}}\right)^{1 / 2}
\end{aligned}
$$




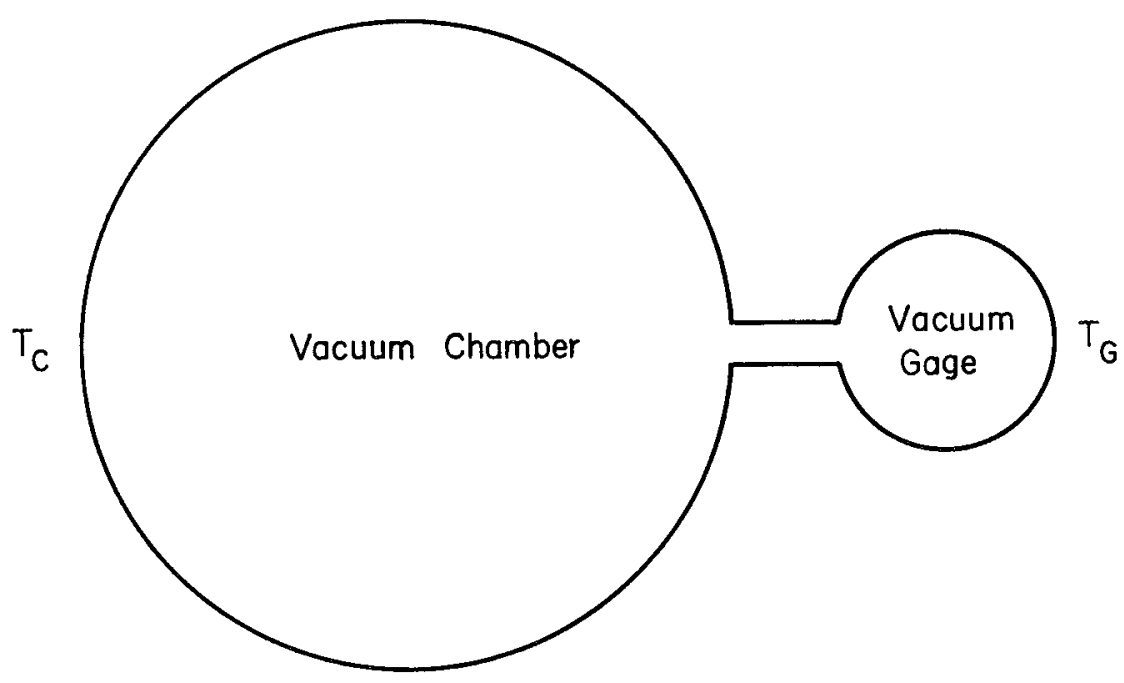

Figure 2.4.-Molecular flow between two evacuated volumes connected by a tube, where each chamber itself is uniform in temperature but the two temperatures are not the same.

The concentration and pressure in the chamber cannot be the same as that in the gage if the flux density is to remain constant. These differences in pressure and molecular concentration characterize the phenomenon called thermal transpiration. According to equations (2.19), (2.20), and the principle of flux uniformity, a concentration gage (i.e., ionization gage), or pressure gage (i.e., McLeod gage) operating at high vacuum produces a reading proportional to the product $(\Phi m)^{1 / 2}$, regardless of chamber temperature or temperature uniformity.

\section{APPLICATION TO SPACE SIMULATION}

Since various observations indicate that the vacuum level in space is extremely high, it is obviously very difficult to obtain conditions in a ground facility which simulate space vacuum. However, the simulation of space conditions is not the same as the duplication of those conditions. Fortunately, it is often far easier to simulate an environment than to duplicate it. The first question to be asked before embarking on simulation experiments concerns what aspects of space or space vacuum must be simulated. For example, to simulate the almost complete absence of thermal conductivity of a gas in space, it is necessary only to produce negligible gas thermal conductivity relative to radiation heat transfer. Such a condition usually is achieved at $10^{-4}$ to $10^{-5}$ torr for ambient temperature test items. If the simulation of the electrical insulating properties of space at voltages of several kilovolts is desired, a pressure near the value of $10^{-5}$ torr provides good simulation of a much higher vacuum with respect to this property. 


\section{Vacuum Characteristics of Space}

Considerable information has been accumulated on the conditions existing in space, particularly in the vicinity of the Earth. In addition to the ARDC Model Atmosphere, 1962, references 11 to 14 discuss the present knowledge of this subject. The following paragraphs will present a summary of the information. The data will be discussed here in terms of concentration or flux rather than pressure. Pressures reported in the

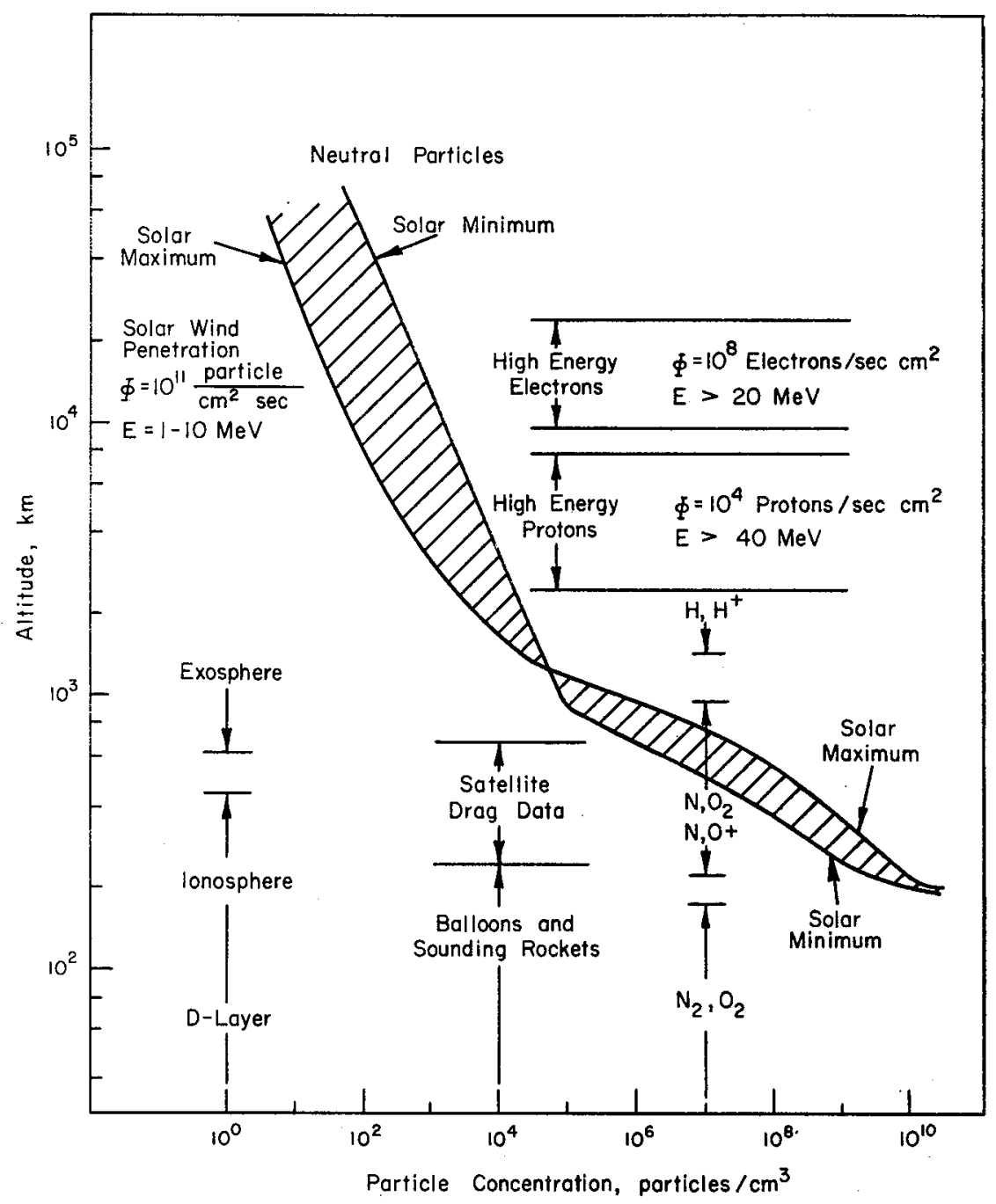

Figure 2.5.-Characteristics of the high-altitude atmosphere surrounding the Earth, to an altitude of $10^{5}$ kilometers. 
literature, which uses pressure rather than concentration or flux, are those experienced by a stationary object.

In actuality, the average spacecraft, especially in earth orbit, travels at a velocity considerably in excess of the average gas molecule. Thus, the pressure is actually determined principally by spacecraft velocity and gas concentration, and is highly directional.

Some of the main features of the high-altitude atmosphere surrounding the earth are shown in figure 2.5. The gas molecule particle concentration is estimated to fall in the shaded area of the figure. It is important to realize that the particle density varies with the time of day and the amount of solar activity. At an altitude below 200 kilometers, the atmosphere is essentially air, a mixture of $\mathrm{N}_{2}$ and $\mathrm{O}_{2}$. Upper portions of this zone may be appreciably ionized. Between 200 and 1000 kilometers, the atmosphere is principally atomic nitrogen and oxygen, which may be largely ionized at periods of solar maxima. There is some evidence of an appreciable amount of helium comprising the atmosphere at about a 700 - to 1000 -kilometer altitude. Above an altitude of 1500 kilometers, the atmosphere consists of neutral atomic hydrogen, protons, and electrons.

At an altitude of 450 to 650 kilometers, depending upon conditions, the important transition from ionosphere to exosphere takes place. The exosphere is that region is which negligible molecular interactions occur. Molecules in the exosphere are assumed to have the same kinetic energy throughout. Their temperature is estimated to vary from $900^{\circ} \mathrm{K}$ at night to $1450-1600^{\circ} \mathrm{K}$ during the day (ref. 12). Satellite drag measurements have indicated a considerable variation in density with time of day and solar activity in a zone extending from an altitude of 300 kilometers to about 700 kilometers.

Tables 2.1 and 2.2 present data on the average molecular velocities and orbital velocities of spacecraft, thus permitting a comparison to be made.

It is apparent that spacecraft velocities are 5 to 8 times the average molecular velocity at altitudes below 700 to 1000 kilometers where hydrogen ceases to predominate. The resulting pressure on the forward part of the spacecraft can be estimated by neglecting the molecular

TABLE 2.1.-Average Velocities of Various Molecules at $1500^{\circ} \mathrm{K}$

\begin{tabular}{|c|c|}
\hline Gas molecule & Velocity, km/sec \\
\hline $\mathrm{H}$ & 6.1 \\
$\mathrm{O}$ & 1.5 \\
$\mathrm{~N}$ & 1.6 \\
$\mathrm{O}_{2}$ & 1.08 \\
$\mathrm{~N}_{2}$ & 1.15 \\
\hline
\end{tabular}


TABLE 2.2.-Orbital Velocities of Spacecraft in Earth Orbit at Selected Altitudes

\begin{tabular}{|c|c|}
\hline Altitude, $\mathrm{km}$ & Orbital velocity, $\mathrm{km} / \mathrm{sec}$ \\
\hline 300 & 8.1 \\
1000 & 7.6 \\
5000 & 6.2 \\
10000 & 5.2 \\
\hline
\end{tabular}

velocities and assuming that the collisions of molecules with the spacecraft are elastic. The flux density is

$$
\Phi=n v_{s}
$$

where $v_{s}$ is the spacecraft velocity. The average incident velocity of molecules relative to the spacecraft is equal to $v_{s}$ and for molecules reflected diffusely, the average departure velocity is $v_{s} / 3$. The pressure then becomes

$$
P=n m v_{s}\left(v_{s}+\frac{v_{s}}{3}\right)=\frac{4}{3} n m v_{s}^{2}
$$

The pressure $P_{0}$, on a stationary vehicle (from eq. 2.11), is

$$
P_{0}=\frac{n m u^{2}}{3}
$$

Thus,

$$
\frac{P}{P_{0}}=4\left(\frac{v_{8}}{u}\right)^{2}
$$

As a result, peak pressures are from 100 to 250 times those values usually quoted.

The pressure and flux density experienced by a spacecraft are more nearly equal to stationary gas values at altitudes above 1000 kilometers where atomic hydrogen predominates.

Another class of particles, called corpuscular radiation because of the high-energy levels, or velocities of the particles, impinges upon a spacecraft. In this class, protons, with energies greater than $40 \mathrm{MeV}$, in the Van Allen belt in the Earth's atmosphere are confined mainly to an altitude zone of 2500 to 8000 kilometers. It is thought that lower energy protons exist at higher altitudes. High-energy electrons in the Van Allen belt are found mostly at altitudes ranging from 5000 kilometers to between 25000 and 35000 kilometers. The quantity of electrons present at the highest altitudes is quite dependent on solar activity. Both protons and electrons are trapped in the magnetic field of the Earth and travel back and forth along magnetic field lines in helical paths. The flux densities of high-energy particles, protons above $30 \mathrm{MeV}$ and 
electrons above $1.6 \mathrm{MeV}$, are about $10^{4}$ particles $/ \mathrm{cm}^{2}$ sec. Electrons of lower energy are present with flux densities of $10^{7}$ to $10^{8}$ particles $/ \mathrm{cm}^{2} \mathrm{sec}$ in zones ranging to an altitude of 40000 to 50000 kilometers. Since these lower energy particles are traveling with comparatively large velocities, the orbital velocities of spacecraft are insignificant in comparison, thus permitting the discussion to be carried out in terms of flux and pressure on a stationary object.

Protons may be emitted in large quantities during a solar flare. The spectrum of these particles indicates a maximum flux density which appears to be approximately inversely proportional to the energy level. That is, the flux density ranges from $10^{12}$ protons $/ \mathrm{cm}^{2}$ sec at $300 \mathrm{eV}$ to 1 proton $/ \mathrm{cm}^{2} \mathrm{sec}$ at $10 \mathrm{BeV}$. These data hold true in the case of a major flare. Apparently, less intensive flares emit protons with energies below a few hundred $\mathrm{MeV}$. The particle flux duration at low-energy values, a matter of 1 to 2 days, is greater than that of high-energy particles which disappear within a few hours. Here again, spacecraft velocities are negligibly small relative to the solar particle velocities. However, the high-energy particles have an essentially unidirectional motion. Particles with energies below a value of about $10 \mathrm{MeV}$ behave more like a continuous plasma and presumably do not have a highly directional velocity distribution. The term "solar wind" has been applied to the particles at the low end of this spectrum, and they are thought to be present much of the time in varying amounts.

In conclusion, there is an interplanetary atmosphere which most probably consists of hydrogen with some helium, at a temperature of about $10^{\circ} \mathrm{K}$ and a concentration of about 600 particles $/ \mathrm{cm}^{3}$. The flux density on a spacecraft in this atmosphere is about $5 \times 10^{9}$ particles $/ \mathrm{cm}^{2}$ sec and is distributed as if the spacecraft were stationary.

It has been shown that the spacecraft vacuum environment varies as a function of altitude and time with respect to flux density and distribution, molecular or ionic species and energy level; i.e., temperature. In addition, the spacecraft velocity may be comparable to, or even much larger than, molecular velocities. This condition gives rise to pressures and flux levels quantitatively different from those on a stationary object. It appears from these considerations that the pressures in space usually cited in the literature are at best an oversimplification of space-vacuum conditions.

\section{Vacuum Characteristics of Space Simulators}

In the foregoing section, the vacuum characteristics of space were discussed in terms of the incidence of the space atmosphere on a spacecraft. The outgassing of the vehicle and components of the atmosphere arising from the presence of the vehicle were not considered. Thus, it is that portion of the vacuum environment which impinges on the 
surfaces of the vehicle or test item that must be controlled to achieve the simulation of space vacuum. As comments are made about differences between space vacuums and those existing in simulators, it should be understood that a judgment as to the significance of such differences depends on the nature and objectives of the individual test performed under the simulated conditions.

A wide variety of systems have been proposed for the simulation of various aspects of the space environment (chapter 10 discusses a few of these configurations). In the high-vacuum-system class, two principal types may be distinguished. There are systems in which the gas impinging on the test item is essentially the same as that given off by the test item, and there are systems in which the impinging gas is substantially different. Due to the long mean free paths of molecules in space, the space environment resembles the latter type of system more than it does the former. In either case, the gas incident on a test item mounted in a simulator is likely to differ considerably from that on a spacecraft in space, both in flux density and distribution, in the gas species and state of ionization, and in the energy level. It is evident that there is some difficulty in describing these parameters by a single measurement of pressure, in the sense of gage reading.

The more advanced space simulators usually incorporate some form of cold wall or shroud which has the ability to condense certain kinds of gases. The composition of the gas environment is shifted in favor of the noncondensable gases. The cold wall and shroud act as pumping surfaces and tend to produce highly directional gas-flow patterns in the gas species which are condensed, and also tend to lower the temperature of the gas environment incident on the test item to approximately the wall temperature. This directional pressure effect is discussed in chapter 4 and also has undergone an extensive analysis in reference 8 . Pressure measurements in directional pressure environments are discussed in chapter 3.

The vacuum characteristics actually achieved in a simulator depend on the nature of the gas load which originates principally as outgassing from the test item, a phenomenon discussed further in chapter 8. Table 2.3 compares various parameters for the two environments, the space simulator and space itself.

Conclusions drawn from the above data and from the previous discussion include:

(1) Molecular flux densities on test items in high- and ultra-highvacuum space simulators may be comparable to those in space environments.

(2) The kinetic energies of molecules in simulators are of completely different orders of magnitude than those in space. 
TABle 2.3.-Comparison of Pressure, Gas Species, Flux Density, and Energy for a Space Simulator and the Space Environment

\begin{tabular}{|c|c|c|c|c|}
\hline \multicolumn{5}{|c|}{ Space Simulator } \\
\hline $\begin{array}{l}\text { Pressure at test } \\
\text { location, torr }\end{array}$ & \multicolumn{2}{|c|}{$\begin{array}{l}\text { Probable gas } \\
\text { species }\end{array}$} & $\begin{array}{l}\text { Flux density, } \\
\text { molecules } / \mathrm{cm}^{2} \text { sec }\end{array}$ & $\begin{array}{l}\text { Temperature } \\
\text { (energy), }{ }^{\circ} \mathrm{K}\end{array}$ \\
\hline $\begin{array}{l}1 \times 10^{-8} \\
1 \times 10^{-8} \\
1 \times 10^{-10} \\
1 \times 10^{-12}\end{array}$ & \multicolumn{2}{|c|}{$\begin{array}{c}\mathrm{H}_{2} \mathrm{O} \\
\mathrm{H}_{2} \mathrm{O}, \mathrm{N}_{2}, \mathrm{H}_{2}, \mathrm{CO} \\
\mathrm{H}_{2}, \mathrm{CO}, \mathrm{CH} \\
\mathrm{H}_{2}, \mathrm{CO}, \mathrm{He}\end{array}$} & $\begin{array}{l}5.0 \times 10^{14} \\
5.0 \times 10^{12} \\
1.4 \times 10^{12} \\
1.4 \times 10^{9}\end{array}$ & $\begin{array}{c}300 \\
80-100 \\
80-100 \\
4-20\end{array}$ \\
\hline \multicolumn{5}{|c|}{ Space Environment } \\
\hline Altitude, km & $\begin{array}{c}\text { Probable gas } \\
\text { species }\end{array}$ & $\begin{array}{r}\text { Maxi } \\
\text { pa }\end{array}$ & $\begin{array}{l}\text { mum flux density, } \\
\text { rticles } / \mathrm{cm}^{2} \mathrm{sec}\end{array}$ & $\begin{array}{l}\text { Equivalent energy } \\
\text { (temperature), }{ }^{\circ} \mathrm{K}\end{array}$ \\
\hline $\begin{array}{r}200 \\
700 \\
1500 \\
50000 \\
\text { Interplanetary }\end{array}$ & $\begin{array}{c}\mathrm{N}_{2}, \mathrm{O}_{2} \\
\mathrm{~N}, \mathrm{O} \\
\mathrm{H}, \mathrm{H}^{+}, \mathrm{e}^{-} \\
\mathrm{H}^{+}, \mathrm{e}^{-} \\
\mathrm{H}^{+}, \mathrm{e}^{-}\end{array}$ & & $\begin{aligned} & \approx 10^{18} \\
& 10^{12}-10^{13} \\
& \approx 10^{11} \\
& \approx 10^{11} \\
& 5 \times 10^{9}\end{aligned}$ & $\begin{array}{c}6 \times 10^{4} \\
3 \times 10^{4} \\
\approx 3000 \\
1-10 \mathrm{MeV}(\text { solar wind) } \\
10^{5}\end{array}$ \\
\hline
\end{tabular}

(3) The gas species present in simulators are usually different from those in space.

The preceding discussion has attempted to define pressure and discuss what it means in vacuum and space-simulation technology. In addition, some other properties of the vacuum environment have been reviewed and their relationship to pressure indicated. The space environment has been briefly described in the terms introduced and has been compared with the typical simulator environment.

\section{REFERENCES}

1. American Vacutm Society, Inc., Committee on Standards: Glossary of Terms Used in Vacuum Technology. Pergamon Press, 1958.

2. Newton, G. P.; Pelz, D. T.; Miller, G. E.; and Horowitz, R.: Response of Modified Redhead Magnetron and Bayard-Alpert Gauges Aboard Explorer XVII. Trans. of the Tenth National Vacuum Symposium, G. H. Bancroft, ed., The Macmillan Co., 1962, pp. 208-212.

3. JEANs, J. H.: An Introduction to the Kinetic Theory of Gases. The Macmillan Co., 1940.

4. KenNard, E. H.: Kinetic Theory of Gases With an Introduction to Statistical Mechanics. First ed., McGraw-Hill Book Co., Inc., 1938.

5. Loeb, L. B.: Kinetic Theory of Gases. Second ed., McGraw-Hill Book Co., Inc., 1934.

6. Dushman, S.: Scientific Foundations of Vacuum Techniques. Second ed., J. M. Lafferty, ed., John Wiley \& Sons, Inc., 1962. 
7. Prutton, C. F.; and Maron, S. H.: Fundamental Principles of Physical Chemistry. Second ed., The Macmillan Co., 1951, ch. 1.

8. Tsonis, C. A.: Molecular Flux Distribution in an Aerospace Chamber. Analysis of Gas Kinetics-Summary Report. (Prepared under Contract AF40(600)954), AEDC-TDR-63-88, Apr. 1963.

9. Jones, D. W.; Tsonis, C. A.; ANd Hol,keboer, D. H.: Theoretical Investigation of Oil Backstreaming Through a Vacuum Trap. AEDC-TDR-63-149, July 1963.

10. McAdams, W. H.: Heat Transmission. Third ed., McGraw-Hill Book Co., Inc., 1954.

11. Anon.: Satellite Environment Handbook. F. S. Johnson, ed., Stanford University Press, 1961.

12. Williams, N. A.; and Christensen, D.: Rockets in Space Environment, vol. II. The Space Environment, RTD-TDR-63-1050, Feb. 1963.

13. Crapman, S.: Interplanetary Space and the Earth's Outermost Atmosphere. Proc. Roy. Soc. (London), ser. A, vol. 253, no. 1275, Dec. 29, 1959, pp. 462-481.

14. Anon.: Space Research. Proc. of the First International Space Science Symposium, Hilde Kallmann-Bijl, ed., North-Holland Pub. Co. (Amsterdam), 1960. 


\section{3 \\ Pressure Measurement}

\section{INTRODUCTION}

$\mathrm{T}$ HE TERM “PRESSURE MEASUREMENT" describes a variety of techniques for the measurement of the degree of vacuum existing in a component or system. It was pointed out previously that pressure, as used in vacuum technology, has other interpretations in addition to its definition as a force per unit area on a surface. Similarly, many of the methods of pressure measurement are actually measurements of some related gas property rather than what is defined as pressure. When gage readings are discussed in terms of the pressure unit, the torr, it should be noted that the term is being used in one of its imprecise senses.

Various measurement principles used in high-vacuum gages, including examples of appropriate gages, are discussed in this chapter as well as the characteristics and problems of ionization gages. Gage-calibration techniques and recommendations on the application of ionization gages to space simulation are also presented.

\section{TYPES OF GAGES}

The difficulties of measuring high-vacuum levels have given rise to a wide variety of gage types (refs. 1 to 5 ). Since it is not possible to discuss all the gage types in existence, the several principles which formed the basis for many of these gages will be discussed with particular emphasis on commercial high-vacuum gages which are commonly used.

Considering the molecular flow regime only, pressure gages may be divided into enclosed or tubulated gages and exposed or nude gages. Tubulated gages are characterized by a sensing zone located in an enclosing envelope which in turn communicates with the vacuum space to be measured. The gas entering the gage may be modified by the envelope which may adsorb part of it, add desorbed material, or change the temperature of the gas. Generally, the tubulated gage can be regarded as a means of measuring the incident flux density of molecules entering the gage mouth. Since the temperature of the gage enclosure affects the concentration and pressure in the gage but does not affect the flux density, the above assertion can be made. There are differences 
between nude and tubulated gages which are very significant in spacesimulator applications. For this case, the gas in the vacuum space may consist of several populations of molecules from different sources, where each population possesses a directional pressure pattern and a characteristic temperature.

Nearly all vacuum measurement gages are of the tubulated type. In addition to this division, high-vacuum gages may be classified on the basis of their particular operational mode:

(1) Force measuring

(2) Compression

(3) Transport property

(4) Radioactive ionization

(5) Discharge ionization

(6) Thermionic ionization

(7) Gas analysis ionization

\section{Force-Measuring Gages}

These gages respond to pressure changes within the gage envelope, and are used primarily for differential pressure measurements. One type, the liquid manometer, which uses mercury or oil, has been used for laboratory work (references 6 to 15 ), but is rarely used on vacuum systems for high-vacuum measurements.

The mechanical measurement of forces by thin diaphragms, ordinarily limited to pressures above $10^{-5}$ torr, has been used experimentally and in several commercial gages (refs. 16 to 23). Gages of this type are relatively expensive due to the complexity of the readout equipment required to detect the very small motion of the diaphragm at low pressures. Additionally, hysteresis in the diaphragm is a problem.

\section{Compression-Type Gages}

This second type of pressure measurement gage is characterized by the McLeod gage (refs. 24 to 32 ), which is illustrated in figure 3.1. The McLeod gage operates by the compression of a known volume of gas to a much smaller known volume, where the pressure after compression is measured, and Boyle's law is used to compute the initial pressure. Compression occurs when a liquid, usually mercury, is raised in the gage to trap a sample of gas in a bulb of an accurately known volume.

The liquid is raised further to compress the gas sample into a compression capillary whose cross-sectional area is also known accurately. If the height of the closed end of the gage is taken as a reference point, the compression ratio is 
where

$$
\begin{aligned}
R_{c} & =\frac{V_{1}}{V_{2}} \\
& =\frac{V_{1}}{A h_{c}}
\end{aligned}
$$

$V_{1}$ the volume of the bulb and capillary

$V_{2}$ the volume of the sample after compression

$h_{c}$ the distance of the liquid in the closed capillary from the closed end

$A$ the cross-sectional area of the closed capillary

The pressure of the sample after compression is obtained by measuring the difference in height between the liquid in the closed and comparison capillaries, and the density of the liquid. If the pressure is expressed in terms of the height of the mercury,

where

$$
P_{2}=\frac{\rho}{\rho_{M}}\left(h_{c}-h_{o}\right)
$$

$P_{2}$ the pressure of the sample after compression

$\rho$ the density of the liquid

$\rho_{3 f}$ the density of mercury (standard conditions)

$h_{o}$ distance of liquid in comparison or open end capillary from the closed end of compression capillary

Under the conditions of an ideal gas and a constant temperature, the initial pressure of

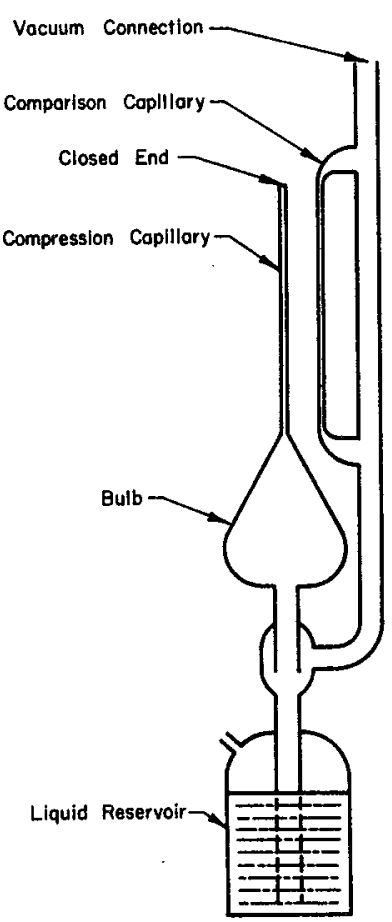

Figure 3.1.-The McLeod gage operates by the compression of a known volume of gas to a much smaller known volume where the pressure after compression is measured and Boyle's law is used to compute the initial pressure. the sample is

$$
P_{1}=\frac{V_{2}}{V_{1}} P_{2}
$$

where $P_{1}$ is the pressure of the sample before compression.

Therefore

$$
P_{1}=\frac{A}{V_{1}} h_{c}\left(h_{c}-h_{o}\right)
$$

The gage is normally operated such that $h_{o}=0$.

There are certain problems inherent in the approach characterized by the McLeod gage. First of all, there will be deviations from ideal gas behavior, notably in the case of water vapor which is a common gas in vacuum systems (chapter 8 ). Since the measured pressure is interpreted as that of the permanent gases, any saturated vapors present initially 
do not affect the gage reading. However, errors will arise if there is an unpredictable change in the pressure of a gas constituent due to adsorption or condensation during the compression.

A second error arises from the fact that the volume after compression is calculated on the premise that the closed capillary is a perfect cylinder with flat ends. When $h_{c}$ is small, the presence of a fluid meniscus and imperfections in the closure constitute an error, while nonuniformity of the capillary diameter introduces an error when $h_{c}$ is large.

Another problem arises from the capillary effect on the fluid, which may be larger than the pressure even after compression has taken place. This effect may be highly variable from point to point and from time to time. A typical depression of $1 \pm 0.1$ centimeter due to capillary action may be found using mercury and a 1-millimeter-diameter capillary. The comparison capillary does not effectively cancel the capillary depression effects, but actually tends to add to the uncertainty (ref. 5).

The final problem concerns mercury which, if used in the gage, produces a vapor stream which tends to pump out the gage. The pumping effect is strongly dependent on room temperature (refs. 33 and 34), and is serious only for the heavier gases such as nitrogen or argon.

Generally, the McLeod gage is not used directly on a vacuum system for high-vacuum measurement. Rather, it is treated as a highly accurate standard of reference. However, considerable care is required to achieve accurate results with the $\mathrm{McLeod}$ gage. Depending on the compression ratio of the gage, readings below $10^{-3}$ to $10^{-4}$ torr are likely to be of limited accuracy.

\section{Transport Property Gages}

These gages measure vacuum in terms of the transport properties of the gas; i.e., viscosity or thermal conductivity. Those gages which measure thermal conductivity are more commonly used. Thermal gages are usually nonlinear, and are sensitive to gas type, variations in ambient temperature, and gas concentration. Thermal gages are divided into three classifications: thermocouple, Pirani, and thermistor.

Thermocouple gages utilize a wire filament which is heated electrically at a value of power which is usually constant and the resulting temperature is measured. These gages ordinarily measure the range from 1 to $10^{-3}$ torr, although extensions of each end of the range by more than one order of magnitude have been demonstrated.

The heated wire itself is used as the temperature indicator in Pirani gages, and the resistance of the wire is measured in a bridge circuit. A permanently evacuated compensating gage is sometimes used in Pirani-type gages. With these modifications, the sensitivity has been extended to the $10^{-5}$ torr range.

The third classification, the thermistor gage, is similar in principle 
to the Pirani gage, except that the heated element is a semiconductor bead instead of a metal wire. Thermistor gages are normally used as one arm in a bridge circuit, and a permanently evacuated compensating gage frequently forms a second arm of the bridge. The pressuremeasuring range of thermistor gages is similar to that of the Pirani gage.

All thermal gages are limited by the fact that the pressure-dependent heat transfer becomes smaller than the heat transfer due to radiation as the vacuum level improves. Small changes in the radiation cooling of the heated element produce output variations which tend to mask the pressure effects, thus imposing a lower limit for pressure measurement.

\section{Radioactive Ionization Gages}

Most high-vacuum gages operate by ionizing the gas and then measuring the ion current collected. The ionization in radioactive gages is produced by alpha radiation from radium within the gage (refs. 35 and 36). Because of the fact that alpha particle sources of sufficient intensity to produce an easily measurable ion current at very-highvacuum values are either dangerous or too large, radioactive gages are limited in range. The alpha radiation gage yields readings in the $10^{-4}$ range, and the experimental tritium gage extends the readings to the $10^{-5}$ range. These gages can be used at high pressures, up to $1 \mathrm{~atm}$.

\section{Glow Discharge Ionization Gages}

Direct observation of a glow discharge in a strong electric field has been. used to estimate vacuum levels from 10 to $10^{-2}$ torr. The addition of a fluorescent screen enables the gages to be used at pressures as low as $10^{-5}$ torr (ref. 4). Ordinary glow discharges tend to be extinguished when the pressure falls below $10^{-3}$ to $10^{-5}$ torr.

Several types of cold-cathode-discharge gages which use a magnetic field to sustain the discharge have been constructed for high- and ultrahigh-vacuum applications. Figure 3.2 illustrates the operation of such a gage. A high voltage of 1 to 10 kilovolts is applied between the cylindrical anode and the flat plate cathodes. At the same time, a magnetic field is applied parallel to the axis of the anode. The discharge is initiated by a few electrons which must be supplied from another source such as cosmic ray particles, field emission, or perhaps an auxiliary filament. After the discharge starts, electrons are supplied from the ionization of the gas molecules, and by secondary emission as ions impact on the cathodes. These electrons are constrained to take helical paths along magnetic field lines, and cannot readily reach the anode without undergoing collisions with gas molecules or other particles, further ionizing the gas and releasing additional electrons. The gas ions are not appreciably restrained by the magnetic field and are collected at the cathode. A measurement of the current between the anode and the cathode indicates the degree of vacuum. 
Magnetic Field

$1-2$ kilogauss
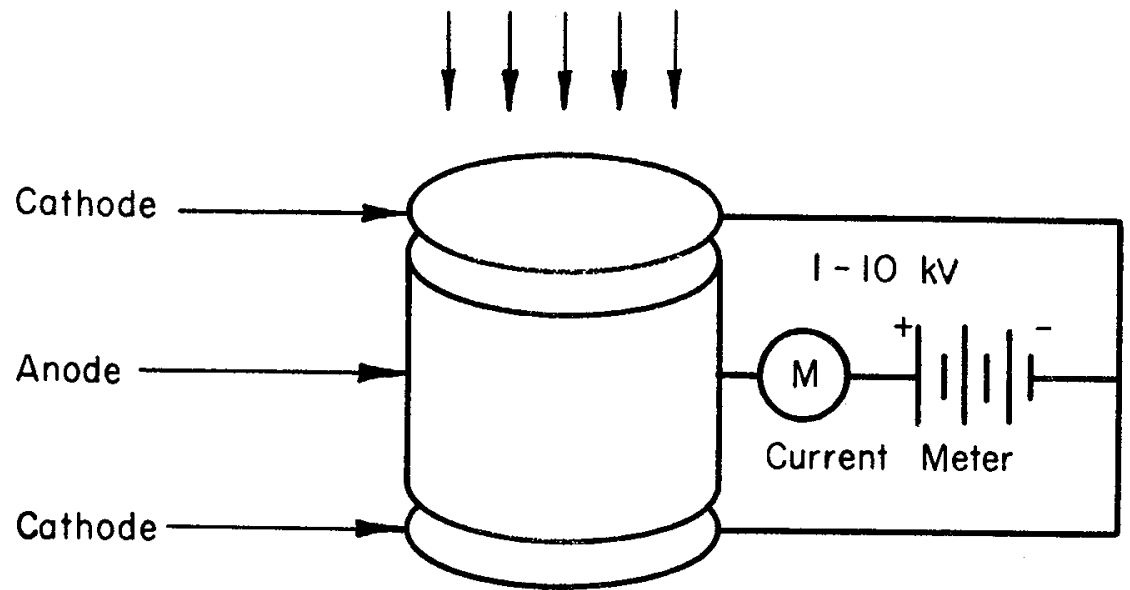

Figure 3.2.-A cold-cathode discharge gage uses a magnetic field to sustain the discharge. To initiate the discharge, a few electrons must be supplied initially from an outside source, perhaps an auxiliary filament.

An early cold cathode gage design, known as a Penning or Philips gage, which used a loop of wire as the anode, operated on the above principle (refs. 37 and 38). Some of the disadvantages of these gages are that the discharge may be unstable under some circumstances, and it is difficult to maintain the discharge at pressures below $10^{-5}$ torr. Modified versions of the.Penning gage are commercially available in which the discharge is almost completely enclosed in a cylindrical anode. If the anode voltage and the magnetic field strength can be properly selected, the discharge can be stabilized to permit operation in ultrahigh vacuum. Another design, the Redhead gage, incorporates the features mentioned above, plus a perforated anode cylinder (refs. 39 to 41). Auxiliary cathodes are used as guard rings to prevent field emission currents from contributing to the measured cathode current. One form of the cold-cathode gage is called an magnetron gage. In this gage, the anode is a rod located on the axis of a cylindrical cathode such that the d.c. field is radial and is perpendicular to the magnetic field. The magnetron-gage discharge may tend to oscillate. However, there is little reported data on the effect of this phenomenon on gage readings.

Cold-cathode-gage characteristics vary as a function of geometry and operating conditions (refs. 42 and 43). These gages possess a high sensitivity ranging from 1 to $10 \mathrm{amp} /$ torr, and a high pumping speed. In fact, ion pumps are basically ganged assemblies of Penning-type gages. Figure 3.3 illustrates the so-called sensitivities of a series of 


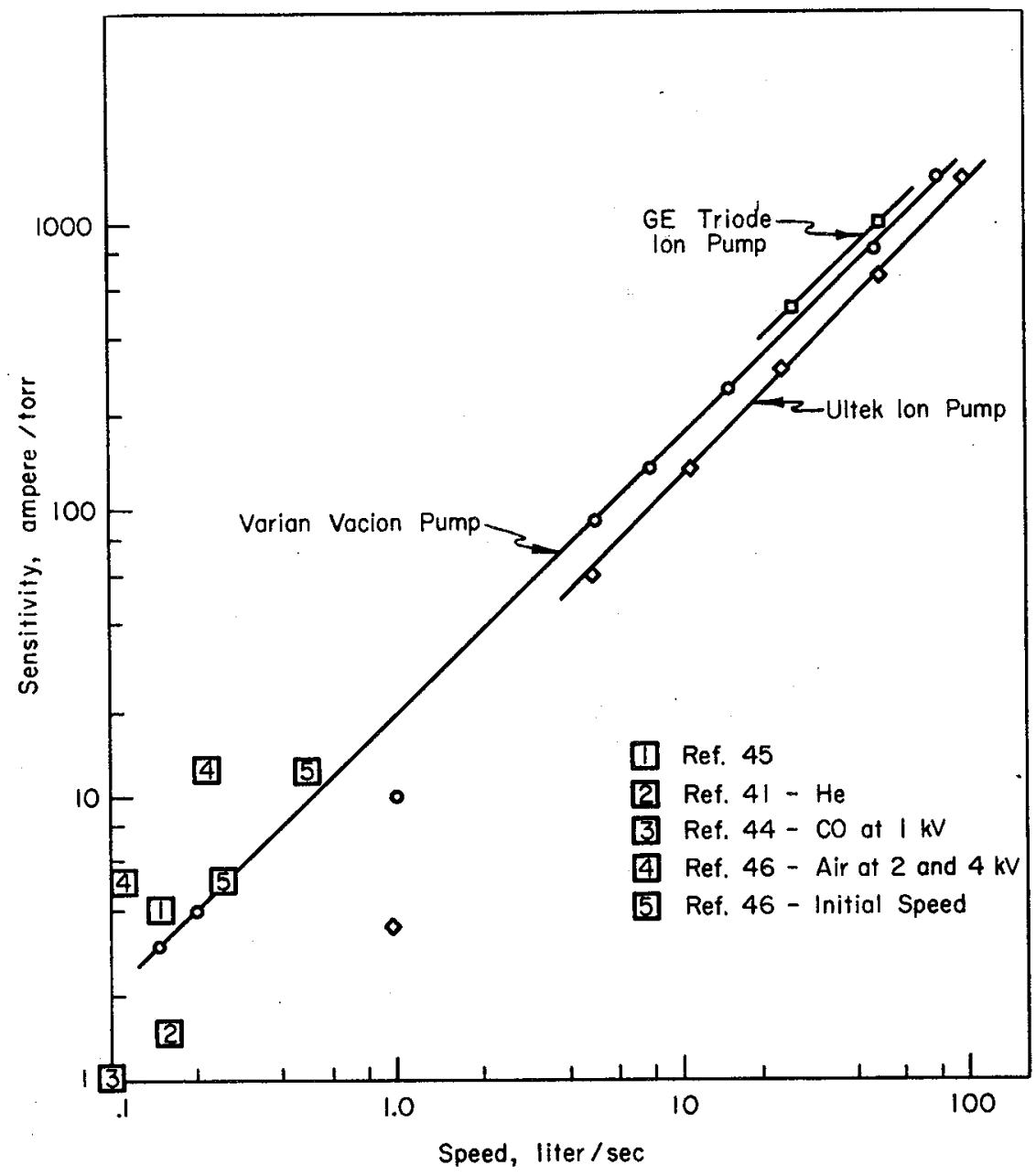

FicURE 3.3-Sensitivities of a series of commercial ion pumps treated as vacuum gages.

commercial ion pumps treated as vacuum gages. Values of the steadystate pumping speed range from 0.1 to $0.5 \mathrm{liter} / \mathrm{sec}$ for air at sensitivities of 2 to $10 \mathrm{amp} /$ torr. References 5, 40, 43, 44 and 45 contain information on some actual gage pumping speeds.

Of course, there are some differences between the pumping mechanism in ion pumps and in gages. That is, gages seldom contain an active metal such as titanium which is intentionally sputtered to promote pumping, as in the case of the ion pump. In addition, the quoted pumping speeds are somewhat dependent on the condition and history of the gage (refs. 5 and 45), and are widely variable functions of sensitivity. One 
reason for the discrepancies in the quoted data may be due to the relatively long saturation process reported by Leck (ref. 5) for a gage pumping air. It has been estimated that at a pressure of $1 \times 10^{-9}$ torr, the time to reach saturation will equal 20000 to 30000 hours. During this time period, the pumping speed decreases by a factor of about 2 .

The response of cold-cathode gages generally is not a linear function of pressure. The output current $I$ is related to the pressure $P$

$$
I=P^{n}
$$

where $n$ varies from 1.1 to 1.4 , and is a function of gas type, magnetic field strength, voltage, gage geometry, and materials of construction. The exponent $n$ has been reported to show a pressure dependence in the Redhead gage by changing from a value of approximately 1.0 to $1.5-1.7$ as the pressure is reduced below $2 \times 10^{-10}$ torr (ref. 40).

\section{Thermionic-Ionization Gages}

Thermionic- or hot-cathode-ionization gages ionize the gas in the gage with electrons obtained from a heated filament. For a specific electron emission current, the ion current reaching the collector is taken as a measure of pressure. These gages are in general use in the inverted Bayard-Alpert form shown in figure 3.4 , or in a modification of this basic arrangement. The central electrode of a Bayard-Alpert gage is the ion collector which operates at ground potential, while the filament is maintained at 25 to 50 volts above ground potential, and is heated to produce electron emission at a desired current level, often about 10 milliamperes. The electrons so produced are accelerated through an additional potential of about 150 volts. Subsequently, the electrons are collected on the electron collector which is usually called the grid.

There are several important features of the Bayard-Alpert type of thermionic gage. Due to its relatively open construction, the gage can be used as a nude gage, and it need not appreciably modify the gas temperature in the sensing zone. Again, the open construction causes the electrons to make a number of passes through the grid area prior to being collected. The resulting increased path length increases the probability of ionization and thus increases the sensitivity of the gage. In addition, the Bayard-Alpert gage was designed to reduce the X-ray limit, and the small collector serves this function. The $\mathrm{X}$-ray limit refers to a spurious output current which is superimposed on the ion current and is indistinguishable from it. When soft X-rays generated by the impact of electrons on the grid strike the ion collector, electrons are ejected from it photoelectrically to form the output current. The $\mathrm{X}$-ray current limits the pressures that can be measured, and is equivalent to a pressure reading in the $10^{-11}$ torr range in a Bayard-Alpert-type gage. Earlier gages which had a cylindrical collector outside the grid experienced an X-ray limit of about $10^{-8}$ torr. 


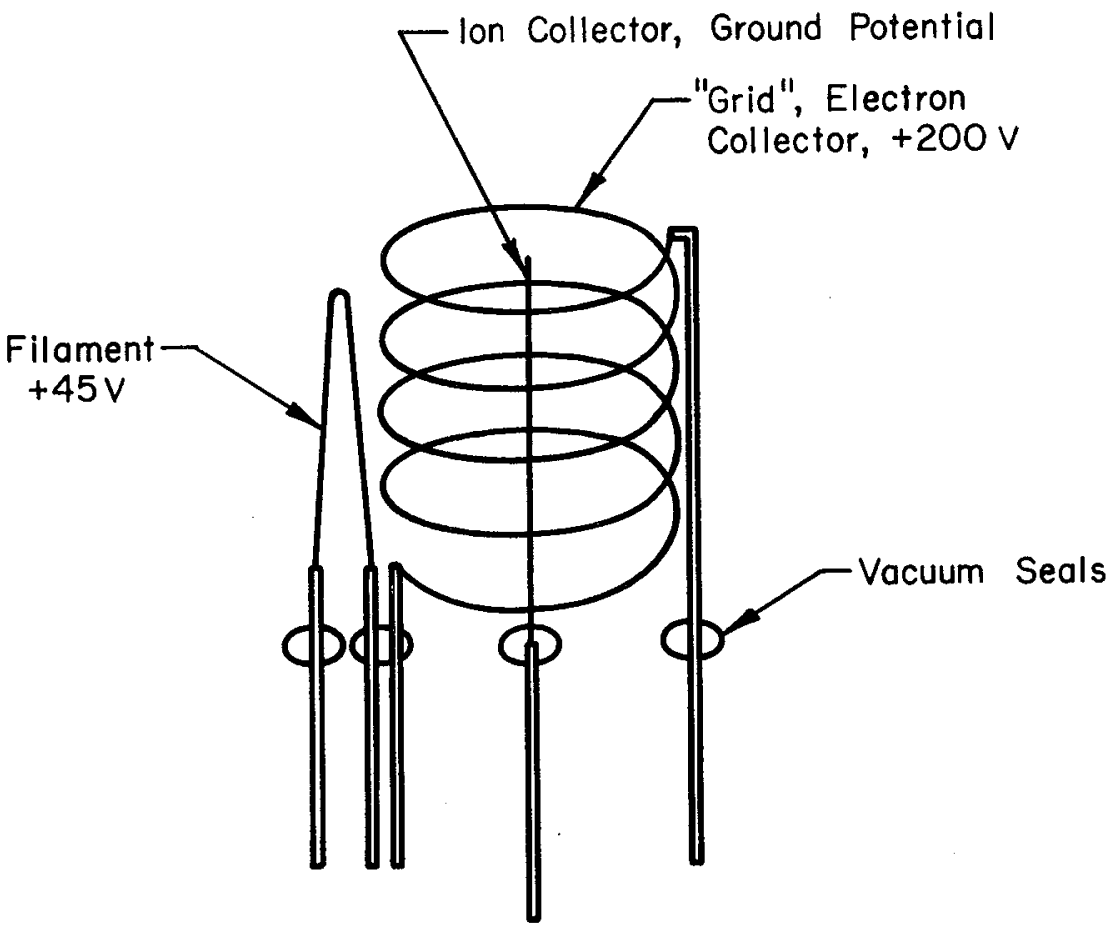

Figure 3.4.--Inverted Bayard-Alpert form of the thermionic or hot-cathode ionization gage which operates by ionizing the gas in the gage by electrons obtained from a heated filament; the measure of pressure is the ion current reaching the collector at a specific electron emission current.

Vacuum technology uses the term "X-ray limit" in two different senses. The term therefore requires some qualification. In the first case, the X-ray limit may refer to the lowest pressure indication which may be obtained in a gage when all the output current is due to X-ray-induced photoemission and there is an absence of gas. The present discussion will confine itself to this first meaning. The second meaning which is sometimes used is that the X-ray limit refers to the pressure level at which the X-ray current is a substantial part, for example, 10 percent, of the total ion collector current.

A number of modifications have been made in the Bayard-Alpert gage. Filaments, which are usually made of tungsten, have been fabricated of thoriated iridium, and rhenium. Both of these substances will briefly withstand the oxidation accompanying accidental release to atmospheric pressure, although thoriated iridium tends to poison in the presence of hydrocarbon vapors. Operational stability has been improved with the addition of an outer grid or shield surrounding the gage structure to establish a fixed wall potential (refs. 46 and 47). 
Another modification introduced closed grid ends and increased sensitivity by a factor of 2.5 to 5 (ref. 46). In addition, a modulator electrode has been incorporated to permit separate measurements of the ion current and X-ray current (refs. 5, 48, and 49). The modulator is an additional electrode adjacent to the ion collector, and can be switched at will from grid potential to ground potential. When the modulator potential is switched, the ion and X-ray photoelectric currents are both changed by fixed, but different, ratios. The observed change in total current following switching may be analyzed to determine the ion current percentage of this total current.

A number of experimental hot-cathode gages have been developed which differ to various extents from the Bayard-Alpert gage. The modifications usually attempt to reduce the X-ray limit, or the effect of the hot filament, or both. The location of an additional electrode which is negatively biased may suppress the photoelectron current from the $\mathrm{X}$-rays by repelling the photoelectrons and forcing them to return to the ion collector (refs. 50 and 52). Since photoelectrons ejected from the suppressor can reach the ion collector and appear as a negative $\mathrm{X}$-ray current, the suppressor must be shielded from the X-rays. A second modification used a magnetic field to increase the electron path length, which in turn increases the sensitivity of the gage (refs. 53 and $55)$.

\section{Gas-Analysis-Ionization Gages}

Gages which distinguish between the various chemical species in a sample of gas are known by a number of names: partial pressure gages, residual gas analyzers, and mass spectrometers, plus a variety of trade names. All of these devices detect gas atoms by ionizing the gas atoms through electron bombardment. It should be noted that this is the same process employed by the ionization gage. A heated filament is usually the source of the bombarding electrons.

A variety of techniques have been developed by which the ions can be separated according to mass, or the mass-to-charge ratio. Three common types-magnetic deflection spectrometers, cycloidal spectrometers, and omegatrons-require a magnetic field with a strength of 1000 to 5000 gauss. Additional commonly used types are the radio frequency ( $\mathrm{rf}$ ) spectrometer, the time of flight spectrometer, and the quadrupole-monopole designs, all of which require a high-frequency a.c. field with an accurately controlled amplitude. The omegatron employs both the magnetic field and the high-frequency electric field.

Among the important differences between the various types of spectrometers are sensitivity and resolution. Sensitivity is the amount of output current produced at a given partial pressure. Most gage types produce $10^{-4}$ to $10^{-5} \mathrm{amp} /$ torr without an electron multiplier, and from 
0.1 to $10 \mathrm{amp} /$ torr with the addition of a multiplier. Readout of the current is accomplished with the vacuum tube electrometer, which is capable of indicating 2 or $3 \times 10^{-13}$ amperes full scale, or the vibrating reed electrometer which can be used at $10^{-15}$ amperes full scale. The combination of a nonmultiplier tube and vacuum tube electrometer can be expected to indicate partial pressures in the $10^{-10}$ torr range. When the electron multiplier is added, the partial pressure readings may be extended to the $10^{-14}$ torr range quite readily. Unfortunately, electron multipliers are subject to poisoning by oil vapors and work well only in very clean systems. Since multipliers cannot operate in a magnetic field, they cannot be employed readily in cycloidal- and omegatron-type spectrometers.

Differences in resolution are also an important factor for the various spectrometer types. Resolution is the ability to separate ions of similar mass, and is defined in terms of the largest mass at which a given criterion is met. There are several criteria which are in common use.

(1) A 1-percent valley means that the level in the valley between adjacent mass numbers is 1 percent of the adjacent peak height.

(2) A 1-percent interference means that one peak contributes 1 percent of its height to an adjacent mass number.

(3) The peak width means that the width of a peak at one-half its height is equal to the separation of adjacent mass numbers.

The relationship between these criteria depends on the shape of the peak, except that criterion 1 is twice the separation of criterion 2. Most types of spectrometers offer resolutions varying between 10 and several hundred according to criterion 2, a 1-percent interference.

The cost of the various types of spectrometers is a function of the degree of resolution; the greater the resolution, the greater the cost. Commercially available mass spectrometers vary considerably in size, convenience of installation, and cost. The cost of an operable device varies from $\$ 2000$ to $\$ 20000$. As a general rule, the more expensive devices require more care in use, but provide better performance.

In conclusion, mass spectrometers are similar to ionization gages in that detection of gas components is accomplished by ionizing the gas atoms and recording the current collected. Most of the difficulties attendant on operation of ionization gages, with the exception of the $\mathrm{X}$-ray limit and the unknown sensitivity due to unknown gas types, are also present in mass spectrometers. In addition, the sensitivity and resolution of spectrometers may be affected by contaminant films on the focusing electrodes, by magnet misalinement, and by the condition of the multiplier. In addition, the output currents in mass spectrometers are about four decades smaller than those in ionization gages with the result that severe current measurement problems may arise. However, 
the various types of gas-analysis-ionization gages have many advantages in the measurement of system pressures. The identification of the type of residual gas not only provides a correction to the true pressure reading but it also provides an insight into the operation of the system for the purpose of troubleshooting, or engineering analyses of various gas loads. Spectrometers possess the additional advantage of being able to indicate the presence of leaks and locate them.

\section{IONIZATION-GAGE CHARACTERISTICS}

The following discussion of the characteristics and problems pertinent to the ionization gage is centered on the hot-cathode gage, although many of these characteristics will apply to any ionization gage, including mass spectrometers, cold-cathode gages, and radioactive gages. It will be obvious when particular characteristics do not apply to certain gages.

The division of the gage characteristics into electrode effects, gassurface reactions, gas-species effects, and residual current effects will facilitate the discussion.

\section{Electrode Effects}

The electrodes of a gage tube establish the electric fields which direct the motions of electrons and ions, and collect currents in the gage. Improper potentials on the electrodes can produce faulty operation. If an electrode such as a spare filament is left floating, it assumes a potential which is dependent on the relative number of electrons and positive ions incident upon it, or, in other words, a potential which is a function of vacuum level. Similar conditions exist with respect to insulated surfaces, such as glass walls in an ionization gage. Some gages are constructed with a conductive coating on the walls, or with a screen grid by which a definite wall potential can be established (ref. 46). The potential variation occurs mainly at high pressures, about $10^{-5}$ torr.

In another case, oil vapors frequently result in the formation of electrically insulating deposits on the electron collection or ion-collection surfaces. These deposits result in a change of the field configuration and sometimes affect the collection rate. In the case of mass spectrometers, the focusing and resolution may be altered.

The output of the gage tube is affected by changes in the physical position of electrodes, particularly the filament in a Bayard-Alpert gage. In reference 56 , it is reported that a 1-millimeter shift in filament position can result in a 20-percent error in output.

\section{Gas-Surface Reactions}

The gas in an enclosed ionization gage may be altered in quantity or type by reactions with the surfaces of the gage. Outgassing within the gage causes readings which are erroneously high. If a pumping process 
occurs, low readings are produced. Gas-exchange reactions may also exist and will affect gage readings.

Gage surfaces will exhibit outgassing or desorption of gases in a manner similar to that for other surfaces exposed to vacuum (chapter 8). Since outgassing is temperature dependent, changes in filament power or air circulation tend to affect the outgassing load. The outgassing in a nonbaked gage will produce a typical pressure reading of about $3 \times 10^{-7}$ torr (ref. 57). This reading can be lowered to about $10^{-8}$ torr by degassing. The outgassing of the tubulation alone yields a similar value. The base plate of a nude gage has an outgassing rate which can result in readings in the $10^{-9}$ torr range. Pressures such as these can be attained in a few hours in a space simulator due to the high pumping speeds possible with cryogenic pumping. Therefore, a substantial part of the gage reading may be due to outgassing within the tube itself.

To reduce the outgassing in a gage to a negligible level, the process of degassing is usually employed. That is, a large current of 5 to 10 amperes is passed through a spiral grid, or electron bombardment with an emission of approximately 100 milliamperes and a grid voltage of 400 volts or more is used. The electron bombardment process heats the grid to incandescence, also the gage enclosure, with the result that a large pressure burst occurs. This procedure, however, does not effectively degas the gage tubulation. An indication of subnormal pressures often occurs after the termination of outgassing (ref. 58).

The subnormal pressures mentioned above are sometimes observed after degassing and are an example of gage pumping. There are several mechanisms by which pumping takes place. For very clean surfaces, chemically active gases are adsorbed directly to a coverage of about $10^{15} \mathrm{molecule} / \mathrm{cm}^{2}$, or approximately a monomolecular layer. A chemical pumping effect in Bayard-Alpert gages was reported in reference 59. The effect saturated after pumping $10^{15}$ molecules of nitrogen at a pumping speed of 2 liter/sec. Pumping effect readings for a typical glass-tubulated gage will be 20 to 50 percent lower during the period of chemical pumping. The duration of the effect may be of the order of 4 hours at $1 \times 10^{-9}$ torr, and is inversely proportional to pressure. Chemical pumping does not occur significantly in the case of noble gases (ref. 60).

Another pumping mechanism is termed "electronic" or "ionic pumping." Even the noble gases may be pumped ionically (ref. 60). The pumping speed of nitrogen has been reported to be about 0.1 to 0.2 liter/sec at 8- to 10 -milliampere emission, and is proportional to emission. The pumping of noble gases occurs at about one-tenth of this speed. The ionic pumping effect saturates after $10^{16}$ to $10^{17}$ molecules have been pumped. This कf frocess requires pumping approximately 3 months at $1 \times 10^{-9}$ torr. This saturation most probably results from the desorption of previously pumped gas at a rate equal to the pumping 
speed. In a manner similar to chemical pumping, ionic pumping is at least partially reversed when the surface is heated.

The most common remedy for pumping effects is to provide a passage of large conductance between the gage tube and the vacuum space. For example, a connection $7 / 8$ inch inside diameter by 2 inches long has a conductance of about 20 liter/sec for nitrogen. At a pumping speed of $0.2 \mathrm{liter} / \mathrm{sec}$ (ionic pumping), the density of gas in the gage is reduced approximately 1 percent by the impedance offered by the connecting tube. In comparison, a 10-percent error could occur in a freshly degassed gage from chemical pumping on clean surfaces. The latter effect may be reduced to a negligible level if the gage is nude.

A third mechanism of pumping in gages involves chemical reactions with the hot filament. A number of gases such as oxygen, nitrogen, water vapor, and hydrogen have been shown to react with tungsten or the carbon present in tungsten. Oxygen pumping rates of 0.2 to 0.6 liter/sec have been reported in reference 5 . Reactions of nitrogen and hydrogen are much slower. The rates of these reactions are dependent on filament temperature, and are low enough that serious errors in measurement can be avoided when high-conductance connections are used. In small systems, however, the change in gas composition may be significant.

Tubulated gages may fail to sense hydrocarbon vapors, such as those from pump oils. This phenomenon is known as the Blears effect (refs. 61 and 62), and, although the mechanism of the effect is not completely understood, the practical result is a difference between the tubulated and nude gage readings (the nude gage will read higher) as the ultimate pressure is approached in a system containing oil vapors.

An important point to consider is that the net effect of adsorption and desorption processes depends on the temperature. A gage tends to come to equilibrium with the gas in the attached vacuum chamber, so that it is neither a source nor a sink for the gas. However, the equilibrium can be disturbed by a temperature or pressure change, and some time will be required to bring the conditions back into balance.

\section{Gas-Species Effects}

Ionization gages respond in varying ways to different chemical species in gases. The relative sensitivities for various gases are not the same for all types of gages, and may differ as much as \pm 20 percent for so-called identical gage tubes. As a result, the values given in the table below are somewhat approximate and should be regarded as typical.

Mass spectrometers also exhibit differing sensitivities for various gas types. Due to the ion analyzer characteristics of spectrometers, the sensitivity usually decreases with mass number, whereas the sensitivity ordinarily increases with mass number in the ionization gage. 
TABLE 3.1.-Ionization-Gage Sensitivity Relative to Nitrogen for Various Gases

\begin{tabular}{|c|c|c|c|}
\hline Gas type & $\begin{array}{l}\text { Bayard-Alpert } \\
\text { hot-cathode } \\
\text { gauge }\end{array}$ & $\begin{array}{l}\text { Cold-cathode } \\
\text { gage (GE) }\end{array}$ & $\begin{array}{c}\text { Radioactive } \\
\text { gage } \\
\text { (alphatron) }\end{array}$ \\
\hline Oxygen & 0.8 & & \\
\hline Hydrogen & $.4-.5$ & 0.48 & 0.23 \\
\hline Carbon monoxide & $1.03-1.05$ & & \\
\hline Carbon dioxide -..-_-_- & $1.3-1.6$ & & 1.5 \\
\hline Water vapor & $1.1-2.0$ & & .88 \\
\hline Argon & $1.2-1.5$ & 1.2 & 1.2 \\
\hline Neon & $.25-.32$ & .24 & .5 \\
\hline Helium _- & $.14-.21$ & .16 & .21 \\
\hline Mercury vapor & $2-3$ & 10 & \\
\hline
\end{tabular}

\section{Residual Current Effects}

This term is applied to several phenomena in ion gages which yield currents not directly dependent on vacuum level. The $\mathrm{X}$-ray effect is a current due to electrons emitted following the impact of soft X-ray photons upon various surfaces in the gage. The X-rays are generated by the bombardment of the electron collector by the arriving electrons. Photoelectrons emitted from the ion collector appear as a current indistinguishable from an ion current. In addition, photoelectrons may be ejected from other surfaces, such as glass walls, at potentials enabling them to reach the ion collector and produce negative currents. The emission of photoelectrons from a metal shell surrounding a gage has been reported to yield a negative residual current which, in effect, lowers the X-ray limit (ref. 64).

The magnitude of the residual current in a gage has been found to be quite dependent on the gas species present. For example, the residual current may be a decade higher than normal in the presence of oxygen. Anomalous background currents of this type tend to be most prominent at low-emission levels (ref. 63).

Ionization gages, particularly the hot-cathode type, may have readings affected in the $10^{-8}$ torr range (for the Bayard-Alpert gage) by possible residual currents. The existence of a substantial X-ray current can be detected by varying the electron accelerating potential. A 10-percent increase of grid-to-filament voltage normally causes a decrease of a few percent in true ion current and a 15- to 20-percent increase in X-ray current.

\section{VACUUM-GAGE CALIBRATION}

Vacuum technology, and its application to space technology, has created new demands for the calibration of high- and ultra-high-vacuum gages. Calibrated gages must be used when experiments are performed 
that require accurate knowledge of the vacuum level. Where similar experiments are performed in several laboratories, results may differ if uncalibrated gages are used. Another application for a calibration system is that it may be used as a convenient means of investigating vacuumgage characteristics under carefully controlled conditions.

The following sections discuss the requirements of a vacuum-gagecalibration system, and the common methods of calibration are described, particularly the steady-state technique.

\section{Requirements for a Vacuum-Gage-Calibration System}

To calibrate vacuum gages, a readout of the gage under a quantitatively known vacuum condition is obtained or, preferably, a series of readouts are obtained at a variety of vacuum levels. If ionization gages are considered, the creation of a known vacuum condition is more complex than just the establishment of a known pressure. That is, ionization gages resspond differently to differing chemical species of gases. Therefore, a gage-calibration system must produce a gas atmosphere of a known composition. The predominant gas in the system must be the gas introduced by the experimenter or, in other words, the background level of residual gas must be well below the working level of the system. Under normal conditions, to permit calibration in steady-flow systems at pressures below $10^{-6}$ torr, the system must be baked to reduce the background gas level. For the attainment of the lowest pressure levels, the entire high-vacuum section including gages should be baked at a uniform temperature of 400 to $450^{\circ} \mathrm{C}$, preferably in an enclosing oven.

The most common gases used for calibration are nitrogen and air. However, since noble gases such as helium, argon, or neon may also be used, a calibration system must be able to handle any of these gases. On the other hand, some gases of interest are quite reactive in a vacuum system and cannot readily be used for calibration. Examples are water vapor, which is frequently the predominant gas in vacuum systems and strongly adsorbs on clean system surfaces, and oxygen which reacts with hot-cathode-gage filaments and adsorbs on system surfaces to the extent that it may even displace other adsorbed gases. Hydrogen and carbon monoxide, on the other hand, may be used for calibration purposes. It is recommended that a small mass spectrometer be placed on the calibration chamber to be certain of producing the desired gas composition.

In addition to establishing a gas atmosphere of known composition, the calibration system must produce a known vacuum level. The term "vacuum level" is used instead of pressure, due to the fact that ionization gages do not measure pressure directly, even though the readings are indicated in pressure units. Rather, a gage responds to the concentration of molecules within its ionizing zone. In a steady-flow system, or in any 
system where the gage enclosure is only a small part of the high-vacuum zone, the concentration of molecules in the gage is inversely proportional to the square root of the absolute temperature of the surfaces enclosing the gage sensing element. Therefore, the gage should be calibrated at normal operating conditions. If this cannot be achieved, a temperature correction should be made. In the case of the usual glass-tubulated gage, normal temperature requires operation in the normal position, with normal airflow over the gage at normal room temperature. Since gages are not necessarily linear, it is desirable to perform calibrations near the level at which the gage will be operating. The standard by which the vacuum level is established must therefore be adapted to this pressure level.

The design of the calibration system must also consider the characteristics of the auxiliary equipment used to operate the gage tube. There are two alternate approaches which may be used. The first approach holds that gage tubes may be calibrated with a particular control unit and are thereafter used only with this unit, while the second approach says that gage tubes and control units may be calibrated separately against reference standards and then used in any combination.

\section{Methods of Gage Calibration}

The simplest calibration system design is the direct-comparison type in which a standard gage is used to measure the vacuum level to which the test gages are exposed. The standard reference gage may be a previously calibrated gage, such as a McLeod gage, which has the advantage that its calibration can be derived from the accurate and stable physical measurements of length and volume. However, its operation is time consuming and requires considerable care. Effects which tend to limit the accuracy of the McLeod gage in the lower part of its operating range are that a large part of the mercury column depression is due to capillary effects which are quite variable. The mercury vapor streaming from the gage to the cold trap tends to pump the gas out of the gage and yields an erroneous low reading (the magnitude of this effect is highly dependent on room temperature and mercury cleanliness), and the measured volume of gas after compression becomes so small that there is an appreciable uncertainty in the measurement itself. However, the most serious objection to calibration using direct reference to a McLeod gage arises from the fact that the region of accurate operation occurs at pressures which are too high for accurate, linear behavior of high- or ultra-high-vacuum gages (ref. 29). As a result, it is highly desirable to be able to relate the test pressure to another higher pressure level which can be measured by a McLeod gage or some other acceptable reference standard. Calibrations are sometimes carried out using either an ionization gage or a mass spectrometer as a 
standard. These methods have proved useful in special cases which required a technique for the study of behavior under unusual conditions such as those which exist in extrahigh vacuum. As a general rule, the results of an approach of this type are doubtful, and are likely to be less accurate than absolute measurements.

There are two principle classes of measurement techniques by which a relatively high pressure may be obtained to use as an index of a lower test pressure. One class comprises expansion or compression methods, and the second class consists of steady-flow methods. Expansion techniques may be carried out in a static system where an accurately measured small volume is loaded with a test gas at a measured pressure and is expanded into a larger, evacuated volume which constitutes the test space. The volume of the test space and that of the gage to be calibrated must be known accurately. In most cases, multiple stage expansions are required to reach suitable pressures. Alternately, dynamic expansion or compression, for which the test gas is pumped out or leaked into the test space at a known rate to obtain the rate of change of pressure, may be used. The principal advantage to be gained from the use of expansion methods is that the calibration of the standard depends on physical measurements of volume, while reference pressures are measured at a level where their accurate determination is readily accomplished. Among the disadvantages is the necessary assumption that the surfaces of the system and gages are neither gas sources nor sinks. A severe sorption problem does exist, as can be seen by the fact that at pressures below about $10^{-4}$ torr, a monomolecular layer of gas adsorbed on the surface of a vacuum chamber constitutes a greater quantity of gas than that in the vacuum space. At high- or ultra-high-vacuum conditions, a 1-percent change in surface coverage may involve thousands of times more gas than the assumed amount of test gas. The condition of a baked, all-glass system used at pressures above $10^{-6}$ torr is the best answer to the requirements that gas is not desorbed or adsorbed when the pressure is changed, and is not pumped to any significant extent by the ionization gage.

Steady-flow methods avoid, to a large extent, the serious problems of gas adsorption or desorption and can be applied to lower pressure levels. The steady-flow approach requires a pumping system of known speed or an orifice of known conductance, and a calibrated inlet gas supply. The pressure existing in the calibration chamber is then defined by the relation

$$
P=Q / S
$$

where $Q$ is the inlet gas flow and $S$ is the system pumping speed. Equation (3.6) is valid only if all the temperature considerations have been examined, and pressure variations within the calibrating region do not exist. A number of variations in the application of the gas-flow calibra- 
tion procedure can be used. The degree of variation is a function of the method used to calibrate the inlet gas flow, the use of multiple orifices or cascade systems, and the decision as to the type of primary standard to be used.

Figure 3.5 illustrates one configuration of the orifice-gas-flow calibration system. Gas is introduced into the calibration chamber through the inlet gas restriction. Variations in pressure $P_{2}$ control the flow rate by means of throttle valves leading to the gas supply and the auxiliary

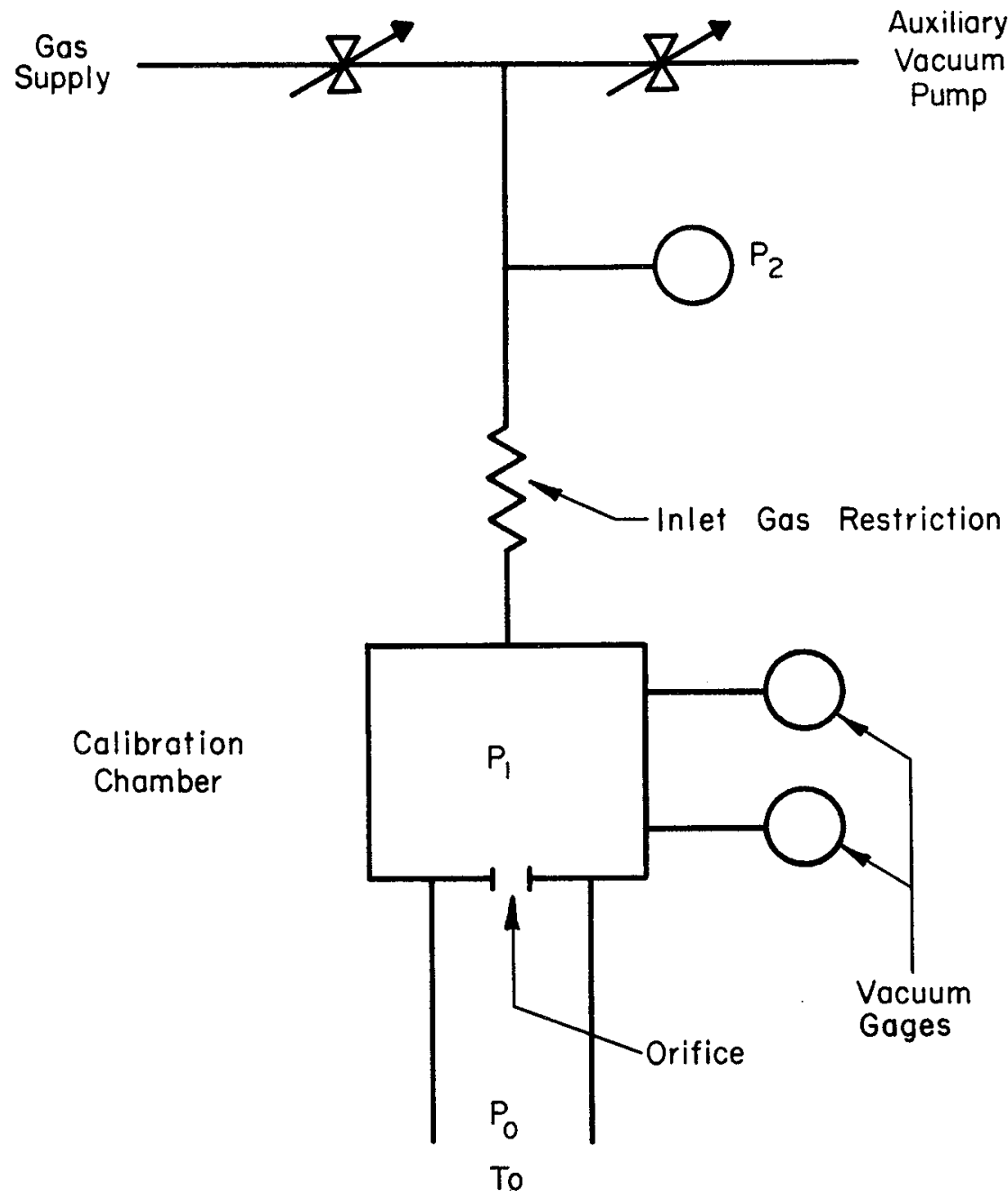

Figure 3.5.-Schematic of one form of the orifice-gas flow calibration and high-vacuum pumping system where the gas flow through the inlet restriction is $Q=K\left(P_{2}-P_{1}\right)$. 
vacuum region. The gas flow through the inlet restriction is given by the relationship

$$
Q=K\left(P_{2}-P_{1}\right)
$$

where the factor $K$ depends on the flow type prevailing, geometry of the restriction, temperature, and gas type. If the downstream pressure $P_{1}$ is very small compared to the high side pressure $P_{2}$, equation (3.7) reduces to

$$
Q=K P_{2}
$$

The gas then passes through the orifice leading to the pumping system and causes a pressure drop, which is described by the relationship

$$
Q=K^{\prime}\left(P_{1}-P_{0}\right)
$$

If the orifice is sufficiently small relative to the system pumping speed, $P_{0}$ may be ignored relative to $P_{1}$ and the equation reduces to

$$
Q=K^{\prime} P_{1}
$$

Since the same flow is going through both restrictions, it is possible to equate equations (3.9) and (3.10). Thus

$$
K P_{2}=K^{\prime} P_{1}
$$

Equation (3.11) illustrates that a definite and repeatable pressure relationship exists between $P_{2}$ and $P_{1}$. This relationship depends on the factors $K$ and $K^{\prime}$, even though they may not be true constants. Calibration of the system may be carried out in several different ways. One method permits calibration by reference to a primary gage standard such as a McLeod gage. The gage is placed on the chamber at pressure $P_{1}$ and is used to determine the relationship existing between $P_{2}$ and $P_{1}$ by a point-to-point calibration. Thus, the calibration is in effect transferred from the McLeod gage to the system. Alternately, both the inlet restriction and the limiting orifice may be calibrated individually and the pressure determined from the relationship of equation (3.11). The orifice calibration may be obtained from physical measurements applied to the known expression for gas flow through an orifice in the freemolecular-flow region. The inlet gas restriction may be calibrated by actual measurement of the quantity of gas flowing through the leak.

A variable leak may be used as a variation on this technique. The leak will draw gas from a supply at atmospheric pressure. The volume of gas consumed per unit time can be measured and the gas flow calculated from equation (3.8). Flow measurements must be made for each calibration point. A disadvantage to this technique is the difficulty of measuring the extremely small quantities of gas which a reasonably sized pump can handle at ultrahigh vacuum. Points in favor of this method are that the system is relatively simple and considerable confidence can be placed in the results. 
The basic double restriction method can be refined considerably if both the system-limiting orifice and the gas inlet restriction are in molecular flow throughout their entire operating pressure. As a result, the flow through either restriction can be expressed as

$$
Q=K P\left(\frac{T}{M}\right)^{1 / 2}
$$

where

$K$ a true constant which depends only on the dimensions of the restriction

$T$ temperature of the gas flowing in the restriction, ${ }^{\circ} \mathrm{K}$

$P$ high side pressure, torr

$M$ molecular weight of the gas

If the expressions of the gas flow through each restriction are equated, both from equation (3.12), it becomes apparent that the ratio of the pressure at the inlet manometer to the true system pressure is a constant for any pair of restrictions. In addition, if the temperature of the gas is made to be the same at both restrictions, the pressure ratio is independent of the absolute value of the temperature. Similarly, the molecular-weight term cancels out and the pressure ratio is independent of the gas type used. Therefore, it is not necessary to recalibrate the system for different gas types. In the case of a double molecular impedance calibration system, it may be calibrated by determining the ratio of the pressure at the inlet to that in the system at a single-vacuum level for any gas type, and at any common temperature. Thereafter, the pressure ratio thus determined remains a constant for other pressures, gas types, or temperatures.

There are a number of molecular-flow impedances which are in common use in calibration systems. Among these are orifices, glass frits, and porous plugs. For glass frits and orifices, molecular flow is confined to relatively low gas supply pressures which permit the use of a McLeod gage to measure the supply pressure. When an orifice impedance is employed, several stages of pressure reduction are required to reach ultrahigh vacuum. At each stage, a known fraction of the gas, perhaps 99 percent, is pumped off with an auxiliary pumping system, and the remaining 1 percent is passed on to the next stage. In this way, a chamber at $10^{-3}$ or $10^{-4}$ torr can be connected through several stages to a test chamber operating at $10^{-9}$ torr.

In a gas-flow system, a method of pumping at constant speed is required. Getter ion pumps may be used, although there are qualifications to their use. That is, they have widely variable pumping speeds for different gases, with a low speed for noble gases. In addition, they have limited life at the high pressures required during a part of the calibration. The ion pump may exhibit a memory effect resulting from 
sputtering in the pump. This memory effect may release gases used in previous calibrations and change the composition of the calibrating gas. The remaining limitation is that the discharge within the getter ion pump causes chemical reactions to occur which change the composition of some of the calibrating gas. The use of a pump, large relative to the crifice size, will minimize these limitations.

In an oil-diffusion-pumped system, special precautions are necessary to avoid generating trace amounts of hydrocarbons which would seriously interfere with the calibration. If a completely bakable system, including a liquid nitrogen trap, is used, and the proper operating techniques are followed, this objective will be accomplished and at the same time the low background pressure necessary for accurate calibration will be achieved. It is necessary to keep the pumping speed of the system constant so that the pressure does not drift during the admission of the calibrating gas. To do this, the pumping speed is controlled with a limiting orifice of a condustance that is small relative to the speed of the diffusion pump.

In addition to pumping requirements, the configuration of the section where the gages are mounted is important. For example, system restrictions other than the limiting orifices must be negligibly small relative to the orifice to avoid pressure drops in the system. Such a requirement eliminates the use of small-diameter manifolding and suggests the use of a plenum chamber system which has the advantage that all gages can be mounted in symmetrical locations. When a limiting orifice is used with a plenum chamber, time constant and system outgassing considerations must be carefully examined. That is, a pumping speed of only $1 \mathrm{liter} / \mathrm{sec}$ on a large volume would result in both high ultimate pressures and a long time constant for the establishment of equilibrium. In addition, the gas admission port design should insure that the gas is introduced with a uniform distribution, and no pressure peaks exist in the calibrating region. A large-diameter orifice requires a very large pumping system. Calculations suggest that the minimum diffusion pump size should be 6 inches in diameter.

Since organics and elastomers cannot be tolerated in an ultra-highvacuum calibration system, all system seals must be either welded, brazed, glass to metal, or metal gasketed, including those by which the gages are mounted. Glass-tubulated gages can be mounted by sealing them to glass to metal seals, which in turn are welded into flanges for attachment to the vacuum system. Many flange- or pipe-mounted gages cannot be sealed to the system without the use of O-rings, gaskets, or some type of sealing compound. Such an arrangement is incompatible with the system bakeout procedure, cleanliness requirement, and ultrahigh vacuum. If O-rings or threads must be used, the system user must be willing to accept a higher system ultimate pressure. 


\section{SPACE-SIMULATION GAGES}

In most vacuum systems of the past, the total amount of the chamber wall which was pumping was sufficiently small that a random gas flow could be assumed everywhere in the chamber, except at the throat of the pump itself. However, for modern space-simulation systems in which large quantities of cryogenic pumping arrays are used, a large amount of the vacuum-chamber wall acts as a pump. A highly directional gas flow from the test item to the chamber wall results from the pumping effect. This characteristic is particularly true of water-vapor outgassing which is being condensed on liquid-nitrogen-cooled walls. A major problem created by the existence of a highly directional gas flow is that of measuring the pressures which exist in the system.

While there is no direct relationship between the pressure measured at the test vehicle and the various levels of altitude, it is desirable to have an indication of the incident pressure or molecular flux on the vehicle. Three important problems must be solved before the vacuum level of simulation can be determined. It must be known what the ionization gage reads in terms of the gas properties in the test region, the type of gage best suited for space simulators, and the optimum location of the gages.

The first problem was solved previously. That is, a tubulated ionization gage reads in proportion to the molecular flux incident on the end of the tubulation, while a nude ionization gage reads the molecular density in the test region. Therefore, if it is desired to measure the rate at which molecules are bombarding the surface of the vehicle, some form of tubulated or restricted entrance gage should be used (ref. 30).

Concerning the problem of gage location, since it is apparent that the desired measurement is the flux on the vehicle, gages mounted on the vehicle seem to be the answer. However, there are several drawbacks to this location. First of all, there are problems of electrical connections from the amplifier to the sensing elements. These leads not only may have to be long, but they may also have to be capable of rotation consistent with the vehicle rotation. Serious problems of electrical pickup and high noise level in ion-current measurements exist due to the above factors. In many vehicles, there may not be mounting locations available for the desired gages. In addition, the presence of the gage may interfere with the thermal balance of the test item or, conversely, the test item may result in changes of the ion gage temperature with corresponding variations in calibration. For these reasons, a wallmounted gage would appear to be a more practical solution. The major problem for this configuration is how to make a wall-mounted gage read the same as if it were mounted on the test vehicle. Any wall-mounted gage or combination of gages capable of interpretation at the vehicle surface is referred to as a "space-simulation-ionization gage." 
The literature has reported two different types of space-simulationionization gages. Type $I$ is a pumping gage (ref. 65) in which the gage element is subjected to a reduced pressure of condensable gases by incorporating a condensing surface in the gage. Figure 3.6 illustrates a

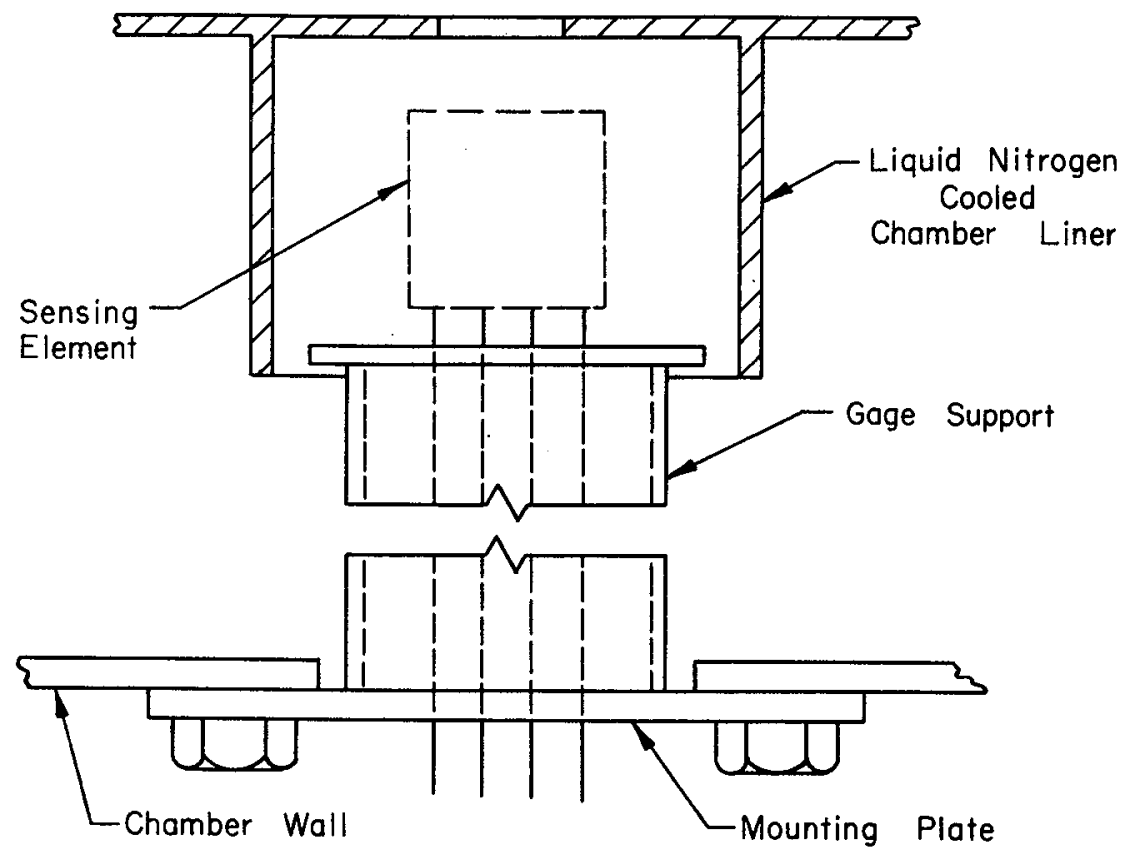

Figure 3.6.-A pumping-type space-simulation gage in which the gage-sensing element is subjected to a reduced pressure of condensable gases. The gage reads normally for permanent gases.

possible configuration. When the correction for the cold-shell temperature is made, the gage reads normally for permanent gases. A flux of condensable gas on the gage mouth is almost totally condensed by the cold surfaces and contributes a greatly reduced reading. By proper design, the reduction in reading can be made the same as the reduction in gas incident on the test vehicle due to cryogenic pumping in the test chamber.

The Type II space-simulation-ionization gage (ref. 65) represents a different approach to the same problem. The test item is subjected to a background pressure of gas molecules which come from the surrounding surfaces. Therefore, gages may be placed as necessary to measure the intensity of the molecular flux leaving these surrounding surfaces. One possible configuration has a tubulated gage, which faces 
toward the chamber wall, supported on a strut extending from the wall. The reading of the gage will be proportional to the average molecular flux density leaving those chamber surfaces within the view of the gage. A similar effect can be achieved using a baffle as shown in figure 3.7.

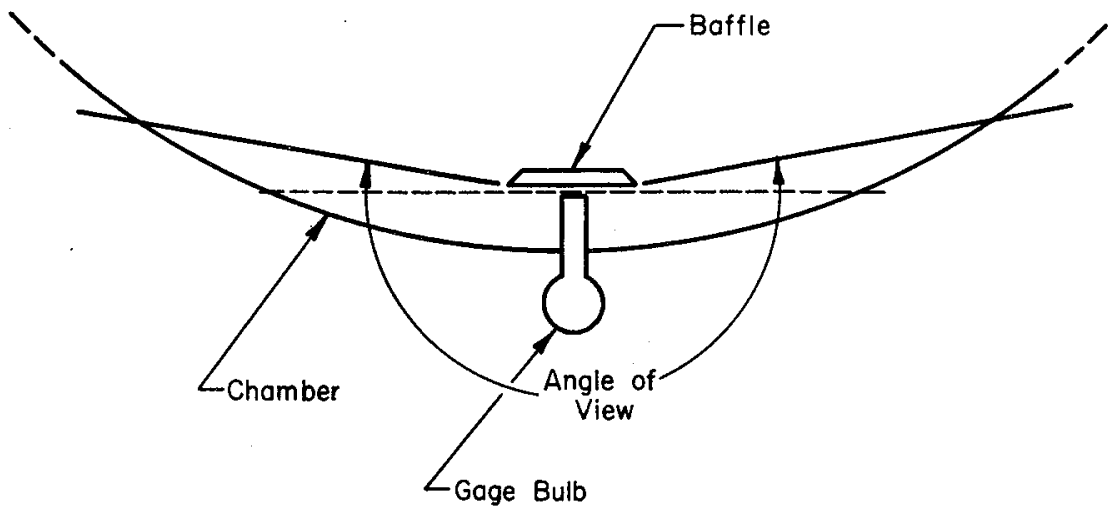

Ficure 3.7.-A Type II space-simulation gage which is so placed in the vicinity of the vacuum chamber as to measure the intensity of the molecular flux leaving the surrounding surfaces.

Several Type II space-simulation gages are required to obtain full coverage of the vacuum-chamber surfaces. The readings of these gages can be used to determine quantitatively the gas-emission pattern of the chamber. Using the probability equation for the flow of gas molecules from the chamber to a vehicle, the pattern of gas incidence on the vehicle may be calculated.

The Type I gage has the advantage of a low outgassing background level relative to the Type II gage. The principal disadvantage is that it operates correctly only when the vehicle is a strongly predominant source of outgassing. Type I and Type II gages may be partial-pressure gages (mass spectrometers) as well as total-pressure gages. In a directional-pressure environment, the mass spectrometer is subject to the same orientation effects that appear in a conventional gage. Nude gages possess low outgassing levels and exhibit a rapid approach to equilibrium in high and ultrahigh vacua. However, they do not respond to the directional properties of the gas flow and cannot distinguish between gas emitted by the vehicle and the environmental background gas incident upon the vehicle.

The type and number of gages to be used in a given case depends on the information desired. In general, it would appear desirable to use several calibrated, directional, partial-pressure gages. 
Chapters 2 and 3 have introduced the concept of pressure and the means available to measure pressure levels. It is now appropriate to examine the remaining two factors in the basic equation governing vacuum technology: gas flow and pumping speed. Chapters 4 through 7 will discuss these factors in detail for various typical geometries and pumping system configurations.

\section{REFERENCES}

1. Dushman, S.: Scientific Foundation of Vacuum Techniques. Second ed., J. M. Lafferty, ed., John Wiley \& Sons, Inc., 1962, pp. 201-352.

2. Steinhertz, H. A.: Handbook of High Vacuum Engineering. Reinhold Pub. Corp. (New York), 1963, pp. 80-109.

3. Guthrie, A.: Vacuum Technology. John Wiley \& Sons, Inc., 1963, pp. 142-197.

4. Pirani, M.; and Yarwood, J.: Principles of Vacuum Engineering. Reinhold Pub. Corp. (New York), 1961, pp. 81-129.

5. LeCk, J. H.: Pressure Measurement in Vacuum Systems. Second ed., Chapman \& Hall Ltd. (London), 1964.

6. Brombacher, W. G.; Johnson, D. P.; and Cross, J. L.: Mercury Barometers and Manometers. NBS Monograph No. 8, U.S. Dept. Com., May 20, 1960.

7. Thomas, A. M.; Johnson, D. P.; and Litte, J. W.: Design of an Interferometric Oil Manometer for Vacuum Measurement. Trans. of the Ninth National Vacuum Symposium, G. H. Bancroft, ed., The Macmillan Co., 1962, pp. 468-473.

8. CARr, P. H.: Problems in Establishing Standards for Vacuum Measurements and in Calibrating Vacuum Gauges. Vacuum, vol. 14, no. 2, Feb. 1964, pp. 37-45.

9. TAYLOR, L. R.: A Tilting Micromanometer With Continuous Sensitivity Control. J. Sci. Instr., vol. 32, no. 5, May 1955, pp. 173-177.

10. Mastach, G. J.: A Precision Differential Manometer. Rev. Sci. Instr., vol. 23, no. 7, July 1952, pp. 367-369.

11. Kramer, A. G.; and Platzman, P. M.: Microwave Manometer. Rev. Sci. Instr., vol. 29, no. 10, Oct. 1958, pp. 897-898.

12. Keмp, J. F.: Liquid Manometer With Electromagnetic Balance Indicator. J. Sci. Instr., vol. 36, no. 2, Feb. 1959, pp. 77-81.

13. Hirsch, E. H.: Precision Pressure Gauge for the Range 0 to $30 \mathrm{~mm}$ of Mercury. Laboratory and Work Shop Notes, J. Sci. Instr., vol. 36, no. 11, Nov. 1959, p. 477.

14. Gaffee, D. I.; and Monroe, A.: Measurement of Small Differential Pressures at Low Absolute Pressures. Nature, vol. 174, no. 4433, Oct. 16, 1954, p. 756.

15. Lynn, S.; Corcoran, W. H.; and Sage, B. H.: Micromanometer of High Sensitivity. Rev. Sci. Instr., vol. 27, no. 6, June 1956, pp. 368-369.

16. Drawin, H. W.: Electrical Capacity-Diaphragm Vacuum Gage. Vakuum Technik, Bd. 7, Heft 8, Dez. 1958, pp. 177-185.

17. Perls, T. A.; Kaechle, W. H.; and Goalwin, D. S.: A Diaphragm-Type Capacitance-Type Micromanometer for Very Low Differential Pressures. NBS Report 2165, U.S. Dept. Com., Jan. 1953.

18. Baxter, I. G.: A Differential Capacitance Manometer. J. Sci. Instr., vol. 30, no. 10, Oct. 1953 , pp. 358-360.

19. ANon.: Diaphragm Micromanometer With Electrical Pick-up. Instruments, vol. 23 , Nov. 1950, p. 1136. 
20. Cook, D. B.; ANd Danby, C. J.: A Simple Diaphragm Micromanometer. J. Sci. Instr., vol. 30, no. 7, July 1953, pp. 238-240.

21. Pressey, D. C.: Temperature-Stable, Capacitance Pressure Gauges. J. Sci. Instr., vol. 30, no. 1, Jan. 1953, pp. 20-24.

22. Becker, E. W.; ANd Stehl, O.: An Electrostatic Differential Manometer of a Sensitivity of $2 \times 10^{-3} \mathrm{~mm} \mathrm{Hg}$ per Scale Division. Z. Angew. Phys., Bd. 4, Heft 1, Jan. 1952, pp. 20-22.

23. De Crescente, M.; And Janz, G. J.: Brass Bellows Gauge for Null-Reading Manometers. Rev. Sci. Instr., vol. 28, no.6, June 1957, p. 468.

24. Jansen, C. G. J.; And Venema, A.: A McLeod Manometer With Prescribed Volumes for Use as a Standard Instrument. Vacuum, vol. 9, no. 3/4, July/Sept. 1959, pp. 219-229.

25. Grifriths, L. J.: A Modified McLeod Gauge for Low Pressure Measurements. Advances in Vacuum Science and Technology, vol. I. E. Thomas, ed., Pergamon Press, 1959, pp. 263-265; Discussion, p. 265.

26. Lotr, P.: Simplified Method for McLeod Gage Design and Calibration. Anal. Chem., vol. 28, no. 2, Feb. 1956, pp. 276-277.

27. Wri,IIE, H. A.: A McLeod Gauge for Measuring a Wide Range of Pressures. J. Sci. Instr., Lab. and Workshop Notes, vol. 33, no. 8, Aug. 1956, p. 317.

28. Goто, M.: On the Standards of Vacuum Installed in the Electrotechnical Laboratory. The Standard McLeod Gauge and the Calibration of Ionization Gauges Against It. Advances in Vacuum Science and Technology, vol. I. E. Thomas, ed., Pergamon Press, 1959, pp. 266-270; Discussion, p. 270.

29. Nottingham, W. B.; and Torney, F. L.: A Detailed Examination of the Principles of Ion Gauge Calibration. Trans. of the Seventh National Sympasium on Vacuum Technology, C. R. Meissner, ed., Pergamon Press, 1961, pp. $117-122$.

30. Rosengera, P.: Design of an Accurate McLeod Gauge. Rev. Sci. Instr., vol. 10, 1939, pp. 131-134.

31. McLind, H.: Apparatus for Measurement of Low Pressures of a Gas. Phil. Mag., vol. 48, 1874, pp. 110-112.

32. Pobquaski, H. H.; and Davis, F. N.: A Precision McLeod Gauge for Volumetric Gas Measurement. Vacuum, vol. 10, 1960, pp. 377-381.

33. Mein Ke, C.; ANd Reich, G.: Vermeidung von Fehlmessungen mit dem System McLeod-Kuhlfalle. Vak. Technik, Bd. 12, Heft 3, 1963, pp. 79-82.

34. IsHir, H.; aNd Nakayama, K.: A Serious Error Caused by Mercury Vapor Stream in the Measurement With a McLeod Gauge in the Cold Trap System (Effect of Diffusion of Nitrogen in the Mercury Vapor Stream). Trans. of the Eighth National Vacuum Symposium and the Second International Congress on Vacuum Science and Technology, vol. I. L. E. Preuss, ed., Pergamon Press, 1962, pp. 519-524.

35. VACCA, R. H.: Recent Advances in the "Alphatron" Vacuum Gauge. Trans. of the National Symposium on Vacuum Technology, E. S. Perry and J. H. Durant, eds., Pergamon Press, 1957, pp. 93-100.

36. Roenrig, J. R.; and Vanderschmidr, G. F.: Advances in the Design of Vacuum Gauges Using Radioactive Materials. Trans. of the Sixth National Symposium on Vacuum Technology, C. R. Meissner, ed., Pergamon Press, 1960, pp. 82-84.

37. Penning, F. M.: High Vacuum Gauges. Philips Tech. Rev., vol. 2, 1937, pp. 201-208. 
38. Pennina, F. M.; And Nienhuis, K: Construction and Application of a New Design of the Philips Vacuum Gauge. Philips Tech. Rev., vol. 11, 1949, pp. 116-122.

39. Redhead, P. A.: The Magnetron Gauge: A Cold-Cathode Vacuum Gauge. Can. J. Phys., vol. 37, no. 11, Nov. 1959, pp. 1260-1271.

40. Rejhead, P. A.: Pressure Measurements at Ultra High Vacuum. Trans. of the Fifth National Symposium on Vacuum Technology, W. G. Matheson, ed., Pergamon Press, 1959, pp. 410-413.

41. Hobson, J. P.; ANd Redhead, P. A.: Operation of an Inverted-Magnetron Gauge in the Pressure Range $10^{-3}$ to $10^{-12} \mathrm{~mm} \mathrm{Hg}$. Can. J. Phys., vol. 36, no. 3, Mar. 1958, pp. 271-288.

42. Kreisman, W. S.: Attainment and Measurement of Pressures Ranging From $10^{-6}-10^{-12} \mathrm{~mm} \mathrm{Hg}$. Bull. Amer. Phys. Soc., ser. II, vol. 8, no, 2, 1963, p. 143.

43. Young, J. R.; And Hession, F. P.: A Cold Cathode Discharge Gauge for UltraHigh Vacuum Use. Trans. of the Tenth National Vacuum Symposium, G. H. Bancroft, ed., The Macmillan Co., 1963, pp. 234-237.

44. Rhodin, T. N.; and Rovner, L. H.: Gas-Metal Reactions in Oxygen at Low Pressures. Trans. of the Seventh National Symposium on Vacuum Technology, C. R. Meissner, ed., Pergamon Press, 1961, pp. 228-237.

45. LECK, J. H.: Sorption and Desorption of Gas in the Cold-Cathode Ionization Gauge. J. Sci. Instr., vol. 30, no. 8, Aug. 1953, pp. 271-274.

46. Notringham, W. B.: Design and Properties of the Modified Bayard-Alpert Gauge. Trans. of the Vacuum Symposium, Committee on Vacuum Techniques, Inc. (Boston), 1955, pp. 76-80.

47. Carter, G.; ANd Leck, J. H.: Bistable Behaviour of the Bayard-Alpert Izonization Gauge. Brit. J. Appl. Phys., vol. 10, Aug. 1959, pp. 364-367.

48. Redhead, P. A.: Modulated Bayard-Alpert Gauge. Rev. Sci. Instr., vol. 31, no. 3, Mar. 1960, pp. 343-344.

49. Appelt, G.: The Measurement of Very Low Pressures with the Bayard-Alpert Modulator Gauge. Vak. Technik, Bd. 11, Heft 6, Sept. 1962, pp. 174-177.

50. Schummann, W. C.: A Photo-Current Suppressor Gauge for the Measurement of Very Low Pressures. Trans. of the Ninth National Vacuum Symposium, G. H. Bancroft, ed., The Macmillan Co., 1962, pp. 428-430.

51. Metson, G. H.: The Physical Basis of the Residual Vacuum Characteristic of a Thermionic Valve. Brit. J. Appl. Phys., vol. 2, Feb. 1951, pp. 46-48.

52. KLopfer, A.: An Ionization Gauge for Measurement of Ultra-High Vacua. Trans. of the Eighth National Vacuum Symposium and the Second International Congress on Vacuum Science and Technology, vol. I. L. E. Preuss, ed., Pergamon Press, 1962, pp. 439-442.

53. LAfferty, J. M.: Hot Cathode Magnetron Ionization Gauge for the Measurement of Ultrahigh Vacua. J. Appl. Phys., vol. 32, no. 3, Mar. 1961, pp. 424-434.

54. Conn, G. K. T.; and Daglish, H. N.: A Thermionic Ionization Gauge of High Sensitivity Employing a Magnetic Field. J. Sci. Instr., vol. 31, no. 11, Nov. 1954, pp. 412-416.

55. Houston, J. M.: New Ultra-High-Vacuum Ionization Gauge. Bull. Amer. Phys. Soc., ser. II, vol. 1, no. 6, June 21, 1956, p. 301.

56. Nottingham, W. B.: Design Parameters' Influence on M.I.T. Bayard-Alpert Gauge Sensitivity. Trans. of the Eighth National Vacuum Symposium and the Second International Congress on Vacuum Science and Technology, vol. I. L. E. Preuss, ed., Pergamon Press, 1962, pp. 494-498.

57. Santeler, D. J.; Pressure Measurement in Non-Baked Ultra-High Vacuum Systems. Trans. of the Eighth National Vacuum Symposium and the Second 
International Congress on Vacuum Science and Technology, L. E. Preuss, ed., Pergamon Press, 1962, pp. 549-554.

58. REICF, G.: The Influence of Adsorption and Desorption on Measurements of Low Pressures. Trans. of the Seventh National Symposium on Vacuum Technology, C. R. Meissner, ed., Pergamon Press, 1961, pp. 112-116.

59. Redhatad, P. A.: Errors in the Measurement of Pressure With Ionization Gauges. Trans. of the Seventh National Symposium on Vacuum Technology, C. R. Meissner, ed., Pergamon Press, 1961, pp. 108-111.

60. Roberts, R. W.; ANd Vanderslice, T. A.: Ultra High Vacuum and Its Applications. Prentice-Hall, Inc., 1963.

61. Blears, J.: Measurement of the Ultimate Pressures of Oil Diffusion Pumps. Proc. Roy. Soc. (London), ser. A, vol. 188, no. 1012, Dec. 31, 1946, pp. 62-76.

62. Haefer, R. A.; AND Hengevoss, J.: Studies on the Blears Effect at Pressure Measurements in the Ultra-High Vacuum Range. Trans. of the Seventh National Symposium on Vacuum Technology, C. R. Meissner, ed., Pergamon Press, 1961, pp. 67-74.

63. Ackley, J. W.; Lorhrop, C. F.; AND Wheeler, W. R.: Anomalous Behaviour of Ionization Gauges Operated at Low Grid Currents. Trans. of the Ninth National Vacuum Symposium, G. H. Bancroft, ed., The Macmillan Co., 1962, pp. 452-455.

64. Hayward, W. H.; JepSen, R. L.; ANd Redhead, P. A.: Reverse X-Ray Currents in Bayard-Alpert Ionization Gauges. Trans. of the Tenth National Vacuum Symposium, G. H. Bancroft, ed., The Macmillan Co., 1963, pp. 228-233.

65. Holkeboer, D. H.: Space Simulation Ion Gauges. (Prepared under Contract AF40(600)-954), AEDC-TDR-63-148, July 1963.

66. HolKeborr, D. H.: Vacuum Measurement Techniques for Space Simulation Chambers. Trans. of the Tenth National Vacuum Symposium, G. H. Bancroft, ed., The Macmillan Co., 1963, pp. 292-296. 



\section{Pumping Speed}

\section{INTRODUCTION}

$\pi^{N Y}$ VACUUM PUMPING SYSTEM functions primarily to exA haust a volume at a required pressure, and to maintain the volume at the operating pressure while pumping a gas load. The basic equation of vacuum technology relates pressure and gas load to the pumping speed of a system by

$$
Q=P S
$$

where $Q$ is the gas load or throughput, $P$ is the system pressure in torr, and $S$ is the pumping speed in liter/sec. Chapters 2 and 3 have defined pressure and discussed means of measuring pressure and its associated phenomena. Pumping speed logically becomes the next item of the triumvirate to investigate in detail.

Generally, equation (4.1) is true for both the steady-state and transient-flow conditions, and in the molecular-flow and continuum-flow regimes. There are, however, qualifications which tend to complicate the equation. In the transient case, $Q$, the gas load arising from leaks, material outgassing, and pumpdown rate, is a complex function of pressure, temperature, and time. (For further discussion, see chapter 8.) In addition, the pumping speed $S$ is a function of gas specie as well as pressure and temperature. The following sections discuss the effects of pumping speed on a vacuum chamber; chapters 5 and 6 deal with the determination of pumping speed.

The factors determining and existing in the steady-state and transient conditions will be investigated next. (For an auxiliary discussion of the concept of pumping speed, see also ref. 1.)

\section{STEADY-STATE CASE}

Gas flow will occur in the molecular regime for high-vacuum systems. The gas molecules will impinge on the wall of the vacuum chamber at a rate proportional to the molecular density and the average molecular speed. The expression for this rate, to be derived in chapter 5 , in units of liter $/ \mathrm{cm}^{2} \mathrm{sec}$, is

$$
f=3.638 \sqrt{T / M}
$$

where $T$ is the gas temperature in ${ }^{\circ} \mathrm{K}$ and $M$ is the gram molecular weight of the gas. A separate computation of $f$ for each gas specie will 
be necessary if a mixture of gases is present. To illustrate the magnitude of $f$, its value for nitrogen at $25^{\circ} \mathrm{C}$ is $11.86 \mathrm{liter} / \mathrm{cm}^{2}$ sec. If a section of the chamber wall were removed to form an orifice communicating with a perfect vacuum, the pumping speed of the orifice would be

$$
S=A f
$$

where $A$ is the orifice area. The idealized condition characterized by equation (4.3) may be termed a perfect pump. Realistically, however, only the fraction $a$ of the impinging molecules in a pumping system will be pumped

$$
S=a A f
$$

The fraction $(1-\alpha)$ will be returned to the chamber. When the pumping probability factor $a$ is applied to the entrance of a pump, it is frequently called the Ho coefficient after the man who introduced the concept (ref. 2). The value of $a$ will vary with the type of pump. That is, for diffusion pumps, based on the gross inlet area, $a$ can range from about 0.20 for a 2 -inch mercury pump to over 0.50 for a 35 -inch pump. Typical values of $a$ in a sputter ion pump range from 0.08 for a 4 -inch size to 0.20 for the 24 -inch pump. If a cryogenic condensing surface is considered, a value of $a=1$ can be approached. Since a pump normally will be separated from a chamber by a trap, baffle, or a length of connecting tube, the speed seen by the chamber is less than the speed measured at the pump entrance. Chapter 5 will consider various methods of calculating net pumping speeds.

Equation (4.4) indicates that the maximum pumping speed of any component in molecular flow is the product Af. Occasionally, however, the literature quotes higher pumping speeds, particularly at the high values of $a$ encountered on cryogenic pumping surfaces (ref. 3). The methods of obtaining the pressure measurements in these cases account for the apparent anomaly in pumping speeds.

It is now appropriate to return to figure 4.1 and examine the significance of the four pressure-gage locations. Note that the chamber is large relative to the pumping port. All the pressure gages would read the same pressure for the conditions of zero pumping speed. If the port were a perfect pump, $a=1$, gages $P_{1}, P_{2}$, and $P_{3}$ would read the chamber pressure, while gage $P_{4}$ would not. In the case of zero pumping speed, equal proportions of molecules impinging on the mouth of $P_{4}$ come from the directions of the chamber and the pump. For the second case, the perfect pump, the molecular flux at the gage mouth is reduced to one-half the original value. As a result, the indicated pressure on $P_{4}$ is also reduced to one-half the value of the nonpumping condition, since gas molecules would not arrive from the direction of the pump. The reading on $P_{4}$ for the general case is 


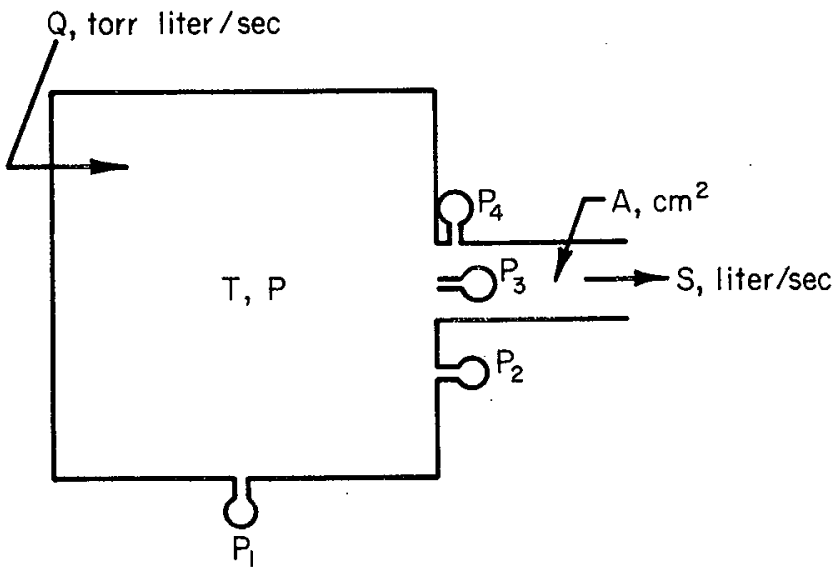

Figure 4.1.-Pressure measurement positions for gas flow in the molecular regime in a high-vacuum system.

$$
P_{4}=\frac{1}{2} P+\frac{1}{2} P(1-\alpha)=P\left(1-\frac{a}{2}\right)
$$

Of course, it is assumed that random molecular flow from the pump and the chamber exists.

A measurement of the speed of a pump is calculated by allowing a metered $Q$ to flow into a chamber and then measuring the resulting $P$. If the true chamber pressure is measured, $S$ becomes

$$
S=\frac{Q}{P}=a A f
$$

whereas the resultant speed for the pressure $P_{4}$ is

$$
S^{\prime}=\frac{Q}{P_{4}}=a^{\prime} A f
$$

The ratio of $S^{\prime}$ and $S$, substituting $P_{4}$ from equation (4.5), is

$$
\begin{aligned}
\frac{S^{\prime}}{S} & =\frac{a^{\prime}}{a}=\frac{P}{P_{4}}=1+\frac{a^{\prime}}{2} \\
& =\frac{1}{1-\frac{a}{2}}
\end{aligned}
$$

The apparent pumping speed approaches twice the actual value when the pressure is measured in the $P_{4}$ position normal to the flow axis and when the pumping probability factor a approaches unity. At lower values of $a$, the effect on pumping speed is less pronounced but may still be significant. The standard method (ref. 4) of determining the published speed of a commercial diffusion pump is illustrated in figure 4.2 . 


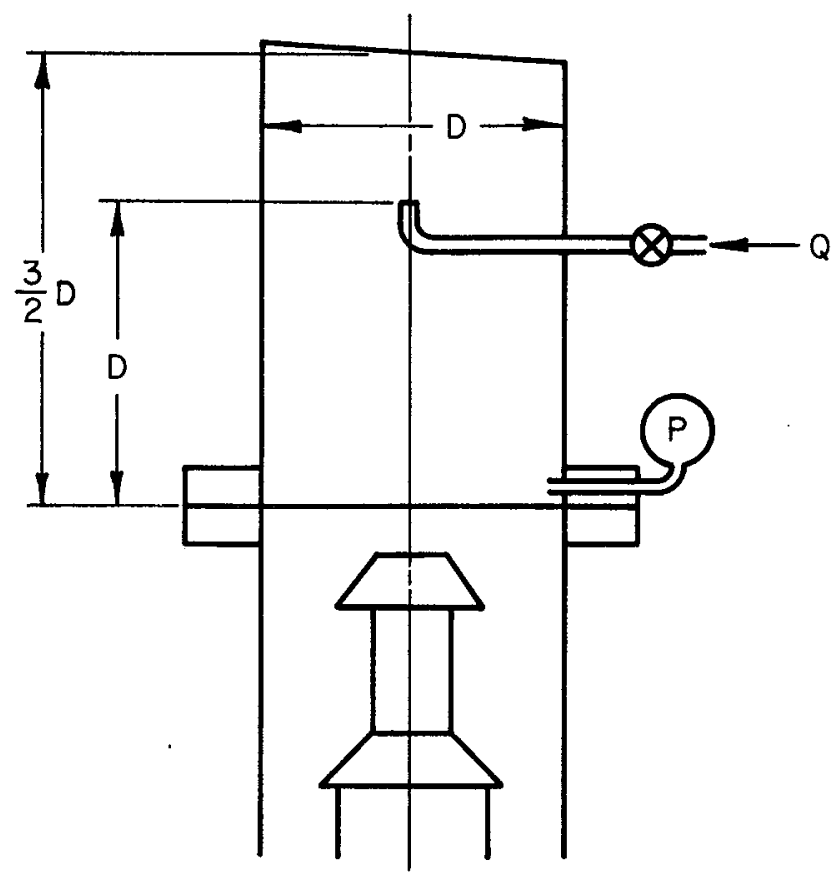

Figure 4.2.-Typical test dome used to determine the published speed of a commercial diffusion pump.

The pressure is measured in a manner similar to that for $P_{\&}$ in figure 4.1. Consequently, the published speed can be corrected to an approximation of the speed defined by equation (4.4), using equation (4.6). Since the gas flow is not random from either the test dome or the pump in figure 4.2, the use of equation (4.6) in this case is not exact. The effect of the nonrandom gas streaming from the test dome on the pressure reading is treated analytically by reference 4 , which shows that a tubulated gage tested in a dome similar to the one in figure 4.2 , and in the position $P_{4}$, can indicate a pumping speed equal to more than twice the $A f$ product obtained when considering cryogenic surfaces. If a standard diffusion pump test dome is used, a comparison of the corrected pumping speed from equation (4.6) and the pumping speed calculated from reference 4 shows that equation (4.6) yields a value which is 5.5 percent higher at $a=0.4$, and 7 percent higher at $a=0.5$. Reference 4 does not consider the molecular reflection pattern from the pump, a factor which could have an important effect on the indicated pumping speed for instances where $a$ is not close to unity. The reflection pattern is a function of the diffusion pump geometry and varies from one manufacturer's model to another. 
The discussion to this point has tried to make the engineer aware of the fact that interpretation of the pumping speed may be complicated by directional effects on pressure gaging. The problem attains its ultimate expression in space-simulation chambers where the walls, or the major portion thereof, may be covered with high-speed cryogenic pumping surfaces. Figure 4.3 illustrates such a chamber. Assume that the

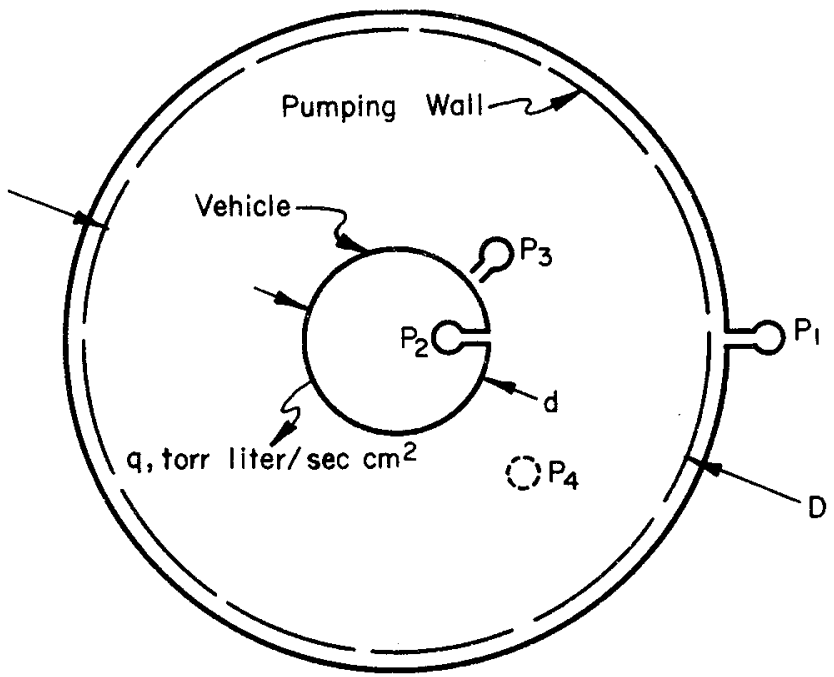

FigURE 4.3.-Pressure measurement positions in a space-simulation chamber where the walls are covered with high-speed cryogenic pumping surfaces, gages $P_{1}, P_{2}$, and $P_{3}$ are tubulated ionization gages, and $P_{1}$ is a nude gage.

chamber is spherically symmetrical with tubulated ionization gages at stations $P_{1}, P_{2}$ and $P_{3}$, and a nude ionization gage at $P_{4}$. If a perfect pumping action were taking place, i.e., if every gas molecule, coming from the vehicle, which reached the walls were condensed, $P_{3}$, the radially placed gage which is sensitive to the molecular flux at the gage mouth, would indicate a pressure which decreased by the square of the radius as it moved from the vehicle surface to the wall position, $P_{1}$. The nude gage, $P_{4}$, sensitive to molecular density, would act in a similar mianner as the radial position changed. Since no molecules return from the chamber, a tubulated gage facing away from the vehicle, $P_{2}$, would read zero at any radial position. Therefore, a pumping speed calculated from $P_{3}$ and $P_{4}$ would increase with the radial position of the gage, while the pumping speed based on $P_{2}$ would be infinite. Since a fraction $Z$ of the outgassing molecules, under actual conditions, return to the vehicle 
before being finally pumped, the pressures indicated at all gages would be higher. However, until $Z$ becomes rather high, the same general results would exist.

The question of how to define a useful pumping speed now arises. It is helpful to remember that the molecular conditions of space are being simulated in an actual chamber. As a result, the flux rate of molecules returning to the vehicle becomes the important parameter. Gage $P_{2}$ in figure 4.3 senses the flux rate. The gage reading provides the basis for the calculation of a useful pumping speed. The following discussion will clarify this statement.

A tubulated ionization gage which has been calibrated in a standard chamber will receive a flux density at its mouth, in units of molecule/ $\mathrm{cm}^{2} \mathrm{sec}$, of

$$
\Phi=\frac{\bar{v} n}{4}=10^{3} \mathrm{fn}
$$

where $\bar{v}$ is the average molecular velocity, $f$ is defined in equation (4.2), and $n$ is the molecular density at the gage mouth in molecule $/ \mathrm{cm}^{3}$. The quantity $f$ is calculated at a standard temperature, usually $25^{\circ} \mathrm{C}$. The basic relationship of kinetic theory relates the flux density to the indicated gage pressure

$$
P=n k T
$$

When this equation is combined with equation (4.7), the resulting $P$ is

$$
P=10^{-3} \Phi \frac{k T}{f}
$$

where $k$ is Boltzmann's constant in torr $\mathrm{cm}^{3} /$ molecule ${ }^{\circ} \mathrm{K}$ and $T$ is the standard temperature. Therefore, a tubulated gage facing the vehicle and also located at its surface will indicate a pressure

$$
P_{3}=\frac{10^{-3} \Phi_{1} k T}{f}
$$

$\Phi_{1}$ for equation (4.9) is the outgassing flux from the vehicle. The $P_{2}$ gage, which looks out from the vehicle skin, will see the flux $Z \Phi_{1}$, so that $P_{2}=Z P_{3}$, where $Z=$ (molecules returning to vehicle wall) $/($ molecules initiating at vehicle wall).

The conventional way of expressing the units of the outgassing rate $q$ at room temperature, $25^{\circ} \mathrm{C}$ or $298^{\circ} \mathrm{K}$, is torr liter $/ \mathrm{cm}^{2}$ sec. The flux $\Phi$, then becomes

$$
\begin{aligned}
\Phi_{1}= & q\left(\frac{\text { torr liter }}{\mathrm{cm}^{2} \mathrm{sec}}\right) \times \frac{1}{760}\left(\frac{\text { atm }}{\text { torr }}\right) \times \frac{1}{22.4}\left(\frac{\text { gram mole }}{\text { std atm liter }}\right) \\
& \times \frac{273}{298}\left(\frac{{ }^{\circ} \mathrm{K}}{{ }^{\circ} \mathrm{K}}\right) \times 6.02 \times 10^{23}\left(\frac{\text { molecule }}{\text { gram mole }}\right) \\
= & \left(\frac{q}{760}\right)\left(\frac{1}{22.4}\right)\left(6.02 \times 10^{23}\right)\left(\frac{273}{298}\right) \text { molecule } / \mathrm{cm}^{2} \mathrm{sec}
\end{aligned}
$$


The apparent pumping speed, defined as $S^{\prime}=Q / P_{2}$ liter/sec, which relates the pressure felt by the vehicle to the outgassing flux from the vehicle becomes

$$
S^{\prime}=\frac{A_{1} q}{P_{2}}=\frac{A_{1} q f}{10^{-3} \Phi_{1} Z k T}
$$

where $A_{1}$ is the vehicle surface area. The basic gas law yields the product $k T$ which is evaluated at $25^{\circ} \mathrm{C}$ and a pressure of 1 atmosphere

$$
\begin{aligned}
k T= & \frac{P}{n} \\
= & 760(\text { torr }) \times \frac{1}{6.02 \times 10^{23}}\left(\frac{\text { gram mole }}{\text { molecule }}\right) \times 22.4\left(\frac{\text { std liter }}{\text { gram mole }}\right) \\
& \times \frac{298}{273}\left(\frac{{ }^{\circ} \mathrm{K}}{{ }^{\circ} \mathrm{K}}\right) \times 10^{3}\left(\frac{\mathrm{cm}^{3}}{\text { liter }}\right)
\end{aligned}
$$

Substituting equations (4.10) and (4.12) into equation (4.11) yields

$$
S^{\prime}=\frac{A_{1} f}{Z}
$$

Chapter 7 derives the fraction $Z$ for spherical symmetry

$$
Z=\frac{A_{1}}{A_{2}} \frac{\left(1-a^{\prime}\right)}{a^{\prime}}
$$

where $A_{2}$ is the chamber wall area, and $\alpha^{\prime}$ is the fraction of gas molecules pumped by the cryowalls on one encounter. A further substitution of equation (4.14) into equation (4.13) yields the final result of

$$
S^{\prime}=\frac{a^{\prime}}{\left(1-a^{\prime}\right)}\left(A_{2} f\right)
$$

The quantity $a^{\prime}$ is evaluated for several common shielded cryogenic array geometries in chapter 7 . When considering unshielded pumping surfaces, such as liquid-nitrogen-cooled surfaces pumping water vapor, $a^{\prime}$ can approach unity and $S^{\prime}$ will approach infinity.

\section{TRANSIENT CASE, PUMPDOWN, AND REPRESSURIZATION}

The following discussion will take up the second case of pumping speed under transient conditions where $Q$, the gas load, is a complex function of pressure, temperature, and time.

The differential equation describing the isothermal pumpdown of a chamber by an exhaust system is

$$
-V \frac{d P}{d t}=S P-Q_{L}
$$

where $V$ is the chamber volume in liters, $P$ is the pressure, $t$ is time, $S$ is the pumping speed of the exhaust system, and $Q_{L}$ is the gas influx, which includes leaks and outgassing, in torr liter/sec. Assuming that 
$S$ and $Q_{L}$ are constants, the integration of equation (4.16) yields

$$
t=\frac{V}{S} \ln \frac{\left(P_{1}-Q_{L} / S\right)}{\left(P_{2}-Q_{L} / S\right)}
$$

where $t$ is the time required to reduce the pressure from $P_{1}$ to $P_{2}$. Since the term $Q_{L} / S$ is equal to the steady-state pressure of the system, and as long as $P_{2}$ is a decade or more greater than the steady-state pressure, the following simplification can be made:

$$
\begin{aligned}
t & =\frac{V}{S} \ln \frac{P_{1}}{P_{2}} \\
& =2.303 \frac{V}{S} \log _{10}\left(\frac{P_{1}}{P_{2}}\right)
\end{aligned}
$$

During a system pumpdown, quasi-steady-state pressures may be encountered. A possible occurrence when a water-contaminated system is pumped down to the vapor pressure of water is the pressure which is generated, 23.7 torr at $25^{\circ} \mathrm{C}$. This pressure will be maintained until the free water is eliminated. In addition, the pumping speed of a system varies for different gas species so that the pumping speeds for hydrogen and nitrogen might vary by a ratio of $3: 1$ in diffusion pumps. Again, the pumping speed for water vapor in a liquid nitrogen-trapped and diffusion-pumped system will be greatly augmented due to vapor condensation on the trap surfaces. Due to variations in pump speed and system conductance as a result of pressure variations, the pumping speed $S$ is not a constant, even with a given gas specie. The pump performance criteria can be obtained from the manufacturer's data. Chapter 5 of this text will discuss conductances. The calculation procedure which is generally accepted breaks down the exhaust process into various pressure ranges. An average value of $S$ is next assigned to each pressure range and equation (4.18) is then applied to each range. The use of a plot of system speed as a function of pressure will aid the calculation. Reference 5 describes a graphical computation process which also proves extremely useful in calculations. During any examination of transient processes, the term "time constant" is frequently encountered in at least three forms. One form is $\tau$, the time required to reduce the pressure to $1 / \epsilon$, or 38.2 percent of its original value; another form is $\tau_{1 / 2}$, the half-life or the time to reach one-half of the original value of pressure; and the third form is $\tau_{1 / 10}$, the time required to reduce the pressure by a decade. These three time constants can be evaluated from equation (4.18).

$$
\begin{aligned}
\tau & =\frac{V}{S} \\
\tau_{1 / 2} & =0.693 \frac{V}{S} \\
\tau_{1 / 10} & =2.303 \frac{V}{S}
\end{aligned}
$$


In a discussion of time constants, first consider a volume $V$, which is being exhausted by a pump through a series of restrictions; i.e., pipes, valves, or similar plumbing. Some authors hold that the time constant of this type of system is the sum of the time constants of the pump and each of the several restrictions,

$$
\begin{aligned}
\tau & =\tau_{P}+\tau_{1}+\tau_{2}+\ldots+\tau_{n} \\
& =\frac{V}{S_{P}}+\frac{V}{F_{1}}+\frac{V}{F_{2}}+\ldots+\frac{V}{F_{n}}
\end{aligned}
$$

where $S_{P}$ is the speed of the pump, and $F_{n}$ represents the conductances of the several series restrictions. These incremental time constants are for arithmetical convenience only, and result from the fact that the net pumping speed of the above system is

$$
\frac{1}{S}=\frac{1}{S_{P}}+\frac{1}{F_{1}}+\frac{1}{F_{2}}+\ldots+\frac{1}{F_{n}}
$$

The simple summation of equation (4.21) is valid only under certain restrictions which will be discussed in chapter 5 .

Figure 4.4 illustrates the typical pumpdown curves for two diffusion

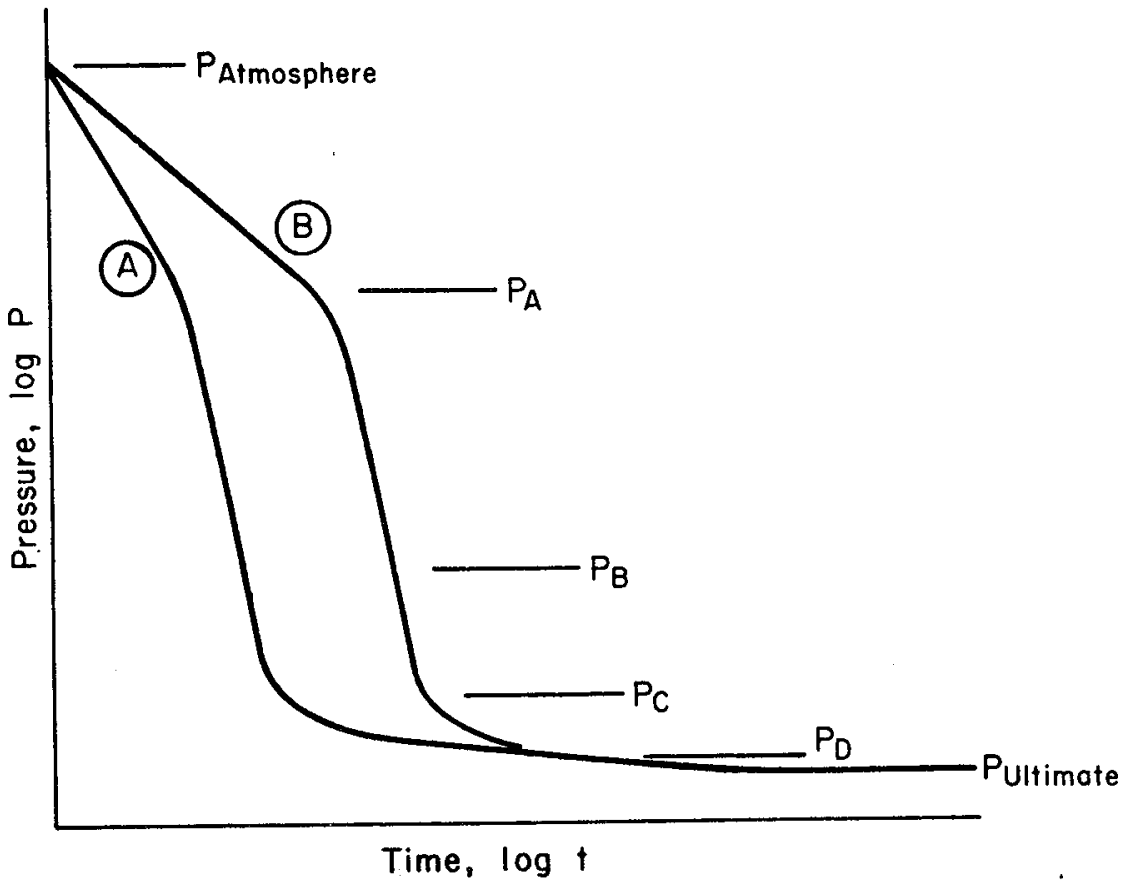

FigURE 4.4.-Typical pumpdown curves for two diffusion pump systems, $A$ and B, where system $A$ has a larger mechanical pump than system $B$. 
pump systems. The systems differ only in that system $B$ has a mechanical pump of the minimum size necessary to back the diffusion pump, while system $\mathrm{A}$ has a larger mechanical pump. $P_{A}$ is the pressure at which the diffusion pump begins to operate, and $P_{C}$ is the pressure at which a simple outgassing process begins to dominate the exhaust process. Equation (4.18) can be used to compute the exhaust time if the required system pressure is greater than $P_{c}$. If lower pressures are desired, the extent of an additional exhaust time depends on the cleanup rate of the outgassing source. Since the cleanup rate is a complex function of material type and temperature history, it will be treated in detail in chapter 9.

While the initial system exhaust rate is nearly proportional to the size of the mechanical pump which is employed, a small mechanical jump can be almost as effective as a much larger pump if a very low system pressure is required. Figure 4.4 will help to clarify this statement. When the required pressure is $P_{B}$ or greater, the use of a larger mechanical pump can decrease the cycle time greatly. However, if a pressure $P_{D}$ or less is required, the minimum-sized mechanical pump will achieve substantially the same result at a lower cost due to the fact that the desired pressure is controlled by system outgassing and diffusion pump speed. Reference 6 shows in detail how useful the analysis of experimentally determined exhaust curves, such as those in figure 4.4, can be in the study of such phenomena as the outgassing characteristics of materials and gages, the gettering action of various materials, and the determination of leak flows.

The assumption of isothermal conditions for the derivation of equation (4.17) presupposes a system of average speed. In the case of a large system with a very high exhaust rate, however, the heat transfer from the chamber walls to the gas being exhausted will not be sufficient to maintain the gas temperature, and adiabatic conditions will be approached. Such conditions are likely to exist in a space-vehicle test chamber being exhausted at a rate approximating that obtained during a rocket launching. During the initial exhaust period, when continuum flow holds, the temperature of the chamber gas tends to follow an isentropic expansion

$$
\frac{T_{2}}{T_{1}}=\left(\frac{P_{2}}{P_{1}}\right)^{\frac{\gamma-1}{\gamma}}
$$

where $\gamma$ is the ratio of specific heats of the gas, and $T$ and $P$ are the absolute temperature and absolute pressure of the gas. The resultant temperature drop increases the mass flow rate over the isothermal case. If a programed exhaust schedule is desired, the temperature drop must be taken into account.

System repressurization, the inverse of the exhaust process, generally 
is slow enough to be considered an isothermal process. In some instances, however, the process is so fast that it approaches adiabatic conditions. An example is the emergency dump of a man-rated space chamber. One possible result of this action is a substantial temperature rise of the incoming gas. This temperature rise is caused by the flow work being done on the gas by the pressure of the atmosphere or of the storage tank, if one is used. The temperature rise can be readily calculated for either type of system. For the case of the tank-storage repressurization system with the assumption of an ideal gas and adiabatic conditions, the energy equation is

$$
w_{1} T_{1} c_{v}=w T c_{v}+\left(w_{1}-w\right) T^{\prime \prime} c_{v}
$$

where $c_{v}$ is the specific heat at a constant volume of the gas, $w$ is the mass of gas in the reservoir, $T$ is the absolute temperature of the gas in the reservoir, and $T^{\prime}$ is the absolute temperature of the gas in the chamber being repressurized. Subscript 1 indicates the initial storage tank conditions. An additional assumption made for equation (4.23) is that the gas entering the chamber mixes thoroughly with the gas already in the chamber. The equation is valid at any time during the process. As the gas leaves the storage tank, the remaining gas will expand isentropically to result in a temperature drop in the remaining gas

$$
\frac{T}{T_{1}}=\left(\frac{\rho}{\rho_{1}}\right)^{\gamma-1}=\left(\frac{w}{w_{1}}\right)^{\gamma-1}
$$

where $\rho$ is the gas density in the storage tank. A substitution of equation (4.24) into equation (4.23) yields a final result of

$$
T^{\prime}=T_{1} \frac{1-\left(\frac{w}{w_{1}}\right)^{\gamma}}{\left(1-\frac{w}{w_{1}}\right)}
$$

Equation (4.25) makes it clear that the temperature of the gas in the vacuum chamber, $T^{\prime}$, is a function of the initial storage temperature $T_{1}$, and the fraction of the gas remaining in the storage tank $w / w_{1}$. The temperature of the gas entering the chamber initially will be high, but as the fraction $w / w_{1}$ approaches zero, $T^{\prime \prime}$ will approach $T_{1}$. The limit of equation (4.25), taken as $w / w_{1}$ approaches one, describes the initial blowdown temperature as well as the chamber temperature obtained when an infinite reservoir, i.e., atmospheric blowdown, is used. Thus, in the limit

$$
T^{\prime}=\gamma T_{1}
$$

Therefore, if a chamber is vented to atmosphere rapidly at $300^{\circ} \mathrm{K}$, the temperature of the air, with $\gamma=1.4$, in the chamber will be $420^{\circ} \mathrm{K}$ or $147^{\circ} \mathrm{C}$. The initial blowdown temperature of a reservoir system will be 
the same as that in equation (4.26). However, as the stored air expands out of the reservoir, the temperature in the vacuum chamber will fall according to equation (4.25). Of course, heat transfer to the chamber walls will lower the temperature (ref. 7), and if large cryogenic surfaces are present, the cooling effect can be quite large. Although an ideal gas was assumed in the derivation of equation (4.25), the Joule-Thompson effect becomes significant in reducing the final chamber air temperature if the repressurization air is stored at pressures higher than about 20 atmospheres.

\section{REFERENCES}

1. Dushman, S.: Scientific Foundations of Vacuum Techniques. Second ed., J. M. Lafferty, ed., John Wiley \& Sons, Inc., 1962.

2. Ho, T. L.: Multiple Nozzle Diffusion Pumps. Rev. Sci. Instr., vol. 3, Mar. 1932, pp. 133-135.

3. Mullen, L. O.; and JacoBs, R. B.: Some Characteristics of a Simple Cryopump. Trans. of the Ninth National Vacuum Symposium, G. H. Bancroft, ed., The Macmillan Co., 1962, pp. 220-226.

4. Stickney, W. W.; and Dayton, B. B.: The Measurement of the Speed of Cryopumps. Trans. of the Tenth National Vacuum Symposium, G. H. Bancroft, ed., The Macmillan Co., 1963, pp. 105-116.

5. SAnteler, D. J.: A Graphical Solution for the Analysis of Vacuum Systems Performance. Trans. of the Vacuum Symposium, Committee on Vacuum Techniques, Inc. (Boston), 1956, pp. 31-41.

6. SANTeler, D. J.: Vacuum Process Evaluation. Proceedings of the First International Congress on Vacuum Techniques, vol. I. Fundamental Problems in Vacuum Techniques, Ultra High Vacuum, E. Thomas, ed., Pergamon Press, 1959, pp. 98-109.

7. Bebcher, N.: Thermal Analysis of Space Simulation Chambers. Trans. of the Ninth National Vacuum Symposium, G. H. Bancroft, ed., The Macmillan Co., 1962, pp. 253-259. 


\section{Gas Flow in Components and Systems}

\section{INTRODUCTION}

W HENEVER A GAS FLOWS through a tube or other system component, it experiences a pressure drop. The vacuum engineer must be familiar with the mathematical relationships describing such system restrictions to properly understand vacuum systems and experiments. An incomplete understanding of the importance of gas-flow restrictions frequently results in less efficient system performance and increased cost.

An example of such inefficiency occurs when the speed of a $\$ 1000$ pump is limited to that of a much less expensive pump by pipe worth a few dollars at most. A piece of pipe, the same size and shape as the diffusion pump to which it is connected, will reduce the speed of the pump approximately one-half its rated value. For example, a $5 \frac{1}{4}$-inchdiameter pipe, 16 inches long, will reduce the speed of a 720-liter/sec diffusion pump to approximately $330 \mathrm{liter} / \mathrm{sec}$. In another instance, the pressure inside a package under test in a vacuum chamber, or in space, might be assumed to equal that of the chamber. Under actual conditions, however, because of the restrictions to the outgassing flow offered by the various passages and orifices in the package, the expected pressure may not be reached for hours or even days.

The gas-flow regime which will occur in tubular flow depends on the size of the tube and the pressure, temperature, and type of the gas under consideration. Free molecular flow prevails at low gas densities where the mean free path or the average distance a molecule travels between successive intermolecular collisions is large compared to the tube cross section. As the mean free path shortens for higher pressure conditions, intermolecular collisions will predominate rather than collisions with the walls of the tube. Such a high-density gas flow is called viscous or continuum flow. The mathematical relationships, which are entirely different from those pertaining to molecular flow, used to describe viscous flow, depend on whether it is laminar, turbulent, incompressible, compressible, or critical. A complex transition region existing between the molecular and viscous regimes can be described by empirical expressions, although the region is little understood. Several of the continuumgas-flow regimes have essentially no application to vacuum technology and will not be discussed further in this text. 
The development of gas-flow technology has proceeded from the viscous, incompressible region into the molecular region. Many of the observations and criteria of low-pressure gas flow have been reported as extensions of the relationships originally developed for the higher pressures of viscous flow. However, from the vacuum engineering viewpoint, molecular flow is the more basic case, while viscous flow represents the modified extension. The kinetic-theory approach to calculating the various relationships appears to be more consistent with actual operating conditions than the historical gas-flow equations, due to the fact that the calculations encountered in vacuum applications are made in the molecular- or transition-flow regimes. Even when dealing with viscous flow, the condition of critical flow is far more prevalent than the laminar or Poiseuille flow more usually discussed in the literature.

\section{MOLECULAR FLOW THROUGH AN ORIFICE}

A convenient starting point for the discussion of gas flow in tubes is the consideration of an orifice which connects two large chambers each containing a very low pressure gas. Since the mean free path of the gas molecules will be very large compared to the orifice diameter, molecular flow will prevail. Figure 5.1 illustrates this situation. Every molecular

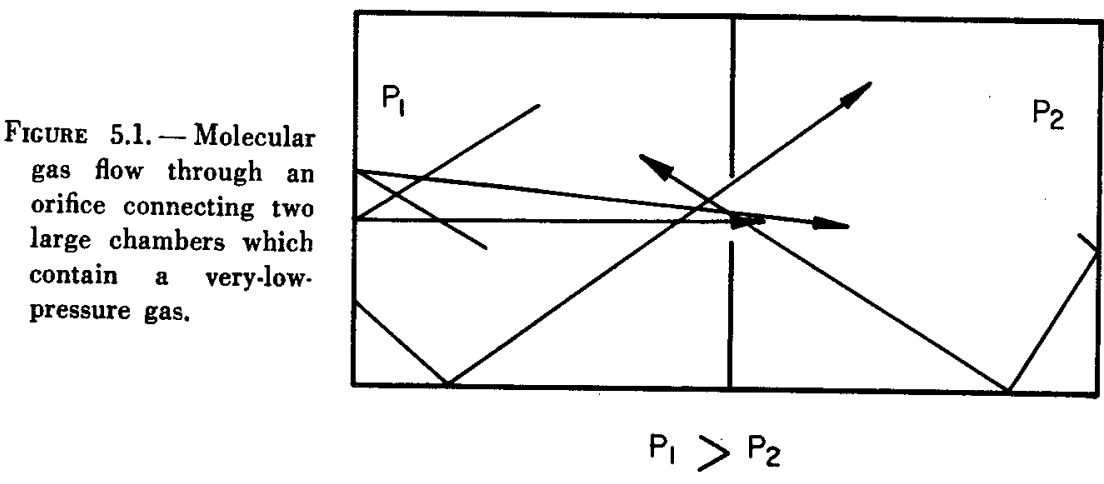

specie arriving at the orifice will pass through with a flow rate proportional to the pressure of the gas and the area of the orifice, due to the fact that the pressure for a particular molecular specie at constant standard temperature is proportional to the molecular incidence rate per unit area. Thus, the basic equation describing molecular flow is, at the orifice

$$
Q=K P A
$$

where $P$ is the pressure in torr and $A$ is the orifice area, $\mathrm{cm}^{2}$.

$K$ has been derived from kinetic theory (ref. 1), while $Q$ may be expressed as the number of molecules per unit time, or in terms of 
pressure-volume units per unit time. It is more convenient in the subsequent discussion to express $Q$ in torr liter/sec. As a result, $K$ equals (ref. 1) $3.638 \sqrt{T M}$, where $T$ is the absolute gas temperature in ${ }^{\circ} \mathrm{K}$, and $M$ is the molecular weight. Equation (5.1) for molecular flow through an orifice becomes

$$
Q=3.638 P A \sqrt{T / M}
$$

It is not always possible to assume the low side pressure equals zero. However, as the molecular flows in each of the two directions through the orifice do not interfere with each other, $P$ of equation (5.2) can be replaced by $\left(P_{1}-P_{2}\right)$

$$
Q=3.638\left(P_{1}-P_{2}\right) A \sqrt{T / M}
$$

Equations (5.2) and (5.3) are strictly valid only when both sides of the orifice look into large volumes. More will be said of this in a later discussion of the combining of flow restrictions.

Figure 5.2 depicts the logarithmic plot of the orifice-flow relationship among other items. The relationship $Q=K A P$ is shown as a $45^{\circ}$ straight line whose position depends on the orifice area, the gas type, and the temperature. Once a line for a given set of conditions has been graphed, the values of $Q$ can be determined for any value of pressure. If the downstream pressure is unequal to zero, the net flow can be obtained by the principle of superposition which assumes a forward flow $K A P_{1}$ and a reverse flow $K A P_{2}$. The difference between the two flows is the true flow, $Q=Q_{1}-Q_{2}$.

\section{MOLECULAR FLOW THROUGH A TUBE OF CIRCULAR CROSS SECTION}

Another pattern of gas flow considers molecular flow through a tube which may be regarded as an orifice with length added. Of the quantity of molecules which pass through the tube opening, a number will collide with the walls. There are two explanations as to what occurs during such a collision. In one case, it is assumed that the gas molecule is adsorbed on the wall for some finite period of time, and is subsequently reevaporated according to a cosine distribution law. That is, the probability of a molecule leaving the wall in any given direction is proportional to the cosine of the angle formed between the velocity vector and the normal to the wall.

For the second case, it is assumed that the molecule is reflected diffusely from the wall on collision, and the net reflection also has a cosine distribution due to the surface roughness of the wall. One important common point stands out in these two differing theories on the collision process. That is, a molecule leaving the wall after a collision has an equal probability of continuing its forward direction down the tube or 


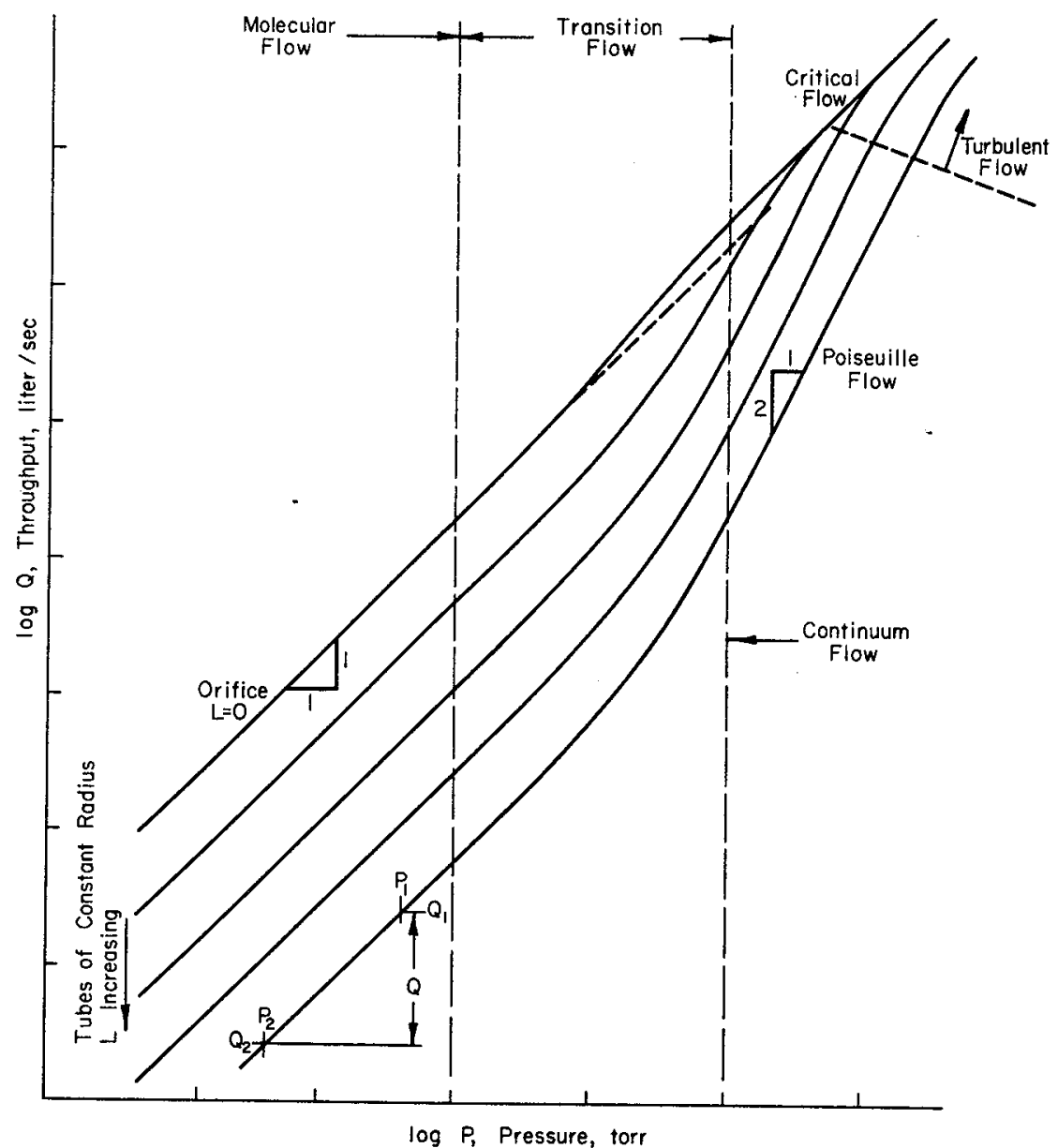

Ficure 5.2.-The behavior of gas flow in the various flow regimes.

returning toward its initial starting point. Therefore, a quantity of the molecules entering the tube will not pass through, but will be returned to their starting point. As a result, the flow of the gas through a tube is reduced relative to orifice flow.

Assume that the low side pressure equals zero. A qualitative study of the flow requires a consideration of the two momenta associated with the molecule. One is the total kinetic energy of the molecular random velocity, and the other is that component of momentum or velocity which is directed down the tube. Any molecule which collides with a wall loses its directional momentum, since it has an equal probability of traveling in either direction after impact. The probability of a molecule leaving either end of the tube depends on its relative distance 
from either end. In the case of very short tubes, the probability depends primarily on a solid angle defined by the tube radius $a$ and length $\ell$. If a molecule can negotiate this solid angle, it can pass through the tube without striking a wall. As the tube increases in length, single, double, and multiple collisions become more prevalent. However, the probability of a successful passage still remains a function of the ratio $\ell / a$ which defines an attenuation factor applicable to the directional energy incident on the tube entrance. Reference 1 has shown that the attenuation factor equals $\frac{8}{3} \frac{a}{\ell}$ for very long tubes of circular cross section. Thus $Q$, torr liter/sec, for long tubes is

$$
\begin{aligned}
Q & =\frac{8}{3} \frac{a}{\ell} Q_{\text {orifice }} \\
& =30.48 \frac{a^{3}}{\ell} \sqrt{T / M} P
\end{aligned}
$$

where $a$ and $\ell$ are in centimeters. Clausing (references 1 and 2) has defined the relationship between the ratio $\ell / a$ and the attenuation factor for tubes of any length as

$$
\begin{aligned}
Q & =K^{\prime} Q_{\text {orifice }} \\
& =11.4 K^{\prime} a^{2} P \sqrt{T / M}
\end{aligned}
$$

where the units of $Q$ are torr liter/sec and $K^{\prime}$ is a complex, analytically determined function of $\ell / a$. Values of $K^{\prime}$ are tabulated in table 5.1 and plotted in figure 5.16 for various $\ell / a$ ratios.

According to Dushman, the flow restriction of a short tube can be approximated as the linear combination of the tube and an entrance orifice of the same diameter. If this simplifying assumption is used, the value of $K^{\prime}$ in equation (5.5) becomes

$$
K^{\prime} \approx \frac{1}{1+\frac{3}{8} \frac{\ell}{a}}
$$

Equation (5.6) proves to be exact for orifice flow and very long tubes. However, in the case of short tubes, the approximation is too high as illustrated in figure 5.16. The approximation reaches a maximum error of about 12 percent at an $\ell / a$ ratio of 1.5 . A further discussion of this approximation appears later in the chapter.

The flow equation for a tube is also shown in figure 5.2 as a straight $45^{\circ}$ line, since it is still a linear relationship between flow and pressure. A family of such lines is plotted for tubes with a constant radius, and with length increasing toward the bottom of the graph. It must be noted that the upper curve, which illustrates orifice flow, defines the number of molecules which are incident on the end of the tube for every line in the family. 
TABLE 5.1.-Values of Clausing's Factor $\mathrm{K}^{\prime}$ as a Function of the $\ell / a$ Ratio for Long Tubes

\begin{tabular}{|c|c|c|c|c|}
\hline \multicolumn{2}{|c|}{$\begin{array}{c}\text { Ratio, } \\
\ell / a\end{array}$} & $K^{\prime}$ & $\begin{array}{c}\text { Ratio, } \\
\ell / a\end{array}$ & $K^{\prime}$ \\
\hline 0 & --- & 1 & 3.2 & 0.4062 \\
\hline .1 & $-\cdots$ & .9524 & 3.4 & .3931 \\
\hline .2 & $\ldots$ & .9092 & 3.6 & .3809 \\
\hline .3 & $\ldots$ & .8699 & 3.8 & .3695 \\
\hline .4 & - & .8341 & 4.0 & .3589 \\
\hline .5 & - & .8013 & 5.0 & .3146 \\
\hline 6 & --- & .7711 & 6.0 & .2807 \\
\hline .7 & $-\cdots-$ & .7434 & 7.0 & .2537 \\
\hline .8 & $-\cdots$ & .7177 & 8.0 & .2316 \\
\hline .9 & $-\ldots . .$. & .6940 & 9.0 & .2131 \\
\hline 1.0 & $\ldots$ & .6720 & 10.0 & .1973 \\
\hline 1.1 & - & .6514 & 12.0 & 1719 \\
\hline 1.2 & $\ldots$ & .6320 & 14.0 & .1523 \\
\hline 1.3 & - n.... & .6139 & 16.0 & . 1367 \\
\hline 1.4 & $-\cdots$ & .5970 & 18.0 & . 1240 \\
\hline 1.5 & --- & .5810 & 20.0 & .1135 \\
\hline 1.6 & ---- & .5659 & 30.0 & .0797 \\
\hline 1.7 & -----. & .5518 & 40.0 & .0613 \\
\hline 1.8 & ---- & .5384 & 50.0 & .0499 \\
\hline 1.9 & --.-- & .5256 & 60.0 & .0420 \\
\hline 2.0 & ----- & .5136 & 70.0 & .0363 \\
\hline 2.2 & - & .4914 & 80.0 & .0319 \\
\hline 2.4 & $-\cdots$ & .4711 & 90.0 & .0285 \\
\hline 2.6 & ---- & .4527 & 100.0 & .0258 \\
\hline 2.8 & --- & .4359 & 1000.0 & .002658 \\
\hline 3.0 & -..-- & .4205 & & $(8 a / 3 \ell)$ \\
\hline
\end{tabular}

\section{LAMINAR VISCOUS FLOW IN A LONG CIRCULAR TUBE}

Up to this point, the discussion has dealt with gas flow at pressures low enough such that molecule-to-wall collisions predominate. As the pressure increases and molecular fow disappears, the basic character" of the flow equations changes, as illustrated in figure 5.3.

The end of a circular tube is shown, for which the inner surface is one mean free path distant from the wall surface. When a gas molecule collides with another gas molecule, there is no net change in the momentum vector. Molecules which enter the center section of the tube have little chance of colliding with anything other than another molecule, and thus have a small likelihood of losing momentum. However, as molecules enter the outer section of the tube, there are more opportunities for them to collide with the wall and lose their directed momentum. As the mean free path decreases, the restriction offered by the tube should also decrease. This effect may be examined by first considering 


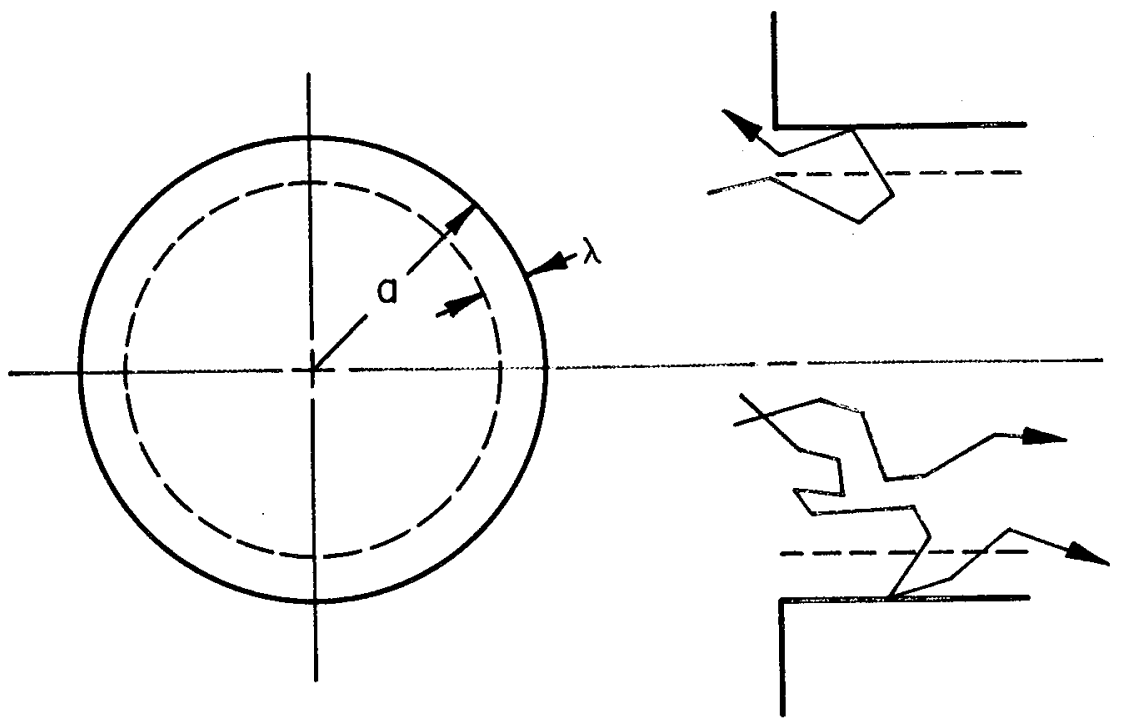

Figure 5.3.-Transition flow through a tube of circular cross section.

the equation for molecular flow through a tube. Those molecules which collide with the wall react in the same manner as in the molecular-flow condition and have no effect on the equation. Molecules which collide only with other molecules do not lose their directional momentum, and tend to amplify the molecular-flow equation. If the equation is multiplied by a factor proportional to the ratio of the number of molecules in gas-to-gas collisions to the number in gas-to-wall collisions, the resulting expression should have the proper form for describing viscous flow. Figure 5.3 shows that the number of molecules making gas-to-gas collisions is proportional to $\pi(a-\lambda)^{2}$, while the number making gas-to-wall collisions is proportional to $\pi a^{2}-\pi(a-\lambda)^{2}$. The ratio of these quantities, when $\lambda<<a$, is $a / 2 \lambda$. Since the mean free path is inversely proportional to pressure, the multiplying factor becomes $C a \bar{P}$, where $\bar{P}$ is the average pressure in the tube, and $C$ is a general constant introduced to account for other factors which are not considered in this discussion.

The equation, which is called the Poiseuille equation, for laminar viscous flow through a long circular tube does exhibit the form predicted by the considerations above (ref. 1):

$$
\begin{aligned}
Q & =\frac{\pi a^{4}}{8 \eta \ell}\left(\frac{P_{1}+P_{2}}{2}\right)\left(P_{1}-P_{2}\right) \\
& =C a \bar{P}\left(Q_{\text {molecular }}\right)
\end{aligned}
$$


where

$\bar{P}=\frac{1}{2}\left(P_{1}+P_{2}\right)$, torr

a tube radius, $\mathrm{cm}$

$\ell$ tube length, $\mathrm{cm}$

$\eta$ gas viscosity, poise

Table 5.2 lists the room-temperature viscosities of several common gases.

TABLE 5.2.-Viscosity of Several Common Gases at Room Temperature, $300^{\circ} \mathrm{K}$

\begin{tabular}{|c|c|}
\hline Gas & Viscosity, $\eta$, micropoise \\
\hline Air $-\cdots---$ & 187 \\
$\mathrm{~N}_{2}-\cdots---$ & 178 \\
$\mathrm{O}_{2}-----$ & 207 \\
$\mathrm{H}_{2}-\cdots--$ & 89 \\
$\mathrm{Ar}-\cdots--$ & 226 \\
$\mathrm{He}-\cdots--$ & 197 \\
\hline
\end{tabular}

Although the Poiseuille equation was derived from a continuum concept of viscosity, the form of the variables in the case of molecular considerations is exactly the same as that inferred from the foregoing simplified approach.

The four assumptions which govern the derivation of the Poiseuille equation are listed below:

(1) An incompressible gas

(2) Fully developed flow; that is, the velocity profile is the same at any station along the tube length

(3) The flow is laminar rather than turbulent

(4) Velocity at the wall equals zero

An extensively used measure in the field of fluid mechanics to establish the degree of compressibility in a gas-flow problem is the Mach number, $v / c$, where $v$ is the flow velocity and $c$ is the local acoustic velocity. Equation (5.7) is valid according to compressibility considerations if $v / c$, evaluated at the tube exit, is less than 0.3 , and $c$, expressed in $\mathrm{cm} / \mathrm{sec}$, is

where

$$
c=8700 \sqrt{\gamma T / M}
$$

$$
\begin{array}{cl}
\gamma & \text { ratio of specific heats } \\
T & \text { temperature }{ }^{\circ} \mathrm{K} \\
M & \text { gram molecular weight }
\end{array}
$$

Therefore, the flow of room-temperature air in a tube should not exceed $Q / a^{2} P=31$. If higher Mach number flows cannot be avoided, 
texts on compressible flow, such as Shapiro (ref. 3), are available and should be consulted.

Because a certain entrance length of tube must be negotiated before fully developed flow is established, equation (5.7) must be corrected for short tubes. The correction factor is a function of the Reynolds number of the flow (ref. 1). Short tubes frequently lead to compressible flow and to critical or choked flow which is described in a later section.

The criterion that determines whether the flow will be laminar or turbulent is the Reynolds number which is usually defined for the case of tube flow, as

$$
R_{e}=\frac{2 v a_{\rho}}{\eta}
$$

where $\rho$ is the fluid density. The dimensionless Reynolds number for air at $25^{\circ} \mathrm{C}$ is

$$
R_{e}=5.4 \frac{Q}{a}
$$

where the units for $Q$ and $a$ are torr liter/sec, and $\mathrm{cm}$, respectively. If the Reynolds number is less than 1000, laminar flow will exist in smooth tubes where the entrances are well rounded. $R_{e}$ values greater than 2000 will indicate that turbulent flow will exist. Turbulent flow will occur for a short time in a vacuum system during the initial highpressure portion of a pumpdown cycle. This flow phenomena will not be treated in the present discussion, since the literature concerning it is quite extensive (ref. 4).

The assumption that for the Poiseuille equation, gas velocity equals zero at the wall is satisfied as long as the ratio $a / \lambda$ is large.

The next discussion logically follows from the above considerations. It has been mentioned previously that there is a transition-flow region between the molecular and viscous regions. The transition occurs over a range of two or three decades of pressure where the molecule-to-wall collision pattern decreases and gives way to the intermolecular collision conditions which characterize viscous or continuum flow. In long, circular tubes the transition essentially reaches completion in the range $0.1>\frac{a}{\lambda}>100$. Unfortunately, the flow in this region is not well understood despite the amount of experimental work which has been accomplished (refs. 1, 5, and 6). For the present, an empirical expression first formulated by Knudsen describes the region adequately

$$
Q=Q_{\text {viscous }}+Z Q_{\text {molecular }}
$$

Equation (5.8) is a linear combination of the viscous and molecular 
flows described in equations (5.5) and (5.7). From reference 1, $Z$ in long tubes of circular cross section becomes

$$
Z=\frac{1+2.507\left(\frac{a}{\lambda}\right)}{1+3.095\left(\frac{a}{\lambda}\right)}
$$

Table 5.3, as given in reference 1 , illustrates equation (5.9):

TABLE 5.3.-Transition Flow in a Long Tube

\begin{tabular}{|c|c|c|}
\hline$\underset{a / \lambda}{\text { Ratio, }}$ & $Z$ & $\begin{array}{l}Q_{\text {transition }} / Q_{\text {niol ocular }} \\
(\text { eq. }(5.8) / \text { eq. }(5.4))\end{array}$ \\
\hline $10^{3}$ & 0.810 & 148 \\
\hline $10^{2}$ & .810 & 15.53 \\
\hline 10 & .816 & 229 \\
\hline 5 & .822 & 1.56 \\
\hline 1 & .857 & 1.00 \\
\hline 0.5 & .885 & .959 \\
\hline . & .905 & .952 \\
\hline $2 \quad \ldots$ & .933 & .962 \\
\hline 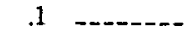 & .955 & .970 \\
\hline . 05 & .974 & .981 \\
\hline . 01 & .995 & .997 \\
\hline 0 & 1.000 & 1.000 \\
\hline
\end{tabular}

The minimum value of flow evident in table 5.3 tends to disappear in short tubes and porous media (ref. 7).

To permit the viscous flow to be plotted on the molecular-flow equation graph, one of the pressure values in equation (5.7) must be eliminated. If the equation is considered to be a linear combination of two equations, then

$$
\begin{aligned}
Q & =Q_{1}-Q_{2} \\
& =\frac{\pi a^{4} P_{1}^{2}}{16 \eta \ell}-\frac{\pi a^{4} P_{2}^{2}}{16 \eta \ell}
\end{aligned}
$$

Once the flow has become completely viscous, the slope of the curve is $2: 1$ as the relationship between $P$ and $Q$ indicates. Figure 5.2 shows the transition- and viscous-flow portion of one of the molecular curves added to the molecular-flow equation graph. Although the tube length has attenuated the flow in the molecular region, the higher pressure and accompanying flow regime change have amplified the flow back toward the upper $45^{\circ}$ line which represents the molecular gas flow incident on the end of the tube. Since the flow through the tube cannot exceed the amount of gas entering the end, there is an upper limit on the useful range of the Poiseuille equation. That is, when the viscous-flow curve 
reaches this upper limit, it must transfer and follow a $45^{\circ}$ slope. The upper viscous-flow limit is somewhat higher than that of molecularorifice flow due to the streaming and acceleration of a gas before passing through the tube or orifice entrance. The maximum continuum flow which an orifice can pass is called the critical or choked flow. Before critical flow can be said to exist, several conditions must be satisfied. The Mach number must equal unity at the throat of the tube, and the pressure ratio across the orifice must be equal to or greater than the so-called critical pressure ratio, which is defined as

$$
\left(\frac{P_{1}}{P_{2}}\right)=\left(\frac{\gamma+1}{2}\right)^{\frac{\gamma}{\gamma-1}}
$$

where $\gamma$ is the ratio of specific heats. The critical pressure ratio equals 1.90 for diatomic gases such as air and nitrogen for which $\gamma=1.4$. Helium, argon, and other monatomic gases, for which $\gamma$ equals 1.66, exhibit a critical ratio equal to 2.05 . The critical orifice flow $Q^{*}$, expressed in conventional vacuum units, torr liter/sec, becomes

$$
Q^{*}=28.6 C\left[\frac{\gamma T}{M}\left(\frac{2}{\gamma+1}\right)^{\frac{\gamma+1}{\gamma-1}}\right]^{1 / 2} P a^{2}
$$

where $C$ is a dimensionless orifice coefficient. The ratio of critical orifice flow to molecular orifice flow can be obtained if equation (5.11) is divided by equation (5.3)

$$
\frac{Q^{*}}{Q_{\text {mole }}}=R^{*}=2.5 C\left[\gamma\left(\frac{2}{\gamma+1}\right)^{\frac{\gamma+1}{\gamma-1}}\right]^{1 / 3}
$$

As a result, $R^{*}=1.72 C$ for a diatomic gas. The orifice coefficient, $C$, for critical flow varies from approximate values of unity, in the case of a well-rounded entrance, to 0.75 . The substitution of values of $C$ determined by the entrance condition in equation (5.12) yields a range of values of $R^{*}$ for a diatomic gas, $1.3<R^{*}<1.7$. For the conditions of critical viscous flow, a rounded entrance yields a higher flow than a sharp opening of the same orifice area, while the opposite condition exists in molecular flow. Equation (5.11) and the associated discussion are approximately true for short tubes as well as orifices. As a tube is lengthened under the conditions of critical flow, the sonic choke moves downstream to the tube exit. The added friction yields lower values of critical flow than is indicated in equation (5.12) (ref. 3).

The complete curve representing all the regions of flow is shown in figure 5.2. There is no readily available equation to describe the transition region between viscous and critical flow. The curvature illustrated in the figure was obtained from measurements carried out on different tubes for which the exhaust pressure essentially equaled zero. There are two separate flow rates which determine the net flow in 
both the viscous- and molecular-flow regimes when neither pressure equals zero. This superposition principle is valid in the molecular-flow region as the forward and reverse flows are actually occurring. The mathematical properties of Poiseuille flow validate the application of the superposition principle in the viscous region. However, the superposition method cannot be applied in the critical flow region where the flow is independent of the downstream pressure if the critical pressure ratio is exceeded.

Critical flow and its associated phenomena are extremely important considerations to the vacuum engineer, while Poiseuille flow seldom exists under the conditions of normal vacuum operation. The flowpressure curves for pipes of various diameters are plotted in figure 5.4. The radii of the pipes are 1 centimeter and 5 centimeters, with $\ell / a$ ratios of $0,100,1000$. At an $\ell / a$ ratio of 100 , there is an overlapping of the critical- and transition-flow regimes. Thus, very little of the curve can be described by the 2:1 slope of the Poiseuille equation.

An $\ell / a$ ratio of 1000 still results in only one pressure decade coming under the jurisdiction of the Poiseuille equation. Since a vacuum system rarely requires a pipe with an $\ell / a$ ratio as great as 100 , viscous Poiseuille flow seldom exists in normal vacuum applications. As a result, equation (5.7) may be neglected for a majority of vacuum applications.

A common error made in the design of vacuum pumping systems is that a roughing line is sized on the basis of viscous flow which means that the pump is supposedly burdened with a small, calculated restriction. Under actual conditions, critical flow exists, and the roughing line may represent an appreciable restriction to the pump.

\section{FLOW CHARACTERISTICS OF COMPLEX SHAPES- MOLECULAR FLOW}

The gas flow through a vacuum component may be considered analogous to the current flow through an electrical circuit element. The conductance $F$ is defined as

$$
\begin{aligned}
F & =\frac{Q}{P_{1}-P_{2}} \\
& =\frac{Q}{\Delta P}
\end{aligned}
$$

where the units of $F$ are liter/sec. The conductance of an orifice from equation $(5.2)$ is

$$
F=3.638 A \sqrt{T / M}
$$

The molecular conductance of a vacuum component is a function of its shape and is proportional to the flow area and the gas property, $\sqrt{T / M}$. Table 5.4 makes use of the factor $\sqrt{T / M}$ to compare the air conductance of a component in molecular flow with the conductances of 


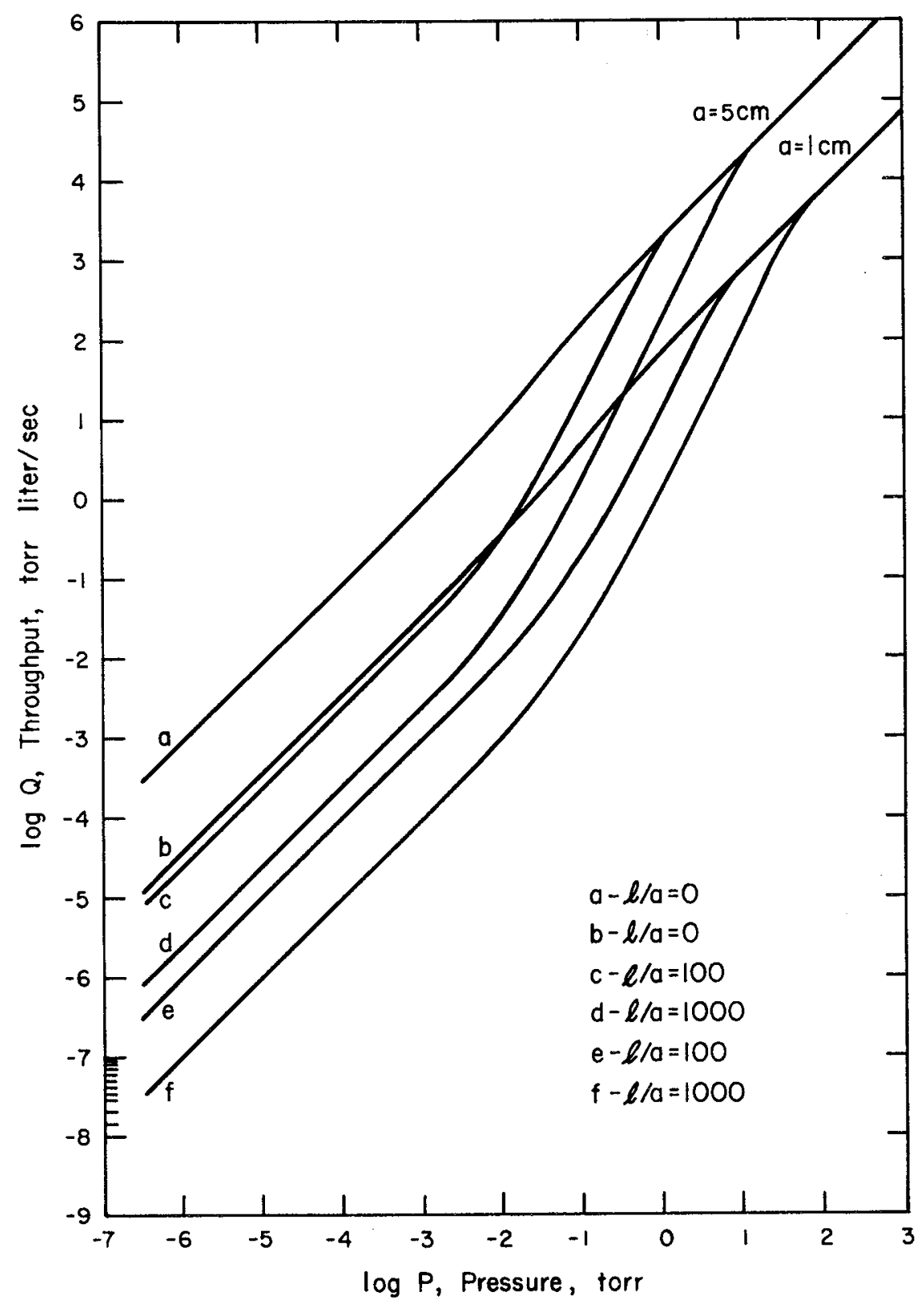

FigURE 5.4.-Flow-pressure curves for pipes with radii of $1 \mathrm{~cm}$ and $5 \mathrm{~cm}$ and $l / a$ ratios of $0-1000$, and extending into several flow regimes. 
several other common gases at $T=300^{\circ} \mathrm{K}$. The orifice conductance for air is equal to $11.7 \mathrm{liter} / \mathrm{cm}^{2}$ sec.

TABLE 5.4.-Comparison of Conductances for Several Common Gases at $300^{\circ} \mathrm{K}$

\begin{tabular}{|c|c|}
\hline \multirow{2}{*}{ Gas } & Molecular conductance for gas \\
\cline { 2 - 2 } $\mathrm{H}_{2}-\cdots-\cdots-$ & Molecular conductance for air \\
$\mathrm{He}$ & 3.8 \\
$\mathrm{H}_{2} \mathrm{O}-\cdots--$ & 2.7 \\
$\mathrm{CO}_{2}$ & 1.3 \\
& .81 \\
\hline
\end{tabular}

The restriction of a component in molecular flow may also be expressed as the probability $a$, that a molecule falling on the entrance orifice will emerge from the downstream end. The probability $a$, for an orifice, equals 1 , and $\alpha=K^{\prime}$ for a tube, as described in equation (5.5). If it is desired to know the conductance, the probability multiplied by the conductance of an orifice having an equal entrance area yields

$$
F=3.638_{a} A_{1} \sqrt{T / M}
$$

where $a$ is a function of the component shape only. The flow restriction of vacuum system components may be calculated from the basis of either $F$ or a, and each method has its advantages. These advantages will be analyzed, but first, the conductances of a variety of common geometries will be examined. The conductance values will be given for air at $300^{\circ} \mathrm{K}$, length and area measurements will be in $\mathrm{cm}$ and $\mathrm{cm}^{2}$, respectively; $F$ is in units of liter/sec.

Orifice connecting two large volumes

$$
F=11.7 A
$$

and

$$
a=1.0
$$

Long cylindrical tube of circular cross section (no end correction)

$$
F=98 \frac{a^{3}}{\ell}
$$

and

$$
a=8.44 \frac{a}{\ell}
$$

Long tube of rectangular cross section (ref. 8) (no end correction)

$$
F=31.2 \frac{a^{2} b^{2}}{(a+b) \ell} K
$$


and

$$
a=2.66 \frac{a b K}{(a+b) l}
$$

where $a$ and $b$ are the dimensions of the long and short sides, respectively, of the rectangular tube. Various values of the shape factor $K$ are listed in table 5.5.

TABLE 5.5.-Shape Factor $\mathrm{K}$ from

Equation (5.17) for Various b/a Ratios

\begin{tabular}{|c|c|}
\hline$b / a$ & $K$ \\
\hline 1 & 1.108 \\
$2 / 3$ & 1.126 \\
$1 / 2$ & 1.151 \\
$1 / 3$ & 1.198 \\
$1 / 6$ & 1.297 \\
$1 / 8$ & 1.400 \\
$1 / 10$ & 1.444 \\
\hline
\end{tabular}

Circular cross-section tube of any length

This geometry is represented by the Clausing equation, equation (5.5). Conversion to air at $300^{\circ} \mathrm{K}$ yields

$$
F=11.7 K^{\prime} A
$$

and

$$
a=K^{\prime}
$$

where the shape factor $K^{\prime}$ for equation $(5.18)$ is listed in table 5.1 (ref. 1).

Tube of narrow rectangular cross section with slits

Clausing has computed the molecular flow in tubes of cross-section $a b$ where $a>>b$ and $a>>l$

$$
F=11.7 \mathrm{Kab}
$$

and

$$
a=K
$$

where the shape factor $K$ for equation (5.19) is listed in table 5.6 (ref. 1).

\section{Baffle assemblies}

References 9 and 13 investigate the transmission probability factors for the many complex geometries which are typical of vacuum baffles and other assemblies. Figures 5.5 through 5.11 illustrate several of 
TABLE 5.6-Shape Factor K from Equation (5.19) for Various 1/b Ratios [Ref. 1]

\begin{tabular}{|c|c||c|c|}
\hline$\ell / b$ & $K$ & $L / b$ & $K$ \\
\hline 0 & 1.0000 & 1.5 & 0.6024 \\
.1 & .9525 & 2.0 & .5417 \\
.2 & .9096 & 3.0 & .4570 \\
.4 & .8362 & 4.0 & .3999 \\
.8 & .7266 & 5.0 & .3582 \\
1.0 & .6848 & 10.0 & .2457 \\
& & $\infty$ & $(b / \ell) \ln (\ell / b)$ \\
\hline
\end{tabular}

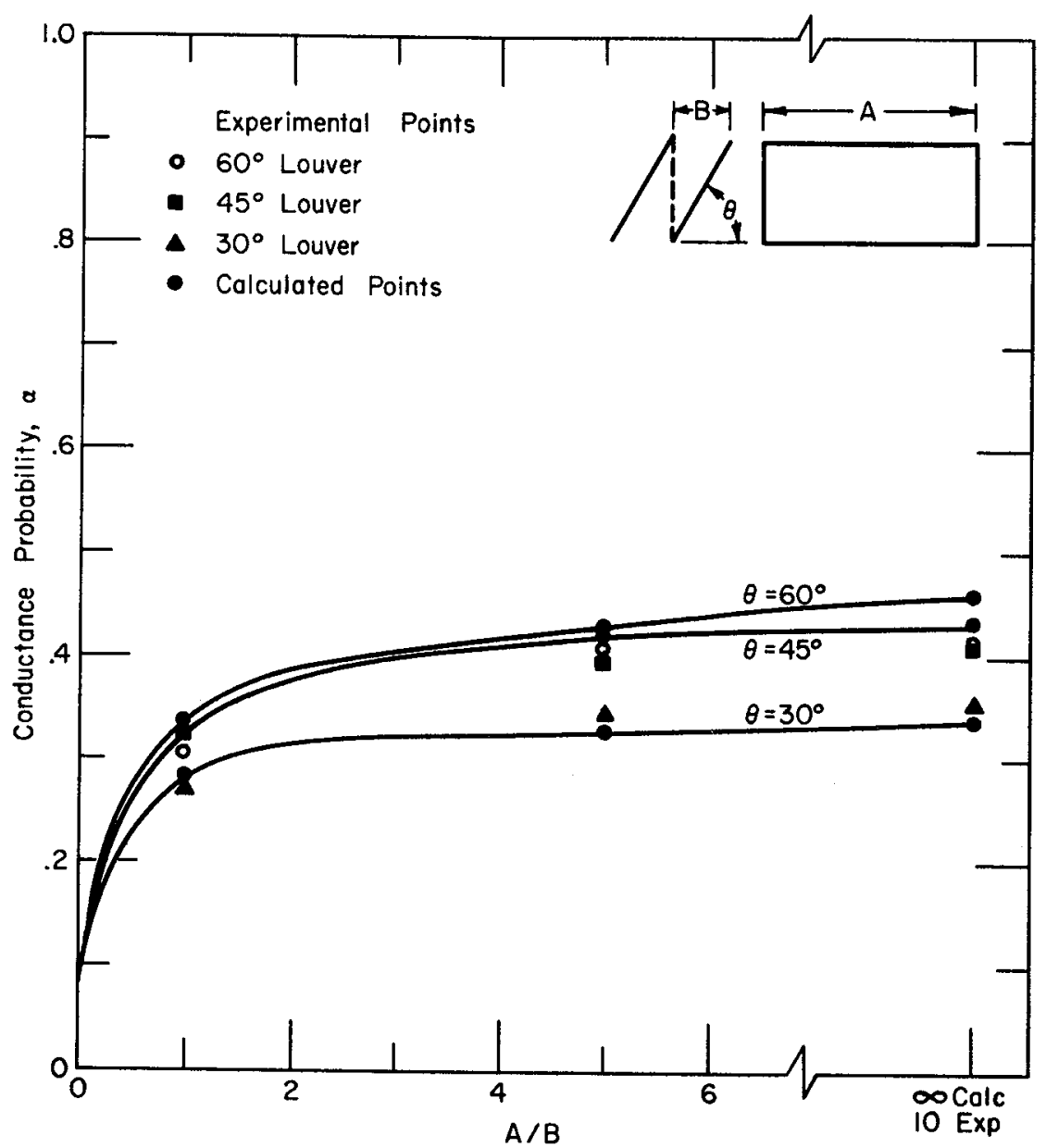

Ficure 5.5.-Transmission or conductance probability factor $a$ for a vacuum baffle assembly in a louver geometry. The distribution of molecules on the entrance orifice of the configuration is assumed to be random. 
the theoretical and experimental results for complex geometries. The data shown are based on an assumed random distribution of molecules on the entrance orifice of the configuration. If the area of an entrance orifice, $A_{1}$, differs from the area of its exit orifice, $A_{2}$, then

$$
\mathrm{a}_{12} A_{1}=\mathrm{a}_{21} A_{2}
$$

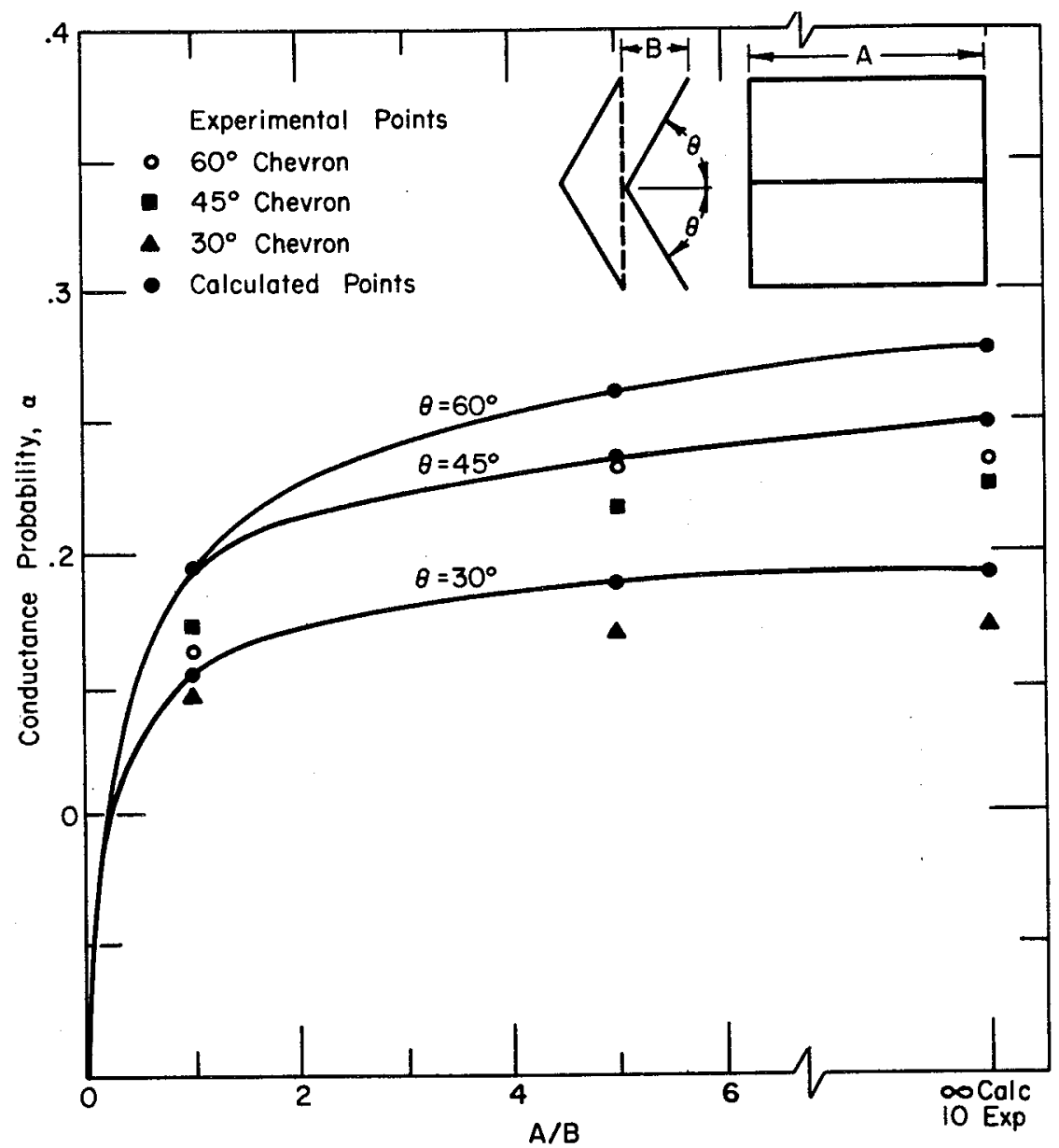

Figure 5.6.-Transmission or conductance probability factor a for a vacuum baffle assembly in a chevron geometry. The distribution of molecules on the entrance orifice of the configuration is assumed to be random. 


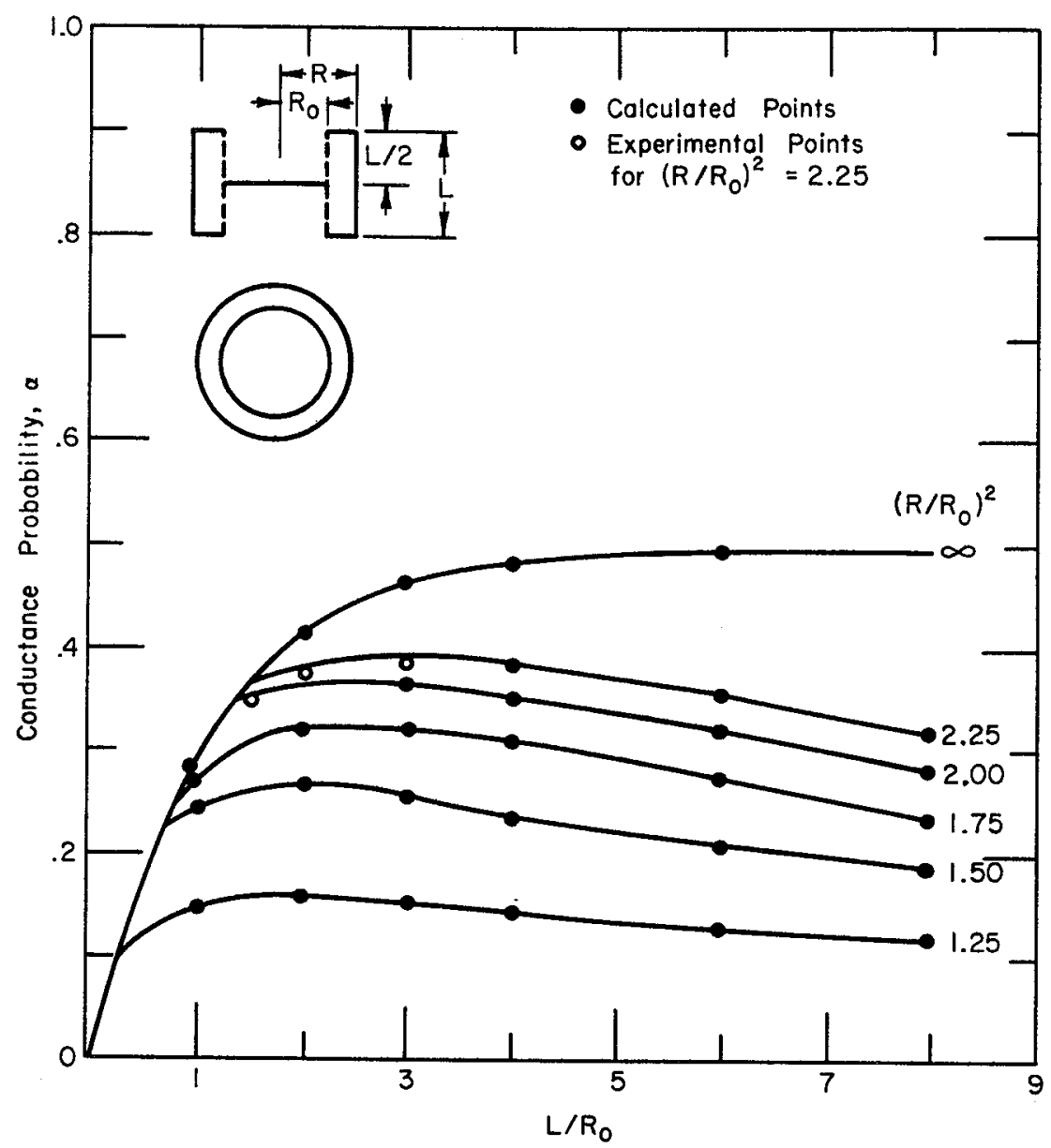

Figure 5.7.-Transmission or conductance probability factor a for a straight cylinder with two restricted ends and a circular blocking plate. The distribution of molecules on the entrance orifice of the configuration is assumed to be random. 


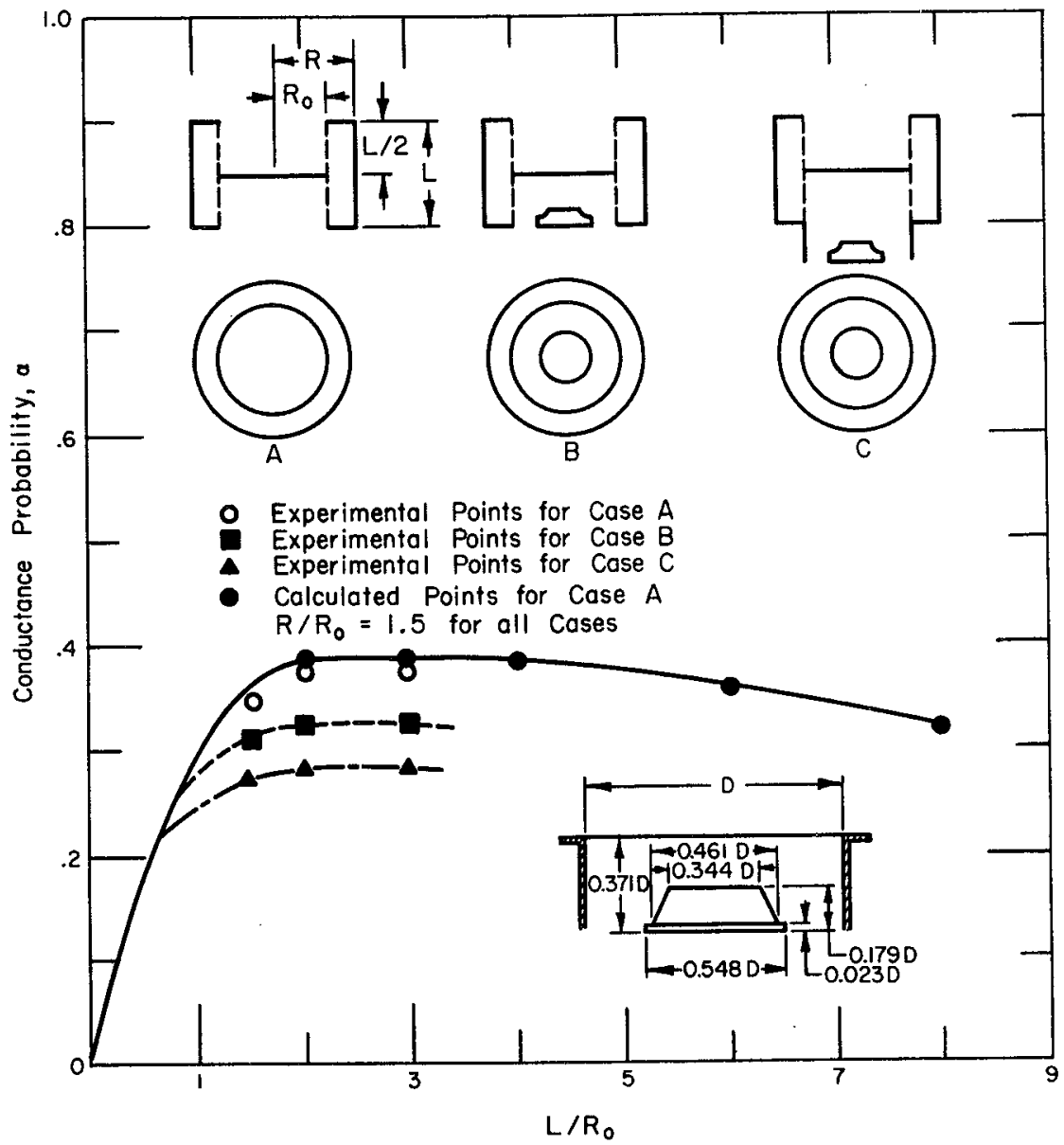

Figure 5.8.-Transmission or conductance probability factor a for a straight cylinder with two restricted ends and a circular blocking plate in diffusion pump system. The distribution of molecules on the entrance orifice of the configuration is assumed to be random. 


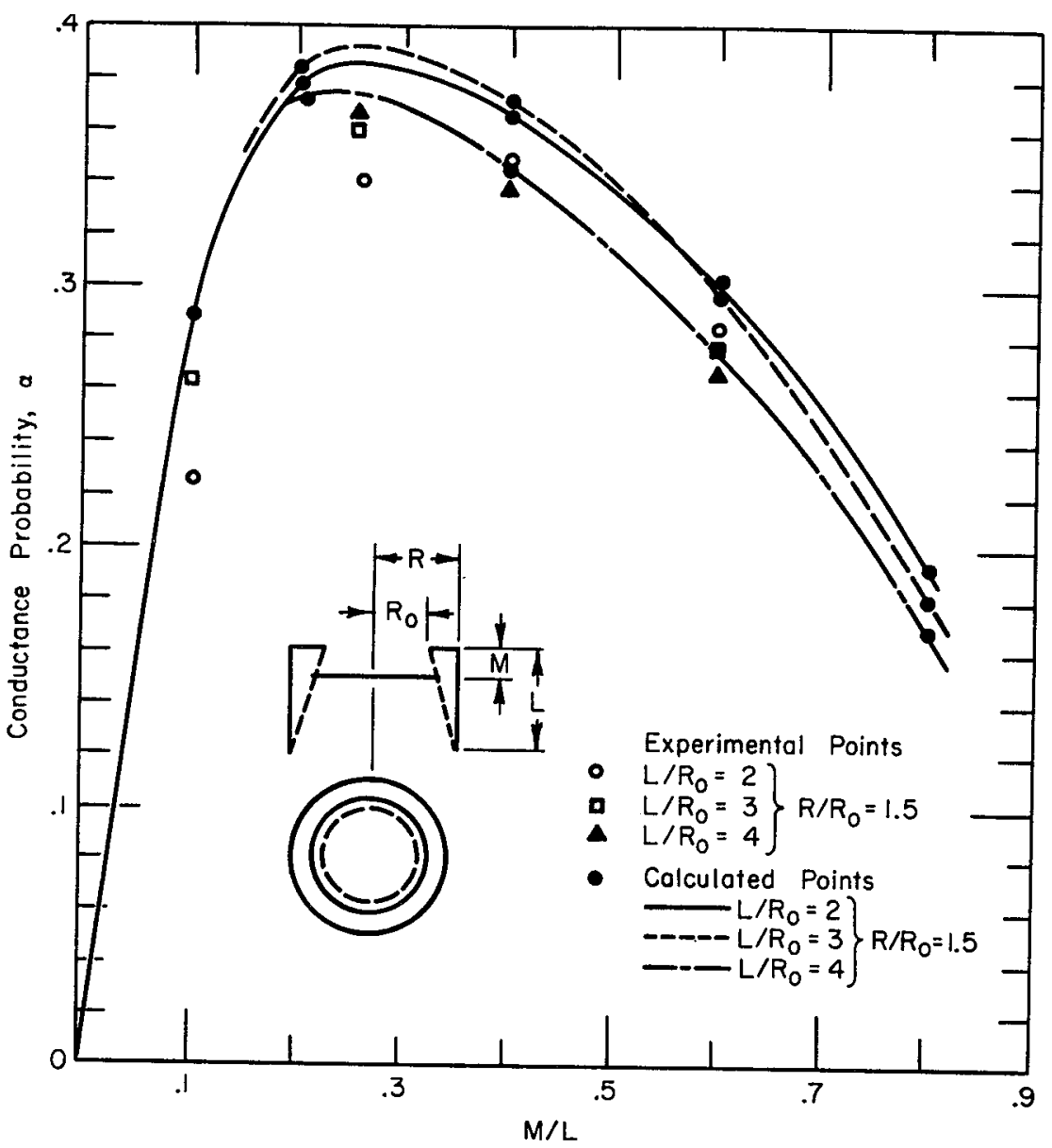

Figure 5.9.-Transmission or conductance probability factor a for the small end of a straight cylinder with one restricted end and a circular blocking plate. The distribution of molecules on the entrance orifice of the configuration is assumed to be random. 


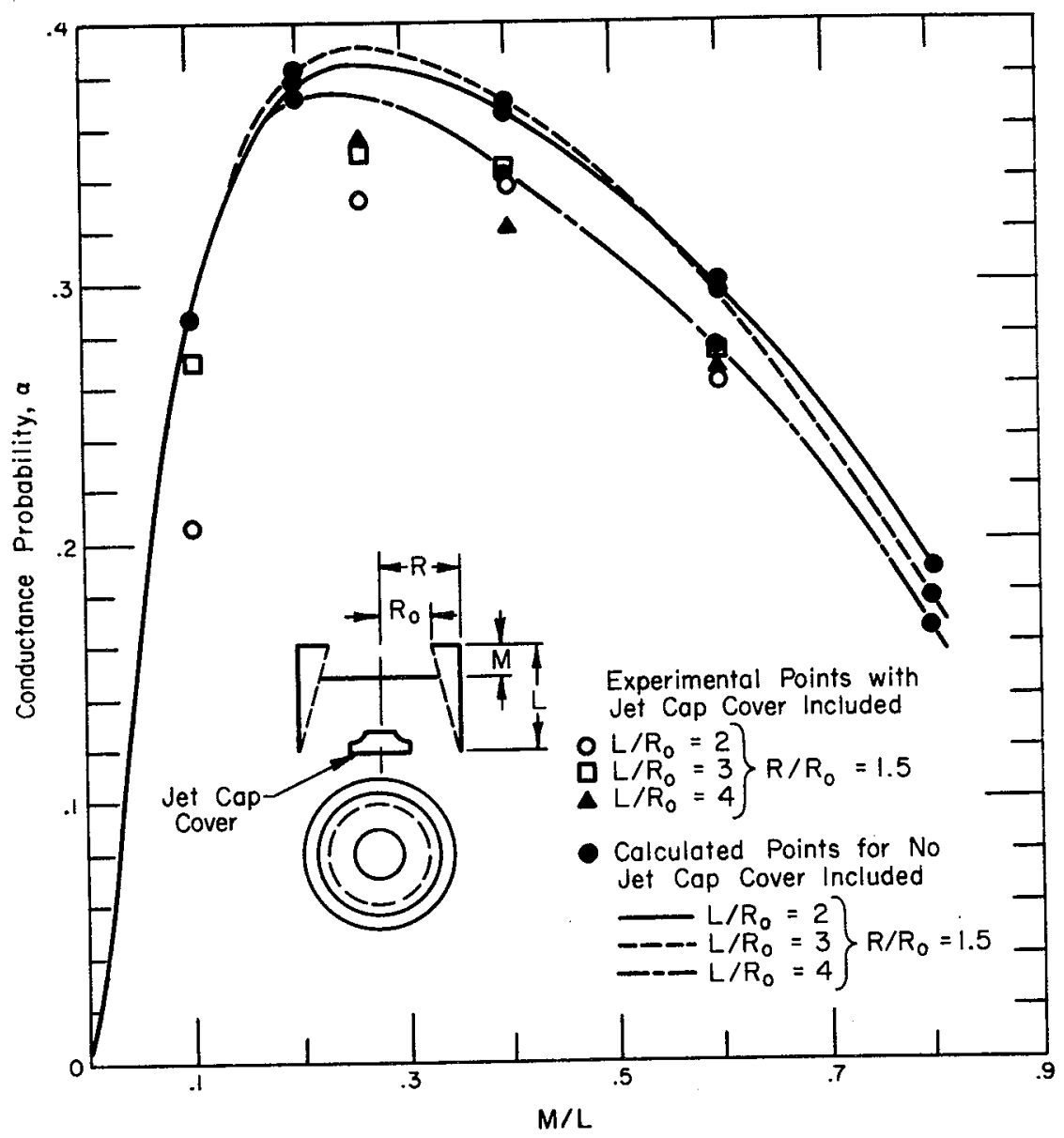

Figure 5.10.-Transmission or conductance probability factor $a$ for the small end of a straight cylinder with one restricted end and a circular blocking plate in diffusion pump system. The distribution of molecules on the entrance orifice of the configuration is assumed to be random. 


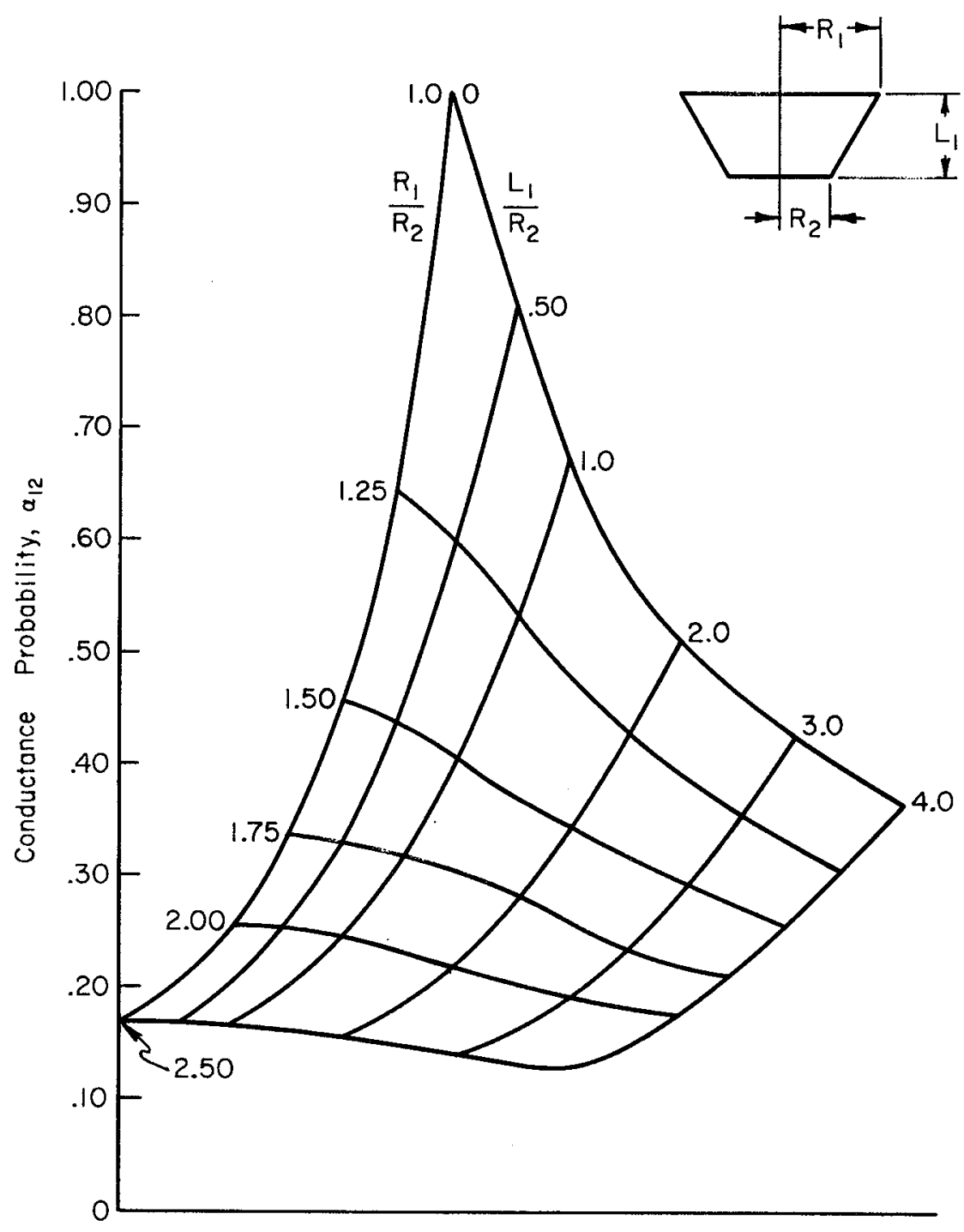

Figure 5.11.-Transmission or conductance probability factor $a$ for a frustum. 


\section{FLOW CHARACTERISTICS OF COMPLEX SHAPES- TRANSITION FLOW}

The conductance definition for the molecular-flow regime remains valid for molecular- to viscous-transition flow. Table 5.3 lists values of the ratio of transition to molecular flow. This ratio is equal to the ratio of transition to molecular conductance for long circular tubes. Reference 6 presents data for long tubes of annular and rectangular cross sections. At the present time, data are not available for short tubes or for more complex geometries.

\section{FLOW CHARACTERISTICS OF COMPLEX SHAPES- LAMINAR VISCOUS FLOW}

Laminar viscous flow conductance is inversely proportional to the gas viscosity (table 5.2).

\section{Long Circular Duct}

The conductance for air at $300^{\circ} \mathrm{K}$ for this case is

$$
F=2.80 \times 10^{3} \frac{\bar{P} a^{4}}{\ell}
$$

Long Annular Duct (Ref. 1)

$$
F=2.80 \times 10^{3} \frac{\bar{P}}{\ell}\left[b^{4}-a^{4}-\frac{\left(b^{2}-a^{2}\right)^{2}}{\ln _{\mathrm{e}}\left(\frac{b}{a}\right)}\right]
$$

where $a$ and $b$ are the inner and outer radii of the duct, respectively.

Long Rectangular Duct (Ref. 1)

$$
F=260 K \frac{a^{2} b^{2} \bar{P}}{\ell}
$$

Table 5.7 lists values of $K$ from equation (5.23) for various $a / b$ ratios.

TABLE 5.7.-Values of Shape Factor K for Various $\mathrm{a} / \mathrm{b}$ Ratios

\begin{tabular}{|r|r|}
\hline$a / b$ & $K$ \\
\hline 1.0 & 1.00 \\
.9 & .99 \\
.8 & .98 \\
.7 & .95 \\
.6 & .90 \\
.5 & .82 \\
.4 & .71 \\
.3 & .58 \\
.2 & .42 \\
.1 & .23 \\
\hline
\end{tabular}


The streaming action which is present at an entrance orifice during viscous or transition flow precludes the proper application of the probability factor $a$.

\section{FLOW CHARACTERISTICS OF COMPLEX SHAPES- CHOKED OR CRITICAL FLOW}

In the strict sense of the term, the concept of conductance does not apply to critical flow which is a function of $P_{1}$ only. If the downstream pressure $P_{2}$ is defined as zero, $\Delta P$ equals $P_{1}$ and the conductance is defined. In the case of a choked orifice, the conductance is

$$
F=11.7 \mathrm{KA}
$$

where $K$ varies from 1.3 to 1.7 as the orifice varies from a sharp-edged to a rounded entrance configuration.

\section{Combination of Vacuum Elements}

The concept of conductance $F$ was introduced by the analogy of vacuum to electrical circuit elements. If this analogy is extended, the net conductance of a parallel group of vacuum elements becomes

$$
F=F_{1}+F_{2}+\ldots+F_{n}
$$

The conductance of a series group of vacuum elements is

$$
\frac{1}{F}=\frac{1}{F_{1}}+\frac{1}{F_{2}}+\frac{1}{F_{3}}+\ldots+\frac{1}{F_{n}}
$$

and for a pumped system

$$
\frac{1}{S}=\frac{1}{S_{P}}+\frac{1}{F}
$$

where $S$ is the resulting system speed of a pump with speed $S_{p}$ which is exhausting through a system of conductance $F$.

The above relationships are true only if the vacuum circuit elements are linear and not a function of either the flow or the driving potential, and the conductance of an element is not affected by the presence of adjacent elements. The condition of linearity does not hold in the case of viscous flow due to the fact that the conductance is proportional to the average pressure in the element. If a pressure level, which can be corrected after the first iteration, is assumed, the difficulty regarding linearity can be overcome. The same procedure can be applied to pump speeds which also vary with changes in the pressure level.

The condition of a conductance unaffected by adjacent elements is seldom satisfied in vacuum systems. The many vacuum lines and other circuit elements such as traps, baffles, and pumps, which are normally in the order of 1 diameter in length, may be thought of as being principally in an entrance condition. As a result, the performance of such 
elements will be very sensitive to adjacent elements, both upstream and downstream.

The remainder of this chapter will deal with the determination of the overall conductance values for practical vacuum system configurations in the molecular-flow regime.

Ho (reference 11) laid the foundation for the concept of the probability factor a, while Harries (reference 12) and Oatley (reference 13) published, apparently independently, a concept which effectively used the Ho approach to calculate the conductances of short tubes and orifice combinations. Their concept was the major advance in the development of the probability approach. In 1960, Levenson, Milleron, and Davis (reference 10) used a Monte Carlo solution on a computer to solve for the transmission probability of important geometric shapes, several of which are presented in figures 5.5 through 5.10. As a result, for the first time, the necessary data for many cases of complex conduction became available. Pinson and Peck (reference 14) made further advances to the basic approach and, in addition, used the Monte Carlo technique to compute other configurations.

A redefinition of the term "probability" and its relationship to conductance is appropriate at this time prior to discussing various probability techniques.

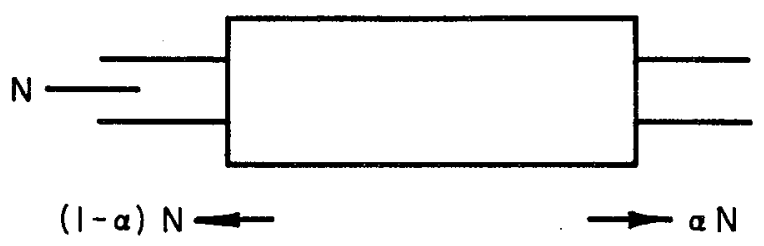

Figure 5.12.-Black-box flow element containing two separate apertures where $N$ molecules enter one aperture, $a N$ molecules leave the other aperture, and gas flow through the box is governed by $Q=K A a P$.

Figure 5.12 illustrates a black box which contains two separate apertures. If $N$ molecules enter one aperture, $a N$ molecules eventually will leave through the other aperture, while $(1-a) N$ will return to the original aperture. The fraction $a$, the transmission probability, may depend on the directional distribution of the incoming molecules. In many configurations the incoming molecules will be uniformly distributed over all viewing angles. If it is assumed that both uniform distribution of molecules and molecular flow exist, the incident $N$ molecules will be proportional to both the pressure and the inlet area. The flow of gas through the black box will be governed by

$$
Q=K A a P
$$


The flow of gas through a component is related by conventional vacuum usage to the pressure differential across the component; i.e.,

$$
Q=F \Delta P
$$

where $F$ is the conductance.

If it is assumed that the gas flows in a single direction for which the exhaust pressure is zero, a comparison of the above equations indicates that conductance is proportional to the product of probability and inlet area. Since probability is independent of the inlet area, the basic expressions describing it can be written as functions of geometrical shape only. As a result, probability is a more basic consideration than conductance and is better understood in terms of the phenomena occurring in molecular collisions.

\section{FLOW OF GAS THROUGH CYLINDRICAL TUBES}

Reference 13 describes a method of combining the probabilities $a_{1}$ and $a_{2}$ of two tubes in series as illustrated in figure 5.13. A portion of this description states:

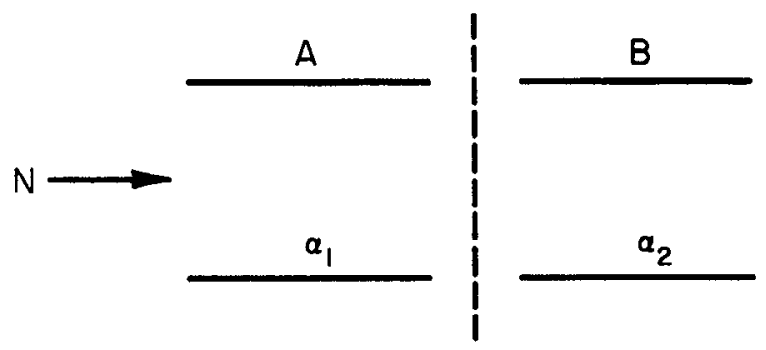

Figure 5.13.-Determining the conductance probability fac. tor $a$ of two tubes in series experiencing molecular flow;

$$
\frac{1}{a}=\left(\frac{1}{a_{1}}+\frac{1}{a_{2}}-1\right)
$$

Of the $N$ molecules entering $A, N a_{1}$ pass into $B$ and $N\left(1-a_{1}\right)$ are turned back to the reservoir from which they came. Of the $N a_{1}$ entering $B, N a_{1} a_{2}$ emerge from the right-hand end of $B$, while $N a_{1}\left(1-a_{2}\right)$ return to $A$. Of these, $N a_{1}\left(1=a_{2}\right)$ $\left(1-a_{1}\right)$ enter $B$ once again and $\mathrm{Na}_{1} a_{2}\left(1-a_{1}\right)\left(1-a_{2}\right)$ emerge from the right-hand end of $B$. Proceeding in this way, we see that the total number of molecules passing right through the tube from left to right is given by the infinite series

$$
\begin{aligned}
N a & =N a_{1} a_{2}\left[1+\left(1-a_{1}\right)\left(1-a_{2}\right)+\left(1-a_{1}\right)^{2}\left(1-a_{2}\right)^{2}+\ldots\right] \\
& =\frac{N a_{1} a_{2}}{a_{1}+a_{2}-a_{1} a_{2}}
\end{aligned}
$$


whence

$$
\frac{1}{a}=\left(\frac{1}{a_{1}}+\frac{1}{a_{2}}-1\right)
$$

A comparison of equations (5.28) and (5.26) with the correct results obtained by Clausing will permit the determination of the probability of transmission for two short tubes in series. Table 5.8 presents the results of this comparison.

TABLE 5.8.-Comparison of Calculated Values of a From Clausing, Reference 13 and Equation (5.26)

\begin{tabular}{|c|c|c|c|c|c|c|c|c|}
\hline \multirow{2}{*}{$\ell_{1} / a_{1}$} & $\ell_{2} / a_{2}$ & \multicolumn{3}{|c|}{ Values from Clausing } & \multicolumn{2}{c|}{$a$ calculated } & \multicolumn{2}{c|}{ Percent error } \\
\cline { 3 - 8 } & & $a_{1}$ & \multicolumn{1}{c|}{$a_{2}$} & \multicolumn{1}{c|}{$a$} & Eq. (5.26) & Eq. (5.28) & Eq. (5.26) & Eq. (5.28) \\
\hline 0.5 & 0.5 & 0.801 & 0.801 & 0.672 & 0.401 & 0.669 & 40.3 & 0.5 \\
1.0 & 1.0 & .672 & .672 & .514 & .336 & .507 & 34.6 & 1.4 \\
4 & 4 & .359 & .359 & .232 & .180 & .219 & 22.4 & 5.6 \\
8 & 8 & .232 & .232 & .137 & .116 & .131 & 15.3 & 4.4 \\
20 & 20 & .114 & .114 & .061 & .057 & .060 & 6.6 & 1.7 \\
4 & 1 & .359 & .672 & .315 & .234 & .305 & 25.7 & 3.2 \\
4 & 4 & .359 & .359 & .232 & .180 & .219 & 22.4 & 5.6 \\
4 & 8 & .359 & .232 & .172 & .141 & .164 & 18.0 & 4.6 \\
4 & 16 & .359 & .137 & .114 & .099 & .110 & 12.7 & 3.1 \\
\hline
\end{tabular}

It is evident that the error does not exceed 6 percent for any pair of tubes for equation (5.28).

The concept of probability can be used directly to calculate the values of $a$ for any tube length (figure 5.14). A tube of length $\ell$ is divided

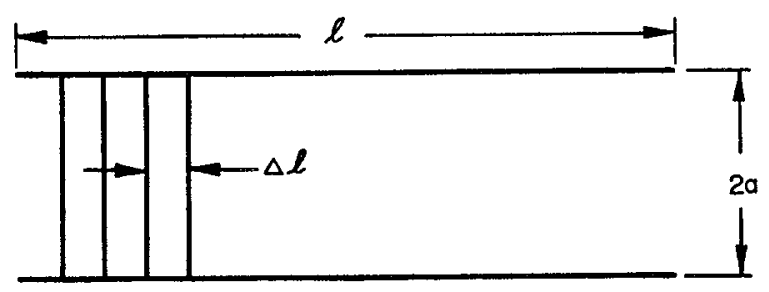

FIGURE 5.14.-Configuration by which the concept of probability can be used directly to calculate the values of a for any length of tube.

into $n$ increments each of length $\Delta \ell$, where $\Delta \ell$ is very small relative to the radius $a$. At this point, it is necessary to obtain a probability expression for the combination of a large number of identical elements and an expression for the individual probability of each element. 
Initially, if $a_{1}$ equals $a_{2}$, equation $(5.28)$ becomes

$$
\alpha=\frac{\alpha_{1}}{1+\left(1-a_{1}\right)}
$$

With the addition of a third element, the expression becomes

$$
a=\frac{a_{1}}{1+\left(1-a_{1}\right)^{2}}
$$

Therefore, for $n$ elements

$$
a=\frac{a_{1}}{1+\left(1-a_{1}\right)\left(n-a_{1}\right)}
$$

Since $\Delta \ell / a$ is very small, $a_{1}$ will approach unity as $n$ becomes very large and the equation becomes

$$
a=\frac{1}{1+\left(1-a_{1}\right) n}
$$

Equation (5.29) is the desired expression for many identical elements in series.

The expression for the probability of the individual elements can be obtained using the configuration given in figure 5.15, where a tube of

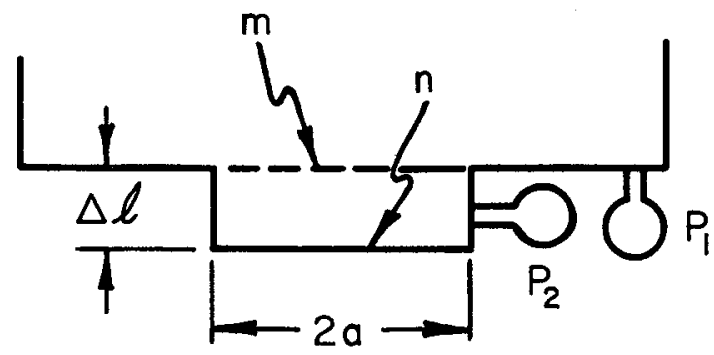

Figure 5.15.-Contiguration for determining the prob. ability of the individual elements of figure 5.14, where a tube of radius $a$ and length $\Delta l$ is connected to a very large reservoir;

$$
a=\frac{1}{1+\frac{l}{2 a}}
$$

radius $a$ and length $\Delta \ell$ is connected to a very large reservoir. When the gas flow equals zero, the pressure is uniform throughout the system; i.e., $P_{2}=P_{1}$. At any point in such a system, molecules are arriving in equal quantities from any direction. Therefore, when $\Delta \ell$ is very small relative to $a$, half of the molecules arriving at surface $\Delta \ell$ must be coming directly through surface $m$, and half of the molecules striking surface $\Delta \ell$ must be coming from surface $n$. If surface $n$ is assumed to be a perfect pump, pressure $P_{2}$ must be reduced to $P_{1} / 2$. 
To compute the probability that a molecule crossing surface $m$ will be pumped by surface $n$ rather than being returned to the reservoir, let $\Phi$ equal the rate of molecules per unit area striking surface $m$. This value of $\Phi$ corresponds to pressure $P_{1}$. The incidence rate of molecules on surface $m$ equals

$$
\phi=\pi a^{2} \Phi
$$

The incidence rate per unit area on surface $\Delta \ell$ is

$$
\phi_{\Delta \ell}=2 \pi a \Delta \ell(\Phi / 2)
$$

The difference between $\phi$ and $\phi_{\Delta \ell}$ is the rate of molecules which are directly transmitted to surface $n$. Half of the molecules which are diffusely reflected in a cosine distribution upon striking $\Delta \ell$ will pass through both surface $m$ and surface $n$. The combination of the expressions for $\phi$ and $\phi_{\Delta \ell}$ yields the rate of molecules transmitted

$$
\phi a=\phi-\phi_{\Delta \ell}+\phi_{\Delta \ell} / 2
$$

Therefore, the fraction of molecules transmitted, or the probability of transmission is

$$
\begin{aligned}
a_{\Delta \ell} & =\frac{\phi a}{\phi} \\
& =1-\frac{\Delta \ell}{2 a}
\end{aligned}
$$

The combination of the expression for $a_{\Delta \ell}$ and equation (5.29) for many elements in series for which

$$
n=\ell / \Delta \ell
$$

yields the transmission probability of the tube

$$
a=\frac{1}{1+\frac{\ell}{2 a}}
$$

Figure 5.16 is a plot of equation (5.30) as a function of $\ell / a$. The plot also contains a graph of Clausing's data for the same conditions. It can be seen that for the condition of short tubes, the agreement between the two sets of values is quite good. However, as $\ell / a$ becomes greater than 2, an error begins to appear. It was assumed for this discussion that the probability of each element in series is the same as the probability for any other element.

Dayton (reference 15) and others have commented that the beaming effect of gas passing through one element will affect the transmission probability of the gas through the next element.

The beaming effect is illustrated by figure 5.17 in which a gas is flowing through a tube in the presence of a pressure gradient. If an observer were placed at point $P$ at the tube inlet and looked back into the cham- 


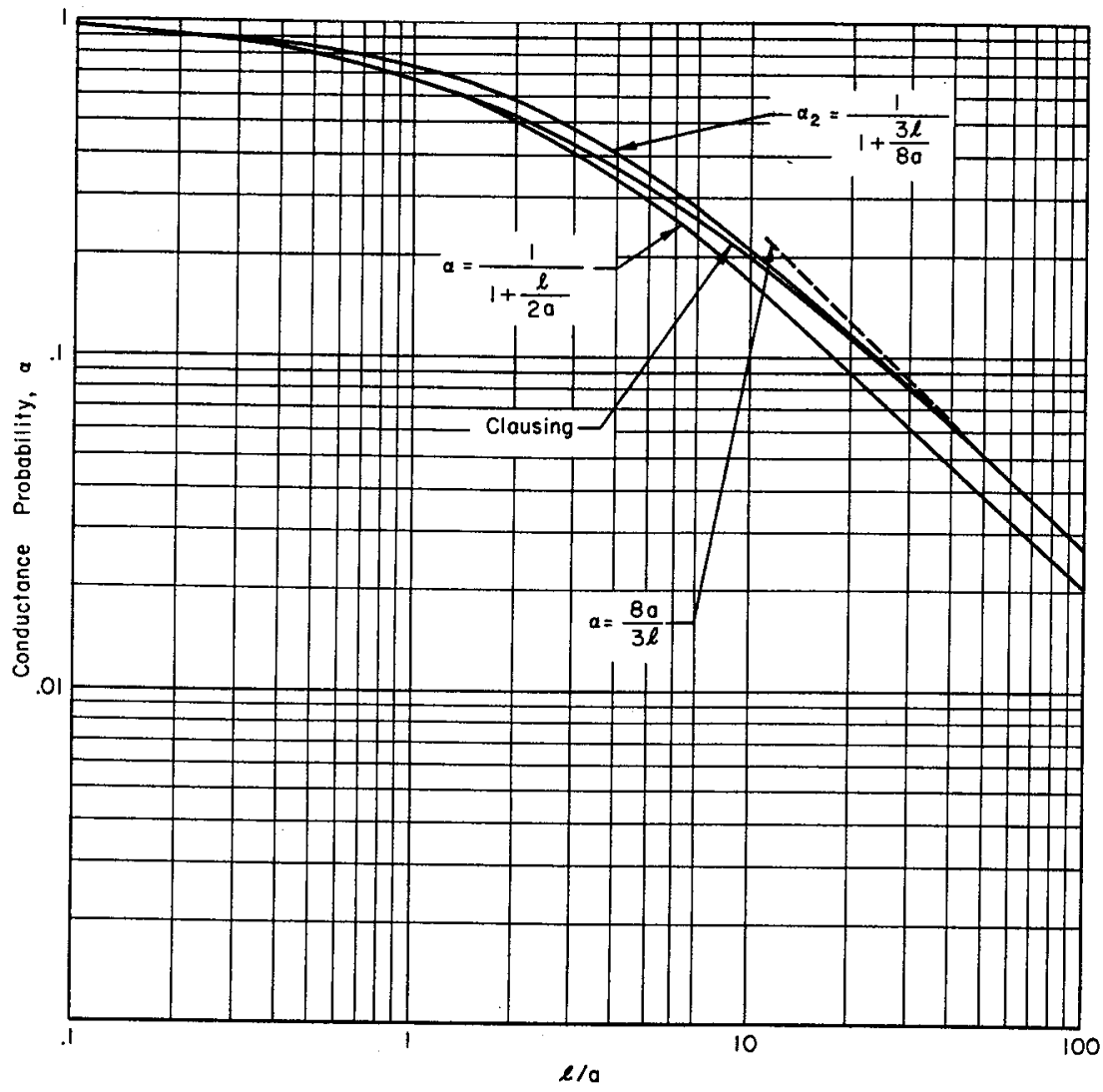

Figure 5.16.-Plot of equation (5.6), $a=\frac{1}{1+\frac{3 \ell}{8 a}}$, and equation (5.30), $a=\frac{1}{1+\frac{\ell}{2 a}}$ both approximations to Clausing values for short tube conductance probabilities.

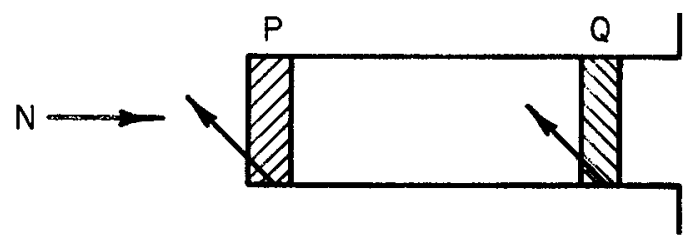

Figure 5.17.-Beaming effect in a tube at a point $Q$ further down the tube and looking in the direction of a reservoir. $a$ for point $Q$ differs from $a$ at point $P$ due to a pressure gradient. 
ber, he could see molecules arriving at equal rates from any given solid angle. The derivation above was made for this condition. As the observer moves to point $Q$ further down the tube and again looks toward the main reservoir, he will note a change in the condition. Due to the pressure gradient, molecules will not arrive in equal quantities from all directions. The molecular flux per unit angle within the solid angle subtended by the walls of the tube must decrease with the pressure along the tube. As a result, the probability of transmission at element $Q$ is composed of uniformly distributed molecules from the solid angle subtended by the inlet and nonuniformly distributed molecules from the pressure gradient along the wall of the tube. In a manner identical to the previous derivation, an integration over the tube length will yield the number of molecules which hit the tube at point $Q$ as well as along an incremental wall area at point $Q$. This procedure would duplicate the results obtained by Clausing.

Equation (5.6)

$$
\begin{aligned}
a & =K^{\prime} \\
& =\frac{1}{1+\frac{3}{8} \frac{\ell}{a}}
\end{aligned}
$$

is a popular approximation of Clausing's findings. Equation (5.6) frequently is used to compute the conductance of short tubes. However, it becomes asymptotic to the Clausing results only for long tubes. A comparison of equation (5.6) to equation (5.30) for a long tube terminating in a perfect pump indicates that the pressure on the tube surface of the last incremental area is only three-eighths of the pressure incident on the exit plane of the tube, rather than the factor of one-half derived for the inlet. Figure 5.18, which is a plot of both equations, shows that the two expressions are asymptotic to the Clausing data at large and small values of tube length. Molecules which have just passed through the inlet orifice, where the distribution is uniform over the entire solid angle, govern the gas flow for small values of $\ell / a$. At large values of $\ell / a$, the flow is related to the molecules reflected from the wall where a linear pressure gradient exists.

It is often and erroneously believed that gas flow in the molecular regime is comprised of two contributions: an entrance loss and a tube drop. Since the expression $3 \ell / 8 a$ in the denominator of equation (5.6) is identical to the drop for long tubes, it is associated with the tube drop. A value of unity in the denominator is associated with the so-called entrance loss. The literature frequently states that the use of the expression

$$
\frac{1}{F}=\frac{1}{F_{1}}+\frac{1}{F_{2}}
$$


yields erroneous results for short tubes due to the fact that the loss has been included twice. Dayton has pointed out that while the modified form of the expression

$$
\frac{1}{F}=\frac{1}{F_{1}}+\frac{1}{F_{2}}-1
$$

eliminates the duplication of entrance loss, it is still not accurate due to the beaming effect of gas leaving the first tube. This effect modifies the gas-flow pattern through the second tube (figure 5.18).

The true Clausing findings do not follow an expression of the form

$$
a=\frac{1}{1+C\left(\frac{\ell}{a}\right)}
$$

Therefore, the flow cannot be divided into an entrance loss and a tube drop. Rather, the plot of Clausing's data is a transition from the derived

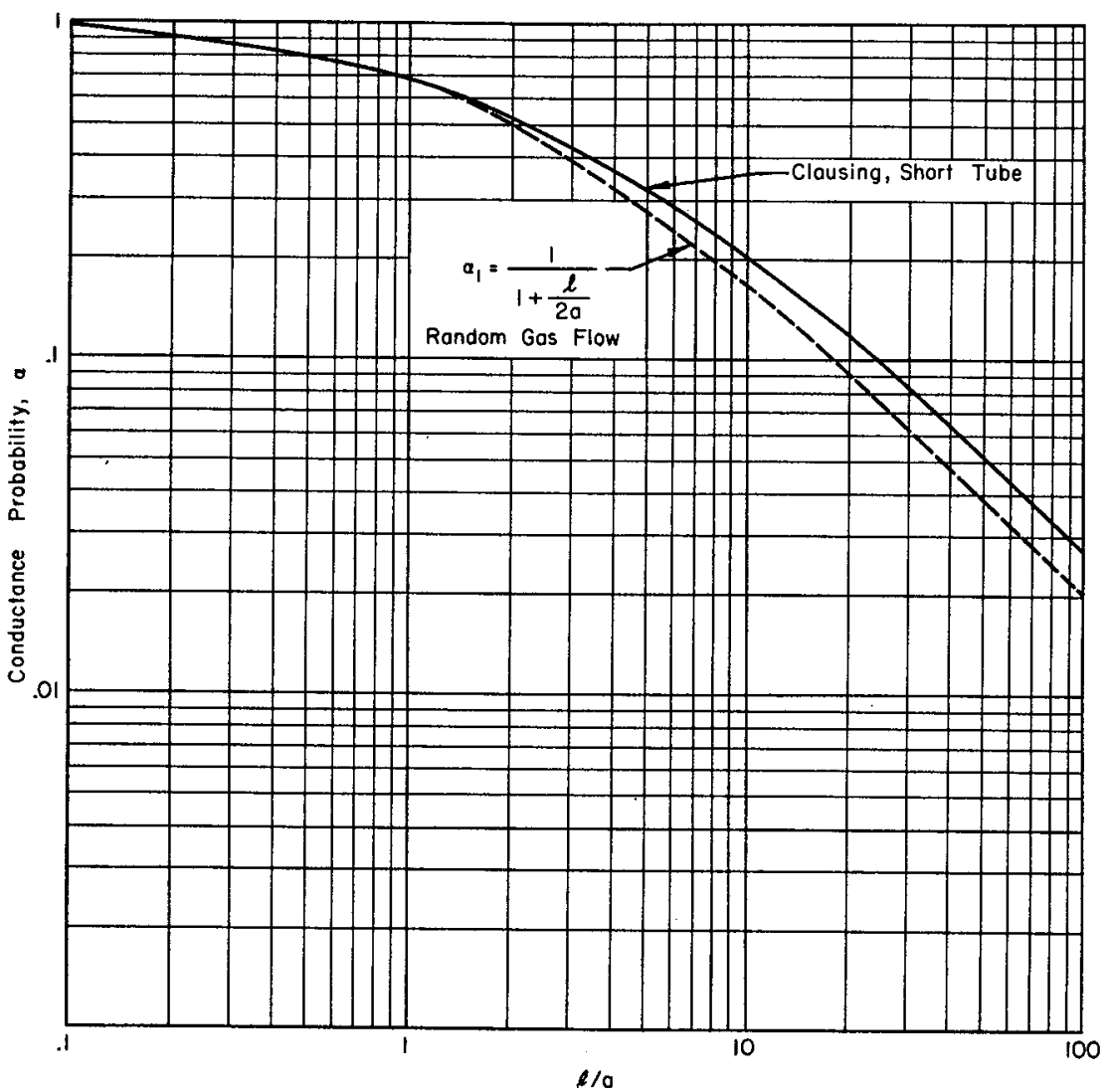

FiguRE 5.18.-Comparison of molecular conductances for short tubes, where equation (5.30) is plotted versus $\ell / a$. 
probability expression for short tubes and random gas to the expression for a long tube for which the molecular flux pattern is controlled by the existing pressure gradient along the tube.

These considerations will assist in the calculation of more complex configurations involving randomizing elements in tubes or the combining of elements which exhibit intermediate-flow conditions.

Figure 5.19 illustrates an example of a more complex configuration; i.e., a tube containing two angled-fin arrays. If it is assumed that each

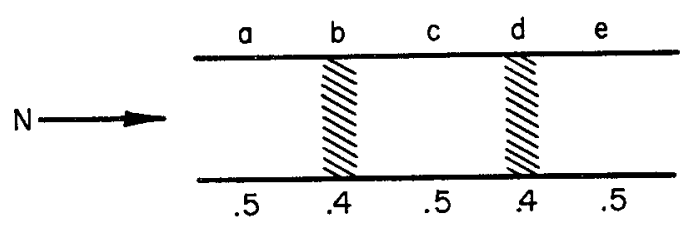

Figure 5.19.-Determination of a for a tube containing two angled fin arrays, where equation (5.28) gives the conductance probability for each section and the total $a$ is given by

$$
\frac{1-a}{a}=\frac{1-a_{1}}{a_{1}}+\frac{1-a_{2}}{a_{2}}+\ldots+\frac{1-a_{n}}{a_{n}}
$$

of the tube sections a, c, and e have a length equal to the diameter, equation (5.28) will yield a transmission probability for each section of 0.5 . A value of 0.4 is chosen for the transmission of each of the fin arrays. The conventional solution of adding the reciprocals of the conductance yields a net probability of 0.09 . Alternately, the three tube sections could be added together, using the Clausing value for the $\ell / a$ ratio of 6 in place of 2 . To this sum is added the resistances due to the two angled fins. As a result, the tube would have a probability of 0.28 , while the two fin sections would have a combined value of 0.2 for a net probability of 0.116 . However, it can be seen from the previous discussion that the fin sections randomize the flow. Therefore, there are five individual segments in figure 5.19 , each of which may be treated as having an inlet flow of uniform distribution. That is, an observer at a point in section e, looking back toward the inlet, will see a uniform distribution of molecules coming from the fins at $d$. A generalization of equation (5.28) yields

$$
\frac{1-a}{a}=\frac{1-a_{1}}{a_{1}}+\frac{1-a_{2}}{a_{2}}+\frac{1-a_{3}}{a_{3}}+\ldots+\frac{1-a_{n}}{a_{n}}
$$

Equation (5.31) yields an answer of 0.143 to the above problem. This value is considerably greater than either of the two previous answers. Unfortunately, there are no experimental data or Monte Carlo calculations available to check these results, but as the last calculation more 
properly accounts for the actual conditions, it merits the greater confidence. Always exercise care to interpret what gas distribution exists in order to determine the proper approach to the solution of the problem.

Another common problem which is almost impossible to solve from normal conductance approaches is the resistance to flow of an elbow in a tube section. However, this problem may be readily understood by reference to the composite curve of figure 5.18. The Clausing data are applied to the inlet tube to the elbow. Since the elbow randomizes the flow, the second half of the elbow must be treated as a new pipe. Thus, the Clausing data can be used again for the second half of the elbow. The two sections can then be combined, using the expressions given in reference 13. The amount of restriction offered by the elbow depends on the length of the two sections of tube; i.e., on how much directional gas flow accumulated before the elbow caused the randomization.

A more complicated configuration is the case of a number of elbows connected in series. An observer looking back toward the inlet from any section sees the side wall of the previous tube rather than the pressure gradient along the tube. In this sense, each section sees a random gas inlet. The net probability of transmission through the group of short elbows is given by

$$
a=\frac{1}{1+\ell / 2 a}
$$

where $\ell$ is the total length of tube as measured along the tube axis. A tube of the same length, with no elbows, will have a probability given by the Clausing curve. The ratio of probabilities (or conductances) for very long sections will be $8 / 3$ to 2 , or a net discrepancy of 4 to 3 . In a similar fashion, a tightly coiled tube will not follow the Clausing curve, but will follow the curve for random gas flow.

\section{TRAPS AND BAFFLES}

Many modern traps and baffles contain elements composed of angled fins, chevrons, and herringbone sections. Reference 10 presents an analysis of the fin and chevron arrangements for angles of $30^{\circ}, 45^{\circ}$, and $60^{\circ}$, using the computerized Monte Carlo approach (figs. 5.5 and 5.6). The probability of two-element chevrons and three-element herringbone arrays can be determined from equation (5.31), on the basis of the transfer probability of angled fins.

$$
\begin{aligned}
& a_{\text {cher }}=\frac{a_{\text {fin }}}{\left(2-a_{\mathrm{fin}}\right)} \\
& a_{\text {herr }}=\frac{a_{\mathrm{fin}}}{\left(3-2 a_{\mathrm{fin}}\right)}
\end{aligned}
$$

Table 5.9 lists chevron transfer probabilities calculated from equation (5.33), using $a_{\text {fin }}$ from figure 5.5. The transfer probability is compared 
with the Monte Carlo values from figure 5.6, and the values calculated from a simple addition of conductances, equation (5.29). The table indicates that equation (5.33) yields the better of the two approximations to the Monte Carlo solution. In this case, the Oatley approach has overcompensated for the error of equation (5.26).

Table 5.9.-Chevron Transfer Probabilities From Equation (5.33)

\begin{tabular}{|c|c|c|c|c|c|c|}
\hline \multirow[b]{2}{*}{$\begin{array}{c}\text { Fin angle, } \\
\text { deg }\end{array}$} & \multirow[b]{2}{*}{$\begin{array}{c}a_{\text {fin }} \\
\text { (fig. 5.5) }\end{array}$} & \multicolumn{3}{|c|}{ Chevron probability } & \multicolumn{2}{|c|}{ Percent error } \\
\hline & & $\begin{array}{l}\text { Monte } \\
\text { Carlo }\end{array}$ & Eq. $(5.33)$ & Eq. $(5.26)$ & Eq. (5.33) & Eq. (5.26) \\
\hline $30 \ldots$ & 0.34 & 0.19 & 0.205 & 0.17 & +8 & -10.5 \\
\hline 45 & .43 & .25 & .274 & .215 & +9.5 & -14.0 \\
\hline 60 & .46 & .278 & .298 & .23 & +7.5 & -17.5 \\
\hline
\end{tabular}

Chevron traps provide another example where misunderstanding can lead to erroneous results. Figure 5.20 represents a chevron section in a

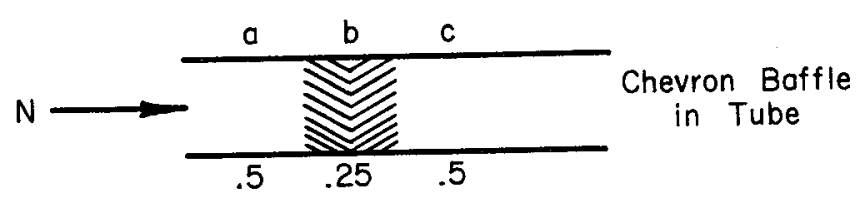

Figure 5.20.-Determination of $a$ for a chevron baffle section in a tube using equation (5.31).

tube where the length-to-diameter ratio equals unity for each of the sections, the probability of transmission equals 0.5 for each section, and chevron section probability equals 0.25 . The combined net probability is 0.16 . If the arrangement is examined, it is apparent that the limiting item is the chevron section. Figure 5.21 illustrates the enlargement of

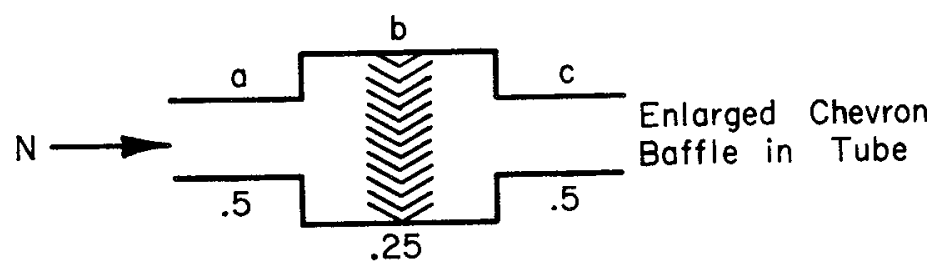

Figure 5.21.-Determination of $a$ for an enlarged chevron baffle in a tube. This configuration does not substantially alter the net probability of flow through the combination from the value obtained for figure $\mathbf{5 . 2 0}$. 
the chevron section in the tube. The enlarged chevron section has a higher conductance as an individual item. Its probability as an individual item can be determined by reference to the system of figure 5.20 where molecules which reach the end of the tube necessarily must enter the chevron array. In the expanded arrangement, a certain fraction of the molecules reaching the end of the tube may not enter the chevron because of collisions with, and return from, the outer wall containing the chevrons. Those molecules which do enter the ehevron still have the 0.25 probability of transmission. However, all the 0.25 molecules transmitted to the chevron do not enter the exit tube nor does all the 0.75 fraction reflected backward enter the original inlet tube. While the detailed mathematical treatment is quite complex, it should be apparent that increasing the size of a fixed geometrical array (limited by a smaller size inlet and outlet aperture) does not alter substantially the net probability of flow through the combination. A more realistic situation for vacuum systems in general, and large space simulators in particular, is the case where only one end of a geometrical array is limited by a smaller size aperture. Pinson covered this situation in part, and figure 5.22 illustrates this case. An optically tight elbow trap con-

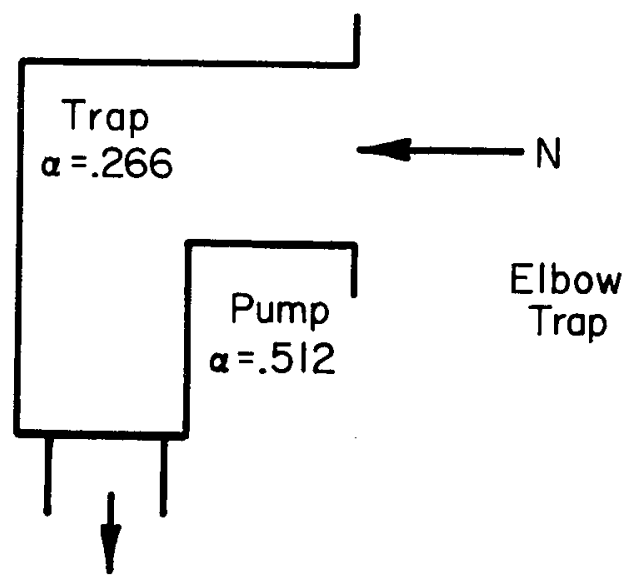

Figure 5.22.-Determination of a for an optically tight elbow trap which connects a large vacuum chamber and a diffusion pump, where only one end of this geometrical array is limited by a smaller size aperture.

nects a large vacuum chamber and a diffusion pump. The transmission probability of each leg of an optically tight trap should be 0.42 from Clausing's curve. If the two trap sections are combined, the transmission probability is 0.266 . This value applies regardless of the size of the trap. 
Since the pump may be regarded as a section of tube with a particular transmission probability, the solution may be obtained for two segments of different areas. To obtain a numerical solution, the pump probability must be known. A 35-inch diffusion pump with a rated speed of 50000 liter/sec will have an apparent pumping probability of 0.688 ; i.e., the ratio of the speed to the speed of an orifice of the same area. However, since commercial pumps are rated by means of a pressure measurement normal to the gas flow, a correction must be applied to obtain the true pumping probability. Such a correction may be derived by probability (chapter 4 )

$$
a=\frac{a^{\prime}}{1+\frac{a^{\prime}}{2}}
$$

where $a^{\prime}$ is the apparent probability and $a$ is the true probability. The true probability for the 35 -inch pump is reduced to 0.512 . The net speed (trap and elbow) can be computed by combining the true pump probability and that of the optically tight elbow trap with the proper corrections made for the area reduction on leaving the trap and entering the pump, per Oatley's method. Figure 5.23 presents these data in two curves. The lower curve is the value of speed available for exhausting a large chamber, while the upper curve is the speed which would be measured by a vacuum gage located at the entrance to the trap, normal to the gas flow. The curves indicate that increasing the trap size does

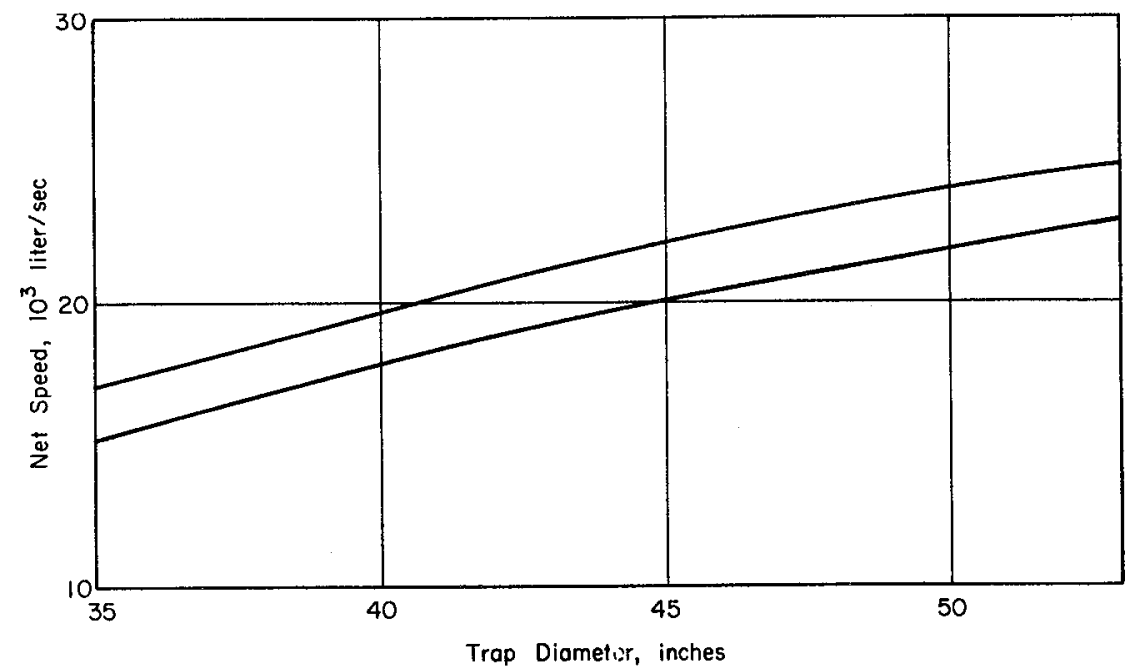

FiguRE 5.23. - Net pumping speed for a 35-inch pump, rated at 50000 liter/sec, and an elbow trap obtained by combining the true pumping probability, equation $(5.35)$ and that of the optically tight elbow trap. 
result in a large increase in the net speed, if the trap has only one limiting aperture; i.e., that entering the pump. In addition, the relative values of the numbers are of interest when it is realized that there is a large reduction in system speed for what might appear to be reasonably sized traps, and that the overall speed is significantly less than the rated speed of the pump alone.

Now that the basic relationships governing gas flow in system components have been presented and explained, the groundwork has been laid for a discussion of pumping systems to follow in chapter 6 .

\section{REFERENCES}

1. Dushman, S.: Scientific Foundations of Vacuum Techniques. Second ed., J. M. Lafferty, ed., John Wiley \& Sons, Inc., 1962.

2. Clausing, P.: Über die Strömung sehr Verdünnter Gase durch Röhren von beliebiger Länge. Ann. Physik, Bd. 12, Heft 8, 10 März, 1932, pp. 961-989.

3. Shapiro, A. H.: Dynamics and Thermodynamics of Compressible Fluid Flow. Ronald Press Co. (New York), 1953.

4. Bird, R. B.; Stewart, W. E.; and Lrghtroor, E. N.: Transport Phenomena. John Wiley \& Sons, Inc., 1960.

5. Kennard, E. H.: Kinetic Theory of Gases With an Introduction to Statistical Mechanics. First ed., MeGraw-Hill Book Co., Inc., 1938.

6. Dong, Walter: Vacuum Flow of Gases Through Channels with Circular, Annular, and Rectangular Cross Sections. UCRL 3353, U.S. Atomic Energy Commission Report, Apr. 1956.

7. Dong, Walter; and Bromlex, L. A.: Vacuum Flow of Gases Through Channels With Circular, Annular, and Rectangular Cross Sections. Trans. of the Eighth National Vacuum Symposium and the Second International Congress on Vacuum Science and Technology, vol. II. L. E. Preuss, ed., Pergamon Press, 1962, pp. 1116-1132.

8. Pollakd, W. G.; and Presentr, R. D.: On Gaseous Self-Diffusion in Long Capillary Tubes. Phys. Rev., vol. 73, no. 7, Apr. 1, 1948, pp. 762-774.

9. Turnbuli, A. H.; Barton, R. S.; and Rivière, J. C.: An Introduction to Vacuum Technique. John Wiley \& Sons, Inc., 1963.

10. Levenson, L. L.; Mrtheron, N.; and Davis, D. H.: Optimization of Molecular Flow Conductance. Trans. of the Seventh National Symposium on Vacuum Technology, C. R. Meissner, ed., Pergamon Press, 1961, pp. 372-377.

11. Ho, T. L.: Multiple Nozzle Diffusion Pumps. Rev. Sci. Instr., vol. 3, Mar. 1932, pp. 133-135.

12. Harries, W.: Molecular Flow of Gases Through Tubes in Series. Z. Angew. Phys., Bd. 3, Heft 8, Aug. 1951, pp. 296-300.

13. Oatrey, C. W.: The Flow of Gas Through Composite Systems at Very Low Pressures. Brit. J. Appl. Phys., vol. 8, Jan. 1957, pp. 15-19.

14. Pinson, J. D.; And Peck, A. W.: Monte Carlo Analysis of High Speed Pumping Systems. Trans. of the Ninth National Vacuum Symposium, G. H. Bancroft, ed., The Macmillan Co., 1962, pp. 406-410.

15. Dayton, B. B.: Gas Flow Patterns at Entrance and Exit of Cylindrical Tubes. Trans. of the Third National Symposium on Vacuum Technology, E. S. Perry and J. H. Durant, eds., Pergamon Press, 1957, pp. 5-11. 
16. Moone, R. W.: Cryopurnping in the Free-Molecular Flow Regime. Trans. of the Eighth National Vacuum Symposium and the Second International Congress on Vacuum Science and Technology, vol. I. L. E. Preuss, ed., Pergamon Press, 1962, pp. 426-438.

17. Hamilton, D. C.; and Morgan, W. R.: Radiant-Interchange Configuration Factors. NACA TN 2836, 1952.

18. SANTELER, D. J.: A Graphical Solution for the Analysis of Vacuum System Performance. Trans. of the Second Vacuum Symposium, Committee on Vacuum Techniques, Inc. (Boston), 1956, pp. 31-41.

19. Bescher, N.: Thermal Analysis of Space Simulation Chambers. Trans. of the Ninth National Vacuum Symposium, G. H. Bancroft, ed., The Macmillan Co., 1962, pp. 253-259. 



\section{6}

\section{Pumping Systems}

\section{INTRODUCTION}

7 HIS CHAPTER presents a comprehensive examination of the various classes and types of vacuum system pumps, including the basic characteristics of each type. In addition, the grouping of pumps to form pumping systems is investigated, with advantages and disadvantages noted for various applications.

There are several basic pumping methods which can be employed to produce and maintain vacuum conditions. It is a common procedure in the design of ultra-high-vacuum systems to use more than one of these pumping methods in the same system. The following list briefly describes the basic pumping methods commonly employed.

\section{Positive Displacement Pumps}

Positive displacement pumps operate by mechanically isolating the gas from the vacuum system. The gas is then compressed. Examples of this type of pump are the rotary-oil-sealed pumps and Roots blowers.

\section{Vapor Stream Pumps}

In this application, gas molecules are forced to a higher pressure level by gaining added momentum through collisions with a high-velocity jet. For high-vacuum applications, the jet is composed of a condensable vapor with a high molecular weight, such as oil or mercury. Water vapor can be used when only a rough vacuum is required. Diffusion pumps and ejectors fall into the vapor-stream- pump classification.

\section{Chemical Pumps}

The gas is chemically combined with an active getter, such as barium or titanium, to form a low-vapor-pressure compound.

\section{Ion Pumps}

In this case, the gas molecules in the system are ionized and electrically accelerated into an anode in which they are buried or chemically trapped. The chemical- and ion-type pumps have been combined in the device known as the sputter-ion pump. 


\section{Adsorbent Pumps}

The gas molecules are removed from the system through adsorption on a surface-active material such as activated charcoal or zeolite. Adsorption is greatly enhanced if the temperature of the adsorbent is lowered.

\section{Cryogenic Pumps}

In this method, the gas molecules are removed from the system through condensation to a low vapor pressure on a cold surface. Cryogenic temperatures are required for the effective pumping of the noncondensable gases such as nitrogen and oxygen.

\section{Molecular Pumps}

The last method applies to high-vacuum mechanical pumps which provide added momentum to the gas molecules through molecular collisions with a high-speed surface or vane. Examples of this type of pump are the classical Gaede pump and the modern Welch-Pfeiffer turbomolecular pump (refs. 1-3).

Not all of the pumps which can be placed in the above categories are suitable for space-simulation applications. Among the acceptable pump types, rotary-oil-sealed pumps, Roots blowers, diffusion pumps, getter ion pumps, and adsorption pumps are discussed further in this chapter. Chapter 7 is devoted to the subject of cryogenic pumping.

\section{ROTARY OIL-SEALED PUMPS}

This type of positive displacement pump is used extensively for initial system exhaust and for backing other types of lower pressure pumps which are not capable of exhausting directly to atmospheric pressure. Commercially available rotary oil-sealed pumps may use rotary vanes, an eccentric rotor and vane, or an eccentric rotor and piston (ref. 4). Despite variations in design details, all of these pumps operate on the principle that gas which is taken into the chamber is compressed as the rotor advances. In addition, oil is admitted to the chamber in order to seal the sliding surfaces, and to minimize the clearance volume, thus to provide a large compression ratio. The gas pressure in the final stages of compression exceeds atmospheric pressure, and in conjunction with the oil is swept out of the chamber through some form of check valve.

Since the compression ratio is very large, a single-stage pump which is exhausting to atmosphere can produce a blankoff pressure of about 10 millitorr, while a two-stage pump can reach $10^{-4}$ torr. The rotary oil-sealed pumps normally operate at speeds of several hundred revolutions per minute. They are air cooled in the smaller sizes and water 
cooled in the larger sizes. Pumps with capacities ranging from a few tenths to over a thousand cubic feet per minute are available commercially. Price information as a function of pumping speed is given in figure 6.1, while typical performance curves are shown in figure 6.2 .

Rotary oil-sealed pumps are constant-volume-displacement-type pumps due to the fact that a fixed volume is swept out by the pump in each revolution. An increased gas-handling ability can be obtained by increasing the speed. However, a greater speed will cause more friction and wear, and will result in additional maintenance and a shorter pump life.

Pumping speed has been defined as the volume of gas at inlet pressure which passes through the pump per unit time. Although the physical volume which the pump rotor sweeps out per second is constant, the pumping speed is not constant and decreases with decreasing inlet pressure. Interior leakages in the pump contribute to the decreasing speed. These leaks may be in the form of gas dissolved in the pump oil, and leaks at the various sealing surfaces. Imperfect oil filling of the clearance volume, or a pressure drop in the inlet passages also contribute to decreasing pump speed.

When gases other than air are being pumped, the theoretical displace-

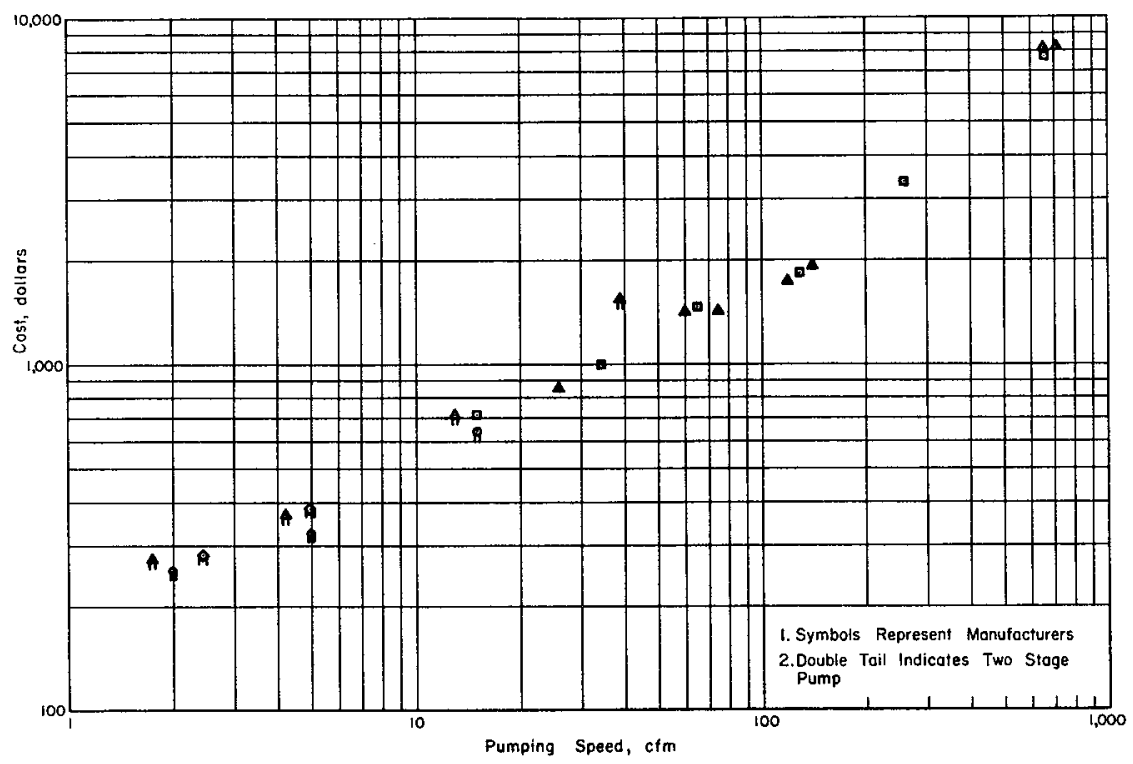

Ficure 6.1.-Cost data for rotary oil-sealed, positive displacement pumps, 1965 figures, plotted as a function of pumping speed, cubic feet per minute. 


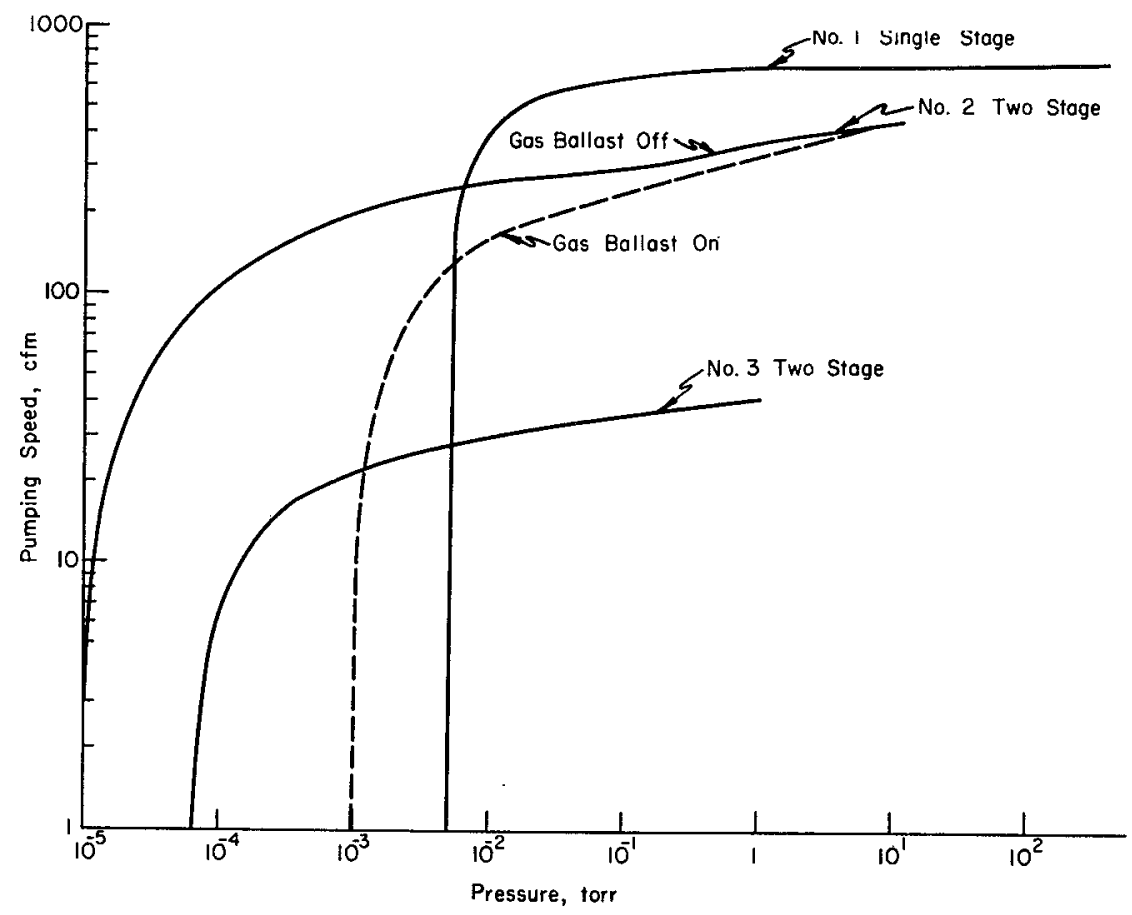

Figure 6.2.-Typical pumping-speed performance curves at selected pressures for some common rotary oil-sealed pumps.

ment remains the same. However, variations in solubility and internal leakage for the different gases will result in modifications of the pumping speed curve. Pump design differences determine the relative importance of the above factors for various pumps. Since there are no general speed corrections available, it may be assumed that the pumping speed for permanent gases is similar to the speed for air. The problems associated with condensable or highly soluble gases will be discussed in a later section.

The system ultimate pressure, which is defined as the lowest pressure that can be obtained in the absence of an external gas flow, is limited by real or virtual leakage, the vapor pressure of the pump oil and its decomposition products, and the presence of contaminants in the pump oil. Real leakage may be the result of poor shaft seals, improper inlet connections, or leakage within a stage such that gas is allowed to pass from the exhaust to the inlet during compression. Virtual leakage results from either outgassing of the inlet pump parts or the oil. Air has some degree of solubility in oil, and will be brought into the high-vacuum 
region with the oil. Reference 5, which discusses temperature and friction effects on the ultimate pressure, concludes that a considerable portion of the residual pressure is due to oil decomposition products produced by the intense local heating of friction and high contact pressure.

It is possible to measure two different ultimate pressures, depending on whether the permanent or the total gas pressure is considered. Since the pump manufacturer measures only the permanent gas pressure using a trapped McLeod gage, the result is a very low ultimate pressure. This is a true indication of the ultimate pressure which can be obtained when liquid nitrogen traps are used on a very clean system. When traps are not used, the vapor pressure of the pump fluid, its condensable decomposition products, and the existing contaminants of the oil must also be considered, since these factors result in a considerably higher ultimate pressure. In general, two-stage pumps should not be planned for use below pressures of 10 millitorr, while single-stage pumps should not be used below 100 millitorr. However, untrapped ultimate pressures of 2 and 20 millitorr, respectively, can sometimes be obtained.

\section{Pump Problems}

There are a number of areas where pumps may face a variety of problems. For example, it is frequently observed that when power is applied to a mechanical pump, the pump will not start. Possible causes for the failure to start are oil in the pump chamber and freezeup. In some commercial pumps, oil will enter and fill the chamber if the pump is stopped while under vacuum, or if it is allowed to sit for prolonged periods at atmospheric pressure. The rate of oil leakage back to the chamber varies with the pump type and may even vary between pumps of the same type. Some pumps are observed to fill with oil after being off for only a few seconds while under vacuum. The pump can be restarted by turning the power off and on quickly several times, or by hand rotating the pulley to discharge the oil. In some instances, the oil not only enters the pump chamber but passes through the pump and into the vacuum system due to the atmospheric pressure on the exhaust. If there is no reservoir available to catch the oil, it will continue to rise throughout the vacuum system. Since this problem does exist, a mechanical oil-sealed pump should never be stopped for an appreciable period under vacuum. The pump should be vented back to atmosphere as soon as it is stopped.

During unattended operation, this situation may unavoidably occur due to power failure, motor burnout, or V-belt breakage. Several dif- 
ferent methods can be employed which will provide protection against power failure. In pumps with a separate oil reservoir, a normally closed solenoid valve will shut off the oil supply. Solenoid valves can also be used to close off the pump from the rest of the system and to vent it to atmospheric pressure if the power is turned off. In some cases, it is advisable to add a reset relay to prevent the pump from restarting when the power returns. Complete protection can be provided by installing a sensing device on the pump shaft or pulley. Thus, if the pulley fails to rotate for any reason, the sensing device shuts off power to the pump, closes the pump from the system, vents the pump to atmosphere, and shuts off any additional parts of the system in order to bring about a safe condition.

Freezeup of the pump may occur if an improper oil does not provide adequate lubrication and allows the pump to overheat and expand. In addition, freezeup can also occur from chemical cracking of the oil or from alien materials entering the pump. Oil cracking will produce a varnish-type sludge which will cause binding if it deposits on the walls of the pump.

The difference between oil in the chamber and freezeup can be detected by rotating the pump pulley. True freezeup will usually require a complete pump overhaul. Alien materials in the pump may cause the cylinder or vanes to be scratched with the subsequent appearance of pump leakage and the loss of ultimate pressure. Inlet filters are added whenever glass particles or abrasive-type materials may be present. A rotary piston and plunger type of pump will withstand the action of abrasive materials better than the rotary vane pump. However, this advantage is offset by the increased vibration of the pump.

The most severe problem which normally faces mechanical pumps is that of contamination. The pump will contaminate the system, and vice versa. The contamination from the pump results from hydrocarbon pump fluids, where their vapor pressure and that of their cracking products at room temperature is in the range of several millitorr. The pump fluids will migrate from the pump back to and throughout the vacuum system, particularly at low pressures when the pump line is in the molecular flow region. The amount of hydrocarbon contamination transferred to the system is a function of the length of time that the pump line is connected directly to the system. Hydrocarbon contamination can be minimized with liquid nitrogen or absorbing traps, or by means of a gas bleed (chapter 10).

When the pump is handling vapors which are normally liquid at the pump temperature, the vapors may condense during the compression 
segment of the cycle and mix with the pump oil. The condensed liquid will circulate through the oil system back to the inlet high-vacuum region where it will reevaporate during the intake stroke. This action reduces the available pumping speed of the pump and raises its ultimate pressure. The contamination will continue to increase until the pressure is elevated to the partial pressure of the contaminating source. The pump has failed completely at this point and will continue to compress and evaporate the vapor only. When noncondensables which are directly soluble in the oil from the vapor phase are being pumped, the same type of contamination will occur. The most common source of pump contamination arises from water vapor originating from outgassing or dehydration processes in the vacuum system.

The basic determinant of whether a vapor will condense and contaminate the pump is a function of the ratio of the permanent gas to condensable vapor. Little or no contamination will result during compression if the total gas pressure exceeds atmospheric before the condensable vapor reaches its equilibrium vapor pressure at the prevailing temperature. It is apparent that when vapors such as water are being pumped, the pump must be protected. One method of protection is to heat the pump in order to raise the equilibrium vapor pressure to the point where there can be no condensation. A special oil is required which will assure proper viscosity at the elevated temperature. For higher temperatures, however, a separate purifying system may be required to handle the greater cracking and increased hydrocarbon contamination. Such a system removes the contaminated oil from the pump, circulates the oil through a stripping unit, and returns it to the inlet of the pump. The stripping unit can be used as a continuous process unit in line with the pump or as a batch-process unit. In the batch process, contaminated oil is removed and is processed on the separate stripping unit to remove the contamination. Another method of handling condensables such as water vapor calls for a trap in front of the pump to prevent the vapor from entering the pump. A third method is a permanent gas purge which may utilize one of three methods.

The Knox method involves blowing dry air into the discharge pipe between the exhaust valve and the separator tank to remove the contamination from the oil. A contaminated pump can be cleaned by a flow of dry gas or air into the inlet and through the pump. In many pumps, the nature of the oil circulation is such that a stagnant pool of oil will remain in the side of the reservoir and will remain contaminated even after a good vacuum level has been attained. In this case, a much better vacuum level will result immediately after the gas flow is turned 
off. However, as the pump continues to operate, the pressure will rise as the contaminated oil interdiffuses with the clean oil. This effect provides a means of testing for pump contamination. For example, if a pump will not pump below $30 \mu$, it is desirable to know whether this limit is due to leakage or contamination. Air is allowed to bleed into the inlet to raise the operating level of the pump. The gas is turned off after a few minutes of bleeding and the pressure is observed as a function of time. If the difficulty is due to a leak, the pressure will level at the previously observed value. If contamination is the cause of the difficulty, the pressure will overshoot to a much lower value and will rise to the previous blankoff pressure.

A similar situation exists in many manufacturing uses of mechanical pumps. That is, during the initial evacuation, the high flow of air through the pump prevents contamination. However, the permanent gas flow becomes negligible as the end of the process approaches, and water vapor is the predominant gas entering the pump. Condensation occurs at this time, and the pump begins to become contaminated. During the next cycle the high-air flow again attempts to remove the contamination. The pump eventually reaches an equilibrium state where the amount of water removed at the beginning of each cycle is just equal to that which is added at the end of the cycle. In some instances, the high-air-flow rate is not sufficient to remove the vapor added, and the pump slowly increases in contamination to the point that its pressure is too high for the particular application. When this occurs, it is necessary to change the oil or to remove the contamination by one of the previously discussed methods.

A better purge method is to introduce the gas into the pump chamber or to the interstage vacuum of a two-stage pump rather than into the pump inlet. The first method, which was developed by Gaede, is known as gas ballast and is available on many commercial pumps. The Gaede method will be discussed in a following section. The second method, the introduction of the gas into the interstage vacuum, can be accomplished on most two-stage pumps by removing a plug which connects to this region or by drilling a hole through the wall of the pump. A filter must be added to the air intake of the pump to avoid the intake of small particles which may score the cylinders or vanes. The latter method is not as efficient as the gas ballast or Gaede method, but non-gas-ballast pumps can be readily converted to a semiballast condition. A converted pump will run at a reasonably high-air-purging rate with a pressure increase at the inlet of only a few millitorr.

Gas ballast permits the prevention or removal of vapor contamination in a mechanical pump by means of a gas purge introduced directly into 
the pump chamber. The gas is taken into the pump chamber through the inlet. As this volume of gas moves through the pump, a check valve opens, prior to the start of compression, to admit atmospheric gas which mixes with the gas being pumped. As a result, the ratio of condensable to noncondensable gas is changed. No condensation occurs during the compression stroke due to the more favorable ratio.

The following example illustrates the discussion. It is assumed that the vacuum system pressure is 20 millitorr, and is composed of equal proportions of nitrogen and water vapor (table 6.1). The gas mixture is taken into the pump chamber where it must be compressed to a total pressure of approximately 1000 torr before it can be exhausted to the atmosphere. The equilibrium water vapor pressure is assumed to be 50 torr at the pump temperature. To obtain a total of 1000 torr, the nitrogen pressure must be 950 torr. These ratios indicate that most of the water vapor has condensed. Table 6.1 also illustrates the same initial conditions with the addition of gas ballast and an additional pressure of 980 millitorr.

TABLE 6.1.-Pressures of Water Vapor and Nitrogen for the Initial and Final Conditions of a Pump With and Without Gas Ballast

\begin{tabular}{|c|c|c|c|}
\hline Condition & Water vapor & Nitrogen & $\begin{array}{l}\text { Total } \\
\text { pressure }\end{array}$ \\
\hline \multicolumn{4}{|c|}{ Without gas ballast } \\
\hline $\begin{array}{l}\text { Initial } \\
\text { Final }\end{array}$ & 10 millitorr & 10 millitorr - & $\begin{array}{l}20 \text { millitorr } \\
1000 \text { torr }\end{array}$ \\
\hline \multicolumn{4}{|c|}{ With gas ballast } \\
\hline $\begin{array}{l}\text { Initial } \\
\text { Ballast }- \\
\text { Total } \\
\text { Final }\end{array}$ & $\begin{array}{l}10 \text { millitorr } \\
20 \text { millitorr } \\
30 \text { millitorr } \\
30 \text { torr }\end{array}$ & $\begin{array}{l}10 \text { millitorr }- \\
960 \text { millitorr }- \\
970 \text { millitorr } \\
970 \text { torr }-\end{array}$ & $\begin{array}{l}20 \text { millitorr } \\
980 \text { millitorr } \\
1000 \text { millitorr } \\
1000 \text { torr }\end{array}$ \\
\hline
\end{tabular}

Since this gas contains only about 2 percent water vapor, there is a considerable shift in the composition ratio in the chamber prior to compression; i.e., 30 millitorr of water vapor and 970 millitorr of nitrogen. After a compression of $1000: 1$, the final pressure of the water vapor remains at 30 torr when the total pressure in the chamber reaches the exhaust level, thus preventing condensation. The amount of gas ballast which is required will depend on the partial pressure of water being pumped and the nominal temperature of the pump. In addition, the effectiveness of gas ballast is related to the amount of oil in the pump. Therefore, a small reservoir will contaminate quickly, but it will also 
clean up quickly when gas ballast is applied. A large reservoir is slow to contaminate and is slow to clean up. When the gas ballast is turned on, it is apparent that the pump chamber is at the high exhaust pressure for a longer amount of each cycle. This results in a greater leakage and a corresponding reduction in the ultimate pressure. The increase in ultimate pressure is directly related to the amount of gas ballast applied. Commercial pumps nominally have an adjustable control on the gas ballast to regulate for different levels of vapor. Statements of the ultimate pressure under full gas ballast should also include the waterhandling capacity at full ballast. Single-stage pumps operating with full gas ballast will have a high ultimate pressure ranging from 10 to 100 times the normal ultimate. This higher pressure sometimes prohibits their use in backing diffusion pumps. Since the compression ratio in the first stage of a two-stage pump is small, it is only necessary to gas ballast the second stage. The resulting increase in interstage pressure is only slightly reflected back to the inlet. As a result, double-stage pumps can operate with a very high gas ballast and still maintain low ultimate pressures.

Both the air stripping and gas ballast methods of attacking the water contamination problem have been successfully used. Although the latter method does increase the ultimate pressure obtainable, it has the advantage of being less expensive to apply. That is, the gas ballast is built into the pump by the manufacturer, and no auxiliary equipment is required.

\section{Installation}

A number of different items should be considered when a pump is first installed. Large pumps should be bolted to the floor, particularly those which are not dynamically balanced. In most applications, the pump should be vibration isolated from the remainder of the system. Isolation can be accomplished with metal bellows or a vacuum hose. If the $V$-belt is in an accessible position, a $V$-belt guard should be added. A safety shutoff on the electrical power, or located directly on the pump shaft or pulley, is a desirable feature which would prevent damage from power or pump failure.

If the pump is operating in a normal work area, an exhaust line should be added to transfer the obnoxious vapors out of this area. The exhaust line should be adequately sized to prevent backpressure at full gas flow, and should not contain any valves or restrictions which could easily be clogged. The layout of the exhaust line should avoid any manometer effects which could trap liquid oil, while horizontal runs should be set to drain condensed oil back to the pump. If an external exhaust line is not used, an exhaust trap may be added to condense the oil droplets and 
vapor out of the gas stream and return the oil to the pump. However, the problem of obnoxious vapors, while reduced by the above process, still is not completely eliminated.

A leaktight connection to the pump proper should be provided on the inlet side of the pump. The addition of an oil reservoir volume or a solenoid valve to the inlet line will prevent oil from backing up into the system.

An additional consideration is the line size on the inlet side of the pump. Generally, the line should be no smaller than the fitting provided by the manufacturer. More will be said on the subject of line sizes further on in the text.

If there is a possibility of broken glass or abrasive materials entering the pump, some form of inlet trap should be installed.

After the pump operation installation has been completed, the only normal requirement for operation is to apply power to the motor. If the motor starts but the pump fails to turn over, the power should be immediately removed. This failure to turn over indicates an oil-filled pump or a frozen cylinder. The pump should be manually operated, or the power applied in short increments, until the oil is discharged from the chamber. Pumps which have separate oil reservoirs may require the adjustment of the oil-control valves per the manufacturers' directions. However, if instructions are not available, open the control valves one full turn and adjust for best ultimate pressure after the pump has warmed up. The manufacturer's data on horsepower and tolerable temperatures will provide information for the maintenance of continuous operation at high pressure. Most mechanical pumps, however, are not designed to operate continuously at high pressure.

If the ultimate pressure rises after the pump has been operating for a period of time, the condensables may be removed by turning on the gas ballast. If gas ballast is not available, admit gas to the inlet at a pressure of a few tenths of a torr and allow the gas to flow until the pressure recovers to normal ultimate when the gas bleed is shut off. The inlet side of the pump should be released to atmospheric pressure when the pump is shut off.

\section{Test and Maintenance}

A routine procedure for the checkout of a pump should include at least the minimum items given below. The $V$-belt should be checked for wear or slippage. If any signs of wear are apparent, the belt should be replaced. The oil level should be examined while the pump is running, and oil added as required. The pump should be checked for indications of oil leaks at the shaft seals and the seals replaced as required. The ultimate pressure should be examined with the pump blanked off from the system. 
High ultimate pressures may result from vapor contamination, oil which has been used too much, leaks in the system, or a damaged pump. Contamination may be the cause if the ultimate pressure overshoots to a very low value immediately after running at high pressure. The addition of gas ballast or gas bleed until the ultimate pressure recovers to a normal value will correct the condition. If a high ultimate pressure persists, all the inlet connections should be leak checked, and if leaks are not detected, the oil should be changed. Complete removal of contaminated oil may require several flushes with a lower grade oil. If the high ultimate pressure persists after fresh oil has been added and the system is leaktight, the pump is probably damaged and will need to be repaired. The draining of the oil from the pump for an oil change follows the following procedure. The oil drain plug is removed with the inlet open to atmosphere. When a hand is placed over the pump exhaust and the pump is turned on, the high pressure developed by the pump will quickly blow the oil from the pump. It is advisable to provide adequate eatch basins for the oil.

\section{POSITIVE DISPLACEMENT BLOWERS}

The rapid pumpdown required by larger vacuum chambers will demand a higher pumping capacity than is practically obtainable from rotary oil-sealed mechanical pumps. The positive displacement blower or Roots blower will provide the increased pumping capacity. The blower is a lobe type, discussed in references 4 and 6, which operates without mechanical contact or an oil seal, where the leakage is mini-

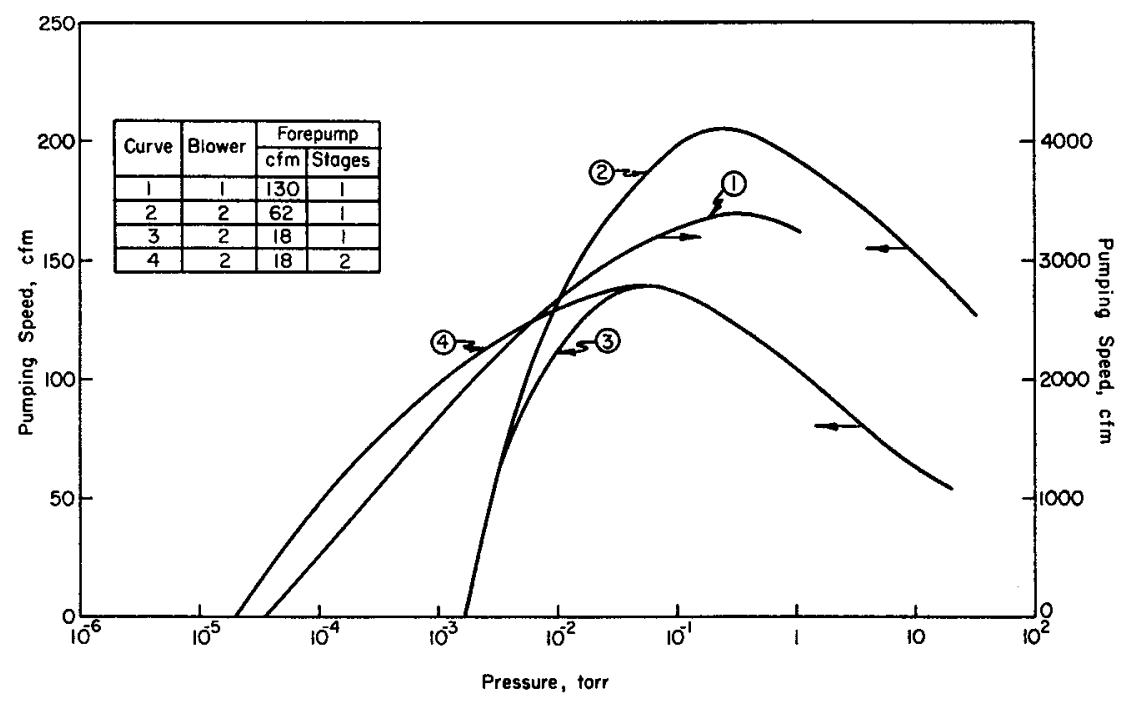

FiguRE 6.3.-Performance curves of some typical positive displacement blower and forepump combinations. 
mized by close clearances. Single units with capacities of about $100 \mathrm{cfm}$ to $15000 \mathrm{cfm}$ are commercially available. The temperature rise will limit the pressure ratios across a Roots blower to values of about $2: 1$ when exhausting to atmosphere, of 10:1 at an inlet pressure of 1 torr, and of 40:1 at an inlet pressure of 0.1 torr (ref. 6). As a result, when a system is being exhausted to atmosphere, the Roots blower must be backed by a rotary oil-sealed pump. Figure 6.3 illustrates typical performance curves of commercial blower-forepump combinations. Reference 6 presents an excellent discussion of the application of blowers to the pumpdown of a large space simulator. The system described is quite complex, as it involves the rearrangement of blowers in parallel and series-parallel groups as the pumpdown proceeds. This procedure can take full advantage of the blower characteristics, but usually cannot be justified on small systems. In the case of smaller systems, the economic advantage frequently lies with the use of a simple rotary pumpRoots blower combination or a larger rotary pump used alone to achieve the required pumpdown time.

\section{DIFFUSION PUMPS}

The diffusion pump, which is essentially a multistage ejector, utilizes the vapor-stream principle to pump gases in the molecular-flow region and is capable of obtaining a maximum pumping speed at inlet pressures lower than $10^{-3}$ torr. The basic components of the diffusion pump are the boiler, jet assembly, casing, and working fluid. References 4 through 12 present detailed descriptions of such pumps. The following discussion treats the performance and operational details of diffusion pumps.

Since the diffusion pump cannot exhaust to atmospheric pressure, it exhausts to a forepressure which is maintained in the range of tenths of a torr by a mechanical pump. While the foreline pressure is maintained below a critical value called the tolerable forepressure, the diffusion pump performance is independent of forepressure. If the tolerable pressure is exceeded, however, the pump performance rapidly deteriorates as the result of the breakdown of one or more jet stages. This rapid deterioration is characteristic of modern highly efficient pump designs which feature a high forepressure tolerance. Older diffusion pump designs, which degrade at a more gradual rate as forepressure increases, have a poorer forepressure tolerance.

Figure 6.4 depicts typical performance curves for a modern diffusion pump. Curves 1 and 4 are plots of the speed in liter/sec and throughput in torr liter/sec of the untrapped pump versus pump inlet pressure. Curve 3 illustrates the reduction of pumping speed when the pump is used in conjunction with a typical trap. The throughput curve is similarly reduced. Curve 6 is the plot of tolerable foreline pressure versus throughput and illustrates that the forepressure requirement is more 


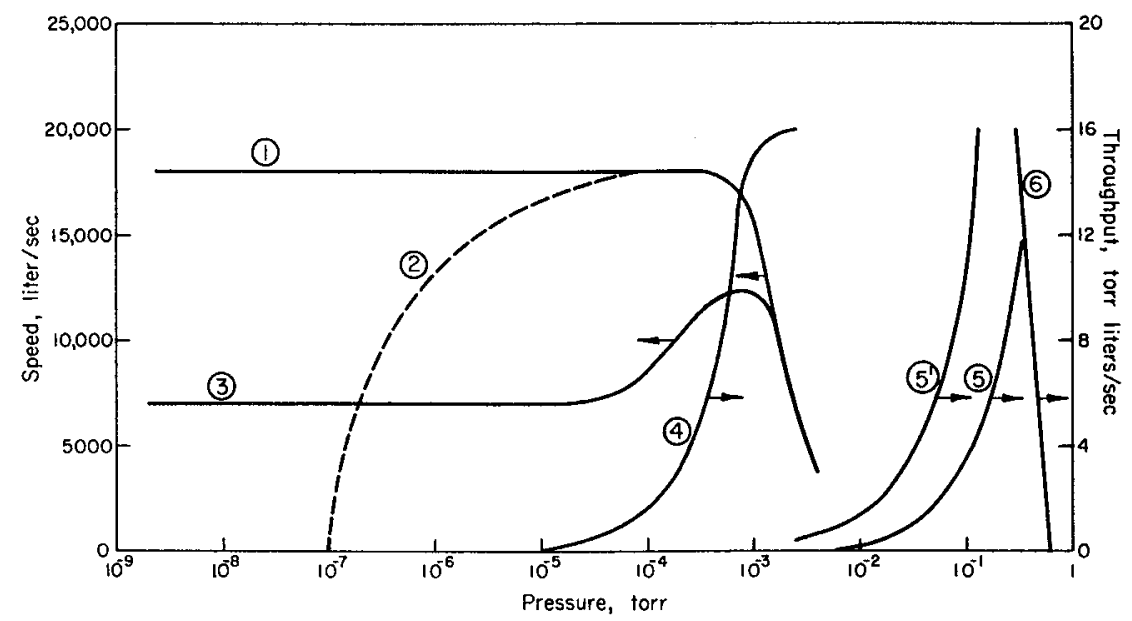

Figure 6.4.-Typical performance criteria of a modern diffusion pump. Curves 1 and 4 are the actual pumping speed and throughput, respectively, of an untrapped pump versus pump inlet pressure; curve 2 is the net pumping speed in the presence of a gas load; curve 3 shows the reduction of pump speed when used in conjunction with a typical trap; curves 5 and $5^{\prime}$ represent the throughput-pressure characteristics for two sizes of mechanical forepump; and curve 6 represents the relationship of tolerable foreline pressure to throughput.

severe at the higher throughput level. Curves 5 and $5^{\prime}$ are not diffusion pump performance curves, but represent the throughput-pressure characteristics of two sizes of a mechanical forepump. It can be seen that the throughput is the same for a diffusion pump and its accompanying forepump at steady-state conditions due to the fact that $Q$ is essentially a mass flow rate. An examination of curves $5,5^{\prime}$, and 6 will show that the smaller forepump of curve 5 can provide a foreline pressure less than the tolerable forepressure only up to a throughput of about 12 torr liter/sec, while the larger forepump of curve $5^{\prime}$ insures stable operation over the entire throughput range. Larger forepump sizes provide faster system pumpdown, while smaller sizes are adequate to hold the diffusion pump once it has been pumped down to pressures corresponding to the low throughput range. Chapter 11 discusses the necessary factors to consider when choosing the proper size of forepump for a system.

Diffusion pumps may be rated as to the ultimate pressure attainable, although this method is less commonly used now. It has been recognized that the attainment of ultimate pressure in a test dome is not due to the cessation of pumping action, but rather it is due to the increase of the volume of flow from parasitic gas sources such as the vapor pressure of the pumping fluid, surface outgassing, leaks, and gas from the pump fluid (either from contamination or decomposition). As a result, the 
performance of a pump is not described properly by curve 2 of figure 6.4 , but rather by curve 1 . The pumping efficiency as measured by the Ho coefficient, or the ratio of actual pumping speed to the theoretical maximum, is maintained at a high value. For air, the pumping efficiency measured at the flange of a modern pump is approximately 0.5 , down to indeterminately low pressures.

\section{Effect of Gas Type}

The manufacturers' claims for the performance of a diffusion pump are usually based on air as the gas being pumped. The pumping speed for gases of similar molecular weight, nitrogen, oxygen, carbon monoxide, carbon dioxide, and argon is very similar to that for air which has a molecular weight of 29 . Two gases commonly encountered in a highvacuum system are helium and hydrogen with molecular weights of 4 and 2 , respectively.

Chapter 5 concluded that the molecular conductance of a short tube, which in this case is the pump opening, varies inversely with the square root of the molecular weight of the gas specie. Therefore, the lighter gases possess a greater pumping speed than air by the above ratio, if it is assumed that the light molecules are pumped by the jet with the same efficiency as the heavier molecules. Unfortunately, this assumption cannot be made. The discussion of reference 13 shows that the capture efficiency of helium at the first-stage jet of a tested diffusion pump is about one-half of that for nitrogen. The net result is that the speed for the light gas is only slightly higher than that for air in a pump of modern high efficiency design. Figure 6.5 compares the pumping speed of air and hydrogen for a large pump of modern design. Other pump designs attain hydrogen pumping speeds as little as 20 percent higher than the pumping speed for air. Some of the older pump designs had a greater speed ratio of light gas to air, at the expense, however, of the pumping speed for air due to a greater length of casing from the inlet flange to the first-stage jet.

Another diffusion pump parameter is the pressure ratio which a pump can maintain between the inlet and foreline. Although this ratio is limited by the back diffusion of gas through the vapor jet, it is seldom a problem with air or similar gases, since its value is generally about $10^{\mathbf{1 0}}$. If this value is insufficient, the addition of a smaller diffusion pump in series with the first pump can be used to reduce the forepressure. For hydrogen and helium, however, pressure ratios as small as $10^{3}$ have been reported (refs. 14 and 15). Other experimenters have reported helium pressure ratios as high as $10^{10}$ (ref. 16), and have attributed the high value to the high density of the jet vapor stream in the pump under consideration. It can be seen that considerations of the light gas 


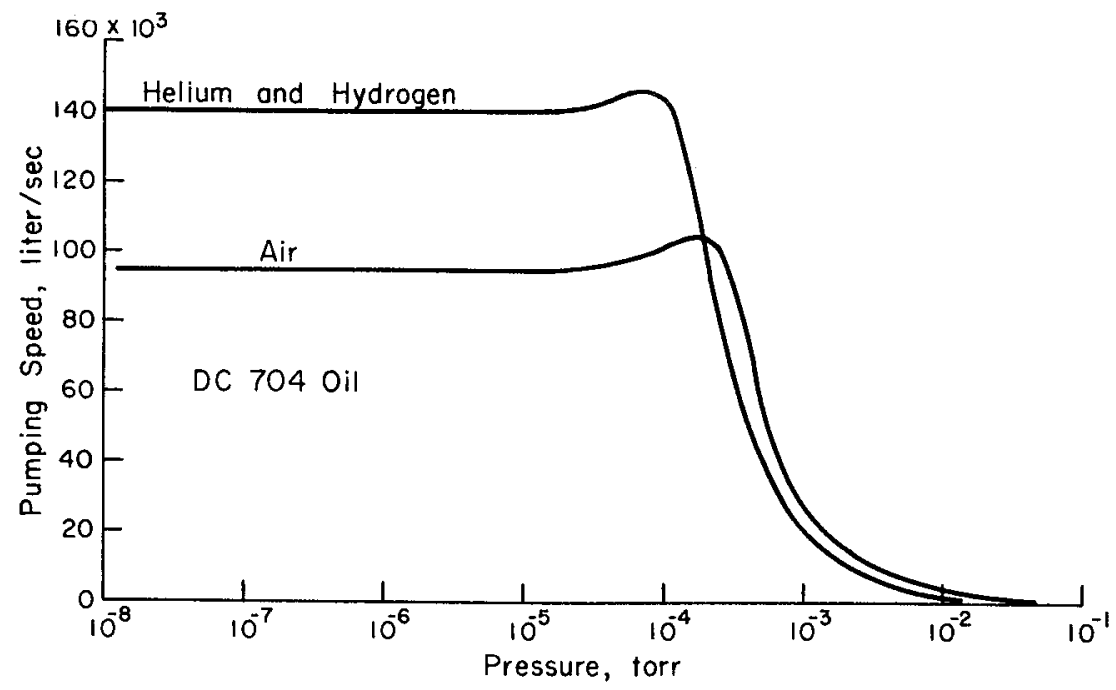

Figure 6.5.-Comparison of pumping speed for helium and hydrogen and air for a 52-inch ID diffusion pump using DC 704 oil.

pressure are factors when a pump for a particular system is being decided upon.

Figure 6.4, curve 3, illustrates that the presence of a trap will reduce the net pumping speed for permanent gases. The speed reduction can be computed as a function of gas type using the methods of chapter 5 . The primary reason for the addition of the trap to the diffusion pump of an ultra-high-vacuum system is to prevent the pump fluid from entering the system. In addition, a liquid-nitrogen-cooled trap is an extremely efficient pump for condensables. Since water vapor is the predominant gas load of many ultra-high-vacuum systems, the pumping speed of a trapped diffusion pump for water vapor will generally be several times the speed of the pump for noncondensables.

\section{Diffusion Pump Fluids}

The original diffusion pumps utilized mercury as the working fluid. Today, however, various types of oil have largely displaced mercury in pumps, although mercury still offers several advantages over oil. For one, mercury does not decompose, and it does not contribute to the noncondensable gas load of a system. Since species of unknown molecular weight are not introduced into the system, mass spectrometer analysis is not complicated. Another advantage is that mercury does not react with most other gases or with hot filaments.

Some of the disadvantages of mercury when compared to oil are that mercury has a smaller molecule and is less effective as a pump fluid, it 
has a high vapor pressure and therefore must be used with a refrigerated trap, it amalgamates with many metals, and it is toxic.

As a result of these considerations, mercury is used for some specialized applications. Oil, however, remains the predominant diffusion pump fluid. The extensive use of oil has been made possible through the development of relatively stable oils, and by the design of the pump boiler. Many boilers are of the fractionating type which separates the higher and lower vapor pressure fractions of the oil and supplies the top jet with the lower fractions. Another design feature involves the separation of dissolved gases from the condensed oil returning to the boiler and ejects them into the foreline. Efficient design of the heating elements is also necessary in order to eliminate hot spots which tend to promote oil decomposition into high vapor pressure fractions and gases such as methane and hydrogen which cannot be readily trapped.

The general tendency in the development of diffusion pump oils has been toward the attainment of oils of lower vapor pressure and greater resistance to oxidation. Figure 6.6 shows that oils with room temperature vapor pressures in the range of $10^{-10}$ torr are available. This property is not particularly important in an ultra-high-vacuum system having adequate trapping. For example, at liquid nitrogen temperatures,

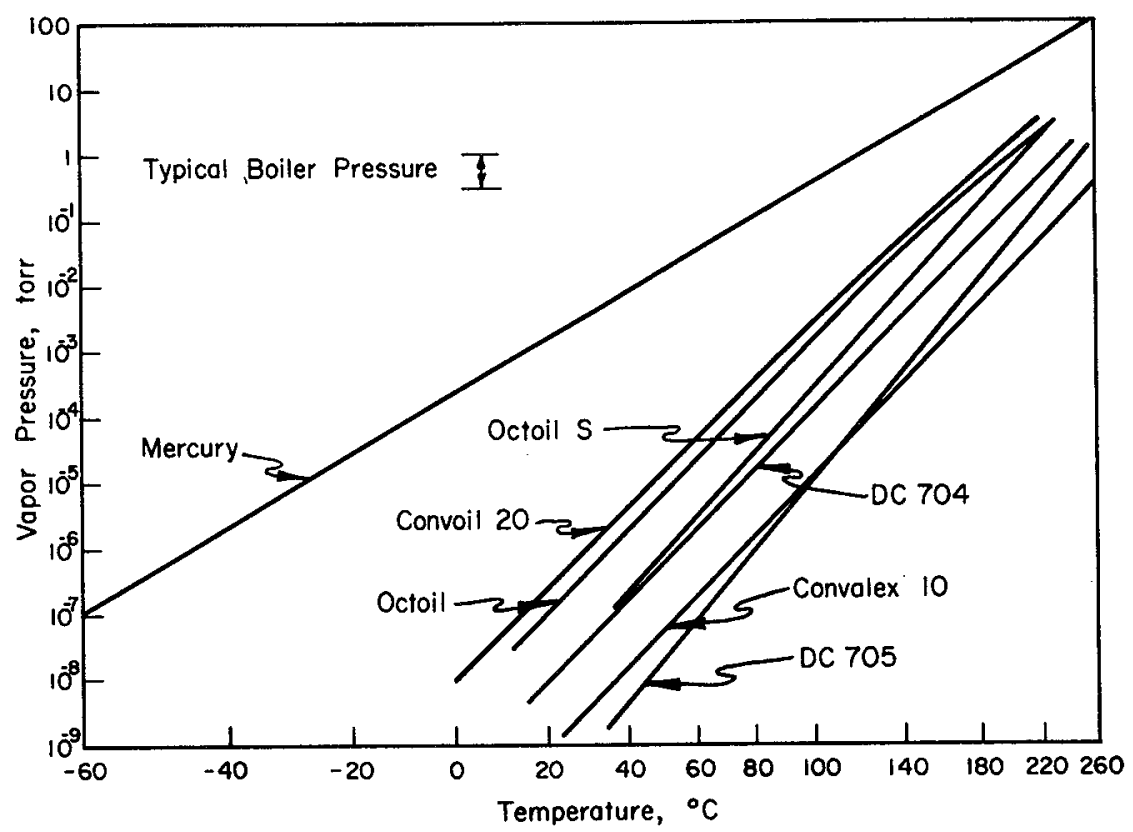

FigURE 6.6.-Vapor pressure versus temperature curves for several common diffusion pump fluids. 
the vapor pressure of Convoil 20 is extremely low and the fluid is many times less expensive than other oils. Convoil 20 has been used in welltrapped systems which have reached the low $10^{-11}$ torr pressure range without additional cryogenic pumping. For this type of system, the vapor pressure of the pump fluid is less important than its chemical stability. That is, an oil having a low vapor pressure, but which contributes noncondensable hydrogen to the system by decomposition may well have a higher ultimate pressure. The ultimate pressure produced in the system mentioned above, with silicone oil DC 704 in one type of pump, was about one decade higher than when Convoil 20 was used in a different pump design. Silicone oils have operated on a par with Convoil 20 in the latter design of pumps which seem to have a more uniform heating pattern in the boiler. The silicone oils possess a higher resistance to oxidation at elevated temperatures. This factor is a distinct advantage in many applications where the hot oil is exposed to the atmosphere.

Since each oil type has different pressure-temperature characteristics, and since the boiler temperature affects oil decomposition, both the pump heater power and the cooling water flow have an effect on the performance of a pump when any of the mentioned oils are used in a pump. The best performance obtainable from a pumping system operating at low pressures can be brought about only by experimentation with the variables of pump heater power and cooling water flow. General rules for such experimentation do not exist, except that sufficient time must be allowed for steady-state conditions to be established, and the oil should be used enough to sufficiently condition it to drive off impurities and the components more likely to fractionate. In some cases, several days may be required for this process.

The diffusion pump fluid can be continuously contaminated by the mechanical forepump due to the transfer of low vapor pressure mechanical pump oil. This transfer can be eliminated by the use of a trap, or if a smaller diffusion pump is placed in the foreline to act as a barrier. The severity of the mechanical pump oil problem can be greatly reduced by the use of diffusion pump oil in the mechanical pump. No mechanical difficulty was experienced when Convoil 20 was used. As a rule, however, diffusion pump fluids should not be expected to possess the necessary qualities of viscosity and lubricity to operate successfully in a mechanical pump.

Backstreaming, or the transfer of pump fluid beyond the pump flange, is also affected by the type of oil used as well as the pump design. Backstreaming can be effectively stopped by a properly designed trap (chapter 7), but only at the expense of reduced pumping speed (figure 5.23).

Figure 6.7 shows typical costs and sizes of commercially available oil diffusion pumps. 


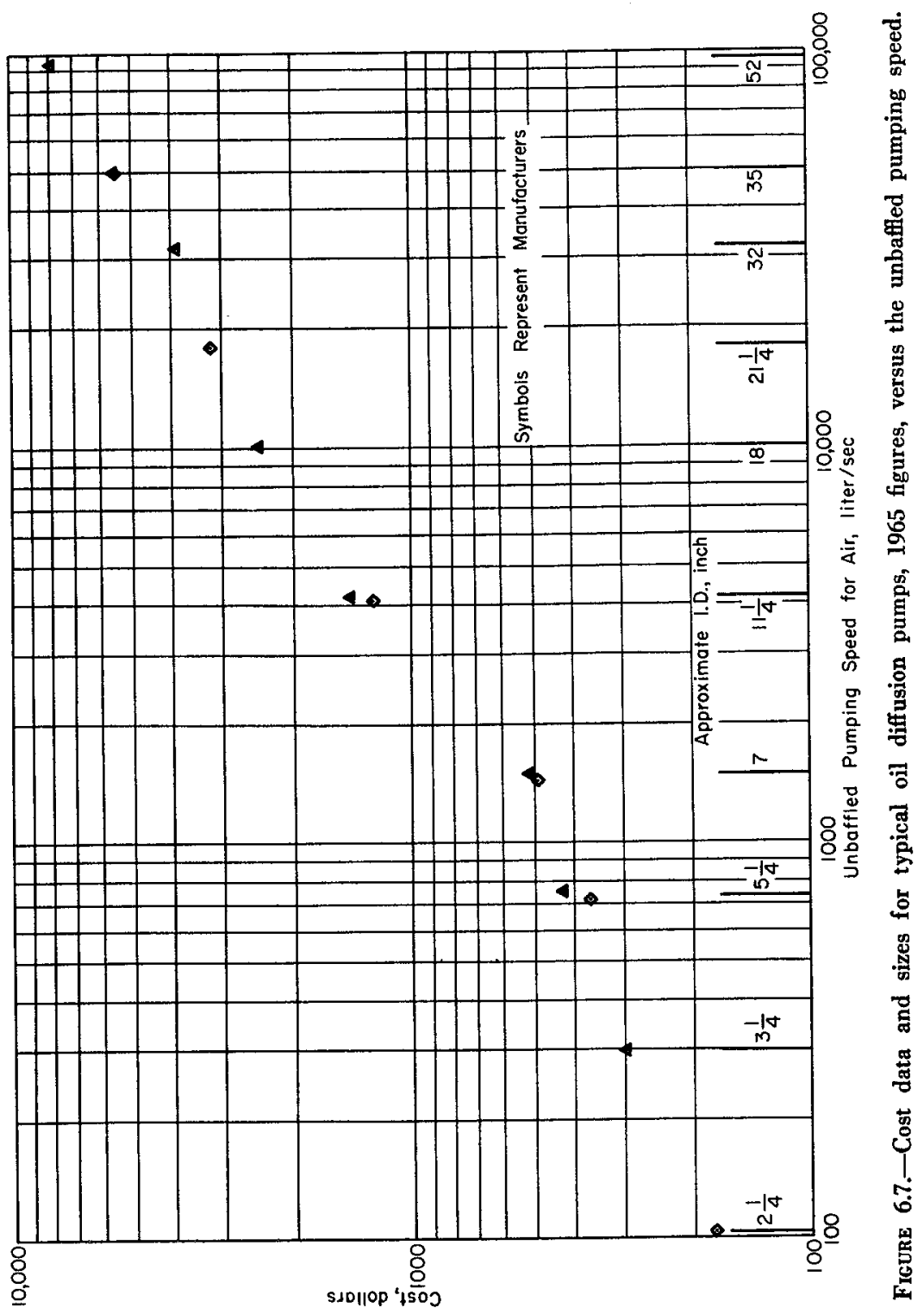




\section{SPUTTER ION PUMPS}

The sputter ion pump is designed such that an electrical discharge occurs between the anode and cathode at a potential of several thousand volts in a magnetic field of a few thousand gauss. Since the magnetic field causes the electrons to follow a flat helical path, the length of their path to the anode is greatly increased. A high efficiency of ion formation down to pressures of $10^{-12}$ torr and less is assured by this long path length. The gaseous ions so formed are accelerated to the titanium anode where they may perform in one of three ways. They may penetrate the anode surface and thus be pumped, or they may combine chemically with the anode material to form a low vapor pressure compound. The third possible occurrence is that they will vaporize some of the anode material (sputtering). The sputtered atoms of metal will in turn condense on other parts of the pump structure and trap gas molecules. Since the ionizing current is proportional to pressure for a given gas type, the pump can serve as an ionization gage. Unlike the diffusion pump, the sputter ion pump does not require a forepump to pump the collected gas up to atmospheric pressure, since the pumped gas is in effect digested. However, an auxiliary pump is needed to reduce the system pressure initially to the range of $10^{-4}$ torr where the ionic pumping action will commence. Mechanical or sorption pumping methods are usually used for the initial pumpdown of a vacuum system.

Due to the complex nature of the pumping action of sputter-ion-type pumps, the pumping speed for various gases varies greatly, depending on the chemical activity, ease of ionization, and the molecular size of the molecule. Table 6.2 (from reference 17) shows a speed variation of nearly 300 to 1 for a simple diode pump. The low pumping speed for argon is frequently of concern, since the atmosphere contains 1 percent argon. In addition to their poor argon pumping speed, simple diode

TABle 6.2.-Relative Pumping Speed for Gases in Flat Cathode Diode Sputter Ion Pump

\begin{tabular}{|l|l|}
\hline Gas & Pumping speed, liter/sec \\
\hline Hydrogen & 270 \\
Deuterium & 190 \\
Light hydrocarbons & 90 to 160 \\
Nitrogen & 100 \\
Carbon dioxide & 100 \\
Water vapor & 100 \\
Oxygen & $\mathbf{5 7}$ \\
Helium & 10 \\
Neon & 4 \\
Argon & 1 \\
\hline
\end{tabular}


pumps exhibit pressure instability such that the pump will regurgitate bursts of argon. The instability occurs when argon previously buried by sputtered anode metal is uncovered. If the pump cathode is slotted, or if a third element, the sputter cathode, is interposed between the anode and the collector (triode pump), the problem of the argon pumping speed is alleviated. Both modifications result in stable pumping. The slotted-cathode method is less complex and increases the argon pumping speed by a factor of 2 or 3 , while the second method increases it by a factor of about 30 . Both methods cause a section of the cathode area to receive a net buildup of sputtered material to form a dependable argon-pumping area.

It is frequently stated that sputter ion pumps are preferable to diffusion pumps because they do not inject hydrocarbon contaminants into a system. This is true when a poorly trapped diffusion pump is used for comparison. However, sputter ion pumps can produce significant amounts of hydrocarbons, while a properly trapped oil diffusion pump can maintain hydrocarbons at less than $10^{-12}$ torr. The hydrocarbons are formed by the reaction of carbon- and hydrogen-bearing gases such as carbon dioxide and water vapor, and also may arise from carbon impurities in the cathode material (references 18 and 19). The predominant compound formed is methane. Hydrocarbons are not normally objectionable, as their pressure is held down by the pumping action. However, if the pump is turned off, the methane partial pressure has been observed (reference 19) to rise more than a decade during a period of several minutes.

Sputter ion pumps do not have an indefinite life due to the fact that active pump material is being consumed during the normal operation of the pump. The usual pump-life limitation is not depletion of the active material, but the buildup of sputtered material to the point where flaking occurs. These flakes frequently cause pressure bursts when they fall into the discharge region, and they can also cause short circuits. Problems such as this which will necessitate pump-element cleaning, may be expected to occur after about 1000 to 5000 hours' operation at $10^{-5}$ torr. Pump life is inversely proportional to pressure.

Figure 6.8 indicates the availability and cost of typical commercial sputter ion pumps.

\section{SORPTION PUMPING}

Gas molecules may be removed from the gas phase if they become attached to a solid surface, or if they penetrate into a solid. The first phenomenon is termed "adsorption" and the second is "absorption." For the case of solid-gas reactions, there is no clear distinction between the two processes and the general term "sorption" applies. "Sorption pumping" refers to such processes when they are used to produce vacuum 


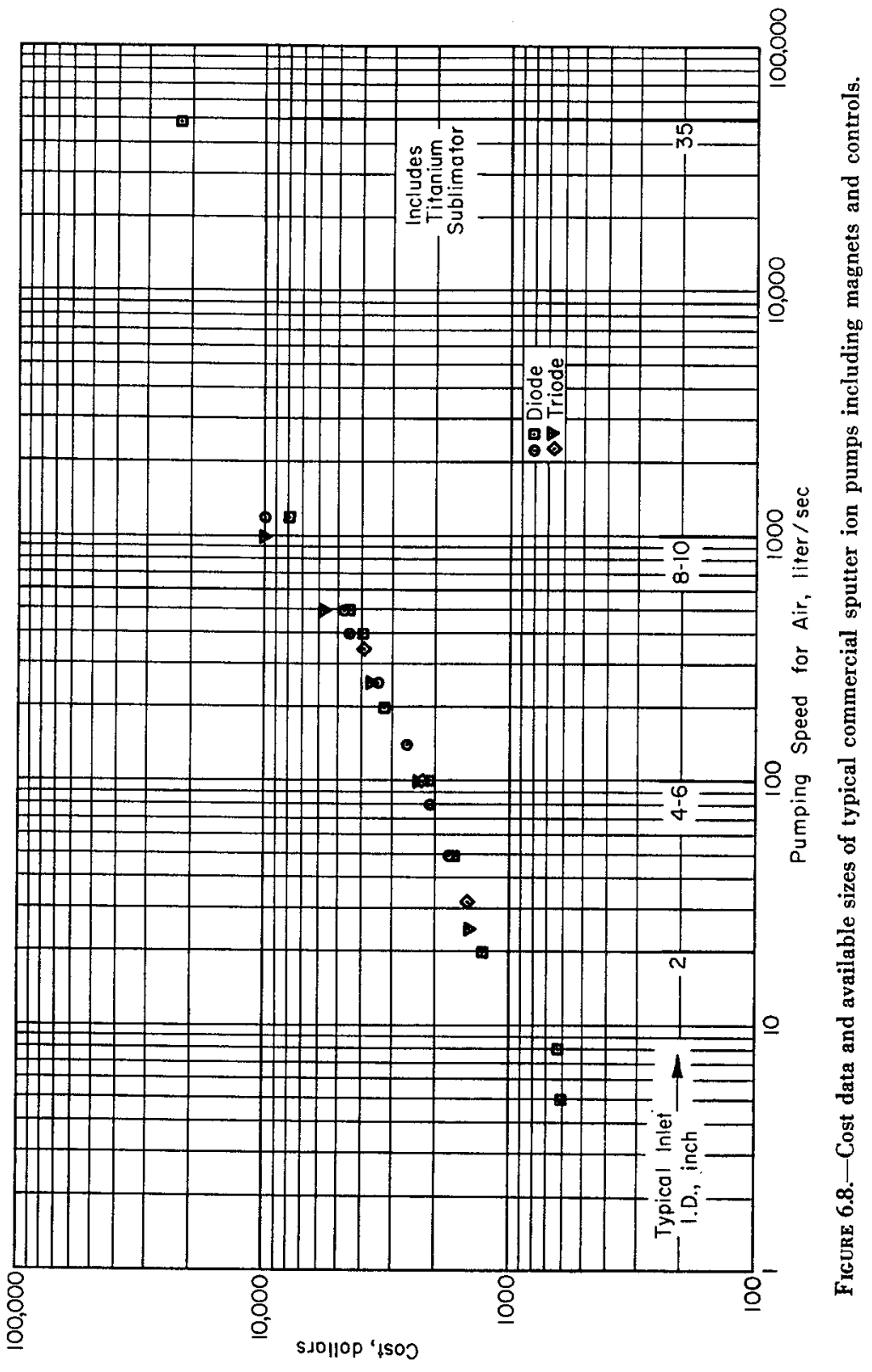


conditions. This pumping method is frequently applied to a sputter-ionpumped system to obtain the initial pumpdown to a pressure range of $10^{-3}$ torr or lower. In this manner, the possibility of system contamination by mechanical pump oil is eliminated.

In its simplest form, the pumping method utilizes a sorption pump which consists of a closed chamber packed with a granulated sorbent or molecular sieve material. Initially, the gases are sorbed on the sieve material to an amount which is in equilibrium at room temperature. The gases may be present in a lesser amount due to the bakeout of the sieve at an elevated temperature. The sorption pump is valved into the system and immersed in liquid nitrogen to cool the sorbent material. As the temperature of the molecular sieve falls, it sorbs more gas from the system to cause a reduction in the pressure.

Reference 7 cites an example of such a pumpdown. An amount of molecular sieve material equal to about 2 liters was used to pump down a 28-liter system from atmospheric pressure to $10^{-1}$ torr in about 15 minutes, with an ultimate pressure of about $2 \times 10^{-2}$ torr. For this case, internal copper fins were used in the sorbent chamber to increase the heat transfer with the liquid nitrogen.

After pumpdown to the required pressure has been accomplished, the valve to the chamber is closed. The sorption pump is then regenerated by venting it to atmosphere and allowing it to return to room temperature. The regeneration process can be speeded up and made more complete by a mild bakeout cycle. If the pump is badly contaminated, with water vapor, for example, a bake of about $300^{\circ} \mathrm{C}$ is required for zeolite.

The use of a more sorbent material per liter of gas to be pumped will enable lower pressures to be achieved. Multistage pumping, which is a much more effective method (references 17 and 20), uses one pump to remove the bulk of gas, which is valved off, and then uses another pump to continue the process. The first stage can be either a sorption or a mechanical pump. Oil contamination from a mechanical pump can be minimized by a trap, or by insuring that the first stage is not pumped beyond the pressure at which viscous flow ceases. The advantage of mechanical pumping in the first stage is that the ultimate partial pressures of neon and helium, which are not well pumped by sorbents, can be greatly reduced.

Both zeolites and activated charcoal are used as sorbents. Zeolites are more commonly used due to their shorter regeneration time, higher capacity, and greater freedom from dusting. However, activated charcoal is lower in cost, has a lower regeneration temperature, and a higher thermal conductivity which allows it to chill faster.

The absorption characteristics of a typical zeolite material are shown in figure 6.9 (reference 20), where the curves are the absorption isotherms for a sorbent with a mean pore size of $5 \AA$. The curves show the 


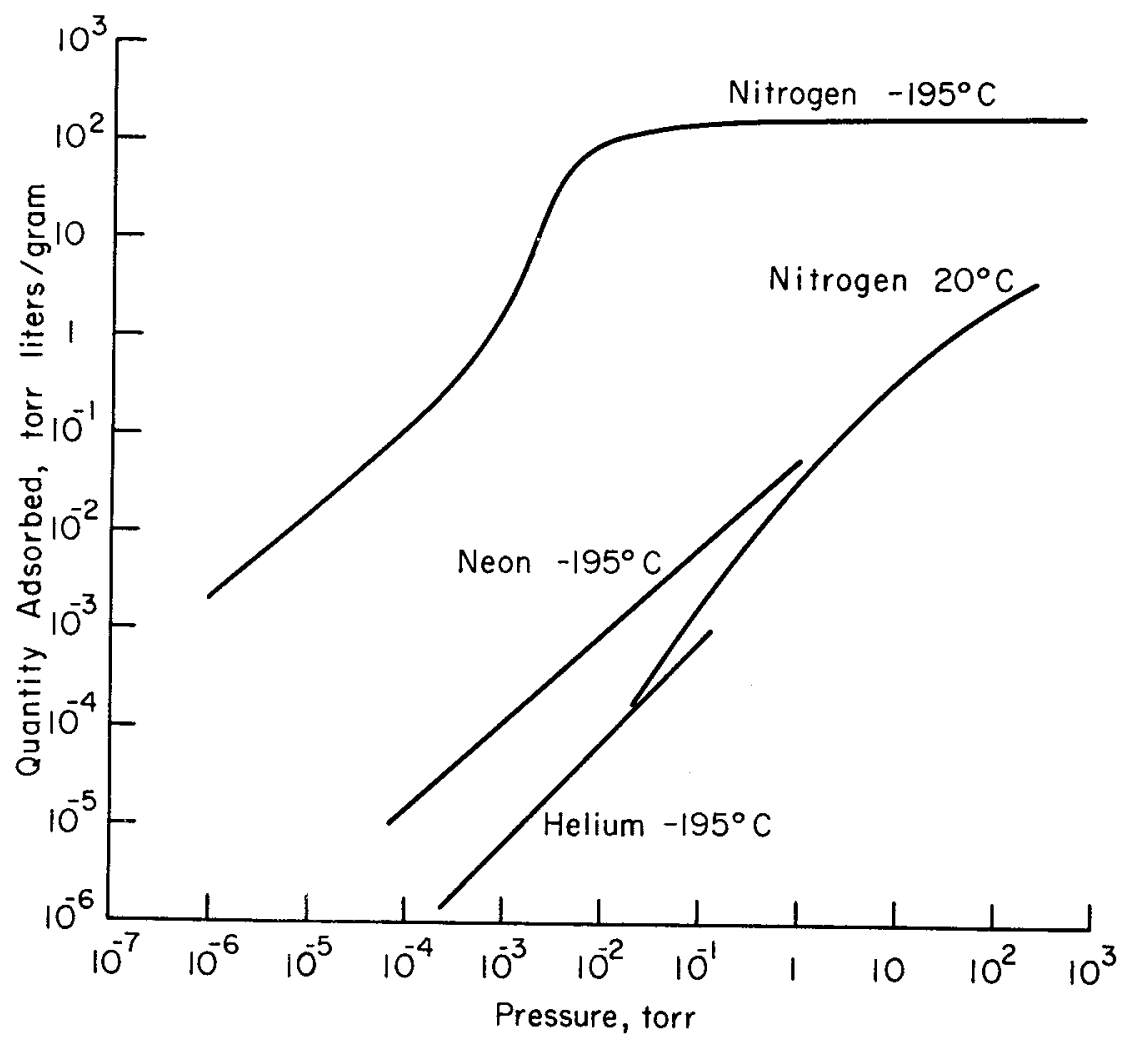

Figure 6.9.-Curves of the adsorption isotherms of nitrogen, neon, and helium on typical zeolite sorbent materials with a mean pore size of $5 \AA$ (ref. 20).

quantity of gas which can be sorbed, in torr liter of gas per gram of sorbent, as a function of gas pressure. It is apparent that as the sieve temperature is lowered, more gas molecules can be sorbed.

Neon and helium, however, are sorbed to a lesser degree than nitrogen. In the Earth's atmosphere, the partial pressures of nitrogen, neon, and helium are $593,1.4 \times 10^{-2}$, and $4 \times 10^{-3}$ torr, respectively. If the sieve is exposed to a volume of atmospheric air, the nitrogen partial pressure will be reduced to a much greater extent than that of neon and helium as a result of the selective sorption mentioned previously. Figure 6.10 (reference 20) illustrates that the nitrogen will be reduced to a lower pressure than the two rare gases for certain ratios of initial gas to sorbent weight. If the volume had been pumped previously with a mechanical pump to a pressure of 0.76 torr, the concentration of the rare gases would already have been reduced by a factor of $10^{-3}$, since all components of the gas would have been pumped equally to that point. 


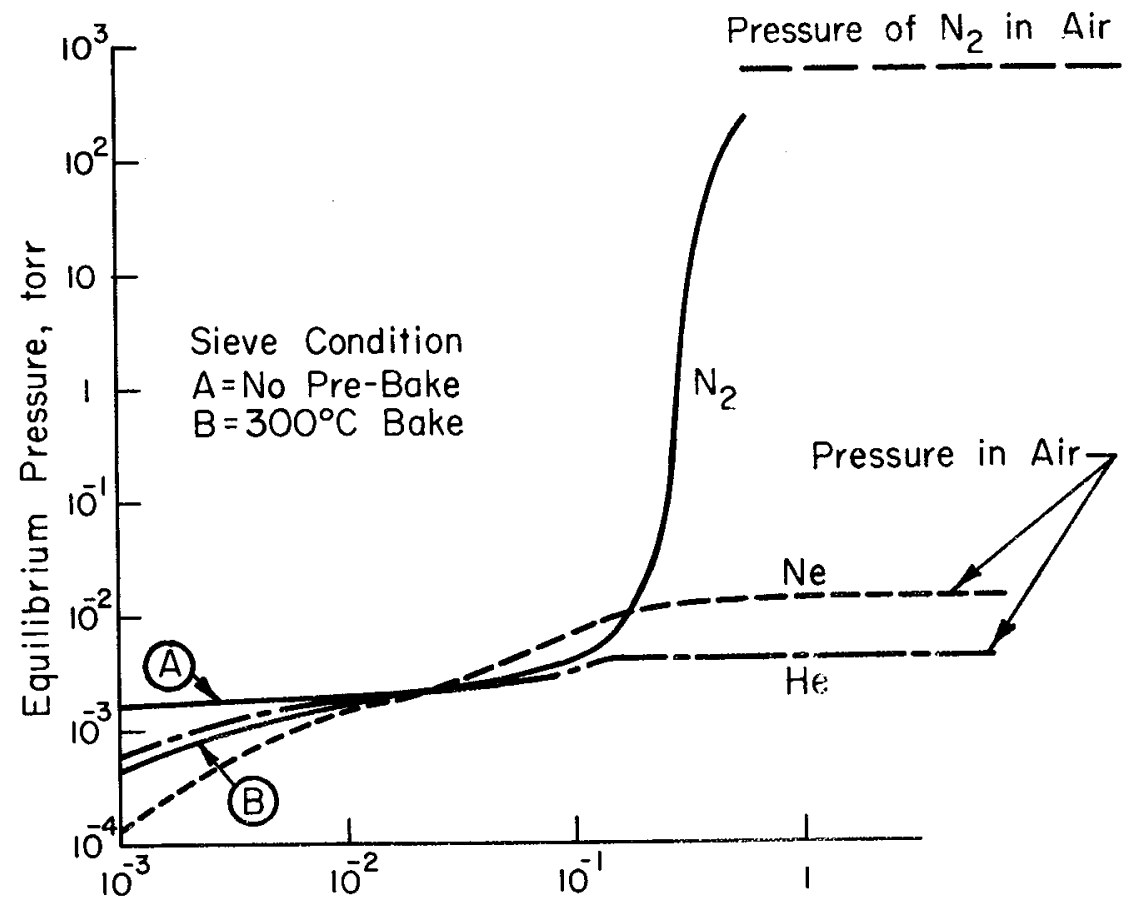

Chamber Volume/Sorbent Weight, liters/gram

Figure 6.10.- Nitrogen is reduced to a lower pressure than neon or helium for certain values of the ratio of initial gas to sorbent weight during the pumpdown of a chamber containing molecular sieve material with a mean pore size of $5 \AA$ (ref. 20).

The other atmospheric gases—oxygen, argon, and carbon dioxide-are readily sorbed.

Sorption pumping has been combined with cryopumping (chapter 7) to yield a process called cryosorption. In essence, cryosorption merely involves lowering the sieve temperature to the region of $20^{\circ} \mathrm{K}$ or less. The geometry of experimental and proposed cryosorption systems is different from the sorption pumping arrangement discussed to this point due to the fact that cryosorption systems strive for very high pumping speeds. To achieve these high pumping speeds, large areas of the sieve are placed directly in the vacuum chamber in a manner very similar to the cryopump arrays described in chapter 7 .

An example will illustrate what cryosorption techniques can accomplish. If a simple cryopanel is cooled to $20^{\circ} \mathrm{K}$, it cannot pump hydrogen gas which has a pressure of $1 \mathrm{~atm}$ at that temperature. At $4.2^{\circ} \mathrm{K}$, the temperature of liquid helium at a 1-atm saturation pressure, the panel can maintain hydrogen at a pressure of $10^{-8}$ torr. However, the cost of 
operating at such a temperature is usually prohibitive. If the cryopanel is covered with a molecular sieve (reference 21 ), the cryopanel will pump hydrogen in the $10^{-15}$ torr region at a temperature ranging from $15^{\circ} \mathrm{K}$ to $20^{\circ} \mathrm{K}$.

In addition to possessing all the problems of the simpler sorption and cryopumping systems, a cryosorption system must also contend with the problem of properly bonding the sieve to the cryopanel. It is necessary to hold the sieve in place and also provide a good thermal contact which will maintain the entire sieve material at the proper temperature. The bond must withstand thermal cycling resulting from excursions from bakeout to cryogenic temperatures. Epoxy bonding of zeolite to stainless steel (reference 21) is successful for short-duration tests only, due to the fact that the adhesive diffuses into the sieve and prevents hydrogen absorption. The direct bonding of the molecular sieve to aluminum plates without an adhesive has proved to be a successful method (ref. 22). The zeolite $5 \AA$ used for this type of bonded molecular sieve has also been filled with aluminum flakes to provide improved thermal conductivity.

Hydrogen sorption for $5 \AA$ molecular sieve material has been measured

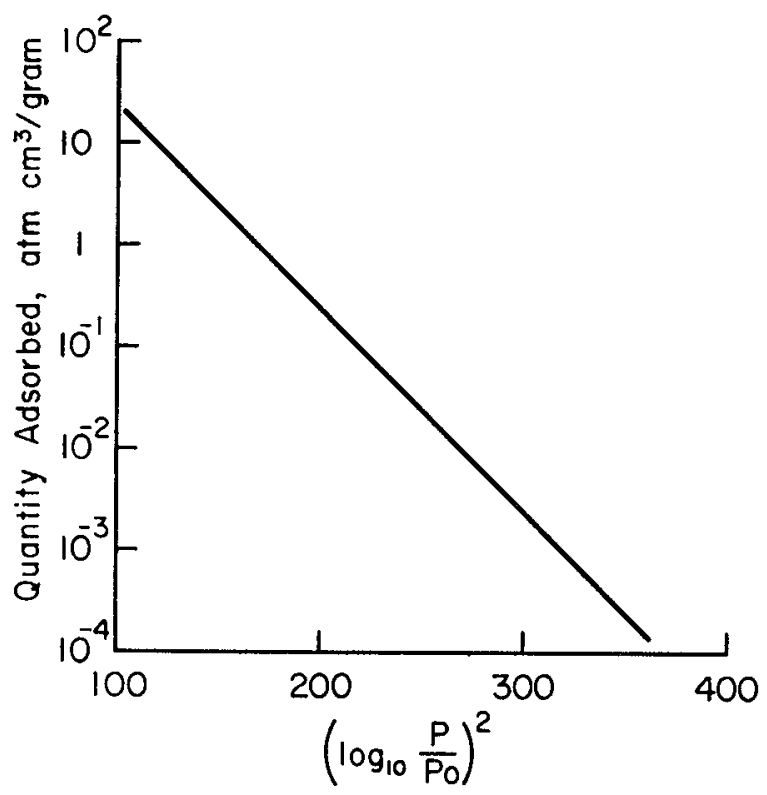

Figure 6.11.--Equilibrium adsorption of hydrogen on $5 \AA$ molecular sieve at $20^{\circ} \mathrm{K}$, as measured at a pressure of $10^{-8}$ to $10^{-10}$ torr and extended theoretically to $10^{-15}$ torr (reference 21). $P=$ equilibrium pressure; $P_{0}=$ vapor pressure of $\mathrm{H}_{2}$ at $20^{\circ} \mathrm{K} \cong 1 \mathrm{~atm}$. 
in the range of $10^{-8}$ to $10^{-10}$ torr (references 22 and 23 ), and has been extended on a theoretical basis to $10^{-15}$ torr (reference 21) as shown in figure 6.11. Reference 21 describes a proposed vacuum system utilizing these techniques.

A basis for the understanding of the general methods of pumping has been laid by chapter 6 . Simple systems were introduced to give a feel for the parameters involved in the choice of a pumping system for a particular application. The logical extension of this discussion involves the more complicated pumping systems required for ultra-highvacuum considerations and, in particular, space-simulation systems. Chapter 7 introduces and more fully discusses the phenomena of cryogenic pumping.

\section{REFERENCES}

1. BECKER, W.: t̛ber eine neue Molekularpumpe. Proceedings of the First International Congress on Vacuum Techniques, vol. 1. E. Thomas, ed., Pergamon Press, 1959, pp. 173-176.

2. BeAms, J. W.: Bakable Molecular Pumps. Trans. of the Seventh National Symposium on Vacuum Technology, C. R. Meissner, ed., Pergamon Press, 1961, pp. 1-5.

3. Kruger, C. H.; and Shapiro, A. H.: Vacuum Pumping With a Bladed AxialFlow Turbomachine. Trans. of the Seventh National Symposium on Vacuum Technology, C. R. Meissner, ed., Pergamon Press, 1961, pp. 6-12.

4. Guthrie, A.: Vacuum Technology. John Wiley \& Sons, Inc., 1963.

5. Hockly, D. A.; AND Butr, C. S.: The Ultimate Vacua of Two Stage Rotary Oil Pumps. Vacuum, vol. 4, 1954, pp. 40-47.

6. Dowling, D. L.; Herrick, D. B.; and Rose, W. E.: Evacuating Large Space Chambers With Positive Displacement Blowers. Trans. of the Eighth National Vacuum Symposium and the Second International Congress on Vacuum Science and Technology, vol. II. L. E. Preuss, ed., Pergamon Press, 1962, pp. 1235-1243.

7. Steinhertz, H. A.: Handbook of High Vacuum Engineering. Reinhold Publ. Corp. (New York), 1963.

8. Dushman, S.: Scientific Foundation of Vacuum Technique. J. M. Lafferty, ed., John Wiley \& Sons, Inc., 1962.

9. Stevenson, D. L.: A New Type of Boiler That Permits Improvements in the Performance of Oil Diffusion Pumps. Trans. of the Sixth National Symposium on Vacuum Technology, C. R. Meissner, ed., Pergamon Press, 1960, pp. 134-139.

10. Hablanian, M. H.; and Landfors, A. A.: Design and Performance of Modern Diffusion Pumps. Trans. of the Seventh National Symposium on Vacuum Technology, C. R. Meissner, ed., Pergamon Press, 1961, pp. 55-59.

11. Bächler, W.; Clary, R.; and Forth, H.: Development of Diffusion Pumps. Trans. of the Tenth National Vacuum Symposium, G. H. Bancroft, ed., The Macmillan Co., 1963, pp. 153-158.

12. Florescu, N. A.: Critical Survey on the Physics of the High Vacuum Vapor Pump. Vacuum, vol. 13, Dec. 1963, pp. 569-578.

13. Milleron, N.; ANd Levenson, L. L.: Discussion on Optimization of Large Oil Pumped Ultra-High Vacuum Systems. Trans. of the Seventh National Symposium on Vacuum Technology, C. R. Meissner, ed., Pergamon Press, 1961, pp. 213-218. 
14. Fujinaga, A.; Hanasaka, T.; And Tottori, H.: Behavior of Residual Oil Vapor and Back Diffusion of Diffusion Pumps. Trans. of the Ninth National Vacuum Symposium, G. H. Bancroft, ed., The Macmillan Co., 1962, pp. 390-394.

15. Hengevoss, J.; AND HUBer, W. K.: The Influence of Fore-Vacuum Conditions on Ultra-High Vacuum Pumping Systems With Oil Diffusion Pumps. Vacuum, vol. 13, no. 1, Jan. 1963, pp. 1-9.

16. Hablanian, M. H.; and Vitkus, P. L.: Residual Gases in Ultra-High Vacuum Systems With Oil Diffusion Pumps. Trans. of the Tenth National Vacuum Symposium, G. H. Bancroft, ed., The Macmillan Co., 1963, pp. 140-146.

17. Barrington, A. E.: High Vacuum Engineering. Prentice-Hall, Inc., 1963.

18. Klopfer, A.; And Ermrich, W.: Properties of a Small Titanium-Ion Pump. Vacuum, vol. 10, no. 1/2, Feb./Apr. 1960, pp. 128-132.

19. Lichtman, D.: Hydrocarbon Formation in Ion Pumps. J. Vac. Sci. \& Tech., vol. 1, no. 1, Sept./Oct. 1964, pp. 23-24.

20. Turner, F. T.; And Feinleib, M.: Performance Criteria for Sorption Pumps. Trans. of the Eighth National Vacuum Symposium and the Second International Congress on Vacuum Science and Technology, vol. I. L. E. Preuss, ed., Pergamon Press, 1962, pp. 300-306.

21. John, J. E. A.; ANd Hardgrove, W. F.: Cryosorption-A Method for Simulating the Space Vacuum Environment. A paper presented at the AIAA Space Simulation Testing Conference, AIAA Publication CP-11, Nov. 1964, pp. 1-7.

22. Hemstreet, R. A.; et al.: The Cryosorption Pumping of Hydrogen at $20^{\circ} \mathrm{K}$. AEDC-TDR-64-100, May 1964.

23. Stern, S. A., et al.: The Cryosorption Pumping of Hydrogen and Helium at $20^{\circ} \mathrm{K}$. AEDC-TDR-62-200, Oct. 1962. 
 \\ Cryogenic Pumping}

\section{INTRODUCTION}

G ASES CONDENSED to the solid state on low temperature surfaces are said to be cryogenically pumped. The concept is not new to vacuum technology, in the general sense of cooling a substance to lower the vapor pressure. Low-temperature traps have been used for many years to pump water vapor and diffusion pump fluid vapors. However, the additional requirement of very high pumping speeds for low density wind tunnels (ref. 1) and space simulators (ref. 2) led to the process of low-temperature pumping of noncondensable gases such as air. Although temperatures of $20^{\circ} \mathrm{K}$ or lower are required for space simulator applications as compared to the $78^{\circ} \mathrm{K}$ temperature in liquidnitrogen-cooled traps, the basic phenomena of cryogenic pumping are the same for the two instances. The major differences between the two applications arise from the increased complexity of the refrigeration equipment required to attain the lower temperature. More extensive thermal shielding is required with a resulting change in the geometry of the pumping surface array.

This chapter will begin the discussion of cryogenic pumping by defining a perfect cryopumping surface and investigating the parameters involved in this process. Several common cryopumping arrays are presented and analyzed in detail. An important factor in high-vacuum systems, the liquid nitrogen trap, is discussed and criteria for its performance are presented, together with illustrations of several common trap designs.

\section{PERFECT CRYOGENIC PUMPING SURFACES}

When every molecule of a gas specie adheres to a surface upon its first contact, that surface is said to be a perfect cryopumping surface. The pumping speed of this surface will be identical to that of an orifice experiencing a zero pressure on the downstream side. The pumping speed, for molecular flow conditions, is

$$
S_{0}=\frac{\bar{v}}{4} \times 10^{-s}
$$

where $\bar{v}$ is the average molecular velocity, $\mathrm{cm} / \mathrm{sec}$

$$
\bar{v}=\left(\frac{8 k T}{\pi M}\right)^{1 / 2}
$$


$S_{0}$, evaluated at $25^{\circ} \mathrm{C}$ for air, is $11.67 \mathrm{liter} / \mathrm{cm}^{2}$ sec. The reemission of condensed molecules can be neglected. Thus, equation (7.1) holds true when the surface temperature is low enough to produce a vapor pressure which is a decade or more lower than chamber pressure. Gases of higher vapor pressure require lower surface temperatures to produce the desired results.

Figure 7.1 illustrates the vapor pressure-temperature relationship for common vacuum system gases. If a sufficiently large pumping surface can be maintained at $20^{\circ} \mathrm{K}$, nitrogen, carbon monoxide, oxygen, and the more condensable gases may be pumped to partial pressures below $10^{-8}$ torr. However, helium, hydrogen, and neon must be handled separately by diffusion pumps rather than by the pumping surface.

It has been stated previously in defining the perfect cryopumping surface, that every molecule striking the surface will adhere to it. Does this process occur under actual conditions? That is, will a molecule striking the cold wall take on the wall temperature and thus adhere to the wall? A surface which possesses a unity accommodation coefficient for a particular gas will completely accommodate the temperature of an impinging gas molecule to that of the wall. The molecular sticking coefficient or the probability that the molecule will adhere to the wall, $a$, is closely related to the accommodation coefficient. It is standard operating procedure in vacuum technology to hold cryogenic surfaces at temperatures which correspond to vapor pressures a decade or more lower than system pressure. Thus, if the accommodation coefficient approaches unity, the sticking coefficient $a$ will also approach unity.

Experimental methods for evaluating $a$ for common gases (refs. 3 to 7) have yielded values ranging from 0.4 to 1.0. However, the more recent experiments (refs. 5 and 6) concur on values close to unity. References 7 and 8 , and chapter 4 of this manual demonstrate that the effects of directional pressure dictate the use of care in the interpretation of $a$ on the basis of measured pumping speed. Several investigators have reported pumping speeds greater than those obtainable from equation (4.4) through neglect of the above considerations. It is not possible to have values of $a$ greater than unity in molecular flow, although there is evidence of values greater than unity (refs. 6 and 7) in the transition region. Sticking coefficients, based on projected area, can be made to approach unity in some applications if honeycomb condensing surfaces are used (ref. 8) to decrease the thickness of the solidified gas and to increase the thermal emissivity. 
Temperature, Degrees Centigrade

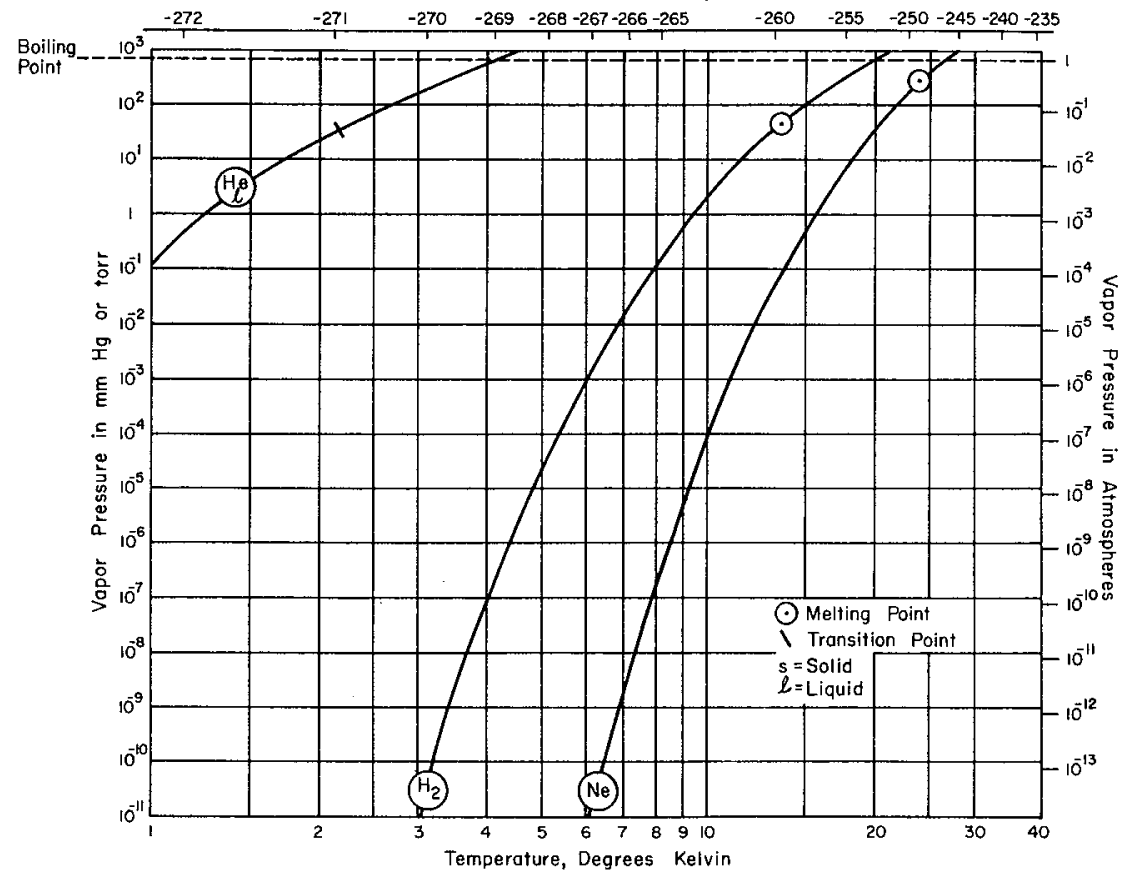

Temperature, Degrees Centigrade
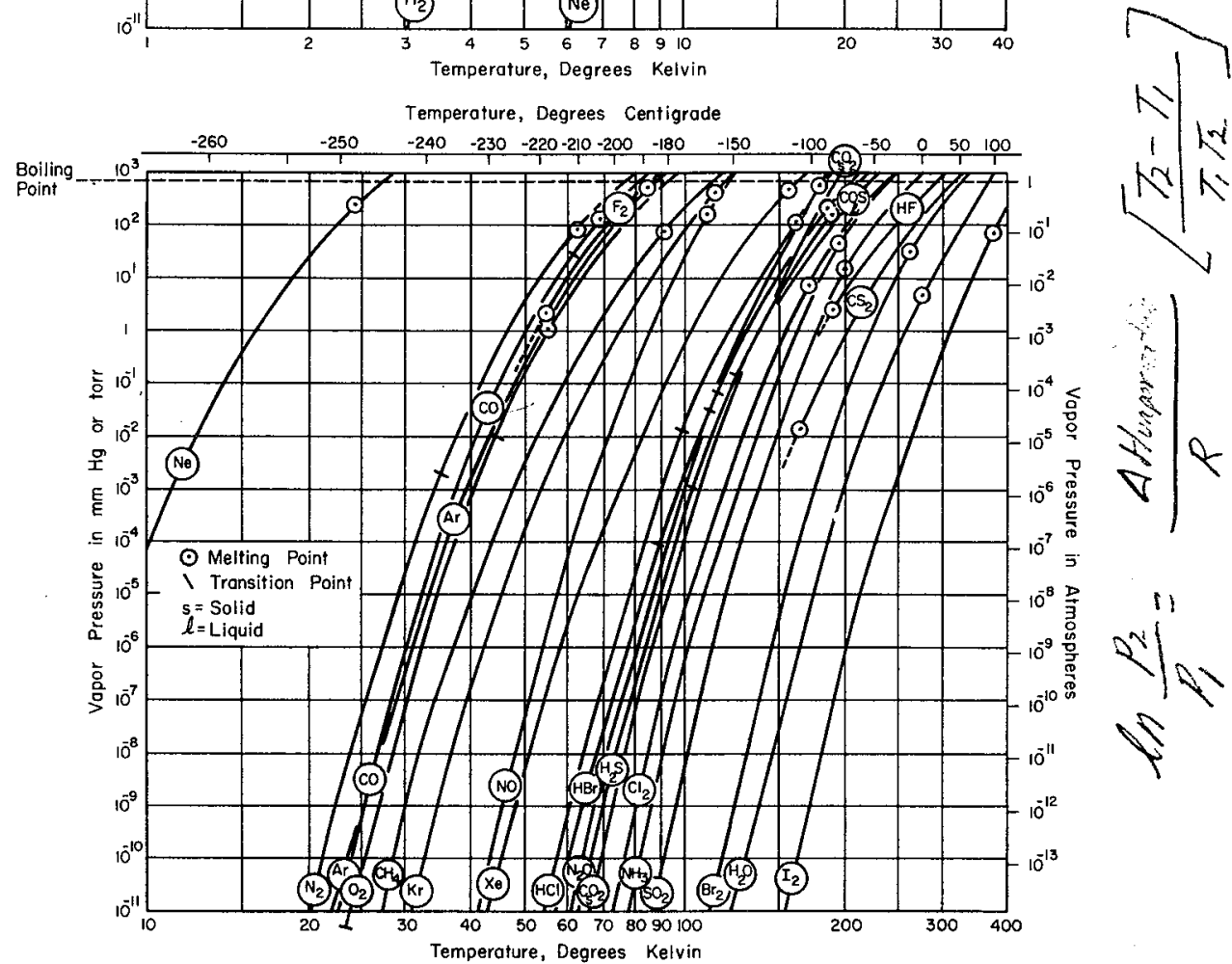

FIGURE 7.1.-Vapor pressure-temperature relationship curves for common vacuum system gases. 


\section{EXTENDED CRYOGENIC PUMPING SURFACES}

Cryogenic pumping provides the only practical way of achieving realistic simulation of the vacuum conditions existing in outer space (ref. 2). Only by distributing the pumping about the walls of the
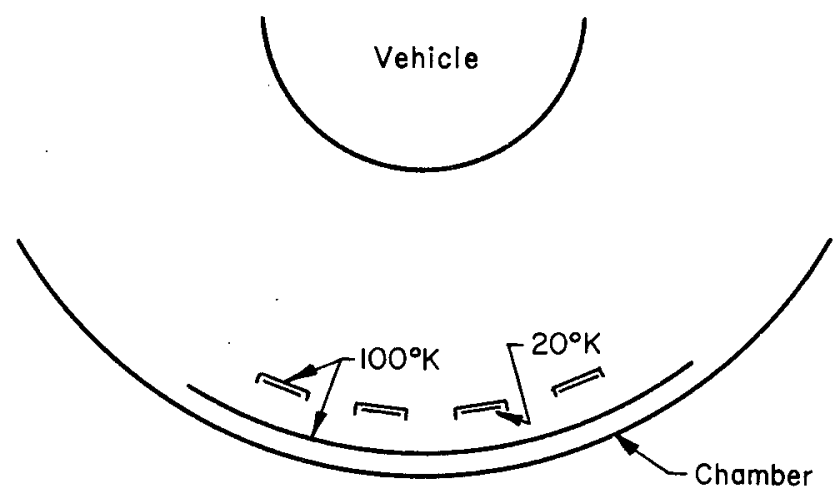

(a)

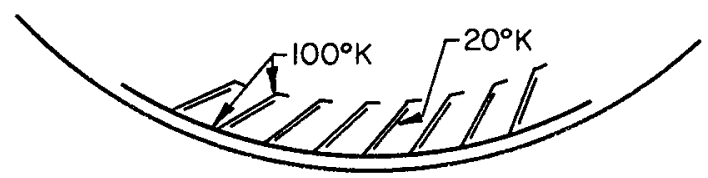

(b)

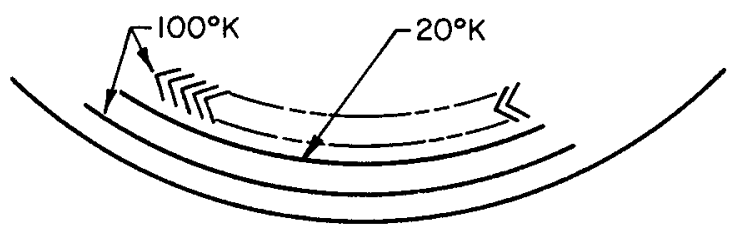

(c)

Figure 7.2.-Cryopumping array schemes, all of which can $b$ applied to large surfaces; $100^{\circ} \mathrm{K}$ surfaces are interposed be tween $20^{\circ} \mathrm{K}$ surfaces and the test specimen and chamber wall: so as to protect the $20^{\circ} \mathrm{K}$ surfaces from radiant energy. 
simulation chamber can the gases originating at the test specimen have a small probability of returning to the specimen.

Figure 7.2 illustrates several common cryopumping array schemes, all of which can be applied to large surfaces. Configurations (a) and (b) have been used in chambers over 30 feet in diameter. The criteria of surface temperature plays an important role in each of the displayed schemes. Temperatures in the range of $20^{\circ} \mathrm{K}$ and lower require more extensive and costly refrigeration equipment, while temperatures in the vicinity of $100^{\circ} \mathrm{K}$ can be produced at a much lower cost using liquid nitrogen. The $20^{\circ} \mathrm{K}$ surfaces must be protected from the radiant energy of the test specimen and the chamber walls, so shields cooled to $100^{\circ} \mathrm{K}$ are interposed. Since the $20^{\circ} \mathrm{K}$ surfaces may receive reflected energy from the shield surfaces, the thermal emissivity, $\epsilon$, of the shield surfaces is made as high as possible. Heat transfer by gas conduction and convection is negligible at space-simulator pressures.

In addition to protecting the $20^{\circ} \mathrm{K}$ surfaces, the liquid-nitrogen-cooled shields serve two other important functions. First, they are efficient pumping surfaces for water vapor which is usually the largest single gas load; and second, they provide the radiative thermal simulation of space. That is, they reflect and reradiate only a very small thermal flux compared to that emitted by a test vehicle near room temperature. (Radiated energy is proportional to the fourth power of absolute temperature.)

It would be possible to duplicate the infinite pumping speed of space if the chamber walls were totally covered with perfect pumping surfaces so that none of the outgassed molecules from the test specimen could return to the specimen. References 2,9 , and 10 describe the effect of nonperfect pumping. A somewhat simplified development of reference 10 will be discussed in the following sections.

Figure 7.3 depicts a spherical vehicle whose surface does not act as a sink for returning molecules. If $\alpha^{\prime}$ is the capture probability of a molecule striking the chamber wall and $B$ is the probability that a molecule leaving the chamber wall will strike the vehicle, consideration of the mass balance at an instant of time will yield

$$
q_{w}=q_{w}^{0}+q_{w}(1-B)\left(1-\alpha^{\prime}\right)+q_{v}\left(1-\alpha^{\prime}\right)
$$

and

$$
q_{v}=q_{v}^{0}+q_{w} B
$$

where

$q_{w}$ total gas flow from the chamber cryowall, torr liter $/ \mathrm{cm}^{2}$ sec

$q_{w}{ }^{0}$ gas flow from the cryowall due to outgassing, torr liter $/ \mathrm{cm}^{2} \mathrm{sec}$

$q_{v}$ total gas flow from the vehicle, torr liter $/ \mathrm{cm}^{2} \mathrm{sec}$

$q_{v}^{0}$ gas flow from the vehicle due to outgassing, torr liter $/ \mathrm{cm}^{2} \mathrm{sec}$ 


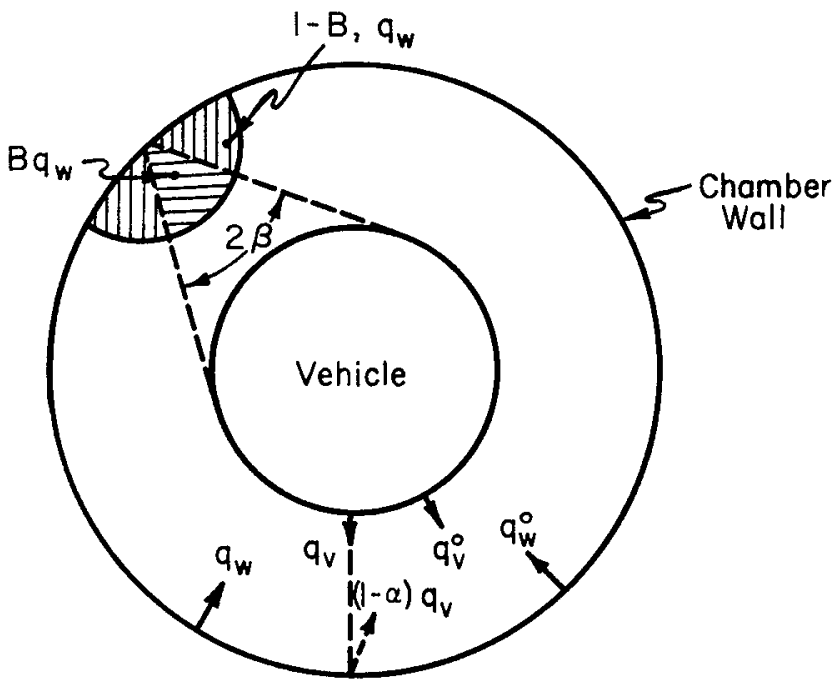

Figure 7.3.-Geometry assumed for the derivation of $Z$, Molecules returning to vehicle wall Molecules initiating at vehicle wall

It can be seen from equation (7.2) that the chamber wall makes three contributions to the total mass flow. These are the outgassing of the wall, that part of the mass flow from the wall which has not struck the vehicle and has not been pumped on one wall encounter, and the total mass flow from the vehicle which has not been pumped on one wall encounter. The total mass flow from the vehicle wall, as given by equation (7.3), is the sum of the outgassing of the vehicle and that portion of the total gas flow leaving the chamber wall which is intercepted by the vehicle. The substitution of equation (7.2) into equation (7.3) yields the total gas flow from the vehicle

$$
q_{v}=q_{v}{ }^{0}\left[\frac{1-(1-B)\left(1-a^{\prime}\right)}{\alpha^{\prime}}\right]+q_{w}^{0}\left(\frac{B}{\alpha^{\prime}}\right)
$$

The effectiveness of a chamber as a space simulator may be measured by the following ratio,

or

$$
Z=\frac{\text { Molecules returning to vehicle wall }}{\text { Molecules initiating at vehicle wall }}
$$

$$
Z=\frac{q_{v}-q_{v}^{0}}{q_{v}^{0}}
$$

If the chamber outgassing has a small value, $Z$ becomes

$$
Z=\frac{B\left(1-a^{\prime}\right)}{\alpha^{\prime}}
$$


$B$, evaluated on the basis of a cosine distribution of the gas leaving a spherical chamber wall, is given as

$$
\begin{aligned}
B & =\frac{\int_{0}{ }^{\beta} \sin \theta \cos \theta d \theta}{\int_{0}^{\pi / 2} \sin \theta \cos \theta d \theta} \\
& =\sin ^{2} \beta \\
& =\frac{a_{1}{ }^{2}}{a_{2}{ }^{2}} \\
& =\frac{A_{1}}{A_{2}}
\end{aligned}
$$

The factor $\alpha^{\prime}$, for perfect pumping surfaces, is the fraction of the chamber wall area covered by such surfaces. If the pumping of water vapor is considered, the configurations of figure 7.2 would approximately illustrate this statement. However, the $20^{\circ} \mathrm{K}$ cryopumping surfaces are shielded from the direct vehicle gas flux, thus complicating the computation of $a^{\prime}$. References 11 and 15 have calculated the capture coefficient $a^{\prime}$ of the shielded surfaces by Monte Carlo techniques. Note that the data in reference 11 are presented in a form which is slightly different from that used in the present discussion. The factor $a^{\prime}$, defined in the present discussion as

$$
\alpha^{\prime}=\frac{\text { Molecules captured by wall in one encounter }}{\text { Molecules incident on wall }}
$$

is replaced by the factor $P$ in reference 11 ,

$$
P=\frac{\left(\begin{array}{c}
\text { Molecules emitted from } \\
\text { vehicle }
\end{array}\right)-\left(\begin{array}{c}
\text { Molecules returned to } \\
\text { vehicle the first time }
\end{array}\right)}{\text { (Molecules emitted from vehicle) }}
$$

where $P$ is not equivalent to the term $Z$ of equation (7.5). That is, when computing $P$, the molecules are traced only until their first return to the vehicle wall if they have not been condensed previously. Factor $P$, as a result, is a step in the computation of $Z$, the factor which describes the simulated molecular flux sensed on the vehicle wall. The relationships among the several variables discussed are

$$
\begin{gathered}
\alpha^{\prime}=\frac{B P}{1-P(1-B)} \\
Z=\frac{B\left(1-\alpha^{\prime}\right)}{a^{\prime}}=\frac{1-P}{P}
\end{gathered}
$$

It has been assumed for equation (7.11) that the cryopanel outgassing rate is negligibly small. Table 7.1 lists pumping probabilities and other pertinent data for various cryopanel configurations.

The data presented in table 7.1 (ref. 11) were calculated for infinitely long, cylindrical cryopanel configurations. The factor $\alpha^{\prime}$ may be determined if the $B$ factor in equation (7.10) is replaced by $B=a_{1} / a_{2}$. 
The rows labeled $a^{\prime}, Z_{\mathrm{cyl}}$, and $Z_{\mathrm{sph}}$ of table 7.1 are not included in reference 11. They have been calculated from equations (7.10) and (7.11) for a cylindrical geometry and a spherical geometry, respectively. $Z_{\mathrm{sph}}$ may be calculated if $a^{\prime}$, the capture coefficient of an array, is assumed to be the same for a cylindrical or a spherical chamber. An average value of $a^{\prime}$ can be obtained for the case of a chamber wall incompletely covered with pumping arrays by multiplying the table value of $\boldsymbol{a}^{\prime}$ and the fraction of wall covered. The sticking coefficient of the surface $a$ was assumed to equal unity for the computations of reference 11. For lower values of $\alpha$, the factor $\boldsymbol{a}^{\prime}$ will also be reduced, but at a rate which is less than proportional to $a$. Once a molecule has penetrated the protective shields to the condensing surface, it has a high probability of returning to the condensing surface before colliding with the vehicle. Thus, if a falls to $0.9, a^{\prime}$ might fall to only about 95 percent of its value in table 7.1.

The quantity $Q_{T} / Q_{1} \alpha_{E}$ of table 7.1 is composed of the ratio $Q_{T} / Q_{1}$, the fraction of vehicle radiated heat flux that is absorbed by the $20^{\circ} \mathrm{K}$ surfaces, and $a_{E}$, the radiant absorbtivity of the cryopanel. The protective shield has an assumed absorbtivity of 0.9 , and diffuse reflections are as-

Table 7.1.-Pumping Probabilities and Heat Loads for Various Optically Tight Cryopanel Configurations

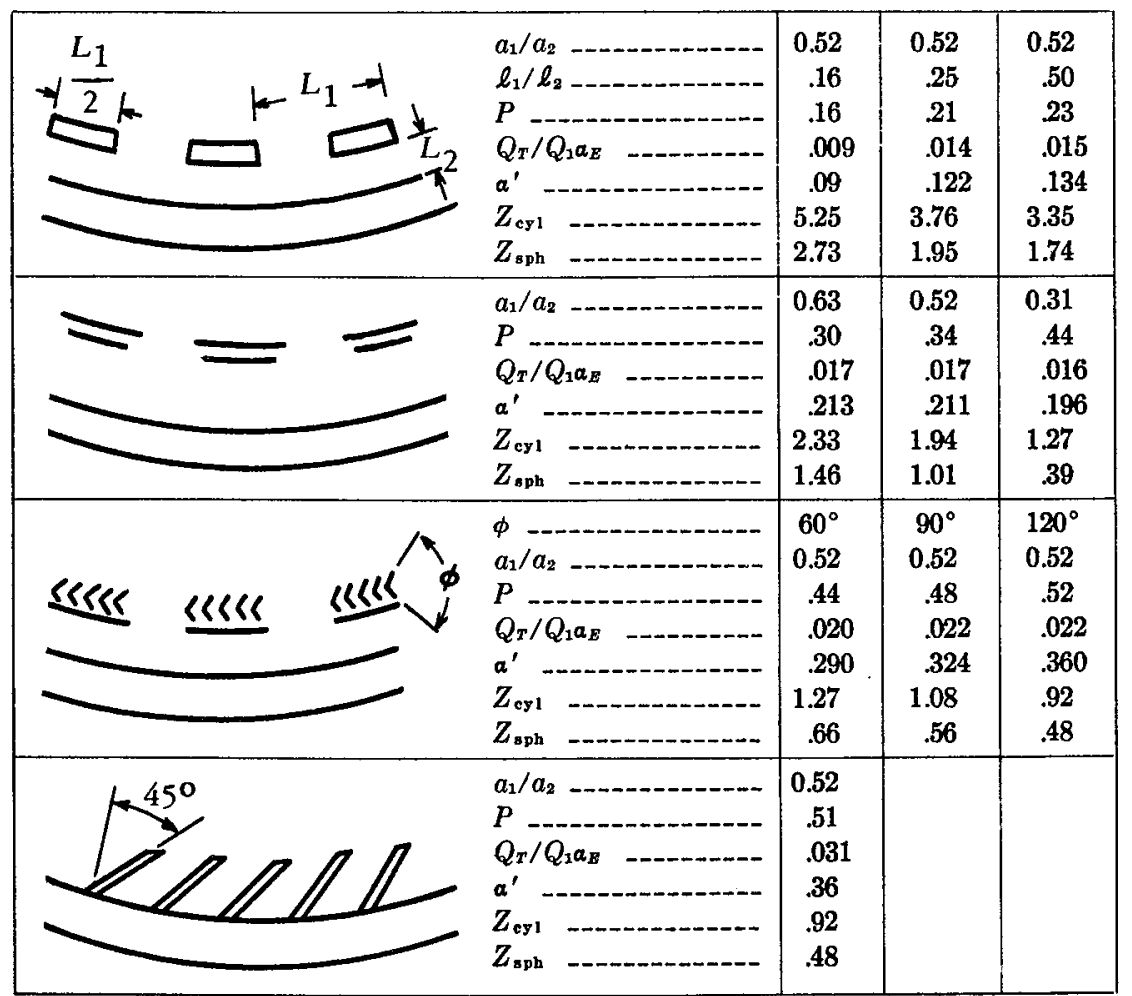




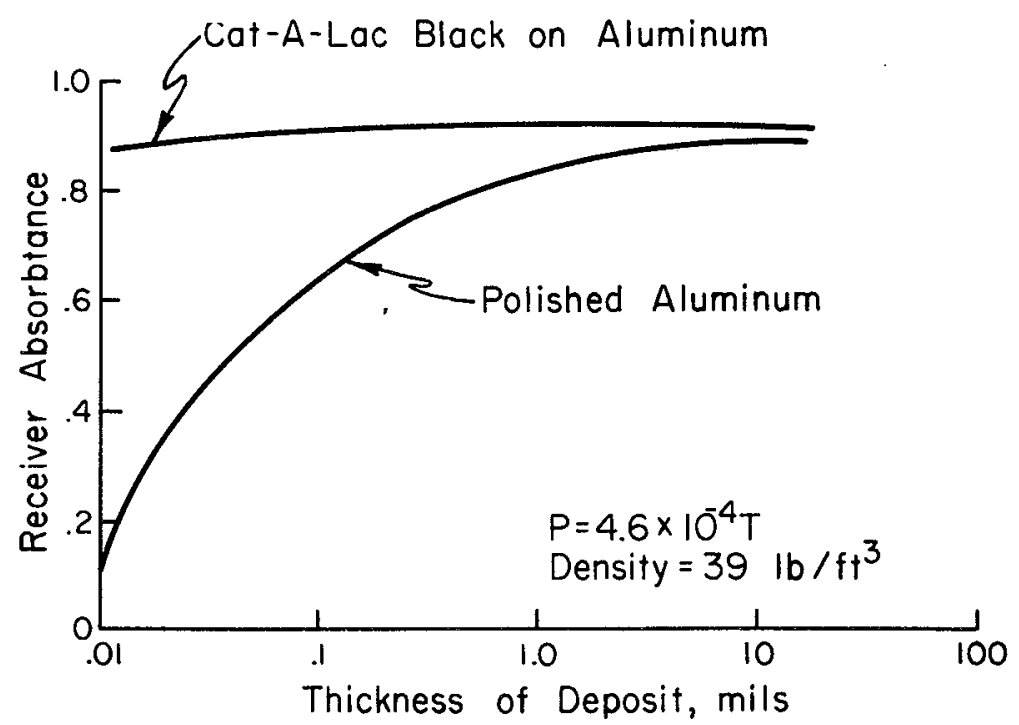

Figure 7.4.-Values of absorbtivity of a water cryodeposit for room temperature blackbody radiation.

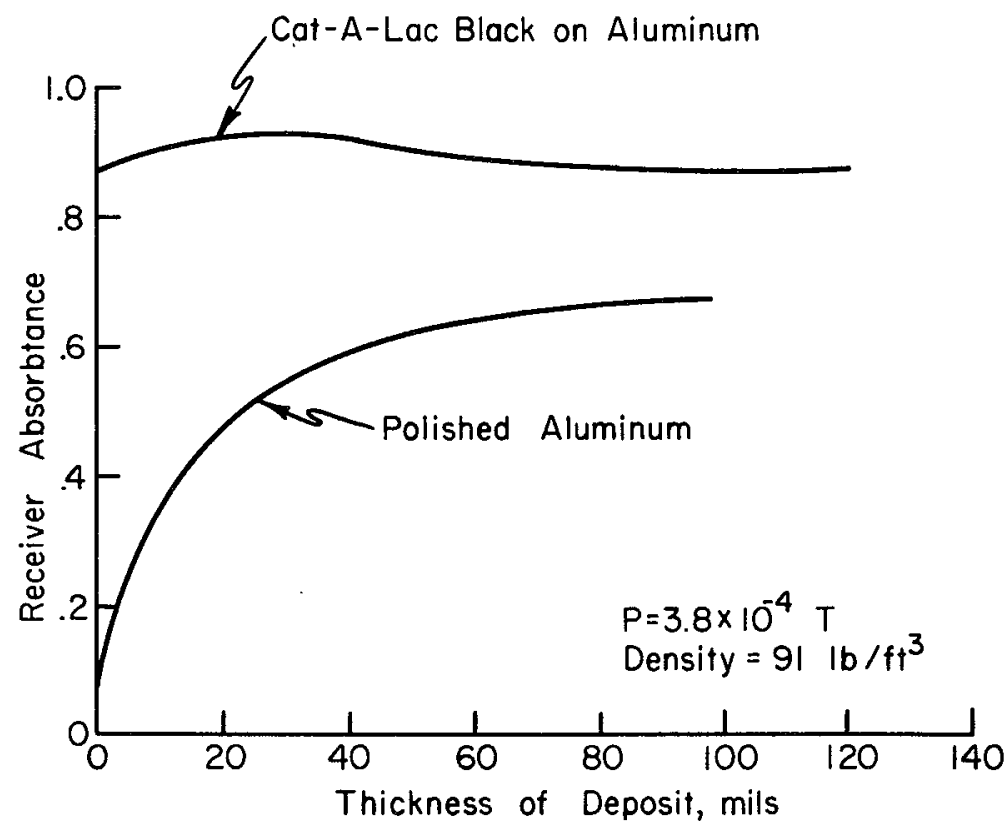

Figure 7.5.-Values of absorbtivity of a carbon dioxide cryodeposit for room temperature blackbody radiation. 


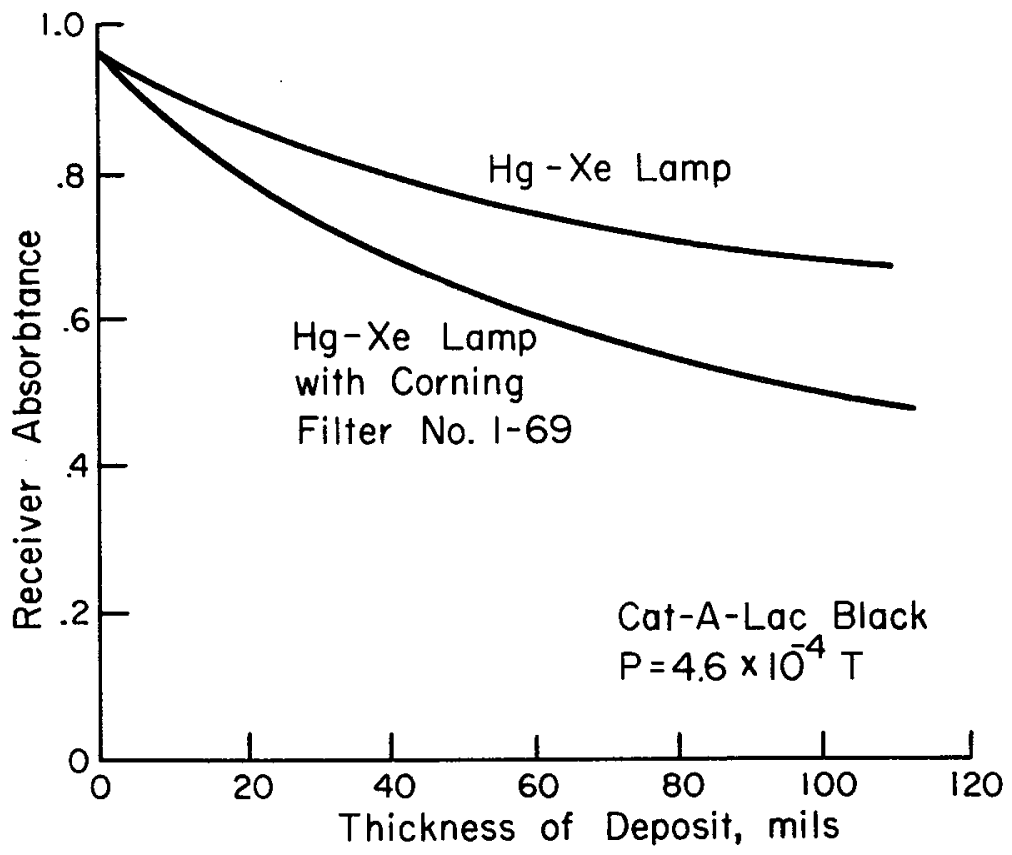

FiguRE 7.6.--Values of absorbtivity of a water cryodeposit for simulated solar radiation.

sumed. The table shows that the thermal shielding of the condensing surface can be made quite effective without destroying the molecular pumping speed.

Figures 7.4 to 7.7 present data from references 12 to 14 on measured values of absorbtivity for several types of cryodeposits. Infrared radiation tends to exhibit higher absorbtance for the cryodeposit values as deposit thickness increases, while the opposite is true for simulated solar radiation. This factor is an important consideration in the design of liquid nitrogen shields for solar-simulating space chambers. For example, liquid nitrogen shields have been covered with blackened aluminum honeycomb materials to maintain high solar absorbtivity.

There are a number of refrigeration system designs which are in use for cooling of the cryosurfaces. A frequently used method for the arrays shown in figure 7.2 incorporates liquid nitrogen and gaseous helium. The liquid nitrogen circulates in the shield panels, while gaseous helium from a gas-cycle refrigerator flows in the $20^{\circ} \mathrm{K}$ panels. The gas-cycle refrigeration units are available commercially in sizes ranging from about 200 watts at $20^{\circ} \mathrm{K}$ to several kilowatts. The $20^{\circ} \mathrm{K}$ temperature is only a nominal value, and lower system ultimate pressures can be maintained with lower temperatures. 


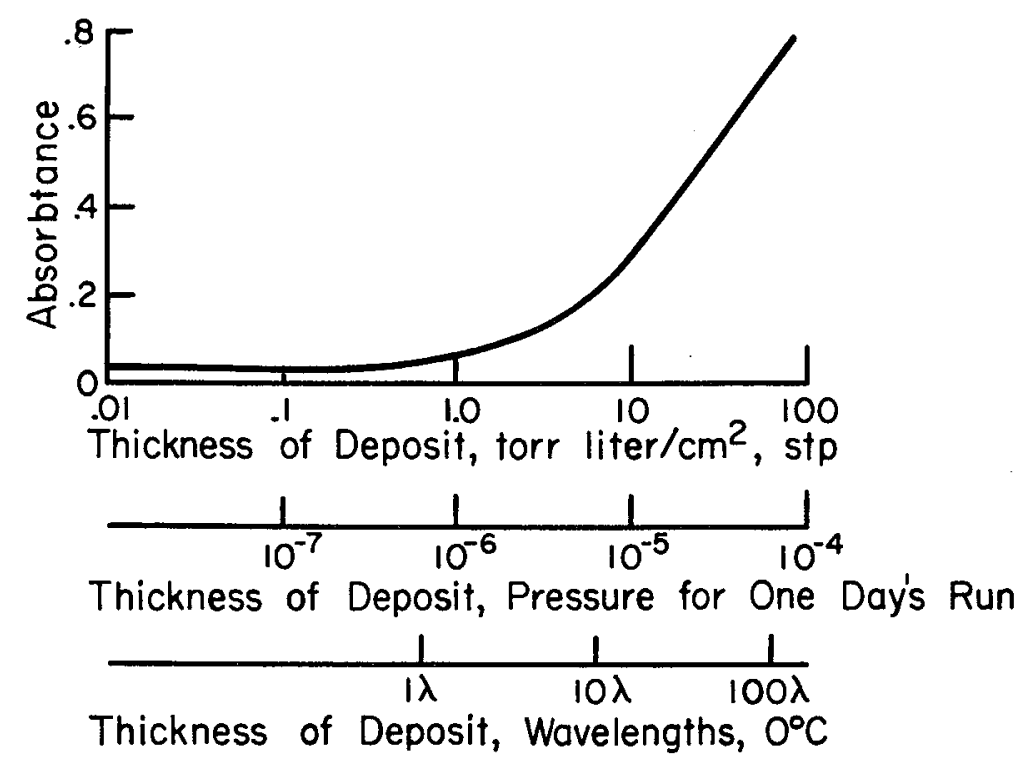

Ficure 7.7.-Values of absorbtivity of a nitrogen cryodeposit for $0^{\circ} \mathrm{C}$ black. body radiation, where the thickness of the cryodeposit is given in torr liter/ $\mathrm{cm}^{2}$, pressure for a run of 1 day, and wavelength.

One common characteristic of all refrigeration cycles is that the capacity decreases as the temperature is lowered. Therefore, it is uneconomical to design for a lower temperature than is necessary for effective pumping. In the case of a gas-circulating system, the cryopanel piping becomes an integral part of the refrigerator. This condition means that the panel-pressure drops must be consistent with the limitations of the rest of the cycle. At the same time, the heat-transfer coefficient between the circulating gas and the panels must be high enough to assure efficient utilization of the available capacity.

Another commonly used cooling system, the subcooled or pressurized circulation system, utilizes the refrigerating effect of liquid nitrogen to cool the shield panels. In this subcooled or pressurized circulation system, the liquid nitrogen is pumped through the panels at a pressure high enough to prevent boiling. In other words, the circulating liquid nitrogen must never attain a temperature as high as the saturation temperature corresponding to the liquid pressure. The piping exit is the most likely site for the occurrence of boiling. At this point, the highest temperature exists, and the pressure is the lowest due to losses in the tubing. As long as two-phase flow is avoided, the hydraulics and heat transfer configuration of the system can be handled by standard design methods. 

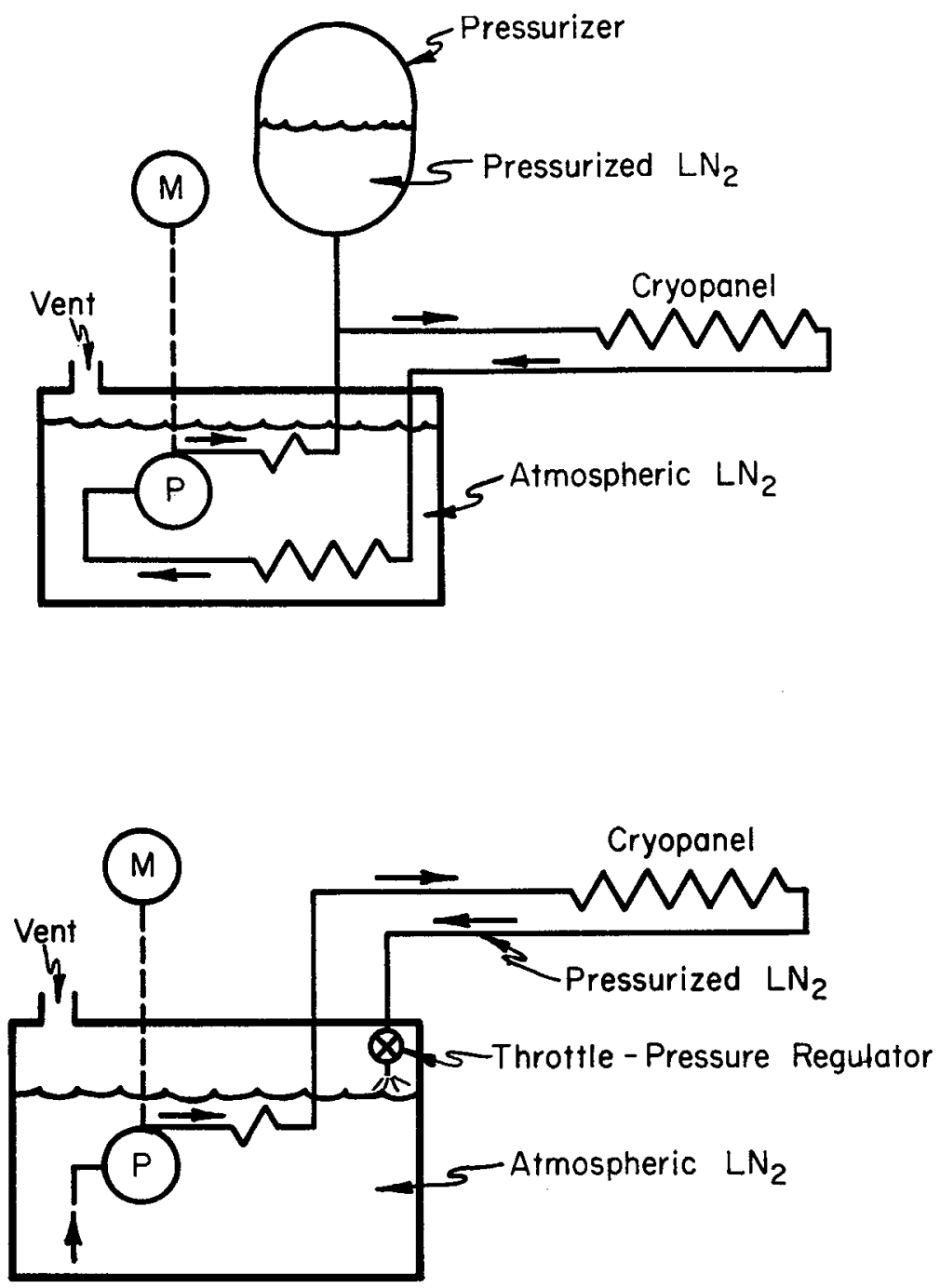

Figure 7.8.-Two types of subcooled liquid nitrogen circulator assemblies used for continuous cooling of cryopanel arrays. Top, closed cycle; bottom, open cycle.

Figure 7.8 illustrates two types of the system just discussed. In the case of the closed system, a pressurizer maintains the average pressure so that the pump supplies only the head required to pump the liquid coolant. Heat absorbed from the panels is removed to atmospheric liquid nitrogen through a heat exchanger, while the heat donated by the pump may or may not be removed by an aftercooler. The second type is an open system where a once-through design specifies that the pump 
must supply the head losses of the system and also maintain the average system pressure. For this configuration, the pressurized liquid nitrogen is flashed back to atmospheric pressure, and after one circulation the latent heat of that portion of the liquid which flashes to gas assumes both the heat absorbed in the panels and the heat from the pump. As in the first type of system, a pump aftercooler may or may not be incorporated. The open-cycle system requires less equipment, but its higher head pump exhibits a greater nitrogen consumption rate than the closed system.

A third system of unpressurized, boiling liquid nitrogen cycles is not used on large systems due to the difficulty of insuring proper distribution of the liquid. Vibration from the boiling action generally tends to dislodge condensed gases to cause chamber-pressure bursts.

The temperature of the outer surface of the condensed gas is the critical temperature of a cryopumping system. If the thermal loads on the cryogenic paneling are small enough to be neglected, the temperature of the outer surface of the condensed gas would be almost identical to the temperature of the heat-transfer fluid. At space-simulation pressures, the latent heat load of the condensing gas is quite small. Several temperature drops between the condensate surface and the incoming heat transfer fluid will occur:

(1) A temperature rise in the heat transfer fluid $\Delta T_{1}$, due to gross heat absorption

(2) A temperature rise between the fluid and the tube wall $\Delta T_{2}$, due to a finite heat transfer coefficient

(3) A temperature rise between tube wall and the fin tip, $\Delta T_{3}$, due to conduction losses in the fin

(4) A temperature rise between the fin and the outer surface of the cryodeposit, $\Delta T_{4}$, due to conduction losses in the solid gas deposit

The bulk temperature rise of the fluid, $\Delta T_{1}$, is given by

$$
\Delta T_{1}=\frac{Q_{H}}{4.186 \dot{m} c_{v}}
$$

where $Q_{H}$ is the heat accumulated by the fluid, or thermal flux, watt, $\dot{m}$ is the mass flow rate, gram/sec, and $c_{v}$ is the specific heat. Temperature rise between the fluid and the tube wall, $\Delta T_{2}$, depends on the fluid heat transfer coefficient which can be calculated by standard methods. References 16 and 17 provide extremely useful information for this calculation. Figure 7.9 illustrates a situation which arises frequently when calculating $\Delta T_{3}$. It can be seen that a temperature drop occurs along a surface which receives uniformly distributed radiant energy. The temperature drop along the length of this fin is

$$
\Delta T_{3}=\frac{q_{H} L^{2}}{2 b k_{\mathrm{fin}}}
$$




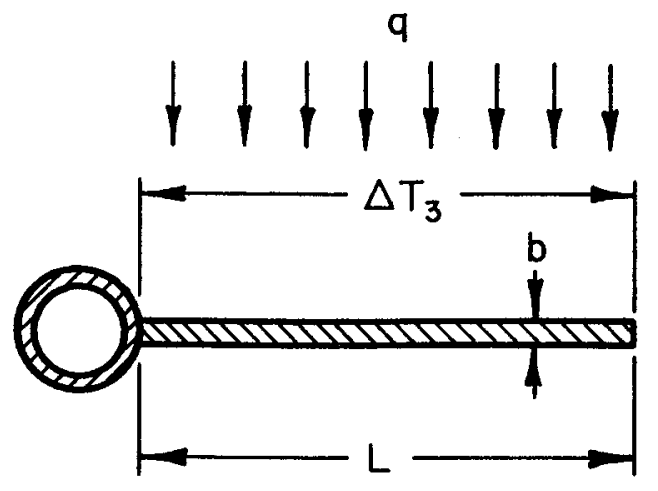

Figure 7.9.-The temperature drop, $\Delta T_{3}$, along a fin which is receiving a uniformly distributed radiant energy.

where

$\Delta T$ temperature drop, ${ }^{\circ} \mathrm{C}$

$q_{H}$ thermal flux density, watt $/ \mathrm{cm}^{2}$

$L$ fin length, centimeters

$b$ fin thickness, centimeters

$k$ thermal conductivity, watt $/ \mathrm{cm}^{\circ} \mathrm{C}$

The fin thickness is instrumental in minimizing cooldown time in that the thickness should not be greater than is necessary to maintain $\Delta T_{3}$ at a reasonable level.

$\Delta T_{4}$ is expressed as

$$
\Delta T_{4}=\frac{q_{H} t}{k_{c}}
$$

where $t$ is the deposit thickness, and the subscript $c$ refers to the condensate. The deposit thickness depends on the amount deposited and the density of the material. For solid nitrogen, reference 18 gives the density as $1.2 \times 10^{3}$ torr liter $/ \mathrm{cm}^{2}$, and a thermal conductivity of $1.67 \times$ $10^{-3} \mathrm{watt} / \mathrm{cm}{ }^{\circ} \mathrm{C}$. Both of these quantities, however, are likely to vary with the pressure and temperature at which the deposit occurs, and with the gas type as well.

Some of the contributions to the heat load on the cryogenic paneling have been mentioned previously. The major sources of this heat load are listed below with a more detailed discussion following:

(1) Radiation from the test vehicle

(2) Radiation, either direct or by reflection from the test vehicle, from albedo and solar simulators

(3) Radiation from the outer chamber wall

(4) Radiation from $100^{\circ} \mathrm{K}$ surfaces to $20^{\circ} \mathrm{K}$ surfaces 
Energy radiated from a test vehicle in a steady-state condition equals the net energy received by the vehicle. This energy may be from electrical power input, conversion of stored chemical or nuclear energy, conversion of intercepted solar simulation, or various other sources. The emissivity of the vehicle and its temperature raised to the fourth power determine the radiant flux density. The order of magnitude for flux densities is indicated by the following example. Consider a black vehicle at $25^{\circ} \mathrm{C}$ where the radiant flux density at the vehicle is $42 \mathrm{watt} / \mathrm{ft}^{2}$, or $0.045 \mathrm{watt} / \mathrm{cm}^{2}$, and the return flux from a black cryosurface at $100^{\circ}$ $\mathrm{K}$ is negligible. Therefore, under the assumption that both the vehicle and chamber are spherical, the flux density received by the cryosurface would be

$$
q_{H}=0.045 \times\left(\frac{\text { vehicle diameter }}{\text { chamber diameter }}\right)^{2}, \text { watt } / \mathrm{cm}^{2}
$$

Of this amount, the fraction received by the $20^{\circ} \mathrm{K}$ surfaces is indicated in table 7.1 for various geometries.

The highest flux densities which cryopaneling may encounter in a space simulator, either directly or by reflection, emanate from solar and albedo simulators. If the additional factors of spillover and reflection are considered while the possibility of a focusing action is neglected, the flux density due to solar and earth albedo simulation for an earthorbiting test vehicle is approximately $200 \mathrm{watt} / \mathrm{ft}^{2}$. It is possible to have much higher flux values, however. For example, the flux density from the heat rejection radiator of a nuclear-power plant equals 13 kilowatt $/ \mathrm{ft}^{2}$ when the plant is operating at $2000^{\circ} \mathrm{F}$ or $1093^{\circ} \mathrm{C}$ with an emissivity of 0.7. Fortunately, such high flux densities generally are confined to a fraction of the chamber wall. As a result, the refrigeration system capacity need only be large enough to absorb the integrated average flux, although the paneling of the arrays must be designed to handle the high flux densities. The $100^{\circ} \mathrm{K}$ circuit completely absorbs radiation from the chamber wall to the outside surface of the cryopaneling. If emissivities of 0.1 and 0.3 are assumed for aluminum paneling and a polished stainless-steel chamber, respectively, the heat load from the chamber wall will amount to $3.4 \mathrm{watt} / \mathrm{ft}^{2}$.

Direct radiation from the $100^{\circ} \mathrm{K}$ to the $20^{\circ} \mathrm{K}$ surfaces may prove to be the largest load on the low-temperature refrigerator even in the presence of solar loads. While the area of solar flux usually extends over a small portion of the paneling, direct radiation is present over the entire paneling. The direct radiation equals $0.53 \mathrm{watt} / \mathrm{ft}^{2}$ on each side of the $20^{\circ}$ fin when the emissivity for both surfaces is assumed to be unity.

Proper design can insure that the heat leaks through mechanical supports are quite small. Thin-walled, stainless-steel members are suitable because of their low thermal conductivity in conjunction with their other desirable features such as relatively high strength and stiffness. Mem- 
bers to be held at $20^{\circ} \mathrm{K}$ temperatures should be mounted from $100^{\circ} \mathrm{K}$ members whenever practical.

\section{LIQUID NITROGEN TRAPS}

The improved design and performance of vacuum components has resulted in the development of large diffusion-pumped vacuum systems capable of maintaining continuous-pressure levels below $10^{-9}$ torr. An important factor in the attainment of these low-pressure levels is the liquid nitrogen trap. The trap functions primarily to prohibit the transfer of pump oil vapor into the vacuum system and to pump, by condensation, water vapors and other vapors which originate in the system. Both of the requirements may be achieved through efficient trap operation. Because of its liquid-nitrogen-refrigerated surfaces, the trap will be treated as a cryogenic device in this discussion.

References 19 to 21 have set forth the basic performance requirements for high-vacuum traps. Well-designed traps will incorporate the features listed below:

(1) The trap offers minimum impedance to the diffusion pump, and to the vapor condensation or cryopumping surface. However, the trap should be effective in keeping pump oil from the chamber.

(2) A constant low temperature of the cryopumping surfaces is maintained to prevent pressure bursts resulting from the reevaporation of condensate.

(3) There is no warm surface path shunting the cold areas which would permit the diffusion pump oil to migrate.

Additional considerations include minimum liquid nitrogen consumption, a trap interior accessible for cleaning, and a water-cooled baffle to prevent holdup of the pump oil on the trap during long-term continuous operation (the condensed oil should be returned to the pump along the trap walls rather than dripping onto the hot jet assembly).

Figure 7.10 illustrates several modern high-vacuum trap designs which meet the above criteria to varying degrees. The first three designs generally are used with pumps of medium and small sizes. An elbow trap may be used on large vacuum systems where a liquid nitrogen circulation system is available. A vacuum system which has been pumped down to its operating pressure range will exhibit molecular flow through the trap for gas and oil molecules. Typical oil molecule trajectories are shown in figure 7.10. It can be seen that for an optically tight trap, an oil molecule must undergo at least one cold-wall collision before entering the vacuum chamber. Ideally, if intermolecular collisions do not occur and the sticking coefficient equals unity, the optically tight trap design should stop all gas-phase oil transfer. Under actual conditions, however, the sticking coefficient is less than unity, and in addition, 


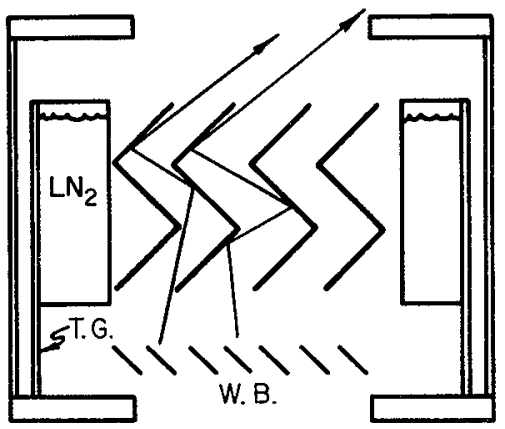

$-a-$

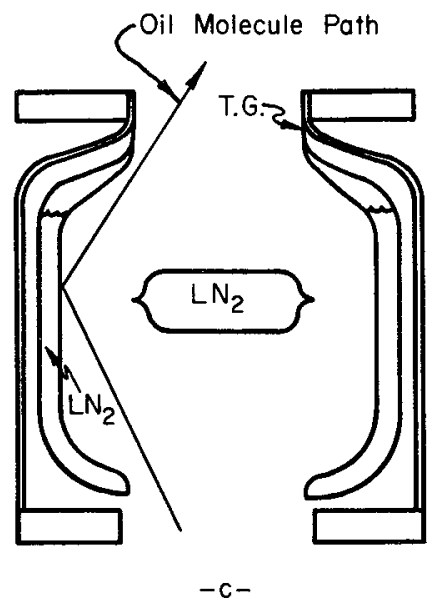

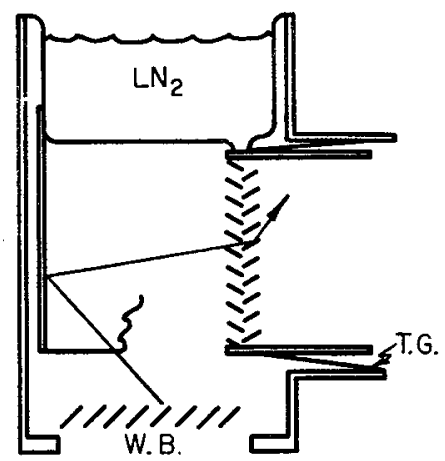

$-b-$

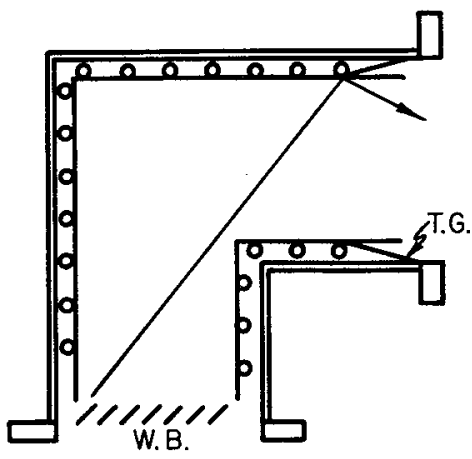

$-d-$

Figure 7.10.-Typical examples of liquid nitrogen trap designs, all of which offer, to varying degrees, minimum impedance to the diffusion pump and cryopumping surface, effective stoppage of pump oil from entering the chamber, a constant low temperature on the cryopumping surfaces, and no warm surface path which shunts the cold areas of the trap. W.B. = water baffle; T.G. = thermal gradient.

oil-to-oil and oil-to-gas molecular collisions occur. Traps can demonstrate more effective trapping action if they are designed such that each oil molecule impinges on the cold surfaces a greater number of times than the minimum of one contact required by simple optical tightness. The first two designs of figure 7.10 illustrate minimum two-contact configurations, while the next two designs are minimum one-contact configurations. Note that figure $7.10(\mathrm{~b})$ satisfies the two-contact requirements with a lower flow restriction than figure 7.10(a).

The results of a study (ref. 22) to determine the oil-backstreaming rate through an optically tight elbow trap with a water baffle are 


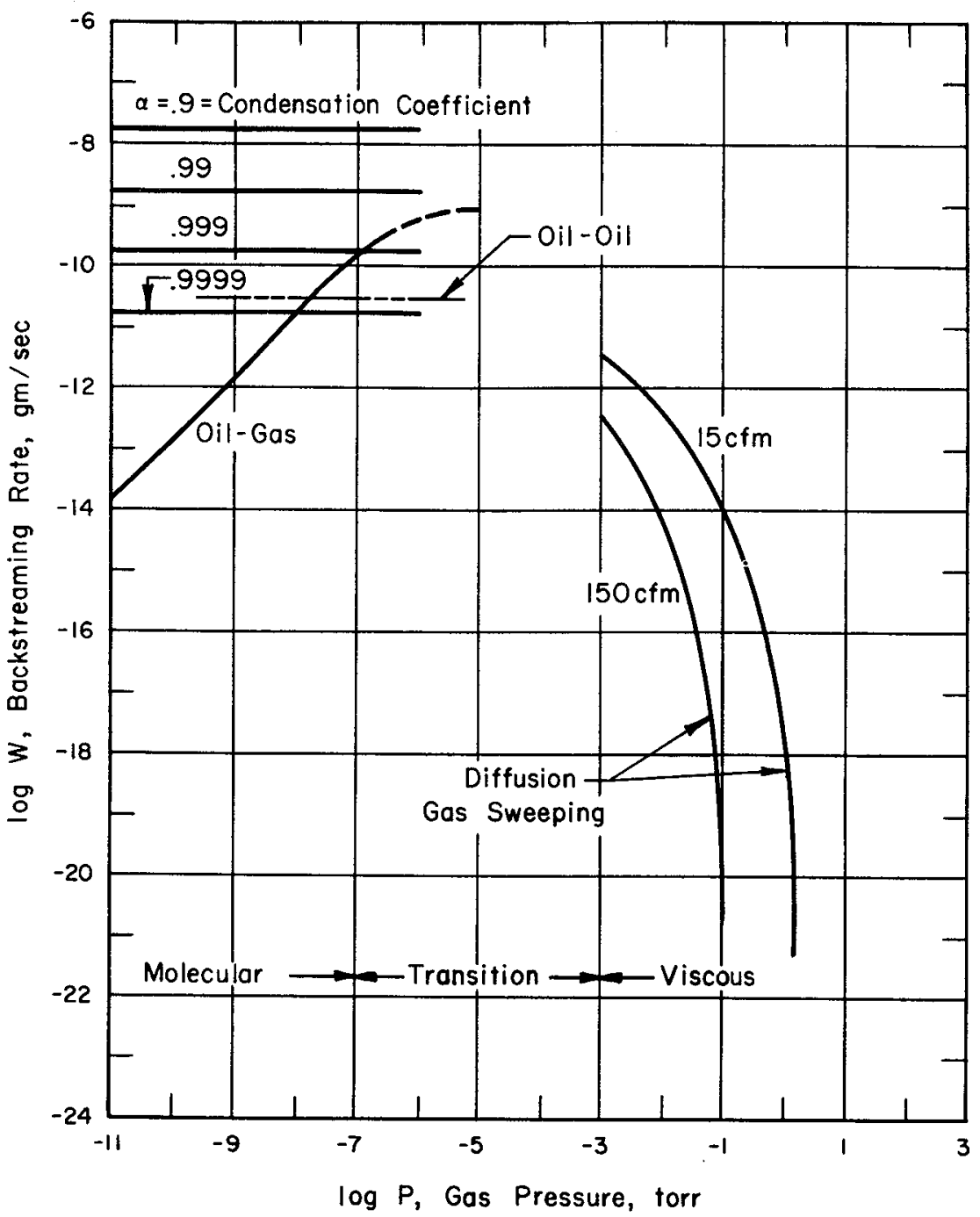

Figure. 7.11.-Composite results of calculating the four modes of oil backstreaming from a 36-inch-diameter elbow trap.

plotted on figure 7.11. The solid horizontal lines represent the backstreaming rate due to a sticking coefficient $a$ unequal to unity, where each line corresponds to a different value of $a$. Unfortunately, $a$ is not accurately known and small differences in its value can cause differences of decades in the backstreaming rate. The interrupted line in the same region of the plot results from oil molecules which are deflected before contact with the condensing surfaces because of oil-to-oil colli- 
sions. This oil-backstreaming mode will be significant only if the sticking coefficient is greater than 0.9999 . The oil-gas collision transfer mode arises with increasing gas pressure and becomes significant only if $a$ exceeds 0.99. Oil transfer can be further minimized by a factor of at least $(1-a)$ if a minimum two-contact elbow trap design is used in place of a simple optically tight trap design. Oil-gas collisions tend to keep the oil molecules from the cold surfaces, thus maximizing oil transfer in the transition flow region (figure 7.11). However, the collisions still have not provided enough sweeping action to prevent the oil from entering the chamber. Traps ordinarily are not meant to operate in the transition region, although conditions existing in the transition region can be expected during pumpdown and other high gas-load situations. The configuration of figure $7.10(\mathrm{~b})$ suppresses this oil-transfer peaking with two regions of trapping which differ significantly in dimension, the skirt and the chevron. As a result, transition flow in the two sections will occur at two different pressure ranges. The oil-transfer peaking, therefore, will tend to spread and be reduced.

Constant low temperatures can be maintained readily on gradient-free cryogenic pumping surfaces. However, regions where the cold surfaces are supported from the ambient temperature surfaces experience temperature gradient shifts resulting from changes in ambient temperature, in the heat load, or in the liquid level or flow rate of the nitrogen. These gradient shifts result in the reevaporation of a part of the condensate. If the reevaporated gas is not retrapped prior to chamber reentry, a pressure rise will occur in the chamber. The configuration of figure $7.10(\mathrm{c})$ has no allowance for retrapping capability. Since the evaporated molecules have a high probability of recondensing before entering the chamber, pressure excursions are suppressed for the other designs of figure 7.10. The amount of gas condensing on the temperature gradient region can also be minimized by shielding these regions from the chamber with cold surfaces (figure 7.10(a), (b), and (d)). Gas condensed on the intermediate temperature areas during the higher pressure phase of a pumpdown will reevaporate at lower pressures and thus cause an increase in pumpdown time. Figures 7.10(a) and 7.10(c) indicate that troublesome temperature gradients may exist on the top of a partially empty liquid nitrogen reservoir when it is in sight of the chamber. If the reservoir walls are designed with a sufficiently high thermal conductivity, this temperature gradient can be avoided.

All the designs of figure 7.10 include an antimigration barrier between the pump and the chamber. That is, surface creepage of the pump oil is prevented, as there are no warm surfaces connecting the inlet and outlet ports.

Traps may receive liquid nitrogen by three methods. The first continuously supplies the nitrogen, throttled from a pressurized source, and 
vents the resultant boiloff to atmosphere. Regulation problems exist for this method which wastes nitrogen due to venting of wet vapor, or causes trap warm spots due to an insufficient supply of nitrogen. The next method, which is quite efficient if the circulation system is available, utilizes the circulation of subcooled liquid with an absence of boiling in the trap. A trap reservoir is not necessary for either method 1 or 2 . For the case of a trap plus a reservoir, intermittent filling, either controlled by a liquid-level controller or by manual operation, can be used. Efficient operation in both methods of filling requires adequately sized reservoirs.

If a diffusion-pumped system is expected to yield clean, ultrahigh vacua, a properly designed and constructed trap is a necessity. In addition, scrupulous attention must be paid to the correct operating procedure for all components of the vacuum system to realize the full potential of the system. Chapter 10 discusses the importance and applications of correct operating procedures.

\section{REFERENCES}

1. Bailey, B. M.; and Chuan, R. L.: Cryopumping for High Vacuum With Low Power. Trans. of the Fifth National Symposium on Vacuum Technology, W. G. Matheson, ed., Pergamon Press, 1959, pp. 262-267.

2. Santeler, D. J.: Pressure Simulation of Outer Space. Trans. of the Sixth National Symposium on Vacuum Technology, C. R. Meissner, ed., Pergamon Press, 1960, pp. 129-133.

3. Hurlbut, F. C.; ANd Mansfiet, R. J.: Calculated and Observed Pumping Speeds of a Shielded Cryogenic Pumping Surface. Vol. 8 of Advances in Cryogenic Engineering, Plenum Press, 1963, pp. 46-56.

4. Dawson, J. P.; Haygood, J. D.; and Col.tins, J. A., JR.: Temperature Effects on the Capture Coefficients of Carbon Dioxide, Nitrogen and Argon. Vol. 9 of Advances in Cryogenic Engineering, Plenum Press, 1964, pp. 443-449; Discussion, pp. 449-450.

5. Buffham, B. A.; Henault, P. B.; and Flinn, R. A.: A Theoretical Evaluation of the Sticking Coefficient in Cryopumping. Trans. of the Ninth National Vacuum Symposium, G. H. Bancroft, ed., The Macmillan Co., 1962, pp. 205-211.

6. Mullen, L. O.; ANd JacoBs, R. B.: Some Characteristics of a Simple Cryopump. Trans. of the Nintn National Vacuum Symposium, G. H. Bancroft, ed., The Macmillan Co., 1962, pp. 220-226.

7. Stickney, W. W.; AND Dayton, B. B.: The Measurement of the Speed of Cryopumps. Trans. of the Tenth National Vacuum Symposium, G. H. Bancroft, ed., The Macmillan Co., 1963, pp. 105-116.

8. Wallace, D. A.; and Rogers, K. W.: Design of Molecular Traps for the High Speed Pumping of Directional Low-Density Nozzle Flow. Vol. 9 of Advances in Cryogenic Engineering, Plenum Press, 1964, pp. 472-480; Discussion, p. 481.

9. Moore, R. W., JR.: Cryopumping in the Free-Molecular Flow Regime. Trans. of the Eighth National Vacuum Symposium and the Second International Congress on Vacuum Science and Technology, vol. 1. L. E. Preuss, ed., Pergamon Press, 1962, pp. 426-438. 
10. Garwin, E. L.: Cryogenic Pumping and Space Simulation. Vol. 8 of Advances in Cryogenic Engineering, Plenum Press, 1963, pp. 37-45.

11. Pinson, J. D.; And Peck, A. W.: Monte Carlo Analysis of High Speed Pumping Systems. Trans. of the Ninth National Vacuum Symposium, G. H. Bancroft, ed., The Macmillan Co., 1962, pp. 406-410.

12. Moore, B. C.: Effect of Gas Condensate on Cryopumps. Trans. of the Ninth National Vacuum Symposium, G. H. Bancroft, ed., The Macmillan Co., 1962, pp. 212-215.

13. Caren, R. P.; Gilcrest, A. S.; and Zierman, C. A.: Thermal Absorptances of Cryodeposits for Solar and $290^{\circ} \mathrm{K}$ Blackbody Sources. Vol. 9 of Advances in Cryogenic Engineering, Plenum Press, 1964, pp. 457-463.

14. Cunningham, T. M.; and Young, R. L.: The Radiative Properties of Cryodeposits at $77^{\circ} \mathrm{K}$. Vol. 8 of Advances in Cryogenic Engineering, Plenum Press, 1963, pp. 85-92; Discussion, p. 92.

15. Ballance, J. O.; Roberts, W. K.; and Tarbeli, D. W.: A Study of Cryopump Configurations in Free-Molecular-Flow Regions. Vol. 8 of Advances in Cryogenic Engineering, Plenum Press, 1963, pp. 57-63; Discussion, pp. 63-64.

16. McAdams, W. H.: Heat Transmission. MeGraw-Hill Book Co., Inc., 1954, p. 532.

17. Sсотт, R. B.: Cryogenic Engineering. D. Van Nostrand Co., 1959.

18. Chuan, R. L.: A Study of the Condensation of Nitrogen Below the Triple Point. Report No. USCEC 56-201, University of Southern California, Feb. 1957.

19. Ullman, J. R.: A Large Metal System Permitting Low Base Pressures. Trans. of the Fourth National Symposium on Vacuum Technology, W. G. Matheson, ed., Pergamon Press, 1958, pp. 95-96.

20. Mrli,kron, N.: Some Component Designs Permitting Ultra-High Vacuum With Large Oil Diffusion Pumps. Trans. of the Fifth National Symposium on Vacuum Technology, W. G. Matheson, ed., Pergamon Press, 1959, pp. 140-147.

21. Henderson, W. G.; Mark, J. T.; and Geiger, C. S.: Evaluation of Large Diffusion Pumps and Traps for the Ultra-High Vacuum System of the Model C-Stellarator. Trans. of the Sixth National Symposium on Vacuum Technology, C. R. Meissner, ed., Pergamon Press, 1960, pp. 170-175.

22. Jones, D. W.; AND Tsonis, C. A.: Theoretical Investigation of Oil Backstreaming Through a Trap. J. of Vac. Sci. \& Tech., vol. 1, no. 1, Sept./Oct. 1964, pp. 19-22. 



\section{Gas Load}

\section{INTRODUCTION}

TT HEN THE GAS is removed from a particular item to produce a vacuum, all phases of the gas-gaseous, solid, and liquid-must be taken into account. The sources of this gas include leaks, capillaries, the vapor of a liquid or solid, the surface of various materials in a vacuum volume, the interior of the materials, and chemical reactions. Each gas source is summed to arrive at the total gas load on the vacuum pump. The determination of the origin of the gas sources and the estimation of their types and rates of evolution at various conditions pose some of the most difficult problems in vacuum technology.

The following sections will investigate the various problems associated with gas load, and will present methods of arriving at conclusive values. It should be realized, however, that this discussion is a simplified treatment of gas sources. The typical vacuum system sources are complex and do not present pressure-time curves as straightforward as those given here which can be obtained only if a single source is placed in the vacuum system and it predominates over other sources.

\section{DEFINITION OF $Q_{\mathrm{L}}$, GAS LOAD ON SYSTEMS}

The basic differential equation of pressure versus time for the exhaust of a volume was given in chapter 4

$$
-V \frac{d P}{d t}=S P-Q_{L}
$$

where $Q_{L}$ is the gas entering the volume from leaks or outgassing, $S P$ is the gas leaving the volume via the pumping system, and $-V(d P / d t)$ is the net gas removed from the volume. If either $S$ or $Q_{L}$ is a function of pressure or time, the solution to equation (8.1) may be extremely complex. In the interest of simplifying the discussion, it is assumed that $Q_{L}$ is either constant, as in the presence of a leak, or is changing very slowly with time such that a quasi-steady-state value may be assumed. In addition, $S$ is assumed to be a constant over the entire range of exhaust pressures. On the basis of these assumptions, equation (8.1) may be simplified to

$$
P=P_{0} e^{(-\Delta t / \nabla)}-\frac{Q_{L}}{S}
$$


Equation (8.2) may be applied to two conditions which are of primary interest and importance to the vacuum engineer. The first occurs during the initial exhaust where $Q_{L}$ is generally very small relative to $P S$, which reduces equation $(8.2)$ to

$$
P=P_{0} e^{\left(-s t / V_{1}\right.}
$$

Equation (8.3), the exponential decay of pressure, is plotted in figure 8.1 as straight-line curves of $\log P$ versus time. The slope of the line is

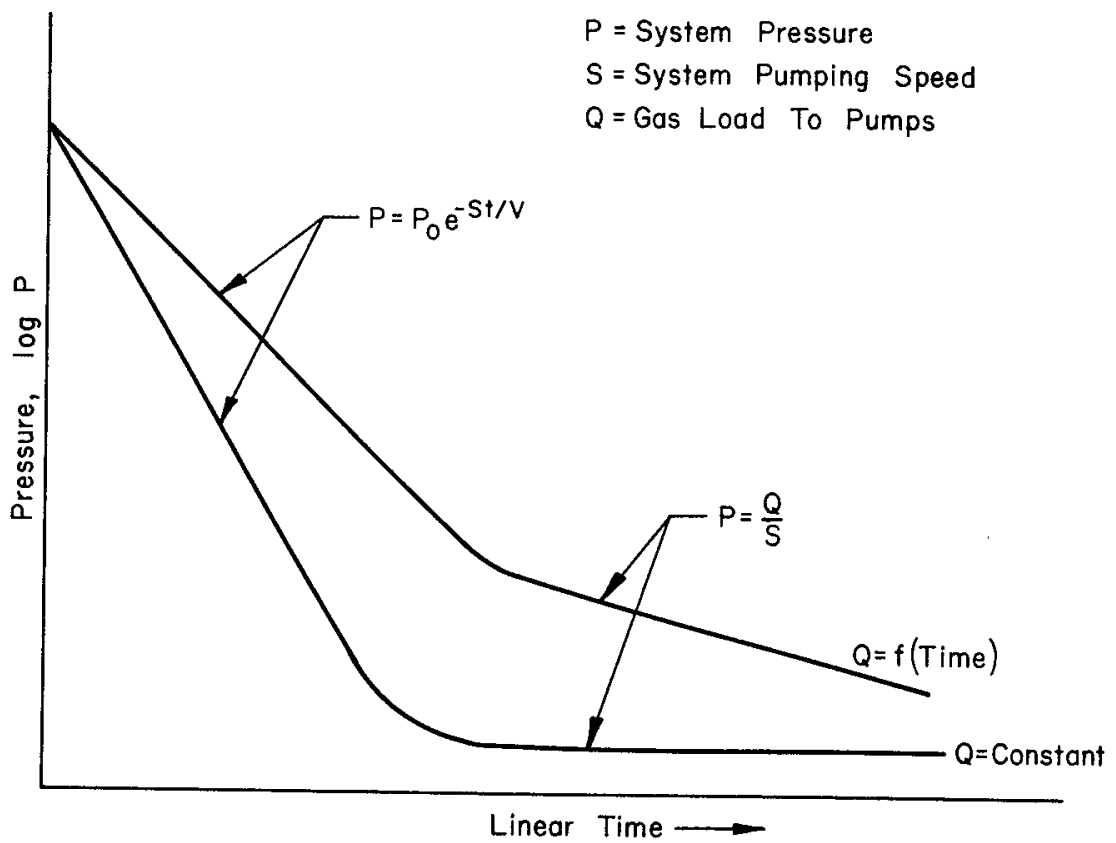

FIGURE 8.1-Exponential decay of pressure where a point is reached, $P=Q / S$, at which the outgassing becomes the controlling factor on system pressure.

determined by the time constant discussed in chapter 4 . In other words, the time to decrease the pressure by one decade is given by equation (4.19)

$$
\tau_{1 / 10}=2.303 \frac{V}{S}
$$

The section on vacuum system operation will cover the application of $\tau$. The second condition occurs farther along in the exhaust process. As the pressure decreases during exhaust, the $P S$ product of gas flow through the pumping system is constantly decreasing and eventually becomes comparable to the existing gas influx $Q_{L}$. The $P S$ decrease will result in 
a departure from the straight-line plot of $\log P$ versus time in figure 8.1. Eventually, a point is reached where the $V(d P / d t)$ segment of equation (8.1), or the exponential term of equation (8.2), is negligible relative to the in-leakage. At this point (the second condition), the outgassing becomes the controlling factor on the system pressure

$$
P=\frac{Q_{L}}{S}
$$

If $Q_{L}$ is a constant value such as a leak, the pressure will remain constant. However, if $Q_{L}$ continually decreases, as in the case for normal outgassing, the pressure also will fall slowly.

\section{SOURCES OF THE GAS LOAD}

\section{I.eaks From an Infinite Source}

If $Q_{L}$ is a true leak in a vacuum system, it is defined by the normal gas-flow equations of chapter 5 . The flow generally associated with large leaks, where the leak rate is greater than about $10^{-5}$ torr liter/sec, occurs in the viscous region. Small leaks of less than $10^{-8}$ torr liter/sec normally will exist in the molecular-flow region.

The exact amount of gas flow through a given physical restriction will depend on the gas type, the driving pressure, and the environmental temperature as defined by the basic gas-flow equations. From a systems viewpoint, the vacuum engineer is concerned only with the presence or absence of a given size leak and not with the mechanics of the gas flow through the leak. As a result, for gas-load considerations, a leak is a steady-state gas load which limits the system ultimate pressure, equation (8.4), and affects the pumpdown curve as shown in figure 8.2. The methods of detecting system leaks are discussed in chapter 11.

\section{Trapped Volumes}

Another and more complicated situation exists if the leak or restriction connects to a limited-volume region rather than to the atmosphere. In this case, the constant removal of gas from the trapped volume reduces the pressure in the volume. The subsequent reduction of the driving force brings about a steady reduction in the leakage value. Figure 8.3 illustrates this situation. A volume $V_{1}$ which is being evacuated by a pump of constant speed $S$, is connected by a conductance $F_{2}$ to a smaller volume $V_{2}$ located within the main chamber. The instantaneous flow of gas from the smaller volume and the gas load on the system is represented by $F_{2}\left(P_{2}-P_{1}\right)$. It is assumed that $F_{2}$ is a constant value, either because the restriction is a sharp-edged orifice, or the flow is always molecular. $F_{2}$ normally is very small relative to the system pumping speed $S$. The relative time constants of the two systems $V_{1} / S$ and $V_{2} / F$ are very important considerations which will be discussed in detail. 


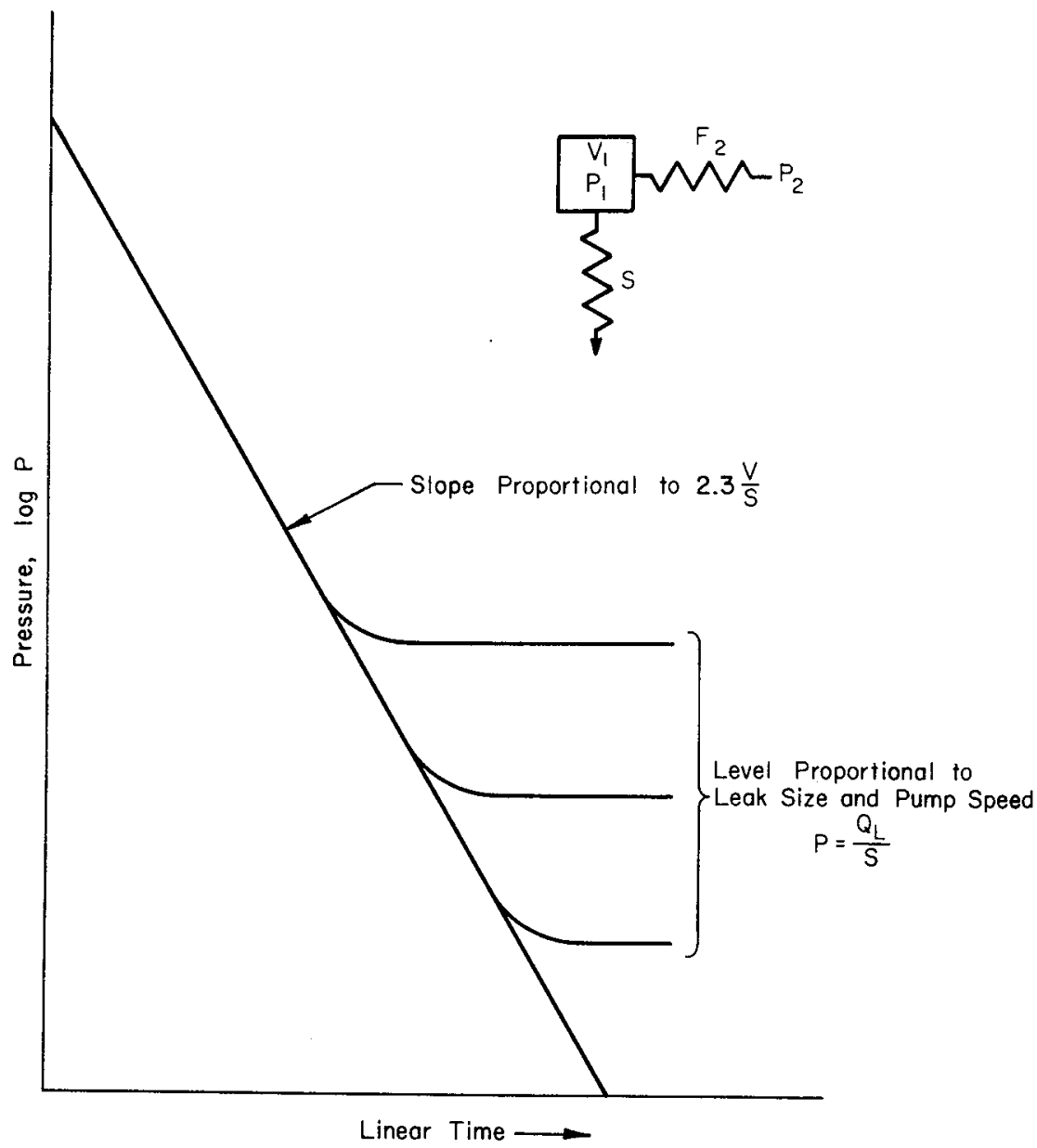

Figure 8.2.-Pressure-time curve for a volume with a constant speed pump and a gas supply from a pressure $P_{2}$ through a conductance $F_{2}$. A leak or steady-state gas load limits the system ultimate pressure.

If $V_{1} / S$ is large relative to $V_{2} / F$, the two volumes will pump down together with only a small lag on the pressure of the smaller interior volume. If the two time constants are of the same order of magnitude, there is no readily available general solution. The case of most interest occurs when $V_{2} / F$ is large relative to $V_{1} / S$ such that the chamber initially pumps down in the conventional manner. A point is reached eventually at which the pressure is limited by the more slowly decreasing gas load from the small volume. The initial pumpdown is governed by equation (8.2)

$$
P=P_{0} e^{-S t / V_{1}}
$$




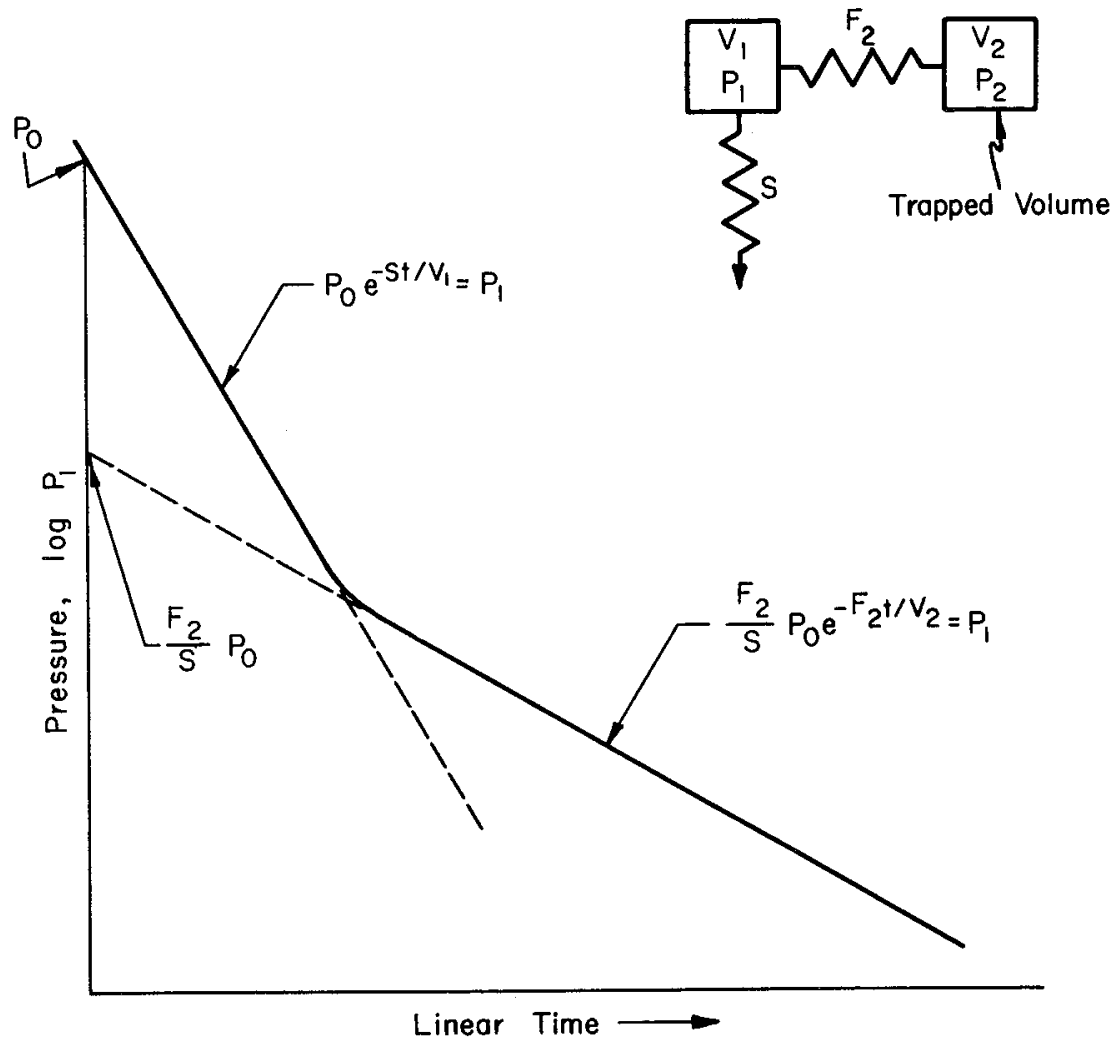

FigURE 8.3.-Effect of a trapped volume, $V_{2}$, on the system pressure, $P_{1}$, as a function of time, when $\frac{V_{1}}{S}<<\frac{V_{2}}{F_{2}}$. Initial pumpdown is governed by equation (8.2), $P_{1}=$ $P_{0} e^{-s t / F_{1}}$, while the later pumpdown is governed by the in-leakage, equation (8.5), $P_{1}=\frac{F_{2}}{S} P_{0} e^{-F_{2} i / r_{2}}$.

while the in-leakage controls the later pumpdown

$$
\begin{aligned}
P & =\frac{Q_{I}}{S} \\
& =\frac{F_{2} P_{2}}{S}
\end{aligned}
$$

During the pumpdown, $P_{2}$ decreases exponentially with time

$$
P_{2}=P_{0} e^{\left(-F_{2} t / \nabla_{2}\right)}
$$

Substituting the expression for $P_{2}$ into equation (8.5) yields,

$$
P=\frac{F_{2}}{S} P_{0} e^{\left(-F_{2} t / \nabla_{2}\right)}
$$


The pressure-time relationship can be approximated by combining the expressions for $P$ at initiation of pumpdown and $P$ further along in the process, and solving for $P$,

$$
P=P_{0} e^{\left(-S t / V_{1}\right)}+\frac{F_{2}}{S} P_{0} e^{\left(-F_{2} t / V_{2}\right)}
$$

Equation (8.7) is illustrated in figure 8.3.

If that part of the curve of figure 8.3 is now considered at which the gas load from the second volume is affecting the pumpdown, there is a least desirable size of leakage path corresponding to a particular time for any given system conditions of volume and speed. This effect is shown in figure 8.4 with curves of the form

$$
P=\frac{F_{2}}{S} P_{0} e^{\left(-F_{2} t / \nabla_{2}\right)}
$$

for various values of $F_{2}$.

The curves of large $F_{2}$ values (steep slopes) are more limiting for short periods of time, while the shallower, longer time constant conditions of small $F_{2}$ values limit more for longer time periods. The least desirable value for a particular value of $F_{2}$ at a particular time may be obtained by differentiating equation (8.7) with respect to conductance and setting it equal to zero; therefore

$$
F_{1 d}=\frac{V_{2}}{t}
$$

Both large and small values of $F_{2}$ at time $t$ give rise to a lower pressure which can be obtained by substituting equation (8.8) into equation (8.7) to yield

$$
P_{\max }=P_{0} \frac{V_{2}}{\text { Ste }}
$$

Equation (8.9) gives the maximum pressure to be expected from the outgassing of a trapped volume $V_{2}$ at time $t$ after the start of the exhaust.

Carelessness and oversight in the design of vacuum systems coupled with unfamiliarity with the subject may frequently result in the phenomena of trapped volumes. Common examples are the bottom of a blind-tapped screw hole where there is no venting or interconnecting flow path to the main chamber, two welds in series with a leak to the intermediate space present on the vacuum side, and nonvented O-rings (fig. 8.5). Good vacuum system design will provide adequate venting of all volumes within the vacuum chamber.

The following example will illustrate the problem of a trapped volume. Assume that a conductance of $10^{-6}$ liter/sec is evacuating a volume 


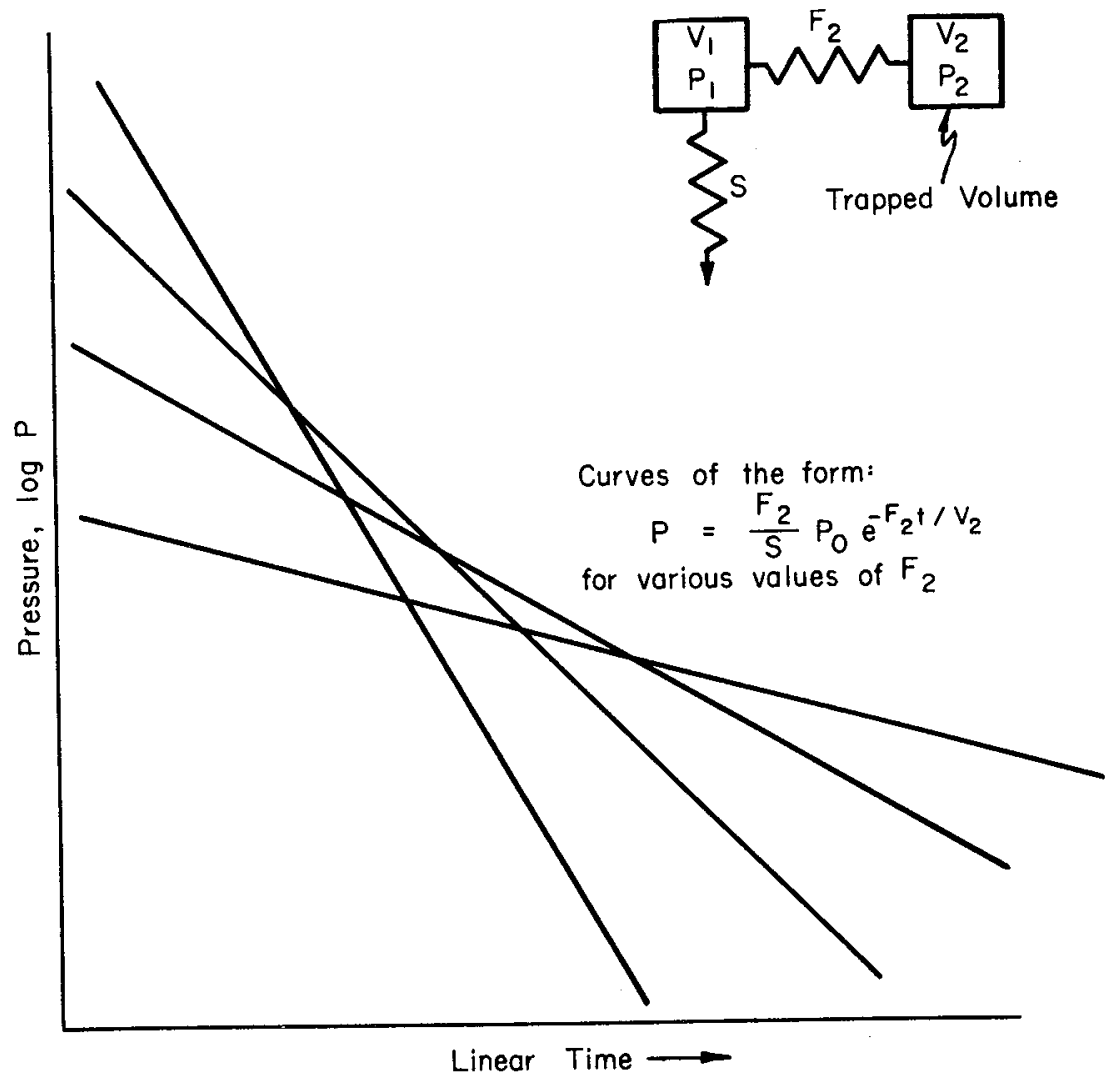

FigurE 8.4.-Effect of various trapped volume conductances, $F_{2}$ on the chamber pressure $P$ as a function of time $t$, when $\frac{V_{1}}{S}<<\frac{V_{2}}{F_{2}}$.

$V_{2}=5 \mathrm{~cm}^{3}$ into a chamber of speed 100 liter $/ \mathrm{sec}$. At the end of 1 hour of pumping, the pressure will be limited to approximately $\left(7.6 / e^{0.72}\right) \times$ $10^{-6}$. This pressure will be quite critical for many high-vacuum applications.

A space vehicle placed in a vacuum system is another example of a trapped volume. The pressure outside the vehicle is described by equations (8.7) and (8.9). However, the principal difference between this case and the previous discussion is that the volume $V_{2}$ may be exceedingly large. If the chamber volume and the vehicle volume are connected by the wrong-size restriction, a serious limitation on the chamber pressure may arise. For example, a large test item of $10^{4}$ liter volume is placed in a chamber with a pumping speed of 50000 liter/sec. The least desirable interconnecting conductance is given by equation (8.8), or a 
Hole Drilled to

Relieve Trapped

Volume

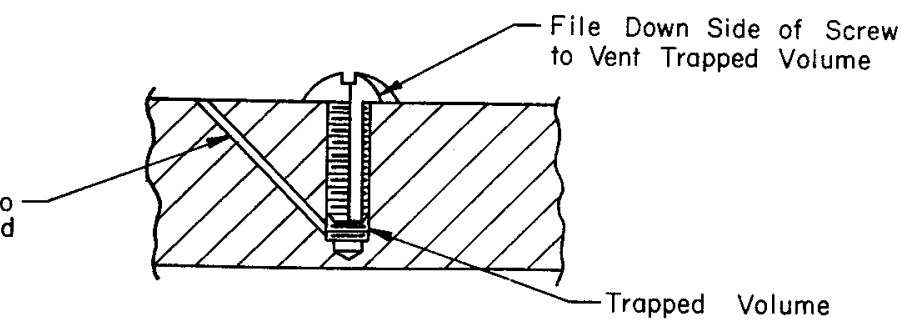

(a)

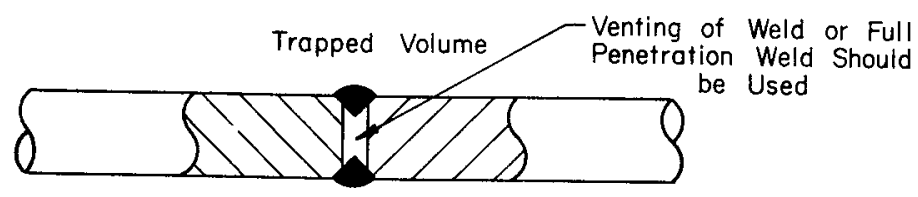

(b)
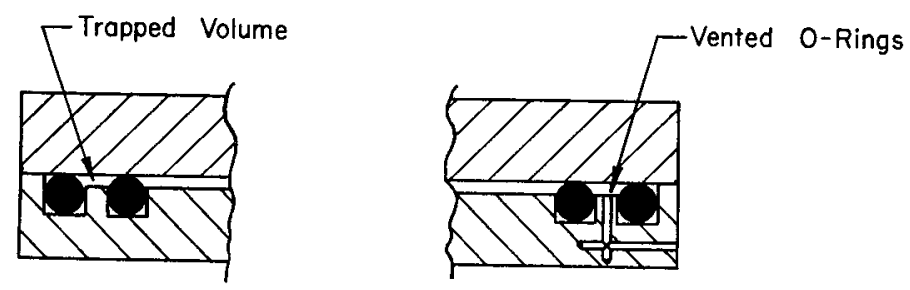

(c)

Figure 8.5.-Examples of some common trapped volumes which are frequently encountered in vacuum systems. (a) Bottom of a blind tapped screw; (b) two welds in series; (c) two O-rings in series.

hole of approximately $0.1 \mathrm{~cm}^{2}$ area. If a test is conducted at the end of $10^{4}$ seconds, the pressure at this time is given by equation (8.9). 


$$
\begin{aligned}
F_{1 d} & =\frac{V}{t} \\
& =1 \text { liter } / \mathrm{sec}
\end{aligned}
$$

For the conditions given, the pressure is $10^{-2}$ torr which is obviously too great a value for space-simulator testing. To prevent the necessity of providing extremely large pumping speeds for vehicle test, large space vehicles must be either leaktight or fully vented. The lack of control over vehicle leakage is one of the principal justifications for the use of high-speed cryogenic pumping in large space-simulator chambers.

Even if the vehicle is fully vented and the rapid pumpdown of the gases within the vehicle can be achieved, the interior components of the vehicle will produce copious quantities of gas. Both the surfaces and the bulk of the components will contribute gas sources to this additional load on the pumping system.

\section{Outgassing}

Leaks and trapped volumes represent only a few of the gas-load sources in a vacuum system. There are also gas forms which originate from the walls of the container and the associated test objects. Among these forms are the gases which are physically sorbed or chemisorbed on the surface, those which are in solution in the materials, those arising from evaporating sources, and decomposition products. Every material exposed to vacuum conditions will liberate gas to varying degrees by one or more of the above processes.

The pumping system which evacuates a chamber also contributes to the gas load of the system. For example, getter ion-type pumps demonstrate a memory effect as the electrical discharge moves over the surface and liberates previously pumped gases. It has been shown that these pumps also generate many gases while pumping others. That is, the reaction of water vapor with the carbon impurities in the surface of the pump generates methane, ethane, and some higher hydrocarbons. A portion of these hydrocarbons is repumped while the remainder reenter the vacuum system.

Diffusion pumps also may contribute to the gas load. Although the working fluid of the pump may be kept from the vacuum chamber by the use of a properly designed liquid nitrogen trap, there are noncondensable gases such as hydrogen and carbon monoxide to consider. These gases, which are the result of the decomposition of the working fluid, cannot be trapped by the liquid nitrogen trap and must be eliminated in a different manner. This difficulty may be resolved in part by operating two diffusion pumps in series. The second pump provides a higher compression ratio, supplies a high pumping speed for the permanent gas decomposition products coming from the first pump, and isolates the mechanical 
pump oil from the boiler of the main diffusion pump. Despite the improvement brought about by the two pumps in series, the hydrogen generation in the diffusion pump is frequently the major limitation on the ultimate pressure in an ultra-high-vacuum system.

The liquid nitrogen trap is a particularly important component in diffusion-pumped systems. If any part of the trap is held at liquid nitrogen temperature during bakeout, it will adsorb gases evolving from the rest of the system and will, in its turn, become a gas source at a later time.

In many ultra-high-vacuum system applications, the principle gas load on the system arises from the vacuum gage used to measure the pressure.

The numerous sources and effects of outgassing pose many difficulties for the designer or operator of a vacuum system. Many technical articles dealing with outgassing have been published. A sampling of the wide range of articles is included in the references listed at the end of this chapter. Reference 1 contains an excellent treatment of the subject.

\section{Adsorption}

The following analysis gives only a physical picture of the desorption process in a vacuum system. It does not permit predictions of experimental data because some assumptions made for the analysis do not hold true for actual conditions. That is, surfaces are not isoenergetic, and the activation energy varies over wide ranges. In addition, since the experimental data generally indicate total outgassing quantities for 100 to 1000 monolayers, the projected area of a surface is not the true area, and surface imperfections result in anomalous behavior.

At any given temperature and pressure, the molecules on a surface are attempting to come into equilibrium with the gases surrounding the surface. At equilibrium conditions, the amount of gas striking and adhering to a surface, $q_{s}$, must equal the amount of gas leaving the surface, $q_{L}$ (torr liter $\left./ \mathrm{cm}^{2} \mathrm{sec}\right)$

$$
q_{8}=q_{L}
$$

The quantity of gas, $s$, will reside on the surface for an average time, $\tau_{R}$, so that the rate of molecules leaving the surface (reference 10) is

$$
q_{L}=\frac{s}{z_{R}}
$$

The number of molecules arriving at the surface is directly proportional to the pressure. If a fraction $a$ adheres to the surface

$$
q_{s}=\alpha K P
$$


where

a sticking fraction, dependent on surface coverage

$P$ gas pressure, torr

$K$ constant calculated from kinetic theory, equals $3.638 \sqrt{T / M}$, equation (4.2)

At equilibrium

$$
\frac{s}{\tau_{R}}=\alpha P 3.638 \sqrt{T / M}
$$

As an illustration of the above discussion, a clean metal surface in a perfect vacuum is assumed. When a gas is introduced into the container at constant pressure, the surface of the container will come to equilibrium with the gas phase. The increase in surface concentration $s$, for a specific gas and temperature, is the product of the molecular incidence $q$, and the time $t$. Equation (8.11) yields

$$
s=3.638 \sqrt{T / M} \text { atP, torr liter } / \mathrm{cm}^{2}
$$

At $T=300^{\circ} \mathrm{K}$ and $M=28$

$$
s=11.8 \mathrm{a} t P
$$

The amount of gas required to form a monolayer is

$$
s_{m}=3 \times 10^{-5} \text { torr liter } / \mathrm{cm}^{2}
$$

and the time to form a monolayer is

$$
t=\frac{2.5 \times 10^{-6}}{a P}, \text { sec }
$$

The times to form a monolayer for various typical pressures and sticking coefficients are given in table 8.1. To obtain the values in the

TABLE 8.1.-Time To Form the Equivalent of 1 Mono. layer on a Surface for Various Pressures and Sticking Coefficients

\begin{tabular}{|c|c|c|}
\hline$a$ & Pressure, $P$, torr & Time, $t$, sec \\
\hline 1.0 & $10^{-6}$ & $2.5 \times 10^{\circ}$ \\
$0.1-\cdots---$ & $10^{-8}$ & $2.5 \times 10^{3}$ \\
1.0 & $10^{-10}$ & $2.5 \times 10^{4}$ \\
0.1 & $10^{-12}$ & $2.5 \times 10^{7}$ \\
\hline
\end{tabular}

table, the initial adsorption rate is extrapolated to the point where an equivalent of one monolayer is assumed to have been deposited. Further assumptions include a sticking coefficient which is independent of surface coverage, and an infinite residence time. 
However, under actual conditions, the residence times are finite, the surface sticking fraction decreases as the available sites are filled, and the time to form a monolayer is considerably longer. In some cases, the deposition of a monolayer may never be attained due to the presence of a balance between the rate of molecules leaving the surface and the rate of molecules being adsorbed.

The gas in the container is then evacuated, and the surface gases immediately attempt to maintain equilibrium with the gas phase. As a. result, there is a net desorption for which the instantaneous rate of change equals the difference between the amount desorbing and the amount being adsorbed. If it is assumed that the amount readsorbed is small in comparison to the amount desorbing, the net outgassing rate is expressed by equation (8.11).

A surface which has a constant residence time $\tau_{R}$ will have an exponential desorption decay rate, and will be analogous to the previous trapped volume situation. The decay rate at a given temperature is a linear function of the number of molecules remaining on the surface. Thus, if the surface coverage is reduced by one-half, the outgassing rate will be reduced by one-half.

The outgassing rate curve for the condition of a constant residence time is

$$
q_{L}=\left(\frac{s_{0}}{\tau_{R}}\right) e^{-t / \tau_{R}}
$$

where $s_{0}$ is the amount of gas adsorbed on the surface at the time $t=0$.

The total amount of gas, torr liter $/ \mathrm{cm}^{2}$, left on the metal surface at any time $t$ can be determined from

$$
\begin{aligned}
s & =q_{L} \tau_{R} \\
& =s_{0} e^{-t / \tau_{R}}
\end{aligned}
$$

A convenient approach to determining $s$ is to define a half-life or the time required to reduce the instantaneous flow rate by a factor of 2 to 1 . For the idealized case, the half-life is a constant. Thus

$$
q=0.693 \frac{s_{0}}{\tau_{1 / 2}}
$$

As a result, the total amount of gas remaining on the surface can be determined by noting the instantaneous outgassing rate and determining the slope of a plot of the log of the outgassing rate versus time.

Both $\tau_{R}$ and the entire outgassing process exhibit a temperature dependency. Figure 8.6 depicts this dependency for the idealized adsorption condition. The curve for $T_{1}$ represents outgassing at a particular temperature. If the temperature is increased to $T_{2}$, the average energy of the surface molecules increases, as does their probability of escape. In addition, a higher initial outgassing rate, and a shorter half-life will 


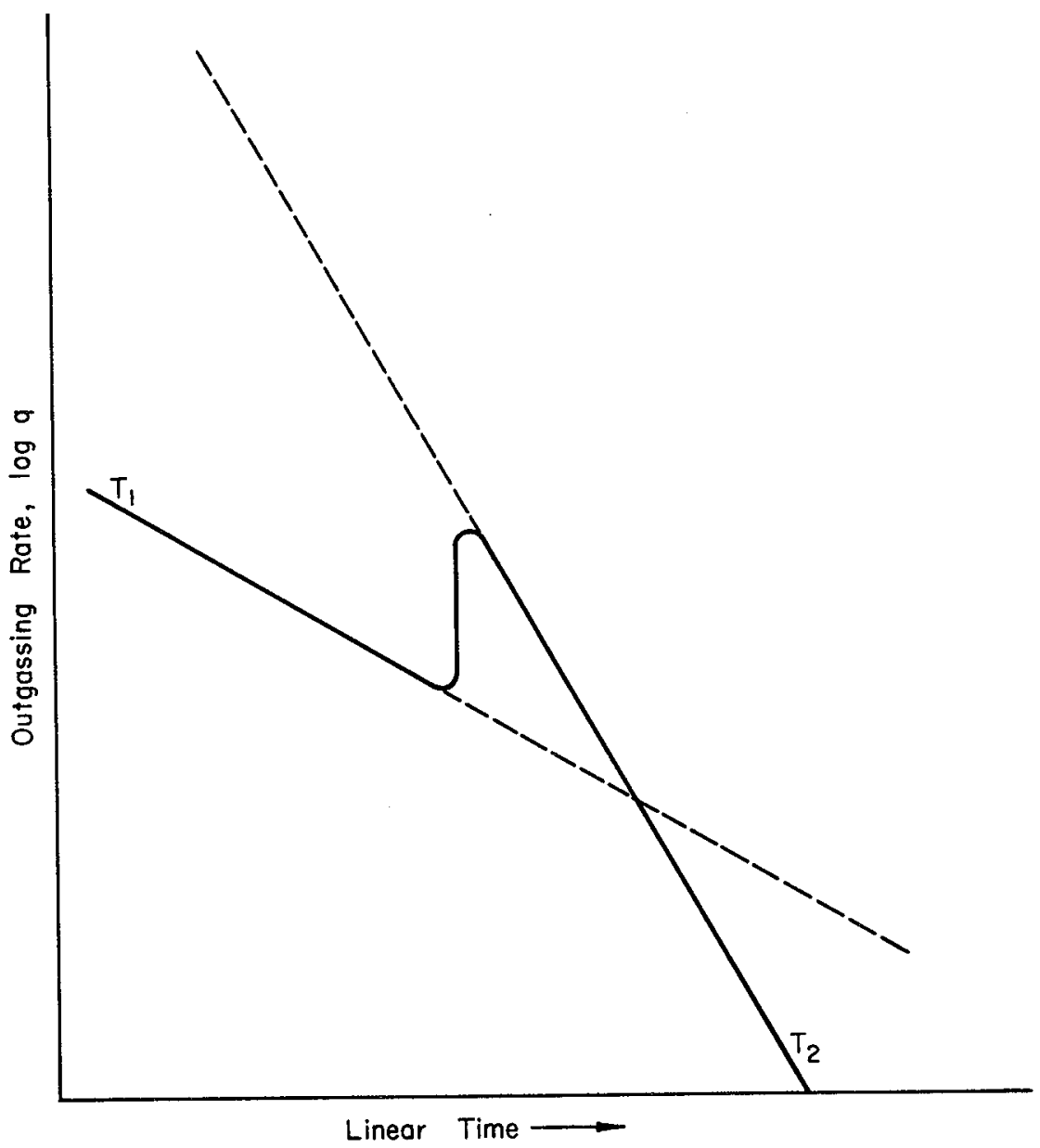

Ficure 8.6.- Illustration of the temperature dependency of activation energy for two different temperatures where

$$
q_{L}=\frac{S}{\tau_{0}} e^{-\Delta E / H T}
$$

$q=$ outgassing rate; $T=$ system temperature where $T_{1}<T_{\mathrm{a}}$.

result from the temperature increase. It has been observed for most outgassing processes that a 10-percent increase in absolute temperature will result in approximately a tenfold increase in outgassing rate and a similar reduction in half-life. To illustrate the foregoing statements, assume that the half-life of adsorbed water at $30^{\circ} \mathrm{C}$ equals 1 hour. The elevation of the temperature to $90^{\circ} \mathrm{C}$ will reduce the half-life to about one-half minute, and will make it possible to obtain a rapid surface cleanup. 
The general rule of the effect of a 10-percent increase in absolute temperature applies best to vapor pressures in the range $10^{-4}$ to 1 torr. The vapor pressure curves of figures $7.1(\mathrm{a})$ and $7.1(\mathrm{~b})$ illustrate this situation. Ideally, the curves should have a constant slope and be parallel to one another. However, as can be seen, the curves have the general appearance of being parallel, but are not of constant slope and do not correspond to the 10-percent rule discussed previously. At pressures of 100 torr the rate of change is closer to 15 percent per decade, while at very low pressures in the order of $10^{-8}$ torr, the rate of change is approximately 5 percent per decade. In the pressure range of $10^{-4}$ to 1 torr, a tenfold rate increase in the temperature sensitive portion of the process does result from a 10-percent change in absolute temperature. Thus, for this particular region, the rule of thumb is applicable and is reasonably accurate. The 10-percent rule permits estimates of processes for which there are no available data, and it is particularly useful in experimental design and short-term process predictions.

The effect of temperature on outgassing can be investigated by means of a different approach, the activation energy concept. Reference 3 relates the activation energy and residence time

where

$$
\tau_{R}=\tau_{0} e^{\Delta E / R T}
$$

$\tau_{0}=10^{-13} \mathrm{sec}$ at room temperature, the vibrational period of the lattice

$\Delta E$ characteristic activation energy, cal/gram mole

$T$ absolute temperature, ${ }^{\circ} \mathrm{K}$

$R$ gas constant

It has been shown that as the temperature increases, the residence time decreases. Therefore, the general equation $q_{L}=s / \tau_{R}$ may be converted to

$$
\begin{aligned}
q_{L} & =\frac{s}{\tau_{R}} \\
& =\left(\frac{s}{\tau_{0}}\right) e^{-\Delta E / R T}
\end{aligned}
$$

The dependency of activation energy on the temperature change is illustrated in figure 8.6 for two different temperatures. At room temperature, the activation energies between 20 and $25 \mathrm{kcal} / \mathrm{gram}$ mole give rise to troublesome outgassing rates. However, lower activation energies yield rates with very short half-lives.

The data presented in chapter 9 will allow a determination to be made of the particular gases which possess troublesome activation energies at various temperatures. That is, the activation energy for simple physical adsorption on most surfaces ranges between one to two times the latent heat of vaporization of the gases. As a result, gases such as 
water and carbon dioxide will be troublesome, while helium and nitrogen will not be.

Activation energies which present problems are $20 \mathrm{kcal} / \mathrm{gram}$ mole at $300^{\circ} \mathrm{K}$, and approximately $40 \mathrm{~K} \mathrm{cal} /$ gram mole at $600^{\circ} \mathrm{K}$. The activation energy of water on metallic surfaces is 22 to $24 \mathrm{kcal} / \mathrm{gram}$ mole.

Under certain conditions, a stronger attraction is exhibited by molecules which possess a strong probability of reacting to form chemical compounds. This particular type of bonding is called chemisorption. For example, carbon monoxide and hydrogen are strongly adsorbed on metals, and for this process the activation energy is quite high, approximately $30 \mathrm{kcal} / \mathrm{gram}$ mole at a system temperature in excess of $300^{\circ} \mathrm{K}$.

The activation energy is a measure of the degree of attraction between an adsorbed molecule and the surface. Therefore, a surface exposed to a mixture of gas types can expect contamination to arise from those types having a higher activation energy; that is

where

$$
\frac{s}{s_{T}}=\frac{P_{1} e^{-\Delta E / R T}}{\sum_{n=1}^{n} P_{n} e^{-\Delta E_{n} / R T}}
$$

$P \quad$ pressure of specific gas, torr

$\Delta E_{n}$ activation energy for the above molecules, $\mathrm{kcal} / \mathrm{gram}$ mole

$\frac{s}{s_{T}}$ fraction of a single gas adsorbed to the total amount of various gas types adsorbed

Values for normal air at $300^{\circ} \mathrm{K}$ are listed in table 8.2 .

TABle 8.2.-Fraction of Gas Adsorbed for Atmospheric Air With Activation Energy Assumed To Be Equal to the Heat of Vapori. zation

\begin{tabular}{|c|c|c|c|}
\hline Gas type & $\begin{array}{c}\text { Activation energy, } \\
\Delta E, \\
\text { cal/gram mole } \\
\text { (ref. 5) }\end{array}$ & $\begin{array}{c}\text { Pressure, } \\
P, \text { torr }\end{array}$ & $\begin{array}{c}\text { Fraction } \\
\text { adsorbed, } \\
s / s_{r}\end{array}$ \\
\hline $\mathrm{N}_{2}$ & 1630 & 592 & $7 \times 10^{-7}$ \\
\hline $\mathrm{O}_{2}$ & 1335 & 152 & $1 \times 10^{-7}$ \\
\hline $\mathrm{H}_{2} \mathrm{O}$ & 9720 & 11 & 1.0 \\
\hline Ar & 1558 & 5 & $6 \times 10^{-0}$ \\
\hline $\mathrm{CO}_{2}$ & 6030 & .23 & $5 \times 10^{-r}$ \\
\hline $\mathbf{H}_{2}$ & 216 & $3.8 \times 10^{-6}$ & $4 \times 10^{-10}$ \\
\hline
\end{tabular}

While the calculation of the fraction of gas adsorbed made use of $\Delta E$, the heat of evaporation, at the normal boiling point, the activation 
energies normally are approximately twice these values. Because of this fact, the adsorption effect for water would be even stronger. Although the calculations of table 8.2 are very approximate, they do confirm the presence of the water-outgassing problem in a vacuum system. The $s / s_{T}$ ratios encountered in actual practice are not identical due to other parameters, such as solubilities and the outgassing of high-temperature regions, which act on systems. The difficulties associated with $\mathrm{CO}$ and $\mathrm{H}_{2}$ actually are more pronounced than the last column of table 8.2 seems to indicate. This apparent anomaly may arise from the fact that water, under actual conditions, will react (ref. 4) in high-temperature regions with other sources such as ionization-gage filaments to form hydrogen and carbon monoxide.

To illustrate the above discussion, consider the calculation of the water outgassing rate of a metal surface, from equation (8.12)

where

$$
q_{L}=\frac{s_{0}}{\tau_{R}} e^{-t / \tau_{R}}
$$

$t=1.5 \mathrm{hr}$ following the start of degassing

$\Delta E=24000 \mathrm{cal} / \mathrm{gram}$ mole, activation energy

$s_{0}=3 \times 10^{-5}$ torr liter $/ \mathrm{cm}^{2}$, concentration for one monolayer at $300^{\circ} \mathrm{K}$

$R=1.987 \mathrm{cal} / \mathrm{gram} \mathrm{mole}^{\circ} \mathrm{K}$

Therefore

$$
q=10^{-9} \text { torr liter } / \mathrm{cm}^{2} \text { sec }
$$

A discrepancy, which may arise from the porous nature of the surface, exists between this value and experimentally determined rates.

\section{Absorption and Diffusion}

When a vacuum is applied to one side of a wall which contains a gas in solution, a diffusion gradient forms within the material and outgassing occurs. As time passes, the outgassing rate decays as shown in figure 8.7 , according to Fick's law of diffusion,

where

$$
q=C_{0} \sqrt{\frac{D}{\pi t}}\left[1+2 \sum_{n=1}^{n=\infty}(-1)^{n} e^{-n^{2} \iota^{2} / D t}\right]
$$

$C_{0}$ initial concentration of outgassing material in a solid, torr liter $/ \mathrm{cm}^{3}$

$D$ diffusion coefficient, $\mathrm{cm}^{2} / \mathrm{sec}$

$\ell$ thickness of material, cm (for outgassing from both surfaces of a material, $\ell=$ half the thickness)

As the outgassing process commences, the bracketed term is approximately equal to 1 , and the outgassing rate decays as $t^{-1 / 2}$. During this initial period, a plot of $\log \tau_{1 / 2}$ versus $\log t$ reveals a linear relationship with a 1:1 slope. As the term $D t / \ell^{2}$ approaches 1 , the bracketed term 


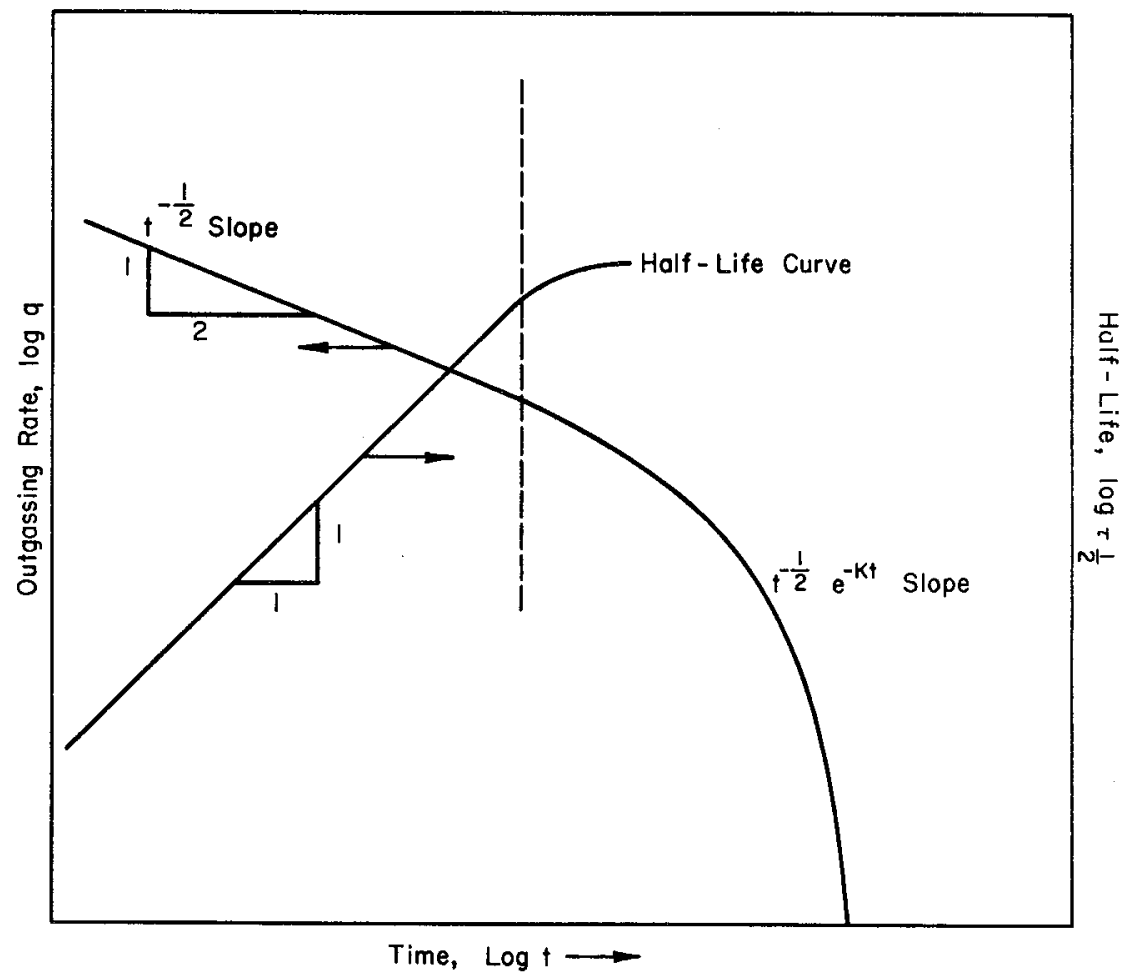

Figure 8.7.--Outgassing rate decay and time constant for diffusion from a semi-infinite slab, where outgassing decays according to Fick's law of diffusion, equation (8.15).

begins to decrease and both terms must be used to determine the outgassing rate at this point.

The initial portion of the curve, where the bracketed term equals 1 , is defined by $q=C_{0} \sqrt{D / \pi t}$. The temperature effect for this initial stage can be estimated if it is assumed that a 10-percent change in the temperature causes a 10:1 change in the temperature-sensitive portion of the process. Since the term most affected by temperature is the diffusion coefficient, a 10-percent change in temperature will cause a 10:1 change in the diffusion constant. However, the same temperature change will result in only a $\sqrt{10}$ change in the outgassing rate at the same time, $t$.

As in the case of adsorption, the effect of temperature on the diffusion process may be evaluated in terms of the activation energy. Therefore, the diffusion coefficient becomes (reference 2)

$$
D=D_{0} e^{-\Delta E / R T}
$$

$D_{0}$ generally varies between 0.01 and $10 \mathrm{~cm}^{2} / \mathrm{sec}$ for solids. 
The solubility of gases in solids is not strongly temperature dependent. For example, when considering metals, only those gases such as hydrogen which react with the base metal are soluble. Inert gases are insoluble and do not diffuse in metals. As a general rule, the total gas content in metals does not exceed 0.1 atom percent, with the exception of hydrogen in zinc, titanium, columbium, and other group B metals (reference 17). In extreme situations in the case of columbium, one hydrogen atom can exist for every metal atom.

Plastics and elastomers will dissolve about 1 percent by weight of various gases, with water vapor the principal gas present. The higher solubility exhibited by plastics results from their low degree of intermolecular bonding which renders them considerably more porous than metals.

A principal gas source in many nonbaked systems is the diffusion of gases from the seals, gaskets, or O-rings, all of which sorb large quantities of atmospheric air, including water, and often are saturated with the plasticizers and lubricants used in their manufacture. It is possible to estimate the outgassing rate of water vapor from an O-ring. Substituting the following values into equation (8.16) and the first part of equation (8.15)

$D_{0}=1.0 \mathrm{~cm}^{2} / \mathrm{sec}$ (a high value due to the porous nature of elastomers)

$\Delta E=13800 \mathrm{cal} / \mathrm{gram}$ mole (obtained from reference 7)

$T=300^{\circ} \mathrm{K}$

$C_{0}=10$ torr liter $/ \mathrm{cm}^{3}$

$t=3600$ seconds

yields

and

$$
D=10^{-10} \mathrm{~cm}^{2} / \mathrm{sec}
$$

$$
\begin{aligned}
q & =C_{0} \sqrt{\frac{\bar{D}}{\pi t}} \\
& =10^{-6} \text { torr liter } / \mathrm{cm}^{2} \mathrm{sec}
\end{aligned}
$$

The $q$ obtained in this calculation closely approximates the experimental value of $1 \times 10^{-6}$ torr liter/sec of outgassing per linear inch of an O-ring after 4 hours.

Figure 8.8 illustrates the effect of changing temperature on the diffusion outgassing. As the temperature increases from $T_{1}$ to $T_{2}$, the outgassing increases to the value which would exist at the new temperature for the same established diffusion gradient, obtained by projecting back along a $45^{\circ}$ line of constant-diffusion gradient. The outgassing then follows the higher temperature diffusion curve. However, since the outgassing starts at a displaced-time increment, the curve becomes a spike tapering into the diffusion curve for temperature $T_{\mathbf{2}}$. 


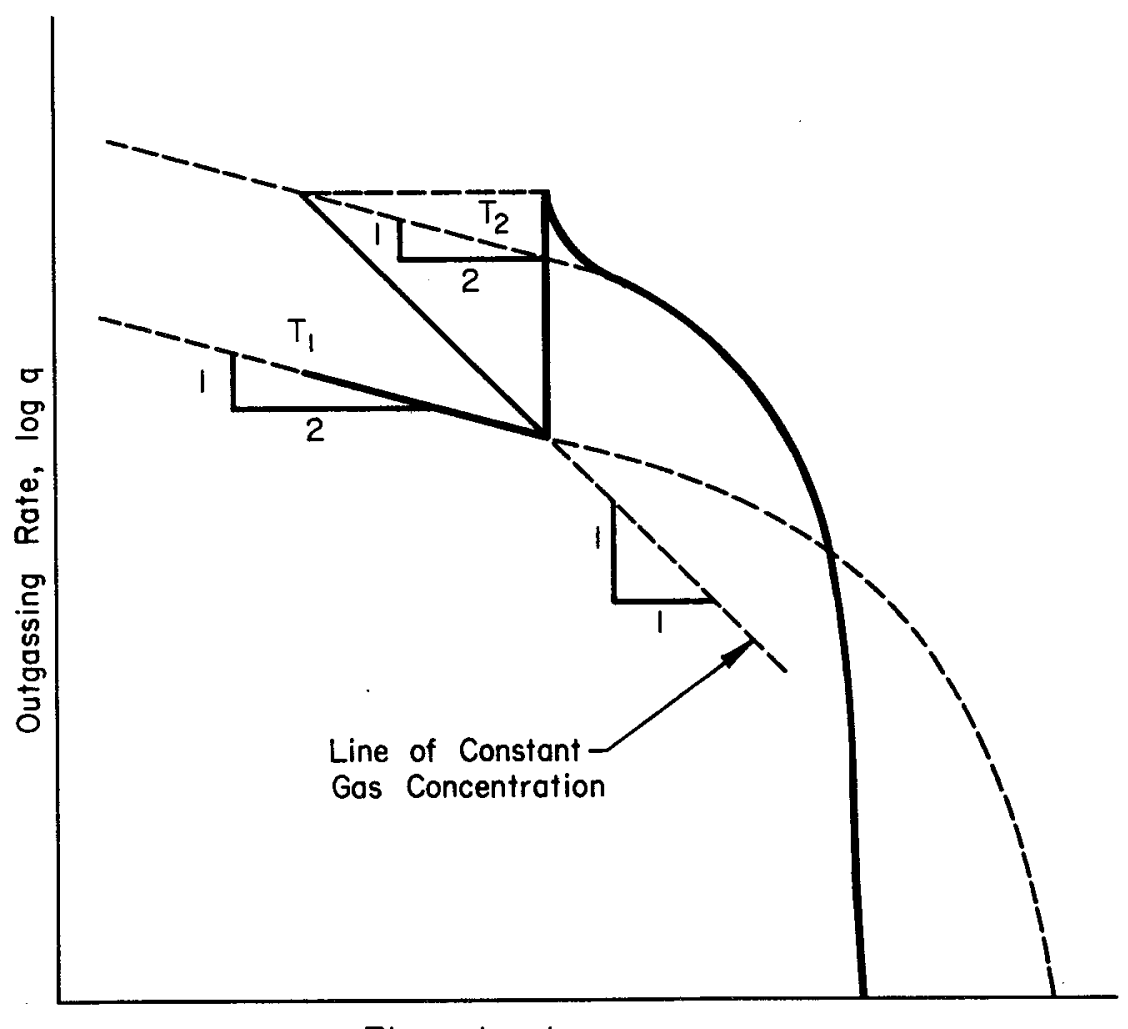

Time, $\log \uparrow \longrightarrow$

Figure 8.8.-Change in outgassing rate for an increase in temperature from $T_{1}$ to $T_{2}$ for a diffusion process.

\section{Permeation}

This process, which combines the actions of solution, diffusion, and dissolution, is another of the sources which contribute to the gas load limiting the ultimate system pressure. Gases which permeate a membrane must first dissolve in the membrane, then diffuse to the opposite side, and finally evaporate.

The units of permeation involve the rate at which a gas travels through a substance. This rate is directly proportional to the permeation coefficient $K_{p}$, and the concentration difference from one side of the system to the other which is inversely proportional to the permeating path length. That is

$$
\begin{aligned}
q & =\frac{K_{p} \Delta P}{\ell} \\
& =\frac{\mathrm{cm}^{2}}{\mathrm{sec}} \frac{\mathrm{atm}}{\mathrm{mm}}
\end{aligned}
$$


The units pose a confusing problem in the calculation of permeation rates. Care should be exercised in determining a constant-permeation value and in noting the units used. It is usually more convenient to express the permeation constant in units of pressure difference in torr, length in $\mathrm{mm}$, flow rate in atm cc/sec, and the cross-sectional area in $\mathrm{cm}^{2}$. The permeation constants listed in chapter 9 have been determined in the units given above.

A typical value for air permeation, principally water vapor, through a neoprene O-ring is $1 \times 10^{-8}$ torr liter/sec per linear inch. That is, a 10-inch-diameter O-ring will permeate air at the rate of $3.1 \times 10^{-7}$ torr liter/sec. There are many techniques which will permit the permeation rate to be lowered. A general rule has evolved which will aid in the selection of an O-ring material: the harder the material, the less permeation it will permit. However, it will take longer for the initial outgassing to decrease to the permeation level.

Another means of lowering the permeation rate calls for two O-rings to be alined in series with a nonpermeating substance between them. The nonpermeable material is generally another vacuum which is inferior in quality to that in the high-vacuum region. If the total pressure is reduced by a ratio of 1000:1, the permeation through elastomers will decrease by the same ratio.

The permeation of diatomic molecules through metals does not behave in the same manner as that of monatomic species. Since gases dissolve in metals atomically, the reaction

$$
X_{2} \rightarrow X+X
$$

takes place at the metal surface. Therefore, the rate of permeation of diatomic gases through metals is proportional to $P^{1 / 2}$ and not $P$. As mentioned previously, nonreacting gases will not permeate through metals.

A section extracted from reference 8 states that-

The permeation of gaseous hydrogen through steel and other metals varies as the square root of the pressure, indicating dissociation to atoms and passage as such through the solid. Recombination occurs on desorption and hydrogen molecules appear on the low side. With glasses and polymers, the gas permeation is proportional to the first power of the pressure for hydrogen and oxygen showing they are permeating in the molecular form.

Hydrogen permeation (reference 8) from the atmosphere through stainless steels occurs at a rate of less than $10^{-18}$ torr liter $/ \mathrm{cm}^{2}$ sec. However, when carbon steel is exposed to water vapor at high temperatures, the iron is oxidized at the surface, which releases atomic hydrogen to cause an effective high hydrogen pressure. This pressure can be prevented by common rust-preventive techniques. 
The temperature effect on permeation is essentially the same as that for diffusion, as illustrated by the diffusion coefficient in the permeation equation. If the temperature is reduced, the permeation will subsequently decrease. Gases of extremely high purities can be obtained by selective permeation at high temperature.

\section{Evaporation}

The last mode of outgassing and source of gas load to be discussed at this time is evaporation, by which a solid or liquid loses material to the gaseous phase.

At the equilibrium condition of zero net flow, there are as many molecules evaporating to the gas phase-as there are being condensed from a gas. Equation (4.2) allows the rate, torr liter $/ \mathrm{cm}^{2} \mathrm{sec}$, at which molecules condense on a surface to be calculated. The sticking coefficient is assumed to equal unity.

$$
q_{L}=3.638 \sqrt{T / M}\left(P_{e q}\right)
$$

where $P_{e q}$ is the equilibrium vapor pressure of the condensed phase. At equilibrium

$$
q_{L}=q_{e}
$$

It can be seen that the maximum evaporation rate is also given by equation (4.2). In a vacuum system which contains both an evaporating source and a pump, the net partial pressure of the evaporant will be reduced to a value $P$ which is less than $P_{e q}$. The net evaporation then becomes

$$
q=3.638 \sqrt{T / M}\left(P_{e q}-P\right)
$$

or, for a specific area, $A_{e}$

$$
Q=3.638 \sqrt{T / M}\left(P_{s q}-P\right) A_{e}
$$

If the pump is a perfect sink of area $A_{p}$, i.e., a cryogenic pump or an equivalent orifice area, the net flow of gas to the pump may be evaluated. This flow must also equal $Q$.

$$
Q=3.638 \sqrt{T / M} P A_{p}
$$

Therefore

$$
P=\frac{A_{e}}{A_{e}+A_{p}} P_{e q}
$$

Equation (8.20) reveals that the pump area must be large compared to the evaporating area for a significant pressure reduction to be obtained.

Figure 8.9 illustrates the effect of the evaporation process on the pumpdown pressure-time curve. Initially there is a rapid transient pressure decrease which continues until the system pressure is determined by the evaporating source pressure. At this point, the pressure remains 


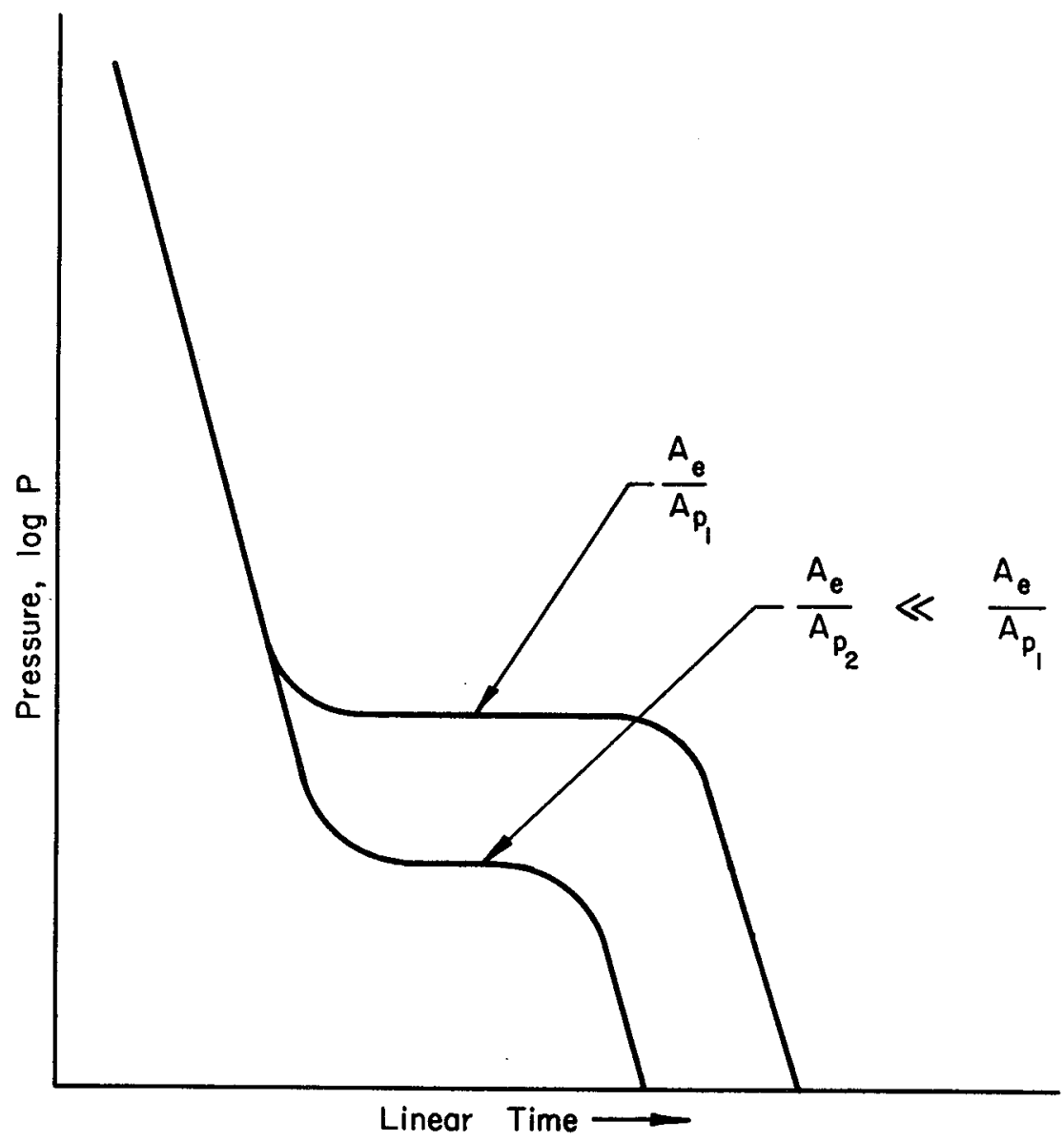

Figure 8.9.-Effect of evaporating area on the pumpdown pressure-time curve. A temperature increase will increase the evaporation rate and thereby decrease the time to remove the evaporating source.

essentially constant until the evaporating surface is depleted to the last molecular layer. The pressure will then fall as previously deseribed in the section on adsorption. Curve (1) of the figure represents a high $A_{e} / A_{p}$ ratio, while curve (2) represents a lower value of the ratio.

An increase in temperature will increase the evaporation rate, and thereby decrease the time to remove the evaporating source.

Trapless diffusion pumps present evaporation problems. The pump oil evaporates and condenses in the chamber such that the ultimate pressure of the system is determined by the vapor pressure of the oil.

The ultimate pressure of trapped diffusion pump systems depends on the efficiency of the trap. A diffusion pumping system utilizing a pump oil of vapor pressure $P_{\boldsymbol{V}}$ will transmit molecules at a rate of

$$
Q=3.638 P_{v} \sqrt{T / M} A(1-\alpha) G
$$


where

$A$ projected area of the trap, $\mathrm{cm}^{2}$

a sticking coefficient

$G$ geometrical factor defining the fraction of molecules which have a single-contact trajectory with the condensing surface. A typical value is 0.01 .

At equilibrium, the pressure on the high-vacuum side of the trap will be $(1-a) G P_{V}$.

If it is assumed that $a=0.99$, the limiting system pressure will be approximately $10^{-4} P_{v}$. The pressure may be reduced considerably below $10^{-4} P_{V}$ if the walls of the chamber are clean. Eventually, however, the walls become contaminated and the pressure will rise to $10^{-4} P_{V}$. Considerable improvement may be obtained in the pressure if one of the more efficient trapping systems of chapter 7 is used.

Liquid nitrogen traps themselves may be sources of carbon dioxide for which the vapor pressure at $77^{\circ} \mathrm{K}$ is approximately $2 \times 10^{-8}$ torr. Carbon dioxide will condense on the walls of the trap if the liquidnitrogen-cooled surfaces are exposed to a high concentration of this gas. However, when the $\mathrm{CO}_{2}$ source is removed, the equilibrium vapor pressure of $\mathrm{CO}_{2}$ is reflected in the high-vacuum section of the system. $\mathrm{CO}_{2}$ can be eliminated by transiently raising the temperature of the liquidnitrogen-cooled surfaces to $130^{\circ} \mathrm{K}$. The carbon dioxide will be removed quickly and the other gases, together with oil and water, will remain bound to the trap.

\section{REFERENCES}

1. Dayton, B. B.: Outgassing Rate of Contaminated Metal Surfaces. Trans. of the Eighth National Vacuum Symposium and the Second International Congress on Vacuum Science and Technology, vol. I. L. E. Preuss, ed., Pergamon Press, 1962, pp. 42-57.

2. Frenkel, J. I.: Kinetic Theory of Liquids. Dover Publications, 1955.

3. De Boer, J. H.: Dynamical Character of Adsorption. Oxford University Press (Toronto), 1953.

4. Hickmotr, T. W.: Interaction of Hydrogen With Tungsten. Bull. Amer. Phys. Soc., ser. II, vol. 4, no. 3, Mar. 30, 1959, p. 139.

5. Honig, R. E.; ANd Hook, H. O.: Vapor Pressure Data for Some Common Gases. RCA Rev., vol. 21, no. 3, Sept. 1960, pp. 360-368.

6 Dushman, S.: Scientific Foundation of Vacuum Technique. J. M. Lafferty, ed., John Wiley \& Sons, Inc., 1962, pp. 536-538.

7. Schiтtкo, F. J.: Measurement of Gas Emission From Solid Surfaces. Vacuum, vol. 13, no. 12, Dec. 1963, pp. 525-537.

8. Norton, F. J.: Gas Permeation Through the Vacuum Envelope. Trans. of the Eighth National Vacuum Symposium and the Second International Congress on Vacuum Science and Technology, vol. I. L. E. Preuss, ed., Pergamon Press, 1962, pp. 8-16.

9. Kraus, T. L.: A Simple Formula for the Pressure-Time Dependence During the Evacuation of Vacuum Systems. Trans. of the Fifth National Symposium on Vacuum Technology, W. G. Matheson, ed., Pergamon Press, 1959, pp. 38-40. 



\title{
Outgassing of Materials
}

\begin{abstract}
HAPTER 8 PRESENTED a general introduction to the subject of the gas load in a vacuum system. The following sections present data and nomographs (refs. 1 to 36 ) to aid in the prediction of actual outgassing rates. The next section discusses the development of the general approach and use of the nomograph. The methods set forth will be used in the presentation of the material on polymers. Additional data pertinent to the discussion of polymers will also be included.
\end{abstract}

\section{METALS}

The outgassing rate of various materials has been summarized by Dayton (refs. 10 to 12), while further compilations by Blears (ref. 13) and Basalaeva (ref. 7) cover much of the significant data available at the present time. The discussion of outgassing mechanisms in chapter 8 is not adequate to describe and explain the data offered in the references. Various outgassing rates of metallic materials for various surface conditions are depicted in figure 9.1. Generally, the data indicates a slope of $1: 1$ on a log-log plot of outgassing versus time. It can be seen from the data that the total amount of gas desorbed from a surface is far in excess of 1 monolayer. Basalaeva indicates that over 100 monolayers will desorb from material in the as-received condition. Dayton discusses this point, relating it to a porous oxide surface on the base metal. In addition, he presents a convincing argument for the 1:1 slope outgassing rate data. This outgassing could arise from at least two sources. One is that the molecules may diffuse from the bulk material with a spectrum of adsorption energies. The adsorption energy may vary in relation to the surface concentration. The other source, by Schittko (ref. 9), proposed that an exponential decrease in outgassing may be found when the pressure in the measuring chamber is proportional to the gas concentration on the surface of the material. Nevertheless, experimental data, with the exception of Basalaeva, gives a strong verification of the $1: 1 \log$ relationship indicated in figure 9.1.

Comparison of the data in reference 13 indicates that approximately the same outgassing rate holds for anodized aluminum, rusty steel, and cast brass (see the uppermost line of fig. 9.1). The data in references 7,10 , and 13 resulted in the curve for material in the as-received condition. The cleaned-sheet curve results from Basalaeva's work. It is 


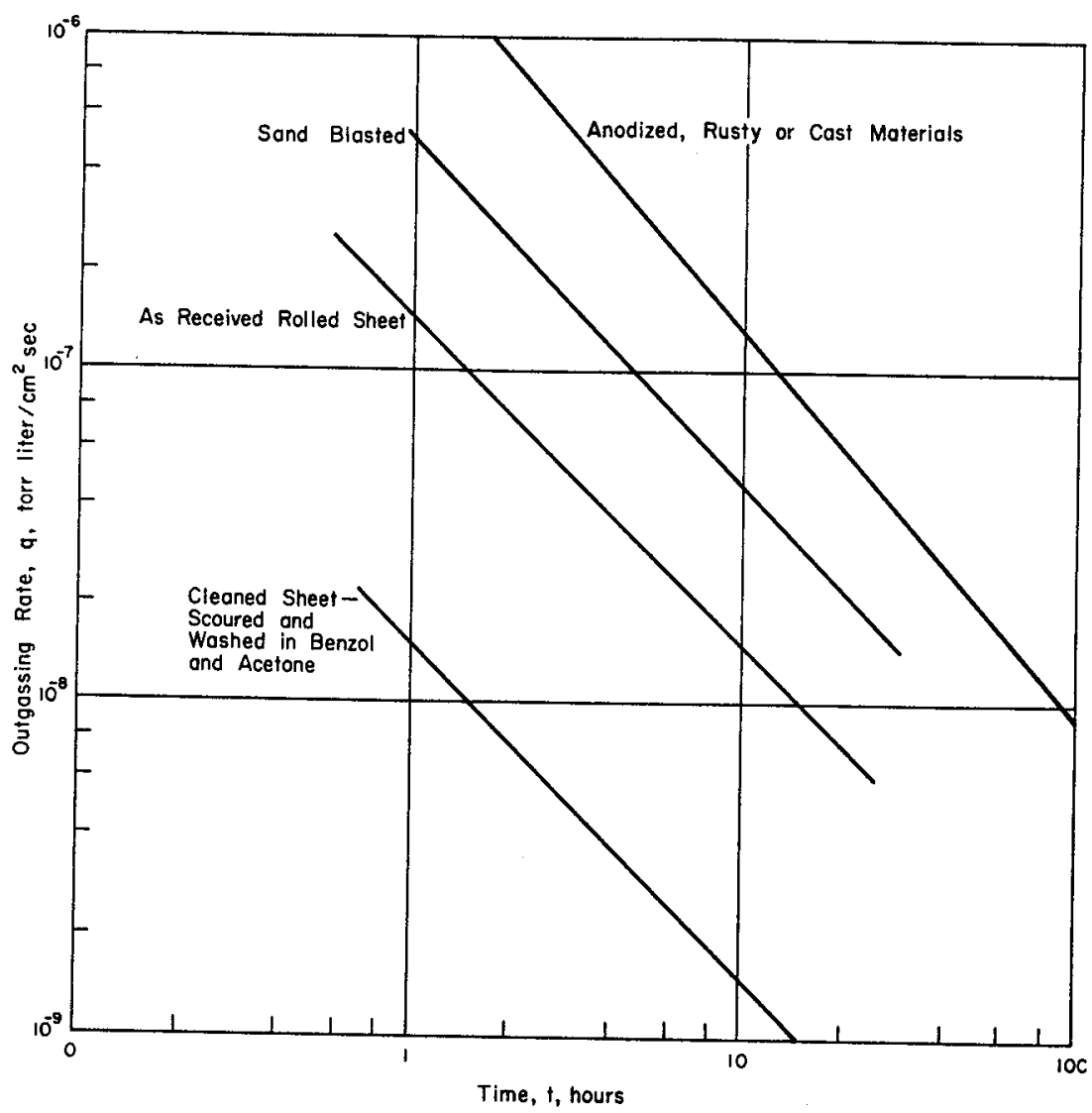

FIGURE 9.1.-Room temperature outgassing rate of metallic materials for various surface conditions as defined in reference 7 .

apparent that the outgassing for metals is more a function of surface conditions than of material type.

\section{Activation Energy}

Often, it is not possible to work at room temperature. Additionally, certain portions of the vacuum system may vary in temperature due to the type of process being performed. The activation energy of the adsorbed gas will determine the effect of temperature on the process.

To illustrate, assume the simple case of an adsorbed phase for which equation (8.12) will apply

$$
q=\left(\frac{q_{0}}{\tau_{R}}\right) e^{-t / \tau_{R}}
$$

where $\tau_{R}$ is the residence time, sec. There is a least desirable case which leads to a maximum outgassing rate at a particular outgassing time. If 
equation (8.12) is differentiated and equated to zero, the result is $\tau_{R}=t$. Since $\tau_{R}=\tau_{0} e^{-\Delta E / R T}$, a relationship between activation energy, temperature, and time can be generated. With $\tau_{0}=10^{-13} \mathrm{sec}$, and $t=10^{4} \mathrm{sec}$,

$$
\Delta E=78.2 T
$$

A plot of equation (9.1) is shown in figure 9.2. Molecules with activation energies close to $23500 \mathrm{cal} /$ gram mole will be troublesome (ref. 12) at room temperature. The outgassing water vapor from metal surfaces has an activation energy between 22000 to $25000 \mathrm{cal} / \mathrm{gram}$ mole. At higher temperatures, hydrogen and carbon monoxide gases are the predominant offenders. Diffusion processes possess lower activation energies for the same time and temperature values as outgassing processes.

A table in reference 14 lists the heats of adsorption and desorption for dilute gaseous layers on several different metals. Table 9.1 summarizes the data of reference 14.

The desorption spectrometer (ref. 15) is of particular value in view of data such as that presented in table 9.1. In this case, the desorption rate is a function of the temperature, heating rate, and the gas properties. It is possible to determine the activation energy of gases sorbed on a tungsten filament, if the wire is heated at a constant rate and the temperature is noted at which a pressure burst or the maximum desorption rate occurs. Experience in handling the data will aid in the determination of gas types. The activation energy can be determined from equation (9.2), which describes the process

$$
\frac{\Delta E}{R T_{P}^{2}}=\frac{1}{\dot{T} \tau_{\tau_{r}}} e^{-\Delta E / R T_{p}}
$$

where

$\tau_{v} \approx 10^{-19} / \mathrm{sec}$, vibrational period of lattice

$\Delta E$ activation energy, cal/gram mole

$R=1.987 \mathrm{cal} / \mathrm{gram}$ mole ${ }^{\circ} \mathrm{K}$

$T_{\mathbf{P}}$ temperature at which maximum desorption occurs, ${ }^{\circ} \mathrm{K}$

$\dot{T}$ heating rate, ${ }^{\circ} \mathrm{K} / \mathrm{sec}$

This technique has been used to determine the activation energy of adsorbed gases and to determine the gas species in a vacuum system.

As discuissed previously, an increase in temperature causes a decrease in $\tau_{1 / 10}$, the 10th life, which is equated to residence time by

$$
\begin{aligned}
\tau_{1 / 10} & =2.3 \tau_{R} \\
& =2.3 \tau_{0}\left(e^{\Delta E / R T}\right)
\end{aligned}
$$

Figure 9.3 depicts the ratio of a process rate predicted by $e^{-\Delta E / R T}$ at a reciprocal temperature difference $\left(\frac{1}{T_{2}}-\frac{1}{T_{1}}\right)$, where $T_{1}$ is the initial tem- 


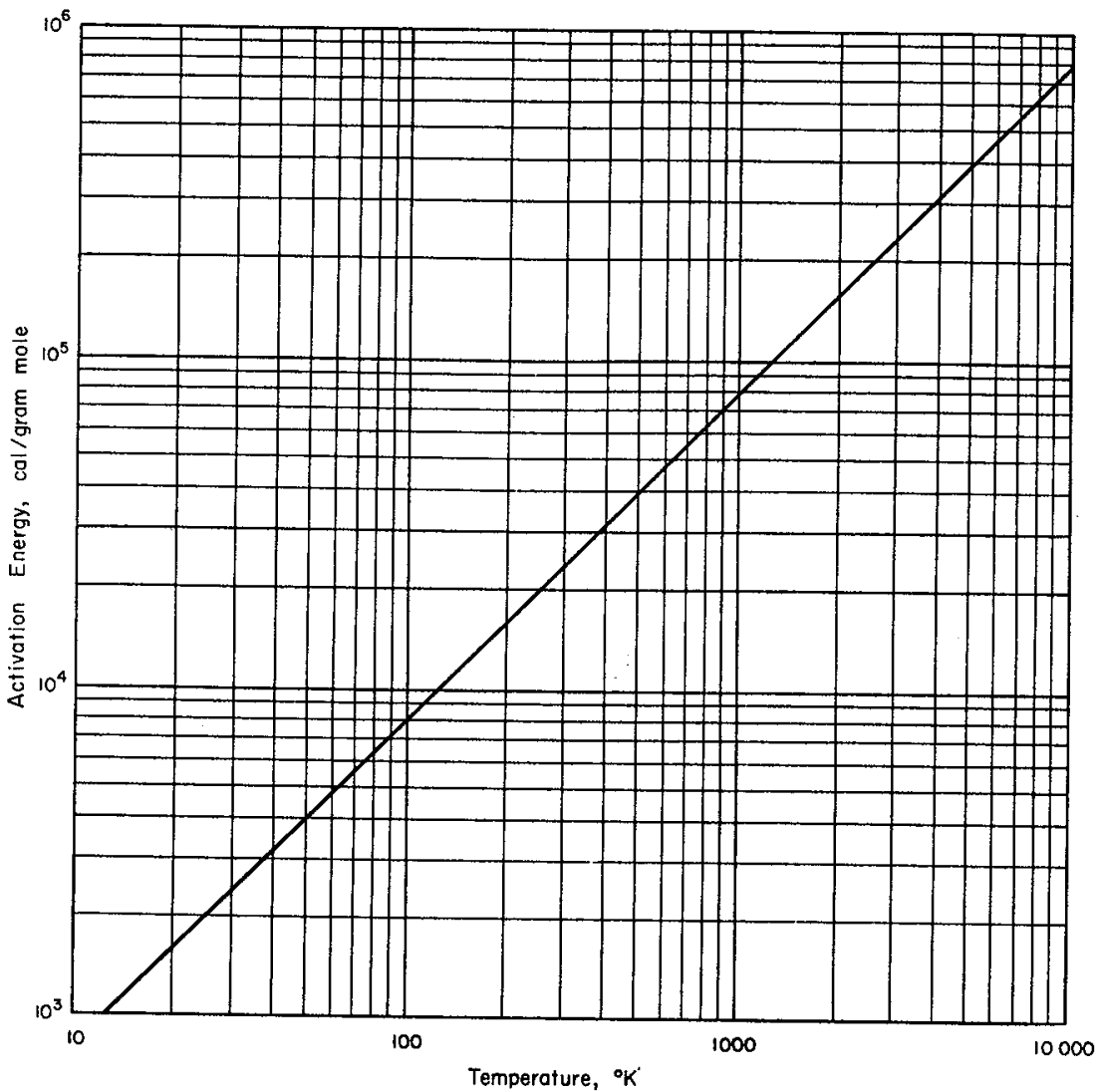

Figure 9.2.-Activation energy versus temperature, per equation (9.1), to result in a maximum outgassing rate after $10000 \mathrm{sec}$ of pumping.

perature and $T_{2}$ is the new temperature. The various slopes are functions of different activation energies; i.e., $80 \mathrm{kcal} / \mathrm{gram}$ mole yields the steepest slope and $5 \mathrm{kcal} / \mathrm{gram}$ mole yields the shallowest slope. Figure 9.4 is a plot of reciprocal absolute temperature, process rate ratio, and process tenth life.

A series of plots similar to those in figure 9.4 enable more accurate predictions of outgassing rates as a function of time to be made. Predictions based on these plots are to be preferred over the rule of thumb that a 10-percent change in absolute temperature will cause a 10:1 change in the outgassing rate. At $300^{\circ} \mathrm{K}$, this rule corresponds to an activation energy, at room temperature, of approximately 12.5 $\mathrm{kcal} /$ gram mole.

Estimations of the vapor pressure of water make use of the plots of figure 9.4. Assuming a vapor pressure of water of approximately 27 torr 
$\mathrm{T}_{\mathrm{ABLE}}$ 9.1.-Heats of Vaporization and Desorption for Dilute Layers of Various Gases on Several Different Metals

[Reference 14]

\begin{tabular}{|c|c|}
\hline Gaseous layers & $\begin{array}{l}\text { Heat of vaporization, } \\
\Delta H, \mathrm{cal} / \mathrm{gram} \mathrm{mole}\end{array}$ \\
\hline $\mathrm{Ar}$ on $\mathrm{Ar}$ & 1558 \\
\hline $\mathrm{CO}$ on $\mathrm{CO}$ & 1444 \\
\hline $\mathrm{N}_{2}$ on $\mathrm{N}_{2}$ & 6030 \\
\hline $\mathrm{O}_{2}$ on $\mathrm{O}_{2}$ & 1630 \\
\hline $\mathrm{H}_{2} \mathrm{O}$ on $\mathrm{H}_{2} \mathrm{O}$ & 9720 \\
\hline $\mathrm{CO}_{2}$ on $\mathrm{CO}_{2}$ & 6030 \\
\hline $\mathrm{He}$ on $\mathrm{He}$ & 20 \\
\hline $\mathrm{H}_{2}$ on $\mathrm{H}_{2}$ & 215 \\
\hline Chemisorption & $\begin{array}{l}\text { Heat of desorption, } \\
\Delta H \text {, cal/gram mole }\end{array}$ \\
\hline $\mathrm{H}_{2}$ on $\mathrm{W}$ & 46000 \\
\hline $\mathrm{O}_{2}$ & 194000 \\
\hline $\mathrm{CO}$ & $\approx 100000$ \\
\hline $\mathrm{N}_{2}$ & 85000 \\
\hline $\mathrm{CO}_{2}$ & 122000 \\
\hline $\mathrm{NH}_{3}$ & 70000 \\
\hline $\mathrm{H}_{2}$ on $\mathrm{Mo}$ & $\approx 40000$ \\
\hline $\mathrm{H}_{2}$ on $\mathrm{Fe}$ & 32000 \\
\hline $\mathrm{N}_{2}$ & 40000 \\
\hline $\mathrm{H}_{2}$ on $\mathrm{Ir}$ & 26000 \\
\hline $\mathrm{H}_{2}$ on $\mathrm{Rn}$ & 26000 \\
\hline $\mathrm{H}_{2}$ on $\mathrm{Co}$ & $\approx 24000$ \\
\hline $\mathrm{H}_{2}$ on $\mathrm{Pt}$ & 27000 \\
\hline $\mathrm{O}_{2}$ on $\mathrm{Pt}$ & 67000 \\
\hline $\mathrm{H}_{2}$ on $\mathrm{Pd}$ & 27000 \\
\hline $\mathrm{H}_{2}$ on $\mathrm{Ni}$ & 30000 \\
\hline $\mathrm{NH}_{2}$ on $\mathrm{Ni}$ & 36000 \\
\hline $\mathrm{CO}$ on $\mathrm{Ni}$ & 35000 \\
\hline $\mathrm{H}_{2}$ on $\mathrm{Cu}$ & 8000 \\
\hline Physiosorption & $\begin{array}{l}\text { Heat of desorption, } \\
\Delta H, \mathrm{cal} / \mathrm{gram} \text { mole }\end{array}$ \\
\hline $\mathrm{Xe}$ on $\mathrm{W}$ & $8000-8000$ \\
\hline $\mathrm{Kr}$ on $\mathrm{W}$ & $\approx 4500$ \\
\hline$A$ on $W$ & $\approx 1900$ \\
\hline $\mathrm{Xe}$ on $\mathrm{W}$ & $\approx 8000$ \\
\hline $\mathrm{Xe}$ on $\mathrm{Ta}$ & $\approx \mathbf{5 3 0 0}$ \\
\hline
\end{tabular}

at $27^{\circ} \mathrm{C}$, and a heat of vaporization of $10440 \mathrm{cal} / \mathrm{gram}$ mole, figure 9.4 indicates a pressure of 270 torr at a reciprocal absolute temperature of $2.88 \times 10^{-4}$, corresponding to a temperature of $74^{\circ} \mathrm{C}$. Actual data from the "Handbook of Chemistry and Physics" gives the vapor pressure as 


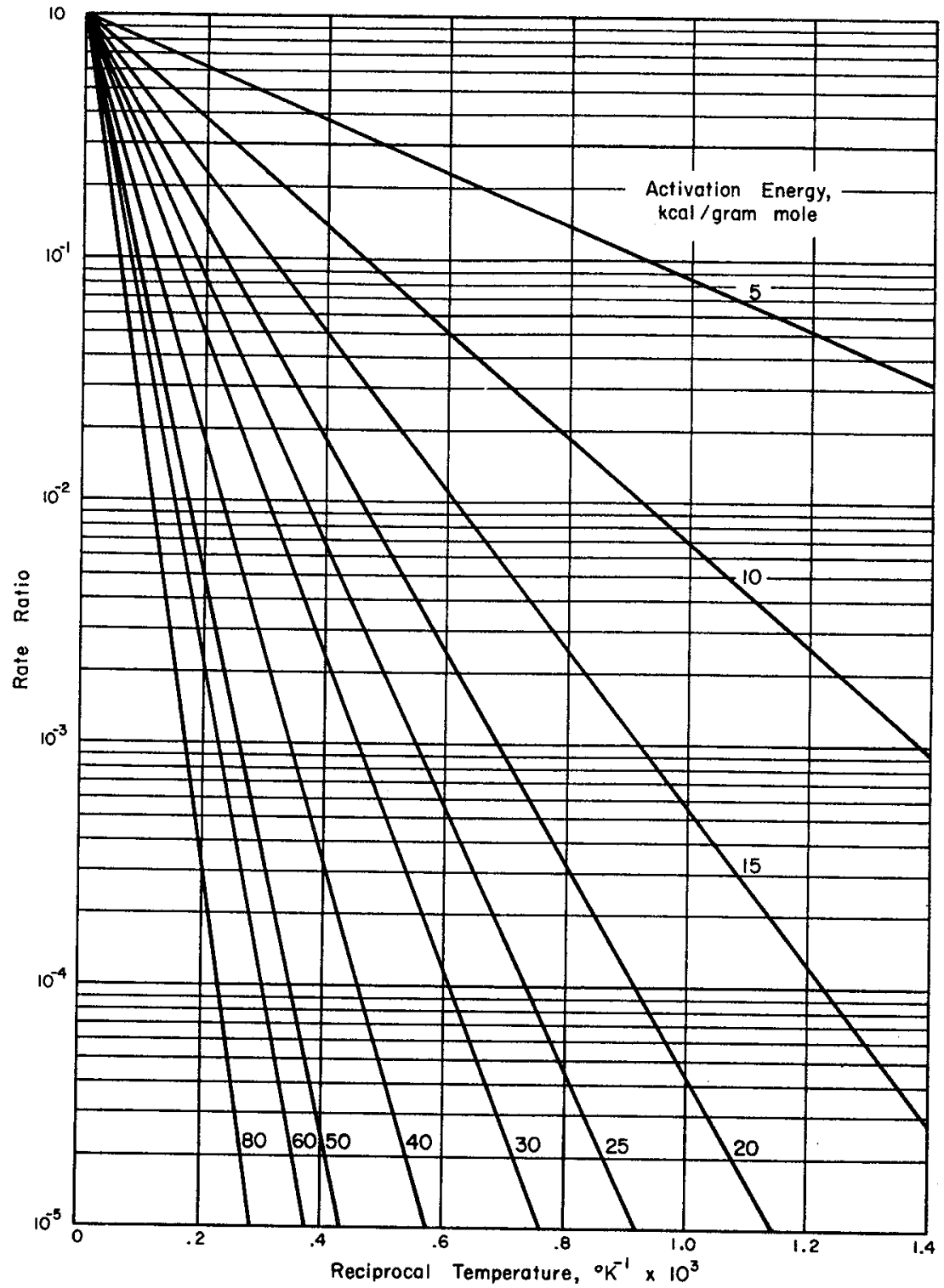

FigunE 9.3.-Plot of the ratio of a process rate predicted by $e^{-\Delta E / R T}$ at a reciprocal temperature difference, $\frac{1}{T_{2}}-\frac{1}{T_{1}}$, where $T_{1}$ is the initial temperature and $T_{2}$ is the new temperature.

277 torr at $74^{\circ} \mathrm{C}$. Similar accuracy can be obtained with other materials if the activation energies are known. Care should be exercised in extrapolating over a number of decades, since the activation energy is 


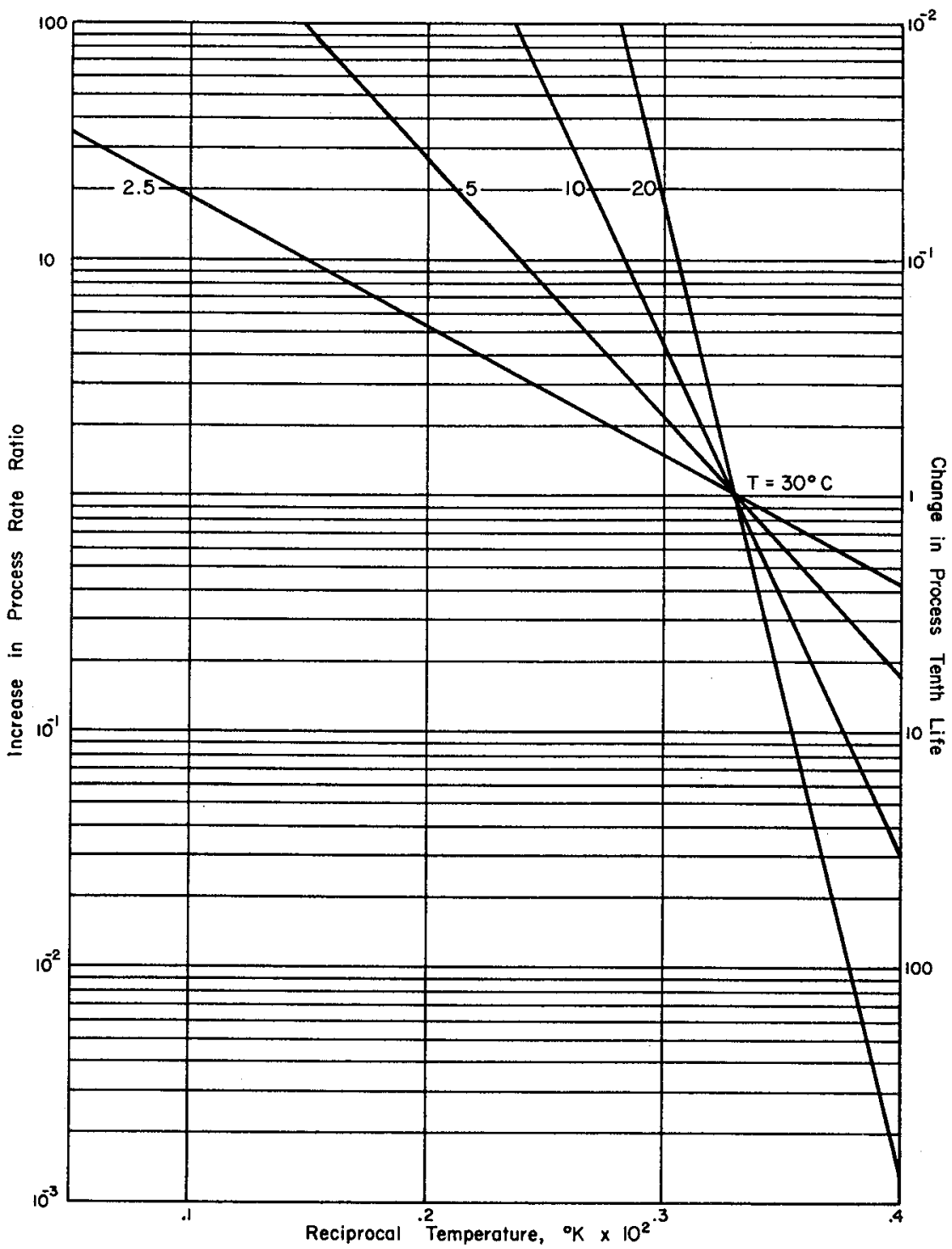

Ficure 9.4.-Plot of the change in process rate ratio and the change in process 10th life as a function of the reciprocal absolute temperature.

a function of temperature and generally decreases with increases in temperature.

\section{Bulk}

Another major gas source originates within the bulk of the material. While this source in metals is not generally a problem, it can be a major 
contributor at high temperatures or very low pressures. Equation (8.15) describes the process.

The following equation determines the rate at which gas is desorbed from the material bulk until approximately 35 percent of the bulk gas is removed, due to the negligible series exponential terms:

$$
q=C_{0} \sqrt{\frac{D}{\pi t}}
$$

where $C_{0}$ is the bulk concentration of a gas, torr liter $/ \mathrm{cm}^{3}$ and $D$ is the diffusion coefficient, $\mathrm{cm}^{2} / \mathrm{sec}$.

While 35-90 percent of the gas is being desorbed from the bulk, the full diffusion equation must be used, equation (8.15). After 90 percent of the gas has been removed, $q$ falls exponentially. A nomograph (figure 9.5), based on equation (8.15), represents a range of values for the diffusion equation. If a particular set of conditions is assumed, an outgassing rate curve can be obtained for various times, temperatures, material thicknesses, and original gas concentration values.

The vertical axis represents the outgassing rate per unit area $q$, divided by the original concentration of dissolved gases $C_{0}$ and covers the range from 10 to $10^{-16} \mathrm{~cm} / \mathrm{sec}$. The units of $q / C_{0}$ are those of velocity. The horizontal axis represents the time divided by the half thickness squared of the material being used, sec $/ \mathrm{cm}^{2}$, where $\ell$ in this instance is the thickness. If one side only of the material is exposed to vacuum, the full thickness is used. To obtain the proper time $t$ for this latter case, the scale must be shifted by $\left(\ell_{\mathrm{act}}\right)^{2}$ for each material. In other words, the scale can be read directly for materials having half thicknesses of $1 \mathrm{~cm}$. The entire family of lines on figure 9.5 represent solutions to the diffusion equation (reference 16). The lines which have slopes of 1:2 represent various diffusion coefficients in a range from $1 \mathrm{~cm}^{2} / \mathrm{sec}$ to $10^{-32} \mathrm{~cm}^{2} / \mathrm{sec}$. The lines with a slope equal to one-half break and begin to fall exponentially when the dimensionless parameter $D\left(t / \ell^{2}\right)=0.1$. At this point, $C / C_{0}$, the bulk concentration divided by the original concentration, equals 0.35 . Each line breaks at exactly the same value. Actually, lines of a negative $45^{\circ}$ slope represent lines of constant concentration or constant $D\left(t / \ell^{2}\right)$.

The following illustration is offered to help clarify the proper method of using the nomograph.

Assume that $C_{0}$ is 1 torr liter $/ \mathrm{cm}^{3}, \ell$ is $1 \mathrm{~cm}$, and $\Delta E$ is $20000 \mathrm{cal} /$ gram mole. The outgassing rate is known to be $3 \times 10^{-8}$ torr liter $/ \mathrm{cm}^{2}$ sec after $10^{4}$ seconds of pumping. Therefore, from the left-hand scale,

and

$$
\frac{q}{C_{0}}=3 \times 10^{-8} \mathrm{~cm} / \mathrm{sec}
$$

$$
\frac{t}{\ell^{2}}=10^{4} \frac{\mathrm{sec}}{\mathrm{cm}^{2}}
$$




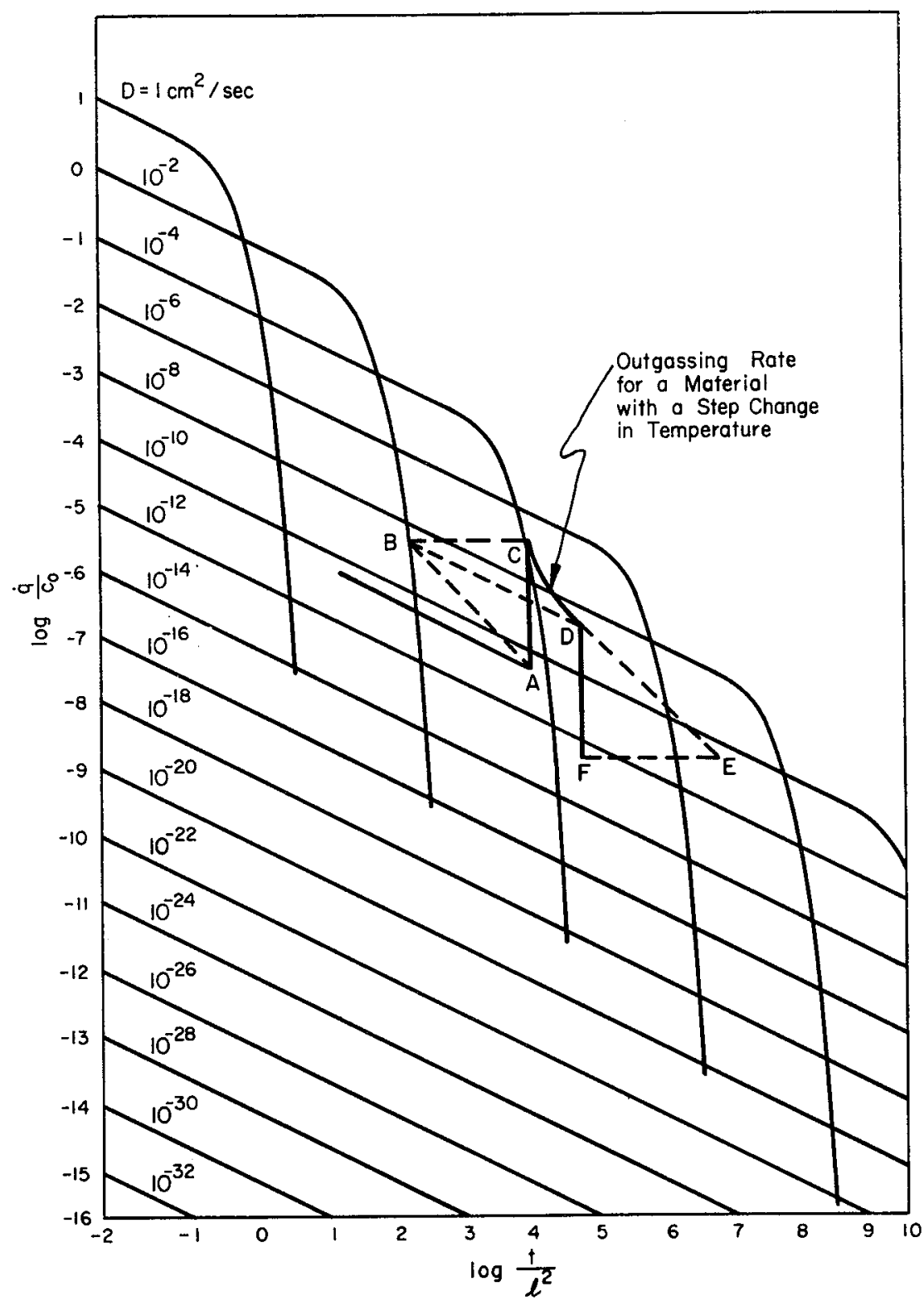

FIGURE 9.5.-Nomograph, based on the diffusion equation (8.15), for determining outgassing rate curves for various times, temperatures, material thicknesses, and original gas concentration values.

with the scale as shown. The diffusion coefficient is determined by locating the point just found on the nomograph plot. At point $A, D=$ $3 \times 10^{-11} \mathrm{~cm}^{2} / \mathrm{sec}$ and the breakpoint of this curve occurs at $10^{9} \mathrm{sec}$. 
If the temperature of the material is increased, the effect on the diffusion coefficient can be seen on figure 9.4. An increase in temperature from $300^{\circ} \mathrm{K}$ to $354^{\circ} \mathrm{K}$ increases the diffusion coefficient 100 times.

The outgassing rate for a material with a step change in temperature can be estimated by starting at point $A$. The increase in temperature causes a vertical change in $D$, the diffusion coefficient, from $3 \times 10^{-11}$ $\mathrm{cm}^{2} / \mathrm{sec}$ to $3 \times 10^{-9} \mathrm{~cm}^{2} / \mathrm{sec}$. Next, move to the left along the constant concentration line, which has a $45^{\circ}$ negative slope, from point $A$ to the line of the new diffusion coefficient, point B. Point B is the equivalent outgassing rate, where

$$
\begin{aligned}
q & =C_{0}\left(\frac{q}{C_{0}}\right) \\
& =\left(3 \times 10^{-6} \mathrm{~cm} / \mathrm{sec}\right)\left(1 \text { torr liter } / \mathrm{cm}^{3}\right) \\
& =3 \times 10^{-6} \text { torr liter } / \mathrm{cm}^{2} \mathrm{sec}
\end{aligned}
$$

after 100 seconds of pumping at the new temperature. This new point actually corresponds to point $\mathrm{C}$ on the correct $t / \ell^{2}$ scale. The outgassing rate at $3 \times 10^{-9} \mathrm{~cm}^{2} / \mathrm{sec}$ moves along line $\mathrm{BD}$ following the additional time at the new temperature. However, the points along BD are transferred. That is, they are shifted horizontally to line $\mathrm{CD}$ which represents the outgassing rate corresponding to the total elapsed time since the start of pumping. For example, after 900 seconds at $3 \times 10^{-9} \mathrm{~cm}^{2} / \mathrm{sec}$, the outgassing rate is determined along BD at time $t=(100+900)=1000$ seconds. Thus, $t / \ell^{2}=1000 \mathrm{sec} / \mathrm{cm}^{2}$. The rate is then transferred to the total elapsed-time scale, $1000 \mathrm{sec} / \mathrm{cm}^{2}+900 \mathrm{sec} / \mathrm{cm}^{2}=1900 \mathrm{sec} / \mathrm{cm}^{2}$, and so on. Assume after a period of approximately 54000 seconds of pumping that the system temperature drops back to $300^{\circ} \mathrm{K}$. The new outgassing rate is found by moving from point $\mathrm{D}$ along a constant concentration line to point $\mathrm{E}$. The rate is determined by reading the vertical axis and multiplying by $C_{0}$

$$
\begin{aligned}
q & =\left(\frac{q}{C_{0}}\right) C_{0} \\
& =\left(1.5 \times 10^{-\theta} \frac{\mathrm{cm}}{\mathrm{sec}}\right)\left(\frac{1 \text { torr liter }}{\mathrm{cm}^{3}}\right) \\
& =1.5 \times 10^{-\theta} \text { torr liter } / \mathrm{cm}^{2} \mathrm{sec}
\end{aligned}
$$

Point $E$ is then transferred to the proper $t / \ell^{2}$ scale by moving horizontally to point $F$, which is determined by noting the total elapsed time and multiplying by $\left(\ell_{\text {act }}\right)^{2}$,

$$
\frac{t}{\ell^{2}}=t(1 \mathrm{~cm})^{2}
$$

Thus, the $t / \ell^{2}$ scale in this case can be read directly as seconds of elapsed time. 


\section{Permeation Rates}

Numerous investigations have been performed to determine the permeation rates of gases through metals. In particular, the process of hydrogen through iron and ferrous alloys has been studied extensively. Reference 3 summarizes the results, with the following conclusions:

(1) Rare gases do not permeate through metals

(2) Hydrogen permeates readily through steel containers

(3) If the steel is unoxidized, the gaseous hydrogen permeates at a maximum rate; oxidized surfaces reduce the rate

(4) Hydrogen permeates chrome steels 10 to 100 times slower than it does iron

(5) Hydrogen permeation varies as the square root of the driving pressure; recombination occurs on desorption

(6) Hydrogen permeation through iron increases with an increasing carbon content (ref. 6)

The amount of free hydrogen in the Earth's normal atmosphere is 0.5 part per million (ppm). Water molecules bind a considerable amount of hydrogen which can be freed by chemical reactions. However, the rusting of steel or iron can be a larger source of hydrogen than the atmosphere. Once a thick oxide layer has formed on a steel surface, hydrogen permeation is greatly reduced. Any process which affects this oxide layer will affect the permeation rate. The process of pickling or abrasion in a damp atmosphere will increase the permeation rate. Electrolytic processes, which yield nascent hydrogen, may produce a hydrogen permeation rate equivalent to thousands of atmospheres of gaseous hydrogen (ref. 3).

The permeation rate of hydrogen through a metal bellows, at $300^{\circ} \mathrm{K}$, equals

$$
K_{p}=10^{-12}\left(\mathrm{~atm}^{1 / 2} \mathrm{~cm}\right)(\mathrm{mm} \text { thick } / \mathrm{sec})
$$

while

$$
q=\left(\frac{K_{p} A}{\Delta y}\right)\left(P_{2}^{1 / 2}-P_{1}^{1 / 2}\right)
$$

Consider the case where

$A$ area, $\mathrm{cm}^{2}$

$\Delta y \quad$ thickness, $\mathrm{mm}\left(5 \times 10^{-3} \mathrm{in} . \times 25.4 \frac{\mathrm{mm}}{\mathrm{in}}=0.127 \mathrm{~mm}\right)$

$P_{2}$ external pressure, atm, $5 \times 10^{-6} \mathrm{~atm}$

$P_{1}$ internal pressure, assume $=0$ 
The outgassing rate $q$ becomes

$$
\begin{aligned}
q & =10^{-12}\left(\frac{\mathrm{atm} \mathrm{cm}}{\mathrm{atm}^{1 / 2} \mathrm{~cm}^{2}}\right)\left(\frac{\mathrm{mm} \text { thick }}{\mathrm{sec}}\right)\left(\frac{1 \mathrm{~cm}^{2}}{0.127 \mathrm{~mm}}\right)\left(0.5 \times 10^{-6}\right)^{1 / 2} \\
& =5.6 \times 10^{-15} \frac{\mathrm{atm} \mathrm{\textrm {cm } ^ { 3 }}}{\mathrm{cm}^{2} \mathrm{sec}} \\
& =4.3 \times 10^{-15} \frac{\text { torr liter }}{\mathrm{cm}^{2} \mathrm{sec}}
\end{aligned}
$$

At $400^{\circ} \mathrm{C}$, the permeation rate is considerably higher

$$
K=10^{-6}\left(\frac{\mathrm{atm} \mathrm{cm}^{3}}{\mathrm{~atm}^{1 / 2} \mathrm{~cm}^{2}}\right)\left(\begin{array}{c}
\mathrm{mm} \\
\mathrm{sec}
\end{array}\right)
$$

and thus increases the total permeation rate to $4.3 \times 10^{-9}$ torr liter $/ \mathrm{cm}^{2}$ sec. The rusting of steel with water vapor present in the atmospheric environment can increase the rate $10^{4}$ times.

The hydrogen permeation rate is highest through palladium and a few palladium-silver alloys. Palladium is often used as a high purity gas source (ref. 1), since hydrogen diffuses through it. In addition, a palladium membrane can act as a hydrogen or an oxygen pump (ref. 2). This metal is also a catalyst for the hydrogen-oxygen reaction. Therefore, as hydrogen permeates through the membrane, any oxygen molecules striking the palladium surface are converted to water vapor by the reaction

$$
2 \mathrm{H}_{2}+\mathrm{O}_{2} \rightarrow 2 \mathrm{H}_{2} \mathrm{O}
$$

Nickel is another material through which hydrogen permeates (refs. 1 and 6 offer discussions of hydrogen permeation through nickel and other alloys). Oxygen permeates through silver, nitrogen permeates molybdenum, and carbon monoxide permeation through iron is indicated in reference 6 . Reference 5 discusses the outgassing of carbon monoxide through nickel and states that it probably originates from bulk atoms of oxygen and carbon recombining at the surface. Figures 9.6 and 9.7 present data for the permeation of gases through metals which are described in tables 9.2 and 9.3. The permeation rate $K_{p}$ is plotted as a function of reciprocal temperature. The units of $K_{p}$ are

$$
\frac{\operatorname{atm} \mathrm{cm}^{3} / \mathrm{sec}}{(\text { atm gas pressure })^{1 / 2}}
$$

for a material with $y=1 \mathrm{~mm}$ and $A_{s}=1 \mathrm{~cm}^{2}$.

\section{CLEANING CHEMICALS}

References 7 and 13 report that the outgassing of aluminum and stainless steel may be decreased by a factor of 10 if the proper cleaning procedures are used prior to placement in a vacuum chamber. The 


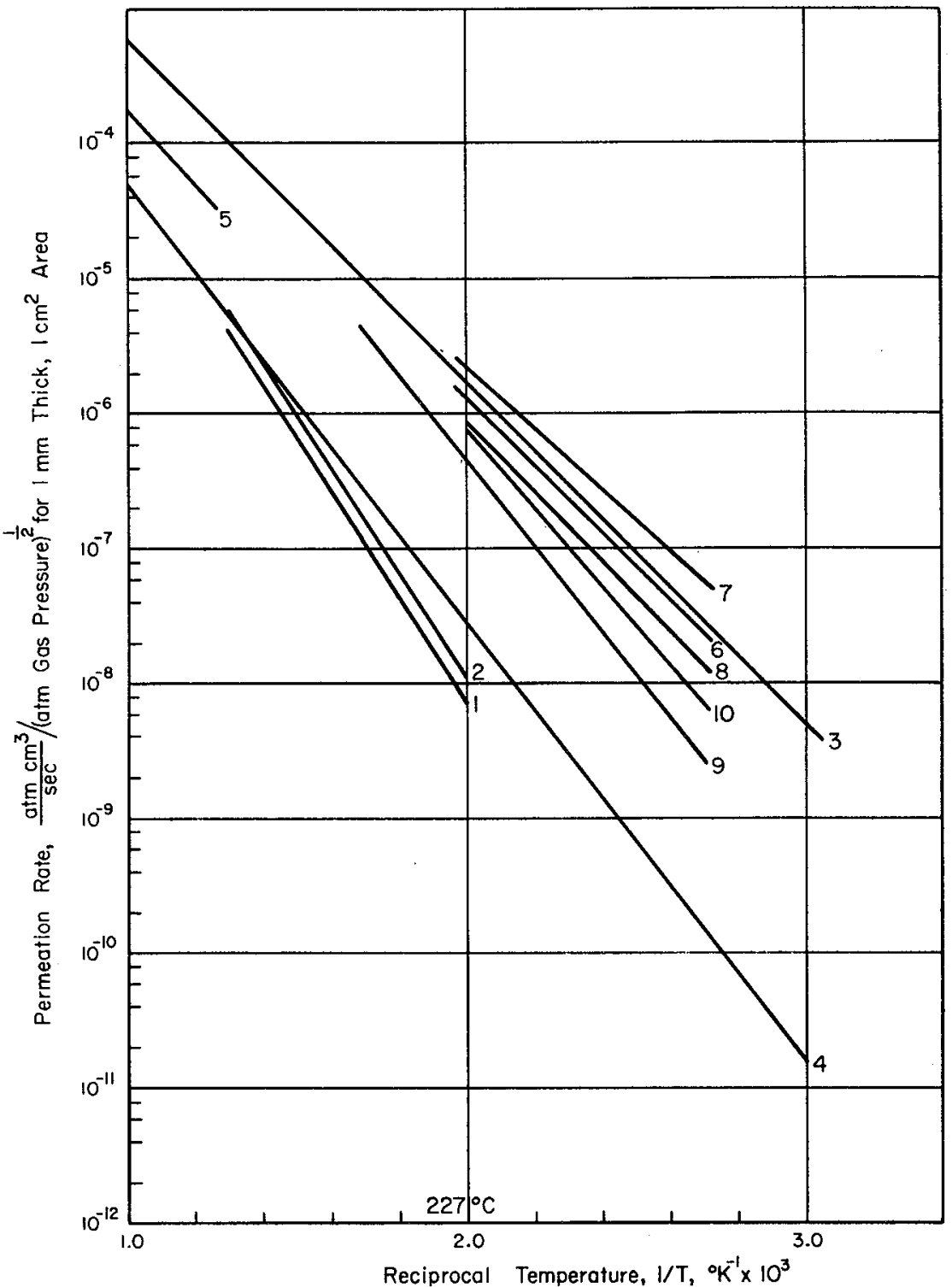

Figure 9.6.-Permeation rates of hydrogen through various ferrous alloys which are described in tables 9.2 and 9.3 .

cleaning techniques used by reference 7 included scouring and washing in benzol and acetone, while reference 13 used stergene. Little detailed data on the techniques are given, although both show vastly improved results. The scouring technique in particular yields excellent results. 


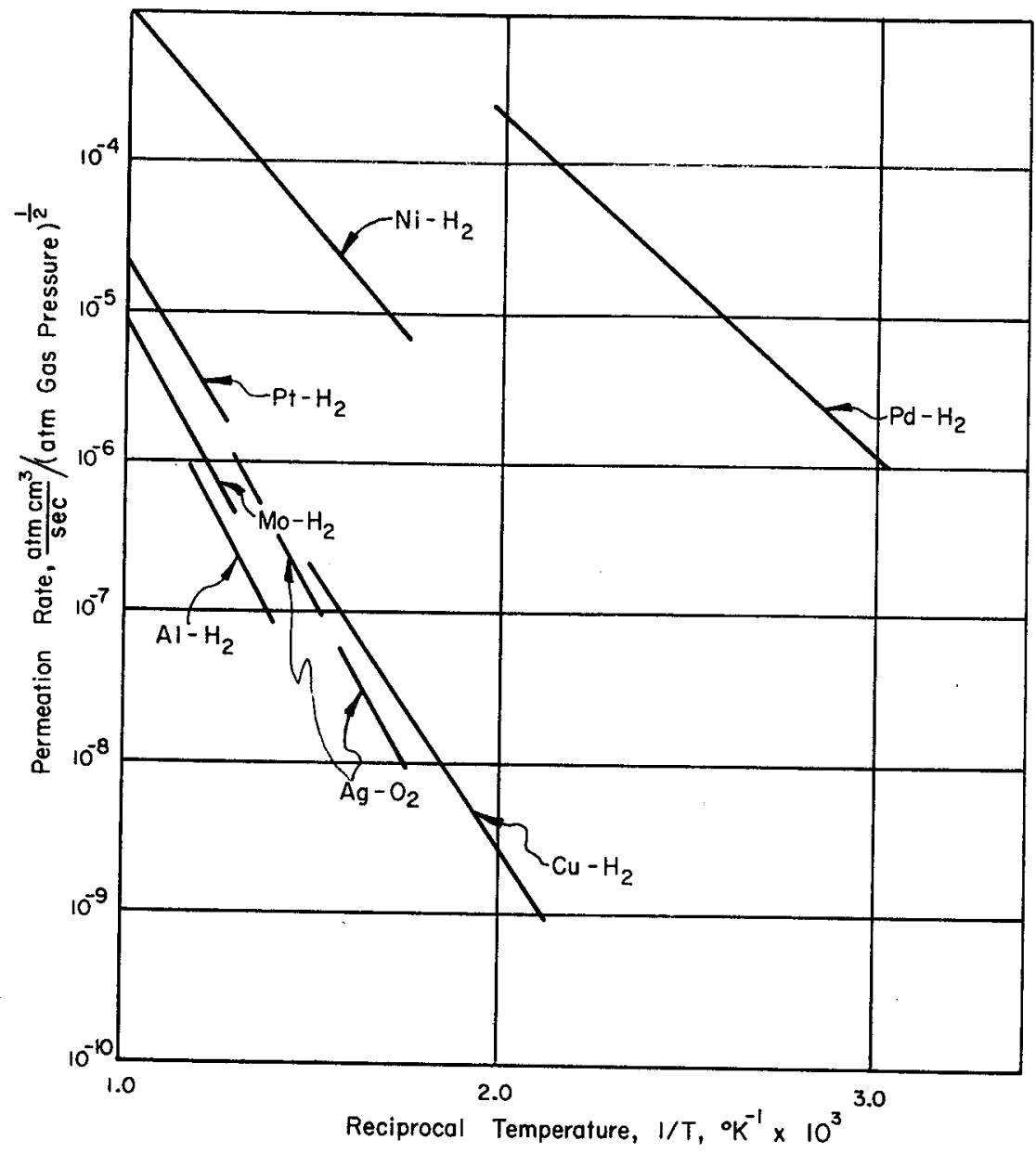

Figure 9.7.-Permeation rates of various gases through metals versus reciprocal temperature, per information presented in references 3 and 6 .

There is a wide variation in the outgassing rate between cleaned materials and those in an as-received condition. As a result, the cleaning technique used just prior to the placement of a material in a vacuum system is much more important than the type of metal used.

\section{HIGH-TEMPERATURE DEGAS}

A previous section discussed the accelerated outgassing process which occurs when the material is heated. A question arises as to the proper temperature and time required to achieve a particular reduction in the outgassing rate (ref. 16). When the dimensionless parameter $D t / \ell^{2}=6.5$, 
TABLE 9.2.-Percentage Composition of Ferrous Alloys Used in Figure 9.6

\begin{tabular}{|c|c|c|c|c|c|c|c|}
\hline $\begin{array}{c}\text { Curve } \\
\text { No. }\end{array}$ & $\mathrm{C}$ & $\mathrm{Cr}$ & $\mathrm{Ni}$ & Mn & $\mathrm{Si}$ & Structure & $\begin{array}{c}\text { Ref- } \\
\text { erence }\end{array}$ \\
\hline 1 & 0.07 & 18.0 & 8.7 & & $-\cdots$ & Austenitic --- & 19 \\
\hline 2 & .07 & 18.0 & 8.7 & -.---- & $-\cdots$ & $\begin{array}{l}\text { Weld ferritic, rest } \\
\text { austenitic. }\end{array}$ & 19 \\
\hline $\begin{array}{l}3 \\
4\end{array}$ & - & $\mathrm{Chr}$ & $\begin{array}{l}\text { Low car } \\
\text { ome ste }\end{array}$ & bon stee & 1 & & 3 \\
\hline 5 & - & $\ldots$ & 50 & 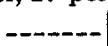 & & & 6 \\
\hline 6 & .12 & & ----- & 0.50 & 0.01 & Ferritic - - & 19 \\
\hline 7 & .09 & & ------- & .51 & .05 & Ferritic - - - - & 19 \\
\hline 8 & .12 & ------- & ------- & .53 & $<.1$ & Ferritic pearlite -- & 19 \\
\hline 9 & .26 & 14.1 & 1.3 & .41 & .46 & Pearlitic & 19 \\
\hline 10 & .11 & $-\cdots-$ & - & .53 & $<.1$ & Ferritic pearlitic - & 19 \\
\hline
\end{tabular}

TABLE 9.3.-Activation. Energy for Ferrous Alloys Described in Table 9.2 and used in Fig. 9.6

\begin{tabular}{|c|c|c|c|c|}
\hline $\begin{array}{l}\text { Curve } \\
\text { No. }\end{array}$ & $\begin{array}{c}\text { Activation } \\
\text { energy for } \\
\text { permeation, } \\
E \text {, cal/gram mole }\end{array}$ & $\begin{array}{c}\text { Activation } \\
\text { energy for } \\
\text { diffusion, } \\
E \text {, cal/gram mole }\end{array}$ & $\begin{array}{c}\text { Activation } \\
\text { energy for } \\
\text { solubility, } \\
E \text {, cal/gram mole }\end{array}$ & $\begin{array}{l}\text { Ref- } \\
\text { erence }\end{array}$ \\
\hline 1 & 17800 & 15600 & 2200 & 19 \\
\hline 2 & 17600 & 15100 & 2500 & \\
\hline 3 & $\approx 12000$ & ----1-- & 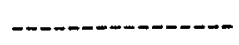 & 3 \\
\hline 4 & $\approx 15000$ & - - - & - & 3 \\
\hline 5 & $\approx 15000$ & 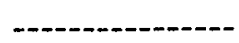 & 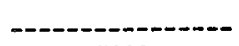 & 3 \\
\hline 6 & 11700 & 6700 & 5000 & $--\infty$ \\
\hline 7 & 10800 & 6400 & 4400 & - \\
\hline 8 & 11700 & 6900 & 4800 & -...-- \\
\hline 9 & 14900 & 13800 & 1100 & ---- \\
\hline 10 & 12800 & 6600 & 6200 & - \\
\hline
\end{tabular}

the diffusion process outgassing rate is $10^{-8}$ that of the rate described by. the equation

$$
q=C_{0} \sqrt{\frac{\bar{D}}{\pi t}}
$$

Table 9.4 lists the values of $D t / \ell^{2}$ required to obtain the ratio of the actual outgassing rate to the outgassing rate as determined from the semi-infinite case. In addition, values of the corresponding fraction of gas left in the material are given. The evaluation of $D$ may pose a problem. Table 9.5 lists some values of $D$ for various materials, under the assumption that

$$
D=D_{0} e^{-\Delta B / R T}
$$


TABLE 9.4.-Outgassing Rate Ratio and Concentration Ratio as a Function of the Dimensionless Parameter $\mathrm{Dt} / \ell^{2}$

\begin{tabular}{|c|c|c|}
\hline \multirow{2}{*}{$D t / \ell^{2}$} & Actual outgassing rate & \multirow{2}{*}{$\begin{array}{c}\text { Gas remaining } \\
C_{a v} / C_{0}\end{array}$} \\
\hline & Outgassing rate for semi-infinite case & \\
\hline 6.5 & $10^{-8}$ & \\
\hline 3.5 & $10^{-8}$ & $4.0 \times 10^{-6}$ \\
\hline 2.5 & $10^{-2}$ & $2.2 \times 10^{-4}$ \\
\hline 1.5 & $10^{-1}$ & $1.2 \times 10^{-2}$ \\
\hline
\end{tabular}

TABLE 9.5.-Values of the Diffusion Coefficient $\mathrm{D}$, When $\mathrm{D}=\mathrm{D}_{0} \mathrm{e}^{-\Delta \mathrm{E} / \mathrm{RT}}$, for Various Gases in Metals

\begin{tabular}{|c|c|c|c|c|c|}
\hline $\begin{array}{l}\text { System of gas } \\
\text { in metal }\end{array}$ & $\begin{array}{l}\text { Diffusion } \\
\text { coefficient, } \\
D_{0}, \mathrm{~cm}^{2} / \mathrm{sec}\end{array}$ & $\begin{array}{c}\text { Activation } \\
\text { energy, } \\
\Delta E, \\
\text { cals/gram mole }\end{array}$ & $\begin{array}{l}\text { Diffusion } \\
\text { coefficient, } \\
D, \mathrm{~cm}^{2} / \mathrm{sec}\end{array}$ & $\mid \begin{array}{c}\text { Temperature, } \\
T,{ }^{\circ} \mathrm{K}\end{array}$ & $\begin{array}{c}\text { Ref- } \\
\text { erence }\end{array}$ \\
\hline $\mathrm{N}_{2}-\mathrm{Fe}$ & 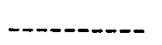 & - & $1.0 \times 10^{-8}$ & 400 & 10 \\
\hline & & & $5.0 \times 10^{-15}$ & 25 & 10 \\
\hline $\mathrm{H}_{2}-\mathrm{Ni}$ & -...-..... & - & $1.16 \times 10^{-8}$ & 85 & 10 \\
\hline & & & $1.0 \times 10^{-6}$ & 25 & 10 \\
\hline $\begin{array}{l}\mathrm{H}_{2}-\mathrm{Fe} \\
\mathrm{Co}-\mathrm{Ni}\end{array}$ & $-\cdots$ & $\cdots$ & $2.6 \times 10^{-8}$ & 20 & 10 \\
\hline $\begin{array}{l}\mathrm{Co}-\mathrm{Ni} \\
\mathrm{O}_{2}-\text { steel }\end{array}$ & $\cdots$ & ----- & $2.5 \times 10^{-8}$ & 700 & 10 \\
\hline $\mathrm{O}_{2}$-steel ----- & & $\cdots$ & $2.0 \times 10^{-20}$ & 25 & 10 \\
\hline $\mathrm{N}_{2}-\mathrm{Fe}$ & 0.003 & & $7.5 \times 10^{-10}$ & 1000 & 10 \\
\hline $\mathrm{O}_{2}-\mathrm{Cb}$ & .0147 & $\begin{array}{l}18200 \\
27600\end{array}$ & --------- & ----- & 17 \\
\hline & .00407 & 24900 & - & 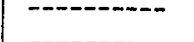 & 17 \\
\hline & .014 & 26600 & - & $-1-1 . .$. & 17 \\
\hline \multirow{5}{*}{$\mathrm{N}_{2}-\mathrm{Cb}$} & 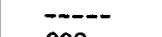 & 22000 & -n--o-- & - & 17 \\
\hline & .098 & 38600 & -------- & --------- & 17 \\
\hline & .0072 & 34800 & ------- & -.n-n-n- & 17 \\
\hline & .061 & 38800 & --------- & ----------- & 17 \\
\hline & $\overline{0215}$ & $\begin{array}{l}35700 \\
19370\end{array}$ & ----- & -------- & 17 \\
\hline $\mathrm{H}_{2}-\mathrm{Cb}$ & .056 & 19200 & ----- & --_-_- & 17 \\
\hline
\end{tabular}

The above data also allow the time and temperature required to degas materials to be evaluated using the same equation

$$
D=D_{0} e^{-\Delta E / R T}
$$

As an example, consider the system $\mathrm{H}_{2}$ in $\mathrm{Cb}$. $D_{0}$ generally varies between 0.0215 and $0.056 \mathrm{~cm}^{2} / \mathrm{sec}$. The present discussion will use a value of $D_{0}=0.0215$. Thus, if $D=D_{0} e^{-\Delta E / R T}$ is substituted in $D t / \ell^{2}$ and the subsequent expression set equal to a value of 4 for a reduction of $10^{-2}$ over the semi-infinite case, then

$$
\frac{t D_{0} e^{-\Delta E / R T}}{\ell^{2}}=4
$$


and

$$
\frac{\Delta E}{R T}=\ln \frac{4}{0.0215} \frac{\ell^{2}}{t}
$$

The system of hydrogen in columbium will have a $\Delta E=19200 \mathrm{cal} /$ gram mole. If it is desired to know the temperature required to reduce the outgassing rate of a piece of $\mathrm{Cb}, 0.1$ centimeter thick to $10^{-2}$ of the semi-infinite case after heating for 16 hours, where $t=5.76 \times 10^{4} \mathrm{sec}$, and $\frac{\Delta E}{R T}=2.3 \log \frac{186 \times 10^{-2}}{5.76 \times 10^{4}}$. Therefore, $T=1880^{\circ} \mathrm{K}$.

\section{LOW TEMPERATURE}

It is also possible to reduce the outgassing rate by reducing the temperature of the gas source. A process used in this case is similar to that which determines the effect of increasing the temperature. Figure 9.4 indicates the variation in process rate as a function of reciprocal temperature. A decrease in temperature causes a decrease in diffusion coefficient. For example, a change in temperature from $300^{\circ} \mathrm{K}$ to $263^{\circ} \mathrm{K}$ causes a drop of two decades in the diffusion coefficient, or, in other words, a two-decade increase in residence time. The diffusion nomograph (fig. 9.5) shows how a temperature change moves the outgassing rate from point $A$ to point $E$ and it shows the subsequent two-decade decrease in outgassing rate which now will remain relatively constant for a long period of time. In effect, room temperature proves an effective bakeout for materials which are to be operated at cryogenic temperatures.

\section{EXPOSURE}

The effect of the exposure of a degassed material to the atmosphere or to contaminating materials is extremely important and has been covered extensively in the literature. Reference 17 presents data on the effect of water vapor in the environment surrounding a work piece. If low outgassing rates are to be maintained, condensable gases must be kept from a degassed surface. The admission of water vapor increases the amount of gas to be desorbed from the surface by several times.

\section{POLYMERS}

Recent years have seen considerable research conducted on the vacuum effects on materials. In particular, the use of plastics in space has been very thoroughly scrutinized, with emphasis on the effects of ultraviolet radiation, temperature, and vacuum.

However, the discussion to follow will analyze the effect of materials on the vacuum, rather than vacuum effect on materials. Data from references $9,12,13$, and 17 are the most frequently quoted in this respect. Reference 9 discusses in detail some of the effects of material properties 
on the permeability rate. Conclusions which seem to be applicable to outgassing as well are:

(1) The permeability constants for the permanent gases such as oxygen, nitrogen, and carbon dioxide are independent of pressure.

(2) In the case of organic vapors, the permeability constant is pressure dependent and generally increases with increasing pressure due to a plasticizing effect of the vapor.

(3) The diffusion coefficient increases with increasing temperature.

(4) The solubility increases as temperature increases for permanent gases, and decreases for condensable gases; this effect is small.

(5) Permeation increases with increasing temperature.

(6) The logarithm of the solubility is generally a linear relationship of the boiling point of the gas.

(7) Increasing crystallinity in the polymer decreases the solubility.

(8) Diffusion through a polymer takes place through the tangled mass of polymer chains and holes. The diffusing molecules move from hole to hole by a temperature-activated process, although there is a non-temperature-activated process in loosely bound systems. Polymers, such as polyethylene and rubber, would be expected to yield different results; i.e., polyethylene should result in an extremely temperature sensitive diffusion coefficient. Rubber, on the other hand, should yield higher diffusion coefficients with less sensitivity to temperature.

(9) In hydrophobic materials such as Teflon and polyethylene, the rate of gas transmission is independent of water-vapor pressure.

(10) In hydrophobic films, the rate of gas transmission increases with increasing relative humidity.

The activation energies for these various outgassing processes do not always agree with the physical picture. For this reason the data should always be used in preference to estimates.

Tables 9.6 to 9.8 list activation energies for various materials for the diffusion, solubility, permeation, and outgassing processes. Of course, it should be realized that the data presented in the following tables are only a small portion of the total amount of data available.

\section{Gas Types}

The bulk of the data does not enumerate the types of gases involved in the outgassing process. Typical gases are water vapor, carbon dioxide, and air, in decreasing order. Other gases desorbed from polymers in the as-received condition are the plasticizers used in their manufacture. An interesting point is that once a material has been degassed and the plasticizer removed, the gas-sorption capability of the material drops to about one-half its original value. 
TABLE 9.6.-Diffusion and Solubility Constants for Various Polymers

\begin{tabular}{|c|c|c|c|c|c|c|c|c|c|}
\hline Polymer & Ref. & $\underset{{ }^{\circ} \mathrm{C}}{\text { Temp., }}$ & $\begin{array}{c}\text { Solubility, } S \text {, } \\
\text { at room temp. } \\
\text { and pressure } \\
\mathrm{cm}^{3} \text { gas } / \mathrm{cm}^{8} \text { solid }\end{array}$ & $\begin{array}{c}\text { Solubility, } \\
S_{0}, \\
\mathrm{~cm}^{8} \text { gas } / \mathrm{cm}^{8} \text { solid }\end{array}$ & $\begin{array}{c}\text { Activation } \\
\text { energy, } \\
E_{s}, \\
\text { cal } / g \text { mole }\end{array}$ & $\begin{array}{c}\text { Diffusion } \\
\text { coefficient, } \\
D, \\
\text { at room temp., } \\
\mathrm{cm}^{2} / \mathrm{sec}\end{array}$ & $\begin{array}{c}\text { Diffusion } \\
\text { coefficient, } \\
D_{0} \\
\mathrm{~cm}^{2} / \mathrm{sec}\end{array}$ & $\begin{array}{c}\text { Activation } \\
\text { energy, } \\
\boldsymbol{E}_{D}, \\
\mathrm{cal} / \mathrm{g} \text { mole }\end{array}$ & Ref. \\
\hline 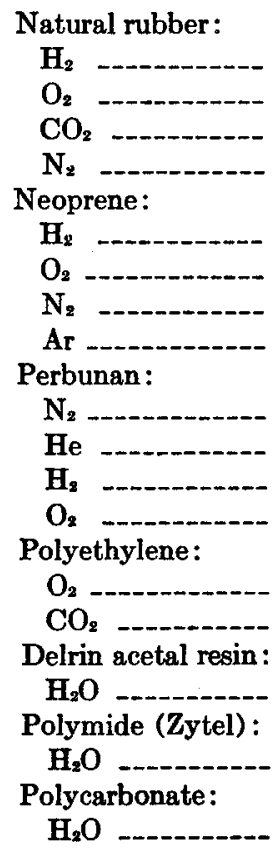 & $\begin{array}{l}10 \\
10 \\
10 \\
10 \\
10 \\
10 \\
10 \\
10 \\
\\
10 \\
10 \\
10 \\
10 \\
\\
20 \\
20\end{array}$ & $\begin{array}{l}25 \\
25 \\
25 \\
25 \\
\\
25 \\
25 \\
25 \\
36 \\
\\
25 \\
-- \\
25 \\
25 \\
\\
30 \\
30\end{array}$ & $\begin{array}{l}3.8 \times 10^{-5} \\
9.87 \times 10^{-5} \\
9.1 \times 10^{-5} \\
5.17 \times 10^{-5} \\
2.9 \times 10^{-5} \\
9.5 \times 10^{-5} \\
3.57 \times 10^{-5} \\
1.52 \times 10^{-4} \\
3.5 \times 10^{-5} \\
2.81 \times 10^{-5} \\
7.6 \times 10^{-5} \\
\\
-3.8 \\
4.84\end{array}$ & $4.94 \times 10^{-6}$ & 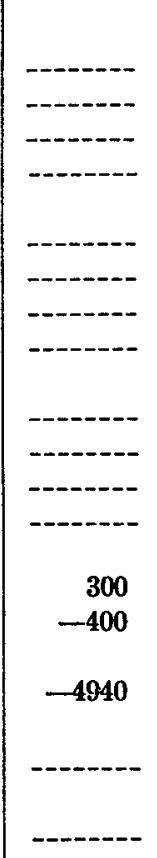 & & $\begin{array}{c}0.23 \\
.57 \\
2.9 \\
.28 \\
3.1 \\
9.3 \\
7.2 \\
85 \\
1.8 \\
1.0 \\
-\ldots--\end{array}$ & $\begin{array}{r}5900 \\
7500 \\
8700 \\
6600 \\
9400 \\
10300 \\
10200 \\
7300 \\
9200 \\
9300 \\
---\end{array}$ & $\begin{array}{l}-- \\
-- \\
-- \\
-- \\
-- \\
-- \\
-- \\
-- \\
-- \\
-- \\
-- \\
-- \\
-- \\
-- \\
--\end{array}$ \\
\hline
\end{tabular}


TABle 9.7.-Permeation Rate for Various Polymers

$\left[\frac{\mathrm{atm} \mathrm{cm}^{3}}{\mathrm{sec} \mathrm{cm}^{2}}\right.$ for a $1 \frac{\mathrm{atm}}{\mathrm{mm}}$ pressure gradient $]$

\begin{tabular}{|c|c|c|c|c|c|c|}
\hline Polymer & $\begin{array}{l}\text { Permeation } \\
\text { rate, } K_{p}\end{array}$ & $\begin{array}{c}\text { Temp. } \\
{ }^{\circ} \mathrm{C}\end{array}$ & $\begin{array}{c}\text { Ref- } \\
\text { erence }\end{array}$ & $\begin{array}{c}\text { Activation } \\
\text { energy } E_{p}, \\
\text { cal/gram } \\
\text { mole }\end{array}$ & $\begin{array}{c}\text { Pressure, } \\
P_{0}, \text { torr }\end{array}$ & $\begin{array}{c}\text { Ref- } \\
\text { erence }\end{array}$ \\
\hline \multicolumn{7}{|l|}{ Buna S: } \\
\hline $\mathrm{CO}_{2}$ & $9.4 \times 10^{-5}$ & 30 & 20 & $-\ldots-$ & & -- \\
\hline $\mathrm{N}_{2}$ & $4.8 \times 10^{-7}$ & 30 & 20 & -...... & & -- \\
\hline \multicolumn{7}{|l|}{ Butyl rubber: } \\
\hline $\mathrm{CO}_{2}$ & $3.9 \times 10^{-8}$ & 30 & 20 & ---- & & -- \\
\hline $\mathrm{N}_{2}$ & $1.8 \times 10^{-8}$ & 30 & 20 & $-\cdots-$ & ------o- & -- \\
\hline $\mathrm{O}_{2}$ & $9.9 \times 10^{-8}$ & 30 & 20 & -...-- & -- & -- \\
\hline \multicolumn{7}{|l|}{ Cellulose acetate: } \\
\hline $\mathrm{CO}_{2}$ & $5.9 \times 10^{-8}$ & 30 & 20 & $-\cdots-$ & $-\cdots$ & -- \\
\hline $\mathrm{N}_{2}$ & $2.1 \times 10^{-8}$ & 30 & 20 & $----\infty$ & - & -- \\
\hline $\mathrm{H}_{2} \mathrm{O}$ & $5.7 \times 10^{-4}$ & 25 & 23 & -......-- & --.-...- & -- \\
\hline \multicolumn{7}{|l|}{ Ethyl cellulose: } \\
\hline $\mathrm{N}_{2}$ & $6.4 \times 10^{-7}$ & 30 & 20 & -...-- & & -- \\
\hline $\mathrm{O}_{2}$ & $2.0 \times 10^{-8}$ & 30 & 20 & - - - & & -- \\
\hline $\mathrm{H}_{2} \mathrm{O}$ & $9.9 \times 10^{-4}$ & 25 & -. & -........ & $-\cdots$ & -- \\
\hline \multicolumn{7}{|l|}{ Hycar OR 15: } \\
\hline $\mathrm{CO}_{2}$ & $5.7 \times 10^{-8}$ & 30 & 20 & ------ & & - \\
\hline $\mathrm{N}_{2}$ & $1.78 \times 10^{-8}$ & 30 & 20 & --n-- & - & - \\
\hline $\mathrm{O}_{2}$ & $7.3 \times 10^{-8}$ & 30 & 20 & - - - - & - & -- \\
\hline \multicolumn{7}{|l|}{ Hycar OR 25: } \\
\hline $\mathrm{CO}_{2}$ & $1.4 \times 10^{-6}$ & 30 & 20 & ------ & -- & -- \\
\hline $\mathrm{N}_{2}$, & $4.6 \times 10^{-8}$ & 30 & 20 & -..-- & -n-n-n-n & - \\
\hline \multicolumn{7}{|l|}{ Methyl methacrylate: } \\
\hline $\mathrm{H}_{2} \mathrm{O}$ & $1.06 \times 10^{-4}$ & 25 & 23 & ------ & & -- \\
\hline \multicolumn{7}{|l|}{ Methyl rubber: } \\
\hline $\mathrm{CO}_{2}$ & $5.7 \times 10^{-2}$ & 30 & 20 & $---\infty--$ & -- & -- \\
\hline $\mathrm{N}_{2}$ & $3.6 \times 10^{-8}$ & 30 & 20 & -n--- & - & -- \\
\hline $\mathrm{O}_{2}$ & $1.6 \times 10^{-7}$ & 30 & 20 & - & - n-n- & $\ldots$ \\
\hline \multicolumn{7}{|l|}{ Natural rubber: } \\
\hline $\mathrm{H}_{2} \mathrm{O}$ & $1.8 \times 10^{-4}$ & 25 & 23 & --.-.-- & --- - & -- \\
\hline $\mathrm{CO}_{2}$ & $9.9 \times 10^{-6}$ & 30 & 20 & ------- & -......... & -- \\
\hline $\mathrm{N}_{2}$ & $6.1 \times 10^{-7}$ & 30 & 20 & 9300 & $6.2 \times 10^{-8}$ & 10 \\
\hline $\mathrm{O}_{2}$ & $1.77 \times 10^{-6}$ & 30 & 20 & 6600 & $1.1 \times 10^{-3}$ & 10 \\
\hline $\mathrm{H}_{2} \ldots$ & -..-- - - & $\ldots$ & $-\ldots-\ldots$ & 6900 & $6.0 \times 10^{-4}$ & 10 \\
\hline $\mathrm{He}$ & -- - - - - - & ---- & $\ldots-\cdots$ & 6500 & $1.8 \times 10^{-4}$ & 10 \\
\hline \multicolumn{7}{|l|}{ Neoprene: } \\
\hline $\mathrm{CO}_{2}$ & $1.9 \times 10^{-6}$ & 30 & 20 & ---- & - & -- \\
\hline $\mathrm{N}_{2}$ & $9.0 \times 10^{-8}$ & 30 & 20 & ------- & - & - \\
\hline \multirow[t]{3}{*}{$\mathrm{N}_{2}$} & $1.06 \times 10^{-7}$ & 27.1 & 10 & --- - - & --_-- & -1. \\
\hline & $1.75 \times 10^{-7}$ & 35.4 & 10 & -......- & - & _. \\
\hline & $1.67 \times 10^{-6}$ & 84.7 & 10 & 10600 & 5.62 & 10 \\
\hline \multicolumn{7}{|l|}{ Neoprene (vulcanized) } \\
\hline \multirow[t]{3}{*}{$\mathrm{H}_{2}$} & $6.85 \times 10^{-7}$ & 18.2 & 10 & 8100 & .912 & 10 \\
\hline & $9.87 \times 10^{-7}$ & 26.9 & 10 & -- - - - & - & - \\
\hline & $4.03 \times 10^{-6}$ & 63.7 & 10 & -......- & - & - $\mid--$ \\
\hline
\end{tabular}


Table 9.7.-Permeation Rate for Various Polymers-Continued

$\left[\frac{\mathrm{atm} \mathrm{cm}^{8}}{\mathrm{sec} \mathrm{cm}^{2}}\right.$ for a $1 \frac{\mathrm{atm}}{\mathrm{mm}}$ pressure gradient $]$

\begin{tabular}{|c|c|c|c|c|c|c|}
\hline Polymer & $\begin{array}{l}\text { Permeation } \\
\text { rate, } K_{p}\end{array}$ & $\underset{{ }^{\circ} \mathrm{C} p}{\text { Temp., }}$ & $\begin{array}{c}\text { Ref- } \\
\text { erence }\end{array}$ & $\begin{array}{c}\text { Activation } \\
\text { energy } E_{p}, \\
\text { cal/gram } \\
\text { mole }\end{array}$ & $\underset{P r e s s u r e,}{P_{0} \text {, torr }}$ & $\begin{array}{c}\text { Ref- } \\
\text { erence }\end{array}$ \\
\hline $\mathrm{He} \ldots \ldots$ & $1.67 \times 10^{-7}$ & 0 & 10 & ------- & & -- \\
\hline & $5.92 \times 10^{-7}$ & 30.4 & 10 & - & & - \\
\hline & $7.15 \times 10^{-6}$ & 101.3 & 10 & 7520 & 0.167 & (calc) \\
\hline $\mathrm{O}_{2} \ldots$ & & & & 9900 & 5.4 & 10 \\
\hline Ar - & $5.17 \times 10^{-7}$ & 36.1 & -- & $\ldots$ & & - \\
\hline & $5.0 \times 10^{-6}$ & 86.2 & 10 & 10000 & 5.64 & (calc) \\
\hline Perbunan: & & & & & & \\
\hline $\mathrm{CO}_{2}$ & $2.35 \times 10^{-8}$ & 30 & 20 & & & -- \\
\hline $\mathrm{N}_{2}$ & $8.05 \times 10^{-8}$ & 30 & 20 & 11000 & 1.06 & -- \\
\hline $\mathrm{He}$ & & & & 7000 & .121 & -- \\
\hline Pliofilm (NO): & & & & & & \\
\hline $\mathrm{CO}_{2}$ & $1.98 \times 10^{-8}$ & 30 & 20 & ------ & $m-m--$ & -- \\
\hline $\mathrm{N}_{2}$ & $6.06 \times 10^{-10}$ & 30 & 20 & --_- & - - - - n- & -- \\
\hline $\mathrm{H}_{2} \mathrm{O}$ & $6.0 \times 10^{-6}$ & 25 & 23 & -- & & -- \\
\hline Pliofilm $P_{4}$ : & & & & & & \\
\hline $\mathrm{CO}_{2}$ & $1.38 \times 10^{-6}$ & 30 & 20 & $---\ldots-$ & & -- \\
\hline $\mathrm{N}_{2}$ & $4.61 \times 10^{-8}$ & 30 & 20 & & & -- \\
\hline Polyamide (nylon): & & & & & & \\
\hline $\mathrm{CO}_{2}$ & $1.21 \times 10^{-8}$ & 30 & 20 & -- & & -- \\
\hline $\mathrm{N}_{2}$ & $7.6 \times 10^{-10}$ & 30 & 20 & $\ldots$ & -..-- & - \\
\hline $\mathrm{H}_{2} \mathrm{O}$ & $5.3 \times 10^{-5}$ & 25 & 23 & ----- & $\ldots$ & -- \\
\hline Polybutadiene: & & & & & & \\
\hline $\mathrm{CO}_{2}$ & $1.05 \times 10^{-5}$ & 30 & 20 & $---\infty-$ & -----n-- & - \\
\hline$N_{2}$ & $4.9 \times 10^{-7}$ & 30 & 20 & -...-- & - - - - & -- \\
\hline $\mathrm{O}_{2}$ & $1.45 \times 10^{-9}$ & 30 & 20 & ----- & - & -- \\
\hline Polyethylene: & & & & & & \\
\hline $\mathrm{N}_{2}$ & $1.6 \times 10^{-7}$ & 30 & 20 & 11700 & .47 & 20 \\
\hline $\mathrm{O}_{2}$ & $5.2 \times 10^{-7}$ & 30 & 20 & 9500 & .02 & -. \\
\hline $\mathrm{CO}_{2}$ & $2.1 \times 10^{-6}$ & 30 & 20 & 8900 & .03 & -- \\
\hline $\mathrm{H}_{2} \mathrm{O}$ & $6.0 \times 10^{-8}$ & 25 & 23 & ------- & ---------- & -- \\
\hline Polyester (Mylar): & & & & & & \\
\hline $\mathrm{N}_{2}$ & $3.8 \times 10^{-10}$ & 30 & 20 & - - & --- & -- \\
\hline $\mathrm{O}_{2}$ & $1.67 \times 10^{-6}$ & 30 & 20 & - - & 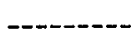 & -- \\
\hline $\mathrm{CO}_{2}$ & $1.16 \times 10^{-8}$ & 30 & 20 & - - & -n- & -- \\
\hline $\mathrm{H}_{2} \mathrm{O}$ & $9.9 \times 10^{-6}$ & 25 & 23 & $-\cdots--$ & - - & -- \\
\hline $\begin{array}{l}\text { Poly (chlorotrifluoro- } \\
\text { ethylene) } \\
\text { (Kel-F) : }\end{array}$ & & & & & & \\
\hline $\mathrm{O}_{2}$ & $4.26 \times 10^{-8}$ & 30 & 20 & -- & - & $-\infty$ \\
\hline $\mathrm{N}_{2}$ & $9.87 \times 10^{-8}$ & 30 & 20 & - - - - & - - & - \\
\hline $\mathrm{H}_{2} \mathrm{O}$ & $2.2 \times 10^{-8}$ & 25 & 23 & ----- & $----\cdots$ & - \\
\hline Polystyrene: & & & & & & \\
\hline $\mathrm{H}_{2} \mathrm{O}$ & $9.1 \times 10^{-5}$ & 25 & 23 & --- & - & $\cdots$ \\
\hline Polyvinyl acetate: & & & & & & \\
\hline $\mathrm{H}_{2} \mathrm{O}$ & $7.6 \times 10^{-4}$ & 25 & 23 & ------ & $-\cdots$ & - \\
\hline
\end{tabular}


TABLE 9.7.-Permeation Rate for Various Polymers-Continued $\left[\frac{\mathrm{atm} \mathrm{cm}^{3}}{\mathrm{sec} \mathrm{cm}^{2}}\right.$ for a $1 \frac{\mathrm{atm}}{\mathrm{mm}}$ pressure gradient $]$

\begin{tabular}{|c|c|c|c|c|c|c|}
\hline Polymer & $\begin{array}{l}\text { Permeation } \\
\text { rate, } K_{p}\end{array}$ & $\underset{{ }^{\circ} \mathrm{C}}{\text { Temp. }}$ & $\begin{array}{l}\text { Ref- } \\
\text { erence }\end{array}$ & $\begin{array}{l}\text { Activation } \\
\text { energy } E_{p}, \\
\text { cal/gram } \\
\text { mole }\end{array}$ & $\begin{array}{c}\text { Pressure, } \\
P_{0}, \text { torr }\end{array}$ & $\begin{array}{c}\text { Ref- } \\
\text { erence }\end{array}$ \\
\hline \multicolumn{7}{|l|}{$\begin{array}{l}\text { Polyvinylidene } \\
\text { chloride (Saran): }\end{array}$} \\
\hline $\mathrm{CO}_{2}$ & $2.2 \times 10^{-9}$ & 30 & 20 & --.---- & ------ - - & -- \\
\hline $\mathrm{N}_{2}$ & $7.17 \times 10^{-11}$ & 30 & 20 & --.----- & --_--_--.- & -- \\
\hline $\mathrm{O}_{2}$ & $4.04 \times 10^{-10}$ & 30 & 20 & -------- & ------_--- & -- \\
\hline $\mathrm{H}_{2} \mathrm{O}$ & $1.06 \times 10^{-7}$ & 25 & 23 & ------ & -.on---- & - \\
\hline \multicolumn{7}{|l|}{ Vulcaprene: } \\
\hline $\mathrm{O}_{2}$ & $1.15 \times 10^{-7}$ & 30 & 20 & --n--- & ------ & -- \\
\hline $\mathrm{N}_{2}$ & $3.7 \times 10^{-8}$ & 30 & 20 & -...... & $-\cdots$ & -- \\
\hline \multicolumn{7}{|l|}{ Delrin (acetal resin): } \\
\hline $\mathrm{O}_{2}$ & $4.8 \times 10^{-6}$ & 22.8 & 34 & $-\cdots--$ & $-\cdots-$ & -- \\
\hline $\mathrm{CO}_{2}$ & $9.27 \times 10^{-8}$ & 22.8 & 34 & $-\ldots---$ & -------- & -- \\
\hline $\mathrm{H}_{2} \mathrm{O}$ & $1.7 \times 10^{-6}$ & 22.8 & 34 & -.....- & $-\ldots-\ldots$ & -- \\
\hline
\end{tabular}

\section{Decomposition}

The decomposition of polymers in a vacuum system is currently under investigation in many laboratories. References 21,23 to 26 , and 30 are just a few of the papers which have been published.

The decomposition of diffusion pump fluids has been a problem of more general interest for a long period of time and is discussed in references 13 and 31 to 33 . Typical vacuum systems which utilize liquid nitrogen traps are concerned with pump fluid decomposition. which would not be condensed on the $77^{\circ} \mathrm{K}$ surfaces.

This decomposition occurs at different rates depending upon the temperature and materials used. Equal fractions of the decomposition products of hydrogen and methane typify silicone oil cracking, while the predomination of hydrogen suggests that organic compounds are breaking down.

\section{Techniques for Reducing the Gas Load}

The outgassing rate for polymers is not decreased when solvents are used for cleaning. Experimenters reported in reference 20 that attempts to clean Viton with isopropyl alcohol had little success. The best outgassing rates are obtained in the as-received condition.

Better results were obtained by a number of workers (refs. 20, 21,26 , and 27) when a high-temperature degas process was applied to the polymeric compound as a means of reducing the outgassing effect. 
TABLE 9.8.-Outgassing Rate for Various Polymers

\begin{tabular}{|c|c|c|c|c|c|c|c|}
\hline \multirow{2}{*}{ Polymer } & \multicolumn{4}{|c|}{ 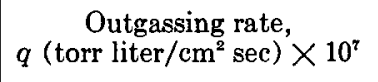 } & \multirow{2}{*}{$\begin{array}{l}\text { Ref- } \\
\text { erence }\end{array}$} & \multirow{2}{*}{$\begin{array}{c}\text { Activation } \\
\text { energy, } \\
\Delta E_{o} \\
\text { cal/gram } \\
\text { mole }\end{array}$} & \multirow{2}{*}{$\begin{array}{l}\text { Ref- } \\
\text { erence }\end{array}$} \\
\hline & $1 \mathrm{hr}$ & $4 \mathrm{hr}$ & $10 \mathrm{hr}$ & $40 \mathrm{hr}$ & & & \\
\hline Buna $\mathbf{S}$ butyl rubber--- & 20 & 6 &.-- & $-\ldots$ & 10 & --o---- & -- \\
\hline $\begin{array}{l}\text { Cellulose nitrate } \\
\text { (celluloid) }\end{array}$ & 86 & & & & 10 & & \\
\hline Convaseal & 10 & 5 & $\ldots \ldots$ & -- & 10 & --_---- & -- \\
\hline Epicote & 25 & 12.5 & ---- & $--\cdots$ & 10 & ----- & -- \\
\hline $\begin{array}{l}\text { Epoxy (Araldite, } \\
\text { epoxy) }\end{array}$ & 20 & & & & 10 & 9500 & 9 \\
\hline Hostafion & .55 & .3 & ---- & ---- & 9 & 16300 & 9 \\
\hline $\begin{array}{l}\text { Hycar H50 (ASTM } \\
\text { Spec. SB-510) }\end{array}$ & 130 & 72 & & & 10 & & _- \\
\hline $\begin{array}{l}\text { Methylmethacrylate, } \\
\text { poly (Plexiglas, } \\
\text { Lucite) }\end{array}$ & 19 & 10 & & & 10 & 6800 & 9 \\
\hline Natural rubber gum -- & 12 & 6.0 & & & 10 & --- - - & -- \\
\hline $\begin{array}{l}\text { Neoprene }(35 / \mathrm{Ne} 746) \\
\text { (B) }\end{array}$ & 26 & 8 & 3.8 & & 9 & $--\infty-\infty$ & -- \\
\hline Nitrate (Buna N) & 80 & 20 & 10 & & 24 & - - - & -- \\
\hline $\begin{array}{l}\text { after } 4 \text {-hr bake at } \\
100^{\circ} \mathrm{C}\end{array}$ & 10 & .6 & .4 & -- & 24 & -...-- & -- \\
\hline Perbunan & 31 & 15 & 9 & $\ldots$ & 9 & $-\cdots-n$ & -- \\
\hline Polyamide (nylon) & 26 & 12 & $---\cdot$ & $-\ldots$ & 9 & $---n---$ & -- \\
\hline Polycarbonate (Lexan) & 7.2 & 3.9 & $-\cdots$ & - & -- & 8700 & 9 \\
\hline Polyethylene & 2.3 & 1.15 & $-\ldots--$ & --- & 10 & - - - & -- \\
\hline Polymethane & 4 & 1.8 & $-\cdots-$ & $-\cdots$ & 9 & -..--- & -- \\
\hline $\begin{array}{l}\text { Polymonochlorotri- } \\
\text { fluoroethylene } \\
\text { (Kel-F) }\end{array}$ & .4 & .17 & $\ldots$ & $--\ldots$ & 10 & ------- & -- \\
\hline Polystyrol & 15 & 7.5 & ----- & $---\cdots$ & 10 & ----- & -- \\
\hline $\begin{array}{l}\text { Polytetrafluoroethyl- } \\
\text { ene (Teflon) }\end{array}$ & 2 & .75 & $-\cdots$ & ---.-- & -- & 9500 & 9 \\
\hline Polyvinyl carbozol --- & 16 & 8 & ----- & $-\cdots-$ & 10 & $-\cdots-$ & :-- \\
\hline Polyvinyl chloride -..-- & 5 & 2.8 & $-\ldots--$ & $-\cdots$ & - & 11600 & 9 \\
\hline Pyrophyllite & - & ---- & ---- & $-\cdots$ & -- & 9200 & 9 \\
\hline Polyester --- & 25 & 8 & $-\cdots$ & $-\cdots$ & 10 & 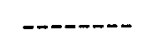 & -- \\
\hline Silicone rubber & 70 & 20 & $-\cdots$ & - & 9 & $\begin{array}{c}7300 \\
T>87^{\circ} \mathrm{C} \\
13900 \\
T<87^{\circ} \mathrm{C}\end{array}$ & $\begin{array}{l}9 \\
9\end{array}$ \\
\hline Silastic 55-74 -- & 28 & 6 & $-\ldots$ & - & 10 & ------ & -- \\
\hline Ultramide & 8.2 & 3.8 & -- & $-\infty$ & 9 & ------ & -- \\
\hline Viton: & 4 & 18 & & & 9 & 13800 & 9 \\
\hline $\begin{array}{l}\text { Processed by baking } \\
\text { in air } 4 \mathrm{hr} \text { at } \\
150^{\circ} \mathrm{C}\end{array}$ & .01 & .003 & .002 & & 24 & & -- \\
\hline Vulkollen & & 80 & 42 & $---\cdots$ & 9 & --- & -- \\
\hline
\end{tabular}


Reductions in outgassing in this case appear to be permanent, since readsorption is less than one-half the original amount after a long exposure to the atmosphere. This effect is probably due to the removal of the plasticizer and the subsequent rearrangement of the polymer molecules to a more tightly packed lattice. Both the outgassing and permeation rates are affected. Reference 27 reports a reduction of more than 100 in the helium-permeation rate for several elastomer-sealing materials after a 100 -hour bake at $200^{\circ} \mathrm{F}$.

A lowering of the temperature of the gas source has been shown to produce a reduction in the outgassing rate. This reduction can be predicted from the nomographs, if a nominal activation energy of $10 \mathrm{~K}$ cal/gram mole is assumed. A decrease in temperature from $27^{\circ} \mathrm{C}$ to $-18^{\circ} \mathrm{C}$ would cause a decrease of two decades in the outgassing rate. References 28 and 29 have used this technique to manufacture ultrahigh-vacuum chambers with standard sealing materials.

A final method, mentioned previously in chapter 8 , is the doublepumping approach which is applicable to organic polymer-sealing techniques. This approach assumes that permeation through the sealing material is the major limitation, rather than outgassing. The advantage of this technique over cooling of the elastomer is that oils or greases are not condensed in the O-ring region. A disadvantage is the long pumping time required to obtain a small outgassing rate.

\section{REFERENCES}

1. Young, J. R.; And Whetten, N. R.: Techniques for the Admission of High Purity Gases to a Vacuum System. Trans. of the Eighth National Vacuum Symposium and the Second International Congress on Vacuum Science and Technology, vol. I. L. E. Preuss, ed., Pergamon Press, 1962, pp. 625-627.

2. Young, J. R.: A Palladium Diaphragm Hydrogen Pump. Class 1 Report, 63 RL-3771E, General Electric Co., July 1963.

3. Norton, F. J.: Gas Permeation Through the Vacuum Envelope. Trans, of the Eighth National Vacuum Symposium and the Second International Congress on Vacuum Science and Technology, vol. I. L. E. Preuss, ed., Pergamon Press, 1962, pp. 8-16.

4. Dushman, S.: Scientific Foundation of Vacuum Technique. J. M. Lafferty, ed., John Wiley \& Sons, Inc., 1962, pp. 491-500, 509-511, 570-587.

5. Koнl, W. H.: Materials and Techniques for Electron Tubes. Reinhold Pub. Corp. (New York), 1960.

6. Waldschmidt, E.: Liberation of Gas and Gas Permeability of Metallic Structural Materials for Vacuum Apparatus. Metallurgy, vol. 8, Oct. 1954. pp. 749-758.

7. Basalaeva, N. IA.: Evolution of Gas in Metals Used in Vacuum Technology. Soviet Physics, vol. 3, no. 5, May 1958, pp. 1027-1031.

8. Schram, A.: Investigation of the True Desorbing Area of Solids in Vacuum. Trans. of the Ninth National Vacuum Symposium, G. H. Bancroft, ed., The Macmillan Co., 1962, pp. 301-306.

9. Schitтко, F. J.: Measurement of Gas Emission from Solid Surfaces. Vacuum, vol. 13, no. 12, Dec. 1963, pp. 525-537. 
10. Dayton, B. B.: Relations Between Size of Vacuum Chamber, Outgassing Rate and Required Pumping Speed. Trans. of the Sixth National Symposium on Vacuum Technology, C. R. Meissner, ed., Pergamon Press, 1960, pp. 101-119.

11. Dayton, B. B.: The Effect of Bakeout on the Degassing of Metals. Trans. of the Ninth National Vacuum Symposium, G. H. Bancroft, ed., The Macmillan Co., 1962, pp. 293-300.

12. Dayton, B. B.: Outgassing Rate of Contaminated Metal Surfaces. Trans. of the Eighth National Vacuum Symposium and the Second International Congress on Vacuum Science and Technology, vol. I. L. E. Preuss, ed., Pergamon Press, 1962, pp. 42-57.

13. Blears, J.; Greer, E. J.; and Nightingale, J.: Factors Determining the Ultimate Pressure in Large High-Vacuum Systems. Advances in Vacuum Science and Technology, vol. I. E. Thomas, ed., Pergamon Press, 1959, pp. 473-480.

14. Ehrlich, G.: Molecular Processes in Adsorption on Metals. Trans. of the Eighth National Vacuum Symposium and the Second International Congress on Vacuum Science and Technology, vol. I. L. E. Preuss, ed., Pergamon Press, 1962, pp. 126-143; Discussion, pp. 144-145.

15. Redhead, P. A.: The Desorption Spectrometer as an Analytic Tool in UltraHigh-Vacuum Investigations. Trans. of the Sixth National Symposium on Vacuum Technology, C. R. Meissner, ed., Pergamon Press, 1960, pp. 12-15.

16. Carslaw, H. S.; and Jaeger, J. C.: Conduction of Heat in Solids. Second ed., Clarendon Press (Oxford), 1959.

17. Wilcox, B. A.; And Huggins, R. A.: Strain Aging Effects in Columbium due to Hydrogen. Columbium Metallurgy, vol. 10 of Metallurgical Soc. Conferences, D. L. Douglas and F. W. Kunz, eds., Interscience Publishers (New York), 1960, pp. 257-278; Discussion, p. 278.

18. SANTEl,zR, D. J.: Outgassing Characteristics of Various Metals. Trans, of the Fifth National Symposium on Vacuum Technology, W. G. Matheson, ed., Pergamon Press, 1959, pp. 1-8.

19. Eschbach, H. L.; Gross, F.; and Schulien, S.: Permeability Measurements With Gaseous Hydrogen for Various Steels. Vacuum, vol. 13, no. 12, Dec. 1963, pp. 543-547.

20. Rogers, C.; Meyer, J. A.; Stannett, V.; and Szwarc, M.: Studies in the Gas and Vapor Permeability of Plastic Films and Coated Papers, Part I. TAPPI (J. Tech. Assoc. Pulp \& Paper Ind.), vol. 39, no. 11, Nov. 1956, pp. 737-741; Part II, pp. 741-747.

21. Rogers, C.; Meyer, J. A.; Stannett, V.; and Szwarc, M.: Studies in the Gas and Vapor Permeability of Plastic Films and Coated Papers, Part III. TAPPI, vol. 40, no. 3, Mar. 1957, pp. 142-146.

22. Rogers, C.; Meyer, J. A.; Stannett, V.; and Szwarc, M.: Studies in the Gas and Vapor Permeability of Plastic Films and Coated Papers, Part IV. TAPPI, vol. 41, no. 11, Nov. 1958, pp. 716-720.

23. Crawley, D. J.; and De Csernatony, L.: Degassing Characteristics of Some O-Ring Materials. Vacuum, vol. 14, no. 1, Jan. 1964, pp. 7-9.

24. Munchrausen, H.; ANd Schittko, F. J.: Investigation of the Outgassing Process of Silicone Rubber. Vacuum, vol. 13, no. 12, Dec. 1963, pp. 548-553.

25. Markley, F.; Roman, R.; and Vosecak, R.: Outgassing Data for Several Epoxy Resins and Rubbers for the Zero Gradient Synchrotron. Trans. of the Eighth National Vacuum Symposium and the Second International Congress on Vacuum Science and Technology, vol. I. L. E. Preuss, ed., Pergamon Press, 1961, pp. 78-84; Discussion,. J. Suhorsky and A. Fisher, p. 85. 
26. Podlaseck, S.: The Behavior of Organic Materials at Elevated Temperatures in a Vacuum. Trans. of the Ninth National Vacuum Symposium, G. H. Bancroft, ed., The Macmillan Co., 1962, pp. 320-323.

27. FulK, M. M.; ANd Horr, K. S.: Sublimation of Some Polymeric Materials in Vacuum. Trans. of the Ninth National Vacuum Symposium, G. H. Bancroft, ed., The Macmillan Co., 1962, pp. 324-333.

28. Rivera, M.; Fassbll, W. M.; and Jensen, J.: The Low-Pressure Gas Desorption of Some Polymeric Materials. Trans. of the Ninth National Vacuum Symposium, G. H. Bancroft, ed., The Macmillan Co., 1962, pp. 342-348.

29. Adpiss, R. R.; PEnsak, L.; ANd Scott, N. J.: Evaluation of a New Fluoroelastomer as a Gasketing Material for High Vacuum Systems. Trans. of the Seventh National Symposium on Vacuum Technology, C. R. Meissner. ed., Pergamon Press, 1961, pp. 39-44.

30. Jordan, J. R.: New Developments in Vacuum Sealing. Trans. of the Eighth National Vacuum Symposium and the Second International Congress on Vacuum Science and Technology, vol. II. L. E. Preuss, ed., Pergamon Press: 1962, pp. 1302-1308.

31. Miller, T. M.; AND Getger, K. A.: Performance of Environmental Chambers for the $10^{-10}$ Torr Range Using Oil Diffusion Pumps. Trans. of the Ninth National Vacuum Symposium, G. H. Bancroft, ed., The Macmillan Co., 1962, pp. 270-272.

32. FARKass, J.; Gould, P. R.; And HoRn, G. W.: $1 \times 10^{-11}$ Torr in Large Metal Chambers, Its Attainment and Application Results. Trans. of the Ninth National Vacuum Symposium, G. H. Bancroft, ed., The Macmillan Co., 1962, pp. 273-277.

33. Boebel, C. P.; Mackie, N. A.; and Quaintance, C. C.: Outgassing Studies of Space Materials. Trans. of the Ninth National Vacuum Symposium, G. H. Bancroft, ed., The Macmillan Co., 1962, pp. 307-310.

34. Bachler, W.: Elimination of Pressure Fluctuations Above Oil Diffusion Pumps. Trans. of the Ninth National Vacuum Symposium, G. H. Bancroft, ed., The Macmillan Co., 1962, pp. 395-398.

35. Kennedy, P. B.: A Hypothesis Concerning Limitations of Diffusion Pumps. Trans. of the Eighth National Vacuum Symposium and the Second International Congress on Vacuum Science and Technology, vol. I. L. E. Preuss, ed., Pergamon Press, 1962, pp. 320-326; Discussion, p. 326.

36. Hickman, K. C. D.: High Vacuum With the Polyphenyl Ethers-a SelfContained Technology. Trans. of the Eighth National Vacuum Symposium and the Second International Congress on Vacuum Science and Technology, vol. I. L. E. Preuss, ed., Pergamon Press, 1962, pp. 307-314; Discussion, p. 314. 


\section{Systems}

\section{INTRODUCTION}

$\mathrm{T}$ HE PREVIOUS CHAPTERS have treated the basic parameters of vacuum technology-pressure, speed, and gas load. The discussion to follow will marry this information to yield a statement of the concept of the total system, its requirements, and its design for space-simulation applications. Initially, space simulation will be defined, and the types of vacuum systems applicable to various space-simulation conditions will be specified. The remainder of the chapter will be devoted to a discussion of the fabrication, operation, and maintenance of vacuum systems.

\section{SPACE SIMULATION}

Due to the rapidly expanding range of uses of space-simulation techniques, the treatment of the subject in recent literature has also increased considerably. References 1 to 18 are typical reports dealing with various aspects of the space-simulation problem.

The entire subject of space simulation hinges upon the fact that the environment of outer space is so different from the environment found on the Earth. The spatial environment is characterized by such factors as extreme low pressures, unfiltered radiation, high-energy radiation from charged particles, micrometeorites, and weightlessness. Chapter 3 presents a more detailed definition of the space-vacuum environment. Since the duplication of this alien environment in a ground-based simulator is impossible, the scientist must select the particular environmental factors to be simulated and the degree to which they are to be simulated must be determined.

Before carrying the discussion further, it is necessary to distinguish between the terms "duplication" and "simulation." The spatial environment cannot be duplicated from the standpoint of gravity, meteorites, electromagnetic radiation or vibration, nor can the thermal environment be duplicated, especially that portion of the environment due to Earth reflections and radiation. In the case of the low-pressure environment, the molecular types, molecular energy, and directional distribution cannot be duplicated. At best, in each of the above categories, only certain effects of the environment can be simulated. As a result, there is a considerable difference between an attempt to duplicate the spatial environ- 
ment and the simulation of the particular effects which are essential to performance or reliability testing. The effects which must be simulated are a function of the test item and the purpose of the test. It is quite possible that these effects will vary between materials testing and system testing.

Despite the engineering limitations, simulation testing must be undertaken to prove engineering designs, to achieve reliability, particularly in those applications involving man, and to obtain new information on basic phenomena and interactions. Space-simulation testing has been applied to such diverse areas as the study of thermal balance and vehicle skin temperature, thermal vacuum life testing, the study of electromagnetic phenomena, arc and glow discharge, systems and components reliability testing, the interaction of system components, the change in surface characteristics, friction, wear and cold welding, material studies, outgassing rates and material integrity, and metallurgical studies, creep and fatigue.

These tests are conducted under space-simulation conditions which vary widely. Test chambers range from very small laboratory-type chambers to system chambers with diameters of over 100 feet. Pressures range from $10^{-2}$ to $10^{-14}$ torr, and the amount of auxiliary simulation equipment varies from none to large complex multienvironment facilities. The question to be asked at this time concerns what vacuum levels are necessary for the various types of simulation.

If the gross pressure effects of outer space are to be simulated, then it is necessary only to reduce the pressure to about 1 percent of atmospheric, or in the range of a few torr. However, at this pressure, the thermal conductivity of the gas is nearly the same as that at ambient pressure. Good simulation of the thermal environment of outer space can be attained if the pressure is reduced to the point where the thermal conduction of the gas is small relative to the radiant heat transfer. The pressure level required in this case depends on the distance between the test item and the cold walls, and also on the temperature of the test item. Test of vehicles operating at normal ambient temperature conditions will require a pressure of about $10^{-5}$ torr. Of course, it is also necessary to supply a source of simulated solar radiation and cold, black walls to simulate the spatial thermal-absorbing capacity which is essential to realistic thermal balance testing. If a well-collimated, uniformly bright sun with a good spectral match is used in conjunction with a cold black adsorbing wall, the surface temperature of the test vehicle in the simulation chamber will be very nearly the same as that which will exist in outer space. Discussions of the limitations and accuracy of this method of directly measuring the skin temperature will be found in many of the cited references. 
The substitution of infrared heating lamps in place of a good sun results in an extreme extrapolation and a rather meaningless test for thermal balance due to the variation in surface absorbtivity with wavelength. The effects of the lack of collimation and uniformity are also disadvantages of this method. It can be seen that infrared suns are best used in those space-simulation applications for which the surface temperature is known, and life or performance tests are to be run at established temperature conditions. Under these conditions, the infrared sun is a direct and inexpensive means to force the skin temperature to the required value. When an infrared sun is used, the cold outer chamber wall is not always a necessary part of the thermal system, but may be required to provide high-speed cryogenic pumping of the outgassing products. An alternate approach to the infrared sun and cold-wall situation uses thermally controlled shrouds to force the predetermined skin temperature of the vehicle. This approach generally is more appropriate to the testing of internal vehicle components.

At a pressure of $10^{-5}$ torr, arc or glow discharge phenomena may still present difficulties. A reduction to a level of about $10^{-6}$ torr pressure permits voltages in the kilovolt region to be used without complieations. The nature of electrical discharge phenomena at low pressures is extremely complex. Each problem requires a separate evaluation and remedy.

Another important consideration is the outgassing of materials in a manner equivalent to that of outer space. There are two significant points to be noted. First, the gas flow in a space-environment simulator is in the free molecular-flow region at pressures in the $10^{-5}$ and $10^{-6}$ torr range. As a result, each gas type acts independently of all others present. The second point is that outgassing is a net process which represents the difference between the gas given off, temperature dependent only, and the gas being collected, pressure dependent. Thus, to attain good outgassing simulation, it is only necessary to reduce the partial pressure of any gas type being evaluated to the point where the gas being taken up again is small relative to the outgassing. The following example illustrates the above discussion.

The effect of an independent gas flow in the molecular-flow region can be seen when the rate of evaporation of a fluid in a spatial environment is measured. If a particular fluid is placed in an ultra-high-vacuum system operating at a very low pressure, say $10^{-10}$ torr or lower, the initial evaporation rate will be essentially equal to the theoretical maximum rate, which would also be observed in space. This rate is due to the clean walls of the vacuum chamber which act as a condensing surface for the molecules leaving the fluid. However, after a period of time, the walls will become contaminated with the fluid and will act as evaporating sources to return a part of the fluid molecules back to the source. There- 
fore, a considerable reduction in the net evaporation rate results. The rate may be reduced by as much as 100:1, depending upon the size and nature of the pumping system. It is obvious that such a reduction may give rise to false conclusions about the evaporating nature of the fluid.

Consider a different experimental setup for which the residual pressure is only $10^{-5}$ torr, but a cold-wall-condensing surface is located directly above the evaporating fluid. At $10^{-5}$ torr, the mean free path of the molecules is approximately $508 \mathrm{~cm}$. As a result, only a very small percent of the evaporating molecules collide in the gas phase on their way to the condensing surface. If the temperature of the condensing surface is well below that of the fluid sample, the evaporation rate from the condensing surface will be very small. relative to the rate from the fluid. Therefore, the number of molecules returned to the fluid will be only a small fraction of the amount evaporated. For this condition, an essentially full evaporation rate will be maintained to simulate the pumping capacity of space.

The outgassing of materials provides a more difficult situation to analyze. The previous discussion of the outgassing of materials indicates that various materials will exhibit many types of outgassing. For example, outgassing which deals with multilayer adsorption, outgassing through pores, or bulk outgassing by diffusion will permit essentially full outgassing if the environmental pressure is only a decade or two below the instantaneous surface equilibrium pressure. Thus, a material which has been in equilibrium with the atmosphere initially will outgas at its full rate at a pressure of only a few torr. As the outgassing continues, the apparent surface equilibrium will continue to drop and the environmental pressure must be reduced to a corresponding degree. In time, a large fraction of the original gas will have been removed from the material such that all the physical aspects of the bulk outgassing will have occurred. That is, consider the case of water-vapor solubility in a polymer, where the equilibrium pressure in the atmosphere may have been about 10 torr. After a prolonged exposure of the polymer in a high vacuum of $10^{-8}$ torr, the equilibrium pressure will be approaching an equivalent pressure of $10^{-6}$ torr. The internal solubility, as a result, will be reduced by over $10^{6}$ to 1 . In general, the known physical effects of outgassing on the properties of embrittlement, insulation resistance, and shear strength will have occurred long before this time. It is difficult to justify the need for ultrahigh vacuums in the study of bulk-outgassing phenomena.

Another situation concerns the effect of surface outgassing from the last monolayer of adsorbed or chemisorbed gas. Since this gas is very strongly bound to the surface, the equilibrium condition at a residual pressure of $10^{-6}$ torr is generally a complete molecular layer of gas coverage. Due to the fact that clean surfaces can be maintained only 
at extremely low pressures, those physical phenomena dealing with clean surfaces, such as dry bearings or cold welding, must be investigated at ultra-high-vacuum conditions. Two alternate approaches are available. It is possible to obtain a clean surface when the vacuum level is sufficiently low so that the equilibrium condition is a small part of a monolayer. Although this procedure requires an extremely high vacuum, it allows for infinitely long test periods. The second approach is to obtain a clean surface in a vacuum environment which is low enough to allow the performance of the test before the surface forms a contaminating layer. At a pressure of $10^{-10}$ torr, several hours are required to form a monolayer when it is assumed that all gas molecules will stick upon collision with the surface.

The attainment of an initially clean surface is an important consideration. The removal of the last layer of contaminating gas from a surface in a reasonable period of time requires the addition of energy to the molecules on the surface. In space, this energy is provided by the bombardment of the surface by high-energy protons and electrons, and in part from the high relative velocity of the space vehicle itself. In a space-simulation chamber, some equivalent energy source must be used. In some instances the nature of the test item will allow the performance of a high-temperature bakeout to provide the initially clean surface. Other methods of providing the clean surface include glow discharge, electron bombardment, or ion bombardment. Specific component tests such as those on gears or bearings frequently require extremes in ultrahigh-vacuum conditions. These experiments generally are performed in small research vacuum chambers where pressures of $10^{-10}$ to $10^{-14}$ torr can be obtained. At the present time, it is not possible to duplicate these pressure levels for large-scale system testing. Although the problems inherent to this application could be solved, the question of economic feasibility versus justifiable applicability requires careful consideration. Large facilities now in operation simulate pressures in the range of $10^{-5}$ to $10^{-\theta}$ torr which are obtained by the use of high-speed cryogenic pumping, particularly liquid nitrogen panels for the condensation of water vapor. The actual operating pressure for any given test is dependent primarily on the outgassing characteristics of the test item. The pressure level indicated above provides for free molecular flow, complete outgassing of the bulk gas, adequate reduction of the gas thermal conductivity, and relative freedom from electrical discharge phenomena, all at an economical cost relative to the attainment of an ultrahigh vacuum in large baked-out chambers.

In all space-simulation experiments, a careful distinction must be made between the chamber ultimate pressure and the chamber operating pressure. The ultimate pressure of a clean, dry, and empty chamber represents the limit of pressure which can be obtained. Since the test 
item generally liberates far more gas than the test chamber, the actual operating pressure during a test is considerably higher than the chamber ultimate pressure. Therefore, the pumping speed of a particular chamber usually is more important than its ultimate pressure. All too frequently it happens that chambers with ultimate pressure capabilities of $10^{-10}$ torr are forced to operate in the $10^{-5}$ to $10^{-6}$ torr range due to an insufficient pumping speed for the gas load originating from the test item. For example, at a pressure of $10^{-8}$ torr a system speed of 1000 liter/sec can handle a gas load of only $10^{-6}$ torr liter/sec. This gas load is equal to the initial outgassing rate of 1 square centimeter of an insulating- or polymeric-type material.

A widely held concept of space-simulation conditions for nonbakable test items calls for the use of high-speed cryogenic pumps, and accepts operating pressures in the range of $10^{-5}$ to $10^{-7}$ torr. Such nonbakable chambers will readily obtain ultimate pressures in the $10^{-9}$ torr range. Bakeable ultra-high-vacuum chambers, due to economic and engineering considerations, are used primarily for research on materials or components rather than for life or reliability testing of systems or subsystems.

A common fallacy in space-simulation technology is the attempt to correlate the system ultimate pressure or the system operating pressure with a given altitude. While it is true that an increase in altitude brings about a corresponding decrease in the pressure, there is an additional change in both the composition of the atmosphere and the energy of the molecules. At high altitudes, the atmosphere is composed primarily of ions of hydrogen and helium. The residual gas is not random, and the relative velocity of the spaceship results in a different collision frequency on the leading edge than on the trailing edge. In addition, extremely high-energy particles from the solar corona will be present. Duplication of high altitudes requires that all the molecules leaving the test item be captured. Also, molecules of the gases existing at particular altitudes and possessing approximate energies must be added. This addition must take into account the relative velocity of the vehicle, as well as the high-energy particles which exist at the simulated altitudes.

In a typical chamber, the residual gas may be nitrogen, oxygen, water vapor, or hydrogen, depending upon the nature of the test vehicle and chamber. The gas will be randomly moving in the chamber at average temperatures which will depend on the temperature of the chamber walls. Ions do not exist, nor are there high-energy particles or the relative directional effects due to the vehicle velocity. Frequently, there is a different directional effect which is characteristic of the chamber but not of space. Such an effect occurs for condensable gases such as water vapor when discontinuities exist in the chamber pumping system. A typical discontinuity is the presence of a nonpumping solar simulator of large area in a chamber containing liquid-nitrogen-cooled walls. The 
reflections of the water-vapor molecules from the solar simulator yields a higher gas flux on the vehicle side facing the sun than on the side facing away from the sun. Calculations of this effect indicate that the ratio of the molecular fluxes at these two points may vary from 10:1 to over $1000: 1$. Thus, it is very difficult to determine what altitude has been simulated.

A pressure of $10^{-5}$ torr may simulate adequately the effect of negligible heat transfer by the gaseous conduction of an outer space vacuum of $10^{-16}$ torr. Conversely, a pressure of $10^{-12}$ torr does not begin to simulate the molecular flux of outer space at any defined altitude. The practice of rating a chamber as a simulator of a given altitude, based only on its ultimate pressure or even its operating pressure, is meaningless. An exception occurs for low-altitude chambers operating in the 1- to 100-torr region where the direct physical effects of pressure as a force are important. A more meaningful rating involves a statement of the vehicle size, and the gas load which can be handled and still maintain certain simulation characteristics of outer space. In conclusion then, a space-simulation chamber simulates particular aspects of phenomena of outer space without regard to altitude; it does not simulate any particular altitude. There is no meaningful relationship between altitude and chamber pressure.

\section{TYPES OF VACUUM SYSTEMS}

The type of vacuum system required for space simulation, in terms of size, pressure, and speed, depends upon the design aims. It must be realized that a universal vacuum system which meets the requirement of every situation does not exist. Therefore, a good design for one application may be a very poor design for another application. When it is desired to obtain a high vacuum, good vacuum design practice calls for a minimum quantity of $O$-rings and valves. However, these components are desirable features when a high degree of flexibility is required and a poorer vacuum level can be tolerated. The following sections discuss the types of vacuum systems which are available and, in addition, discuss relative merits, advantages and disadvantages of each type.

\section{Mechanical Pumping Systems}

The simplest vacuum system consists of a mechanical pump, a volume, an interconnecting tube, a vacuum gage, and a method for gas or air admission. Such a system is illustrated in figure 10.1. The vacuum gage generally used in this application is a thermocouple, Pirani, Alphatron, or McLeod gage. A single-stage mechanical pump is sufficient for vacuums down to $10^{-1}$ torr, while a two-stage mechanical pump is needed for $10^{-2}$ to $10^{-1}$ torr. The mechanical pumping system is not recom- 


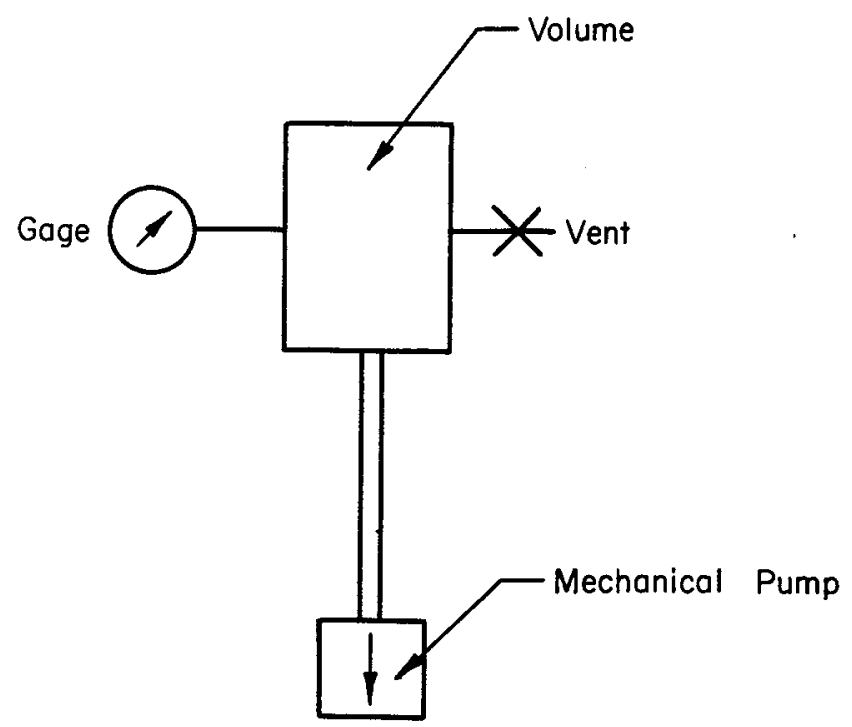

Figure 10.1.-Simplest vacuum system consisting of a mechanical pump, a volume, an interconnecting tube, a vacuum gage, and a method for gas or air admission.

mended for pressures below $10^{-2}$ torr. The system is inexpensive for low pumping speeds; however, different system types are more economical for very high pumping speeds.

There are two main problems inherent in this simple system: it is possible for the pump oil to contaminate the volume, while the volume outgassing may contaminate the pump. When water vapor is the predominant gas at the end of exhaust, a gas-ballast type of pump should be used. Unless the ballast gas is turned off at the end of exhaust, the ultimate pressure will increase. Gas ballast, however, will not prevent system contamination from the pump oil.

A trap, either a chemical or refrigeration type, should be placed between the system and the pump to provide protection for the system from the pump, or vice versa (fig. 10.2). Due to the fact that chemical traps are difficult to clean, they are seldom used. Refrigerated traps generally are cooled by means of a mechanical refrigerator, Dry Ice, or liquid nitrogen. A two-stage mechanical pump may be used for applications as low as $10^{-3}$ torr if a trap is added. There is little advantage in using liquid nitrogen in mechanical pumping systems, and it may be difficult to justify the expense. One disadvantage in the use of the trap is that whenever the system is open to air, moisture will condense on the inside of the trap. Usually, a valve is added between the trap and the volume to prevent this condensation. However, the valve is an addi- 


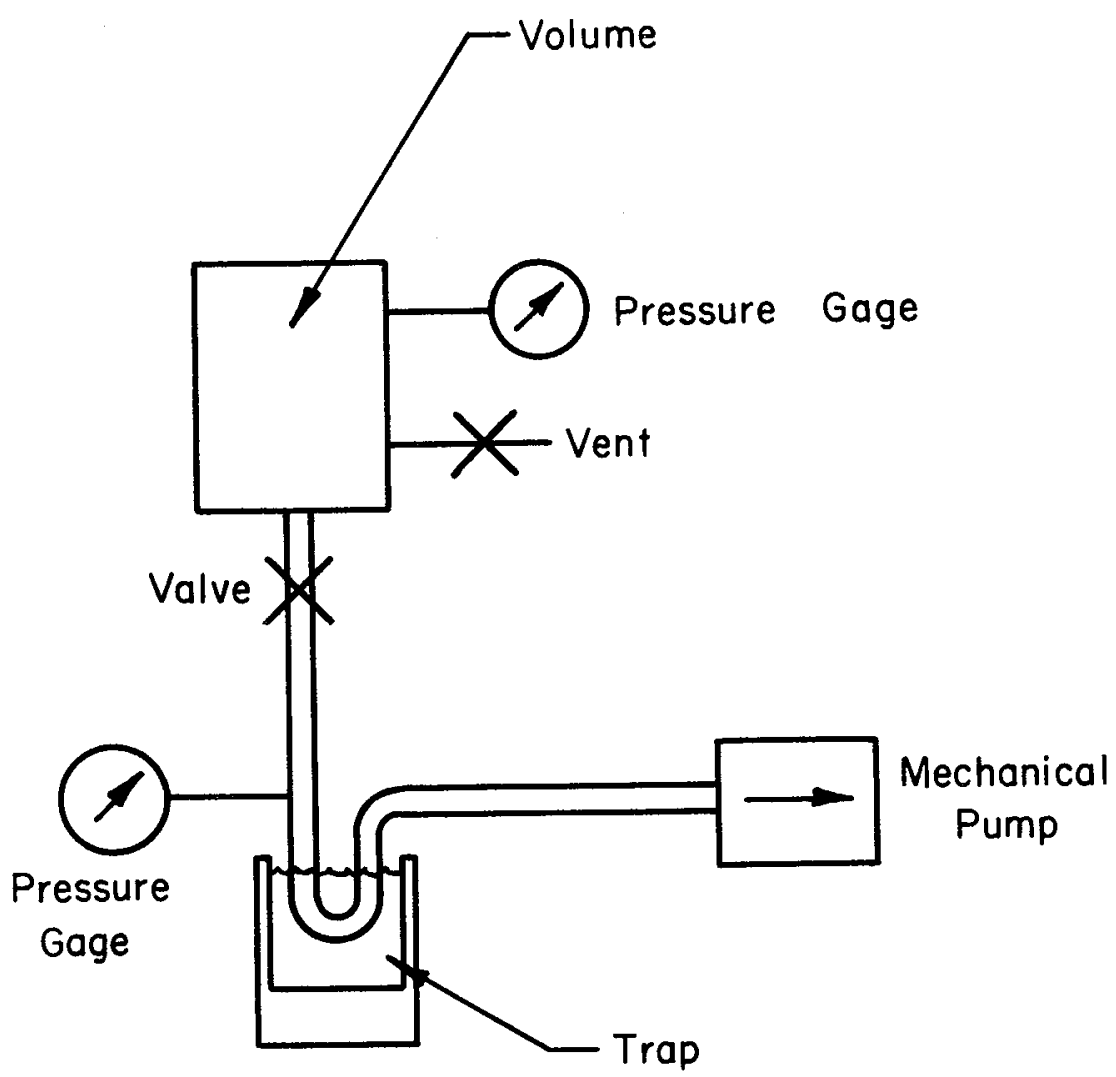

FIGURE 10.2.-Simple mechanical pumping system with a trap inserted between the volume and the pump to prevent either the pump oil from contaminating the volume or the volume outgassing from contaminating the pump.

tional potential leak, and it provides more material to act as outgassing sources. While two vacuum gages can be used, if only one gage is available it should be placed on the volume side of the valve so that it can be used to check the rate of rise of the pressure in the volume. The second gage provides a ready means of checking the ultimate pressure of the pumping system with the volume shut off. In some applications, it may be desirable to place a gage between the pump and the trap to determine if the gas is either a permanent or condensable type. It is important that the gage readings for this type of system be interpreted carefully, as there is a different pumping speed present for the permanent gases and for the condensable gases. The question of the interpretation of the gage readings becomes more complicated when the pressure is in the transition or viscous flow range. When the end of the exhaust process approaches, it is not uncommon for the gage between the pump 
and trap to read the highest pressure, due to the fact that it sees part of the decomposition products of the pump oil. When higher speeds or lower pressures are required, it is necessary to add different pump types to the system.

\section{Booster Pumping System}

A booster pump is used primarily to provide high-speed, economical systems in the pressure range above $10^{-3}$ torr (fig. 10.3). Two types of

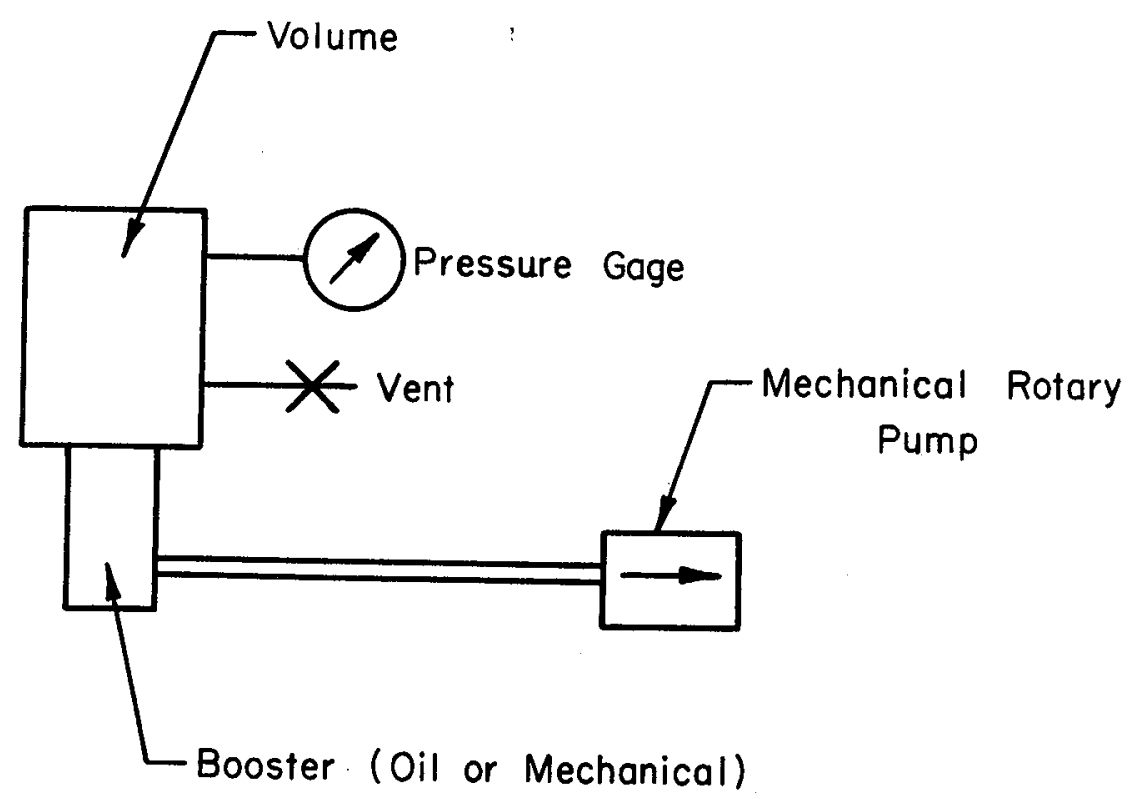

Figure 10.3.-Simple booster pumping system consisting of an oil or mechanical booster pump, a backing rotary pump, a volume, an interconnecting line, a vacuum gage, and some method of opening the system to air, used to attain higher vacuums.

boosters are available: the mechanical and the vapor. Both types possess ultimate vacuums below $10^{-4}$ torr. Below $10^{-3}$ torr, however, the speed begins to fall off appreciably. Their practical operating range, therefore, is considered to be from $10^{-3}$ torr up. The basic differences between the two types of booster pumps are cost and the high-pressure operating limit. While the mechanical booster is priced considerably higher than an oil booster pump of an equivalent size, the mechanical booster has a much better operating range. It will pump at pressures as high as 15 torr. The vapor booster, however, is limited to a pressure of about $10^{-1}$ torr if it is to operate in its high-speed range.

Booster pump systems are used for the exhaust of large volumes, for processes which require a high outgassing rate to be handled at relatively 
low pressures, or for processes requiring a vacuum better than that which can be obtained from a mechanical pumping system. Both pump types will transfer some degree of hydrocarbon or mercury contamination to the volume being exhausted unless they are isolated with a trap. The booster pumps must be backed with a mechanical oil-sealed rotary pump which has a speed about one-tenth that of the boosters. The minimal requirements of systems discussed previously indicate that it is necessary to turn the pump off prior to opening the system to air to allow the oil to cool. This is true if an oil-booster pump is being used. In a similar fashion, the pump must be turned on again when the exhaust commences. As a result, time will be lost while the oil is reheating to the operating temperature. Two solutions are available to overcome the disadvantages of waiting for the pump to cool and rewarm. These are: a more expensive mechanical booster can be used, or a valving system which will allow the pump to remain hot at all times can be added.

Three different methods which are discussed in the references can be used to achieve the latter solution. For example, a rotary pump may be used for both exhaust and for backing the booster pump. However, when the volume is being exhausted, the pressure on the booster exhaust may rise to the point that the pump breaks down. This problem can be minimized if a ballast volume is added between the booster pump and its backing valve. Another arrangement uses two mechanical pumps, each with its own valve. Although this system is quite effective, it is expensive to use two large mechanical pumps for large volumes. An alternate solution uses one large mechanical pump both for the exhaust and for backing the booster pump. During exhaust, the booster pump is handling a very small gas load and can be backed by a small holding pump. Occasionally, a booster pump is called for in an application in which it is necessary to minimize hydrocarbon contamination. Therefore, a trap may be added between the booster pump and the valve.

The only gage necessary on simple vacuum systems is the one located on the volume. However, to establish that the system is performing properly, other gages placed on the system to act as monitors are desirable. In addition, these gages may be used as a method of troubleshooting in the event that difficulties develop. The gage between the booster pump and the high-vacuum valve enables the ultimate pressure of the booster pump to be tested, while the gage located between the two pumps indicates if the rotary pump is performing properly. Although the preferred type of gage for the above applications is one of the thermal conductivity types, such as the Pirani or the thermocouple vacuum gage, an Alphatron or a McLeod gage may be used. The gage in the volume will depend on the particular application involved, and the pressure range at which the system is operating. 


\section{HIGH-VACUUM SYSTEMS-GENERAL CONSIDERATIONS}

Systems with pressures much below $10^{-3}$ torr and definitely below $10^{-4}$ torr require some form of high-vacuum pump. The three principal forms of pumps are the oil or mercury diffusion pump, the getter ion or sputter ion pump, and the molecular or molecular drag pump. Chapter 6 discusses in detail the pump proper. All three pump types may be used as the principal pump on systems which operate in the range from $10^{-3}$ torr to less than $10^{-12}$ torr. The ultimate pressure in a given application generally depends on system design factors such as material selection, the use of auxiliary pumping and trapping mechanisms, and whether or not a system bakeout is used. The basic vacuum equation defining the operating pressure of a complete vacuum system is

$$
P=P_{0}+\sum_{i=1}^{i=n} \frac{Q_{i}}{S_{i}}
$$

where

$P_{0}$ base pressure of the pump proper under a no-load condition, torr

$Q$ system gas load, torr liter/sec

$S$ net system pumping speed for a particular $Q$, liter/sec

Generally, both $Q$ and $S$ are summations of various gas types which must be treated independently.

Since all pumps are gas sources to some degree, even when used in an application where there is no gas load, there will be a lower pressure limit $P_{\mathbf{0}}$ which the pump system can obtain. Chapter 6 discusses this situation. Table 10.1 summarizes estimated practical values of $P_{0}$ for different high vacuum pumping systems.

TABLe 10.1.-Typical Values of Estimated Pumping System Base Pressure P。

\begin{tabular}{|l|l|}
\hline \multicolumn{1}{|c|}{ Pump type } & \multicolumn{1}{|c|}{ Pressure range, torr } \\
\hline Molecular pump & $10^{-10}$ \\
Diffusion pump - & $10^{-7}$ to $10^{-10}$ (depends on pump oil) \\
Diffusion pump, plus trap & $10^{-11}$ \\
$\begin{array}{l}\text { Sputter ion pump - } \\
\text { Sputter ion pump, plus sublimation } \\
\text { Any of the above, plus cryogenics } \\
\text { (less than } 20^{\circ} \mathrm{K} \text { ) }\end{array}$ & $10^{-10}$ \\
\hline
\end{tabular}

In each instance, the base pressure is the equilibrium point between the pump gas source and its pumping speed for the particular gas. Lower pressures of $P_{0}$ have been reported in specific experimental research applications. An actual vacuum system seldom operates at the above pressures due to the various forms of outgassing. 
It is more helpful to examine high- and ultra-high-vacuum systems from the viewpoint of the type of gas loads present rather than the type of pump employed. The diffusion pump will be used as the representative pump in the following discussions, since it is the most widely used pump for vacuum system applications. Essentially, all the factors to be discussed apply equally well to the two other pump types. In those instances where pertinent performance differences exist, a clarification or comparison will be given. There are also differences in the initial cost, operating cost, maintenance procedure, life, and ease of operation between the various pump types. These differences generally are the governing factors in pump selection. The discussion of a particular limiting pump pressure and the specific capability of the pump for handling the system gas load is relatively independent of pump type. Due to the fact that outgassing, which is temperature dependent, is the principal limitation of the ultimate system pressure, the following discussion of high-vacuum-system types will be divided into two separate sections covering nonbaked high- and very-high-vacuum systems, and bakable very-high and ultra-high-vacuum systems. Nonbaked vacuum systems operate in the pressure range from $10^{-3}$ to $10^{-9}$ torr, while the baked systems operate in the range from $10^{-9}$ to $10^{-14}$ torr.

\section{Nonbaked High- and Very-High-Vacuum Systems}

The simplest form of diffusion pumping system involves a diffusion pump, its backing rotary pump, the volume being exhausted, an interconnecting line, a vacuum gage, and some method of opening the system to air. This system is the same as that shown in figure 10.3 for the case of booster pumps. Since there are problems in cooling and heating the pump, a high-vacuum valve and bypass line may be added (fig. 10.4). Unless the exhaust is quite rapid, or the pump can be exposed to high pressures while hot, some means of holding the foreline pressure must be provided. To hold the diffusion pump, a holding volume may be used while a set of valves transfers the rotary pump to the volume for initial exhaust. This method is applicable only for fast cycles or very clean systems. Otherwise, two rotary pumps connected in the two different ways shown on figures 10.5 and 10.6 are required. In one instance, a completely separate mechanical pump is used for exhaust and is sized on the basis of the required exhaust time. In the other case, the same rotary pump is used both for exhaust and for backing the diffusion pump, but a smaller rotary pump is used as the holding pump for the diffusion pump during the time the regular rotary pump is being used for exhaust. Normally, the former of these two systems is preferred, but for very large systems the latter method is more economical.

The selection of the number of gages to be used on the vacuum system depends on the economics of initial cost versus the cost of trouble- 


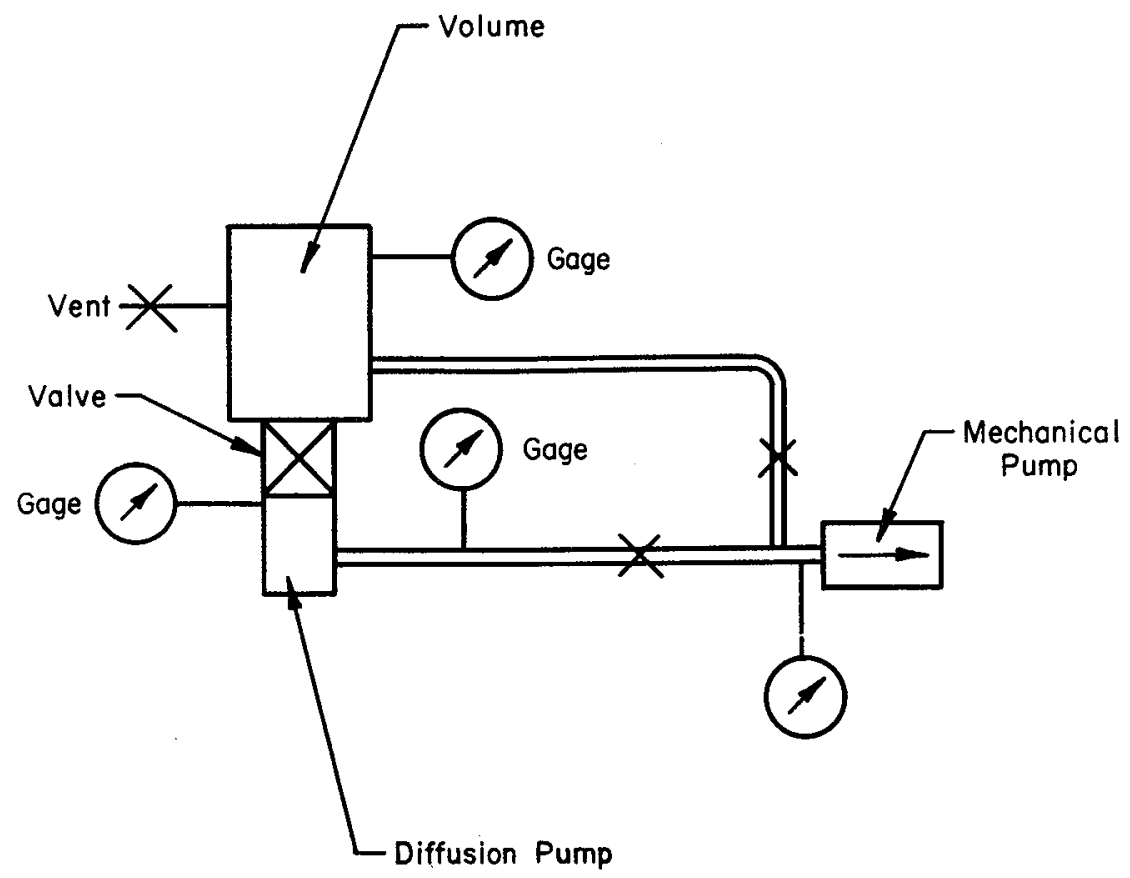

FIGURE 10.4.-Pumping system with a high-vacuum valve and bypass line added to facilitate rapid cycling.

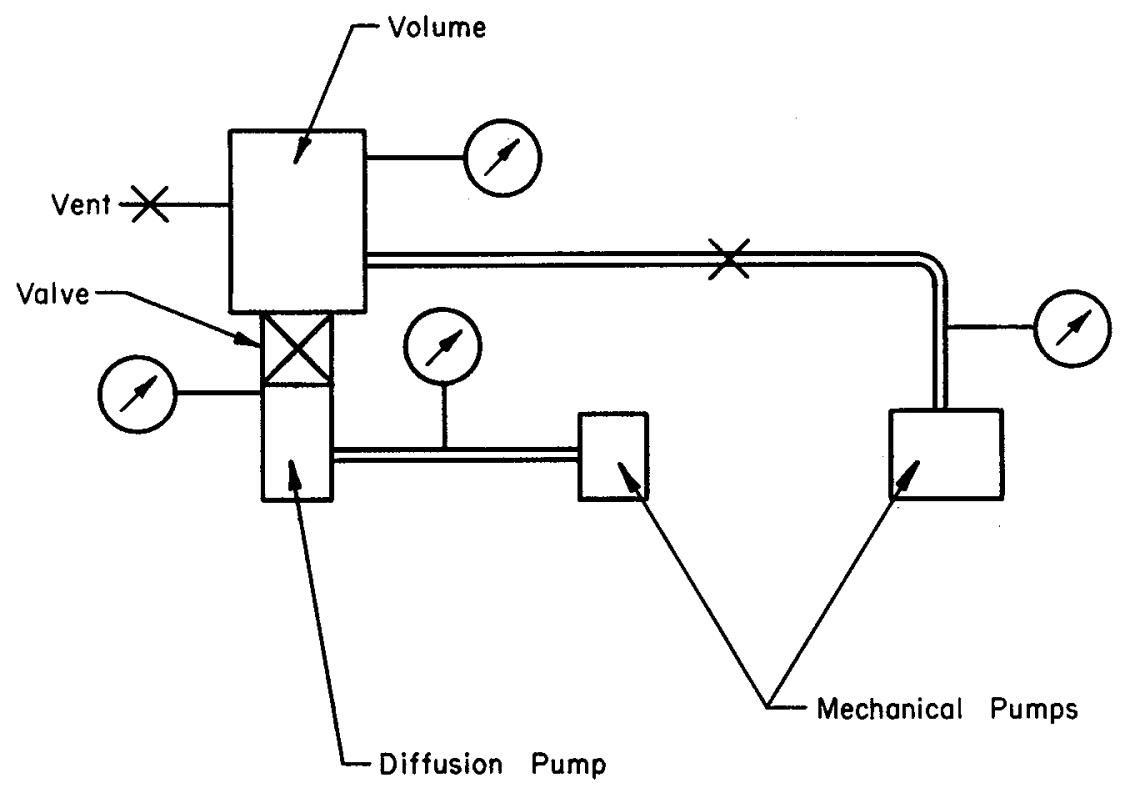

FiguRE 10.5.-Pumping system where a completely separate mechanical pump is added for exhaust. 


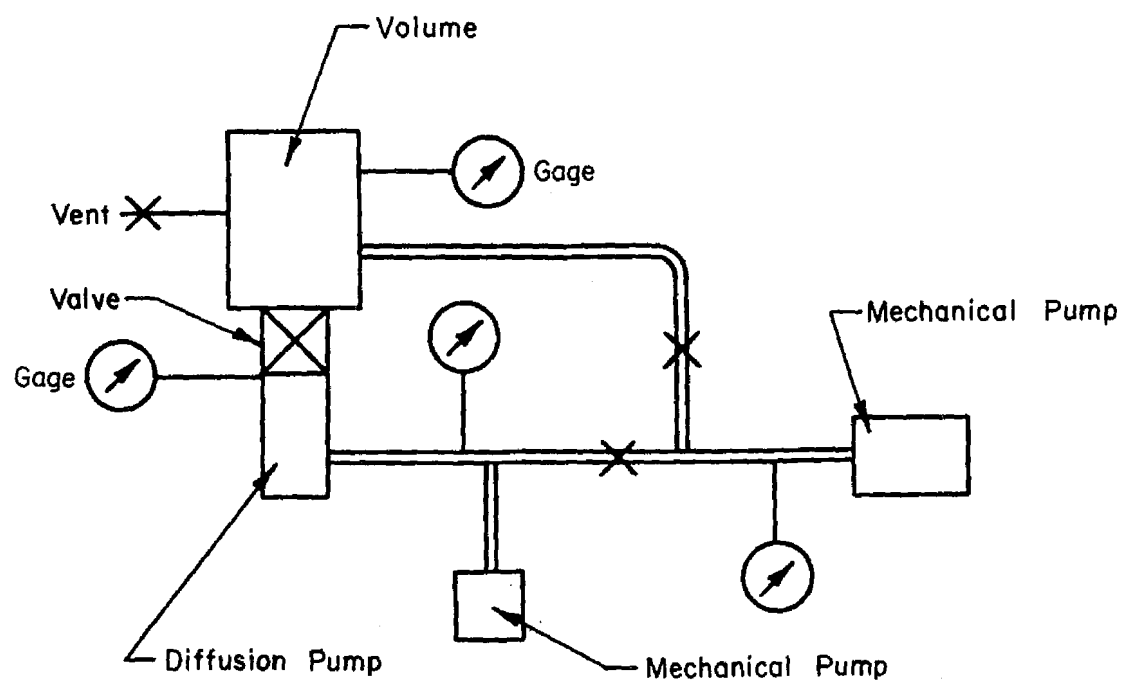

Ficure 10.6.-Pumping system in which the same rotary pump is used for both exhaust and backing the diffusion pump, but a smaller pump is used as a holding pump for the diffusion pump while the regular rotary pump is being used for exhaust.

shooting. Generally, a high-vacuum gage is required on the volume being exhausted. The addition of another high-vacuum gage between the pump and the valve is advantageous when the system performance is being checked, and when leak testing or troubleshooting the system. In a similar fashion, the gage located between the two pumps is also advantageous. The high-vacuum gages are usually of the ionization type, either hot cathode or cold cathode, while the gage in the roughing or foreline is a Pirani or thermocouple vacuum gage.

When very large systems are being considered, a suitable mechanical rotary pump may be excessively expensive or even unobtainable. Therefore, a booster pumping system frequently is used to replace the mechanical rotary pump. A number of different booster arrangements are possible. Figure 10.7 illustrates a system which contains a diffusion pump backed by a booster pump which in turn is backed by a mechanical rotary pump. Any of the pumps may be used to exhaust the volume to its optimum pressure region if the valves are properly operated. At all times, the vapor pumps are on and are protected by the small rotary holding pumps. Since this system is obviously complex, the valving should be interlocked, or the operators well trained to prevent an improper operating sequence.

Nontrapped vacuum systems such as those described above are capable of maintaining pressures between $10^{-5}$ torr and $10^{-7}$ torr, depending on the pumping capacity, the type of oil, the chamber size and material of construction, the test load, and various other design details. The principal pressure limitations are water vapor from outgassing, and pump 


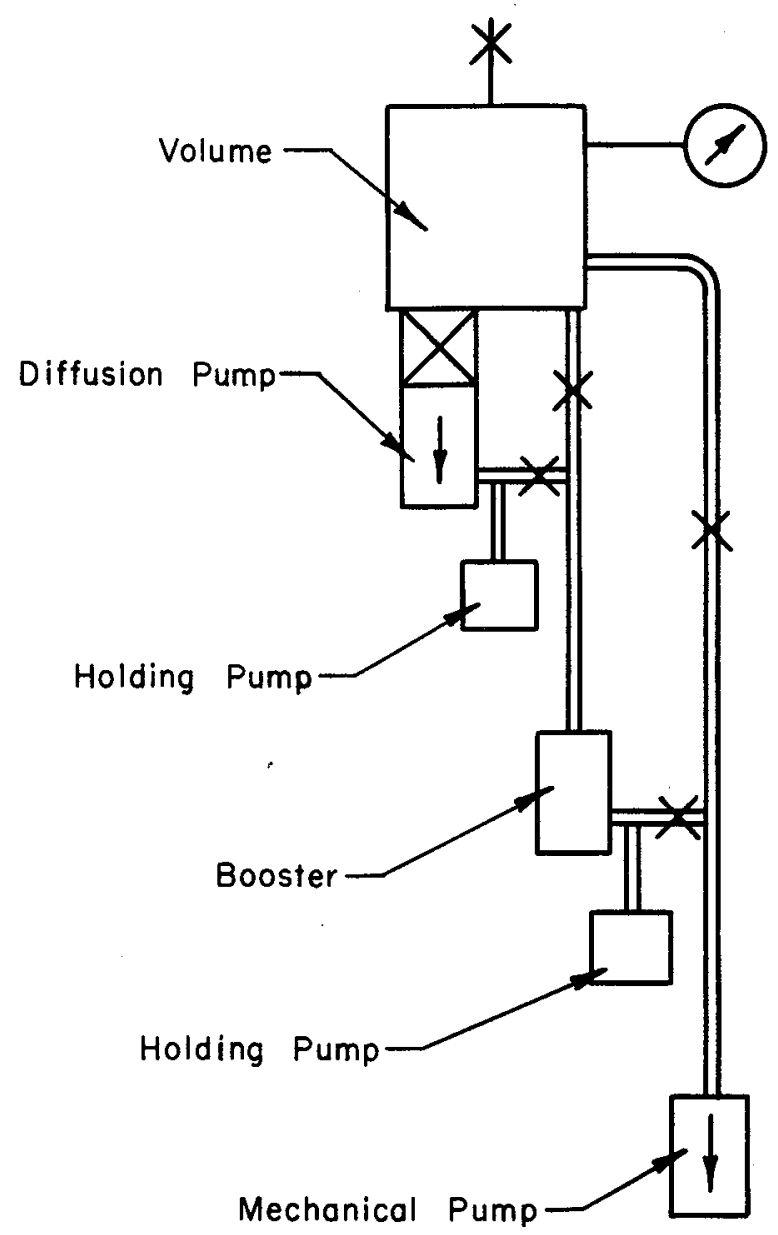

Figure 10.7.--Pumping system, containing holding pumps, in which a diffusion pump is backed by a booster pump which in turn is backed by a mechanical rotary pump. Any of these pumps may be used to exhaust the volume in its optimum pressure region.

oil or cracked oil products from the diffusion pump. Since the two subjects of water-vapor outgassing and hydrocarbon contamination are so pertinent to the further development of high-vacuum systems, the next sections present a detailed discussion of these phenomena.

The surfaces of metals may contain hundreds of molecular layers of water vapor trapped in the surface oxides and pores. The amount of this contamination will vary greatly with the surface characteristics and the amount of treatment accorded to the surface. Well cleaned and polished stainless-steel wall construction is preferred and generally will 
have a gas load 10 times lower than less well-designed systems. The outgassing of the various polymeric-type materials used as seals is frequently a larger gas load than the much larger area of the metal surface. All organic-type sealing materials have a high solubility for water. This water is released when the material is exposed to a vacuum. After prolonged pumping, an $O$-ring which separates the vacuum from atmospheric pressure will contain a diffusion gradient which allows water and other atmospheric gases to permeate the sealing member. This permeation is principally of water vapor and is frequently the major gas load on a vacuum system. The temperature and humidity dependency of permeation frequently accounts for the daily variations in ultimate pressure which are observed for vacuum systems. The permeation gas source may be reduced or even eliminated if the seals are cooled, or by the use of two seals and a guard vacuum, or if all metal seals are used in place of organic seals. In addition to water, most organic materials also contain various solvents, binders, and surface lubricating oils which become further gas loads on the system and are sources of hydrocarbon contamination. These sources may be removed by a vacuum bakeout of the organic seal prior to use.

To overcome the problems of water-vapor outgassing and hydrocarbon contamination, a liquid nitrogen trap can be used between the diffusion pump and the vacuum chamber. If the liquid nitrogen trap has a large area, and is closely coupled to the chamber, the increase in pumping speed for water vapor and hydrocarbons may be of the order of 10 to 1 . In addition, the trap will drastically reduce the amount of diffusion pump backstreaming. In nonbaked systems, where many other hydrocarbon sources exist, almost any optically tight liquid nitrogen trap will keep the diffusion pump oil to a negligible fraction of the total hydrocarbon contamination. Ultraclean bakable chambers demand that particular attention be paid to the proper selection or design of liquid nitrogen traps to assure complete oil stoppage. Molecular pumps or sputter ion pumps on nonbaked systems generally experience the same amount of hydrocarbon contamination from the chamber and its components. One of the principal hydrocarbon sources, that due to the mechanical pump during the initial exhaust, can be eliminated almost entirely by proper operation of the system. The discussion on operation will continue the examination of these considerations. However, at this time it should be stated that untrapped mechanical pumps must not be allowed to pump on a system below a pressure of about $10^{-1}$ torr. The common practice of pumping to a few microns prior to opening into the diffusion pump probably is the principal cause of hydrocarbons in vacuum systems.

A well-trapped, valveless, nonbaked vacuum system using properly designed seals for minimum outgassing and permeation should be able 


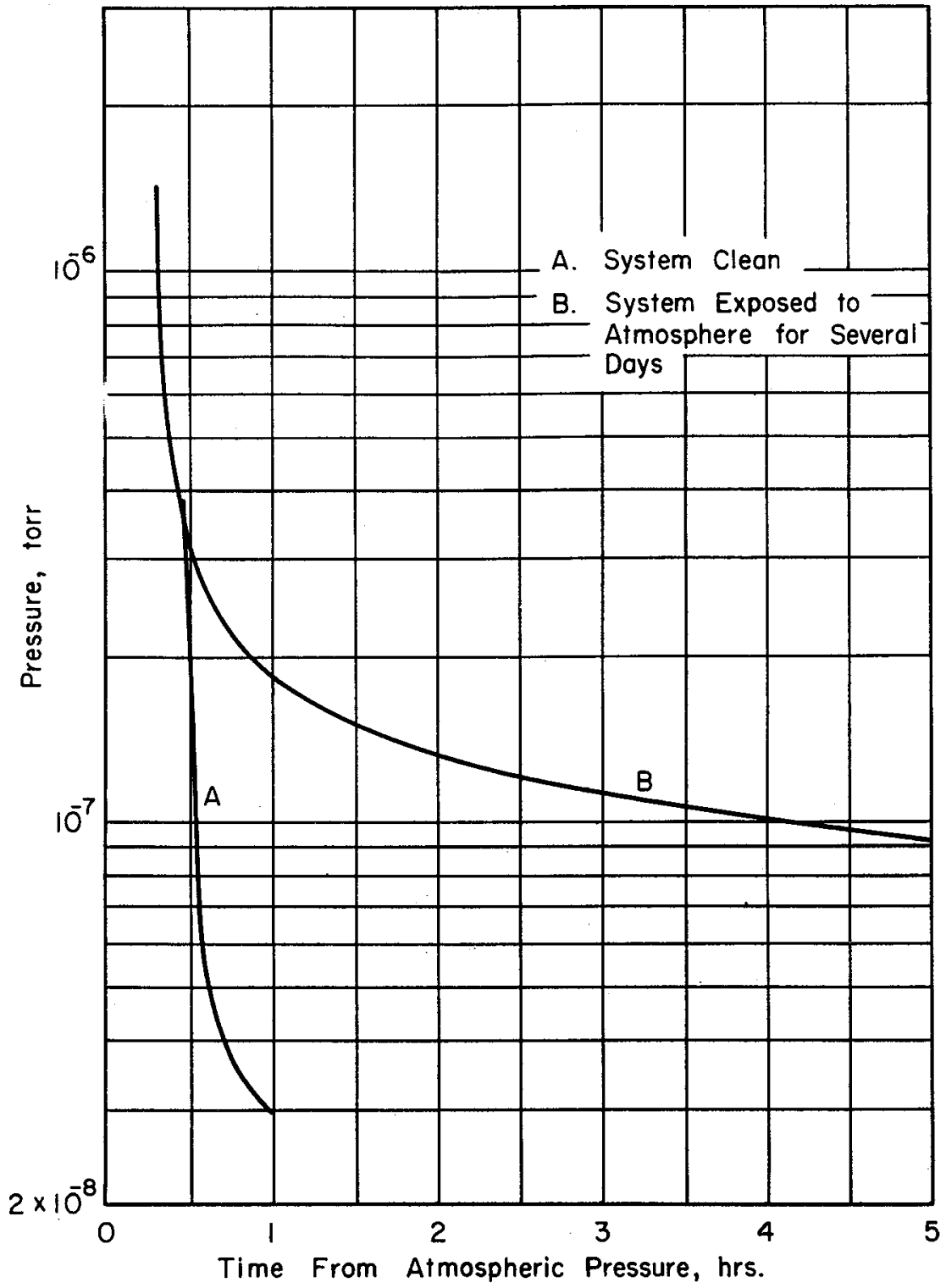

Figure 10.8.-Typical pumpdown of an 18-inch-diameter chamber with a 6-inch liquidnitrogen-trapped diffusion pump. Two cases are illustrated: one treats a clean system and the other treats a system which has been exposed to the atmosphere for several days.

to pump to the low $10^{-7}$ torr range in a very short time, and should have an ultimate system pressure of less than $1 \times 10^{-8}$ torr. A typical pumpdown of an actual system is shown in figure 10.8. The system illustrated 
consists of an 18-inch-diameter chamber, 24 inches high, with a double O-ring main seal and several small single O-ring sealed ports. The pumping system is a 6-inch liquid-nitrogen-trapped diffusion pump. At the start of pumpdown, the diffusion pump is at ambient temperature. It should be noted that the pumpdown time of curve $\mathrm{A}$ in figure 10.8 is considerably shorter than that of curve $B$ due to the fact that for curve A the system had been purged for several days with a dry nitrogen purge at about 0.5 torr and then vented to 1 atm before pumpdown. For curve B, the system had been exposed to the atmosphere for several days and then pumped down from air.

A high-vacuum valve has an important effect on high-vacuum systems. For nontrapped pumps, or for systems which are capable of operating in the $10^{-5}$ torr range, there are no serious problems. In the case of high-vacuum systems operating in the $10^{-7}$ or $10^{-8}$ torr range, the valve introduces two problems. One is that the valve acts as a restriction between the trap and the chamber, and thus reduces the pumping speed for water vapor by threefold to tenfold. The second problem is that most valves are extreme gas sources. That is, the combination of cast surfaces, multiple $\mathrm{O}$-rings, and lubrication for the valve parts frequently results in a gas load which is greater than that of the rest of the vacuum system combined. The addition of a high-vacuum valve may well result in a tenfold increase in system operating and ultimate pressure. However, this increase must be accepted in those instances where a rapid operating cycle is required. Since very few applications in space simulation are of the rapid-operating-cycle type, the higher vacuum, valveless systems are preferred.

One of the most important improvements which can be made in the valveless nonbaked vacuum system, whether the system uses diffusion, sputter ion, or molecular pumps, is the replacement of all organic seals with metal seals. Generally, this simple action will reduce the system ultimate pressure from the high $10^{-9}$ range to the high $10^{-10}$ range, even though the system has never undergone a bakeout. When organics must be used on a vacuum system, it is advisable to give them a vacuum bakeout prior to use to remove the various hydrocarbon impurities in solution. The seal may be used dry if the metal surface has a good finish. Any lubricant is a contaminant, and if a lubricant is required, it should be carefully selected.

In many space-simulation applications, a well-designed high-vacuum system which has a reasonable operating pressure in the $10^{-8}$ torr range is not sufficient, since extremely high outgassing materials or components must be tested. The subsequent high outgassing load could result in system operating pressures above $10^{-4}$ torr. Most of the outgassing from materials is water vapor. Therefore, if a liquid nitrogen shroud is placed inside the main chamber, it will yield a large pumping speed for the 
condensable gases. The net result is that high outgassing rate test items can be maintained at very low pressures. The liquid nitrogen shroud also serves to reduce the normal chamber ultimate pressure due to its high local pumping speed for the outgassing products from the chamber and its seals.

In some instances, the outgassing rate of the test item is not due to water vapor and the maintenance of a low operating pressure still requires a high pumping speed. This occurs commonly when the test item is a sealed container which possesses a leak. If the leak is quite large; the pressure inside the test item reduces rapidly and eventually is limited by the internal outgassing of the test item and the pumping speed of the leak. The main chamber pressure is controlled by both the internal and external outgassing sources. If the leak is quite small, it may represent a negligible load on the pumping system such that the internal vehicle pressure hardly changes in value from the initial loading pressure. Between these two extremes, there are leak sizes which produce a very large gas load for prolonged periods of time. To obtain the extremely high pumping speed which is required to handle a noncondensable gas load, it is necessary to use low-temperature cryogenic pumping systems (chapter 7). The addition of a hidden-panel cryogenic pump to a nonbaked vacuum chamber has little effect on the system ultimate pressure. The principal function of this pump is to provide the. high speed required for particular tests.

Vacuum systems used in space simulation range in size from a few feet to over 100 feet in diameter. When the vacuum systems are leaktight, they have demonstrated ultimate pressures in the ranges previously indicated. Leakage, particularly in cryogenic fluid transfer lines, frequently is the principal limitation on system ultimate pressures. In small chambers, the leaks may be found and repaired readily. However, it is more difficult to both leak-check and repair larger chambers. The initial design of these systems should incorporate leak-detection capability. Chapter 11 will examine the problems and approaches to leak detection.

\section{Baked Very-High and Ultra-High-Vacuum Systems}

While the foregoing nonbaked, valveless systems with supplementary cryogenic pumping have become the workhorses of the space-simulation industry, numerous applications exist where better vacuum levels are required. Most of these applications deal with some form of surface phenomena such as bearing lubrication and wear or cold welding. To obtain the lower pressures required, two alternate approaches are avail- 
able. The entire vacuum system may be cooled to low temperatures to reduce the outgassing of all the sources, or the entire vacuum system may be subjected to a bakeout. In both approaches, the important factor which permits a pressure reduction is the extreme temperature dependency of all forms of outgassing.

A theoretical consideration of the temperature dependency of outgassing for various activation energies indicates that for typical gas sources, a twofold reduction in the absolute temperature will reduce the outgassing rate by approximately seven decades. There are a number of ways in which this relationship can be applied to vacuum systems. For example, if a system is to operate at a normal ambient temperature of $300^{\circ} \mathrm{K}$, and it is baked at $600^{\circ} \mathrm{K}$ until the pressure falls to $1 \times 10^{-7}$ torr followed by a cooldown to ambient temperature, the pressure should fall to the $10^{-14}$ torr range. In a similar manner, if the system is pumped to a pressure of $1 \times 10^{-7}$ torr at $300^{\circ} \mathrm{K}$ and then cooled to $150^{\circ} \mathrm{K}$, the pressure should fall approximately the same seven decades. However, in normal applications, such extensive pressure ratios are not obtained. The subsequent discussion will point out two limiting factors which prevent the attainment of the theoretical pressures, and it will indicate what can be done to overcome the limitations so that the ultimate in clean, ultrahigh vacuum can be obtained.

The first factor to be considered is that of transient adsorptiondesorption reactions. This particular problem occurs when different parts of the vacuum system are subjected to different temperature histories. If it is assumed that 99 percent of the system is subjected to a bakeout, it is immediately apparent that while 99 percent of the system undergoes a phenomenal reduction in outgassing, the remaining 1 percent is unaffected. As a result, the net system outgassing is reduced only by a factor of 100 rather than the desired factor of $10^{7}$. The logical extension of this observation is that a total system bakeout is required to assure good system design. That is, all parts of the system must undergo a twofold change in absolute temperature between bakeout and operation. For example, an ambient temperature of $300^{\circ} \mathbf{K}$ provides an effective bakeout for a surface which is to be operated at liquid nitrogen temperature, $78^{\circ} \mathrm{K}$.

In addition to the temperature ratio between bakeout and operation, equally important factors are the rate of change of temperature and the time at which the change occurs. For example, assume that a hypothetical vacuum system at bakeout conditions has two regions of different temperature, and that both temperature regions will undergo a twofold reduction in their absolute temperature. The absolute values of the temperature are not important. Also, the duration of the bakeout is assumed to be sufficiently long so that the two surfaces are in a quasisteady-state condition of outgassing with respect to each other and with 
the pressure defined by the ratio of their total outgassing and the speed of the pumping system. If one of the surfaces is allowed to drop in temperature from bakeout conditions to the twofold lower temperature for operation, two conditions immediately occur which reduce the system pressure. Initially, the net influx of gas is reduced as the one outgassing rate is dropped to negligible levels as a result of the temperature change. This cooled surface which is out of pressure equilibrium with the system pressure then becomes a pump for the gases evolving from the yet uncooled second surface. The net effect is that the cooled surface begins to contaminate by surface adsorption. If this temperature condition is allowed to remain long enough, the cooled surface eventually will contaminate to equilibrium with the system pressure. At this point, the pumping action of the surface will begin to decrease and the system pressure will rise. Later, when the second surface is cooled to reduce its outgassing, the contamination collected on the first cooled surface becomes a gas source which will prevent the attainment of the expected ultimate pressures. The solution to this condition is that all surfaces of the vacuum system must undergo a temperature reduction after bakeout. In addition, the temperature reductions should occur simultaneously. The cooling of one surface followed by the cooling of other surfaces may give rise to surface contamination and limited ultimate pressure.

Assuming that proper system design and operation have reduced to negligible levels those gas sources due to adsorption and absorption from the walls of the vacuum system, there is still the problem of the gas source from the pump. It has been stated previously that all pumps are gas sources in one manner or another. The limitations of cryogenic pumps arise from the equilibrium vapor pressure of different gas species. Getter ion pumps liberate gas as a result of their memory effect as the electrical discharge moves over the surface and releases previously pumped gases. In addition, the getter ion pumps generate many gases while pumping others. For example, the reaction of water vapor with the carbon impurities in the surface of the pump results in the generation of methane, ethane, and various other higher hydrocarbons, a part of which are repumped and a part of which reenter the vacuum system.

Getter ion or sputter ion pumps should be supplemented with a titanium sublimation pump for ultra-high-vacuum applications. The sublimation pump has the capacity to minimize the gas load on the sputter ion pump and to trap the sputter ion pump regurgitation so as to prevent it from entering the vacuum system. This combination pumping action has yielded system ultimate pressures in the low $10^{-11}$ torr range.

Diffusion pumps also have their limitations. The working fluid may be kept from the vacuum chamber if a properly designed liquid nitrogen trap is used. An optically tight trap is not adequate for long-term opera- 
tion at ultra-high-vacuum conditions. One of the most important ways that oil may pass through a liquid nitrogen trap and enter the vacuum system involves the nonunity sticking coefficient. An approximate fraction of $0.01(1-a)$ of the oil incident on the trap from the pump side will pass through the trap and enter the vacuum system. If the sticking coefficient $a$ is approximately 0.99 , one out of every $10^{4}$ molecules will pass through the trap. This ratio indicates the reduction of the apparent vapor pressure of the oil at the trap surface from the true vapor pressure. For modern diffusion pump oils with vapor pressures in the range of $10^{-7}$ torr to $10^{-9}$ torr, the system ultimate pressure will be in the range of $10^{-11}$ torr to $10^{-13}$ torr. Initially, the oil is trapped by the clean, freshly baked walls of the vacuum system. The chamber pressure due to oils is reduced another tenfold by the ratio of the chamber wall area to the trap area. Eventually, the oil flow produces a monomolecular layer of oil on the chamber wall, and the chamber pressure increases to the apparent vapor pressure of $10^{-11}$ torr to $10^{-13}$ torr. The time required for this to occur is about 1 month for an oil with a vapor pressure of $10^{-7}$ torr, or 1 year for an oil with a vapor pressure of $10^{-8}$ torr, if it is assumed that the sticking coefficient is approximately 0.99 . While the oil appears to have little effect on the base pressure of the system, the fact that it is entering the chamber presents a potential source of contamination for clean surfaces which are a critical part of the test. The use of a trap design which is both antimigration and minimum two-contact trajectory, or the use of two traps in series, one of which is an antimigration-type trap, can effectively eliminate pump oil contamination. Such an arrangement provides a reduction of another $0.01(1-a)$ such that the ultimate system oil pressure is further reduced to $10^{-15}$ to $10^{-17}$ torr, while the time required to contaminate is increased to 1000 years for an oil with a vapor pressure of $10^{-7}$ torr.

An additional problem of the diffusion pump is the production of noncondensable gases such as hydrogen, carbon monoxide, and methane radicals from the decomposition of the working fluid. Since these gases cannot be trapped by the liquid nitrogen trap, they must be eliminated in a different manner. A partial solution is obtained if two diffusion pumps are used in series. The second pump not only provides a higher net compression ratio but also provides a high pumping speed for the permanent gas decomposition products produced in the boiler of the first pump, and isolates the mechanical pump oil from the boiler of the main diffusion pump. Despite this improvement, the permanent gas generation in the pump is frequently the major limitation on the ultimate pressure in an ultra-high-vacuum system. There is a considerable difference in the hydrogen production rate of diffusion pumps manufactured by various venders. The variation is a function of the design and operating temperature of the pump boiler regions. The metallic parts of the pump boiler 
and jet assembly also act as hydrogen sources. As a result, these parts should be given an initial bakeout to avoid extremely long run-in times. In a similar manner, different pump oils yield considerable variations in terms of ultimate system performance. The selection of the proper diffusion pump oil for each brand and type of diffusion pump is a key part of the overall vacuum system design for ultra-high-vacuum applications.

Due to the fact that pumping speed and decomposition are functions of the boiler temperature, adjustments of the heater power and pump cooling will generally yield an optimum performance. Commonly reported ultimate pressures for this type of system are in the low $10^{-11}$ torr range. Note that this pressure range is essentially identical to that obtained with combination sputter ion and titanium sublimation pumps.

As in the case of nonbaked systems, further reductions in pressure require the addition of low-temperature cryogenic pumps. In general practice, hidden-panel cryogenic pumps have been shown to reduce the ultimate pressure of either pump type another decade. In addition, cryosorption pumps will supply high pumping speeds for hydrogen gas such that the system ultimate pressure will be reduced. If the interior test region is surrounded by a liquid-helium-cooled shroud, the pressure will be reduced beyond the measuring capability of existing instrumentation.

In connection with the bakeout process, it must be noted that the liquid nitrogen trap and the upper regions of the diffusion pump are a part of the high-vacuum system. It is important that these regions be included in the bakeout process. However, all pumping fluids must be prevented from entering the system region during the bakeout. If any part of the trap is held at liquid nitrogen temperature during bakeout, that part will adsorb the gases which are evolving from the rest of the system, and will become a gas source at a later time. A particularly obnoxious gas to contend with is carbon dioxide which is condensed readily on the trap surfaces as a result of its equilibrium vapor pressure. After bakeout, the system pressure is limited primarily by the reevaporation of the carbon dioxide from the trap surfaces. There are several approaches which can be used to combat this problem. One approach is to connect two traps in series where each trap has a separate bakeout mantle. This configuration requires a careful operating procedure to avoid transmitting the contamination back and forth between the two traps.

An improved technique for combating this problem uses a controlled operating cycle in which the trap is deactivated at the end of bakeout. The deactivation process consists of permitting the refrigerated surfaces of the trap to warm up to a particular value of temperature where all of the intermediate condensables such as carbon dioxide are evolved. However, none of the regular condensable hydrocarbons are released, and there is no hydrocarbon contamination of the test chamber or its com- 
ponents. The carbon dioxide evolved from the trap is not resorbed on the chamber walls as the chamber is still at the bakeout temperature.

An alternate approach utilizes a highly specialized liquid nitrogen trap which has built-in, controlled thermal restrictions. During the system bakeout, the outer body of the trap is baked at $600^{\circ} \mathrm{K}$. The thermal restrictions control the temperature of the trap interior at approximately $200^{\circ} \mathrm{K}$ during the bakeout. Carbon dioxide does not condense at this temperature. The refrigerated trap surfaces undergo the desired twofold temperature reduction after bakeout, yet none of the pump fluid or its condensable fragments pass the trap and enter the chamber. System ultimate pressure using this technique will reach the low $10^{-11}$ torr range without cryogenics. With the addition of cryogenic pumping, pressures in the low $10^{-12}$ torr range have been attained.

An important factor which reduces many of the problems of ultrahigh-vacuum systems is what might be termed the nonrepeatability of the outgassing. During bakeout, the principal gases removed from the system are water and hydrocarbons from the surface, and carbon dioxide, carbon monoxide, and hydrogen from the interior. The surface gases are evolved at a relatively low temperature, $120^{\circ} \mathrm{C}$ to $150^{\circ} \mathrm{C}$. These gases will re-form every time the system is opened to the atmosphere. The carbon oxides and hydrogen from within the bulk of the metallic materials appear by means of diffusion. The diffusion gradient formed is essentially permanent in that the gases do not reenter the metal. The performance of ultra-high-vacuum systems is affected in two ways as a result of the almost permanent diffusion gradient. First, carbon dioxide is seldom a principal gas after the first high-temperature bakeout, and if new material has not been added to the vacuum system, the basic problem of carbon dioxide on the liquid nitrogen temperature surfaces is minimized. Second, each bakeout is cumulative in increasing the diffusion gradient for hydrogen and carbon monoxide. If one long-term, hightemperature bakeout is performed on the system, it can be seen that, in most instances, the system will pump down readily to the same pressure level with only a mild low-temperature bakeout. A common operating bakeout is $200^{\circ} \mathrm{C}$ for a system which has already been processed at a higher bakeout temperature ranging from $400^{\circ} \mathrm{C}$ to $450^{\circ} \mathrm{C}$. Of course, if new material such as a nonbaked test sample is added, a high outgassing rate may require that a complete bakeout cycle be repeated. The problem of carbon dioxide on the liquid nitrogen trap may again arise for this new cycle. The nonrepeatability characteristic of high-temperature outgassing permits ultrahigh vacua to be attained easily in empty chambers. However, caution must be exercised to assure that these pressures can be reproduced with the addition of test loads. 
Two common variables which can be found in many different ultrahigh-vacuum system designs are the seals and chamber cooldown procedure after bakeout.

A general rule in vacuum system technology is that metal seals are preferable to organic seals for ultra-high-vacuum applications. Several commercial seals utilizing copper gaskets are available for small seals which may range from 1 to 10 inches in diameter. For larger seals, copper (ref. 19), gold wire (ref. 20), and aluminum (ref. 23) have been used successfully. The reliability of these seals is well established, and repeated leaktight closures which may be rebaked many times can be obtained. Leaktight metal seals with diameters up to several feet have been used. These large seals require close attention and time to assure proper closure. All the bolts must be carefully torqued down. An alternate approach is the use of refrigerated organic seals (ref. 21), which takes advantage of the extreme temperature dependency of all forms of outgassing. Normal organic O-ring seals are used with the addition of cooling coils in the vicinity of the seal. During bakeout, water cooling or a refrigerant is used to limit the temperature rise of the material. Thus, excessive decomposition or damage to the seal can be avoided. Following the bakeout, the area in the vicinity of the seal is cooled further by refrigeration to the point that the outgassing is a negligible contribution to the system. The principal advantage of this procedure is that the seals can be easily opened and closed, and can be reused. The principal disadvantage is the problem of hydrocarbon contamination. In addition, the bakeout temperature of the chamber wall in the vicinity of the seal is limited by the cooling of the seals so that a part of the chamber never receives a high-temperature bakeout. Both approaches have brought about vacuum levels in the $10^{-11}$ torr region without cryogenic pumping and the $10^{-12}$ torr region with cryogenic pumping.

The cooling of the vacuum chamber after bakeout has been accomplished with water coils and with air. Water-cooling coils initially are an added expense, but they result in faster cooldown cycles. In many instances, the rapid and nonuniform cooldown cycle may be detrimental due to thermal stresses or readsorption. The thermal stresses may open up leaks, while readsorption may limit the ultimate pressure attained. In general, time permitting, air cooling is preferred.

\section{SYSTEM DESIGN}

To discuss the complete design specifications covering all the types of vacuum systems required for various aspects of space simulation would require a long and detailed integration of all the material prior to this chapter. The complete design of a vacuum system should be left to those who are trained in the art. Nevertheless, many basic details can be 
delineated for those who may become involved in the specification of vacuum equipment or its operation.

\section{Basic Requirements}

There are two criteria which must be defined prior to the specification of any other system parameters: chamber size and required operating pressure. Space-simulation chambers may range from less than a foot to over 100 feet in the principal dimension. Although the chamber size is obviously related to the test item, attention must be given to requirements for additional components in the chamber. For example, the use of shrouds or platens will necessitate an increase in the chamber size. Generally speaking, small chambers are cylindrical in shape with hemispherical end caps and a full-opening door. Chambers ranging from 1 to 4 feet in diameter may be either horizontally or vertically oriented. Vertical chambers are more accessible for loading with an overhead crane, while the horizontal chambers are more accessible for manual operation. Chambers from 5 to 20 feet in diameter are almost always horizontally oriented, while large chambers, which tend toward a spherical shape, are generally vertically oriented to allow for a largecapacity crane to permit both removal of the door and chamber loading.

Allowances should be made for a certain amount of future expansion. Therefore, the chamber should generally be larger than the minimum requirement that can be specified definitely. As a guide, the chamber size might be selected such that it is twice the diameter of the largest expected test item. This will allow adequate space for the placement of instrumentation, and will allow a sufficient area so that work can be performed around the test item.

Operating pressure requirements were discussed in the section on "Space Simulation." In general, there is justification for three separate operating pressures. The first pressure of only a few torr can be obtained with mechanical pumps, and is adequate for all tests dealing with mechanical deformation studies of systems and subsystems. For large chambers, mechanical booster pumps are most economical.

The majority of space-simulation testing requires the maintenance of free molecular flow in the chamber. Therefore, operating pressures around $10^{-5}$ torr or $10^{-6}$ torr will provide essentially all the vacuum environment required. The ultimate pressure of the chamber must be well below this operating pressure level. In general, the proper ultimate pressure to define is that which can reasonably be obtained as a function of other system factors. An ultimate pressure of $1 \times 10^{-8}$ torr is both reasonable and desirable for the nominal, valveless space-simulation chamber. This is true if an excessive amount of organic seals is not used. If the system design calls for a valve, the ultimate pressure should be 
backed off to $1 \times 10^{-7}$ torr. This pressure is still adequate to yield the working vacuum level required.

Surface studies or other experiments requiring ultrahigh vacuum will need the more complex bakeable vacuum system. An ultimate pressure of less than $1 \times 10^{-10}$ torr can be obtained readily. The attainment of pressures in the low $10^{-11}$ torr range requires extreme care, both throughout the design stage as well as when the system is operational. To attain pressures below the $10^{-11}$ torr level, cryogenic pumping is normally required.

No specific recommendations can be set forth with regard to the type of pump to be used. All three high-vacuum-pump types, diffusion, molecular, and sputter ion are capable of producing the vacuum levels discussed previously. The specific differences among pumps concern initial cost, operating cost, maintenance, and ease of operation. Costwise, the combination of the diffusion pump and a liquid-nitrogen-trapped system is the most economical, particularly for baked systems where a sublimation pump must be added if a sputtter ion pump is used. The molecular pump is the highest priced of the three types and to date has had limited application.

\section{Gas Load and Pump Size}

Many experimenters have been concerned with the proper determination of pump size with regard to a calculated gas load. If all the materials present in a given test could be known, as well as the previous history of each material, it would be possible to make an exact determination of the gas load. This information is seldom available. Fortunately, in practical applications, this detailed information is seldom required. Experience and a little thought will provide a reasonable answer to the problem.

Diffusion pumps incur a reasonably small amount of the total cost of a vacuum system. As a result, the selection of the largest pump which will fit the chamber will seldom be an extravagance. Of course, the pump and trap should not be larger than the test chamber. A practical rule of thumb is to use a pump which is about one-third the chamber diameter. That is, an 18-inch chamber will be adequately handled by a 6 -inch pump, while a 30 -inch chamber would use a 10-inch-diameter pump. Note that the net system speed for such a combination is just about 1 percent of the theoretical maximum chamber speed if the walls of the chamber are all perfect pumps. While this ratio is to be used as a guide only, it should be noted that even if the pump size is doubled in diameter, there would be only a fourfold increase in the pumping speed. The difference in the operating pressures of the two systems is in the ratio of 4:1. This difference will seldom make an appreciable effect on space simulation applications. 
In situations where the capacity of the main pump is well exceeded, such as when high gas loads are to be handled at very low pressures, various forms of cryogenic pumps should be considered. A liquid nitrogen shroud will supply as much as 100 times the pumping speed of the main high-vacuum pump for normal outgassing water vapor loads. Permanent gas loads such as nitrogen or oxygen can be handled by a $20^{\circ} \mathrm{K}$ hidden-panel, cryogenic-pump type. In a typical installation, the cryogenic pump will have a speed greater than 10 times that of the diffusion or sputter ion pump. Noncondensable gases such as hydrogen, helium, and neon create the greatest problem. While neon is seldom present and helium is present only in the event of a leak in a cryogenic system within the main chamber, hydrogen may arise from a number of sources. High-speed hydrogen pumping has been obtained using either cryosorption techniques, or titanium sublimation pumping ref. 28).

As the size of the chamber increases, a point is reached where the maximum-sized diffusion pump used alone does not provide the desired speed. Currently, 36-inch- and 48-inch-diameter pumps are the largest that can be obtained commercially. For example, a 39-foot spherical chamber needs approximately 25 of the largest pumping systems to yield about 1 percent effective wall pumping. At this point, the cost has become sufficiently high that cryogenic pumping should be considered. Not only is the cryogenic pump competitive in cost but it can provide on the order of 10 times the pumping speed for nitrogen- and oxygentype gases, and 100 times the speed for condensables like water. A small amount of diffusion pumping is still required to handle the noncondensable hydrogen and helium gases.

Another part of the pump-speed problem involves the effect of traps and baffles on the reduction of the available speed of the diffusion pump. Currently available diffusion pumps have a pumping coefficient in the range of 0.5 . The addition of traps and baffles will reduce the speed to yield a net system pumping coefficient of about 0.2 . It is doubtful that the very best trap and baffle designs exceed 0.25 , nor are the poorer ones any worse than 0.15 . This represents a deviation of only 25 percent from the mean system coefficient of 0.2 . In terms of its effect on operating system pressure, this also represents a mean difference of only 25 percent on the pressure. An average system operating at a pressure of $4 \times 10^{-x}$ torr will have a pressure of $3 \times 10^{-x}$ torr for an optimum baffle design and $5 \times 10^{-x}$ torr for a higher restriction design. This variation is within the measuring error of most ionization gages. Since it is apparent that small variations in trap or baffle design do not seriously affect the system pumping speed, it is important to obtain a trap that has good trapping ability for the pumping fluid from the diffusion pump. 
In most space-simulation chamber applications, the initial evacuation of the chamber is accomplished using a mechanical pump of a size which is related more to the diffusion pump size than to the chamber size. This situation occurs since most testing applications represent long-term tests. As a result, when considering the overall operating time cycle, it does not matter whether the initial exhaust is 10 minutes or an hour in duration. In valveless chambers where initial evacuation takes place through the diffusion pump, the pumpdown time from atmosphere to a pressure of a few hundred microns does not have to be shorter than the heatup time of the diffusion pump.

\section{Seals and Penetrations}

The seals used in vacuum systems fall into two principal classes: organic seals which are principally of the O-ring type, and metal seals. Bakeable systems should use metal seals wherever possible. If metal seals are not used, a limited temperature bakeout and limitations in the ultimate pressure may result. It is also advisable to use metal seals in nonbaked, very-high-vacuum systems to reduce the gas load, both from outgassing and from water permeation of the seals. Small-diameter metal seals are available from several commercial sources. The major problem in the use of metal seals is in the larger seal sizes required for the main chamber opening or for flanges larger than 10 inches.

A variety of metal seal designs have been reported in the literature. The sealing materials have included indium, lead, aluminum, copper, and gold. The first two materials possess a temperature limitation and are seldom used in bakeable applications. Indium has been observed to relax and open up a leak following a low-temperature bakeout at $130^{\circ} \mathrm{C}$. The leak can be resealed by retorquing the bolts. When aluminum is used for sealing applications, the material should possess a high purity, 99 to 99.99 percent. The expansion coefficient of aluminum does not match that of stainless steel. The seal is affected when a thin material is subjected to a very high compression (ref. 23). During the bakeout process, the aluminum interdiffuses with the stainless steel and becomes bonded to the flange such that a large force is required to separate the two flanges after bakeout. Generally, some form of jacking bolts should be incorporated for this purpose.

Copper and gold gaskets are the most commonly used seals for bakeable applications. In general, the most satisfactory and economical solution to the seal problem is to use a commercial flange rather than attempting to design an original flange. In all forms of copper seals, the compression is limited to a small area of the copper. Generally, gold seals are made from wire with either a 0.025 -inch or 0.040 -inch diameter, and are compressed to approximately one-half their original diameter. The surface finish of the flange should be in the range of 16 microinches. For both 
gold and copper seals, it is important that the flange thickness and the number of bolts used be sufficient to avoid deformation of the flange between adjacent bolts. A $2 \frac{3}{8}$-inch spacing on a 1-inch-thick flange has been found adequate for flange diameters of several feet. Both copper and gold wire seals must be pried open after a high-temperature bakeout.

Many different organic and polymeric materials have been used for seals. The most common ones are Teflon, Kel-F, Viton A, butyl rubber, Buna $\mathrm{N}$, neoprene, silicone, and natural rubber. Teflon and Kel-F have very low outgassing rates, but exhibit cold-flow characteristics. Lesspronounced, cold-flow characteristics are exhibited by Viton A, which has a low hydrocarbon decomposition rate at elevated temperatures up to $250^{\circ} \mathrm{C}$. Silicone is the most permeable material of the group and resists temperature extremes. Chapter 9 presents outgassing information on these materials. Since the majority of these materials have the same range of solubility, it should be noted that a material which has a high initial outgassing rate will also clean up rapidly, and will have a high permeation rate. In applications where two 0 -rings are connected in series with an intermediate pumpout area, a material such as silicone, which has a high permeation rate and a high outgassing rate, may be preferred to a material with a low outgassing rate. Silicone materials outgas more rapidly to a very low value. Due to the presence of the guard vacuum, permeation is not a problem. This same situation is true for materials which are used within the vacuum chamber.

Various O-ring groove designs are presented in all standard vacuum texts (ref. 24). Materials which do not exhibit cold-flow characteristics should be incorporated in a groove design which permits the entire O-ring or gasket to be contained within the groove without compressing the volume of the material. While these materials may be readily deformed, they are generally incompressible. As a general rule, the groove depth is between 75 to 80 percent of the gasket thickness. A surface finish of 32 microinches is generally recommended. Frequently, however, leaktight seals have been made on surfaces with finishes of 64 microinches and even 125 microinches. There must be no radial scratches on the surface. With a good surface finish, an O-ring or gasket may be sealed without the use of a lubricant. On poorer surfaces, a lubricant is required, but excessive grease or oil should be avoided. All that is required is sufficient grease or oil to produce a shine on the surface. It should be noted that all surface lubricants will produce contamination in the vacuum system. Complete venting of the O-ring or gasket is a highly desirable procedure. A vent port located on the atmospheric side of the O-ring will reduce the problem inherent to leak checking by permitting the helium or other tracer gas to enter directly into the region of the seal rather than being forced to slowly diffuse between two close-fitting flanges. The vent port on the inside allows the rapid evacuation of the 
gas space next to the O-ring and venting of the corner dead pocket of the O-ring. The seal is made on the flat-facing surfaces of the flanges. An additional seal on the side surface is undesirable, since it may form either a dead pocket or a virtual leak. If both the series seals are leaking, a long time constant leak may exist which is exceedingly difficult to locate. For materials which have a high rate of cold flow, it is advisable to use a groove design which has a smaller cross-sectional area than the gasket. However, this seal requires more bolts and a higher flange load, and is made permanent by the actual compression of the material. As a result, the life of such a seal is shorter than the life for those designs which do not require bulk deformation.

Many types of penetrations are commercially available, among them electrical feedthroughs, bellows, rotary motion devices, cryogenic feedthroughs, and a host of specialized fittings. Most are available in a wide range of sizes and ratings.

\section{Other System Accessories}

Since a vacuum system is used to create an environment in which some form of experiment may be performed, it is necessary that other components be incorporated in addition to the chamber and the pumping system. The number of components available is so extensive that a complete listing is impossible. However, certain accessories are used so frequently that they will be mentioned briefly for consideration in the design of a particular system.

A manual leak-detection valve on the foreline of a diffusion pump is an advantage when it is necessary to leak-test the system. If high sensitivity is required, the mechanical pump should be closed off so that the leak detector alone is backing the diffusion pump. This can be accomplished by an additional manual valve, or by a part of the system control circuitry.

A throttling gas inlet valve on the main chamber is a desirable accessory which should be capable of adjusting the system pressure to a value of several hundred microns when the mechanical pump only is running. The valve, which may be either a throttling type or a gate type connected to a fixed-series resistance, is used to provide a continuous sweeping flow of gas through the vacuum system for either standby or cleaning operations. Gas-purge procedures will be discussed in the section on operation.

Refrigeration equipment frequently is required for the thermal control of internal shrouds or platens which are integral parts of the thermalsimulation system. Refrigeration systems which use both single- and double-fluid arrangements have been fabricated to cover a wide range of high and low temperatures. While it is desirable to obtain a high degree of flexibility and a wide operating temperature range, it should be realized that the more extensive the temperature range specified, the 
higher the cost. In addition, precision control of the surface temperature results in a cost increase. Liquid nitrogen control systems for internal shrouds should be evaluated carefully, both in terms of their cost and their ability to provide unattended control.

Automatic liquid nitrogen level controls for traps or other devices are necessary in space-simulation applications where it is necessary to perform long-term unattended tests. It is desirable to have both an automatic control and an additional warning device to signal any failure of the main liquid nitrogen supply which provides the desired refrigeration. This additional failsafe device may be wired into the main system operating controls so that the system may be shut down in the event of liquid nitrogen failure during the period of unattended operation.

When a vacuum system is being designed for space-simulation applications, it is advisable to make allowances for the possible future addition of solar-simulation equipment or vibration equipment. It is far easier to design this future capability into the system initially than to attempt to work it in at a later date.

Additional vacuum gaging, particularly in the foreline, is very helpful when it is necessary to troubleshoot the system. For example, if a thermal conductivity gage is placed on each side of the forepump isolation valve, considerable information on pump performance may be obtained when the response of the two gages is observed after the valve is closed.

One of the most important gages that can be placed on the main chamber is some form of partial-pressure-measuring device, which provides considerable additional information on the system operation during normal testing. In addition, this partial-pressure gage will be an invaluable aid in assessing system problems or malfunctions, including leakage. The use of such a gage for system troubleshooting will be discussed in the maintenance section of this chapter.

\section{Fabrication}

The subject of the fabrication of vacuum systems and their components is very rarely of specific interest to the system user. However, certain techniques are of sufficient importance that a brief discussion of several may be valuable. References 24 and 25 are very good treatments of the areas of fabrication techniques and cleaning procedures.

The majority of space-simulation vacuum chambers are fabricated from 300 -series stainless steel. The desirable properties of stainless steel include strength, weldability, corrosion resistance, low thermal conductivity, and a readily polished surface for low-surface outgassing. Type 304 stainless steel is used most frequently. However, the low carbon content of type $304 \mathrm{~L}$ is far superior in terms of welding properties. The 304 steel frequently exhibits weld cracks and porosities due to carbon precipitation. The stabilized grades of stainless steel, 321 and 347 , are 
sometimes used for bakable vacuum chambers to avoid the problems of carbon precipitation in the grain boundaries, resulting in eventual fatigue and leakage. Unfortunately, the 347-grade stainless steel frequently exhibits weld cracks and may be extremely difficult to weld leaktight initially.

When welding the vacuum chamber, the heavy wall sections of welding should be full penetration. In the event that full penetration welds are impractical, the weld joint should be made on the inside of the chamber where it is accessible for repair. This procedure will minimize cracks and crevices which may collect cleaning fluids or other sources of contamination. When the additional strength of a double weld is required, i.e., in those places where full penetration cannot be obtained, the outermost weld should be intermittent to avoid the double-leak problem, and also to facilitate leak testing. In those cases where thin sections must be welded to thicker sections, i.e., thin-wall tubing welded to a heavy flange, a weld penetration should be machined in the heavier member and fusion-welding techniques used to join the two sections. Optimum welding will result if both sides of the section are exposed to an inert atmosphere such as argon. Small items may be welded in an argon dry box. Chambers or sections which are sealable can be pumped down and backfilled with argon gas. In open sections, the argon environment on the back side of the weld can be obtained if a second person follows the welder's hot spot with a probe using a heavy flow of argon gas. One of the most common causes of leaky welding is surface contamination. Weld joints should be thoroughly cleaned, both mechanically and chemically, prior to welding. All mechanical cleaning should be done with stainless-steel brushes or files which have been used only on stainless steel and which have been kept free of residues of other types of material. Traces of aluminum, brass, or copper transferred to the weld area from contaminated files will yield porous and leaky welds. Grit or stones from grinding wheels, sandblasting, or sandpaper will be equally detrimental to the attainment of a proper weld.

Wherever possible, welding is preferable to brazing, and brazing is preferable to soft soldering. A wide variety of brazing alloys are available for different temperature applications. In terms of high-vacuum applications, the brazing material must be capable of undergoing repeated bakeouts at temperatures up to $450^{\circ} \mathrm{C}$. This implies that it is advisable to avoid cadmium- or zinc-bearing compounds. All brazed and soft-soldered joints should be carefully cleaned by one of the processes given in reference 25 to remove as much as possible of the fluxes added to the system.

Vacuum systems require careful cleaning following the fabrication and assembly stages. In addition, they may need cleaning following various contaminating experiences during operation. Although numerous clean- 
ing procedures are available, all of these procedures include the following basic steps:

(1) Mechanical cleaning to remove surface dust, burs, or alien surface materials

(2) Degreasing to remove shop oils and other hydrocarbons on the surface

(3) Chemical cleaning, when required, to remove oxides

(4) Multiple washings with water

(5) Final washings in such solvents as alcohol or acetone

The process of recleaning the chamber after oil contamination can be carried out using steps (2) and (5), where the solvents commonly used are trichlorethylene and Alconox.

\section{Operation}

Proper operation of a vacuum system makes the principal contribution to good system performance. Improper operation frequently leads to component malfunction, hydrocarbon contamination, and a limited ultimate pressure. There are only a few basic procedures which must be remembered to yield a proper operating sequence. If these are followed conscientiously, the system user should be rewarded with good system performance and minimum maintenance. The following list of Do's and Don'ts is presented as a guide to be used where appropriate. Following the list, examples of startup and shutdown procedures are included for a few typical systems.

(1) When starting a mechanical pump, always observe the rotor to insure that the pump is not frozen, or that the casing is not full of oil.

(2) Always vent a mechanical pump to atmospheric pressure when the power is turned off. The presence of a residual vacuum in the pump frequently will cause oil to be drawn into the casing, which will make the next startup difficult.

(3) Never permit a mechanical pump to exhaust a high-vacuum system below a pressure of a few hundred microns unless the pump is separated from the high-vacuum chamber with a liquid nitrogen trap or another equivalent means of stopping the mechanical pump oil from entering the chamber. At lower pressures, the pump exhaust line is in free molecular flow and the mechanical pump oil will flow readily to the high-vacuum chamber to yield an excessively high vapor pressure hydrocarbon contamination which may require many hours to remove.

(4) Do not run a mechanical pump at excessively high pressures for continuous periods. The motors supplied with most mechanical 
pumps are not sized for continuous operation at pressures of a fraction of an atmosphere. Check the manufacturer's rating.

(5) A diffusion pump should be cooled to a safe intermediate temperature before it is vented to atmosphere. Check with the oil manufacturer for the maximum temperature operating range. Mercury and silicone oils may be vented to atmosphere while at full normal operating temperature; however, excessive carryover of the fluid into the mechanical pump may result.

(6) Always check that a cooling water supply for the diffusion pump is turned on prior to diffusion pump turn-on. In general, all diffusion pumps should be provided with a thermal protection device to turn the diffusion pump off in the event of loss or failure of the cooling water supply.

(7) In liquid-nitrogen-trapped, high-vacuum systems, the liquid nitrogen trap should be cool enough to condense the diffusion pump oil prior to turning on the diffusion pump. Maximum backstreaming of the pump oil occurs during the startup and shutdown of the pump when the jets are not at full working oil flow.

(8) When the diffusion pump is first turned on, observe the foreline pressure to insure that the pressure does not remain excessive or that the pressure does not continue to blurp. A transient pressure rise or blurping is expected as the pump comes into operation, but this should not continue.

(9) Do not vent a liquid nitrogen trap to air while cold, particularly if the trap arrangement is such that condensed liquid oxygen will remain in contact with the trap surfaces. When the pressure is reduced over an accumulated liquid oxygen condensate, the liquid will boil eruptively to result in rapid evaporation and a pressure rise which may exceed atmospheric pressure (on many occasions this has resulted in the explosion of glass vacuum systems).

(10) Remove all liquid water condensate from the reservoir of a liquid nitrogen trap prior to use to avoid water freezeup and damage to the trap.

(11) On the initial pumpdown, it is advisable to partially fill the liquid nitrogen trap, so that the principal vacuum system condensables can be trapped on the lower portion of the trap which will always remain cold. After high vacuum has been reached, the trap should be filled to a higher level and the temperature held at the point where the original condensate material cannot reevaporate. A liquid nitrogen trap which has O-ring seals at the top should not be overfilled, as the freezing of the O-ring may cause leakage. 
(12) If a water baffle is used between the liquid nitrogen trap and the diffusion pump, the water cooling should be turned on prior to trap cooldown. Thermal radiation is sufficient to freeze the standing water in the baffle such that internal buckling and leakage will result.

(13) When the chamber is vented to atmospheric pressure for short periods of time, it is advisable to use a dry, inert gas such as nitrogen to minimize the moisture adsorption on the surfaces of the vacuum system. Venting should always be on the system side of the liquid nitrogen trap; never vent the system from the foreline of the diffusion pump.

(14) A low-pressure, viscous-flow purge of an inert gas should be used for standby or cleanup operations in the vacuum system. A highvelocity flow of gas will pick up contamination from the lines and carry it through the mechanical pump, while at the same time, the backmigration of any of the mechanical pump oil into the system will be prevented.

(15) Ionization gages should not be turned on until it is reasonably certain that the pressure is below $10^{-3}$ torr. On high-vacuum systems, the ion gage should not be operated at high pressure in order to avoid excessive gage contamination. After a base pressure has been reached, the gage should be degassed thoroughly to remove previously adsorbed or pumped contamination. Further degassing is not required unless the system environment changes. Frequent degassing disturbs the thermal and vacuum equilibrium, and results in transient, false pressure readings.

(16) A hot chamber should not be cooled with water rapidly, since any excessive thermal shock may result in damage to either the seals or welds.

(17) In the event that liquid nitrogen is lost and the trap warms up, turn off the diffusion pump and turn on the gas purge to sweep out the condensables which will be evolved from the trap. The purge may be removed as soon as the trap is recooled.

(18) In the event that electrical power is lost, vent the system to atmospheric pressure with dry nitrogen.

\section{System Operation Example 1: Helium Mass Spectrometer Leak Detector}

Figure 10.9 is the schematic of one typical form of helium mass spectrometer leak detector which has an auxiliary mechanical pump and sampling valve. Valve $V_{4}$ and the series restriction leading to the gas nitrogen supply are not a normal part of the leak detection system and must be supplied by the user. The reentrant trap is used to prevent hydrocarbon contamination from entering the mass spectrometer tube from 


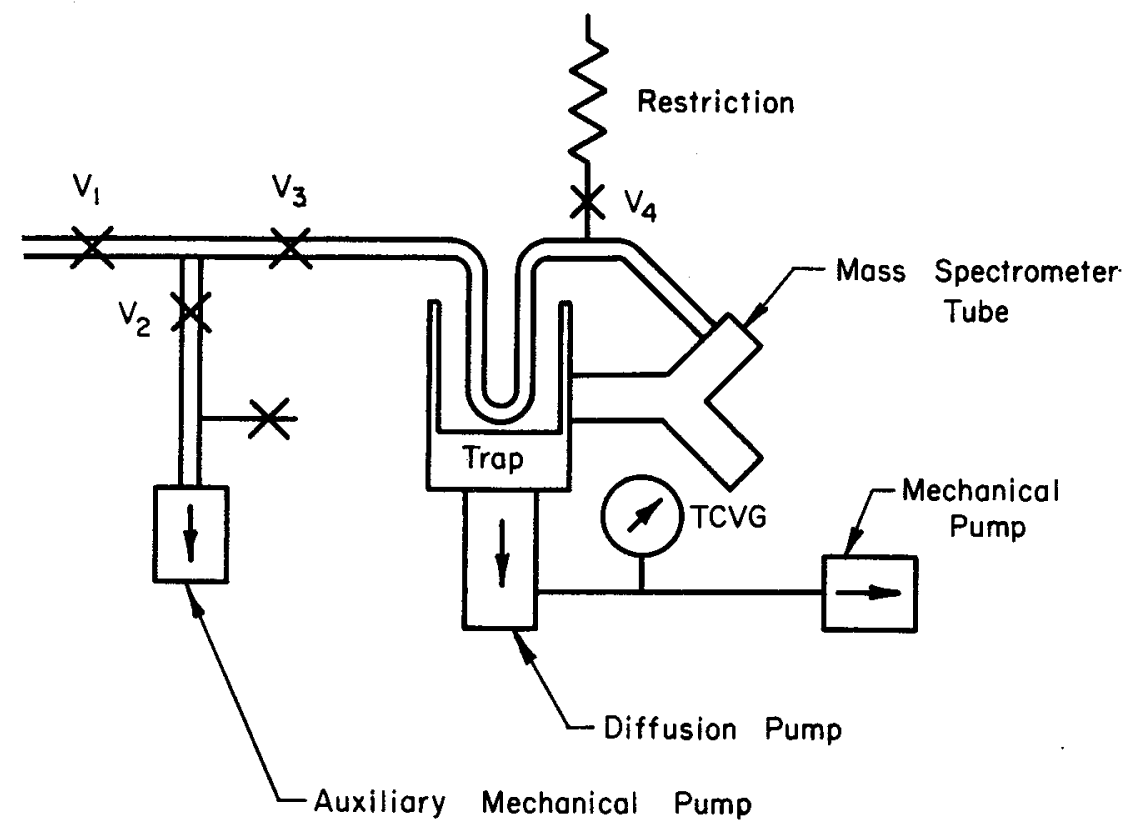

FIGURE 10.9.-Schematic diagram of typical helium mass spectrometer leak detector with an auxiliary pumping system and gas-purge line.

the test item region. For the initial pumpdown, valves $V_{2}, V_{3}$, and $V_{4}$ are opened, and both mechanical pumps are on. The gas flow from the nitrogen leak will maintain a sweeping action which will remove contaminants from the system, and will prevent the mechanical pump oil from backstreaming into the system. To move from the standby mode to a highvacuum condition, liquid nitrogen is added to the trap and the diffusion pump is turned on. After the trap has cooled, the gas purge may be turned off and valve $V_{3}$ closed. The system will pump down quickly.

To accomplish system shutdown, the diffusion pump is turned off first. After approximately 10 minutes of cooling, valves $V_{2}, V_{3}$, and $V_{4}$ are opened to return to the gas-purge standby condition. Condensables in the trap will be removed as the trap warms up. This standby condition may be left in effect for overnight operation. Complete shutdown after the trap has warmed requires the mechanical pumps to be turned off, and the entire system to be vented to atmospheric pressure with dry nitrogen.

\section{System Operation Example 2: Nonbaked, Valveless Space Simulation}

Figure 10.10 illustrates a very common form of space simulator used for thermal vacuum testing. The initial exhaust is accomplished by 


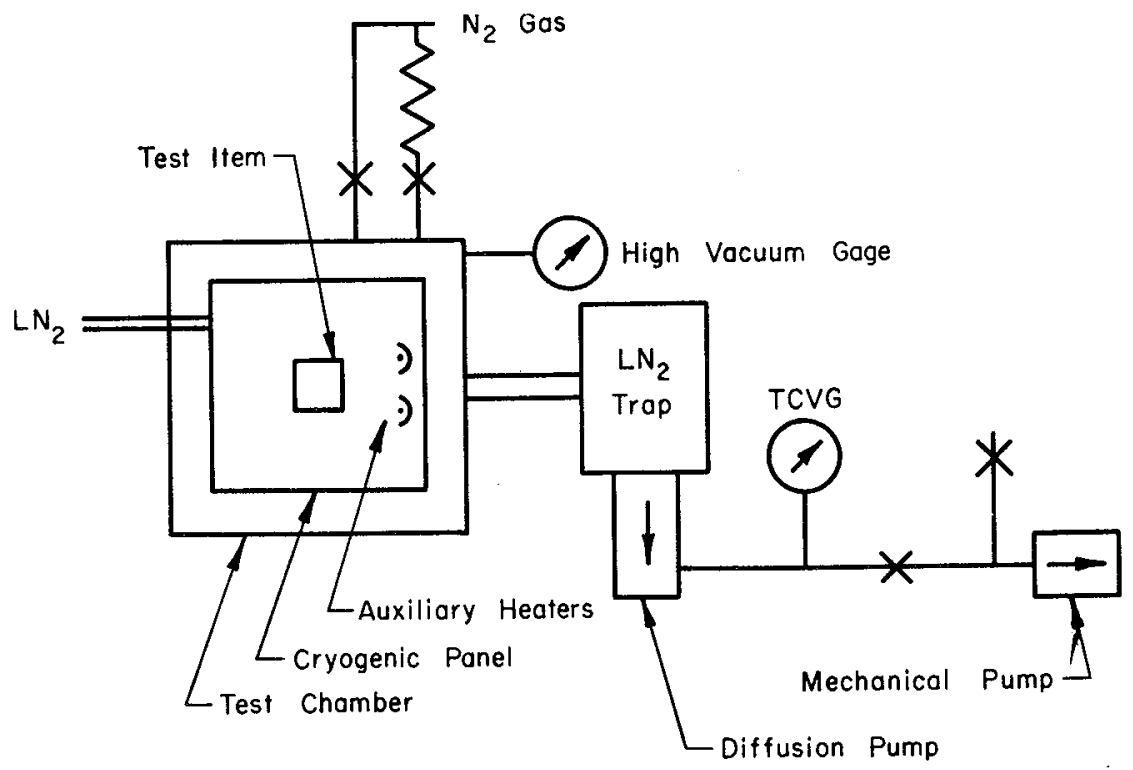

Figure 10.10.-Schematic diagram of a space-simulation chamber commonly used for thermal vacuum testing.

roughing through the diffusion pump. It can be seen that the diffusion pump may be warming up during this exhaust phase. An example of one possible operating cycle requires that the operating times of the various components be known. It is assumed that the system is relatively large, the initial exhaust time to $300 \mu$ is $60 \mathrm{~min}$., the diffusion pump heatup time to operation is $30 \mathrm{~min}$., and the trap cooldown to $135^{\circ} \mathrm{K}$ is $10 \mathrm{~min}$. after liquid nitrogen is added. Figure 10.11 illustrates the operating cycle. The mechanical pump is turned on initially, and 30 minutes later, the diffusion pump is turned on while the pressure is still quite high. At time $t=50$ minutes, the liquid nitrogen trap is cooled. The net result is that at $t=60$ minutes, the mechanical pump has just exhausted the chamber to a pressure of $300 \mu$, the diffusion pump is just up to operating temperature, and the liquid nitrogen trap is cold. The pressure will continue to fall rapidly into the high-vacuum region, depending upon the outgassing load of the warm shroud and the test item. At this point, the shroud may be cooled and the auxiliary heat source turned on.

It can be seen that the starting time for the different components was determined on the basis of their warmup or cooldown times. In many systems, the mechanical pump is sized to pump-down the chamber in the same period of time as that required to warm the diffusion pump. In this case, both pumps are turned on at the same time, and trap cooling is 


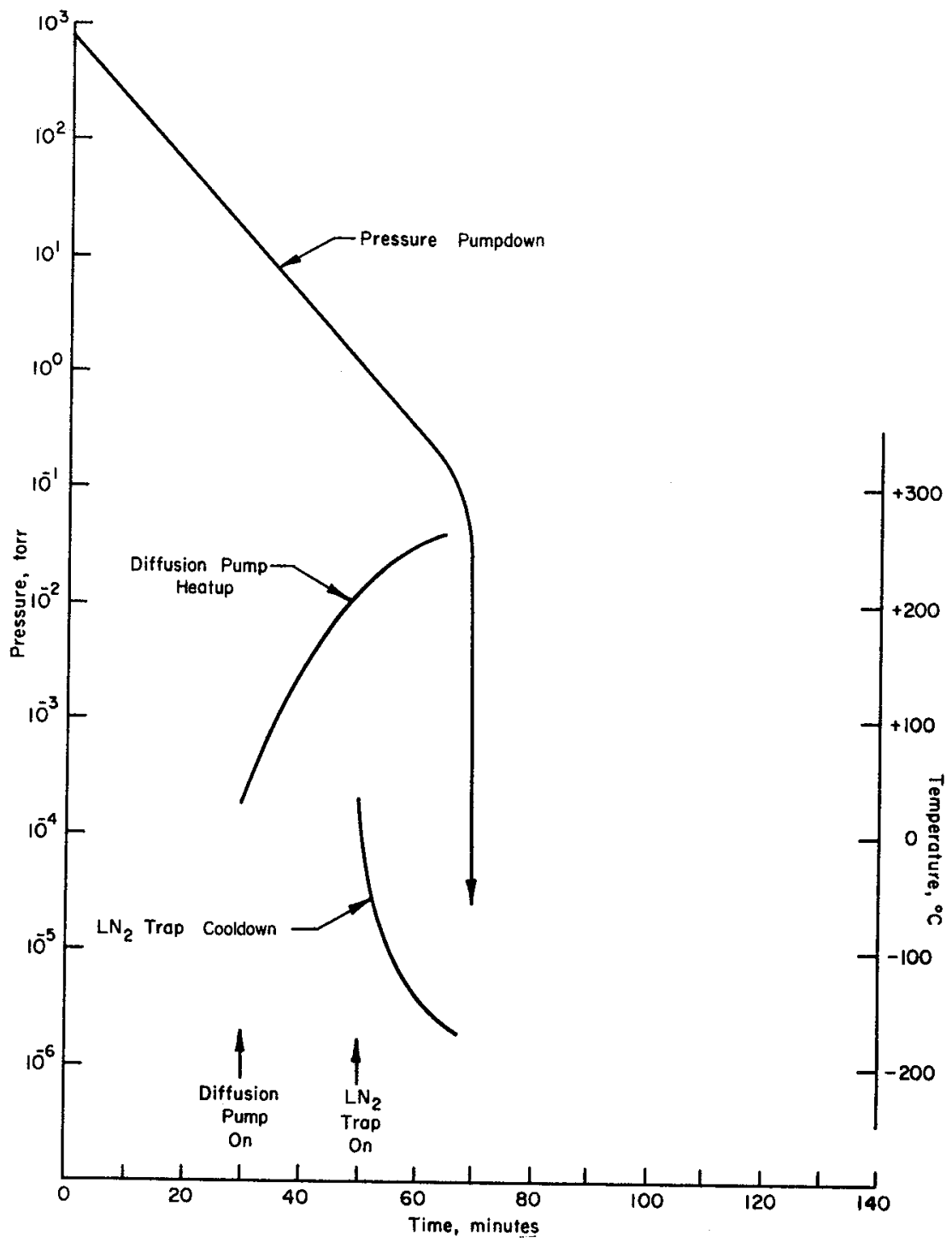

Figure 10.11.--Illustration of scheduled starting of system components to optimize the pumpdown cycle with minimum contamination.

initiated at the proper instant so that the trap is cold before the pressure enters into the free-molecular-flow range.

One disadvantage of the foregoing procedure is that the shroud or the test item may be extensive gas sources, particularly for water vapor. As a result, the trap will receive a large covering of ice in the initial pumpdown. The residual water in the gaseous phase is small relative to the 


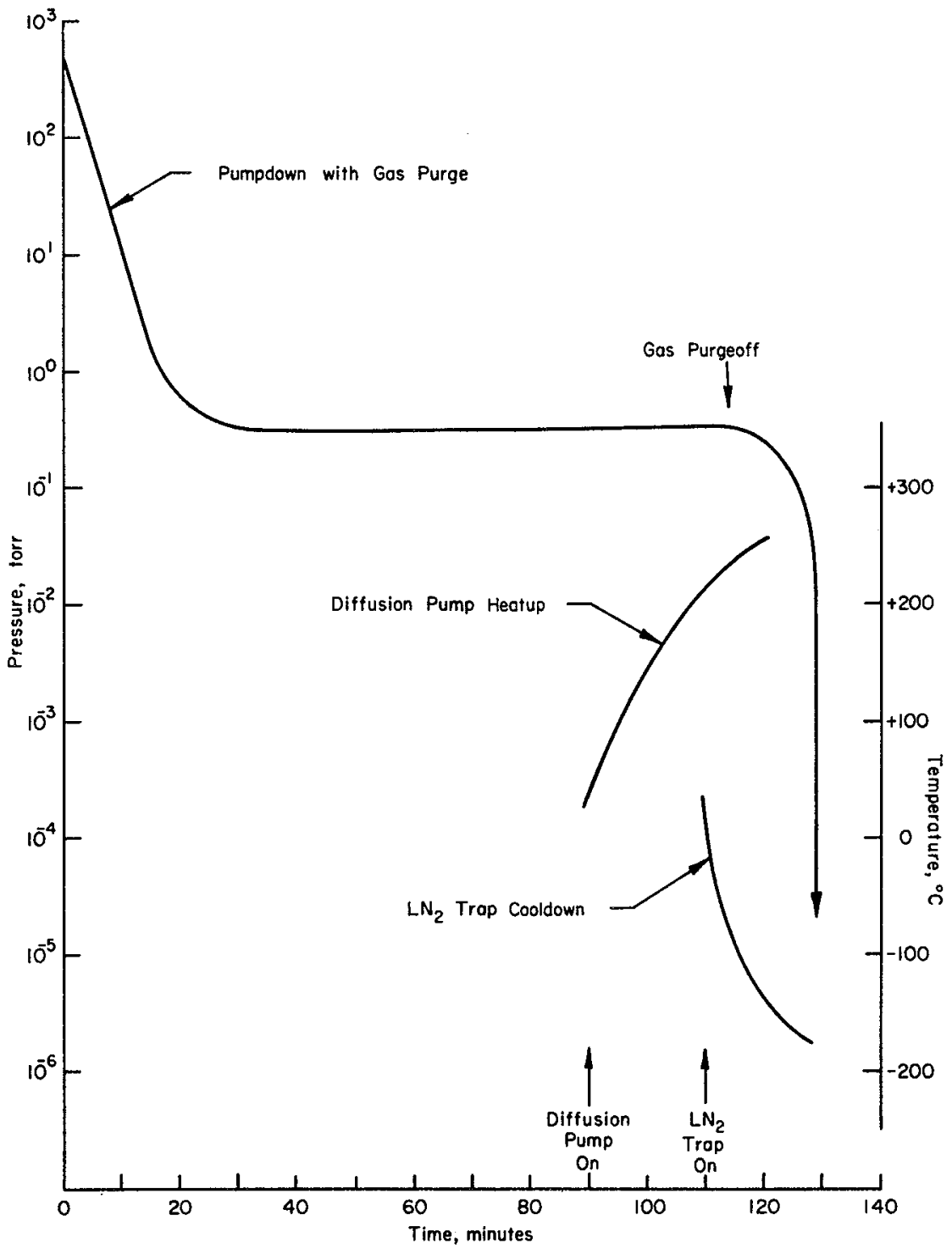

Figure 10.12.-Illustration of the use of the gas purge mode of operation on the initial pumpdown to remove system contaminants, and the use of the operating cycle to transfer to high-vacuum conditions.

water which is coming from the walls of the chamber and the test item. The gas-purge mode of operation may be used to remove an appreciable amount of this moisture which will minimize the amount of contamination built up on the trap. Figure 10.12 illustrates the procedure. The system is pumped down with the mechanical pump while the gas purge 
valve is open. The evaporating moisture from the chamber and the test item is removed by the purge and carried out through the mechanical pump. When the system has dried sufficiently, the trap and diffusion pump are turned on. When the trap is cold, the gas purge may be turned off. The shroud also may be cooled immediately to minimize the amount of water it is evolving.

The procedure for shutting down the system requires that the diffusion pump be turned off first, and allowed to cool while the trap is kept cold. The trap and shroud are then warmed, and the system is vented back to atmosphere. Since gas purge is again a preferable mode of operation, it is turned on as soon as the diffusion pump has reached a safe temperature. The shroud and trap cooling may be removed at this point. As the trap warms up, the evaporating condensables are picked up by the gas purge and carried out of the system. When all surfaces have returned to the ambient temperature, the system is vented to dry nitrogen.

\section{System Operation Example 3: Valved, Nonbaked Vacuum Chamber}

When vacuum systems use high-vacuum and transfer-vacuum valves, a slightly different system-operating cycle is used. A general system schematic is shown in figure 10.13. The high-vacuum pumping system from the holding pump up to the high-vacuum valve may be exhausted initially following the procedure described for nonvalved systems. The same care is required in order to prevent either mechanical pump or diffusion pump oil from passing the trap and entering the diffusion pump side of the high-vacuum valve. As a result, the trap must be cold before the pressure falls into the free-molecular-flow region.

To achieve chamber pumpdown, valve $V_{3}$ is closed and valve $V_{4}$ is opened to allow the main roughing pump to exhaust the chamber. When the pressure reaches a few hundred microns, depending on the chamber and line size, valve $V_{4}$ is closed and valves $V_{3}$ and $V_{1}$ are opened. The chamber exhaust continues through the high-vacuum pumping system. When the chamber pressure has reached a sufficiently low value, valve $V_{3}$ may be closed, and the smaller holding pump is left to back up the diffusion pump. If the large roughing pump is shut down, it should be vented to atmospheric pressure.

The chamber may be vented back to atmospheric pressure if the highvacuum valve is closed. Therefore, the cycle may be repeated rapidly. The presence of the valve will cause the system ultimate pressure to rise as a result of the additional outgassing load and the restricted pumping speed. A complete system shutdown preferably is obtained when the high-vacuum valve is opened and the system is shut down in the manner previously described for the nonvalved systems using gas purge. A separate shutdown of the high-vacuum pumping system with the highvacuum valve closed will generally result in contamination of the diffu- 


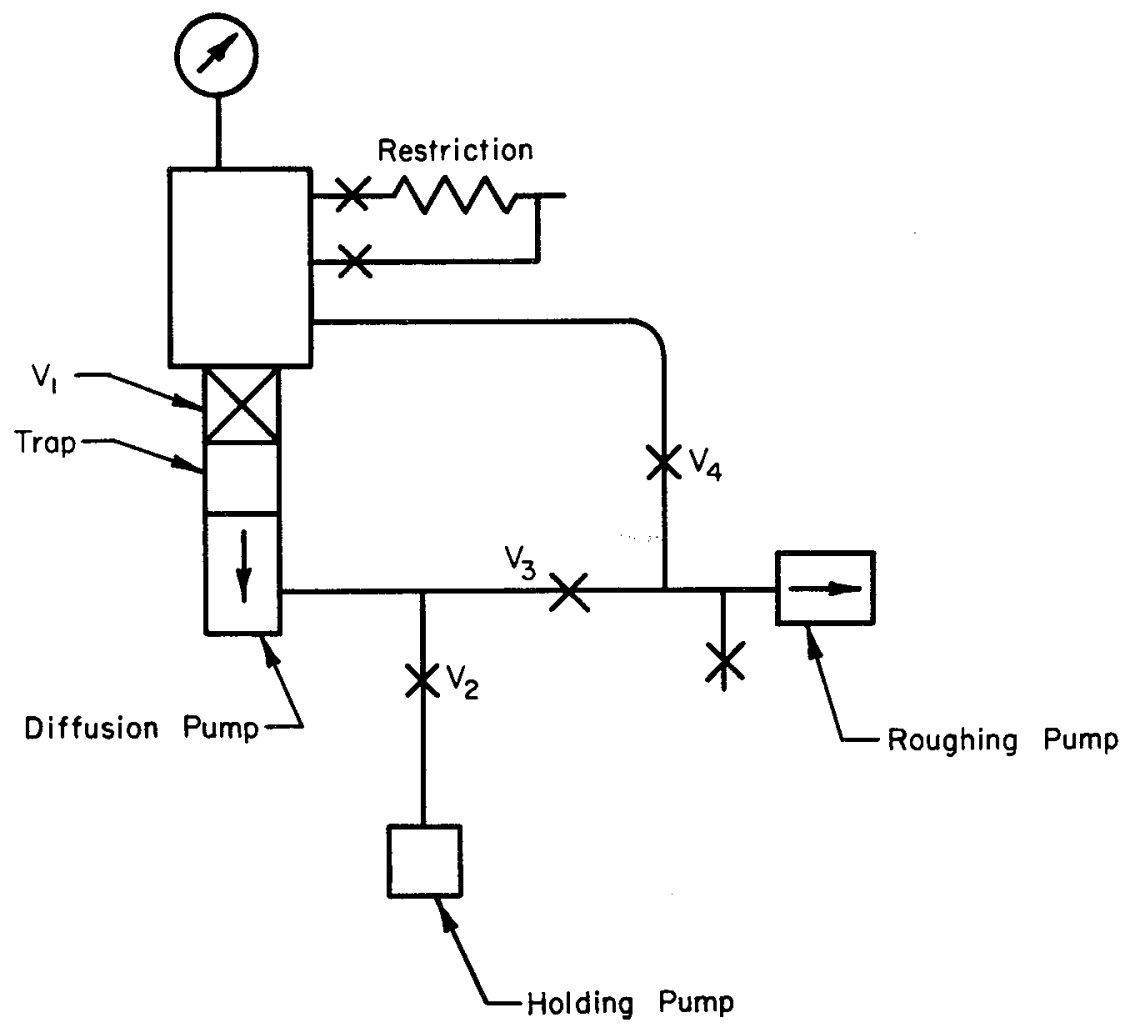

Figure 10.13.-Schematic diagram of a typical nonbaked system using high-vacuum and transfer-vacuum valves.

sion pump side of the high-vacuum valve. This contamination will be transferred to the system during future operating cycles.

\section{System Operating Cycle Example 4: Baked, Valveless System}

Baked-vacuum systems seldom contain a high-vacuum valve, since the longer cycle time required for a bakeout removes the advantage gained with the high-vacuum valve; namely, a very short cycle. The short time required for the diffusion pump warmup is negligible when the resulting advantages of reduced system cost and improved vacuum level are considered. The major drawback of this system is that of the combination of the bakeout and the liquid nitrogen trap. If the trap is not baked out, it will introduce contamination to the system, since it is both a nonbaked portion of the high-vacuum system, and a possible source of contamination, such as carbon dioxide, which it may have collected during the bakeout. If the trap is baked out during the normal system bakeout protection from the diffusion pump oils has not been 
provided. While it is true that the pump oil contaminants can be removed quickly from the system after bakeout, the test item has been exposed to a contaminating environment during the bakeout. As a result, any sensitive surfaces may be modified. Two traps used in series will overcome this difficulty. The trap nearest the system is baked out with the system, while the trap nearest the diffusion pump is kept cold. Unfortunately, the problem still is not totally eliminated, and there is a reduced pumping speed for the noncondensable gases from the system. The following procedure is presented as one method which will attain the desired ultrahigh vacuum.

One of the primary reasons behind a prolonged high-temperature bakeout is the desire to develop a diffusion gradient for hydrogen and carbon monoxide within the metal structure of the chamber walls. Once this gradient has been established, a complete high-temperature bakeout of the entire system is seldom required. The initial bakeout may be accomplished under a gas bleed, by permitting the hydrocarbon contamination to occur while the chamber is empty, or by placing the parts in a larger vacuum furnace. This first high-temperature bakeout, which should include the entire trap, the diffusion pump, and the jet structure within the diffusion pump, will reduce the prolonged break-in time for the pump. A complete bakeout can be obtained if the gas-purge approach is used prior to the addition of oil to the diffusion pump.

For system operation, the chamber is initially exhausted as in the nonbaked vacuum system procedure. Bakeout is instigated while the liquid nitrogen trap is cold. At the end of the bakeout cycle and prior to the start of cooldown, the liquid nitrogen in the trap is removed and the trap is allowed to warm up. The temperature and pressure are carefully monitored. The pressure will rise to a peak value, and will recover as the carbon dioxide is evolved. Other pressure peaks may be observed, depending on what has been condensed on the trap; for example, sulfur dioxide or butane. When the trap reaches a temperature between $135^{\circ} \mathrm{K}$ and $150^{\circ} \mathrm{K}$, the warmup is stopped and the trap is recooled. At this temperature, all critical limiting condensables will have been removed, while all of the diffusion pump hydrocarbons will still remain. Since the main chamber is hot, there will be no readsorption on the walls of the chamber due to the fact that the high-vacuum pumping system is removing the gas. Following the cooldown of the trap, the chamber bakeout is stopped and the system is permitted to return to ambient temperature. This operating cycle should be carefully attended so that the trap will not become too warm with the subsequent contamination of the system. The use of the above trap deactivation cycle at the end of bakeout on a system which has been previously and completely baked out at a high temperature will result in a rapid pressure reduction to the low $10^{-11}$ torr range. The shutdown procedure is the same as for nonbaked systems. 


\section{TROUBLESHOOTING DIFFUSION-PUMPED SYSTEMS}

This outline summarizes many of the diffusion-pumped vacuum system problems which can occur.

I. Malfunction of Mechanical Pumping

A. Mechanical pump does not turn over

1. Control system is malfunctioning-check electrical power to pump motor

2. Motor is defective-motor is too small for pump size

3. Broken V-belts

4. Pump is cold with resulting high oil viscosity-warm the pump up to normal room temperature

5. Pump is oil bound-turn over pump once by hand or jog the motor until the oil is cleared

6. Oil is contaminated thus causing high viscosity-flush pump with clean oil and refill

7. Pump is seized-pump must be repaired

B. Mechanical pump turns over but pressure drops only slightly

1. Foreline valve is closed

2. Mechanical pump oil level is low

3. System has a large leak or a number of large leaks

a. Cover pump exhaust with hand to determine if air is actually being pumped

b. Close foreline valve to find leak position, chamber side or pump side

c. Search for leak by listening; stethoscope is helpful

d. Probe for leaks with helium; leak is indicated by a change in the pump sound

e. Do not overlook the possibility of an open vent valve

C. Pressure drops, but not to the proper level

1. Mechanical pump oil level is low

2. System has a leak or a number of leaks

a. Cover pump exhaust with hand to determine if air is actually being pumped

b. Close foreline valve to find leak position, chamber side or pump side

c. Probe for larger leaks with stethoscope or helium; a change in the pump sound can be distinguished if the oil-mist filter is removed from the pump exhaust

d. Probe for smaller leaks with acetone or helium; watch for deflections on a thermocouple or similar type of gage 
3. Chamber is contaminated with liquid water

a. Characteristic pressure range is 4 to 10 torr

b. Cold spots can sometimes be found on chamber walls where water has collected inside

4. Pump oil is contaminated with water or other condensables

a. Characteristic pressure for water contamination is in the range 0.1 to 1 torr

b. Run pump in atmospheric air for a few minutes and then blank off; if pressure drops to a lower value than before and then rises again, there are probably condensables in the oil

c. Change oil, run pump on gas ballast, or run with dry gas purge at about $\frac{1}{2}$ torr until oil cleans up

5. Gas load in chamber is too high

6. Pressure gage is defective

II. Malfunction of Diffusion Pumping

A. Diffusion pump does not start pumping

1. Pump is not receiving power

2. Foreline or high vacuum valve is closed-foreline may be filled with oil

3. Forepressure is too high-check section I

4. Gas load is too high

a. Fill liquid nitrogen trap; if the load is condensable gas, the thamber pressure will fall

b. Gas leaks must be found with the leak detector, as an ionization gage is usually at too high a pressure to be useful at this stage

5. Diffusion pump oil level is too low

6. Diffusion pump is improperly assembled

B. Diffusion pump starts, but does not pump to a low enough level

1. Pump speed is low

a. If the system has a high-vacuum valve, close it and measure the time rate of pressure rise, $d P / d t$ in the chamber; compute speed from $S=(V / P)(d P / d t)$

where

$S$ speed, liter/sec

$V$ chamber volume, liter

$P$ chamber pressure, torr

$t$ time, seconds

The measured speed should fall somewhere between the baffled pump speed for permanent gases and the trap speed for condensables

b. If the system has no high-vacuum valve, close the foreline valve, measure the time rate of pressure rise in the 
foreline, and compute the speed from $S=(V / P)(d P / d t)$

where

$V$ foreline volume, liter

$P$ foreline pressure, torr

$t$ time, seconds

c. Diffusion pump oil level is low

d. Diffusion pump oil is contaminated or the wrong oil is in pump-broken-down oil can frequently be detected by an acrid, burnt odor

e. Diffusion pump is improperly assembled; nozzle stack loose

f. Diffusion pump cooling water flow is too high or too low

g. Boiler pressure is low

(1) If the oil recommended by the manufacturer is being used, check input power

(2) If oil other than the recommended oil is being used, experiment with heater power and cooling water flow

2. Ionization gage is defective

a. Check zero and emission settings of the ionization gage

b. Check the linearity of the gage reading with emission current-nonlinearity indicates a gassy gage tube or electrical leakage between filament and grid

c. Degas the gage tube

d. Remove the collector lead first from tube and then from the amplifier input. If pressure is indicated, electrical leakage in collector circuit is present

e. Ionization gage can be checked approximately against the forepressure gage by the expression

where

$$
P_{c}=\frac{S_{m}}{S_{d}} P_{f}
$$

$P_{c} \quad$ chamber pressure, torr

$P_{f} \quad$ foreline pressure, torr

$S_{m} \quad$ mechanical pumping system speed, liter/sec

$S_{d} \quad$ diffusion pumping system speed, liter/sec

This expression does not account for condensables pumped by diffusion pump trap. These condensables can cause the ionization gage to read a higher pressure

3. Gas load is high

a. High gas load from test being conducted-wait for outgassing to decrease

b. High gas load from system

(1) Helium can be used to probe for leaks, watching for depression of the ionization gage indicated pressure 
(2) Use leak detector attached to system foreline

(3) Various system parts may be contaminated and outgassing

a. Bake system with or without dry nitrogen bleed

$b$. If a bake is not possible, hold on the system dry nitrogen bleed

c. A badly contaminated system must be opened and cleaned

c. If the system has a partial-pressure analyzer, it should be used to detect the source of the gas load (reference 32). Some detected gases and their possible sources are:

(1) $\operatorname{Air}\left(\mathrm{N}_{2}, \mathrm{O}_{2}, \mathrm{~A}\right)$ : air leak, virtual leak

(2) $\mathrm{N}_{2}$ : leak in liquid nitrogen trap or shroud: leak in vent or purge valve

(3) $\mathrm{H}_{2} \mathrm{O}$ : outgassing, coolant leak

(4) Hydrocarbons: chamber contamination, solvents, pump backstreaming, pump oil decompositions; the presence of equal amounts of $\mathrm{H}_{2}$ and $\mathrm{CH}_{4}$ below $10^{-7}$ torr is an indication of silicone oil decomposition

(5) $\mathrm{CO}_{2}$ : outgassing, if in $10^{-8}$ torr range it could indicate $\mathrm{CO}_{2}$ condensed on the liquid nitrogen trap; let trap warm slightly to drive off the $\mathrm{CO}_{2}$

(6) $\mathrm{H}_{2}, \mathrm{CO}$ : these gases predominate in baked systems, and are produced from hot metal surfaces

(7) He: leaking cryopanels; holdover from helium leak checking; permeation can have long life

\section{Pressure Fluctuations}

A. Fast time constant pressure fuctuations, seconds to minutes

1. Leaks are blurping

a. Leak past greasy or oily O-ring seal

b. Leak in the drain plug of the diffusion pump boiler

2. Diffusion pump boiler is surging

a. Check heater power and cooling water

b. Oil level is low

c. Oil is contaminated or degraded

3. Forepressure is cycling

a. Oil level in mechanical pump is low

b. Oil passages in mechanical pump are clogged

4. Mechanical pump is too small at high throughput rates

5. Ionization gage control is malfunctioning

B. Slower time constant pressure fluctuations, minutes to hours

1. Ambient temperature is changing

a. Outgassing rate of chamber is changing 
b. Outgassing rate of ionization gage is changing; tubulated gages can respond very quickly to drafts

c. Reevaporation from unshielded temperature gradients in traps and cryopumps is occurring (chapter 7)

2. Liquid nitrogen level in trap is changing (see sec. B.1.3)

3. High heat load is being varied or moved on condensation pumping surface; i.e., solar simulation on cryopumping surfaces

\section{Pressure deterioration after a long time}

1. Oil migration

a. Migration of mechanical pump oil into the diffusion pump boiler

b. Migration of diffusion pump oil past trap into the chamber

2. Saturation of pumping surfaces

a. Condensed deposits on the trap and cryopump surfaces have built up until the outer layers are no longer cold

b. A very clean surface such as baked chamber walls; will act as a pump until saturated

c. Surfaces may be cleaned of many contaminants by subjecting them to a dry nitrogen purge of about $\frac{1}{2}$ torr at temperatures a few hundred degrees centigrade above their normal operating temperature

\section{REFERENCES}

1. Reich, H.: High Vacuum Technology in a Space Research Laboratory. Trans. of the Fifth National Symposium on Vacuum Technology, W. G. Matheson, ed., Pergamon Press, 1959, pp. 279-282.

2. Simons, J. C.: An Ultra High Vacuum Chamber for Space Simulation. Trans. of the Sixth National Symposium on Vacuum Technology, C. R. Meissner, ed., Pergamon Press, 1960, pp. 48-54.

3. JAsper, P E..: Vacuum System Requirements for Electrical Space Propulsion Engines. Trans. of the Seventh National Symposium on Vacuum Technology, C. R. Meissner, ed., Pergamon Press, 1961, pp. 155-160.

4. KfLLER, T. A.: NASA Electric Rocket Test Facilities. Trans. of the Seventh National Symposium on Vacuum Technology, C. R. Meissner, ed., Pergamon Press, 1961, pp. 161-167.

5. Santelar, D. J.: Pressure Simulation of Outer Space. Trans. of the Sixth National Symposium on Vacuum Technology, C. R. Meissner, ed., Pergamon Press, 1960, pp. 129-133.

6. Simons, J. C.: Effects of Extreme Altitudes, and the Need for Simulation. Proceedings of the Institute of Environmental Sciences, Institute of Environmental Sciences (Mount Prospect, Ill.), 1960, pp. 5-10.

7. Srmpson, M. H.: Analysis of Hyper Environments and Their Relation to Military Hardware in the Interior of a Space Vehicle. Proceedings of the Institute of Environmental Sciences, Institute of Environmental Sciences (Mount Prospect, Ill.), 1960, pp. 11-18. 
8. Wacholder, B. V.; aNd Fayer, E.: Critical Environments Encountered by High-Altitude Vehicles. Proceedings of the Institute of Environmental Sciences, Institute of Environmental Sciences (Mount Prospect, Ill.), 1960, pp. 19-36.

9. Gelmard, J. C.; and Meyer, S. W.: Design Solution for a Space Environment Simulator. Proceedings of the Institute of Environmental Sciences, Institute of Environmental Sciences (Mount Prospect, Ill.), 1961, pp. 335-347.

10. Camack, W. G.; and Elisis, C. A.: A High Vacuum Orbital Simulator. Trans. of the Eighth National Vacuum Symposium and the Second International Congress on Vacuum Science and Technology, vol. II. L. E. Preuss, ed., Pergamon Press, 1962, pp. 1260-1269.

11. John, J. E. A.; AND Hardgrove, W. F.: Creation of Space Vacuum Utilizing Cryogenic Techniques. Proceedings of the Institute of Environmental Sciences, Institute of Environmental Sciences (Mount Prospect, Ill.), C 1964, pp. 619-624.

12. Youna, K. G.; and Gamble, M. I.: The Development and Operational Characteristics of a Unique Combined Environment Space Simulator. Proceedings of the Institute of Environmental Sciences, Institute of Environmental Sciences (Mount Prospect, Ill.), C 1964, pp. 577-583.

13. Le Vantine, A. D.: Space Thermal Environment Simulation Facilities. Trans. of the Eighth National Vacuum Symposium and the Second International Congress on Vacuum Science and Technology, vol. II. L. E. Preuss, ed., Pergamon Press, 1962, pp. 1270-1278.

14. Le Vantine, A. D.: Thirty Foot Space Thermal Environment Facility With New Solar Simulator. Trans. of the Ninth National Vacuum Symposium. G. H. Bancroft, ed., The Macmillan Co., 1962, pp. 260-265.

15. Lengyri, A.; Marfone, P. A.; And Santeler, D. J.: Design Criteria for a Space Environment Simulator. Trans. of the ASME, paper no. 61-AV-52, 1961.

16. Philisips, W. H.; Quijo, M. J.; ANd Adams, J. J.: Langley Research Center Simulator Facilities for Manned Space Missions. Trans. of the ASME, paper no. 63-AHGT-91, 1963.

17. Kerfoot, H. P.; GaUmer, R. E.; ANd SoKolsky, S.: Environmiental Simulation. Space/Aeronautics, vol. 38, no. 5, pt. 2, Oct. 1962, pp. 15-20.

18. Jones, D. W.; AND Tsonis, C. A.: Theoretical Investigation of Oil Backstreaming Through a Trap. J. of Vac. Sci. \& Tech., vol. I, no. 1, Sept./Oct. 1964, pp. 19-22.

19. Wheeler, W. R.: Theory and Application of Metal Gasket Seals. Trans. of the Tenth National Vacuum Symposium, G. H. Bancroft, ed., The Macmillan Co., 1963, pp. 159-165.

20. Mark, J. T.; ANd Dreyer, K.: Ultra High Vacuum System Developments for the Model C-Stellarator. Trans. of the Sixth National Symposium on Vacuum Technology, C. R. Meissner, ed., Pergamon Press, 1960, pp. 176-180.

21. FArKass, I.; ANd Barry, E. J.: Improved Elastomer Seal Designs for Large Metal Ultra-High Vacuum Systems Permitting Ultimate Pressures in the Low $10^{-10}$ Torr Range. Trans. of the Seventh National Symposium on Vacuum Technology, C. R. Meissner, ed., Pergamon Press, 1961, pp. 35-38.

22. Brothers, C. F.; Tom, T.; and Munro, D. F.: Design and Performance of a 50000 Liter/Sec Pump Combining Cold Cathode Ion Pumping and Active Film Gettering. Trans. of the Tenth National Vacuum Symposium, G. H. Bancroft, ed., The Macmillan Co., 1963, pp. 202-207. 
23. Batzer, T. H.; ANd Ryan, J. F.: Some New Techniques in Ultra High Vacuum. Trans. of the Tenth National Vacuum Symposium, G. H. Bancroft, ed., The Macmillan Co., 1963, pp. 166-169.

24. Turnaull, A. H.; Barton, R. S.; and Riviere, J. C.: An Introduction to Vacuum Technique. John Wiley \& Sons, Inc., 1963.

25. Guthrie, A.: Vacuum Technology. John Wiley \& Sons, Inc., 1963.

26. Ellman, R. P.: Trouble Shooting Vacuum Systems With a Mass Spectrometer. Research/Development, vol. 15, no. 7, July 1964, pp. 45-57.

27. John, J. E. A.; ANd Hardqrove, W. F.: Cryosorption-A Method for Simulating the Space Vacuum Environment. AIAA Publication CP-11, Nov. 1964, pp. 1-7. 



\section{Leak Detection}

\section{INTRODUCTION}

A

LL THE SCIENTIFIC ADVANCEMENTS of modern technology still have not been able to achieve an absolutely leaktight material. Although minute porosities may not be present in a material, there is still the possibility of the existence of the permeation of certain gases through materials such as crystals, polymers, and glasses.

The term "hermetic," which generally applies to the condition of leaktightness, has a multitude of interpretations. Since each interpretation has a particular shade of meaning of the term, such a range of meaning exists that the term has been applied both to a bucket containing water and to an ultra-high-vacuum system. In each case, although the degree of leaktightness depends on the particular requirements, each dictates that the flow be too small to permit measurement. As vacuum technology. applications increased and more complicated systems demanded a greater degree of leaktightness, it became necessary to establish a practical level of leak-detection sensitivity for any given system or component under test.

The establishment of this leak-detection sensitivity level has given rise to two schools of thought on the procedure to be followed. One approach is that it is necessary to establish the leak size for any given test or process which could be detrimental to its successful completion. As a result, the leak-test procedure established would operate at a level which would give a margin of safety better than the required level. The second approach views all leaks as flaws or weaknesses.

In the first approach for an operating vacuum system, the basic vacuum equation $Q=P S$, which relates the pumping speed of the system and the permissible background pressure, can be used to evaluate the tolerable-size leak. A vacuum chamber of a medium size which is operating at an assumed pumping speed of $10^{\mathrm{s}} \mathrm{liter} / \mathrm{sec}$ and a base pressure of $10^{-8}$ torr, could function with a total leakage rate of $10^{-5}$ torr liter/sec. Much larger leaks could be tolerated in very large space-simulation chambers which possess pumping speeds in excess of $10^{5}$ liter/sec. However, in the case of small ultra-high-vacuum research systems which, for 
example, may operate in the $10^{-11}$ torr range with a 100 -liter/sec pumping speed, all leaks greater than $10^{-9}$ torr liter/sec must be eliminated.

Since the second approach views all leaks as flaws or weaknesses, it is thought that the smallest leak, or even flaws which do not exhibit leakage at the time of test, could grow larger and present a potential hazard to system operation. As a result, the leak-detection level based on this consideration would be established at the practical rejection level where the cost of further increased sensitivity could not be justified by the small number of additional leaks which may be found. A helium mass spectrometer leak detector is used for most vacuum system testing, initial testing at least. The test sensitivity may vary between $10^{-9}$ torr liter/sec and $10^{-6}$ torr liter/sec, depending upon the factors of system size and complexity, and background. Since this sensitivity generally is greater than that required by the designed ultimate pressure, the additional sensitivity assures further overall system integrity.

For practical considerations, vacuum engineers merge the two philosophies to achieve a testing program which is applicable to the operation of most types of vacuum systems. Thus, as long as the system ultimate pressure meets the test requirements, leak-detection procedures are not necessary. In the event that excessive pressures exist, the critical leaks are located and repaired until the ultimate pressure requirement is achieved. The recommended vacuum technique calls for a periodic system overhaul during which it is customary to perform a high-sensitivity leak check to locate and repair minute leaks which could have developed in the interim.

The development of leak-detection techniques has produced a variety of terms and sets of units relating to leak-detection procedures and processes. The term of primary concern in any discussion of leak detection is the leakage or mass flow rate which has the dimensions of pressure-volume per unit of time, or torr liter/sec. Conversion factors to other sets of units are presented in chapter 1.

There is some confusion regarding the terms "leak" and "leakage." When a fluid flows through a small orifice, the rate of flow depends on the orifice geometry and the prevailing conditions of fluid pressure and temperature, as well as fluid type. The term "leak" conventionally refers to the physical orifice which exists independently of the quantity of fluid flowing, while the term "leakage" refers to the actual flow or leak rate which is dependent on the gas type and driving pressure. As a result, a minimum detectable leak is the dimensionally smallest hole which can be detected, and the minimum detectable leakage is the minimal fluid flow rate that can be detected.

In connection with leak detection, it is apparent that since the sensing element responds to the quantity of tracer gas present, it will also respond to a flow rate, or leakage, of tracer gas. Leak-detector sensi- 
tivity is synonymous with the minimum detectable leakage. It does not matter to the detector whether this gas flow originates from a small leak with a high driving pressure, or from a larger leak exposed to a lower pressure.

Even though the pressure-dependent nature of gas flow through a leak does not affect the sensitivity of the leak detector in terms of its ability to see a particular minimum gas flow rate, it is apparent that this pressure dependency does have an effect on the sensitivity of a particular leak test. Increasing the driving pressure on a leak will increase the gas flow through the leak, and will permit the detection of smaller physical leaks with a detector of a given sensitivity. Leakage is readily measured in terms of actual gas flow. A question arises as to how the leak size is measured. Since it is generally not possible to measure the physical dimension of a leak, it may be determined by measuring the amount of leakage which it passes under a given set of conditions. These prevailing conditions must be considered in any definition of a leak due to the fact that the leakage rate is dependent upon the conditions. When a leak is described and only the leakage is presented, it must be assumed that the leakage is referred to standard conditions which are defined as dry air at $25^{\circ} \mathrm{C}$, from a pressure of one standard atmosphere to a vacuum (approximately 1/100 of an atmosphere). To summarize the above discussion, the sensitivity of leak-test equipment is synonymous with the minimum detectable leakage or the minimum required flow rate, both of which are independent of the external test conditions. The test sensitivity for a particular application depends on the particular conditions of fluid pressure, temperature, and concentration.

\section{GAS FLOW THROUGH LEAKS}

The gas-flow equations of chapter 5 define the leakage through a true leak or physical restriction as a function of gas type, pressure, and temperature. The use of the equation for a large $\ell / d$ ratio is justified if leaks are considered to be long capillaries. Generally, it is not possible to ascertain the type of gas flow through a leak, whether it is viscous or molecular, due to the fact that the average leak dimensions cannot be evaluated exactly, since the physical leak in the wall of a vacuum chamber may be a material porosity, a thin crack, or a single hole. Numerous observations (references 1 and 2) have shown that leaks with a leakage rate of $10^{-8}$ torr liter/sec or less generally exhibit molecular flow, while leaks with a leakage rate greater than $10^{-5}$ torr liter/sec are in the viscous flow regime.

Many leak-detection techniques require the determination of the leakage changes which occur when a tracer gas is applied to a leak in 
the wall of a vacuum chamber. The following relationship holds for molecular flow:

where

$$
\begin{aligned}
Q_{t} & =Q_{a}\left(\frac{M_{a}}{M_{t}}\right)^{1 / 2} \\
& =Q_{a} \mu
\end{aligned}
$$

$a$ refers to air conditions

$t$ refers to tracer gas conditions

The quantity $\mu$ will signify $\left(M_{a} / M_{t}\right)^{1 / 2}$ in the derivations to follow. Equation (11.1) indicates that a considerable change in flow rate can be obtained if a very light gas such as helium or hydrogen, or a very heavy gas such as a hydrocarbon, is used.

The relationship between air leakage and tracer gas leakage for laminar viscous flow is

$$
\begin{aligned}
Q_{t} & =Q_{a} \frac{\eta_{a}}{\eta_{t}} \\
& =Q_{a^{v}}
\end{aligned}
$$

The quantity $v$ will signify the viscosity ratio of air to tracer gas in the following derivations. When it is necessary to leak-test large viscous leaks, the preferred test gas is one which has a viscosity considerably different from air so that a large change in the flow rate for the tracer gas can be obtained.

If the change in conditions between leak-test procedures and the operation of the system is to involve a change in temperature, pressure, or gas type, the complete molecular or viscous flow equation must be used. Such a situation will be encountered when there is an internal pressure on the vacuum system and a sensing technique such as a halogen leak detector is used. Since pressure techniques in vacuum systems generally are used for gross leak detection, the location of the leak is of interest rather than its size. The nomograph techniques of reference 3 provide an efficient means of converting to different conditions of gas type or pressure. When the flow of gases in leaks is considered, questions will arise concerning unidirectional leaks, pressure-dependent leaks, and temperature-dependent leaks. Generally, since leaks are small tubes, it can be assumed that the gas can pass as easily in one direction as in the other. While there may be small differences in the flow rate for the two different directions due to a change in the entrance-loss configuration, the effect is negligible.

Unidirectional leak effects can be illustrated through the examination of a check-valve effect which involves a small loose particle within the leak. If the particle is free to move, it could constitute a unidirectional shutoff valve. However, this situation requires a good seating arrange- 
ment, and it is seldom encountered in practice. Occasionally, soldered, brazed, or welded joints may exhibit drastic variations in leakage rate when a differential pressure across a system wall is inverted, due to the difference in mechanical deformation of the various joints. However, as a general rule, most leaks do not show a directional-pressure effect. On occasion, moisture plugging may be misinterpreted as a unidirectional leak. It is accepted practice to apply a leak-detection process in either direction.

Both pressure- and temperature-dependent leaks may exhibit little or no leakage until a critical pressure or temperature is exceeded, at which point a large leakage occurs. Further changes in pressure or temperature cause the leakage to behave in the expected manner. If the pressure or temperature conditions are reversed, another critical point is reached at which the leakage again exhibits a large change. In this instance the leakage rate may become too small to be detected. Temperature-dependent leaks such as those which have been observed in liquid-nitrogen-cooled cryogenic panels are generally extremely difficult to locate. However, this type of leak is quite rare. A shock technique employing pressure and temperature variations may be applied to a system to open possible flaws and weaknesses in the structure. If the resulting leak remains once the environmental conditions return to their initial values, the leak can be located. Corrective action can then be taken to increase the overall reliability of the structure or system. This technique is particularly valuable during the construction of a complex system where a particular joint may not be accessible for repair at a later stage in the system construction or checkout.

\section{BASIC EQUATIONS OF STEADY-STATE LEAK DETECTION}

While there are a number of leak-detection methods available for use, only those which involve the effect of a gas flow on some form of sensing element will be treated in this discussion. In addition, the test methods will be further restricted to those which employ a gas flow from atmospheric pressure to a vacuum. Such techniques permit vacuum system leak checking to be accomplished more readily and with a higher sensitivity. While a brief discussion of the pressure methods of leak detection is included in the discussion to follow, principal attention will be directed to the vacuum methods of leak detection.

The following list of subscripts used in the derivations and equations of this chapter specify the various conditions at which the quantities are measured:

$b$ background gas, not related to a particular leak

a air, pertaining to a particular leak

$t$ tracer gas, pertaining to a particular leak

- initial conditions

- no subscript, final conditions 
When steady-state, leak-detection conditions are considered, it is assumed that the speed for a given gas type is constant over the limited range of pressure changes during the course of leak detection. Several gas sources, each divided by its respective pumping speed, comprise the quasi-steady-state pressure existing in a vacuum system. $P_{0}$, the initial pressure condition, is expressed as the sum of the pressure due to the particular leak being detected, plus the pressure due to all other background sources. Thus

$$
\begin{aligned}
P_{0} & =P_{a}+P_{b} \\
& =\frac{Q_{L_{a}}}{S_{a}}+P_{b}
\end{aligned}
$$

If the air on the atmospheric side of the leak is replaced by a tracer gas, the vacuum system pressure signal from the air leak will be replaced by a signal from the tracer gas. Thus, when equilibrium has been established

$$
P=P_{0}-P_{a}+P_{t}
$$

Substituting for $P_{a}$ and $P_{t}$

$$
P-P_{0}=\frac{Q_{L_{t}}}{S_{t}}-\frac{Q_{L_{a}}}{S_{a}}
$$

In general, the sensing elements used for leak detection do not respond to the absolute pressure of the gas, but rather to some physical property of the gas, such as its ionization potential. The gage reading for a particular gas specie is obtained when the pressure is multiplied by a gagesensitivity factor $\sigma$

$$
R_{t}={ }_{\sigma} P_{t}
$$

The value of $\sigma$ for air is assumed to equal unity. Therefore, the gage reading for the case of a tracer gas applied to the leak is

$$
R=R_{0}-R_{a}+R_{t}
$$

where the subscript o refers to the initial conditions of background pressure plus air leakage. If equations (11.6), (11.7), and the basic vacuum relationship $P=P_{L} / S$ are combined, the result is

$$
\begin{aligned}
R-R_{0} & =\Delta R \\
& =\frac{\sigma Q_{L_{t}}}{S_{t}}-\frac{Q_{L_{a}}}{S_{a}}
\end{aligned}
$$

where $\Delta R$ is the change in reading observed on the detector. Two assumptions were made to arrive at equation (11.8). The first is that molecular flow exists in the vacuum system. The second assumption is that there is no interaction of the different gases or, in other words, the detector reading is a simple summation of the individual readings for all gas types present. 
To simplify the discussion further, let $r=S_{a} / S_{t}$, and from equations (11.1) and (11.2), $Q_{L_{t}}=Q_{L_{a}} \mu$ for molecular flow, and $Q_{L_{t}}=Q_{L_{a}} v$ for viscous flow. Combining these expressions with equation (11.8) yields for molecular flow and viscous flow, respectively,

$$
Q_{L_{a}}=\frac{\Delta R S_{a}}{(\sigma \mu r-1)}
$$

and

$$
Q_{L_{a}}=\frac{\Delta R S_{a}}{(\sigma v-1)}
$$

The symbol $\omega$ will be used henceforth to signify either $\mu$ or $v$, depending upon whether the leak under consideration experiences molecular or viscous flow. Hence.

$$
Q_{L_{a}}=\frac{\Delta R S_{a}}{(\sigma \omega r-1)}
$$

If $\Delta R$ is the minimum detectable change in reading for a particular sensing element, then $Q_{L_{a}}$ is the minimum detectable leak size. Equation (11.11) may be used to evaluate practically all of the vacuum methods of leak detection. Therefore, the sensitivity of a given method can be determined if the smallest $\Delta R$ which can be unambiguously detected in the presence of the initial reading $R$ is noted, and if the net effect of the tracer gas on the three factors in the denominator can be evaluated.

It must be possible to evaluate the factor $\Delta R$ on the basis of the initial background reading of the detector. Thus, if an ion gage is used as a detector with a minimum detectable change of 5 percent of full scale, and is reading on the $10^{-5}$ torr scale, $\Delta R$ would be $5 \times 10^{-6}$ torr. A reduction of the system background to the $10^{-9}$ torr scale would result in a $\Delta R$ value of $5 \times 10^{-10}$ torr. The leak detection sensitivity in both cases also depends on the selection of tracer gas $(\sigma \mu r-1)$, and the speed of the system for air, $S_{a}$. Eventually, $\Delta R$ will be limited by instability and noise in the amplifier. An initial inspection of equation (11.11) appears to indicate that increased sensitivity can be obtained by decreasing the pumping speed $S_{a}$. Whether or not this occurs depends upon how the minimum detectable value of $\Delta R$ varies with the background reading. If $\Delta R_{\min }$ is a linear function of the initial pressure reading, and the background pressure increases linearly with a decrease in pumping speed, $\Delta R_{\min }$ increases at the exact rate required to compensate for a decrease in $S_{a}$. That is, $\Delta R_{\min } S_{a}$ equals a constant. Thus, the minimum detectable leakage is a fixed fraction of the total gas influx. This example also illustrates the effect of system pumping speed on sensitivity. Since $\Delta R$ is a constant for any particular pressure scale, a decrease in system speed will cause an increase in the test sensitivity up to the point 
that the background signal is increased to full scale. A pressure-scale change will then result in a 10:1 loss in sensitivity.

When a tracer gas is being selected, for a system test, it is necessary to give careful consideration to each of the tracer gas factors. All three of the factors, $\sigma, \omega$, and $r$, may be greater or less than unity. Therefore, in some types of tests, it is possible for the product $\sigma \omega r$ to be nearly equal to unity, which will result in a negligibly small leak-test sensitivity. The optimum sensitivity is realized when the three factors are either all greater than or less than unity. As $\sigma \omega r$ approaches zero, the limit of sensitivity in one direction is reached, and equation (11.11) becomes

$$
Q_{L_{a}}=-\Delta R S_{a}
$$

The minus sign indicates that the reading will decrease toward zero.

A greater sensitivity is possible if the $\sigma \omega r$ product can be made very large. For example, a helium mass spectrometer leak detector has a factor $\sigma$ of the order of $10^{5}$. Generally, the helium leak detector is calibrated to combine all factors in the form

$$
\begin{aligned}
Q_{L_{a}} & =\left(\frac{\Delta R}{\sigma}\right) \frac{S_{a}}{\omega r} \\
& =\left(\frac{\Delta R}{\sigma}\right) \frac{S_{t}}{\omega}
\end{aligned}
$$

where $\Delta R / \sigma$ is the minimum partial pressure of tracer gas which can be detected in the presence of the background pressure reading. If the factor $\omega$ is deleted from the expression, the flow-rate sensitivity of the leak detector proper can be obtained, rather than the overall test sensitivity. $S_{t}$ is the pumping speed of the leak detector for the tracer gas at the sensing element in the absence of other system pumps.

The differential sorbtion detector, another device which bases its high sensitivity on tracer-gas factors, is governed by the factor $r$ which becomes large when a selectively small $S_{t}$ is used.

When there is no selective pumping speed and the vacuum system gas flow is governed by the molecular-flow equations, $r=1 / \mu$. Therefore, if the leak being detected is also in the molecular-flow region, equation (11.9) will apply and the factors $r$ and $\mu$ will cancel. As a result, equation (11.9) reduces to

$$
Q_{L_{a}}=\frac{\Delta R S_{a}}{(\sigma-1)}
$$

It is evident from equation (11.14) that the gage-sensitivity factor is crucial. Therefore, the oft-quoted advantages of a low molecular weight gas such as hydrogen or helium are really not very beneficial unless the gases also exhibit the necessary high gage-sensitivity factor. 


\section{BASIC EQUATIONS OF TRANSIENT LEAK DETECTION}

The previous analysis was based on the assumption that a steady-state condition is reached after the tracer gas is applied to the suspect leak. The time required to reach the steady-state condition is a function of such factors as the volume and the pumping speed of the system. Frequently, the time is so long that the leak-detection equipment must be expected to work in the transient state. Therefore, it is necessary that the engineer possess a full understanding of vacuum leak detection theory and procedure so that the transient-flow equations can be comprehended.

It can be seen in equation (11.7) that while the actual signal due to air flowing through the leak will decrease with time, the amount of air signal $R_{a}$ to be subtracted from the background pressure increases with time. The expressions of chapter 5 may be used to define this timedependent relationship

$$
\begin{aligned}
R_{a} & =P_{a} \\
& =\frac{Q_{L_{a}}}{S_{a}}\left(1-e^{-B_{a} t / \nabla}\right)
\end{aligned}
$$

where $t$ refers to time and $V$ is the system volume. The speed $S_{a}$ is assumed to remain constant over the limited change in pressure $P_{a}$. The tracer-gas signal $R_{t}$ will increase from a value of zero

$$
R_{t}=\sigma P_{t}=\frac{\sigma Q_{L_{t}}}{S_{t}}\left(1-e^{-S_{t} t / v}\right)
$$

If equations (11.15) and (11.16) are combined in equation (11.17), and the same procedure as that for the steady-state flow is followed, expressions for the detectable leak size as a function of $\Delta R$, the system speed $S_{a}$, the tracer gas parameters $\sigma \omega r$, and time can be obtained

$$
Q_{L_{a}}=\frac{\Delta R S_{a}}{\sigma \omega r\left(1-e^{-S_{t} t / v}\right)-\left(1-e^{-S_{a} t / \bar{V}}\right)}
$$

where $\omega$ refers to the square root of the air to tracer-gas gram molecular weight ratio if the leak is in molecular flow, or to the air to tracer gas viscosity ratio if the leak is in viscous fiow.

The previously stated conditions which concerned the effect of the background pressure on the minimum detectable reading change $\Delta R$, the overall effect of $S_{a}$, and the tracer-gas factors are again applicable in this situation.

When there are two different time constants present, an interesting situation may result. That is, if $S_{t}$ is considerably different from $S_{a}$, the faster speed will determine the initial transient which may be in the opposite direction from the steady-state detector reading change (fig. 11.1). An illustration of this frequently occurs in getter-ion pumped 


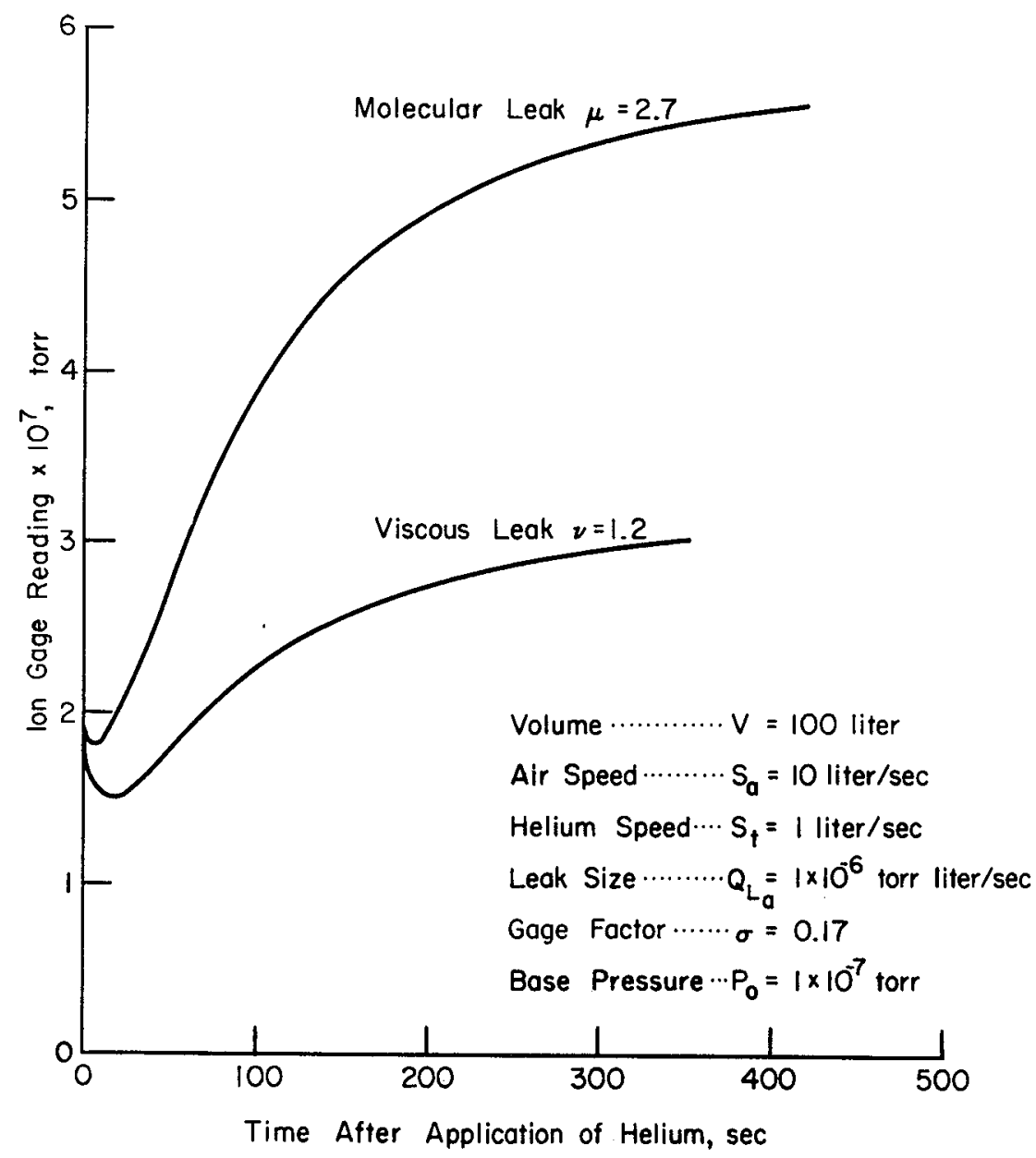

FIGURE 11.1.-Response of sputter ion pumped system to application of helium tracer gas to a leak. Since $S_{t}$ differs from $S_{a}$, the faster speed will determine the initial transient which may be in the opposite direction from the steady-state gage reading change.

systems due to the variable pumping speed for different gases (reference 4).

If it is assumed that the speed of the system for the tracer gas is essentially the same as the speed for air, equation (11.17) may be reduced to

$$
Q_{L_{a}}=\frac{\Delta R S_{a}}{(\sigma \omega-1)\left(1-e^{-S_{a} t / \nabla}\right)}
$$


When the leak detector is sensitive to the tracer gas only, as for example, a helium leak detector or a residual gas analyzer, the air signal decay becomes insignificant and equation (11.17) becomes

$$
\begin{aligned}
Q_{L_{a}} & =\frac{1}{\sigma \omega \eta} \frac{\Delta R S_{a}}{\left(1-e^{-S_{t} t / v}\right)} \\
& =\frac{\Delta R}{\sigma} \frac{S_{t}}{\omega\left(1-e^{-S_{t} t / v}\right)}
\end{aligned}
$$

where $\Delta R / \sigma$ defines the minimum partial pressure of tracer gas which can be detected. At $t=\infty$, equation (11.19) reduces to equation (11.13).

It is appropriate at this time to examine the effect of the time constant on the signal. This effect is quite important in many leak-detection applications and may be examined by rearranging equation (11.17) and solving for $\Delta R$, the change in reading as a function of time for a given leak

$$
\Delta R=\frac{Q_{L_{a}}}{S_{a}}\left[\sigma \omega r\left(1-e^{-S_{t} t / v}\right)-\left(1-e^{-S_{a} t / V}\right)\right]
$$

If it is assumed that $S_{a}=S_{t}=S$, the equation reduces to a single exponential

$$
\Delta R=\frac{Q_{L_{a}}}{S}(\sigma \omega-1)\left(1-e^{-S t / v}\right)
$$

Figure 11.2 illustrates the plot of equation (11.21) versus time.

A useful quantity to know is the time required to obtain 63 percent of the final signal; that is, the time required to obtain a full signal on the

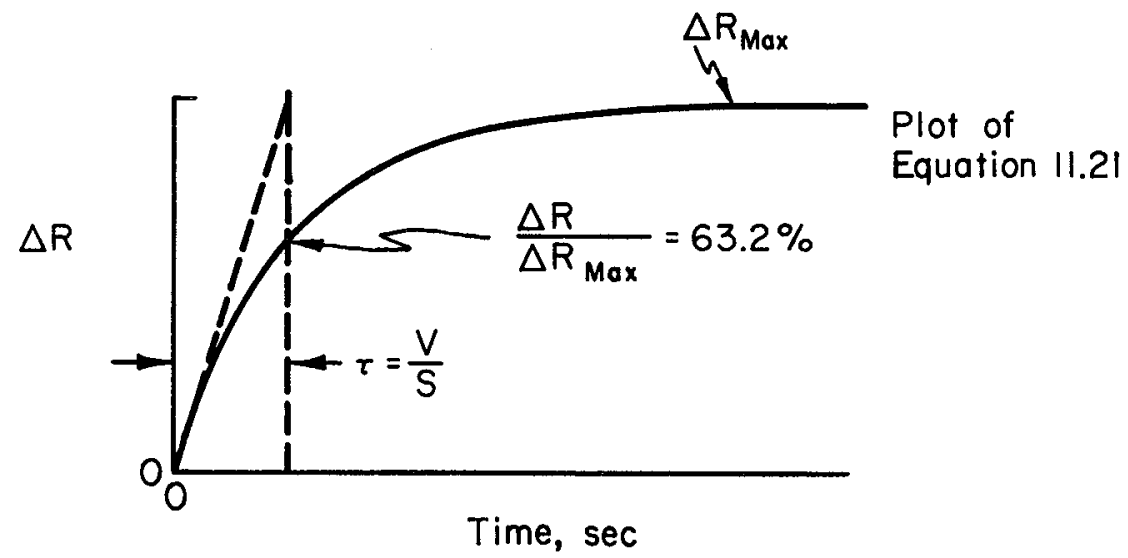

Figure 11.2.-Plot of $\Delta R$, the change in reading as a function of time for a given leak $Q_{L_{\alpha}}$, where. $\Delta R$ is given by equation (11.21). 
basis of the initial slope of the signal change. The time period is defined by $\tau$ and can be obtained from

$$
\tau=\frac{V}{S}
$$

Equations (11.21) and (11.22) show that when it is desired to locate leaks which are well above the minimum detectable value, the minimum detectable reading change will be obtained in a period of time which is small relative to $\tau$. When the leaks being sought are near the minimum detection limit, the tracer gas should be applied to each suspect area for a period on the order of $\tau$.

The following example illustrates the importance of $r$. If 1000 liters volume is being leak tested with a helium leak detector having a pumping speed of 1 liter/sec, the time constant as given by equation (11.22) will equal 1000 seconds. Therefore, the tracer gas must be applied to every suspect area for over 15 minutes to assure the location of all of the minimum detectable leaks. The vacuum engineer frequently encounters this situation when leak testing vacuum systems, particularly those systems which are being exhausted by a single mechanical pump.

Equation (11.21) further indicates that in order to maximize the final signal, the pumping speed $S$ must be minimized. This action contradicts the desire to increase $S$ so that a small time constant may be obtained. As a result, the high-sensitivity leak testing of very large volumes is a difficult problem to resolve. If very short probing times relative to $\tau$ are used, the quantity $\left(1-e^{-s t / v}\right)$ may be approximated by the initial slope of $S t / V$. Equation (11.21) then reduces to

$$
\Delta R=\frac{Q_{L_{a}} t}{V}(\sigma \omega-1)
$$

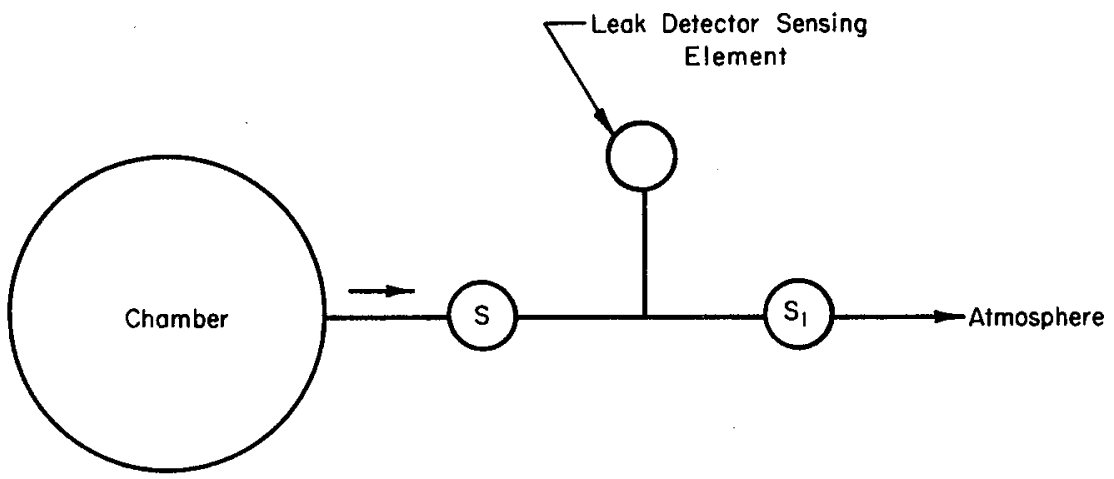

Ficune 11.3.--Use of series pumps for high sensitivity leak detection of large volumes where the leak-detector sensing element is placed in the intermediate region to overcome the difficulty of the need to both reduce $S$ to maximize the final signal and increase $S$ to obtain a small time constant. 
Equation (11.23) demonstrates that the leak-test sensitivity is related directly to the tracer-gas application time for this test condition.

Two pumps used in series (fig. 11.3), and the placement of the leakdetector sensing element in the intermediate region constitutes one method of circumventing the difficulty. When a high-speed diffusion pump is used, the time constant defined by the net speed of the system $S$ may be very short. $S_{1}$, which may be considerably smaller than $S$ defines the steady-state sensitivity. This advantage is maximized when the inherent leak-detector sensitivity is not reduced by the considerably higher total pressure which exists in the foreline, relative to the main chamber.

The time-constant equation is also important when the problem of two leaks in series with an intermediate volume is evaluated (fig. 11.4).

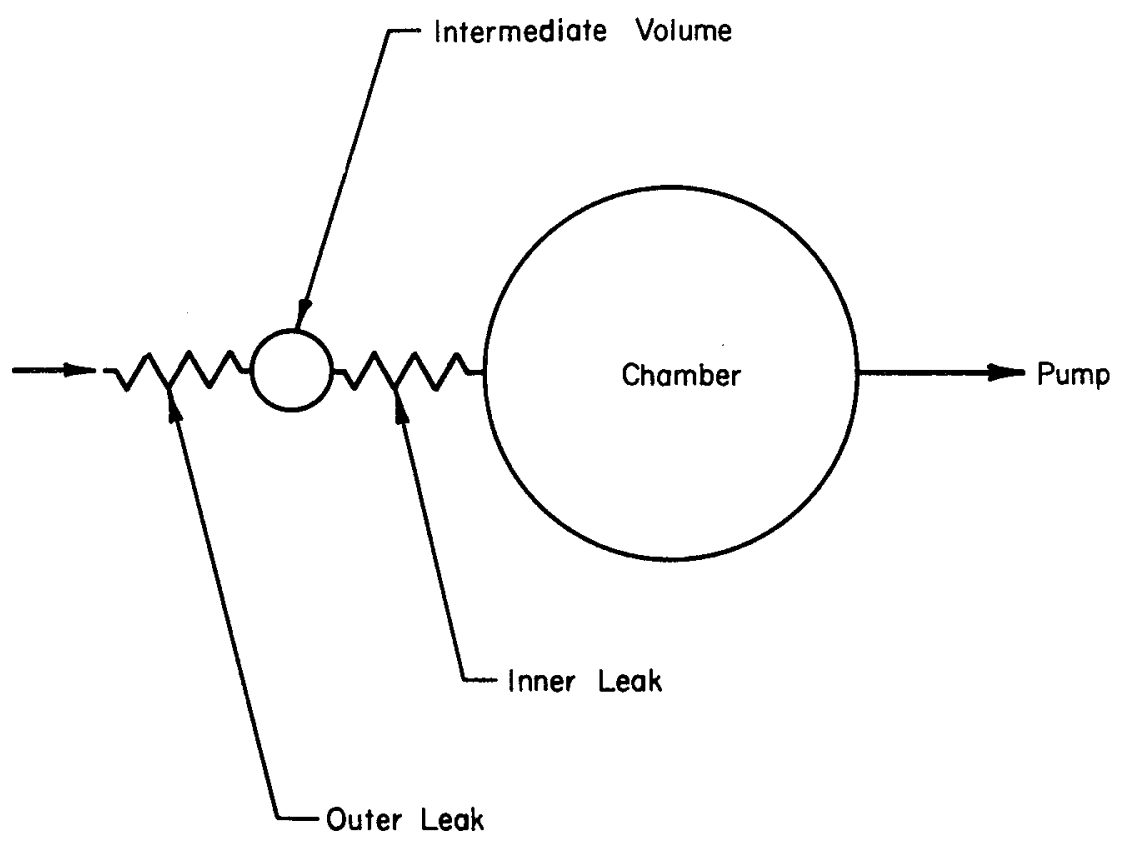

FIgURE 11.4.-Evaluation of time constant, equation (11.22), for two leaks in series with an intermediate volume.

After a tracer gas has been applied to the outermost leak, and before the steady-state, tracer-gas flow prevails through the inner leak, an amount of time is required to accumulate the tracer-gas concentration in the intermediate volume. The time constant for this situation can be evaluated through the use of procedures analogous to those previously 
described. Equation (11.21) defines the accumulation of tracer gas in the intermediate volume where $Q_{L_{a}}$ is the outermost leak size and $S$ is the speed of the inner leak. The time constant is obtained from equation (11.22), where $V$ is the intermediate volume. When extremely small leaks are involved, the speed $S$ may be of the order of $10^{-6}$ liter/sec or less. Therefore, if the intermediate volume is approximately $10^{-3}$ liters, the time constant of the double leak would be $10^{-3} / 10^{-6} \mathrm{sec}=1000$ sec. In most applications, $\tau=1000 \mathrm{sec}$ is larger than the inherent time constant of the main chamber volume and system pumping speed. Since the double-leak time constant cannot be influenced by the system pumping speed, it must be tolerated. Improperly designed vacuum equipment, particularly in cases which are similar to those illustrated in figure 11.5

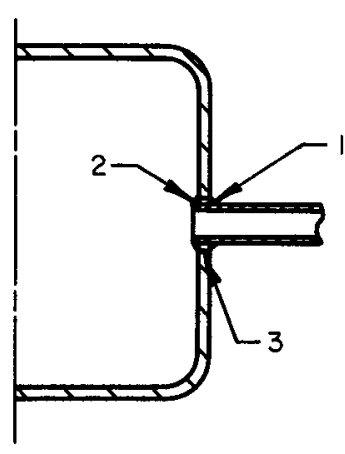

Double Weld

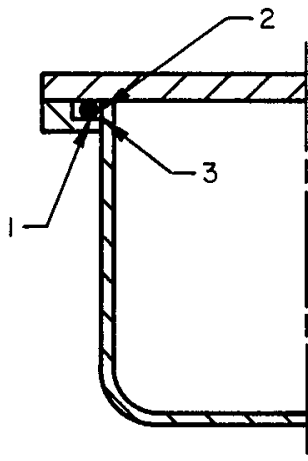

1. Outer Leak

2. Inner Leak

3. Intermediate Volume

Unvented O-Ring

FIGURE 11.5.--Improperly designed vacuum equipment which will frequently exhibit double leaks.

for double welds and unvented O-rings, frequently exhibit double leaks.

Although some leaks may have such a long time constant that it is almost impossible to locate them, they may still be of such a size to provide a serious limitation on the system ultimate pressure.

\section{OTHER ASPECTS OF LEAK DETECTION}

The effect of the concentration of the tracer gas will cause a modification of all of the basic equations. In many leak-detection applications, the concentration of the tracer gas in the vicinity of the leak may not be equal to 100 percent. This situation results from mixing with the surrounding air and necessitates a correction to the equations. If $C$ is the 
tracer-gas concentration such that $C P_{a}$ is the air signal removed, and $C P_{t}$ is the tracer signal added, the correction may be determined from equation (11.4)

$$
P=P_{0}-C P_{a}+C P_{t}
$$

Hence, equation (11.11) becomes

$$
Q_{L_{a}}=\frac{\Delta R S_{a}}{C(\sigma \omega r-1)}
$$

where $C$ may be treated as a multiplying factor on $Q_{L_{a}}$, the leak size being detected.

In a similar fashion, equation (11.21), which is the reduced form of the signal response versus time for a particular leak $Q_{L_{a}}$, becomes

$$
\Delta R=\frac{C Q_{L_{n}}}{S}(\sigma \omega-1)\left(1-e^{-s t / v}\right)
$$

It can be seen from equation (11.26) that the concentration does not affect the value of the time constant $\tau$. Rather, it affects only the linear magnitude of the signal.

The configuration for pressure testing in which an internal pressure forces the tracer gas through the leak is illustrated in figure 11.6.

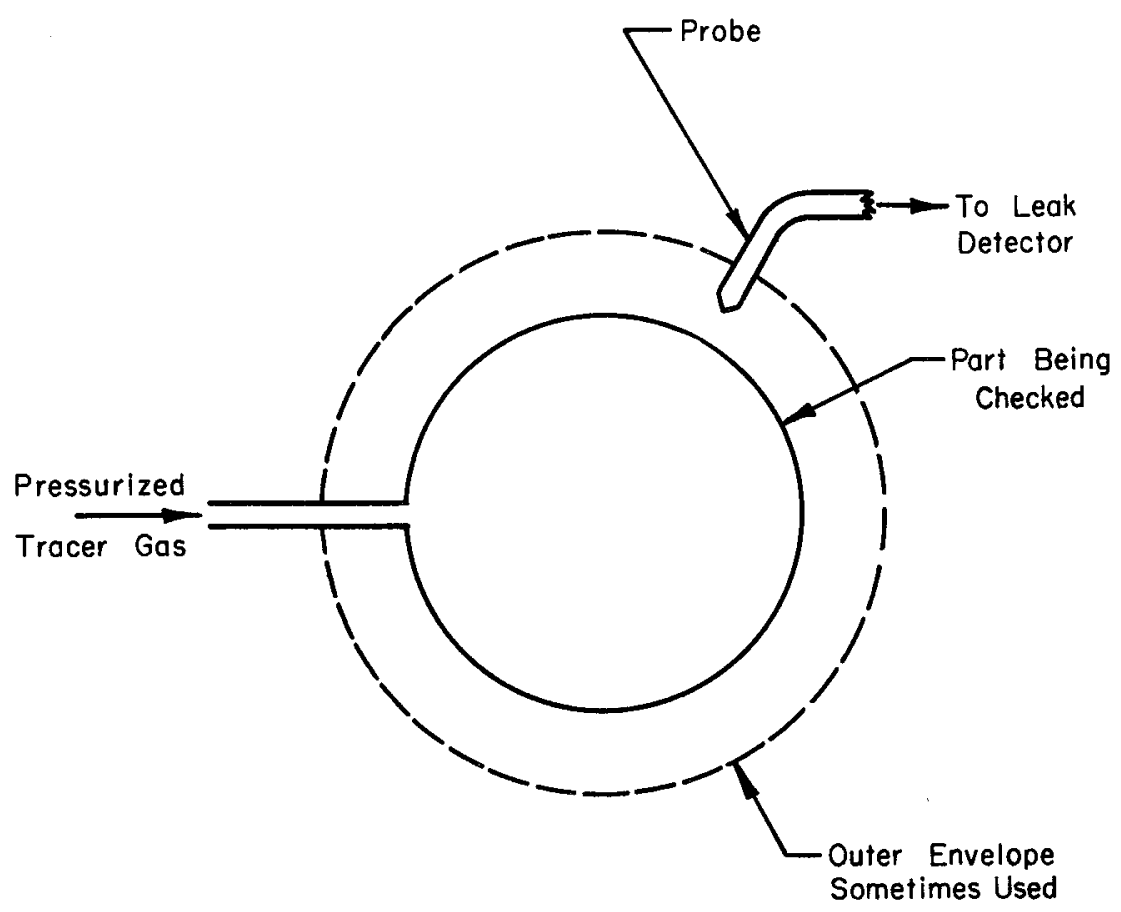

Figure 11.6.-Configuration for pressure testing or leak detection in which an internal pressure forces the tracer gas through the leak. 
Either the actual gas flow or the concentration buildup of the tracer gas provides a means for the detection of the tracer in the atmosphere. Sonic detection, soap bubbles, or air bubbles in water are some methods suitable for detecting the actual gas flow. A sensitive tracer-gas detector such as a halogen leak detector or the reverse-probe helium leak detector is required when searching for small leaks which result in a small concentration of tracer gas in the atmosphere. Either, of the above detectors is capable of detecting the presence of a selected tracer gas in concentrations of a few parts per million.

The tracer-gas flow rate can be derived from the viscous-flow equation due to the fact that the leaks being detected by the internal-pressure methods generally will be large enough to be in the viscous-flow region. If $Q_{L_{a}}$ is assumed to be the leak size in terms of air flowing from a pressure of 1 atmosphere to vacuum conditions, then

$$
Q_{L_{t}}=Q_{L_{a}} \frac{\left(P_{1}{ }^{2}-P^{2}{ }_{\text {atm }}\right) \eta_{a}}{\left(P_{\text {atm }}^{2}-0^{2}\right) \eta_{t}}
$$

The internal pressure is commonly measured in psig, thus

$$
Q_{L_{t}}=Q_{L_{a}} \frac{\left(P_{1}+14.7\right)^{2}-14.7^{2}}{14.7^{2}} \nu=Q_{L_{a}} \nu \frac{P_{1}\left(P_{1}+29.4\right)}{216}
$$

High internal pressures will result in a considerable increase in the flow rate. For example, an internal pressure of 50 psig will cause the flow rate to increase by a factor of 18.4 relative to the air flow from atmospheric pressure to vacuum through the same leak.

During a pressure-type tracer leak test, the tracer-gas flow rate continuously enters the atmosphere at the point of leakage. While the tracer gas subsequently diffuses into the atmosphere, it builds up a concentration gradient. This gradient can be sampled by a low flow-rate leak detector sensing probe which is brought into the vicinity of the leak.

If the flow rate through the sensing probe is small enough so that it does not disturb the gradient accumulation built up by the normal diffusion of the tracer gas into the atmosphere, the test sensitivity will be determined by the capability of the instrument to detect a trace amount of specified gas, as well as the inherent concentration established by normal gaseous diffusion, and the proximity of the sensing probe relative to the leak. If the gas flow drawn through the sensing probe is increased, the inherent sensitivity decreases in favor of response time. The local effect of the gas flow entering the sensing probe is to disturb the normal concentration in the vicinity of the leak. At higher sampling flow rates, all of the tracer gas from the leak will be drawn into the sampling probe, although it will be considerably diluted by air. The sampling concentration for this situation is defined as

$$
C=\frac{Q_{L_{t}}}{\text { Air flow through probe }}
$$


A common method which is used to confine the tracer gas, prevent its diffusion, and thus augment the sensitivity, calls for the test item to be surrounded by a secondary volume. This volume is indicated by the dotted line in figure 11.6. However, care should be exercised in the application of this technique as faulty conclusions may result from improper handling of the various considerations.

If $Q_{L_{t}}$ is assumed to be the rate of flow of the tracer gas into the outer volume $V_{2}$, the tracer partial pressure with no tracer gas loss at any time $\tau$ is

$$
P_{t}=\frac{Q_{L_{t}} t}{V_{2}}
$$

It is apparent that the volume $V_{2}$ is an important factor in the buildup rate of the tracer partial pressure. As an example, consider a test item which is surrounded by a hermetic plastic cover where the volume between the test item and the cover equals 100 liters. In addition, the sensing detector will indicate the presence of $1 \mathrm{ppm}$ of tracer gas in air. Thus, $P_{t}=7.6 \times 10^{-4}$ torr, and a tracer gas flow of $10^{-6}$ torr liter/sec will require a waiting period, as determined from equation (11.30), of

$$
\begin{aligned}
t & =\frac{\left(7.6 \times 10^{-4}\right) 100}{10^{-6}} \mathrm{sec} \\
& =21 \mathrm{hr}
\end{aligned}
$$

This value of $t$ is based on the assumption that there are no tracer-gas losses through the outer container. As a result, it must be concluded that the attainment of a high sensitivity means that the collection volume must be kept to a minimum.

\section{VACUUM-GAGE METHODS OF LEAK DETECTION}

Specialized leak-detection equipment is not available in many vacuum-system applications, and it may prove to be practical to use one of the vacuum gages already installed on the system as a leak detector. The equations given in the above discussion will enable complete solutions to be obtained for these situations.

The following discussion will evaluate the ionization gage, mass spectrometer, and thermal conductivity gage as they are applied for leakdetection situations. Reference 4 presents a detailed treatment of the leak-testing procedure using ion current measurements of a getter ion pump, or an ionization gage connected to a getter ion pumped system. Figure 11.7 illustrates a typical diffusion pumping system which incorporates a main chamber and ionization gage, a diffusion pump, and a backing pump with a thermocouple vacuum gage in the foreline. Various valving arrangements have been deleted for simplicity, and the techniques of closing the valves to determine if a leak exists will be discussed in a later section. 


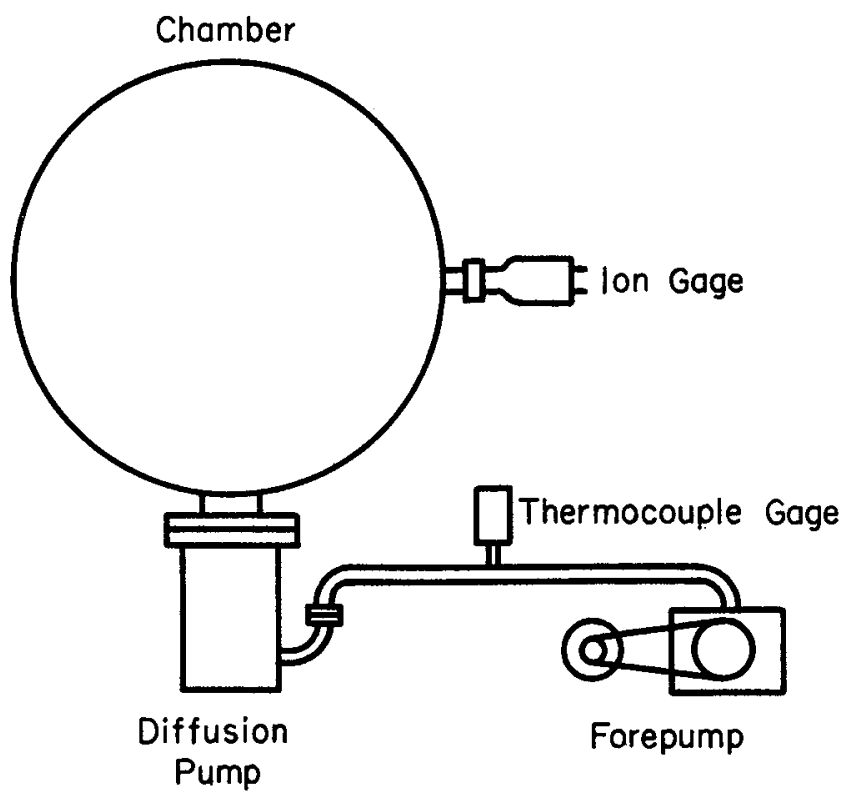

Figune 11.7.-Typical diffusion pumping system which incorporates a main chamber and ionization gage, a diffusion pump and a backing pump with a thermocouple vacuum gage in the foreline.

An additional feature of the system of figure 11.7 is that it may or may not include a liquid nitrogen trap. The analysis may be simplified if it is assumed that leak detection consists of applying a 100-percent tracer gas to each suspect area for a period of time greater than $\tau$. Thus, the steady-state equation (11.11) is applicable.

\section{Ionization Gage as a Leak Detector}

Table 11.1 gives typical values of $\sigma$ for an ionization gage, as well as values of $\mu$ and $\nu$ for a few selected gases.

TABLE 11.1.-Leak Detection Properties of Several Gases

\begin{tabular}{|c|c|c|c|c|c|}
\hline \multirow[b]{2}{*}{ Gas type } & \multirow[b]{2}{*}{$\sigma_{\text {lon gage }}$} & \multirow[b]{2}{*}{$\mu$} & \multirow[b]{2}{*}{$\nu$} & \multicolumn{2}{|c|}{ No trap } \\
\hline & & & & $\underset{(\sigma-1)}{\text { Molecular }}$ & $\begin{array}{c}\text { Viscous } \\
\left(\frac{\sigma \nu}{\mu}-1\right)\end{array}$ \\
\hline $\mathrm{He}$ & 0.17 & 2.7 & 1.1 & -0.83 & -0.93 \\
\hline Ar & 1.3 & .85 & 1.2 & .3 & 85 \\
\hline $\mathrm{H}_{2}$ & 1.5 & 3.8 & .5 & -.5 & -.94 \\
\hline $\mathrm{CO}_{2}$ & 1.4 & 81 & .8 & .4 & .4 \\
\hline $\mathrm{H}_{2} \mathrm{O}$ & .9 & 1.27 & .5 & -.1 & -.72 \\
\hline Hydrocarbons ------ & $3-10$ & $1-0.3$ & $0.6-0.3$ & 2.9 & $1-10$ \\
\hline
\end{tabular}


Generally, it can be assumed when working with ionization gages that molecular flow exists in the vacuum chamber. In addition, $r$ may be assumed to equal $1 / \mu$ for noncondensable tracer gases due to the fact that some form of molecular-flow restriction normally exists between the pump and the chamber. This relation also applies for condensable gases in the absence of a condensing surface. Equation (11.14) thus holds when it is desired to detect small molecular leaks, less than $10^{-8}$ torr liter/sec. For larger viscous leaks

$$
Q_{L_{a}}=\frac{\Delta R S_{a}}{\left(\frac{\sigma v}{\mu}-1\right)}
$$

Table 11.1 lists the tracer-gas factors $(\sigma-1)$ and $\left(\frac{\sigma v}{\mu}\right)-1$ for various gases. The negative signs appearing with several values signify that when the tracer gas is applied, the gage signal will decrease. It follows then that a plus value indicates an increase in the reading. The detection of small leaks requires a maximum absolute value of the tracer-gas expression. An examination of table 11.1 indicates that a hydrocarbon yields the best results. Unfortunately, there is little information available on the exact ionization gage constants for hydrocarbons such as alcohol, trichlorethylene, and acetone. When hydrocarbons make contact with the hot filament of the ionization gage, they exhibit a cracking effect which yields numerous radicals and a subsequent high value of $\sigma$.

Table 11.1 also points out that either helium or hydrogen gas will yield a reasonable sensitivity. Water may be used for the detection of viscous leaks, although it is very insensitive to molecular leaks when there is no condensing type of trap, such as a liquid nitrogen trap, on the system. If a liquid nitrogen trap is added to the system, the value of $S_{t}$ for condensable gases will increase on the order of $S_{t}=10 S_{a}$. As a result, the tracer-gas factor for water for molecular flow will increase from -0.1 to -0.9 . For viscous flow, the tracer factor will increase from -0.72 to -0.95 . Water thus becomes a very good tracer gas when a high-speed trap is part of the pumping system. The tracer-gas factors for condensable hydrocarbons in the presence of a trap change to approximately -0.7 for molecular flow and -0.8 for viscous flow.

Since it appears that the tracer-gas factor seldom exceeds unity, a reasonable sensitivity may be defined as

$$
Q_{L_{a}}=\Delta R S_{a}
$$

At this point, two different conditions may be experienced. If a conventional ionization gage circuit is utilized, the detectable reading change is a fixed percent of full scale of the reading pressure scale. Therefore, if equation (11.32) is divided by the steady-state system 
background expression, $Q_{b}=P_{b} S_{b}$, and it is assumed that the principal background gas is air from various leaks, such that $P_{b}=R_{b}$, and $S_{b}=S_{a}$, then

$$
\frac{Q_{L_{a}}}{Q_{b}}=\frac{\Delta R}{R_{b}}
$$

The inherent limitation of this ionization gage, with a $\Delta R / R_{b}$ value of 2 percent, becomes 2 percent of the background signal. The factor $\Delta R / R_{b}$ may increase to as large a value as 20 percent if the speed cannot be adjusted to give a reading near full scale. Regardless of this consideration, it is apparent that the ionization gage may be used effectively to locate any leak which is limiting system ultimate pressure.

However, the ionization gage may be used as a leak detector to more advantage if the normal background pressure reading can be nulled and thus cause an increase in the sensitivity of the reading $\Delta R$. The limitation of this configuration will depend upon the noise level of the amplifier or the pressure fluctuations of the pumping system. It is rather optimistic to expect a detectable variation of greater than $1 \times 10^{-10}$ torr for most ionization gage controls. A few controls are available which can detect variations as small as $1 \times 10^{-12}$ torr contingent upon the condition that the pressure must be in this range, or some method must be available to balance the background signal so as to be on the appropriate scale.

If a value of $\Delta R=1 \times 10^{-8}$ torr is assumed, the maximum leakdetection capability, in torr liter/sec, is

$$
Q_{L_{a}}=S_{a} \times 10^{-9}
$$

An ionization gage with a balancing circuit may be used to locate leaks which contribute a pressure on the order of the noise level or pressure fluctuations of the gage. However, this procedure requires extreme care and is neither easy nor straightforward. Other ionization gages, such as the Penning or cold-cathode gage, may be analyzed in a similar manner. The gage-sensitivity factor will vary between the different gage types. There are several types of tracer gases for which the tracer-gas factor approaches unity. In practical applications, a regular control unit can be expected to detect any single leak contributing more than 5 percent to 10 percent of the system pressure. The addition of an amplifierbalancing circuit may considerably increase the sensitivity which is limited principally by drift, pulsations, or other variations indicated on the amplifier.

\section{Partial-Pressure Gages as Leak Detectors}

Due to the great amount of amplifier drift or variation resulting from other system gases, both sensitivity and reliability may be improved considerably if some form of partial-pressure gage or mass spectrometer 
is used in place of the ionization gage. Partial-pressure gages, which use tuning and selective filtering devices, respond primarily to one tracer type only and thus minimize background interference from other system residual gases. A conventional ionization gage with some form of filter or barrier, such as a hot palladium window or a sorbent trap (refs. 5 and 6), at the gage inlet will behave in a similar manner.

Gages which may be tuned to any selected gas specie such as the magnetic sector mass spectrometer, the omegatron, and the time-offlight mass spectrometer are used for most conventional vacuum applications. These devices will scan and identify system gas residuals through the adjustment of a parameter such as voltage, frequency, or magnetic field. Therefore, almost any tracer gas may be used when the device is tuned to the proper setting .

Due to the fact that the reduction of the air signal when the tracer gas is applied to the leak, for both the filtering and selective tuning devices, has little or no effect on the output signal, equation (11.11) reduces to

$$
\begin{aligned}
Q_{L_{a}} & =\frac{\Delta R S_{a}}{\sigma \omega r} \\
& =\frac{\Delta R S_{t}}{\sigma \omega}
\end{aligned}
$$

where $\Delta R$ is the smallest reading change, calibrated in torr of air, and $\sigma$ is the sensitivity factor. Since $\Delta P_{t}=\Delta R / \sigma$ and $\Delta P_{t}$ is the smallest actual tracer-gas partial pressure which may be detected

$$
Q_{L_{a}}=\frac{\Delta P_{t} S_{t}}{\omega}
$$

The minimum detectable value of $\Delta P_{t}$ for nitrogen, which is always given on commercial partial-pressure gages, may vary from $10^{-8}$ torr to $10^{-13}$ torr. Values of $\Delta P_{t}$ for other gas types may be computed if the sensitivity factors are known. As in the case of ionization gages, the value of $\Delta P_{t}$ depends somewhat on the system background. When the total background pressure of the nontracer gas is increased, interference with detection of the tracer gas will occur. This interference is caused by limitations in the resolution, or at higher pressures, the interference is due to coulomb force dispersion, ion recombination, or Aston banding. Mass spectrometers may be designed with smaller slit dimensions to improve resolution, but the sensitivity will be sacrificed in the process. Figure 11.8 illustrates typical mass spectrometer sensitivity as a function of total pressure. It can be seen from the curve of $\log \Delta P_{t} / P$ that the detectable fraction of tracer gas in the background gas depends on the total pressure.

Equation (11.35) illustrates that very small leaks can be found when the system pumping speed for the tracer gas is small. That is, if $\Delta P_{t}=$ 


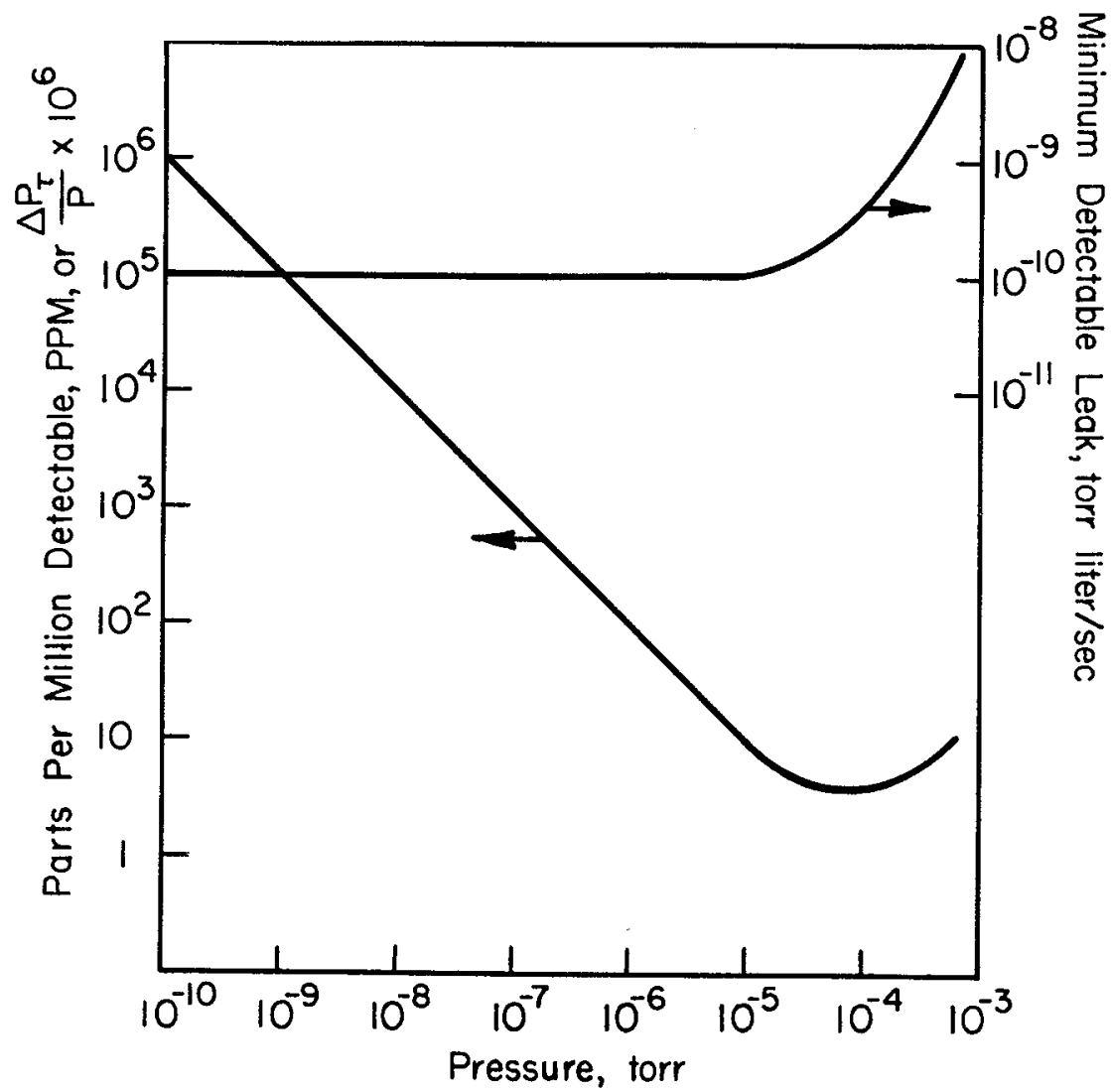

FIGURE 11.8.-Typical mass spectrometer leak detector sensitivity as a function of total pressure, where the detectable portion of the tracer gas in the background gas depends on the total pressure as illustrated by the curve of $\log \frac{\Delta P_{i}}{P}$.

$1 \times 10^{-10}$ torr and $S_{t}=1$ liter/sec, $\omega=1$, leaks as small as $1 \times 10^{-10}$ torr liter/sec can be located. A more important consideration for system applications is that any leak which contributes a pressure greater than the partial-pressure sensitivity of the detector may be found. As a result, a partial-pressure sensitivity in the $10^{-9}$ torr range is adequate for almost all nonbaked vacuum system applications. The more elaborate, baked ultra-high-vacuum systems may require sensitivities of $\Delta P_{t}=10^{-13}$ torr or better.

Not only does a partial-pressure device in the vacuum chamber provide good sensitivity for leak detection, it also initially determines the existence of a leak. A quick scan of the mass spectrum identifies 
the character of the residual gas, whether water vapor, hydrocarbon, nitrogen, air, or some other gas type, and determines if leak detection with a tracer gas is needed. Valuable hours wasted in a search for a nonexistent leak may be saved by the determination of the system conditions.

\section{Helium Mass Spectrometer Leak Detector}

This instrument is a combination of a high-vacuum pumping system and a limited range mass spectrometer, which is designed for high sensitivity and low resolution, where the item under test and the spectrometer system are connected by a sampling line. This arrangement is illustrated in figure 11.9. Any particular spectrometer tube has an inherent mini-

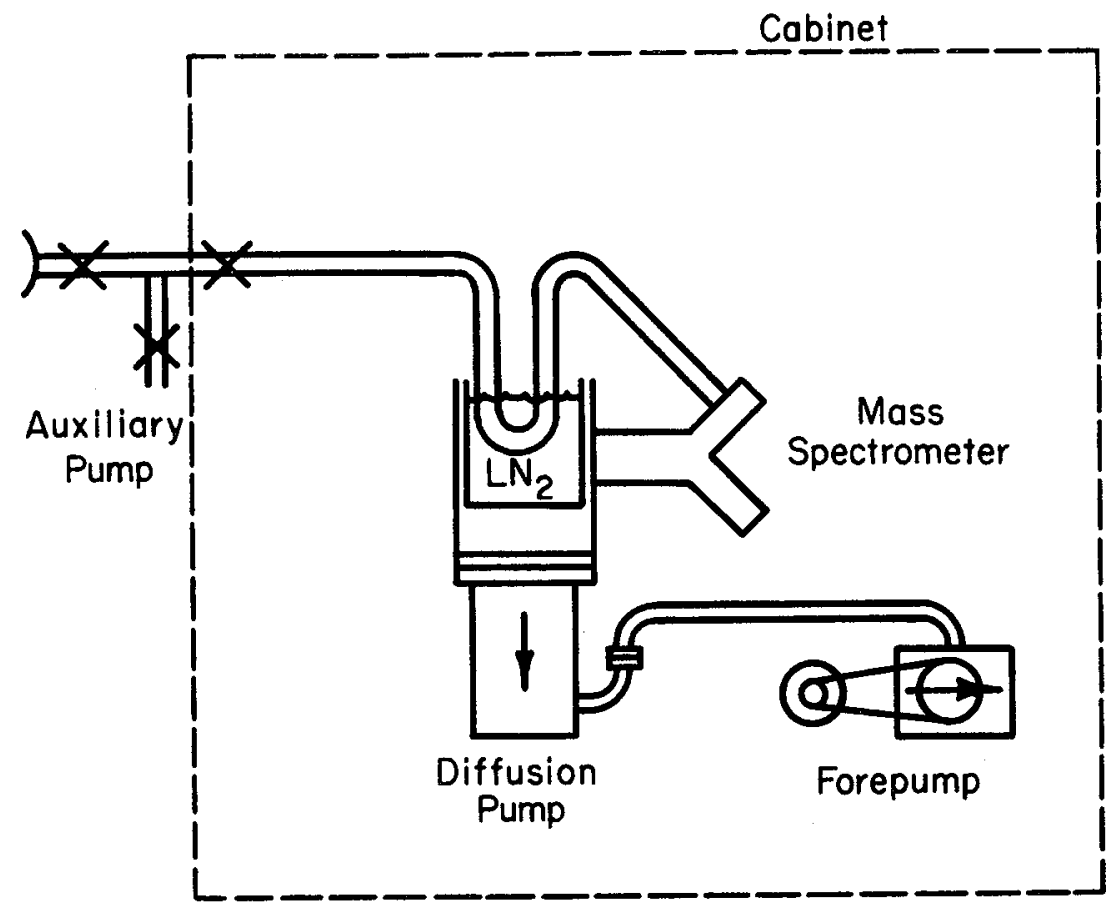

FigurE 11.9.-Typical helium mass spectrometer leak detector which incorporates a pumping system as a part of the sensing device; a sampling line is connected from the test chamber directly to the mass spectrometer pumping system.

mum detectable $\Delta P_{t}$. Therefore, the tracer-gas pressure in the chamber must be greater than $\Delta P_{t}$ so that a differential pressure exists to cause flow through the sampling line. However, the helium mass spectrometer leak detector is seldom evaluated in terms of its $\Delta P_{t}$. Rather, its sensitivity is determined by the value of tracer flow rate $Q_{t}$ which creates a 
minimum pressure change $\Delta P_{t}$ in the presence of the tracer-gas pumping speed $S_{t}$. That is

$$
Q_{t}=\Delta P_{t} S_{t}
$$

Commercial devices may have values of $Q_{t}$ ranging between $10^{-8}$ torr liter/sec to $10^{-12}$ torr liter/sec. To convert these values to minimum detectable air leakage, it is necessary to consider the factor $\omega$ of equation (11.35), and the system factors of time constant and the requirement of additional pumps. Figure 11.10 illustrates a leak detector con-

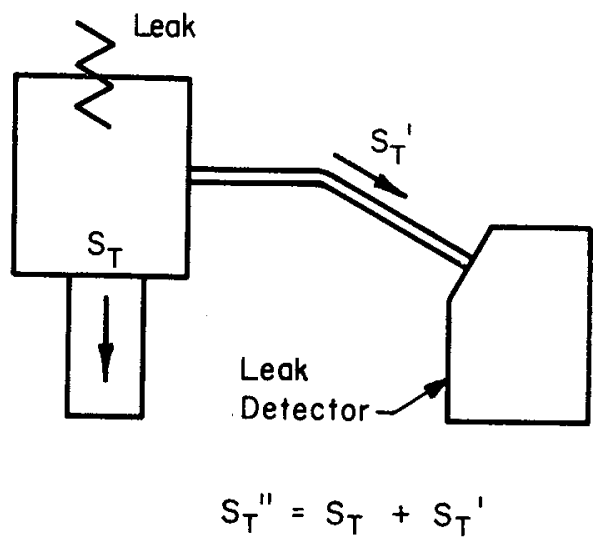

Figure 11.10.-Use of leak detector in parallel with the main system pump.

nected directly to the system chamber. If the main valve is open, the helium gas entering the leak must divide between the two systems in proportion to their speeds. As a result, the available signal for detection is reduced and the test sensitivity is lowered.

When transient conditions prevail, the air signal change upon application of a tracer gas to the leak has a negligible effect for a partialpressure detector. Thus, from equation (11.17) or equation (11.19)

$$
Q_{L_{a}}=\frac{\Delta P_{t} S_{t}}{\omega\left(1-e^{-B_{t} t / V}\right)}
$$

where $\omega=2.7$ for helium if the leak is in molecular flow. If the leakdetector pumping system is the only device pumping on the vacuum system, $S_{t}$, the speed of the leak detector for the tracer gas, defines the time constant $\tau$. Since the above type of leak detector has a limited speed, on the order of 1 liter/sec, the time constant becomes prohibitively long when large volumes are being tested. Pumps connected in parallel will reduce the time constant, but they also reduce the sensitivity. When a high-vacuum pumping system is being tested, the prob- 


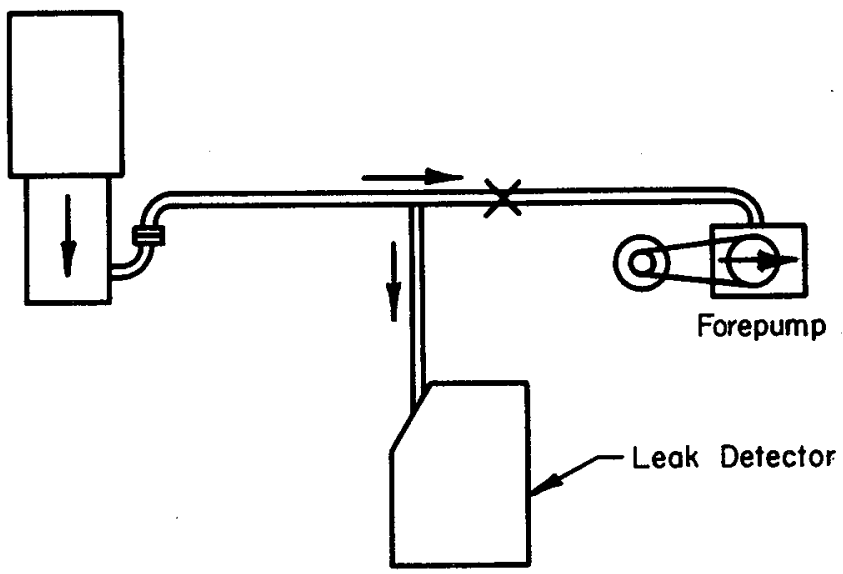

Figure 11.11.-Use of leak detector in series with main system pump to reduce long time constants.

lem of long time constants may be overcome if the mass spectrometer sampling line is connected between the diffusion pump and its backing pump, as illustrated in figure 11.11. There is no loss of sensitivity if the mass spectrometer leak detector can handle the full gas load. However, the time constant is reduced to the system time constant defined by the ratio of the volume to the diffusion pump speed.

In the case of very small volume testing where the time constant is not a problem, it seems to be desirable to reduce the pumping speed of the leak detector to bring about an increase in sensitivity. However, a reduced pumping speed amplifies both the tracer-gas pressure and the background-pressure signal. Every partial-pressure device exhibits a limit for the detection of the lowest concentration of tracer to total gas. A resolution-limited ratio may have its sensitivity affected by the type of background gas present.

The existence of a minimum detectable concentration ratio is illustrated by figure 11.8. Since the minimum value occurs at one operating total pressure only, a total pressure less than this value results in a concentration sensitivity which is limited by a lack of available tracer-gas partial pressure. At higher pressures, the concentration sensitivity is reduced due to the high-pressure effects within the sensing element.

Two principal advantages which emerge from the addition of a selfcontained pumping system to a partial-pressure gage are that it becomes possible to leak-test a component which does not have its own pumping system, and to throttle-in to a pressure region which is too high for normal detector operation. The latter factor yields a reduction in the time constant due to the fact that operation in a diffusion pump foreline is 
permitted. Alternately, it is desirable to have a detector which can be tuned to all mass specie to determine the system gas residuals prior to actual leak detection.

\section{Thermocouple Vacuum Gages}

In the case where a diffusion pump is inoperative due to a very large leak, or a mechanical pump only is being used, the higher pressure reading thermocouple vacuum gage may be used as a leak detector.

The use of the thermocouple vacuum gage reasonably suggests a large leak which can be assumed to be in viscous flow. The flow through the vacuum system, however, may be either viscous or molecular depending upon the total pressure and the line sizes. In addition, the gage-sensitivity factor may vary with the pressure. Such a situation arises when helium is used as a tracer gas. At low pressures, on the order of a few microns, the gage factor in equation (11.10) is of the order of $0.8, v$ is approximately unity for helium, and $r$ in molecular flow is $1 / \mu$, or $1 / 2.7$. The net value of $(\sigma \mathrm{v} r-1)$ is approximately -0.7 . At higher pressures, the gage factor $\sigma$ becomes greater than unity and $r$ becomes $1 / v$. As a result, the net effect of $(\sigma v r-1)$ becomes a positive number. Therefore, at low pressures, helium on the leak will yield a lower reading, while it will cause a higher reading at high pressures. Between the two extremes, however, there is a pressure for which little or no reading change is obtained. If combinations of tracer-gas factors which could give a sensitivity of essentially zero are ignored, a foreline gage may be used to detect a leak which is the principal contributor to the pressure at the gage. In other words, foreline gages should not be used to detect small leaks in the presence of either large leaks or large background outgassing.

\section{LEAK-DETECTION TECHNIQUES FOR SYSTEMS}

It is impossible to define a complete leak-detection procedure for all situations due to the numerous interactions of the parameters of the background and experience of the system operators, the type and complexity of the system, and the equipment available. As a result, this discussion will present the general techniques, factors, and procedures which are frequently applied to a given situation.

The first leak-detection example concerns a system which has no special leak-detection equipment and the main system gage is the detector.

There is a general rule which should be applied to all system operating conditions, and that is Know the system. The knowledge should include the approximate speed of the main pumping system at the location of the vacuum gage for both permanent and condensable gases, and the speed of the forepump at the location of the forepressure gage. Thus, the time constant can be evaluated if the speed of the main pumping 
system is combined with the volume. In most diffusion-pumped systems, the time constant is on the order of a fraction of a second or less and thus will not present any problems. A greater system pressure than normal may indicate a leak. Further information can be obtained by comparing the foreline pressure to that in the chamber. The former should be higher than normal by an amount equal to the product of the chamberpressure increase and the ratio of the two pumping speeds. If the forepressure is too low to account for a greater chamber pressure, a high outgassing rate of condensable gases in the vacuum chamber may be present. Another cause may be an improperly functioning diffusion pump. When the foreline pressure is higher than it should be, relative to the chamber pressure, the sources include a moisture-contaminated mechanical pump or a leak in the foreline.

Any valves present in the system may be used to obtain a considerable amount of initial information. When the main valve is closed, the pressure in the chamber should rise according to the relationship $Q=V(d P / d t)$. Since this relationship also equals the initial pressure times the speed

$$
S=\frac{V}{P_{0}} \frac{d P}{d t}
$$

The speed of the system may be evaluated when the pressure rate of rise is noted. This speed may then be checked against the expected value. Condensing surface effects must be carefully considered in the above comparison.

If it is desired to separate the effects of leakage and outgassing, the chamber walls may be cooled with a spray or refrigerant, or liquid nitrogen may be added to an interior reservoir. As a general rule, the small amount of time spent in system evaluation prior to leak detection will contribute greatly to the avoidance of unnecessary leak detection.

Once it has been determined that a leak is possibly the cause of system malfunction, the available system vacuum gage should be adjusted to the most sensitive region for leak detection. This can be accomplished by throttling the pumping speed, or by adjusting the vacuum gage electrical parameters. At this point, a systematic spraying of all suspect areas with a large flow of tracer gas should be undertaken. The probing speed rate should be compatible with the time constant evaluated previously. The recommended leak-test procedure commences with glass or ceramic feedthroughs and continues through the various weld and braze joints. Particular attention is merited by those areas which could give rise to long leakage time constants, as for example, nonvented O-rings or double weldment areas. If leakage is not evident, the leak-test procedure should continue with the more difficult internal water baffling, liquid nitrogen shrouds, or other interior joints. If there is no leakage 
indication initially, the process should be repeated carefully. The continued absence of a leakage indication, yet with positive evidence that a detectable leak is present, may necessitate the systematic bagging, of various regions in the chamber. A high concentration of tracer gas held at each point for a prolonged period may permit the detection of long time constant leaks. A recorder placed on the output of the vacuum gage frequently proves beneficial.

When a signal is indicated, the flow of tracer gas may be decreased and the exact point of leakage noted. It is recommended that the leakage indication be rechecked to see if it will repeat, thus demonstrating that it is not due to a pressure transient within the system. The question next arises of whether the system should be shut down for proper repair or whether it can be fixed temporarily. In any situation, the best procedure depends on the type of leak, the urgency of system operation, and the system operating time cycle. If the repair can be corrected at a later time, temporary repairs are permissible. However, the reckless use of materials such as Glyptal or putty to temporarily repair leaks in welds and brazes generally results in increased problems. That is, the contamination introduced into a weld must be completely ground out in order to repair the joint at a later time. Whenever time permits, a permanent repair of the joint is the most economical approach. Scotch tape or electrician's plastic tape frequently may be used to cover and temporarily blank off a large leak to permit continued leak detection for smaller leaks. After all located leaks have been repaired, the initial procedures for checking out the system may be repeated to assess the likelihood of further leaks.

Even though the principal system vacuum gage may be used to detect leaks which are limiting the system pressure, it is much faster and more economical to use one of the tracer-gas detectors. Generally, the savings in operating time and man-hours will more than offset the initial investment for the equipment. The most common tracer-gas detectors are the system-mounted partial-pressure gage and the helium mass spectrometer leak detector. Either device will adequately detect system leaks down to their limiting tracer partial pressure. One advantage of the helium mass spectrometer leak detector is that it is able to throttle into a high system pressure to permit the initial detection of the very large leaks. In addition, it can be connected to the diffusion pump foreline to maintain the combination of both sensitivity and time constant, and as a result, it can detect smaller size leaks in a very large system. The advantage of the mounted partial-pressure gage is that it may be used for leak detection, and also for the analysis of system residual gases. Thus, it immediately determines whether leak detection is suitable by determining the presence of any traces of air in the residual system environment. The mounted partial-pressure gage also may be used with any tracer gas, and 
has a sensitivity adequate for the detection of any leak which is capable of producing a pressure contribution greater than the minimum detectable partial-pressure sensitivity of the detector.

The procedures for using a tracer-gas detector are essentially identical to those for leak testing with the system vacuum gage. The higher sensitivity of the tracer-gas detector may result in the observation of drift signals as the tracer gas diffuses through the air and comes near a point of leakage. This action may necessitate a lower flow rate of tracer gas. The ability to locate the exact point of leakage is more accurate with a tracer-gas detector, and may be located to within an eighth of an inch or less. Another advantage of the tracer-detector approach is that very small leaks may be detected in the presence of large leaks. As a result, a complete system leak check may be carried out without shutting down the system and repairing any large leaks detected before beginning to check for small ones. The same factor allows sensitive leak detection to be carried out in the presence of high outgassing rates.

The high sensitivity of tracer-gas detectors poses one particular problem. That is, there may be false leakage indications due to permeation through materials. Helium is a particularly difficult gas in this regard. The permeation of helium through a 1-inch-diameter O-ring is sufficient to produce a readily detectable helium pressure in the test volume. When probing with helium gas, care should be exercised to keep the helium from all O-rings in the system. After all other portions of the system have been leak checked, the individual O-ring seals may be checked. A permeation signal may be separated from a true leak by the rate of the signal response. The response of a true leak is related only to the system time constant if double-leak problems are ignored. Permeation signal response time is related to the time required for the establishment of a diffusion gradient through the material. A typical value of permeation response time for O-ring materials is about 1 hour. Therefore, if helium is applied to an O-ring, a quick response indicates a true leak, while a long-term buildup signifies permeation. The process of permeation is particularly obnoxious due to the fact that it possesses a long cleanup time. Once helium has penetrated through an O-ring, many hours of pumping will be required before the signal cleans up. The presence of only a few O-rings is enough to produce a signal which will completely saturate the leak detector. When large systems are bagged or hooded, it is advisable to isolate the O-rings from the helium atmosphere with a protective gas blanket or by some mechanical means of isolation.

If grease is used too liberally on O-rings, serious problems in terms of long-term cleanup will be created. Unfortunately, it is a common practice to use large quantities of grease in an attempt to make O-rings leaktight. A scratch in the O-ring surface may result in either an intermittent leak or a long-term diffusive leak rather than a clearly defined 
signal. The helium dissolved in the grease may continue to come out for many hours, thus making the leak-test procedure very difficult. Intermittent, or blurping, leaks may also be caused by leaking seals in the diffusion pump drain fitting.

When high-sensitivity leak detection is desired, care should be used to avoid water or other liquids which may plug the point of leakage for many days, yet eventually reopen to permit leakage. Small leaks can be accidentally plugged if a finger is wiped across the point of leakage. The application of heat and vacuum will usually reopen leaks plugged in such a manner.

Generally it can be assumed that the system is leaktight when the partial-pressure gage mounted on the system fails to show any indication of a nitrogen or air signal, or when the helium leak detector fails to show any indication after a prolonged total system hooding with tracer gas. At this point, further reductions in system pressure may be obtained only if the system outgassing is eliminated by a bakeout procedure or cryogenic pumping.

References 7 to 11 present further information on the general methods of leak detection, while references 12 to 18 describe leak detection with vacuum gages.

\section{REFERENCES}

1. Guthrie, A.; and Wakerling, R. K.: Vacuum Equipment and Techniques. MeGraw-Hill Book Co., Inc., 1949.

2. Ockert, N.; and Steckelmacher, W.: The Flow Characteristics of Capillary Leaks. Brit. J. Appl. Phys., vol. 2, Nov. 1951, pp. 332-333.

3. Santeler, D. J.; ANd Moller, T. W.: Fluid Flow Conversion in Leaks and Capillaries. Trans. of the National Symposium on Vacuum Technology, E. S. Perry and J. H. Durant, eds., Pergamon Press, 1957, pp. 29-36.

4. Ackley, J. W.; Barrington, A. E.; Francis, A. B.; Jepsen, R. L.; Lothrop, C. F.; ANd MandoLI, H.: Leak Detection Using Current Changes in Ionization Gauges and Sputter-Ion Pumps. Trans. of the Ninth National Vacuum Symposium, G. H. Bancroft, ed., The Macmillan Co., 1962, pp. 380-383.

5. Ockert, N.; and Steckeimacher, W.: Leak Detection Practice With Particular Reference to the Hydrogen Palladium Method. Vacuum, vol. 2, 1952, pp.
125-136.

6. IshiI, Hiroshi: A Differential Pirani Type Leak Detector With a Charcoal Trap. Advances in Vacuum Science and Technology, vol. 1. E. Thomas, ed.,
Pergamon Press, 1960, pp. 245-247.

7. Pirani, M.; AND Yarwoon, J.: Principles of Vacuum Engineering. Reinhold Pub. Corp. (New York), 1961.

8. Guthrie, A.: Vacuum Technology. John Wiley \& Sons, Inc., 1963.

9. Turnaull, A. H.: The Principles of Leak Detection. Atomic Energy Research Establishment Report G/R 478, United Kingdom Atomic Energy Authority,
1950.

10. Cross, S. H.; and Steckelmacher, W.: Leak Detection by Vacuum Techniques. Research, vol. 9, no. 4, Apr. 1956, pp. 124-131. 11. MiNTER, C. C.: Thermal Conductivity Leak Detector. Rev. Sci. Instr., vol. 29,
no. 9, Sept. 1958, pp. 793-794. 
12. Varićax, M.: Penning Gauge as a Leak Detector. Rev. Sci. Instr., vol. 27, no. 8, Aug. 1956, p. 655.

13. Turnbuli, A. H.: Experiments With a Differential Pirani Gauge Leak Detector. Atomic Energy Research Establishment Report G/R 477, United Kingdom Atomic Energy Authority, 1950.

14. Kenr, T. B.: A Hydrogen Pirani Leak Detector Using a Charcoal Trap. J. Sci. Instr., vol. 32, no. 4, Apr. 1955, pp. 132-134.

15. Schumacher, B. W.: Leak Detector for Hermetic Seals. Electronics, vol. 30, Mar. 1, 1957, pp. 284-294.

16. BecK, A. H.; AND KING, G.: A Sensitive Leak Detector Using Magnetron Ionization Gauges. Vacuum, vol. 4, no. 2, Apr. 1954, pp. 147-158.

17. Botden, T. P. J.: The Silica Gel Leak Detector. Advances in Vacuum Science and Technology, vol. I. E. Thomas, ed., Pergamon Press, 1960, pp. 241-244.

18. Calvi, G.: A Double Pirani Bridge Leak Detector. Vacuum, vol. 10, no. 1/2, Feb./Apr. 1960, pp. 64-67.

A U.S. GOVERNMENT PRINTING OFFICE: $0-799.737$ 